QC852

.C6 no.598
DOD-NAVY-ONR: N00014-91-J-1092

DOD-USAF-OSR: F49620-93-1-0415

NSF: ATM-922-4501

\title{
UNDERSTANDING AND FORECASTING TROPICAL CYCLONE INTENSITY CHANGE
}

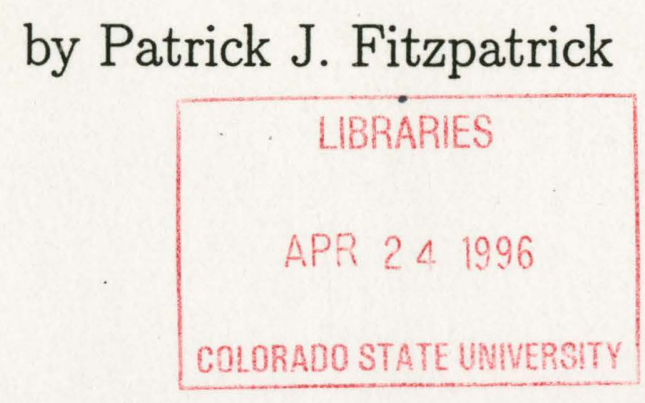

P.I.-William M. Gray

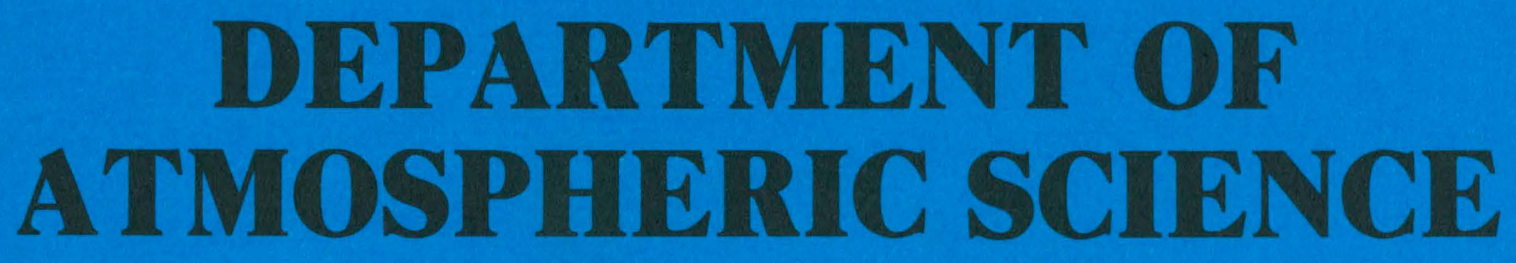

PAPER NO. 598 


\title{
UNDERSTANDING AND FORECASTING TROPICAL CYCLONE INTENSITY CHANGE
}

\author{
By
Patrick J. Fitzpatrick
}

Department of Atmospheric Science

Colorado State University

Fort Collins CO 80523

March, 1996 


\begin{abstract}
This research investigates several issues pertaining to tropical cyclone intensity change. Previous research on tropical cyclone intensity change is reviewed in great detail. The applicability of upper-level forcing theories is questioned.

Inner-core processes related to intensity change are studied, with particular attention on the relationship between the vertical profile of the tangential wind $\left(v_{t}\right)$ field in the eyewall region and future pressure changes. For cases under minimal wind shear and warm SSTs such that vigorous inner-core updrafts exist, the cyclonic circulation should be mostly conserved almost to the upper-troposphere, with the largest vertical $v_{t}$ variation confined near the tropopause. It is hypothesized that a vertically conserved wind profile is conducive to fast intensification.

Observations support this theory. By stratifying inner-core data into fast and slow developers, it is shown that fast developing tropical cyclones contain a more vertically stacked inner-core vortex than slow developers. It is also shown that a direct correlation exists between inner-core upper-level winds and tropical cyclone intensification, with the rate of intensification proportional to the magnitude and symmetry of upper-level $v_{t}$. Diagnostic calculations using the Balanced Vortex equations also support this assertion.

An alternative air-sea interaction theory is presented which incorporates boundary layer cooling. The buoyancy calculations include partial water-loading and ice microphysics, and their relevance to CAPE calculations in the tropics is discussed. It is shown that the lateral extension of the eye, above a sloping eyewall, is the crucial component in maintaining the air-sea interaction despite boundary layer cooling. Implications on the maximum intensity a storm may achieve are discussed.

A multiple regression scheme with intensity change as the dependent variable has been developed. The new scheme is titled the Typhoon Intensity Prediction Scheme (TIPS),
\end{abstract}


and is similar to one used operationally at the National Hurricane Center. However, TIPS contains two major differences: it is developed for the western North Pacific Ocean, and utilizes digitized satellite data. It is shown that the satellite data can distinguish between fast and slow developing tropical cyclones. The importance of other statistical predictors (such as SSTs, wind shear, persistence, and climatology) to intensity change are also clarified. The statistics reveal threshold values useful to forecasters. It is shown that TIPS is competitive with the Joint Typhoon Warning Center. 


\section{DEDICATION}

To my wife Amy, my daughters Megan and Katie, my parents Tom and Sue, and my sister Kelly 


\section{CONTENTS}

1 INTRODUCTION 1

1.1 Overview of Intensity Prediction Accuracy . . . . . . . . . . . . . . 1

1.2 Consequences of Underforecasting TC Intensity . . . . . . . . . . . . 4

1.3 Previous TC Intensity Research . . . . . . . . . . . . . . . 7

1.4 Outline of report ........................ 8

\section{THEORY OF TROPICAL CYCLONE INTENSITY CHANGE BY UPPER-} LEVEL FORCING

2.1 Intensity changes associated with outflow interaction . . . . . . . . 11

2.2 Intensity change induced by dynamic forcing . . . . . . . . . . . 16

2.3 Conceptual view of upper-level forcing on TC intensity . . . . . . . . . 22

2.4 Upper-level forcing and vertical wind shear . . . . . . . . . . . 28

3 TROPICAL CYCLONE INTENSITY CHANGE THEORY NOT REQUIRING UPPER-LEVEL FORCING 38

3.1 Hurricane Andrew - the devastating storm which almost never happened . . . 38

3.2 The Fading of CISK and the Emergence of Air-Sea Interaction (ASI) Theories 46

3.2.1 The Air-Sea Interaction (ASI) process . . . . . . . . . . . . . 46

3.2.2 The role the eye plays in TC intensity change . . . . . . . . . . . 58

3.2.3 Original Conditional Instability of the Second Kind (CISK) . . . . . . . 61

3.2.4 CISK with ASI and Internally Forced Convergence (IFC) . . . . . . . 64

3.2.5 The Carnot Cycle ASI theory . . . . . . . . . . . . . . . . 66

3.3 The debate over eyewall buoyancy ................... 71

\section{TROPICAL CYCLONE INTENSITY FORECAST SCHEMES AND} FOCUS OF THIS STUDY

4.1 Evidence of buoyancy, and the relationship of strong convection to future in-

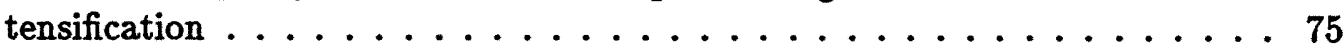

4.2 Satellite TC intensity forecast schemes . . . . . . . . . . . 78

4.2.1 Early research studies on brightness temperature relationship to intensity

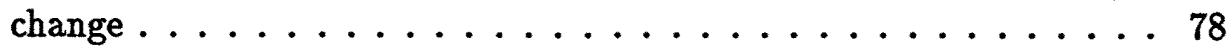

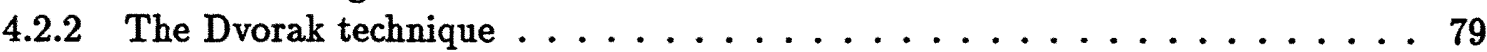

4.2 .3 The Mundell scheme . . . . . . . . . . . . . . . . . . 80

4.2 .4 Analysis of satellite data in this report . . . . . . . . . . . 84

4.3 Subjective TC intensity forecast schemes . . . . . . . . . . 85

4.4 Statistical TC intensity forecast schemes . . . . . . . . . . . 85

4.5 The current state of TC intensity numerical modeling . . . . . . . . 90

4.6 Focus of this study and research approaches . . . . . . . . . . . 91 
5 DATA SOURCES 95

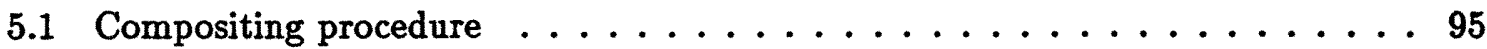

5.2 Inner-core hurricane flight data . . . . . . . . . . . . 97

5.3 Data for statistical analysis . . . . . . . . . . . . . . . . . 99

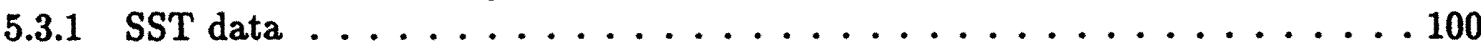

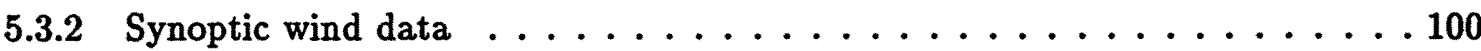

5.3 .3 Satellite data . . . . . . . . . . . . . . . . . 101

5.3.4 Summary of regression sample ................... . . 105

6 INNER-CORE PROCESSES RELATED TO INTENSITY CHANGE 106

6.1 Inner-core temperature profile for intensifying TCs . . . . . . . . . 106

6.2 The progression of the inner-core upper-level winds . . . . . . . . . 108

6.2.1 Generalization of inner-core $v_{t}$ evolution . . . . . . . . . . . . . . 108

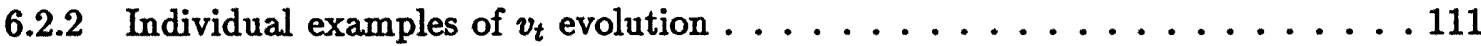

6.3 Thermal relationship to inner-core wind profile . . . . . . . . . . . 113

6.4 Dynamical relationships to inner-core wind profile . . . . . . . . . 126

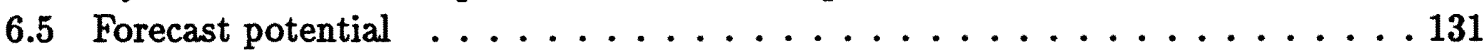

6.5.1 Intensity prediction based on inner-core sounding . . . . . . . . . 132

6.5.2 Intensity prediction based on inner-core upper-tropospheric wind and tem-

perature symmetry . . . . . . . . . . . . . . 138

6.6 Broad-scale composites of fast and slow developing tropical cyclones . . . . 142

6.7 Diagnostic balanced vortex calculations . . . . . . . . . . . 156

6.7.1 Brief review of balanced vortex dynamics . . . . . . . . . . . . 158

6.7.2 Description of the balanced vortex model . . . . . . . . . . . . 160

6.7.3 Methodology of the balanced vortex model . . . . . . . . . . . . 160

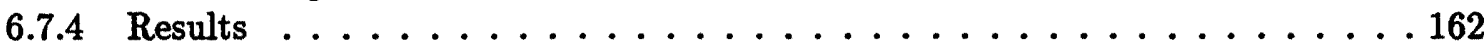

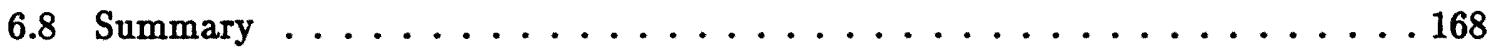

\section{A MODIFIED AIR-SEA INTERACTION THEORY AND NEW MAX-} IMUM POTENTIAL INTENSITY THEORY 170

7.1 Eyewall processes which affect the ASI process . . . . . . . . . . 170

7.1 .1 Evidence of boundary layer cooling . . . . . . . . . . . . . 172

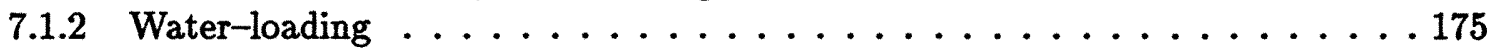

7.1.3 Latent heating by ice processes . . . . . . . . . . . . 176

7.1 .4 Eyewall slope . . . . . . . . . . . . . . . . . . . . 180

7.2 Mathematical treatment of cooling under eyewall, reversible ascent, pseudoadiabatic ascent, and ice microphysics . . . . . . . . . . . . . 181

7.2.1 Representation of surface cooling by adiabatic expansion . . . . . . . . 181

7.2 .2 Representation of water loading . . . . . . . . . . . . . 183

7.2 .3 Representation of ice processes . . . . . . . . . . . . . 184

7.3 Buoyancy results with ice microphysics and water-loading . . . . . . . . 186

7.3.1 Buoyancy comparisons between ice, no ice, reversible, and pseudoadiabatic

calculations ......................... 187

7.3.2 Computational procedure for buoyancy . . . . . . . . . . . . . 190

7.4 Methodology for modified ASI process . . . . . . . . . . . . . . 190

7.4.1 Representation of eyewall warming . . . . . . . . . . . . . . 190

7.4.2 Evidence of boundary layer cooling . . . . . . . . . . . . . . 192

7.4.3 Computational procedure for modified ASI process . . . . . . . . . 193 
7.5 Results of the modified ASI process . . . . . . . . . . . 193

7.6 A new Maximum Potential Intensity (MPI) theory . . . . . . . . . . . 197

7.7 Summary . . . . . . . . . . . . . . . . . . . . . 201

8 THE TYPHOON INTENSITY PREDICTION SCHEME (TIPS) 205

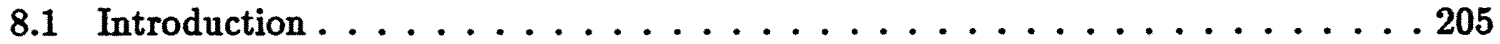

8.2 Possible predictors . . . . . . . . . . . . . . . . 205

8.2 .1 Climatology . . . . . . . . . . . . . . . . . . 206

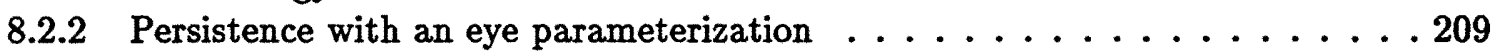

8.2 .3 Sea Surface Temperature . . . . . . . . . . . . . . . 211

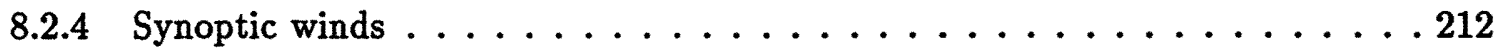

8.2 .5 Satellite data . . . . . . . . . . . . . . . . . . 215

8.2.6 The plausibility of regression development on besttrack data . . . . . 218

8.2.7 Alternatives to averaging along the track . . . . . . . . . . 218

8.2 .8 Summary of possible predictors . . . . . . . . . . . . . . . 219

8.3 Regression methodology . . . . . . . . . . . . . . . . . 219

8.3.1 Validity of linear least squares multiple regression . . . . . . . . . . 222

8.3.2 Screening of potential predictors - the hazards of multicollinearity and ar-

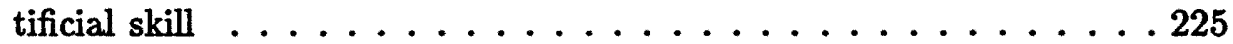

8.3.3 Normalization of regression coefficients ............... 232

8.3.4 Verification of model performance . . . . . . . . . . . . 232

8.3.5 Summary remarks on the regression technique ............ 234

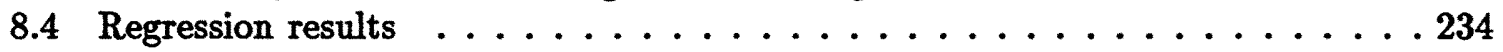

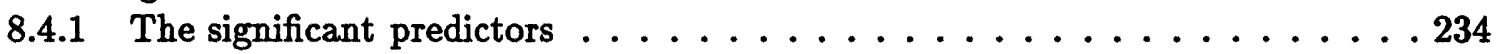

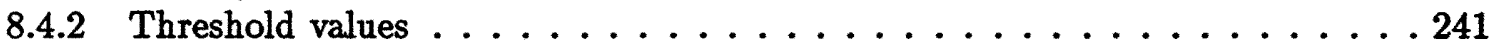

8.4 .3 Complete information on TIPS . . . . . . . . . . . . . . 242

8.4.4 Predictor contributions to intensity change stratifications . . . . . . . 243

8.4.5 Evaluation of TIPS intensity forecasts . . . . . . . . . . . 250

8.4.6 Overall assessment of TIPS compared to JTWC . . . . . . . . . . . 254

8.5 Comments on insignificant predictors . . . . . . . . . . . 257

8.6 Case studies . . . . . . . . . . . . . . . . . . 259

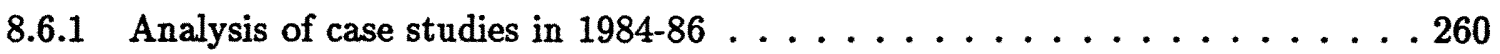

8.6 .2 Analysis of rapidly intensifying case studies in $1983 \ldots \ldots \ldots$

8.7 Miscellaneous results . . . . . . . . . . . . . . . . 272

8.7.1 Experiment with assorted pixel count values . . . . . . . . . . . 272

8.7.2 Comparison of different wind shear parameters . . . . . . . . . . . 280

8.7.3 Comparison of perfect-prog shear forecasts against shear forecasts based on

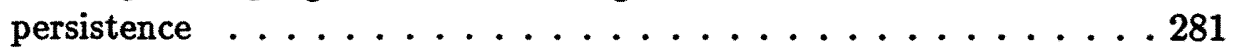

8.7.4 Other sensitivity experiments . . . . . . . . . . . . . . 284

8.7.5 Relationship of Relative Eddy angular momentum Flux Convergence (REFC) to upper-level vorticity advection . . . . . . . . . . 284

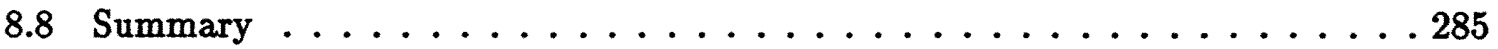

9 CONCLUSIONS AND RECOMMENDATIONS 287

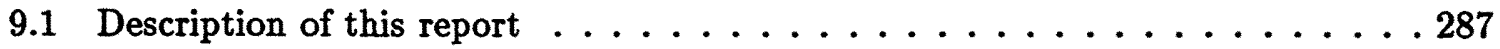

9.2 Summary . . . . . . . . . . . . . . . . . . . . . 290

9.2.1 Rapid intensification theory . . . . . . . . . . . . . . . 290 
9.2.2 Observational study of possible link between inner-core upper-level tropical cyclone structure and intensification . . . . . . . . . . 292

9.2.3 Alternative Air-Sea Interaction (ASI) theory with boundary layer cooling, water-loading, and ice microphysics . . . . . . . . . 293

9.2.4 The increase of CAPE in the eyewall, and a new Maximum Potential Intensity (MPI) theory . . . . . . . . . . . . . . . 295

9.2.5 Statistical analysis of tropical cyclone intensity change . . . . . . . . 297

9.2.6 Forecasting tips and "rule of thumbs" ................ 302

9.3 Recommendations . . . . . . . . . . . . . . . . 304
A SHALLOW WATER DERIVATION OF THE ROSSBY RADIUS OF DEFORMATION
B COMPLETE INFORMATION ON TIPS 


\section{LIST OF SYMBOLS AND ACRONYMS}

$\alpha$ : Specific volume; also the cross-isobaric inflow angle in the boundary layer.

AOML : Atlantic Oceanographic and Meteorological Laboratory

AVHRR : Advanced Very High Resolution Radiometer

ASI : Air-Sea Interaction

BMRC : (Australian) Bureau of Meteorology Research Centre

BV Model : Balanced Vortex Model

Category 1-5 : On the Saffir-Simpson scale, a Category 1 to 5 hurricane contains maximum sustained winds of 33-42, 43-49, 50-58, 59-69, and $>69 \mathrm{~ms}^{-1}$, respectively. MSLPs of Category 1-5 in the Atlantic, east Pacific, and central Pacific are $>980$, 979-965, 964-945, 944-920, and $<920 \mathrm{mb}$, respectively. No official scale exists in the western North Pacific, although efforts are underway to remedy this (see text)

CAPE : Convective Available Potential Energy. This is defined as: CAPE $=\int_{L F C}^{E L} g \frac{T_{v m}(z)-T_{v e}(z)}{T_{v e}(z)} d z$ $C_{D}:$ Drag coefficient

CDO : Central Dense Overcast

CIRA : Cooperative Institute for Research in the Atmosphere

CISK : Conditional Instability of the Second Kind

CLIPER : CLImatology and PERsistence model

$c_{i}:$ Regression coefficient

$c_{i c e}:$ Specific heat of ice at constant pressure; $c_{i c e}=2106 \mathrm{~J} \mathrm{deg}^{-1} \mathrm{~kg}^{-1}$

$c_{p}$ : Specific heat of dry air at constant pressure; $c_{p}=1004 \mathrm{~J} \mathrm{deg}^{-1} \mathrm{~kg}^{-1}$

$c_{p v}:$ Specific heat of water vapor at constant pressure; $c_{p}=1850 \mathrm{~J} \mathrm{deg}^{-1} \mathrm{~kg}^{-1}$

$c_{w}$ : Specific heat of water at constant pressure; $c_{w}=4218 \mathrm{~J} \mathrm{deg}^{-1} \mathrm{~kg}^{-1}$ 
CSU : Colorado State University

$c_{T O T}:$ Total specific heat, defined as: $c_{T O T}=c_{p}+q_{i c e}\left(T^{\prime}\right) c_{v}+\left(q_{l}+q\left(T_{f}\right)-q_{i c e}\left(T^{\prime}\right)\right) c_{i c e}$

D : Dual outflow channel

D3 : Combined intensifying Atlantic depression and tropical storm composite

D1 : Prehurricane cloud cluster composite

deg : Unit of distance, in which $1 \mathrm{deg} \approx 111 \mathrm{~km}=60 \mathrm{n} . \mathrm{m}$. In contrast, the notation ${ }^{\circ}$ is used for both temperature $\left({ }^{\circ} \mathrm{C}\right)$ and direction with respect to a $360^{\circ}$ circle

DIFFPX : PX0270 minus PX2665

DIR : Observed storm direction

DIRAN : Direction anomaly, defined as departures of DIR from average storm direction of $310^{\circ}$, yielding a positive or negative value between 0 and $180^{\circ}$ with respect to $310^{\circ}$. When DIR is between $130^{\circ}$ and $310^{\circ}$, DIRA is positive. Otherwise, DIRA is negative

DMSP : Defense Meteorological Satellite Program

DPXRdTb : 12-h trend of PXRdTb

DRM6RdTb : 12-h trend of RM6RdTb

DRM12RdTb : 12-h trend of RM12RdTb

DRM24RdTb : 12-h trend of RM24RdTb

$e:$ Vapor pressure

$e^{*}:$ Saturation vapor pressure

ECMWF : European Center for Medium-Range Weather Forecasts

EFC : Older notation of Relative Eddy angular momentum Flux Convergence (REFC)

EIR : Enhanced infrared

EL : Equilibrium level

EOF : Empirical Orthogonal Functions

$\theta_{e}:$ Equivalent potential temperature

$\eta:$ Thermodynamic efficiency, defined as $\eta=\frac{T_{\text {sea }}-\bar{T}_{\text {out }}}{T_{\text {sea }}}$ 
EYEPER : Indicator variable that combines persistence with the parameterization of a well-formed, contracting eye

$e_{i c e}:$ Saturation vapor pressure over ice

$f$ : Coriolis parameter

Fast intensification : A generic classification generally encompassing TCs with a 30-mb MSLP decrease or more in a 24 -h period, or an increase in $V_{\max }$ of $20 \mathrm{kts}$ or more in a 24-h period. Rapid intensifiers are a subset of this classification

GMS : Geostationary Meteorological Satellite

GFDL : Geophysical Fluid Dynamics Laboratory

$g$ : Acceleration of gravity

$H$ : Scale height of the atmosphere

$h$ : Sensible heat flux; also used as moist static energy where $h=g z+c_{p} T+L_{v} q$

HRD : Hurricane Research Division

IFC : Internally forced convergence

IMSL : International Mathematical Subroutine Library. FORTRAN subroutines used for statistical and mathematical analysis

JD : Julian date

JDAN : Anomaly from peak onset of rapid deepeners, defined as the absolute value of observed Julian date minus climatological onset of rapid intensifiers

JTWC : Joint Typhoon Warning Center

$\Lambda$ : Dummy variable equal to zero during a pseudoadiabatic process and to 1 during a reversible process

LAD : Least Absolute Deviations

LAT : Initial storm latitude

LCL : Lifting Condensation Level

LFC : Level of Free Convection 
$L_{f}:$ Latent heat of fusion. This is defined as:

$$
\begin{aligned}
L_{f} & =\left(0.3337+0.00216[T-273.15]-0.981 \times 10^{-6}[T-273.15]^{2}\right. \\
& \left.+0.159 \times 10^{-6}[T-273.15]^{3}\right) \times 10^{6} \mathrm{~J} \mathrm{~kg}^{-1}
\end{aligned}
$$

$L_{s}$ : Latent heat of sublimation (deposition); $L_{s}=2.834 \times 10^{6} \mathrm{~J} \mathrm{~kg}^{-1}$

$L_{v}$ : Latent heat of vaporization (condensation); $L_{v}=(2.501-0.00237[T-273.15]) \times$ $10^{6} \mathrm{~J} \mathrm{~kg}^{-1}$

$M$ : Relative angular momentum, defined as $r v_{t}$.

MCAPE : "Modified" CAPE due to inclusion of water-loading. This is defined as: MCAPE $=\int_{L F C}^{E L} g\left[\frac{T_{v m}(z)-T_{v e}(z)}{T_{v e}(z)}-q_{l}\right] d z$

MOT : Coordinate system in which the TC motion is subtracted from the wind observations

MPI : Maximum Potential Intensity for a given SST, defined in kts as: $\mathrm{MPI}=66.5+$ $108.5 \exp [-0.1813(30-\mathrm{SST})]$

MRF : Medium-Range Forecast model

MSLP : Minimum Sea Level Pressure

MUND : Ratio of PX0275 to PX2665

n.m. : Nautical mile, in which $60 \mathrm{n} . \mathrm{m} .=111 \mathrm{~km} \approx 1 \mathrm{deg}$

$N$ : Brunt-Väisälä frequency; also denotes no outflow channel

N1 : Non-developing cloud cluster composite

N3 : Non-developing depression composite

NESDIS : National Environmental Satellite, Data, and Information Service

NHC : National Hurricane Center

NHRL : National Hurricane Research Laboratory

NHRP : National Hurricane Research Project

NMC : National Meteorological Center

NOAA : National Oceanic and Atmospheric Administration

$N S D:$ Normalized Standard Deviation, defined as $[\overline{x c}-\bar{x}] / \sigma$ 
OCS : Outer-Core Strength - an area-weighted tangential wind speed from 1 deg to 2.5 deg with respect to the TC center

$\Omega$ : Earth's angular velocity; $\Omega=7.292 \times 10^{-5} \mathrm{~s}^{-1}$

P : Older notation for POT

PA.ni : Pseudoadiabatic process with no ice microphysics

PA.wi : Pseudoadiabatic process with ice microphysics

$p_{0}: 1000 \mathrm{mb}$

$p_{s f c}:$ Surface pressure

$p_{\text {env }}:$ Environmental surface pressure

$p_{e w}:$ Surface pressure under eyewall

PEFC : Planetary Eddy angular momentum Flux Convergence, defined as $-\overline{f^{\prime} u_{\tau}^{\prime}}$ where a prime denotes the deviation from a wind's azimuthally averaged value

PEFC $_{\text {mid }}$ : 200-mb PEFC averaged between 7 and $10 \mathrm{deg}$

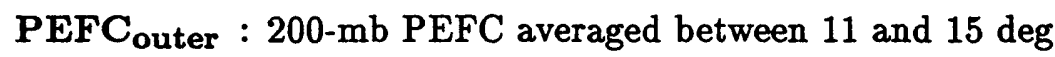

$P_{\text {in }}$ : Significance level for a variable to enter a multiple regression model

$P_{\text {out }}$ : Significance level for a selected variable to stay in a multiple regression model

POS : Principal Outflow Streamline

POT : Maximum Possible Intensity (MPI) for a given SST minus initial intensity $\left(V_{\text {max }}\right)$. MPI is averaged over the forecast track in TIPS

$\theta:$ Potential temperature

PV : Potential Vorticity

PXRdTb : Percent of infrared pixels (PX) in a radial area (Rd) colder than a specified brightness temperature $(\mathrm{Tb})$ at $t=0$. All $\mathrm{Tb}$ are negative. 30 combinations are investigated

PXRdTb**2 : Same as PXRdTb, but squared

$\dot{Q}$ : Diabatic heating rate

$q$ : Mixing ratio 
$q^{*}:$ Saturation mixing ratio

$q_{\text {ew }}: q$ under eyewall

$q_{f}:$ Frozen condensate

$q_{l}:$ Liquid condensate

$q_{\text {ice }}$ : Saturation mixing ratio over ice

$q_{\text {tot }}: q$ for unsaturated air, sum of $q^{*}$ and $q_{l}$ below freezing level for saturated air in a reversible process, and sum of $q^{*}, q_{f}$ and $q_{i c e}$ above freezing level assuming no supercooled droplets in a reversible process. This expression is denoted as qToT above the freezing level in a reversible process. In a pseudoadiabatic process, $q_{l}$ and $q_{f}$ are zero

$R$ : Dry gas constant in a thermodynamic context $\left(R=287 \mathrm{~J} \mathrm{deg}^{-1} \mathrm{~kg}^{-1}\right)$; Multiple regression linear correlation coefficient in a statistical context

$r$ : Single variable linear correlation coefficient in a statistical context; radius in cylindrical coordinates context

$r^{2}$ : Coefficient of determination (variance explained in a linear, one variable regression model)

$R^{2}$ : Coefficient of determination (variance explained in a linear multiple regression model)

$r_{p}:$ Partial correlation

RA.ni : Reversible process with no ice microphysics

RA.wi : Reversible process with ice microphysics

Rapid intensification : Defined as a 42-mb MSLP decrease or greater in a 24-h period, or a satellite inferred increase in $V_{\max }$ of at least $23 \mathrm{~ms}^{-1}$ in a 24-h period

REFC : Relative Eddy angular momentum Flux Convergence, defined as $-\frac{1}{r^{2}} \frac{\partial}{\partial r} r^{2} \overline{u_{r}^{\prime} v_{t}^{\prime}}$ where a prime denotes the deviation from a wind's azimuthally averaged value

REFC $_{\text {mid }}: 200$-mb REFC averaged between 7 and 10 deg

REFC $_{\text {outer }}$ : 200-mb REFC averaged between 11 and 15 deg

$\zeta$ : Relative Vorticity

RH : Relative Humidity 
$\mathbf{R H}_{\text {sfe }}$ : Surface RH

$\mathbf{R H}_{\text {env }}$ : Environmental RH

RI : Rapid Intensification (see definition)

RM6DIFFPX : RM60270 minus RM62665

RM12DIFFPX : RM120270 minus RM122665

RM24DIFFPX : RM240270 minus RM242665

RM6RdTb : Same as PXRdTb, but the pixel counts are a 6-h running mean (RM)

RM6RdTb**2 : Same as RM6RdTb, but squared

RM12RdTb : Same as PXRdTb, but the pixel counts are a 12-h running mean (RM)

RM12RdTb**2 : Same as RM12RdTb, but squared

RM24RdTb : Same as PXRdTb, but the pixel counts are a 24-h running mean (RM)

RM24RdTb**2 : Same as RM24RdTb, but squared

RM6MUND : Ratio of RM60275 to RM62665

RM12MUND : Ratio of RM120275 to RM122665

RM24MUND : Ratio of RM240275 to RM242665

RMW : Radius of Maximum Wind

$\lambda_{R}:$ Rossby radius of deformation

$S:$ Older notation for vertical wind shear (VWS)

$s_{i c e}:$ Ice entropy

$s^{*}:$ Moist entropy

$\mathbf{S}_{\mathbf{P}}$ : Single outflow channel poleward

$\mathbf{S}_{\mathbf{E}}$ : Single outflow channel equatorward

SER : Sub-Equatorial Ridge

SHIFOR : Statistical Hurricane Intensity FORecast model

SHIPS : Statistical Hurricane Intensity Prediction Scheme 
SPD : Observed storm speed

SPDAN : Storm speed anomaly, defined as the absolute value of SPD minus average storm speed of $5 \mathrm{~ms}^{-1}$

SSM/I : Special Sensor Microwave/Imager

SST : Sea Surface Temperature

STIFOR : Statistical Typhoon Intensity FORecast model

$\sigma:$ Standard deviation of a variable

STR : Subtropical Ridge

$\tau$ : frictional stress; also a relaxation time constant in the Kuo cumulus parameterization scheme dependent on moisture availability and the integrated buoyancy difference in a column of air

$T:$ Temperature

$T^{\prime}$ : Temperature resulting from isobaric freezing

$\breve{T}$ : Average temperature of an air parcel at a particular pressure level between the characteristic curves of RA.wi and PA.wi

$T_{b}:$ Brightness (equivalent blackbody) temperature

$T_{f}:$ Temperature at which supercooled droplets freeze

$T_{p}$ : Density temperature, defined as $T_{\rho}=T\left(1+0.61 q^{*}-q_{l}\right)$ below freezing level, and as $T_{\rho}=T\left(1+0.61 q^{*}-q_{f}-q_{i c e}\right)$ above freezing level assuming no supercooled droplets

$\breve{T}_{\rho}$ : Average density temperature of an air parcel at a particular pressure level between the characteristic curves of RA.wi and PA.wi

$T_{e w}: T$ under eyewall

$\bar{T}_{\text {out }}:$ Mean outflow temperature, defined as the entropy weighted average of the outflow temperature in a TC where angular momentum lines are quasi-horizontal

$T_{s f c}:$ Surface temperature

$T_{\text {sea }}: \mathrm{SST}$

$T_{m}$ : Temperature corresponding to a moist adiabatic (constant $\theta_{e}$ value) at a given height

$T_{v}$ : Virtual temperature, defined as $T_{v}=T(1+0.61 q)$; in saturated air, $q^{*}$ is used instead 
$T_{e}:$ Temperature of the environment

$T_{c}$ : Temperature of cloud environment

$T_{m}:$ Temperature of ascending moist air parcel

$T_{v e}:$ Virtual temperature of the environment

$T_{v c}:$ Virtual temperature of cloud environment

$T_{v m}$ : Virtual temperature of ascending moist air parcel

TC : Tropical Cyclone

TIPS : Typhoon Intensity Prediction Scheme

TOMS : Total Ozone Mapping Spectrometer

TUTT : Tropical Upper-Tropospheric Trough

USPD : Zonal component of storm motion

$u$ : Zonal wind

$u_{r}:$ Radial wind

$v:$ Meridional wind

$v_{t}:$ Tangential wind

$\overline{v_{t}}:$ Mean 500 to $200-\mathrm{mb}$ tangential wind

$\overline{v_{t}}(\mathrm{~s}): \overline{v_{t}}$ of a TC with a pressure drop between 10 and $20 \mathrm{mb}$ in the next $24 \mathrm{~h}$

$\bar{v}_{t}(\mathrm{f}): \overline{v_{t}}$ of a TC with a pressure drop $\geq 30 \mathrm{mb}$ in the next $24 \mathrm{~h}$

$V_{\max }: 1-\min$ averaged maximum sustained surface wind speed

VORT2 : 200 -mb vorticity in a $2.5-\mathrm{deg}$ circle over the center of the storm

VORT5 : 200-mb vorticity in a 5-deg circle over the center of the storm

VORTADV : 200 -mb vorticity advection over the center of the storm

VSPD : Meridional component of storm motion

VSST : Older notation for MPI.

VWS : Vertical Wind Shear 
VWSPT : Magnitude of $200-850 \mathrm{mb}$ "single point" vertical wind shear over the storm center. Defined as $\left[\left(u_{200}-u_{850}\right)^{2}+\left(v_{200}-v_{850}\right)^{2}\right]^{1 / 2}$ where the subscripts refer to pressure levels. In TIPS, VWSPT is averaged over the forecast track, and a perfect shear forecast is assumed

VWS2 : Same as VWS5, but averaged over a 2.5-deg circle

VWS5 : Same as VWSPT, but averaged over a 5-deg circle. Let $\Delta u=u_{200}-u_{850}$ and $\Delta v=v_{200}-v_{850}$, then VWS5 is defined as $\left[(\overline{\Delta u})^{2}+(\overline{\Delta v})^{2}\right]^{1 / 2}$ where, denoting the radii $r=2.5 \mathrm{deg} \& 5.0 \mathrm{deg}$ with $i=1 \& 2$, and circle angle $\phi=0,90,180, \&$ $270^{\circ}$ with $j=1$ to $4, \overline{\Delta u}$ is computed as: $\overline{\Delta u}=\frac{1}{9}\left[\Delta u(r=0)+\sum_{i=1}^{2} \sum_{j=1}^{4} \Delta u_{i, j}\right]$ and likewise for $\overline{\Delta v}$

VWSPT $_{\text {per }}$ : Same as VWSPT, but no shear forecast is used. Instead, persistence from the initial shear field is performed along the forecast track

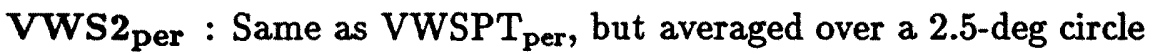

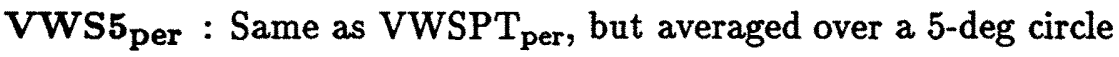

$\omega:$ Vertical velocity in isobaric coordinates $(D p / D t)$

$w_{o}:$ Vertical velocity forced by Ekman pumping

$w_{\max }$ : Maximum vertical velocity due to contributions from CAPE and Ekman pumping, defined as $w_{\max }=\left[2 \mathrm{CAPE}+w_{o}^{2}\right]^{1 / 2}$

$w_{\text {req }}$ : Eyewall vertical velocity required to balance frictional loss and the export of cyclonic momentum from the upper eyewall and still maintain a tropical cyclone's current intensity

WISHE : Wind Induced Surface Heat Exchange

WNP : Western North Pacific Ocean

$\bar{x}:$ Predictor mean

$\bar{y}:$ Dependent variable mean $\left(\overline{\Delta V_{\max }}\right)$

$\overline{x c}:$ Average predictor value for a class 


\section{Chapter 1}

\section{INTRODUCTION}

\subsection{Overview of Intensity Prediction Accuracy}

According to a survey of all tropical cyclone (TC) forecast centers in the world, the overriding research emphasis is placed on track forecasting with little investigation on intensity change (McBride and Holland 1987). As a result, systematic and skillful intensity forecasting techniques have yet to be established, and much is still not understood about TC intensity change. Not surprisingly, large errors occur when forecasting TC intensity. The measure of intensity forecast around the world is the 1-min averaged maximum sustained surface wind speed $\left(V_{\max }\right)$, often tabulated in kts, and often interpolated from upper-levels by subjective wind reduction. Furthermore, except for the Atlantic Ocean, wind measurements are actually averaged every $10-\min$ and then converted to 1-min averages (Elsberry et al. 1992). When observations are not available, empirical relationships are used instead (Atkinson and Holliday 1977). As shown in Table 1.1, the mean 24-h Joint Typhoon Warning Center (JTWC) absolute error is $6.4 \mathrm{~m} \mathrm{~s}^{-1}$ for their intensity forecasts (Mundell 1990). Furthermore, the Australian Bureau of Meteorology Research Center (BMRC) reports that their TC intensity forecasts were not even as accurate as a prediction based on persistence or climatology (Elsberry et al. 1992).

However, the largest errors in TC intensity forecasting occur with fast intensifying systems - especially for TCs undergoing extreme deepening classified as rapid intensification (RI). As will be seen in Chapter 8, large forecast errors are also associated with fast weakening TCs. However, the anticipation of a TC's rapid deepening is of more concern to the forecaster so that proper preparations may be made for a potentially devastating situation. As shown in the next section, unexpected TC intensification can be disastrous. 
Table 1.1: Comparison of JTWC 24-h rapid intensification forecast errors and biases (in $\mathrm{m} \mathrm{s}^{-1}$ ) for the 1972-87 season versus all intensity forecast errors for the $1980-85$ seasons. Bias is defined as observed minus forecast (from Mundell 1990).

\begin{tabular}{|c|c|c|c|c|}
\hline Class & Mean Absolute Error & Bias & Standard Deviation & Count \\
\hline Rap Int (72-87) & 12.4 & 9.7 & 11.2 & 468 \\
\hline All Storms (80-85) & 6.4 & 0.3 & 8.5 & 2891 \\
\hline
\end{tabular}

Holliday and Thompson (1979) define "rapid intensification" as a change in Minimum Sea-Level Pressure (MSLP) of at least $42 \mathrm{mb}$ in a 24 -h period, or a satellite-inferred increase in $V_{\max }$ of at least $23 \mathrm{~m} \mathrm{~s}^{-1}$ per day when no measurement of MSLP is possible. According to Holliday and Thompson, this constitutes $25 \%$ of the cases in 1956-1976 for which MSLP fell in the western North Pacific (WNP). In terms of TC number, $15 \%$ WNP typhoons were RI storms in the 1970s and 1980s (Mundell 1990). An extreme case of a RI storm is shown in Fig. 1.1 for Supertyphoon Vanessa beginning 18 UTC 24 October 1984, in which the pressure fell from $967 \mathrm{mb}$ to $895 \mathrm{mb}$ in $24 \mathrm{~h}$. It should be noted that other authors have used different criteria to identify RI storms (Brand 1972). However, this paper will use the RI definition of Holliday and Thompson.

When forecast intensity errors for all storms are compared to forecast intensity errors for rapid intensifiers, as shown in Table 1.1, it is obvious that there is a lower percentage of accurate forecasts for RI storms (Mundell 1990). Table 1.1 shows that the mean 24-h absolute error for predicting rapid intensifiers during 1972-1987 $\left(12.4 \mathrm{~m} \mathrm{~s}^{-1}\right)$ is nearly twice as large as all forecast errors during 1980-1985. The difference in mean values are significant at the $99.9 \%$ level.

Furthermore, when errors for all storms during 1980-1985 are considered, no bias exists, and in fact the errors are normally distributed (Mundell 1990). On the other hand, the large positive bias of $9.7 \mathrm{~m} \mathrm{~s}^{-1}$ in the RI class exists, resulting in the underforecasting of RI tropical cyclone intensity change just $24 \mathrm{~h}$ later. 


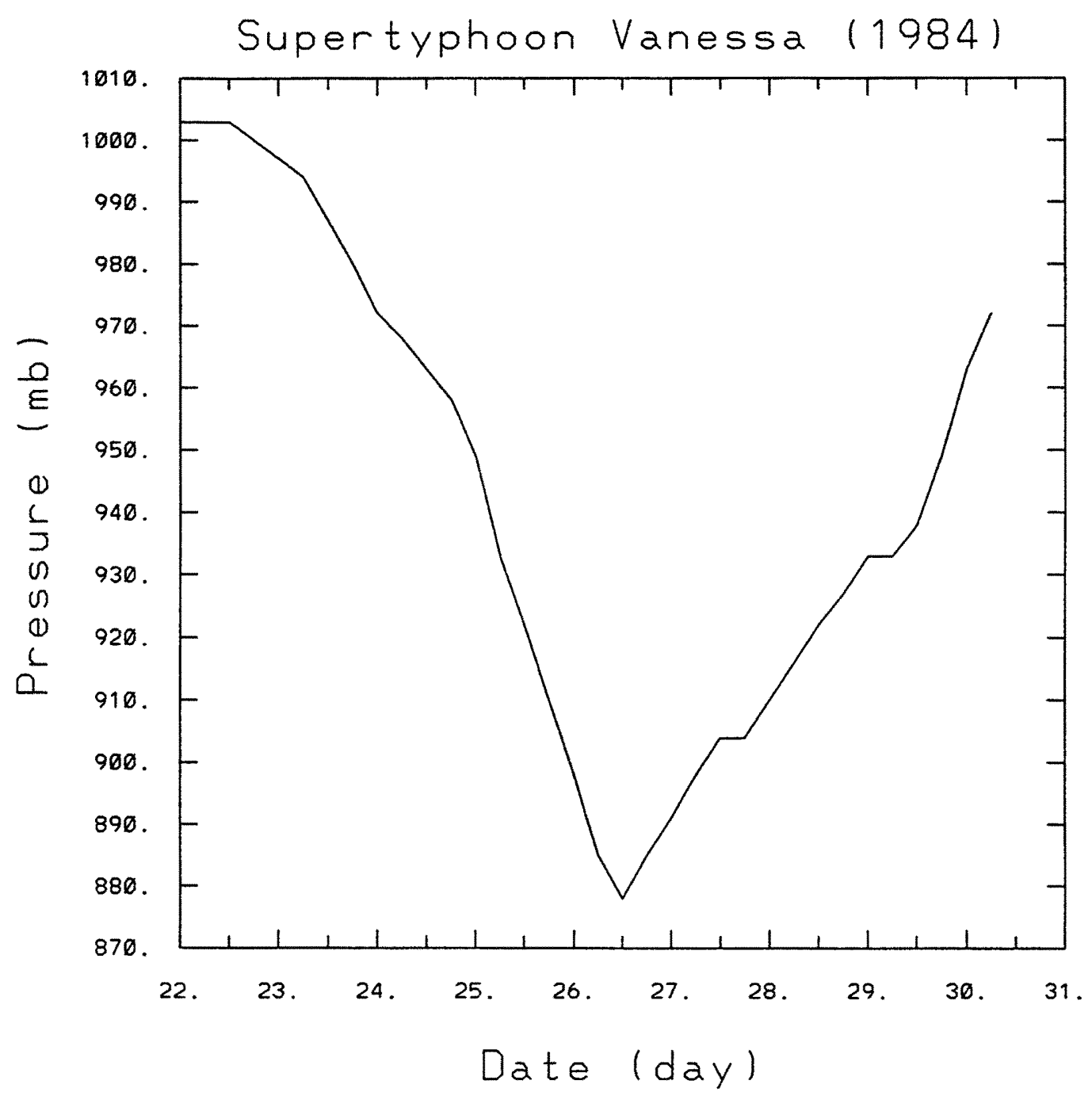

Figure 1.1: Times series of mean sea-level pressure (MSLP) for Supertyphoon Vanessa from 00 UTC 22 October 1984 (22.0) to 06 UTC 30 October 1984 (30.25). Pressure units are in $\mathrm{mb}$. 


\subsection{Consequences of Underforecasting TC Intensity}

Intensity forecast errors can be catastrophic, like the unanticipated rapid strengthening of Typhoon Niña on 24 November 1987. Niña, which was forecast to weaken, abruptly intensified from $90 \mathrm{kts}$ to $150 \mathrm{kts}$ in less than $24 \mathrm{~h}$. Since residents of Samar and southern Luzon (in the Philippines) were not sufficiently prepared for the storm, hundreds died and about half a million were homeless in the aftermath (Mundell 1990; JTWC 1987). On 11 UTC 21 September $1989-12$ h before its South Carolina landfall - Hurricane Hugo contained maximum winds of $49 \mathrm{~m} \mathrm{~s}^{-1}\left(1 \mathrm{~m} \mathrm{~s}^{-1} \text { below Category } 3\right)^{1}$ and were forecast not to change. Local and state officials were advised to prepare for a Category-3 hurricane. However, Hugo strengthened to a Category-4 storm with winds of $60 \mathrm{~m} \mathrm{~s}^{-1}$ at landfall (Sheets 1990).

Two somewhat recent storms epitomize the lack of skill in forecasting RI events. One is the unanticipated strengthening of Supertyphoon Omar from 12 UTC 27 August to 12 UTC 28 August 1992 as it approached Guam. JTWC issued a forecast for Omar to persist at $75 \mathrm{kts}$ during this time frame. Instead, Omar increased to $115 \mathrm{kts}$ with $\Delta p \approx 42 \mathrm{mb}$ (JTWC 1992b). Omar was the most damaging typhoon to strike Guam since Typhoon Pamela in 1976. Omar caused an estimated $\$ 457$ million of damage, destroyed or severely damaged 2158 homes leaving 3000 people homeless, crippled island utilities, and interrupted communications (JTWC 1992b). It is quite possible this damage could have been somewhat reduced had residents prepared for a Category-3 storm instead of a Category 1. Furthermore, two Navy supply ships went aground in Apra Harbor after they broke their moorings. One may assume that these ships would have departed the island had the storm's rapid strengthening been forecasted.

\footnotetext{
${ }^{1}$ On the Saffir-Simpson scale (Simpson 1974), a Category-1 to 5 hurricane contains maximum sustained winds of 33-42, 43-49, 50-58, 59-69, and > $69 \mathrm{~m} \mathrm{~s}^{-1}$, respectively. MSLPs of Category 1-5 in the Atlantic, east Pacific, and central Pacific are $>980$, 979-965, 964-945, 944-920, and < $920 \mathrm{mb}$, respectively (Atkinson and Holliday 1977). No official Saffir-Simpson scale currently exists in the western North Pacific (WNP), but efforts are underway to rectify the situation (C. Guard, personal communication 1995). This new scale is more representative of the WNP, as it incorporates construction materials, building practices, plant life, termites, wood-rot, and the corrosive effects of salt that are common to this tropical region. More categories have been proposed, and in general contains a higher damage potential for weaker TCs compared to a similar Atlantic system of the same intensity.
} 
The final example is Hurricane Andrew which devastated south Florida. As shown in Fig. 1.2, the 24-h forecast valid for 18 UTC 23 August 1992 (23.75 on graph) was an increase from $80 \mathrm{kts}$ (MSLP of $969 \mathrm{mb}$ ) to $95 \mathrm{kts}$; unfortunately, the storm increased to $135 \mathrm{kts}$ (MSLP of $922 \mathrm{mb} ; \Delta p=47 \mathrm{mb}$ ). Subsequent forecasts within $24 \mathrm{~h}$ of landfall (on 09 UTC 24 August) improved, but the storm's rapid strengthening on 18 UTC 23 August was unexpected. And while the MSLP weakened after the RI event, a second pressure drop occurred hours before Andrew hit Florida with a MSLP of $922 \mathrm{mb}$ at landfall (Mayfield et al. 1994).

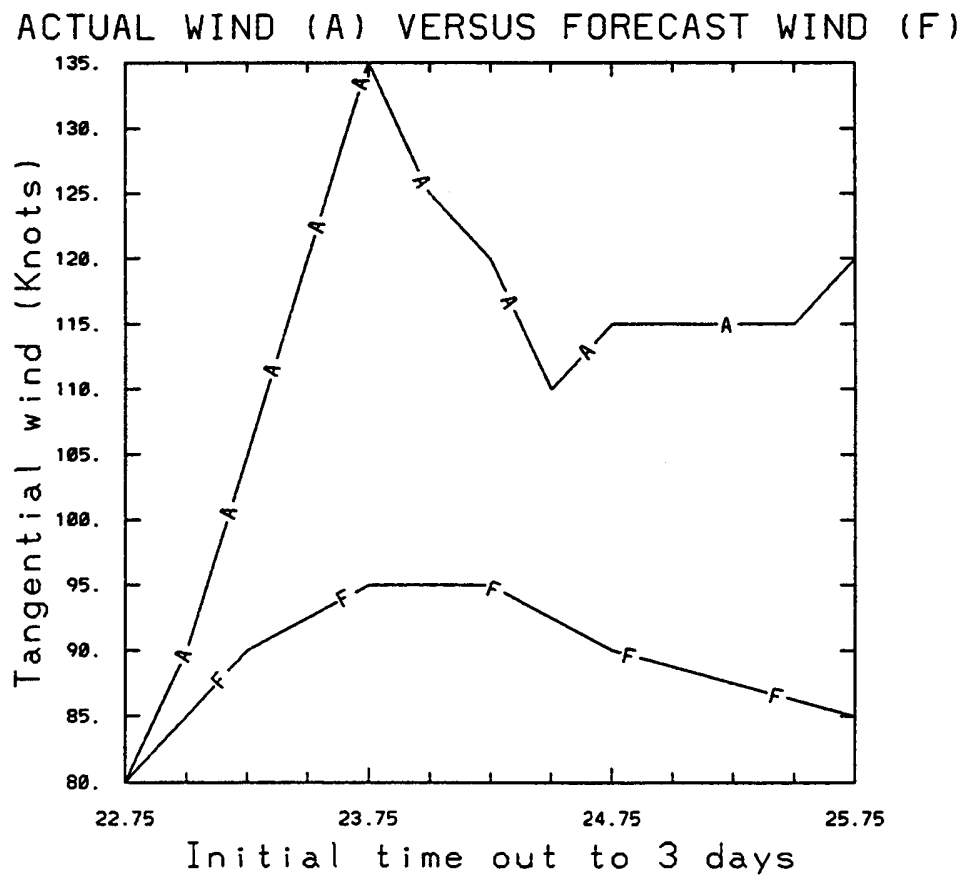

Figure 1.2: Plot of Hurricane Andrew forecast winds (labeled F) initialized at 18 UTC 22 August (22.75) out to 18 UTC 25 August 1992 (25.75) versus actual winds (labeled A).

According to Mayfield, the number of deaths directly attributed to Andrew in south Florida is 15, with 60 to 100 additional (indirect) deaths. Direct property damage is estimated at $\$ 25$ billion, with 25,524 homes destroyed and 101,241 damaged. In addition, revenue depletion due to loss (or disruption) of business is not known. Andrew resulted in the largest economic loss from a natural disaster in United States history, dwarfing previous hurricanes and natural disasters (including earthquakes, such as the devastating 1994 event in Northridge, CA estimated at $\$ 12.5$ billion in insurance losses). According to 
Sheets (1993), total losses adjusted to 1990 dollars are "more than the total of the three previous most costly hurricanes (Hugo in 1989, Betsy in 1965, and Agnes in 1972) or the two most costly hurricanes and the Loma Prieta, CA (1989) earthquake. Insured lossed are now estimated at $\$ 18$ billion..." with eight insurance companies failed. Certainly had south Florida residents known more than a day in advance about the impending fierce winds, many would have prepared sooner and more thoroughly which would have decreased the property damage (although damage would still have been very high). As stated in a National Disaster Survey Report (1993) on Andrew, the critical preparedness period for a Category-4 storm is $36 \mathrm{~h}$. The onset of RI for Andrew was $39 \mathrm{~h}$ before landfall, but residents had only a 24 -h warning of a Category-4 storm. A brief analysis of the environmental conditions leading to the RI of Andrew will be discussed later in Chapter 3.

The National Disaster Survey Report (1993) notes that hurricane intensity forecasts lack skill and lag far behind other forecasting applications such as track prediction. The report urges scientists to "redouble their efforts to develop models and operational techniques to forecast tropical cyclone intensity changes more effectively.” DeMaria and Kaplan (1994b) reached similar conclusions, and noted that official National Hurricane Center (NHC) forecast skill was comparable to a regression forecast based on climatology and persistence, and only slightly better than persistence (Fig. 1.3).

These warnings were recently accentuated by the unexpected rapid intensification of Hurricane Opal (1995) the night before its afternoon landfall near Panama City Beach, FL. Opal's intensity was $100 \mathrm{kts}$ on 03 UTC 4 October, and forecast to increase by 5 kts $9 \mathrm{~h}$ later (12 UTC). Instead, Opal increased to $130 \mathrm{kts}$ in this period. That next morning (October 5), Florida residents, alarmed by the rapid intensification and by an increase in forward motion of Opal, congested the road systems. Traffic clogged Interstate 10 between Mobile, AL, and Tallahassee, FL. Driving from Panama City Beach to Tallahassee (normally a 2-h trip) took $10 \mathrm{~h}$ (J. Stricherz, personal communication 1995). In fact, residents still stuck in traffic at noon were urged by radio reports to return home before Opal made landfall. 


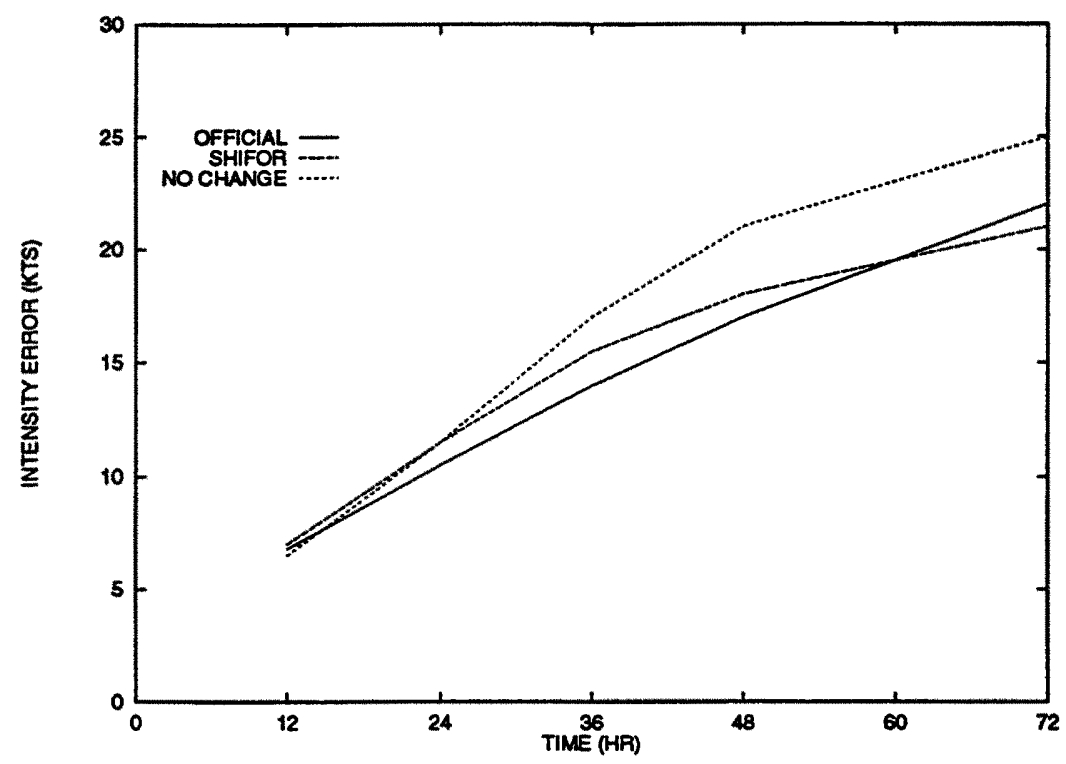

Figure 1.3: The average $12,24,36,48$, and 72-h intensity forecast errors using persistence, the climatology and persistence statistical model SHIFOR, and the official National Hurricane Center intensity forecast. Adapted from DeMaria and Kaplan (1994b).

Traffic had cleared by landfall, but the prospects of more such alarming situations is what motivates this report. The purpose of this paper is to develop more understanding about TC intensity change and to formulate an intensity forecast scheme.

\subsection{Previous TC Intensity Research}

Theoretical work on this subject is confined to two viewpoints: 1) upper-level forcing enhances TC development, with internal convection acting as a passive response; 2) innercore convective processes and Sea Surface Temperature (SST) affect TC intensity change the most, and any positive interaction upper-level forcing is more than offset by the negative influences of vertical wind shear. Vertical wind shear is the vectorial difference of upper-tropospheric and lower-tropospheric winds. As will be discussed throughout this paper, weak wind shear and warm SSTs are fundamental factors in determining TC intensity. In addition to theoretical work, forecasting research on TC intensity is concentrated in five areas, although overlap often occurs: 1) climatology; 2) satellite; 3) EXPERT systems; 4) numerical modeling; and 5) statistical multiple regression. All five approaches are considered in this report for the development of a prediction scheme, with 
particular emphasis on combining climatology, satellite, and synoptic data in a multiple regression scheme. Future theoretical work will utilize some numerical modeling which will be discussed in an upcoming technical report.

\subsection{Outline of report}

This study encompasses an observational, theoretical, and statistical approach to understanding and predicting TC intensity change. Chapters 2-4 describe previous research related to intensity change, with some focus on basic concepts, controversial theories, and forecasting applications. Chapter 2 presents previous research on TC intensity by upperlevel forcing, Chapter 3 discusses previous research on TC intensity change with respect to inner-core processes which do not depend on an external forcing agent, and Chapter 4 addresses the debate over the existence of buoyancy in the TC eyewall, as well as current intensity research and prediction schemes.

The research objectives of this report are also stated in Chapter 4. Details of the research methodology in Chapters 6-8 are postponed until Chapter 4 because necessary background material on TC intensity change needs to be presented first (Chapters 24). Chapters 6-8 are briefly described here. Chapter 6 investigates inner-core processes related to intensity change, with particular attention on the relationship between the vertical distribution of the tangential wind field in the eyewall region and future pressure changes. Chapter 7 presents a modified Air-Sea Interaction theory which incorporates boundary layer cooling. Chapter 8 describes a multiple regression scheme with intensity change as the dependent variable. Chapter 5 describes the data sets used in the research Chapter 9 summarizes this report. 


\section{Chapter 2}

\section{THEORY OF TROPICAL CYCLONE INTENSITY CHANGE BY UPPER-LEVEL FORCING}

One of the most contentious issues in tropical meteorology is whether upper-level forcing is a necessary and positive contributor to TC intensification, or whether TC intensification is more favorable in an environment free of upper-level anomalies since troughs are associated with wind shear. The following sections analyze these arguments.

Observations that upper-level troughs may enhance a TC's development have existed for several decades (Riehl 1948, 1950; Miller 1958a; Colon and Nightingale 1963). However, the idea of TCs being positively influenced by upper-level forcing has mostly gained attention in the last 20 years. While similar theories exist about upper-level forcing of tropical cyclogenesis, this chapter will focus on intensification of an already existing TC. Two primary trough interactions which might intensify a TC have been identified by researchers:

- Enhancement of the storms outflow channels by favorable positioning of the trough.

- Enhancement of the storm's development via upper-level interaction, which can be viewed in the context of eddy angular momentum flux and heat flux convergence or the superposition of fields associated with two potential vorticity anomalies.

Furthermore, one might argue that both these interactions can occur simultaneously.

Composites of intensifying depressions and Atlantic tropical storms have shown a trough to the northwest of the storm at $200 \mathrm{mb}$ (Pfeffer and Challa 1991; Riehl 1948). Figure 2.1 shows these composites which are the motivation for much of the upper-level forcing research on TCs. 

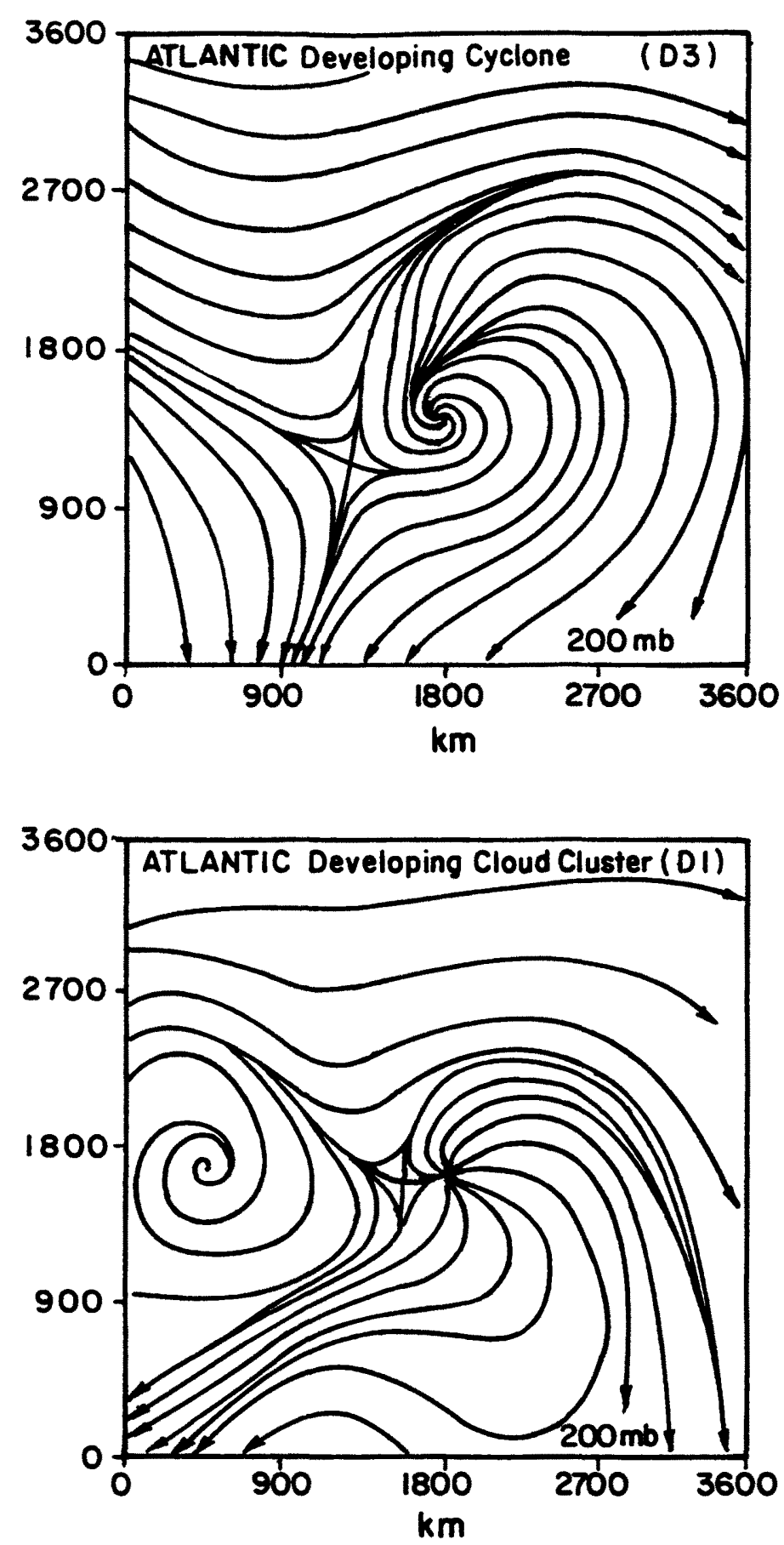

Figure 2.1: Composite 200-mb wind fields for developing Atlantic depression (D1) and intensifying depression or tropical storm (D3). The plots are oriented in the zonal and meridional direction. The tropical systems are located at the center of the figure, and the meridional and zonal scale is $3600 \mathrm{~km}$. Categories are from McBride (1981), and plots are done by Pfeffer et al. (1990). 


\subsection{Intensity changes associated with outflow interaction}

Several scientists have advocated the trough's role as augmenting the TC's outflow channel, thereby increasing mass divergence aloft and, by continuity, mass convergence at the lower levels. Sadler (1978) found for the three mid-season typhoons he investigated, a Tropical Upper-Tropospheric Trough (TUTT) existed to the northwest during their fastest deepening stage. In addition, during these stages the typhoons were centered near the axis of the subequatorial ridge, implying minimum shear as well as an efficient outflow channel to the equatorial easterlies. A similar argument can be made for a favorable positioning of a mid-latitude trough to the northwest with the TC located under the subtropical ridge. A schematic diagram of this dual outflow scenario is shown in Fig. 2.2.
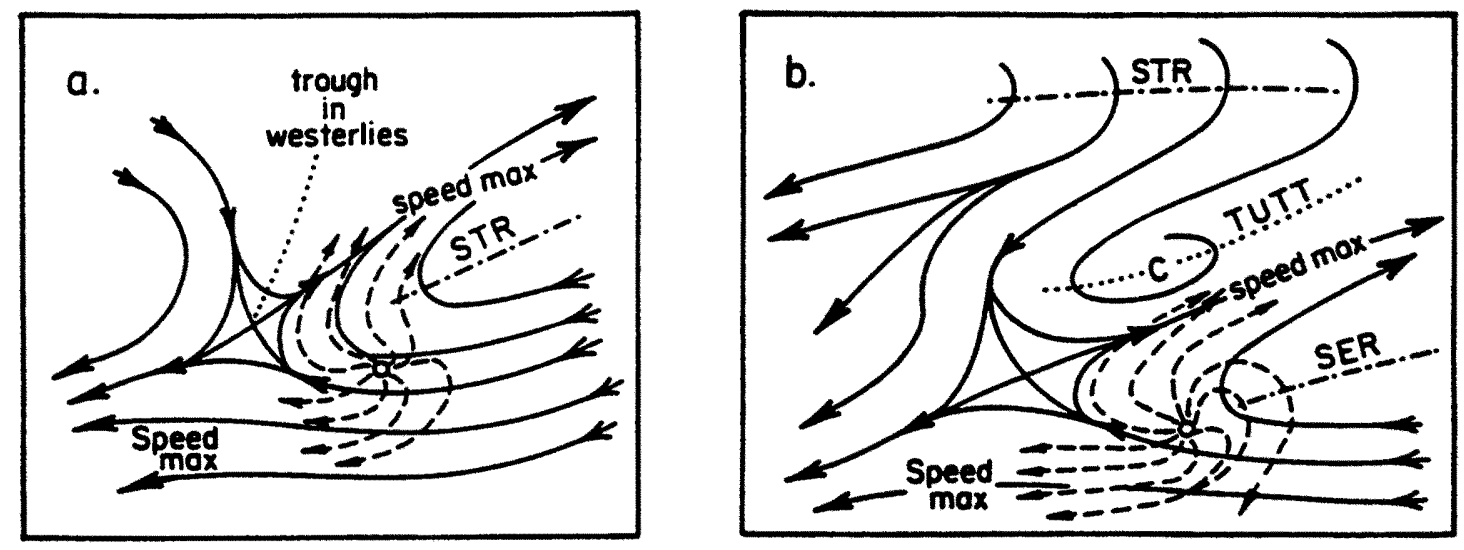

Figure 2.2: Two synoptic models of subtropical upper-tropospheric flow patterns that favor TC intensification in the western North Pacific. STR is the Subtropical Ridge, SER the Sub-Equatorial Ridge, and TUTT the Tropical Upper-Tropospheric Trough. Adapted from Sadler (1978).

This double outflow channel interaction has been partially confirmed by other researchers. Chen and Gray (1986) studied TC intensification over all of the global TC basins during the year-long period of the First GARP Global Experiment (FGGE). They identified six basic types of $200-\mathrm{mb}$ wind field interaction associated with TC intensification (Fig. 2.3):

$I_{1}$ ) Equatorial anticyclone of the opposite hemisphere enhancing a single equatorward outflow channel 


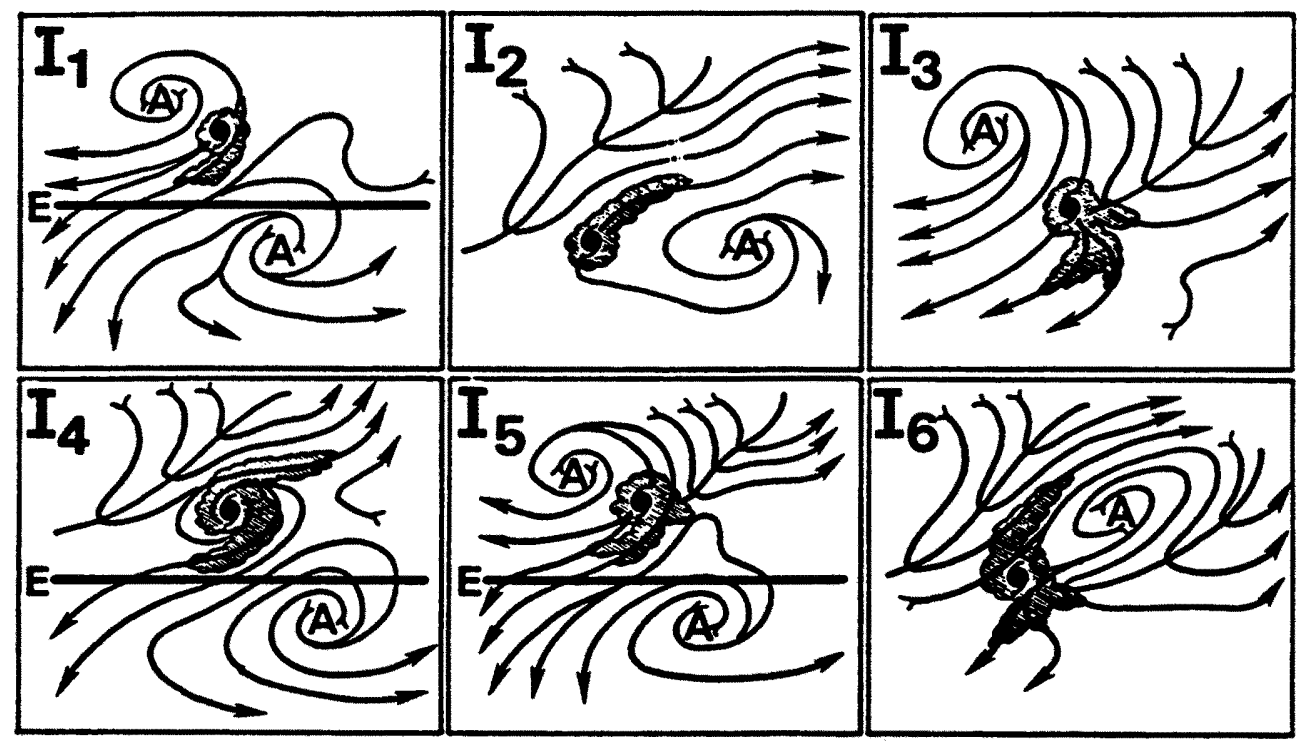

Figure 2.3: Six types of interactions between a TC and its surrounding environment. Symbols are defined in text. Adapted from Chen and Gray (1986).

$I_{2}$ ) Middle latitude trough moving eastward to the poleward and westward side of the TC so as to enhance a single poleward outflow channel

$I_{3}$ ) TC located in rear of a trough (or TUTT) which acts to enhance a single equatorward outflow channel

I4) A trough (or TUTT) and equatorial anticyclone of the opposite hemisphere approaching a TC from different directions and contributing to the establishment of a double outflow channel

$I_{5}$ ) Combined effect of an equatorial anticyclone of the opposite hemisphere and a trough to the northeast enhancing a single equatorial outflow channel

$\left.I_{6}\right)$ TC under an anticyclone flanked by two troughs, establishing a double outflow channel

which can be subdivided into 4 sets: 1) $S_{P}$ denoting single outflow channel poleward, 2) $S_{E}$ denoting single outflow channel equatorward, 3) $D$ denoting dual outflow, and 4) $N$ denoting no channel outflow. Chen and Gray showed that double outflow channels were associated with the largest intensification rates $\left(35 \mathrm{kts}^{-1}\right.$ on average), though they occur 
Table 2.1: Frequency of interaction types in different outflow patterns during the First GARP Global Experiment (FGGE) year. A blank space means no interacting outflow channels were detected with this flow configuration. Total number of observations is 80 . Symbols are defined in text. From Chen and Gray (1986).

\begin{tabular}{|l|c|c|c|c|c|c|c|c|c|c|}
\hline Patterns & \multicolumn{2}{|c|}{$S_{p}$} & \multicolumn{4}{|c|}{$S_{E}$} & \multicolumn{3}{c|}{$D$} & \multicolumn{2}{c|}{$N$} \\
\hline Interaction Type & $I_{2}$ & & $I_{1}$ & $I_{5}$ & $I_{3}$ & & $I_{4}$ & $I_{6}$ & & $I_{1}$ \\
\hline Number & 25 & 3 & 18 & 9 & 2 & 3 & 5 & 1 & 9 & 5 \\
\hline Total & 28 & \multicolumn{3}{|c|}{32} & 6 & 14 \\
\hline
\end{tabular}

with less frequency than single outflow channel interactions (Table 2.1). Chen and Gray also noted that for single channel patterns, equatorial outflow channels on average lead to faster intensification rates than do poleward channel outflows $\left(27 \mathrm{kts}^{-1}\right.$ compared to $18 \mathrm{kts}^{-1}$ on average). It was seen that $89 \%$ of the $S_{P}$ were associated with the approach of a trough. $84 \%$ of $S_{E}$ were associated with favorable positioning of low latitude anticyclones of the opposite hemisphere. Finally, $74 \%$ of the TCs possessed some type of channel, implying that TC and environmental interaction is fairly common during TC intensification. However, the lack of an outflow channel was not necessarily synonymous with non-intensification - Chen and Gray also identified deepening TCs with no outflow channels.

These outflow characteristics had interesting hemispheric and geographic variations as well. In the Northern Hemisphere, $S_{E}$ occurred twice as much as $S_{P}$ (Tables 2.2 and 2.3). In contrast, the occurrence of $S_{E}$ and $S_{P}$ in the Southern Hemisphere was opposite by a factor of three. $S_{E}$ was less common in the Southern Hemisphere because westerly 200-mb winds are closer to the equator than in the Northern Hemisphere.

Chen and Gray also investigated filling storms, and found that as a trough became too close, strong shear would weaken the storm. The majority of the weakening non-landfalling cases (about $87 \%$ ) were associated with too much baroclinic interaction. This implies that environmental interaction with TCs isn't always synonymous with intensification.

Further outflow interaction analysis was performed by (Merrill 1988a). He composited North Atlantic hurricanes using the NOAA National Hurricane Center upper-tropospheric 
Table 2.2: Outflow patterns of the different TC basins of the Northern Hemisphere during the FGGE year with the percentages of each outflow pattern in parentheses. From Chen and Gray (1986).

\begin{tabular}{|l|c|c|c|c|c|}
\hline Outflow Patterns & $S_{p}$ & $S_{E}$ & $D$ & $N$ & Total \\
\hline NW Pacific & $5(16)$ & $16(52)$ & $2(7)$ & $8(26)$ & $31(100)$ \\
\hline N Indian Ocean & $4(67)$ & $2(33)$ & & & $6(100)$ \\
\hline NW Atlantic & $2(22)$ & $1(11)$ & $4(45)$ & $2(22)$ & $9(100)$ \\
\hline NE Pacific & $1(8)$ & $8(67)$ & & $3(25)$ & $12(100)$ \\
\hline \hline N. Hem. Total & $12(21)$ & $27(47)$ & $6(10)$ & $13(22)$ & $58(100)$ \\
\hline
\end{tabular}

Table 2.3: As in Table 2.2 but for Southern Hemisphere.

\begin{tabular}{|l|c|c|c|c|c|}
\hline Outflow Patterns & $S_{p}$ & $S_{E}$ & $D$ & $N$ & Total \\
\hline SW Pacific & $7(64)$ & $4(36)$ & & & $11(100)$ \\
\hline S Indian Ocean & $9(82)$ & $1(9)$ & & $1(9)$ & $11(100)$ \\
\hline \hline S. Hem. Total & $16(73)$ & $5(23)$ & & $1(5)$ & $22(100)$ \\
\hline
\end{tabular}

wind archive, and identified eight patterns for all storms with the outflow channel denoted by a Principal Outflow Streamline (POS). These composites consisted of weakening and intensifying storms combined together, though certain patterns were more associated with intensification and others with weakening. These patterns with the position "counts" are shown in the bottom right hand corner of each drawing in Fig. 2.4. Though Merrill found patterns where a trough (the westerly trough, subtropical jet, and TUTT patterns) occurred ahead of a hurricane in the Atlantic, this constituted less than half of the total storms. Furthermore, the first two patterns were characteristic of hurricanes forming or moving poleward of the upper-level subtropical ridge. Intensification may occur but was often short-lived as the poleward motion of the hurricane brought it under increasing westerly shear and colder waters.

In contrast, a sharp equatorward turning of the POS was far more common, in which over half the patterns were of this type. The type I and II patterns shown at the top of Fig. 2.4 were all associated with intensifying cases. Type I had a POS which "recurves" into the equatorial easterlies, while type II did not, and both had little or no signature of 


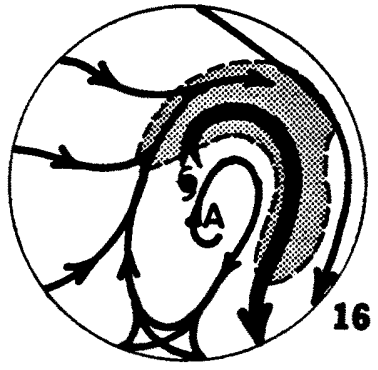

ATLANTIC TYPE II

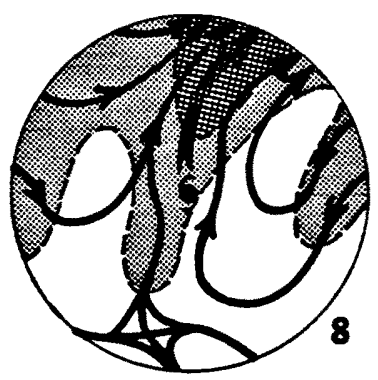

WESTERLY TROUGH

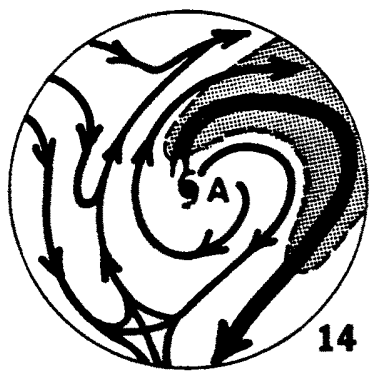

TUTT

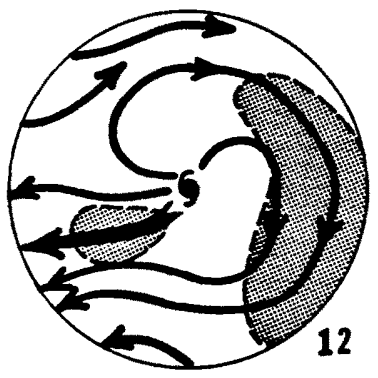

EASTERLY

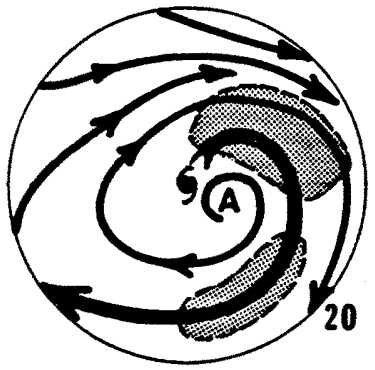

ATLANTIC TYPE I

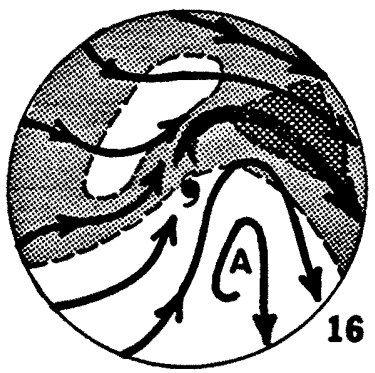

SUBTROPICAL JET

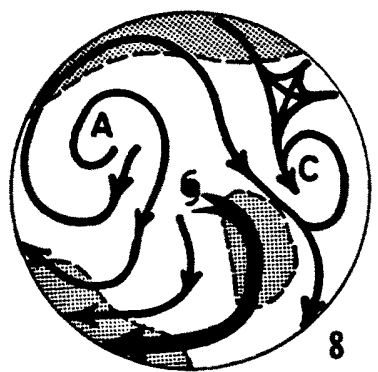

GULF OF MEXICO

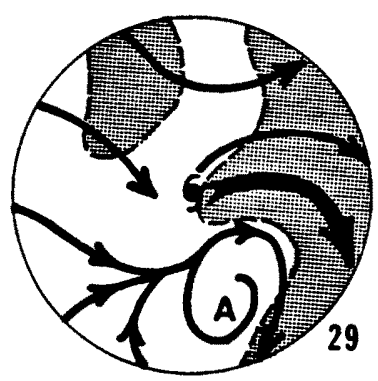

SUBTROPICAL ORIGIN

Figure 2.4: Summary of Atlantic outflow pattern types. Light shading and dark shading denote wind speeds in excess of 15 and $30 \mathrm{~m} \mathrm{~s}^{-1}$ respectively, and the wide arrows shows the location of the principal outflow streamline (POS). The circular domain of the analyses is $2500 \mathrm{~km}$ in radius. Position counts are shown in the bottom right hand corner of each drawing. From Merrill (1988a). 
a trough to the northwest. The remaining two patterns were relatively rare in the Atlantic but are more common in the Pacific. In particular, the easterly pattern was really a double outflow pattern.

Also shown is the subtropical case, which was the most atypical, and indicated that these cyclones are fundamentally different from an "average" hurricane. These were storms classified as subtropical or extratropical during their formative stages and if they exhibit cyclonic curvature and/or cyclonic shear in the outflow layer within $1000 \mathrm{~km}$ of the center. These storms often have a small, symmetric central dense overcast and can have an eye, but often are accompanied by a large cloudband which resembles an occlusion. The lowlevel circulation is larger than most tropical hurricanes, the maximum winds observed are weaker than would be expected for the minimum sea level pressure, and this central pressure seldom falls below $970 \mathrm{mb}$. Therefore, it was not surprising the outflow pattern looks so different than the other seven.

Merrill (1988b) then performed some composite POS work to help differentiate intensifying and non-intensifying hurricanes. He found that intensifying hurricanes had an extending outflow channel, while non-intensifying TCs were associated with a "cut-off" POS, which increased the ventilation over the hurricane and weakened the storm since there was unidirectional flow over the center (Fig. 2.5). The intensifying case usually had an anticyclone to the west, while the non-intensifying had southwesterly flow, implying a trough further west.

\subsection{Intensity change induced by dynamic forcing}

Some researchers view TC evolution through a phase-locking of upper and lower potential vorticity (PV) anomalies. A similar viewpoint is the inducement of low-level ascent by upper-level differential cyclonic vorticity advection, though the PV perspective is preferable since it simultaneously includes thermal anomalies, static stability, and vorticity advection considerations. For example, an upper-level PV anomaly approaching a TC, where the air already has a reduced effective static stability due to saturation, can experience strong deep synoptic ascent - possibly causing the storm to deepen more than 


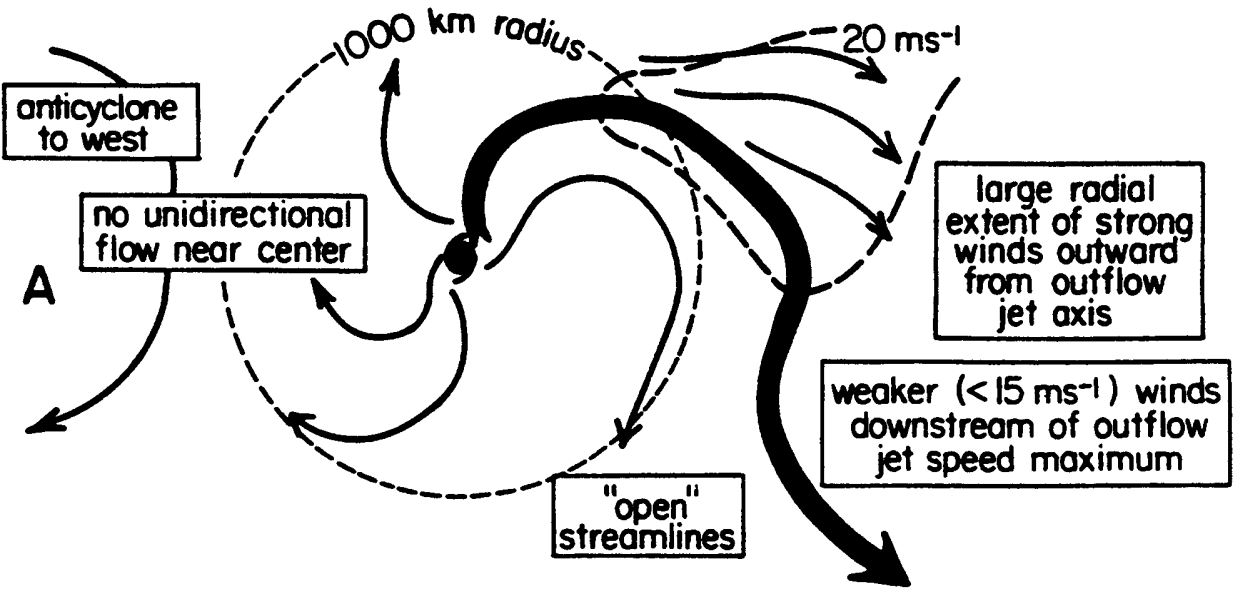

INTENSIFYING

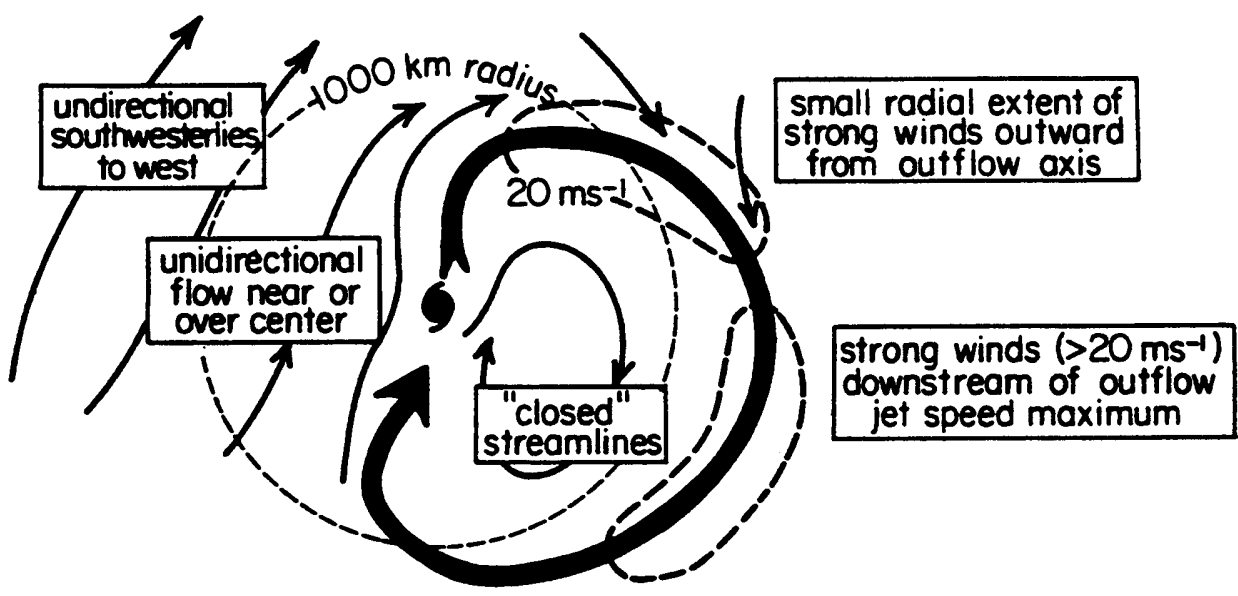

NON-INTENSIFYING

Figure 2.5: Inclusive summary of outflow layer differences between composite intensifying (top) and nonintensifying (bottom) hurricanes. From Merrill (1988b). 
it would without any external processes. (Detailed discussions of PV induction theory are contained in Hoskins et al. (1985) and Bluestein (1993)). Other researchers consider how azimuthally averaged asymmetries (eddy fluxes) will affect the axisymmetric basic-state TC.

Most PV analysis thus far has been restricted to cyclogenesis processes where troughs have acted as a "triggering mechanism" for a low-level disturbance to become a tropical disturbance, whereby an air-sea interaction is initiated in which surface energy fluxes can overcome frictional dissipation, and development can continue even after the upper-level forcing is gone. Several articles discuss this idea through a theoretical (Montgomery and Farrell 1993) and observational (Reilly 1992) framework. The reader is also referred to a paper by Molinari and Vollaro (1993) which describes the intensity change of Hurricane Allen in a PV context. A more recent paper (Shapiro and Franklin 1995) derives a PV analysis of Hurricane Gloria (1985) from Omega dropwindsonde and airborne Doppler radar data.

However, most intensity studies dealing with the TC itself are from the eddy flux convergence of angular momentum perspective. Pfeffer and Challa (1980) derived a "horizontal angular momentum eddy flux" term in cylindrical coordinates, given by $\overline{u_{r}^{\prime} v_{t}^{\prime}}$, which represents the azimuthally averaged asymmetries of the wind field in terms of the radial wind $u_{r}$ and tangential wind $v_{t}$. Pfeffer and Challa utilized the "Balanced Vortex" equations first derived by Eliassen (1951), with the momentum eddy flux term incorporated as a "forcing function" on the axisymmetric flow as:

$$
\frac{\partial v_{t}}{\partial t}=-\frac{1}{r^{2}} \frac{\partial}{\partial r}\left(r^{2} u_{r} v_{t}\right)-\frac{\partial}{\partial p}\left(v_{t} u_{r}\right)-f u_{r} \underbrace{-\frac{1}{r^{2}} \frac{\partial}{\partial r} r^{2} \overline{u_{r}^{\prime} v_{t}^{\prime}}}
$$

where the term with a brace is known as the Relative Eddy angular momentum Flux Convergence (REFC), and the primes indicate eddies. The eddies are computed as the wind observed at different azimuthal locations for a given radius minus the azimuthally averaged wind. The bar over the REFC term indicates $u_{r}^{\prime}$ was multiplied by $v_{t}^{\prime}$ at different azimuthal locations for a given radius and azimuthally averaged. Molinari et al. (1993) show a derivation explaining how REFC and other eddy terms (which have been neglected in this discussion) are integrated into an azimuthally averaged system of equations. 
Convergence of upper-level eddy fluxes $\left(-\frac{1}{r^{2}} \frac{\partial}{\partial r} r^{2} \overline{u_{r}^{\prime} v_{t}^{\prime}}>0\right)$ contributes to an increase of cyclonic $v_{t}$ aloft.

In addition to the $v_{t}$ equation of motion, REFC also appears in a second order linear elliptic operator $\mathcal{L}$. By assuming hydrostatic and gradient wind balance (the "balance" assumption), defining a streamfunction $\psi$ to satisfy the continuity equation, and by algebraically eliminating local time derivatives in the thermodynamic equation and the $v_{t}$ equation, a diagnostic transverse circulation equation may be written as (Eliassen 1951; Ooyama 1969; Sundqvist 1970; Pfeffer and Challa 1980; Holland and Merrill 1984; DeMaria et al. 1993; Krishnamurti et al. 1994):

$$
\begin{aligned}
\mathcal{L}(\psi)= & -r \frac{\partial}{\partial p}(\mathrm{REFC})+\text { diabatic forcing }+ \\
& \text { frictional forcing }+ \text { other possible forcings }
\end{aligned}
$$

Equation (2.2) states that the secondary circulation results from a combination of forcing mechanisms. One of the forcing components is the vertical derivative of REFC. If REFC increases with height up to the outflow layer, this contributes to enhanced outflow and ascent through the TC inner-core, and may be conducive to TC intensification. DeMaria et al. (1993) states that, in this sense, vertical motion forced by REFC associated with troughs is analogous to forcing by $200-\mathrm{mb}$ cyclonic vorticity advection. The similarity of these two forcing mechanisms is addressed in Chapter 8. Balanced vortex theory will be discussed in more detail in Chapter 6, with computations relevant to material covered in that chapter.

When positive REFC is associated with a trough, it often increases with height to a maximum at the tropopause. Therefore, REFC sometimes is only measured at a single level (for example, $200 \mathrm{mb}$ ). But how is a positive REFC value manifested in the wind field? By looking at Fig. 2.1, the relationship is difficult to see, but physically the azimuthal eddies are importing (or exporting) cyclonic (anticyclonic) momentum. A radial crosssection of the combined intensifying Atlantic depression and tropical storm composites in Fig. 2.1 (D3) is shown in Fig. 2.6, and shows calculations of $-r \overline{u_{r}^{\prime} v_{t}^{\prime}}$. It is apparent that there is REFC at $200 \mathrm{mb}$ between 500 and $1000 \mathrm{~km}$ since inward flux eddy flux of 
D1

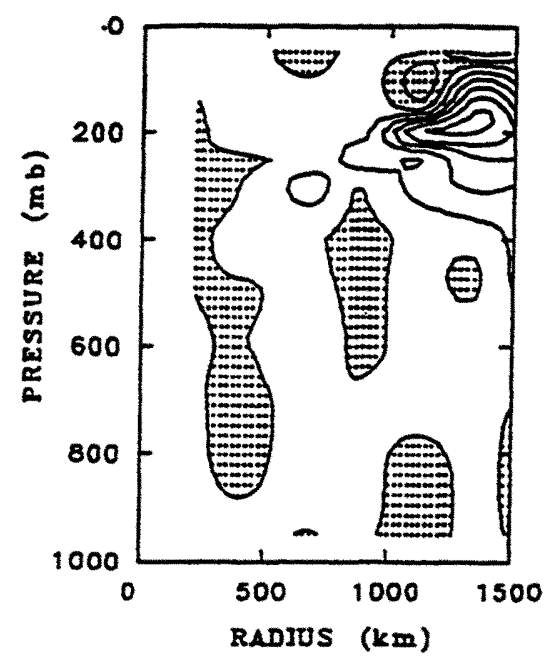

N1

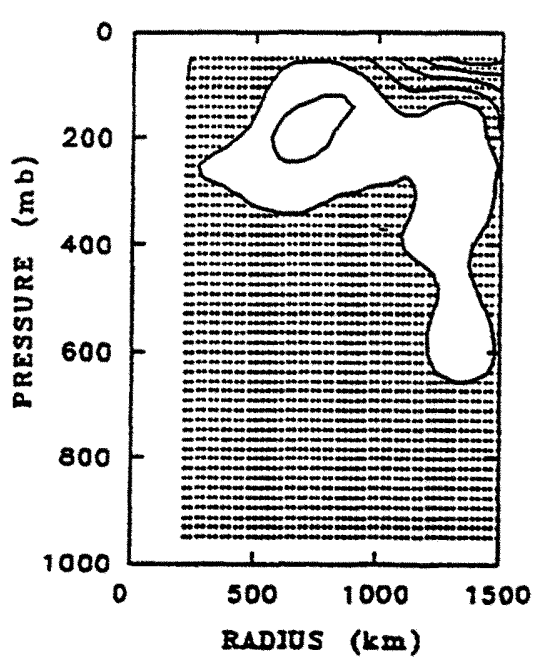

D3

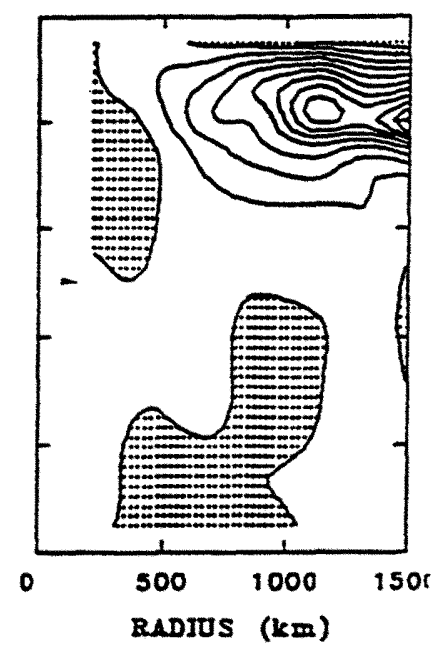

N3

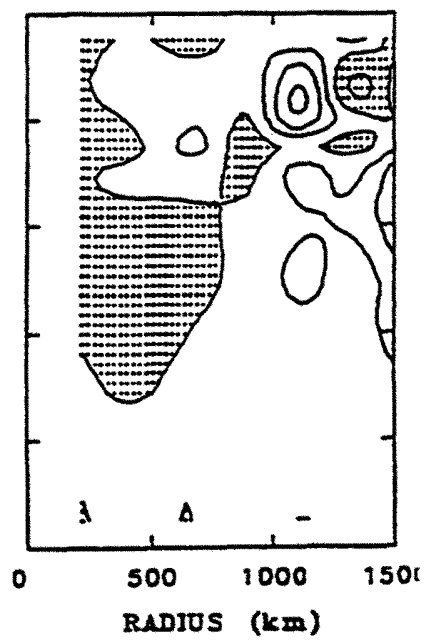

Figure 2.6: Calculations of inward eddy flux of momentum $-r \overline{u_{r}^{\prime} v_{t}^{\prime}}$ for prehurricane cloud cluster (D1), intensifying tropical storm or depression (D3), nondeveloping cloud cluster (N1), and nondeveloping depression (N3). Shading is outward flux. Contour interval is $80 \mathrm{deg} \mathrm{m}^{2} \mathrm{~s}^{-2}$. From Challa and Pfeffer (1990) as classified by McBride (1981). 
momentum is a maximum at $1200 \mathrm{~km}$ and is outward (negative) at $250 \mathrm{~km}$. Interestingly, in the nondeveloping depression (N3) there is little REFC. This suggests that REFC might be needed for intensification to occur. Frank (1977a) reported similar results for composited mature typhoons, although the composite also included weakening storms, hence the relationship to intensity change in his study is not clear.

Since some researchers also believe eddy fluxes can play a role in tropical cyclogenesis, it will be briefly discussed here. A brief review of REFC forcing on genesis also serves to clarify its possible role in TC development. The composites of a prehurricane cloud cluster (D1) and nondeveloping cloud cluster (N1) are shown in Fig. 2.6. The D1 case apparently has REFC while N1 does not, leading some researchers to suggest that upper-level forcing may be necessary to initiate tropical cyclogenesis. Pfeffer and Challa (1980) performed a TC numerical simulation with a balanced vortex model, and later with a three-dimensional model (Challa and Pfeffer 1990). Using the composite of D1 as the initial condition, a TC formed in both models. However, with a N1 initial condition, a TC did not develop in either model. For an additional REFC perspective, Pfeffer and Challa (1993) performed three-dimensional analysis of the D1 wind field. Fig. 2.7 shows the departures of the D1 winds from their azimuthal means, $u_{r}^{\prime}$ and $v_{t}^{\prime}$, as well as $-r^{2} \overline{u_{r}^{\prime} v_{t}^{\prime}}$ One can deduce largescale REFC between the two circles (located at 600 and $1200 \mathrm{~km}$ ). Challa and Pfeffer asserted that the eddy fluxes of momentum exerted an upper-level cyclonic torque on the initially weak vortex, which induced upper level divergence and lower level convergence, increasing the fetch of surface inflow and with it surface heat and moisture fluxes upon which a TC could form and grow.

To examine the reason for a lack of REFC in the N1 and N3 cases, their 200-mb wind fields are shown in Fig. 2.8. The differences between D1 and D3 (as well as N1 and N3) are not obvious, and lends their results controversial since troughs occur in both nondeveloping cases as well. However, the troughs are slightly weaker and somewhat closer to the center of the cloud cluster (or TC) than in the D1 and D3 cases. With this configuration, it is possible that eddies are no longer conducive to positive forcing. This implies to some researchers that REFC is very sensitive to the orientation, strength, and 
D1
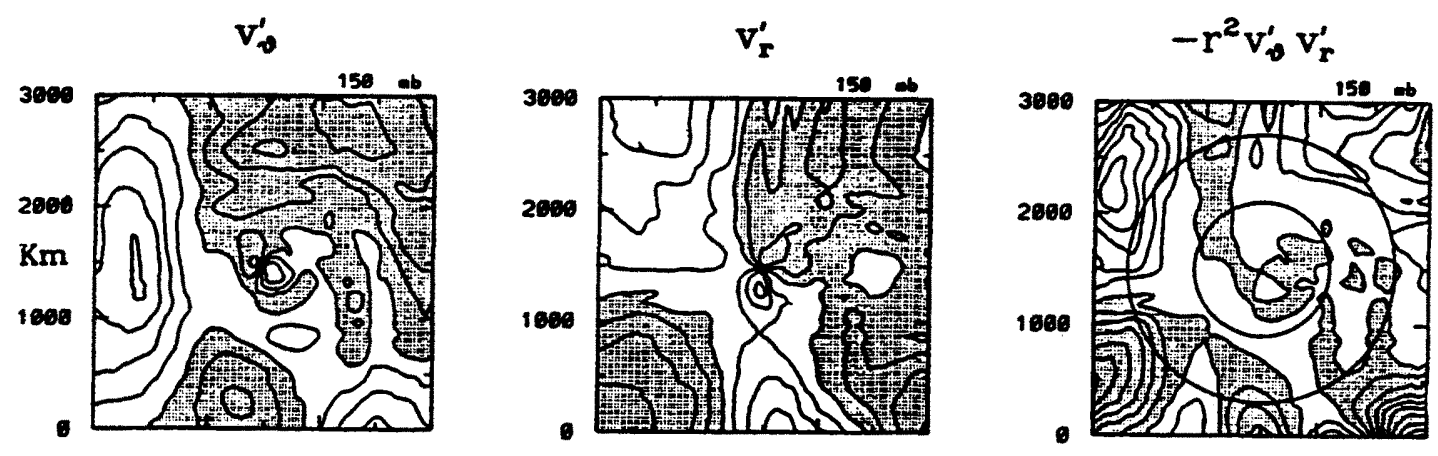

Figure 2.7: Horizontal distributions of $v_{t}^{\prime}, u_{r}^{\prime}$, and $-r^{2} v_{t}^{\prime} u_{r}^{\prime}$ for the prehurricane cloud cluster D1 which is centered in the middle of each box. Contour interval for $v_{t}^{\prime}$ and $u_{r}^{\prime}$ is $4 \mathrm{~m} \mathrm{~s}^{-1}$. Contour interval for $-r^{2} v_{t}^{\prime} u_{\tau}^{\prime}$ is $4000 \mathrm{deg}^{2} \mathrm{~m}^{2} \mathrm{~s}^{-2}$. The shaded region represents anticyclonic tangential velocity, outward radial velocity, and outward eddy flux of angular momentum. From Pfeffer and Challa (1993).

position of a trough. Hence, trough location and magnitude might be important to TC intensity change.

\subsection{Conceptual view of upper-level forcing on TC intensity}

Advocates of upper-level forcing theory usually propose an argument as follows. It is generally known that the inertial stability aloft in the TC is weak due to the anticyclonic outflow (Fig. 2.9). From balanced vortex theory, horizontal displacements of parcels occur more freely in an atmosphere with weak inertial stability (Eliassen 1951). Holland and Merrill (1984) have shown that the response of a balanced TC to an upper-level momentum source is to produce a long, shallow outflow layer at $200 \mathrm{mb}$. This implies that upper-level forcing can directly affect the intensity of the storm by increasing the storm's outflow, further weakening the upper-level inertial stability and enhancing the storm's influence to upper-level forcing. In this manner, a positive feedback may occur between upper-level forcing and TC outflow which could affect TC intensification.

Holland and Merrill presented a conceptual model where an approaching trough enhances TC intensification. For this scenario, the associated cyclonic momentum source and subsequent evolution of $v_{t}$ produces a warmer core, which further enhances the TC 

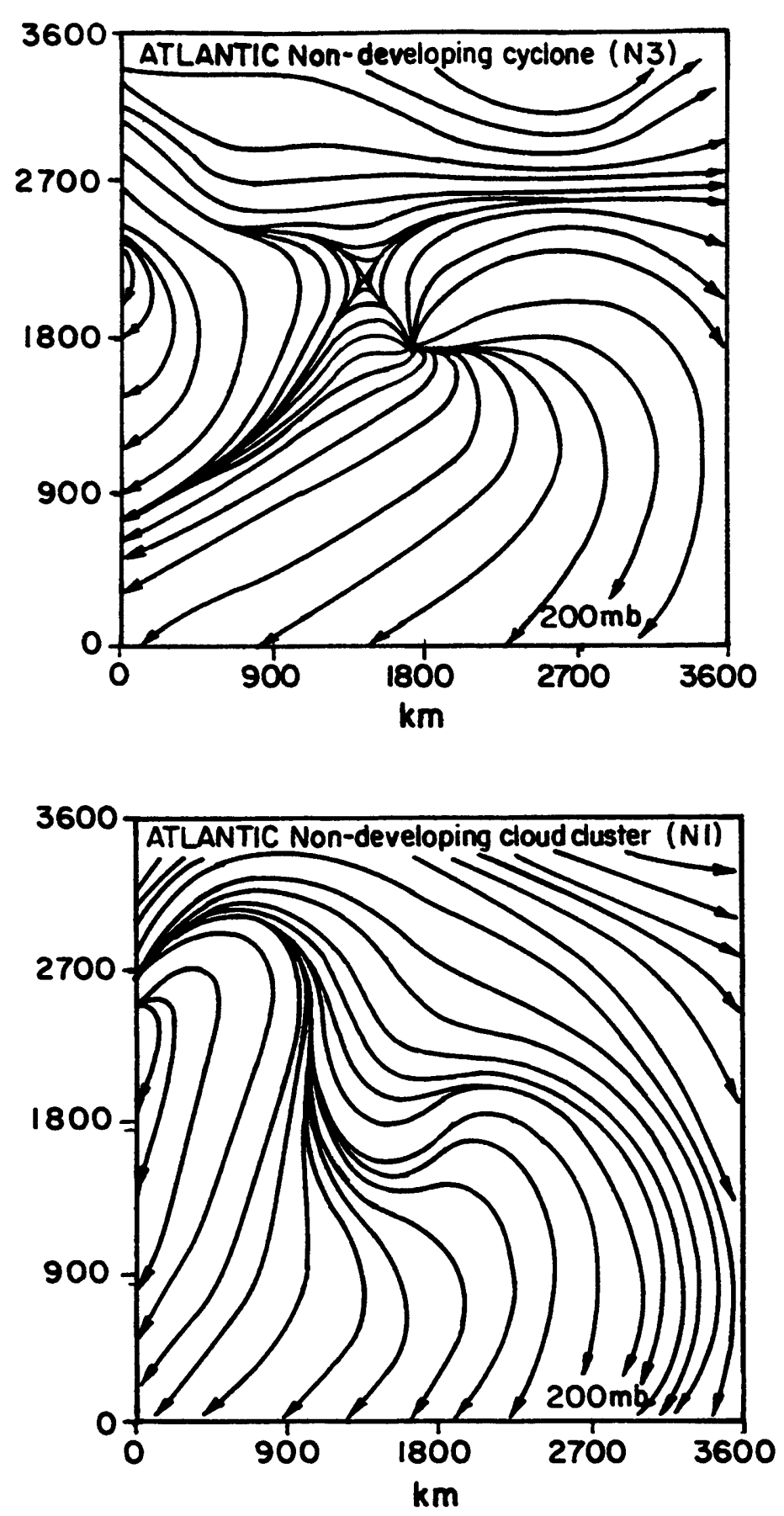

Figure 2.8: Composite 200-mb wind fields for nondeveloping Atlantic depression (N1) and nondeveloping TC composite (N3). The tropical systems are located at the center of the figure, and the meridional and zonal scale is $3600 \mathrm{~km}$. Categories are from McBride (1981), and plots were done by Pfeffer et al. (1990). 


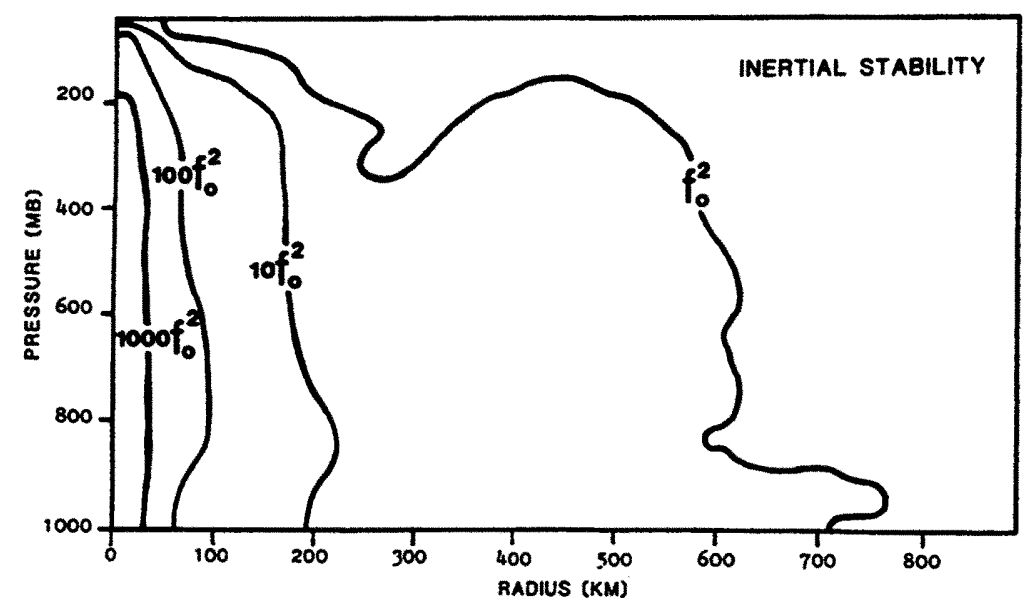

Figure 2.9: Axisymmetric vertical cross-section of inertial stability (in multiples of $f^{2}$ where $f$ is the Coriolis parameter) for an intensifying TC in the South Pacific. From Holland and Merrill (1984).

outflow and weakens its inertial stability more. This coupling of REFC with the storm core also enhances the secondary circulation by interacting with inner-core convection; since saturated ascent already exists, the reduced static stability encourages ascent (Eliassen 1951). It is through this coupling of momentum forcing and the TC through which a positive feedback theoretically occurs that enhances intensification. Extending the outflow channel also allows compensating subsidence to occur over a large area, reducing this drying effect on the boundary layer surrounding the TC so that inflowing air parcels remain nearly saturated. The coupling is of short duration (maybe one to two days) and ceases when the trough moves past and cuts off the outflow channel to leave a slow decaying cyclone, or (more typically) when the westerlies move over the cyclone center and strong shearing followed by rapid decay occurs.

Molinari and Vollaro (1989) investigated this upper-level forcing hypothesis by evaluating a trough's influence on Hurricane Elena's (1985) intensity changes. They calculated radial fluxes (with the storm motion removed) in the following manner:

$$
\frac{\partial M}{\partial t}=-\frac{2 \pi r^{2}}{g} \int_{p_{2}}^{p_{1}} \overline{u_{r}^{\prime} v_{t}^{\prime}} d p
$$

where $M$ is relative angular momentum and $g$ is the acceleration by gravity. These calculations were performed at $200 \mathrm{mb}$ which assumed the winds were constant over a 200-mb 
thickness. Figure 2.10 shows an apparent high correlation between outer radius eddy fluxes computed from Eq. (2.3) at $1500 \mathrm{~km}$ and the pressure tendency of the storm $30 \mathrm{~h}$ later. An inspection of the 31 August wind field (figure not shown) for the 1 September deepening showed that the maximum REFC arose from: 1) the northwest flow behind a trough located to the northwest of Elena, where cyclonic momentum was being added to the storm, 2) the west-southwest flow ahead of the trough, where anticyclonic momentum was being removed from Elena, and 3) in the outflow south of the storm jet, where anticyclonic momentum was being removed.

To further investigate a possible relationship between Elena's development and upperlevel forcing, Molinari and Vollaro (1989) plotted a radius-time section of the 200-mb eddy momentum flux and azimuthally averaged $u_{r}$ (Fig. 2.11). According to Eq. (2.2), a positive eddy flux will produce inflow somewhere below $200 \mathrm{mb}$ (over a depth which depends on the vertical gradient of the eddy flux, which in this case is unknown), and will produce enhanced outflow at $200 \mathrm{mb}$ just inside the eddy flux maximum. Two major outflow events occurred in Elena, and it appears that the second event occurring at $1200 \mathrm{~km}$ on 00 UTC 31 August was inside the large eddy flux maximum as would be qualitatively predicted by the balanced vortex response. In the following two 12-h periods, this outflow maximum shifted inward toward the center, and a period of deepening occurred after the maximum reached the storm core.

For an alternative perspective, a radius-time section of REFC was also computed in Fig. 2.11. On 31 August, an eddy spin-up value corresponding to $25 \mathrm{~m} \mathrm{~s}^{-1} \mathrm{~d}^{-1}$ was located at $650 \mathrm{~km}$, and shifted inward with time, reaching the core at the time of the pressure drop. These results suggest $\mathrm{TC}$ intensification as a result of environmental forcing.

Other studies have found similar results. Davidson et al. (1990) showed that REFC values of about $8 \mathrm{~m} \mathrm{~s}^{-1} \mathrm{~d}^{-1}$ were associated with the interaction of a developing TC and a TUTT. Challa and Pfeffer (1990) found values of about $6 \mathrm{~m} \mathrm{~s}^{-1} \mathrm{~d}^{-1}$ in data composites of developing tropical depressions. These values, along with calculation performed by DeMaria et al. (1993), seem to indicate a REFC value of $10 \mathrm{~m} \mathrm{~s}^{-1} \mathrm{~d}^{-1}$ as a period of significant environmental interaction, and $20 \mathrm{~m} \mathrm{~s}^{-1} \mathrm{~d}^{-1}$ as a period of strong interaction. 

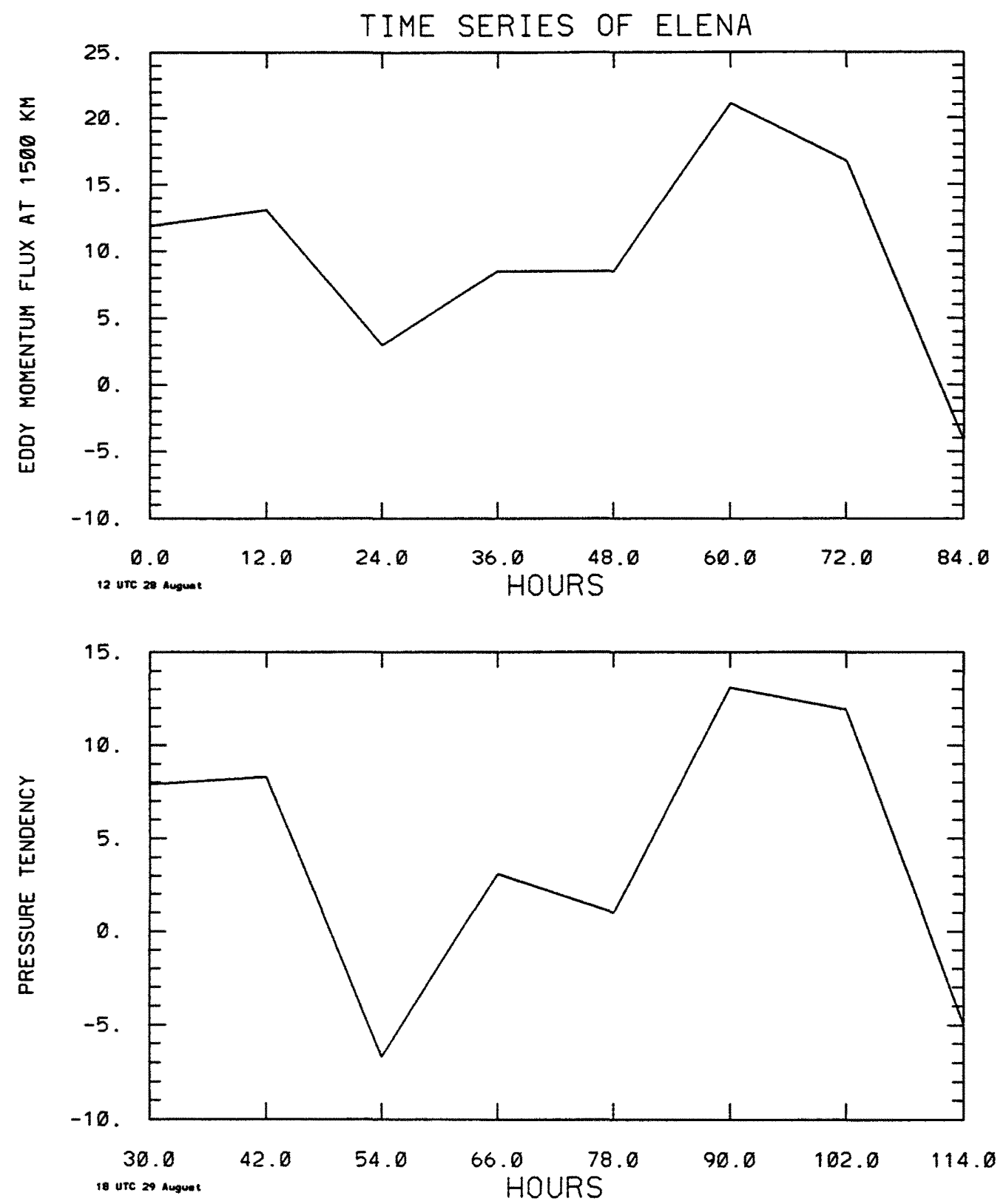

Figure 2.10: (Top) 200-mb eddy momentum flux at the $1500-\mathrm{km}$ radius of Hurricane Elena (1985) with units $1 \times 10^{17} \mathrm{~kg} \mathrm{~m}^{2} \mathrm{~s}^{-2}$. (Bottom) Deepening rate of Elena $30 \mathrm{~h}$ after those in top figure in units $10^{-1} \mathrm{mb} \mathrm{s}^{-1}$. The eddy momentum fluxes are shown from 12 UTC 28 August to 12 UTC 31 August $(0-84 \mathrm{~h})$. The pressure tendency is shown from 18 UTC 29 August to 18 UTC 1 September (30-114 h). A deepening rate (pressure fall) is denoted by a positive sign. Adapted from Molinari and Vollaro (1989). 

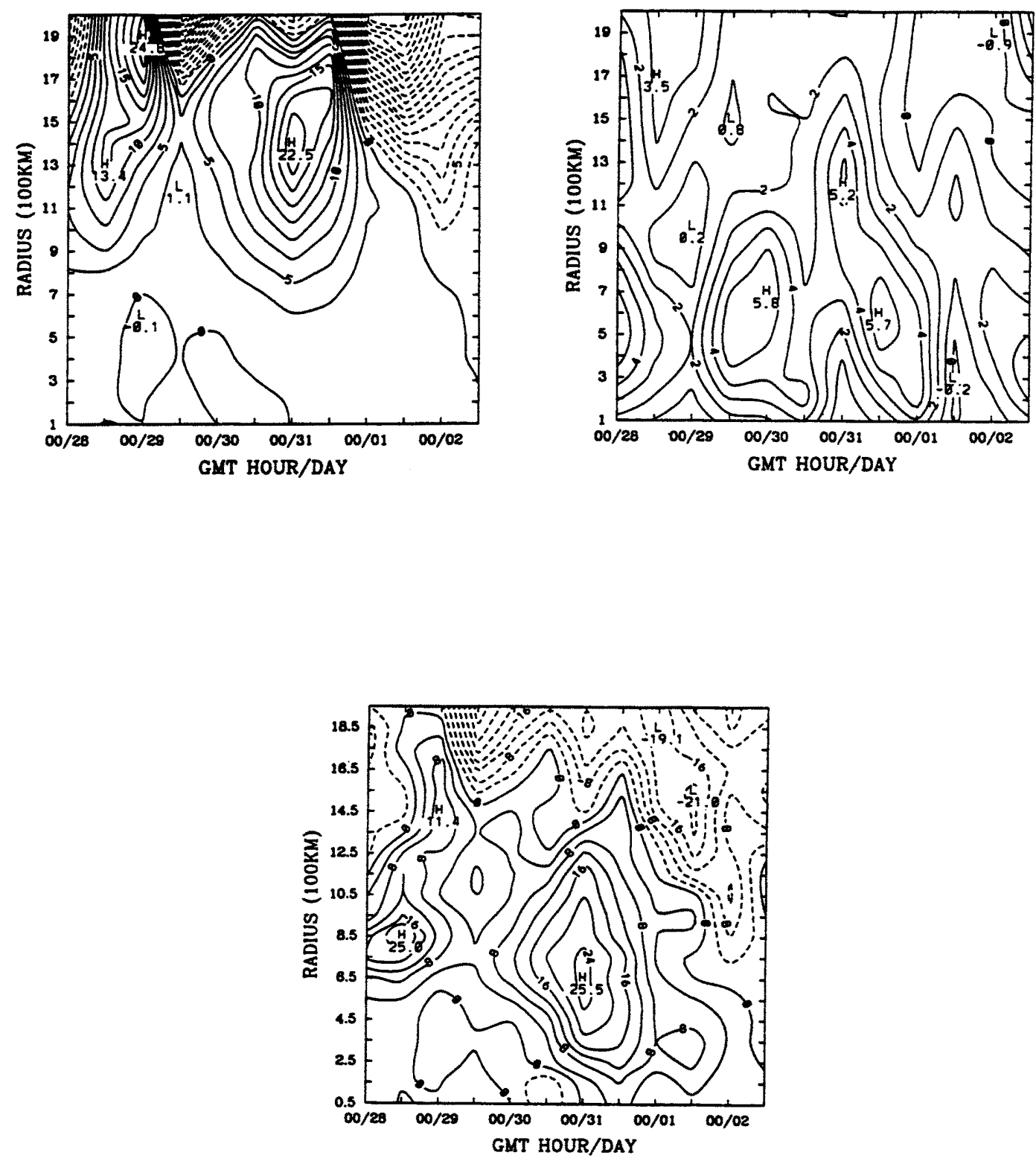

Figure 2.11: 200-mb radius-time section of Elena for: (Top left) eddy momentum flux with contour interval of $2.5 \times 10^{17} \mathrm{~kg} \mathrm{~m}^{2} \mathrm{~s}^{-2}$; (Top right) azimuthally averaged radial velocity with contour interval of $1 \mathrm{~m} \mathrm{~s}^{-1}$; (Bottom) azimuthally averaged eddy flux convergence of tangential velocity with contour interval of $4 \mathrm{~m} \mathrm{~s}^{-1} \mathrm{~d}^{-1}$. From Molinari and Vollaro (1989). 


\subsection{Upper-level forcing and vertical wind shear}

In contrast with these findings, Merrill (1988b) could find no relationship between REFC and intensity change in the composites shown in Fig. 2.5. He speculated that any positive influences of REFC might be offset by the negative influences of increased vertical wind shear associated with a trough. Merrill also stated that, in a general sense, environmental interactions must contribute negatively to intensity change since most TCs rarely reach Category 3 or more. As shown in Fig. 2.12 from Merrill (1988b), most Atlantic hurricanes' intensity occur below the maximum possible intensity based on SST. A more precise empirical intensity bound is described in Merrill (1987). Thus, Merrill argued the environment must be exerting a negative influence. Other researchers have also found that very few TCs reach their maximum potential intensity (DeMaria and Kaplan 1994a; Evans 1993).

Molinari and Vollaro (1993) countered that while a trough's shear may initially hurt the storm, the strong lifting induced by the potential vorticity advection causes strong convection to build, enhancing latent heat release. As a result, the westerlies will flow around the center of the storm as the approaching PV gradient breaks into two separate centers - in effect, the storm builds its own shield from the westerlies as an anticyclone is built aloft due to the strong heating. As an example, Molinari and Vollaro (1989) pointed out that during Elena's first weakening stage shear increased, but the second deepening stage occurred while shear was still strong, so a one to one correspondence between shear and intensity doesn't always exist.

In an attempt to ascertain these differences between vertical wind shear and REFC, DeMaria et al. (1993) performed calculations of these two quantities for 32 named TCs during the 1989-1991 Atlantic hurricane season. Of these, 26 had at least one period of enhanced REFC, but only one-third intensified after the REFC episode. An example is shown in Fig. 2.13 of Hurricane Dean, though it should be noted the areally averaged shear is also low. A vectorial $200-\mathrm{mb}$ minus $850-\mathrm{mb}$ wind calculation less than $20 \mathrm{kts}$ is considered low shear (Zehr 1992). Vector plots in DeMaria et al. (1993) show that a trough to the northwest and an anticyclone to the southeast are influencing the storm. 


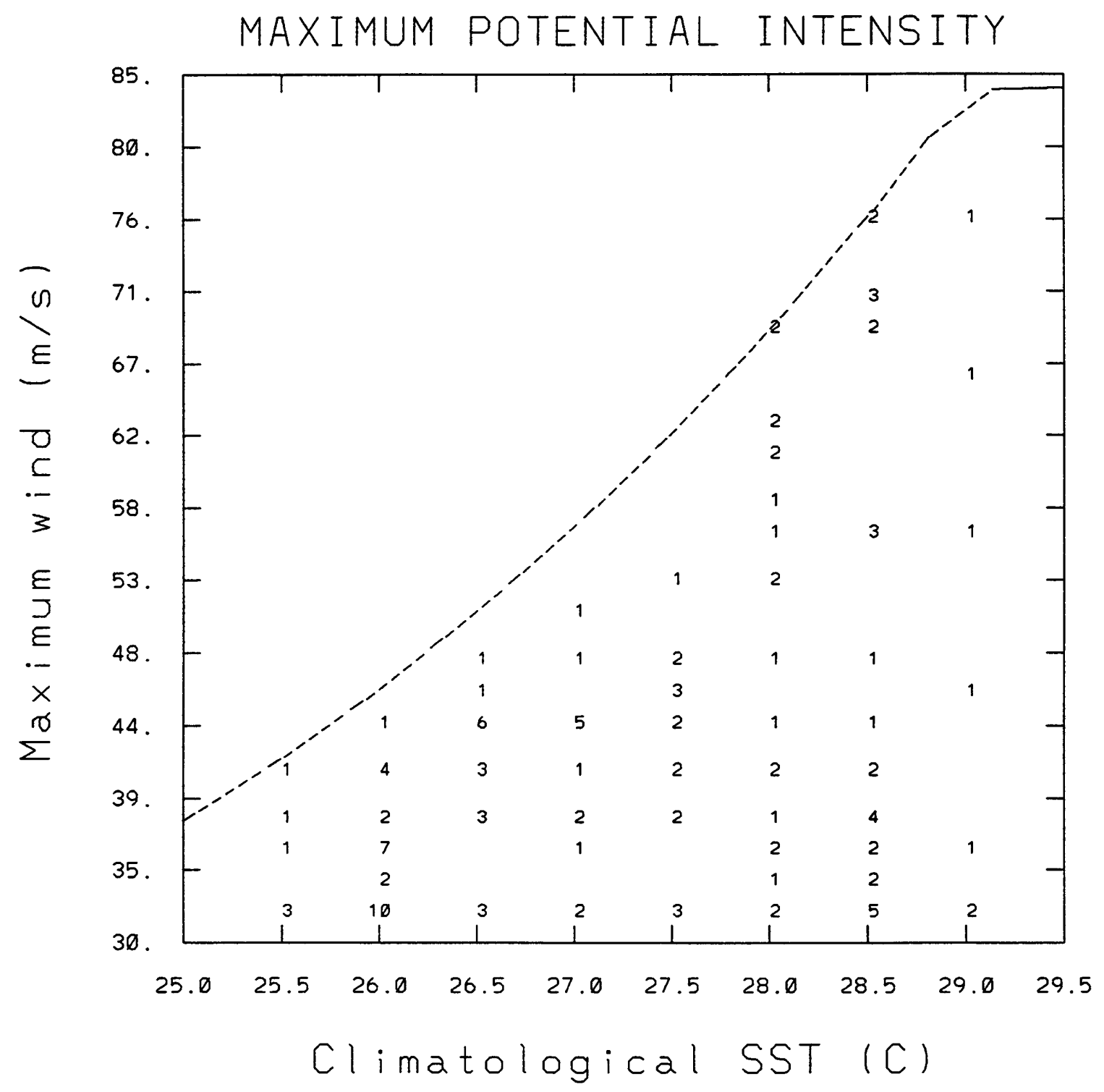

Figure 2.12: The Maximum Potential Intensity (MPI) between climatological SSTs and $V_{\text {max }}$ for a sample of Atlantic hurricanes during 1977-1983. The dashed line is an empirical upper bound on intensity as a function of SST. The numbers indicate how many observations occurred for a given SST and $V_{\max }$. Adapted from Merrill (1988b). 

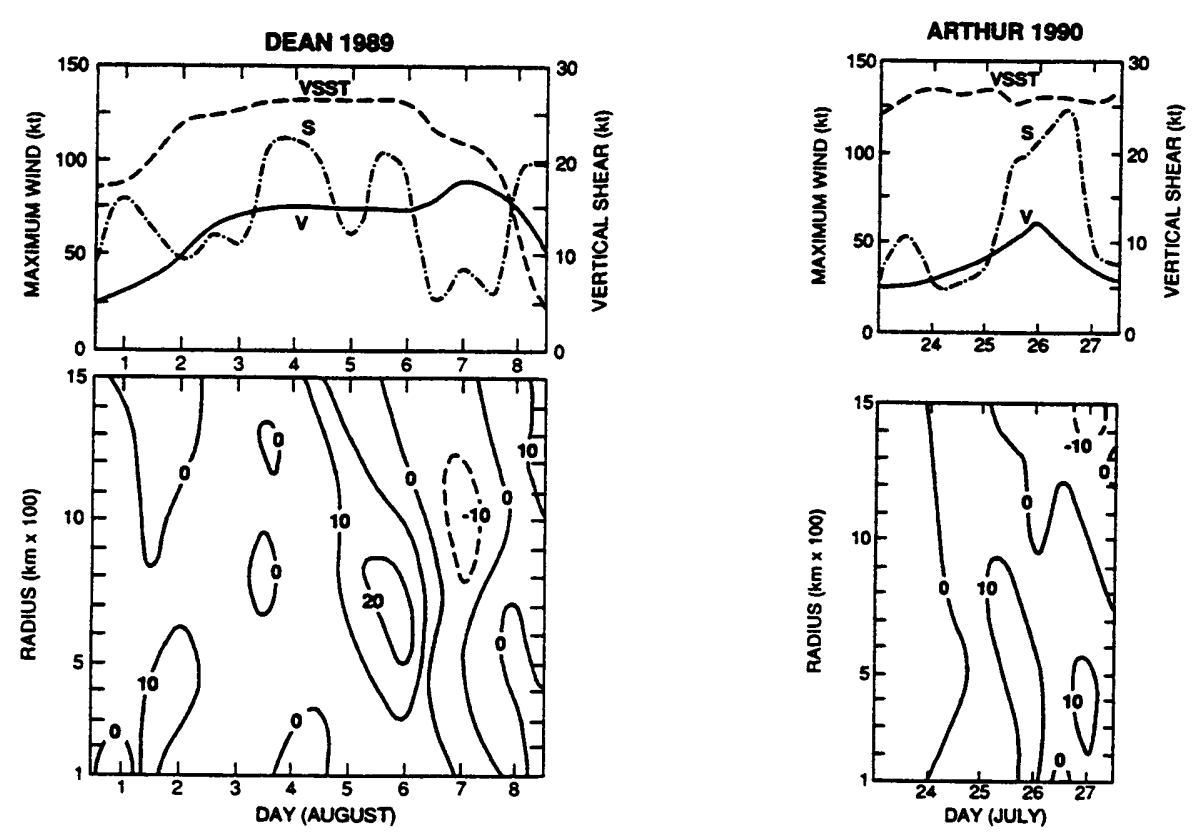

Figure 2.13: Top panel - The time evolution of the maximum wind $(V)$, maximum potential intensity as a function of climatological SST (VSST; see Fig. 2.12), and the areally averaged vertical wind shear $(S$; computed as the vectorial difference between 850 and $200-\mathrm{mb}$ winds, where the winds are averaged within $600 \mathrm{~km}$ of the TC's center). Bottom panel - The time evolution of eddy angular momentum flux convergence in the units of $\mathrm{m} \mathrm{s}^{-1} \mathrm{~d}^{-1}$ as a function of radius and time. Left plot shows Hurricane Dean (1989), the right plot shows Tropical Storm Arthur (1990). From DeMaria et al. (1993).

One should note that the REFC moves radially inward, consistent with Molinari and Vollaro's study of Hurricane Elena.

The other two-third weakened due to strong shear, movement over cold SST's, and/or the storm becoming extratropical. Figure 2.13 shows Tropical Storm Arthur with a significant REFC event on $25 \mathrm{July}$ in association with a TUTT impinging on the northern edge of the storm. However, vector plots in DeMaria et al. (1993) show the storm weakened due to high shear associated with $200-\mathrm{mb}$ westerlies from the TUTT.

An analysis on the 32 TCs showed a statistically significant relationship (at the $95 \%$ level) between the REFC within $600 \mathrm{~km}$ of a storm center and the intensity change during the next $48 \mathrm{~h}$, but only after using a multiple regression technique that accounted for the effects of shear and SST; furthermore, the normalized regression coefficient was a small 0.1 units, indicating that shear and SST were much more correlated to intensity change (Fig. 2.14). The fact the REFC was only correlated near the storm center contradicts the findings of Molinari and Vollaro (1989), who found the relationship to hold at $1500 \mathrm{~km}$. 


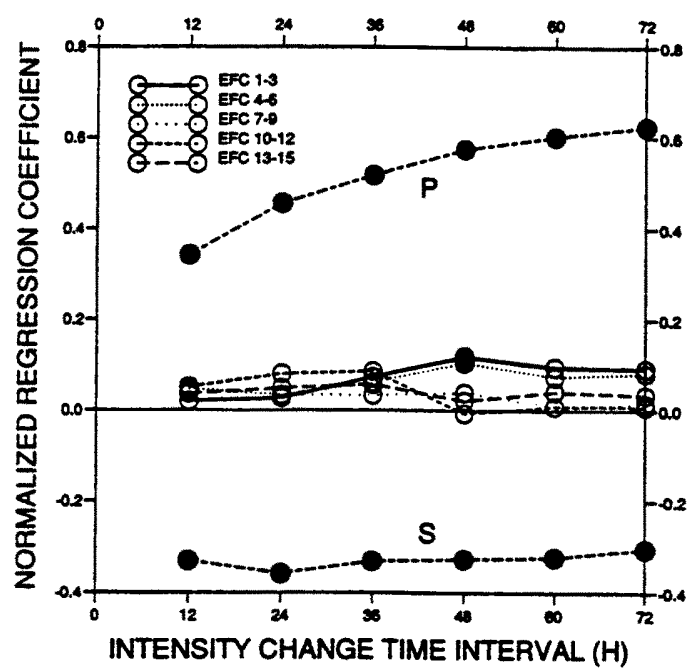

Figure 2.14: Normalized regression coefficients for areally averaged vertical shear (S), a potential intensity change variable based on SST $\left(\mathrm{P}=\right.$ VSST $\left.-V_{\max }\right)$, and the relative eddy angular momentum flux convergence (EFC) in each radial band (1-3 denotes the 100-300- $\mathrm{km}$ average, 4-6 denotes the 400-600- $\mathrm{km}$ average, etc.). A multiple regression was performed that included three independent variables (EFC in a given radial band, $P$, and $S$ ) for each intensity change time interval $(12,24,36,48,60$, and $72 \mathrm{~h})$. The circles are filled for coefficients that were significant at the $95 \%$ level. $\mathrm{P}$ is a significant positive predictor at all time intervals (regression coefficient between 0.4 and 0.6 ), $\mathrm{S}$ is a significant negative predictor at all time intervals (regression coefficient between -0.3 and -0.4 ), and EFC is a slight positive contributor at all time levels and at all radii (regression coefficient between 0 and 0.1 ) but only significant within $600 \mathrm{~km}$ at $48 \mathrm{~h}$. From DeMaria et al. (1993).

Another fact that disputes a relationship between REFC and intensification is that it is generally negatively correlated to $\Delta V_{\max }$ since REFC is often associated with the shearing influences of a trough. DeMaria et al. (1993) found REFC to be negatively correlated with intensity change for nearly all radial bands using linear regression (Fig. 2.15).

As expected, shear had strong negative correlations and SST had strong positive correlations in both Figs. 2.14 and 2.15. DeMaria et al. use a modified SST variable denoted by $\mathrm{P}$ in (DeMaria et al. 1993) and as POT in other papers (DeMaria and Kaplan 1991, 1994b; Burpee and Staff 1994) that measures a storm's maximum potential future intensity change:

$$
\mathrm{POT}=\mathrm{VSST}-V_{\max }
$$

where VSST is the Maximum Potential Intensity (MPI) empirically estimated in terms of a storm's current maximum wind $\left(V_{\max }\right)$ (see Fig. 2.12). MPI is the preferred acronym 
for this empirical relationship (DeMaria and Kaplan 1991, 1994b; Burpee and Staff 1994) and will be used in place of VSST. DeMaria et al.'s regression model will be discussed in more detail in Chapter 4 and Chapter 8.

Of the ten storms in the data set that experienced the largest intensification rates, six were associated with enhanced REFC (for example, Hurricane Bob which was influenced by troughs to its southeast and northwest), while in the remaining four intensification occurred in a low shear environment with no upper-level interaction (for example, Hurricane Hugo). It should also be mentioned that the other six were also in a low shear environment, located at the edge of the upper-level cyclonic features, and never moved under them.

One problem with REFC calculations is the lack of upper-tropospheric data in the tropics. DeMaria et al. (1993) use spline analysis applied to rawinsondes and satellite cloud-track winds for their data. Hence, the calculations may be inaccurate from the interpolation, particularly over the ocean where only satellite estimates are possible at $200 \mathrm{mb}$. Molinari et al. (1992) showed that 200-mb estimates of REFC are accurate to within $50 \%$ using satellite cloud track winds. It is hoped that these estimates are accurate enough to distinguish between periods of trough interaction and noninteraction (DeMaria et al. 1993).

Perhaps a partial answer to upper-level data deficiencies is total ozone mapping as done by Rodgers (1992). Rodgers argued that the Total Ozone Mapping Spectrometer (TOMS) on the polar orbiting Nimbus-7 satellite can better delineate the relative spatial distribution of the tropopause topography associated with upper-tropospheric waves compared to observations from other in situ instruments and remote sensors. One drawback is that, because the sensor uses the ultraviolet part of the spectrum to estimate total ozone, observations are only available at local noon. The distribution of total ozone within subtropical upper-tropospheric waves correspond well with the variation of temperature, thickness, and geopotential height - the higher the total ozone, the deeper the trough (Fig. 2.16). Rodgers, who investigated trough interaction with TCs, provides a plausible summary to this chapter: 


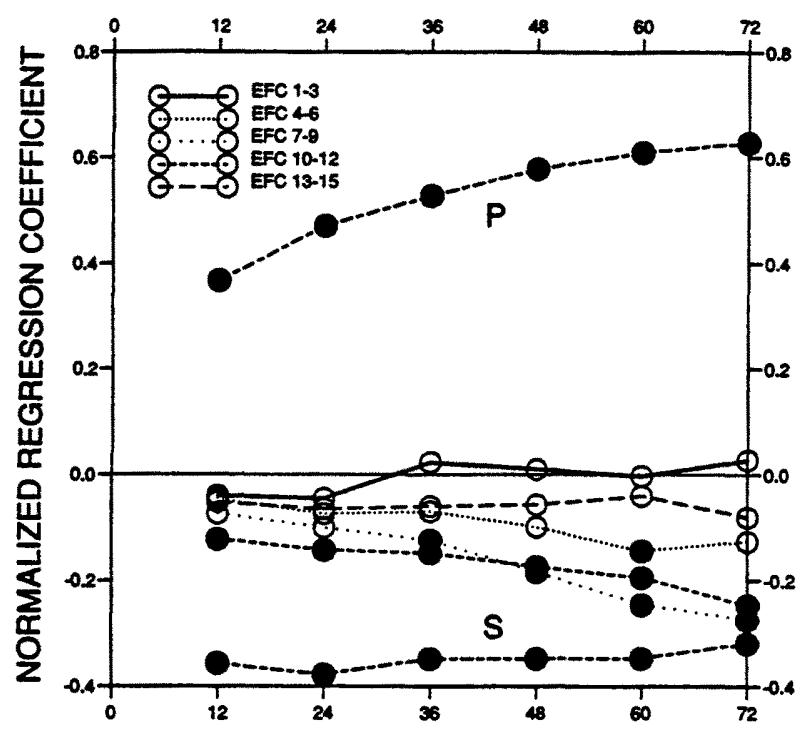

Figure 2.15: Same as Fig. 2.14, except that a separate (linear) regression was performed for each variable and for each intensity change time interval $(12,24,36,48,60$, and $72 \mathrm{~h}$ ). The circles are filled for coefficients that were significant at the $95 \%$ level. Without the combination of $S$ and $P$ to explain TC intensity change, EFC by itself is negatively correlated to $\Delta V_{\max }$. $\mathrm{P}$ by itself is positively correlated, and $\mathrm{S}$ by itself is negatively correlated. From DeMaria et al. (1993).

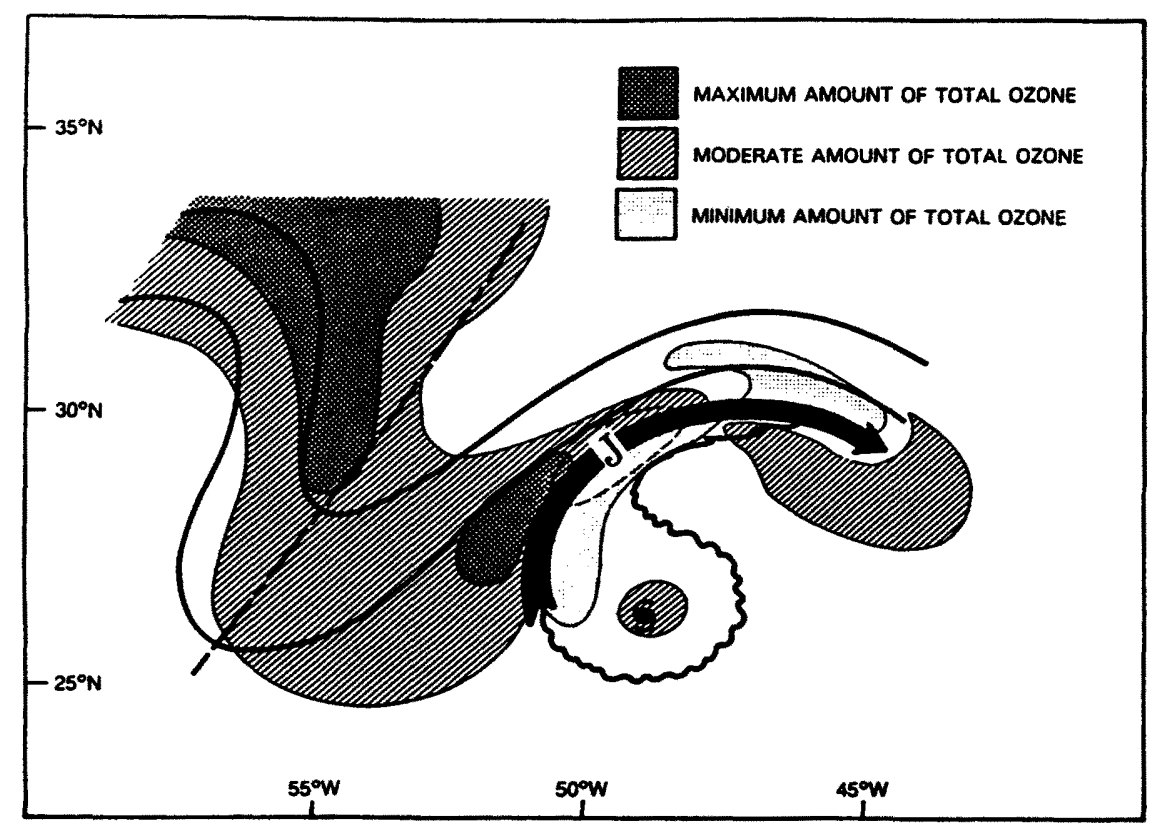

Figure 2.16: The TC total ozone schematic model showing 200-mb contours, TC outflow jet (denoted by "J"), and total ozone distribution as found by Rodgers (1992). Dashed line locates the upper-level trough axis. The unshaded regions delineate the mean total ozone that should be expected in the TC environment. The hurricane symbol locates the hurricane center. Light dashed line depicts maximum velocity region of the jet. From Rodgers (1992). 
- The interaction of high latitude TCs with ozone rich deep mid-latitude troughs inhibit TC deepening due to strong shear. The critical distance is about $10 \mathrm{deg}$.

- However, the juxtaposition of low latitude TCs with moderate total ozone rich shallow troughs favor TC intensification through outflow channel interaction and/or superposition of high PV air. Positive interactions can occur even for distances less than $10 \mathrm{deg}$.

- TCs weaken when ozone rich regions (shallow or deep) pass over the TC center. The depletion of ozone (associated with the anticyclonic outflow jet) extends more radially outwards for the intensifying TCs, implying the outflow channel extends further outwards. Both of these findings are consistent with Merrill (1988b).

The first two findings present new geographical information about high latitude and low latitude storms, but also reaffirm the findings of DeMaria, Holland, Chen and Gray, and others that a trough might have a positive influence on TC intensification as long as the interaction isn't too strong. As stated by Montgomery and Farrell (1993), "There may be a class of admissible shears and PV anomalies...strong enough to promote deep sustained ascent, yet not so strong as to significantly disrupt the cumulus environment (through vertical shear)...that interact favorably" to promote cyclogenesis and/or TC intensification.

This beckons the question - does the presence of a trough within $10 \mathrm{deg}$ of the TC necessarily indicate upper-level interaction? Since a TC doesn't exist in a horizontally homogeneous environment interaction must exist, but when is the interaction from a trough non-negligible? ${ }^{1}$ For example, Mundell (1990) argues that dual outflow channels play an important indirect role in the intensification process by reducing unidirectional shear across the center of the developing TC (Fig. 2.17). Furthermore, downstream of a trough the region is often naturally low-shear (and anticyclonic), with interaction minimized (Fig. 2.18). The location of a trough to the northwest of a TC may just indicate a

\footnotetext{
${ }^{1}$ Dynamicists argue interaction is possible within the Rossby Radius of Deformation - about $10 \mathrm{deg}$ or more in the tropics. However, the actual distance where interaction is non-negligible is unclear.
} 


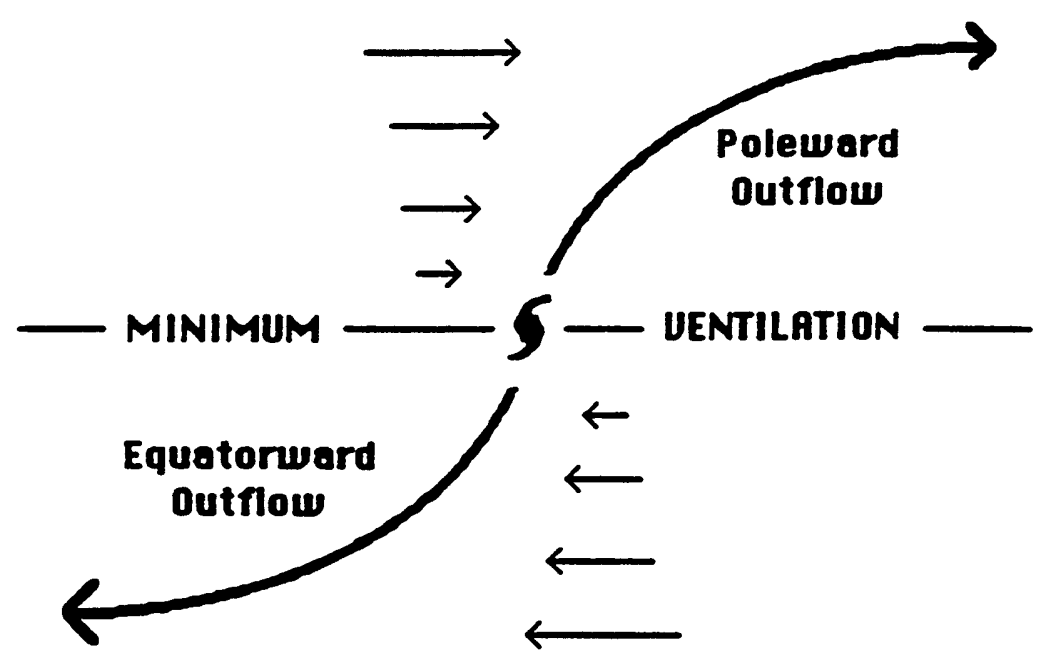

Figure 2.17: Illustration of the effect dual outflow channels (possibly associated with mid-latitude troughs and TUTTs) have in reducing shear (ventilation) across the top of an intensifying TC. From Mundell (1990).

favorable shear environment, with minimal environmental forcing. One must also be careful in discerning "cause and effect" during TC intensification. Three-dimensional models readily produce asymmetric outflow channels in a no-shear environment (Anthes 1972; Tuleya and Kurihara 1975). Hence, dual outflow channels might be a natural consequence of the intensification process, rather than a source of TC development, in many cases.

Additionally, it has been shown that the inner (<1-deg radius) and outer-core (12.5-deg radius) processes of a TC are often decoupled, with the inner-core winds usually increasing much faster than the outer-core winds (Weatherford and Gray 1988a). Furthermore, TC intensity is uncorrelated with the outer-core winds, especially once hurricane strength has been reached (Fig. 2.19). This implies that intensity change is related to inner-core convective processes and not directly linked to environmental conditions at larger radii, such as outflow channels and troughs. REFC might help maintain or expand the outer circulation of a TC, but is probably not related to intensity change. These inner-core and outer-core differences will be reviewed in Chapter 4. This concludes the review of upper-level forcing, and now intensification processes not requiring environmental influences will be discussed in the next chapter. 


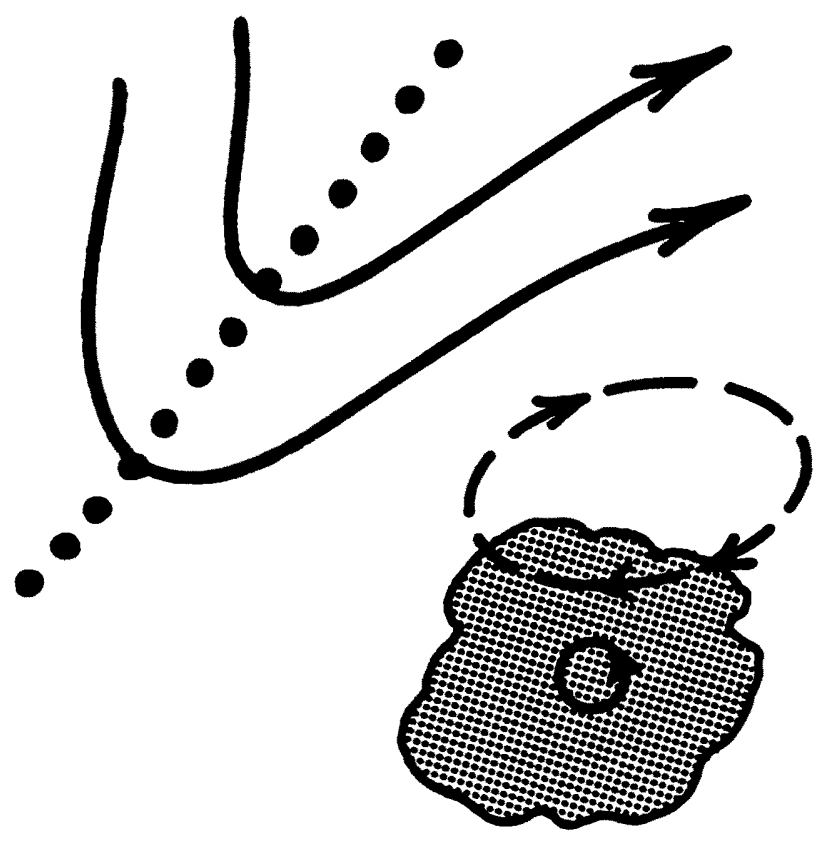

Figure 2.18: Illustration of the minimal shear associated with an anticyclone downstream of a trough. The location of a trough to the northwest of a TC may just indicate a favorable shear environment downstream, with minimal environmental interaction in the vicinity of the TC. 


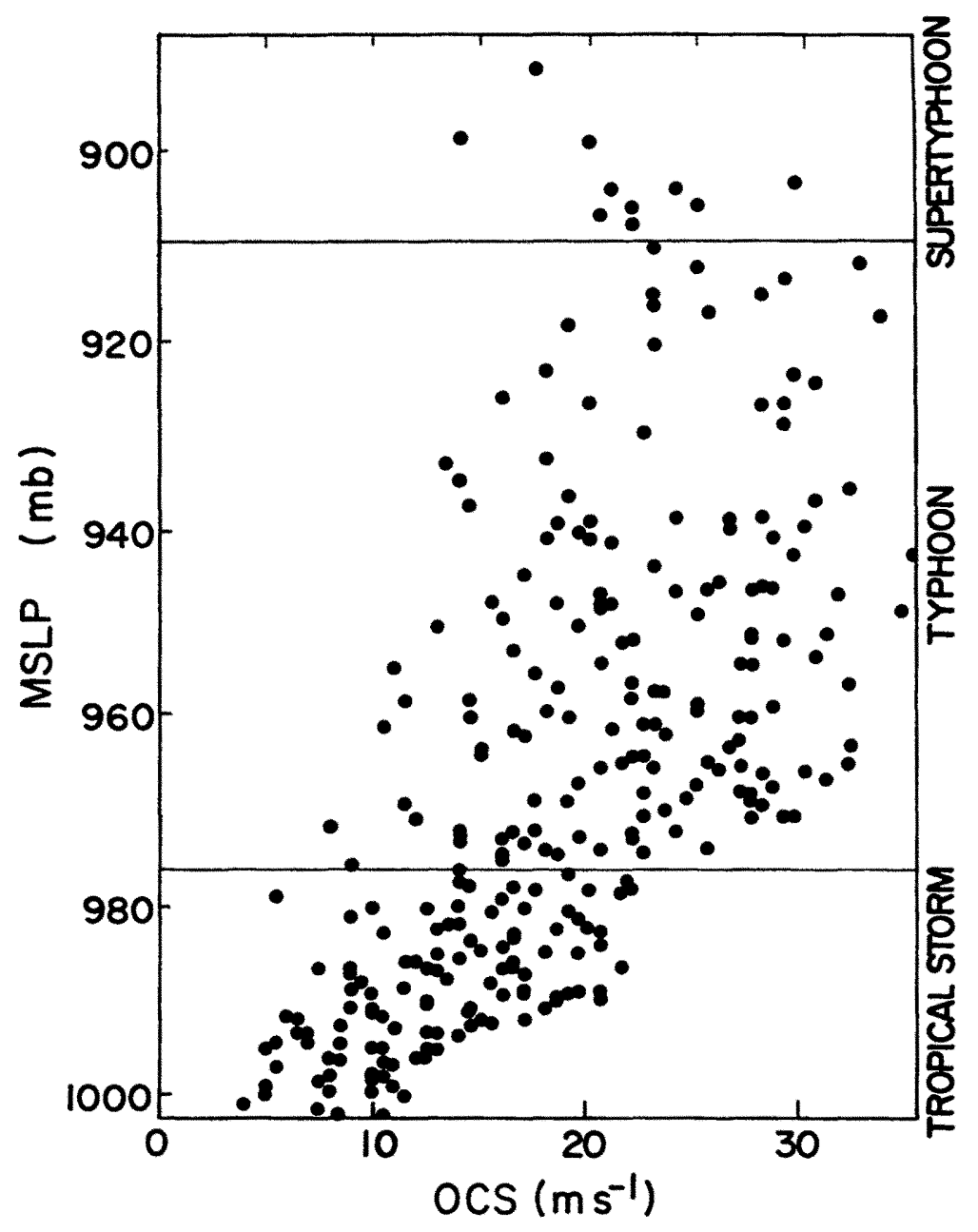

Figure 2.19: Scattergram of intensity (MSLP) versus outer-core strength (mean $v_{t}$ for 1-2.5-deg radius). Taken from Weatherford and Gray (1988a). 


\section{Chapter 3}

\section{TROPICAL CYCLONE INTENSITY CHANGE THEORY NOT REQUIRING UPPER-LEVEL FORCING}

It has been extensively observed that intensification can occur in the absence of upperlevel anomalies as long as wind shear is low. The controversial issue is the following: can fast (even rapid) intensification occur without environmental forcing and, if so, which is the more common case - rapid development with or without external forcing? Hurricane Hugo is an example of a rapidly intensifying storm which required no upper-level forcing and, in fact, strengthened in a flow dominated by easterlies throughout the troposphere which resulted in a low-shear environment.

As an introductory case study, the most famous hurricane of the modern era will be briefly reviewed - Hurricane Andrew, which also intensified in a low-shear region absent of upper-level trough interaction. The factors contributing to Andrew's strengthening will be discussed. These factors will be shown throughout this paper to be the major features correlated to TC intensity change.

\subsection{Hurricane Andrew - the devastating storm which almost never hap- pened}

It is surprising, in retrospect, that Andrew almost completely dissipated before ever reaching the Florida coast. After reaching minimal tropical storm strength (35 kts) on 12 UTC 17 August 1992 at $12.3 \mathrm{deg} \mathrm{N}$ and $42.0 \mathrm{deg} \mathrm{W}$, Andrew strengthened slowly to $45 \mathrm{kts}$ during the next two days as the storm followed a west-northwest path (Fig. 3.1). During 19-20 August, Andrew became influenced by a strong upper-level TUTT which extended northeast from Puerto Rico (Fig. 3.2). At this time, Andrew became embedded in an environment of fast southwesterly $200-\mathrm{mb}$ winds which strongly sheared the storm 


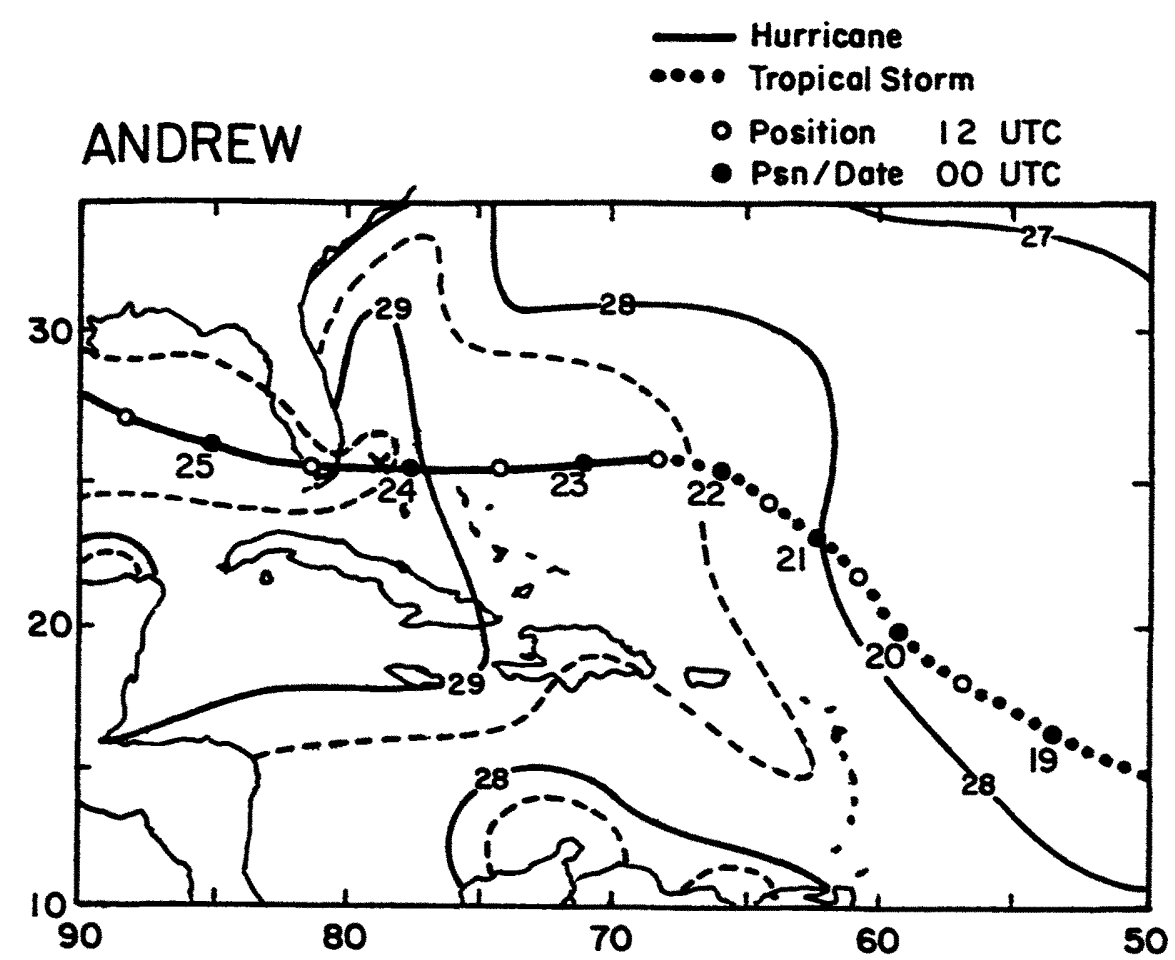

Figure 3.1: SSTs $\left({ }^{\circ} \mathrm{C}\right)$ for August 1992 (Reynolds and Smith 1994). Andrew's path is also shown.

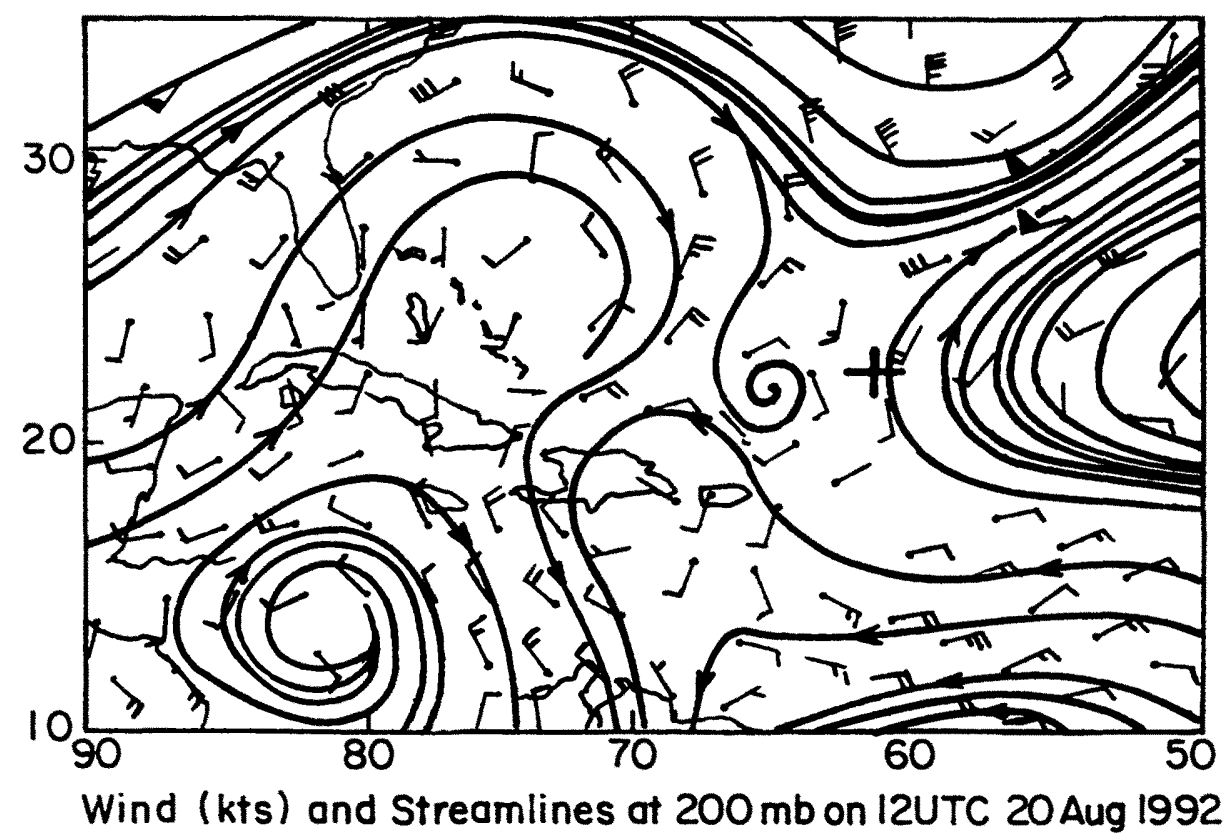

Figure 3.2: Aviation model 200-mb wind barbs and streamlines on 12 UTC 20 August 1992. Andrew is located at + . 
(Fig. 3.3). Traditionally, vertical wind shear (VWS) is computed as the vectorial difference of $u$ and $v$ between 850 and $200 \mathrm{mb}$ over a single grid point (VWSPT) as:

$$
\text { VWSPT }=\left[\left(u_{200}-u_{850}\right)^{2}+\left(v_{200}-v_{850}\right)^{2}\right]^{1 / 2}
$$

Shear values were $17 \mathrm{~m} \mathrm{~s}^{-1}$ over the storm center, and as high as $30 \mathrm{~m} \mathrm{~s}^{-1}$ over Andrew's northern edge. These values are much higher than the $12.5 \mathrm{~m} \mathrm{~s}^{-1}$ threshold defined by Zehr (1992) where shear starts to become detrimental to TC development. ${ }^{1}$ Deep convection

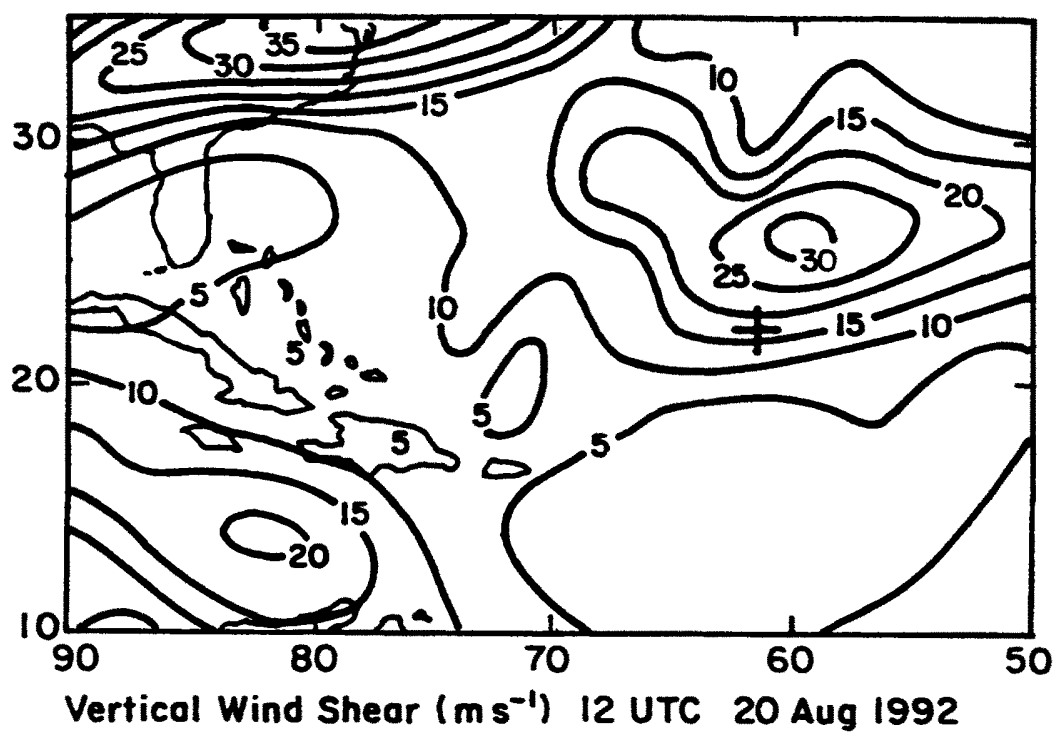

Figure 3.3: Aviation model vertical wind shear (in $\mathrm{m} \mathrm{s}^{-1}$ ) computed as a vectorial wind difference between $850 \mathrm{mb}$ and $200 \mathrm{mb}$ on 12 UTC 20 August 1992. Shear computations are from single grid point wind values of $u$ and $v$. Andrew is located at + .

appeared only in bursts and did not persist as the clouds were advected away from the center (Mayfield et al. 1994). The winds weakened to $40 \mathrm{kts}$ and MSLP rose to $1015 \mathrm{mb}$. Andrew was on the brink of total disintegration. Clearly, upper-level interaction in this instance did not positively enhance intensity and, in fact, almost destroyed Andrew.

Changes in the upper-level environment rescued Andrew. On 21 August the 200-mb trough weakened and retreated northward (Fig. 3.4). At the same time, a strong high pressure cell formed near the U.S. southeast coast. Because of the change in the synoptic

\footnotetext{
${ }^{1}$ Zehr's vertical wind shear criterion was actually defined for tropical cyclogenesis, but is also used as a "rule-of-thumb" by operational forecasters for predicting TC intensification.
} 


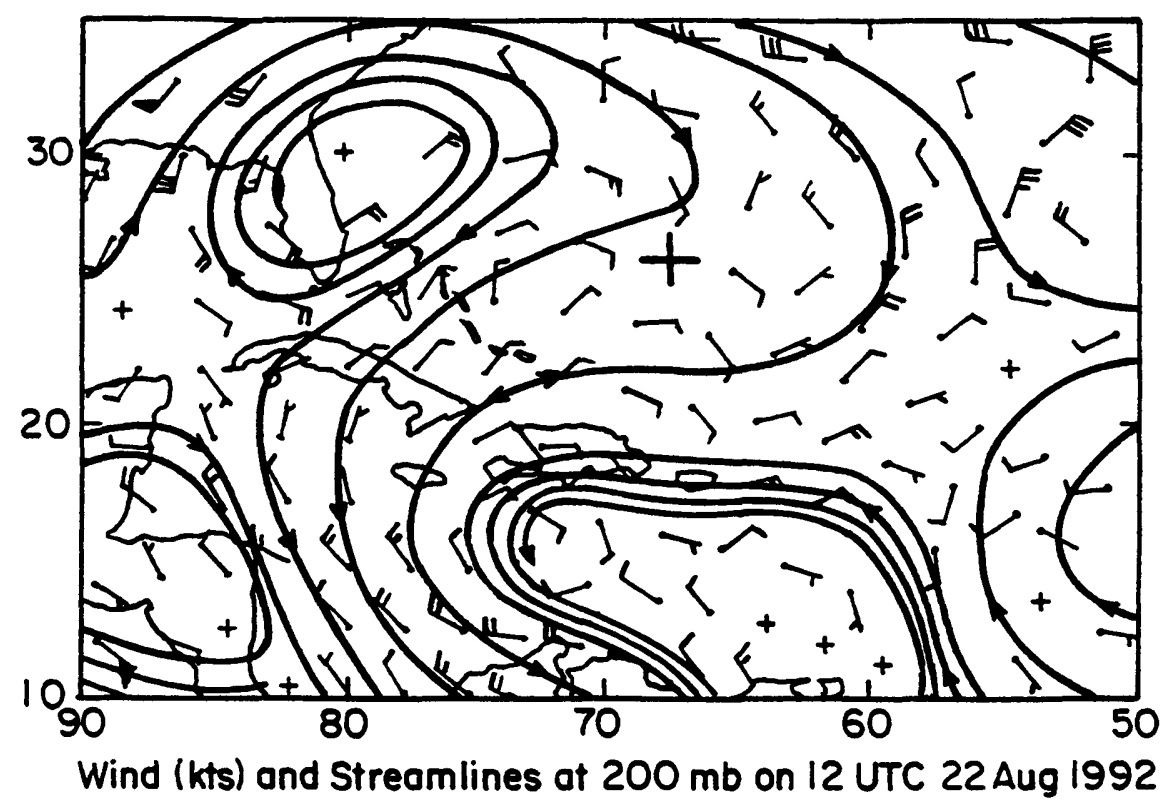

Figure 3.4: Aviation model $200-\mathrm{mb}$ wind barbs and streamlines on 12 UTC 22 August 1992. Andrew is located at + .

regime, Andrew was suddenly embedded in a basic easterly steering flow which turned Andrew to the west and positioned the storm in a low shear environment (Fig. 3.5). Values of VWS were $5 \mathrm{~m} \mathrm{~s}^{-1}$, favorable for strengthening. No trough was in the vicinity at this time.

Another positive influence is the increasing SSTs along Andrew's path (Fig. 3.1). It has been known for many decades that an $\mathrm{SST}>26.5^{\circ} \mathrm{C}$ is required for $\mathrm{TC}$ formation and maintenance (Palmén 1948), although TCs may exist in a weakening state over colder waters after their development. However, Mundell (1990) and Holliday and Thompson (1979) have shown the minimum SST for rapid developers in the western North Pacific is $28.5^{\circ} \mathrm{C}$. This observation is consistent with Fig. 2.12 which shows an exponential relationship between SSTs and maximum potential intensity (MPI). Indeed, the rapid intensification of Andrew occurred when the storm first traveled over SSTs of $28.5^{\circ} \mathrm{C}$ on 18 UTC 22 August. According to Fig. 2.12, the corresponding MPI values for 00 UTC 20-24 August are 134,138,150,155, and $165 \mathrm{kts}$ respectively. When compared to the observed maximum wind values of $40,45,55,90$, and $125 \mathrm{kts}$ respectively, it seems likely Andrew tapped into the extra 31 kts of potential intensity due to increasing SSTs of 28.0, $28.1,28.5,28.7$, and $29.3^{\circ} \mathrm{C}$, respectively, since the $1.3^{\circ}-\mathrm{C}$ increase along the track served 


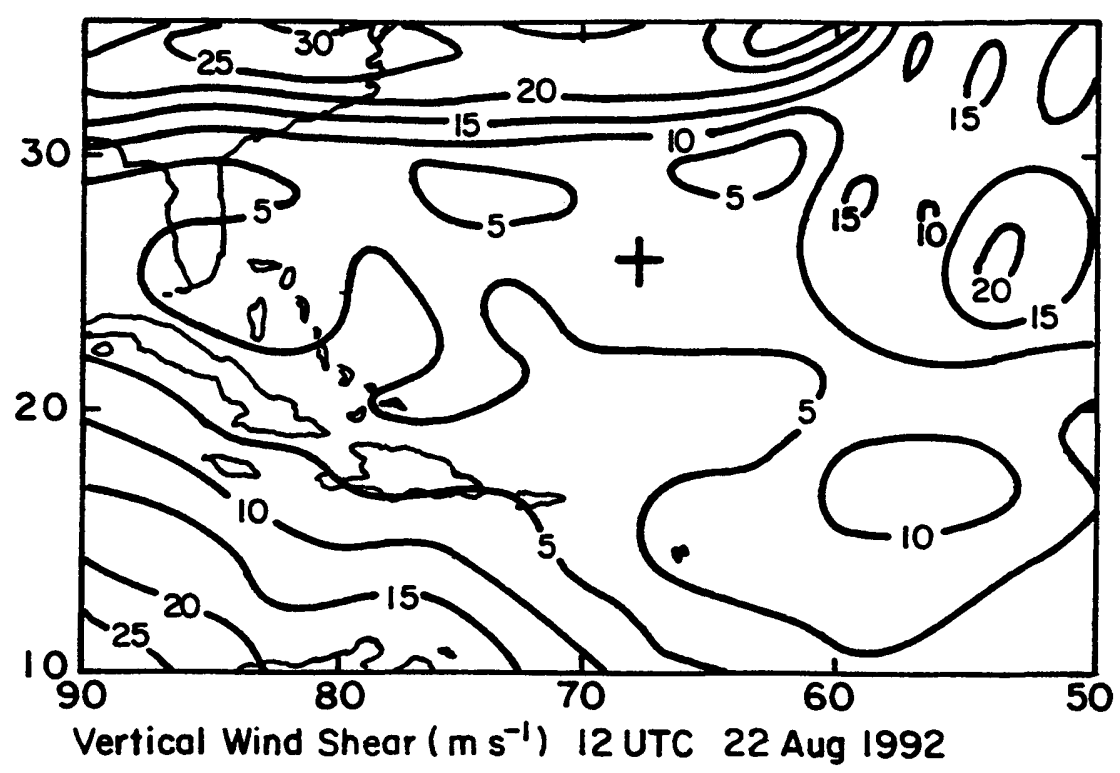

Figure 3.5: Aviation model vertical wind shear (in $\mathrm{m} \mathrm{s}^{-1}$ ) computed as a vectorial wind difference between $850 \mathrm{mb}$ and $200 \mathrm{mb}$ on 12 UTC 22 August 1992. Shear computations are from single grid point wind values of $u$ and $v$. Andrew is located at + .

to increase the rate of intensification. It is also noteworthy that Andrew did not reach its MPI. As noted previously, few TCs are able to utilize their full intensity potential.

A third factor contributing to Andrew's intensification is inner-core convection. Once a storm is in a favorable environment and located over warm water, a positive feedback between convection and the storm favors further intensification. It should be mentioned that some researchers disagree with this idea, and argue that convection is merely a passive response in a TC's development (Emanuel 1986, 1991; Xu and Emanuel 1989; Elsberry et al. 1992). Their concerns will be addressed in Chapter 4.

Figure 3.6 is an infra-red satellite draft of Andrew at the onset of rapid intensification. Three features are most notable: 1$)$ the cold cloud-top temperatures $\left(<-65^{\circ} \mathrm{C}\right)$ within $1 \mathrm{deg}$ of the storm center, representing deep inner-core convection; 2) the general symmetry of the $-55^{\circ} \mathrm{C}$ cloud shield, denoting little VWS; and 3) a newly formed eye (5-h old). It will be shown in Chapter 6,7 , and 8 that these satellite characteristics exhibit intensity prediction skill, and generally portend fast intensification.

Another consequence of a convectively active TC in a low shear environment is that cyclonic momentum is transported aloft in the eyewall. Figure 3.7 shows Andrew's vertical 


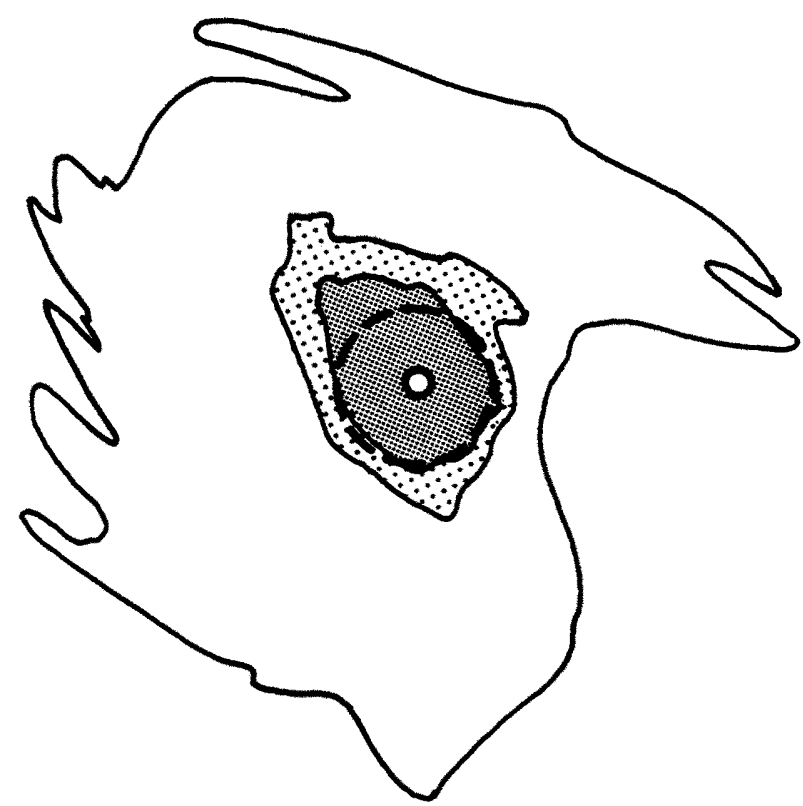

Figure 3.6: Draft of Hurricane Andrew on 21 UTC 22 August 1995. The light shading denotes cloud tops with brightness temperature colder than $-55^{\circ} \mathrm{C}$, and dark shading colder than $-65^{\circ} \mathrm{C}$. The outline represents the edge of the warmer clouds. An eye is also evident inside the cloud canopy. The circle denotes $1 \mathrm{deg}$ radius.

wind structure in the middle of the 24 -h pressure drop of $47 \mathrm{mb}$. The central pressure at this time is $961 \mathrm{mb}$, and the decrease of $v_{t}$ from $2 \mathrm{~km}$ to $12 \mathrm{~km}$ is only one-half. Because the shear is small (Fig. 3.5), Andrew was able to advect significant cyclonic vorticity in the eyewall to the upper-levels. It will be hypothesized that advection of $v_{t}$ to the upper eyewall region furnishes a positive feedback mechanism for continued intensification. It will be shown in Chapter 6 that a vertically stacked vortex (due to low shear) provides more eyewall buoyancy than a sheared TC with a poorly vertically defined vortex. Therefore, if $v_{t}$ is conserved with height such that strong eyewall updrafts are possible, and if the tropical cyclone has not attained its MPI, it is likely that further development will occur perhaps even "rapid" development should the "potential" (defined as MPI minus current intensity) be large.

On 00 UTC 24 August, Andrew experienced a weakening in both the wind and MSLP (which rose to $941 \mathrm{mb}$ ) before reintensifying hours before landfall to $922 \mathrm{mb}$. Such pressure fluctuations are common in TCs. Rappaport (1994) hypothesizes that the lowest surface pressure was embedded in a "small, transitory, dynamically imbalanced perturbation which was orbiting about the eye," and that the aircraft measurement on 00 


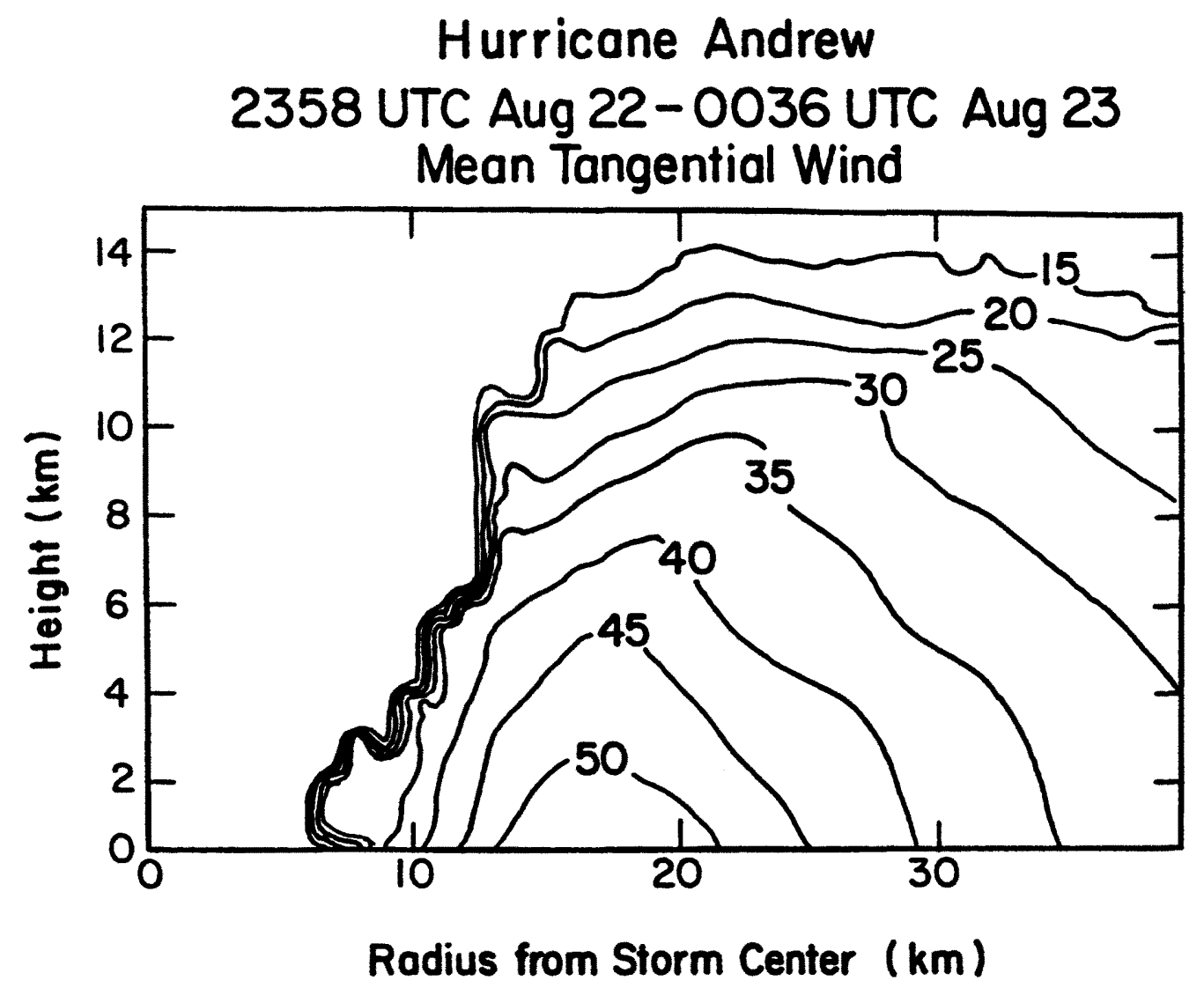

Figure 3.7: Cross-section of Hurricane Andrew inner-core winds on 00 UTC 23 August 1992 during rapid deepening (47 mb pressure drop). Central pressure is $961 \mathrm{mb}$. Wind analysis is obtained from VTD analysis (Lee et al. 1994). Contour intervals are $5 \mathrm{~m} \mathrm{~s}^{-1}$. The tight wind gradient within $10 \mathrm{~km}$ is probably an artifact of the analysis technique. Figure is courtesy of Frank Marks of the Hurricane Research Division. 
UTC 24 August did not intersect this disturbance. ${ }^{2}$ Mayfield et al. (1994) attributes the pressure drop before landfall due to increased low-level convergence related to land friction which enhanced eyewall convection and eye subsidence.

However, according to C. Landsea and H. Willoughby (personal communication 1992), and in this writer's opinion, the likely cause for the second pressure drop is the formation of a second eyewall which contracted and replaced the inner eyewall. Radar, aircraft, and satellite data show a decreasing eye diameter and strengthening eyewall convection before landfall (Rappaport 1994). This inner-core process is known as the concentric eyewall cycle which occurs in many intense TCs. The concentric eyewall cycle is associated with a temporary weakening of the storm over a $24-36-\mathrm{h}$ period, then reintensification as the new eyewall becomes established (Willoughby et al. 1982; Willoughby 1990; Black and Willoughby 1992). The concentric eyewall cycle of Andrew was remarkable in that the whole process occurred in approximately $7 \mathrm{~h}$, the fastest on record. Perhaps the very warm SSTs of $29.5^{\circ} \mathrm{C}$ near the Florida coast helped facilitate the second intensification and sped up the concentric eyewall cycle. ${ }^{3}$ The concentric eyewall cycle is an important intensity change feature that the reader should be aware of, and will be discussed further in Chapter 6.

It should be noted that some researchers believe upper-level forcing played a positive role in Andrew's intensification. As Molinari and Vollaro (1993) write with regard to Andrew's strengthening, "Approaching troughs often disrupt tropical cyclones temporarily, producing unimpressive satellite pictures; but the seeds of intensification are being planted

\footnotetext{
${ }^{2}$ A similar transient mesoscale vortex almost caused the NOAA research WP-3D plane to crash in Hurricane Hugo. One of the plane's engines failed in Hugo's eyewall upon encountering the vortex. The plane, which entered the eyewall at $1200 \mathrm{ft}$, descended quickly before recovering at $350 \mathrm{ft}$, about $6 \mathrm{~s}$ before reaching the ocean surface! The crippled plane slowly circled the eye for one hour before reaching a safe altitude to exit, with the scientists discarding equipment off the injured plane to help lighten the weight. During this period, the plane encountered the vortex nine more times in different locations, indicating that the vortex was orbiting the eyewall. (Marks and Black 1990; Black and Marks 1991; P. Black, personal communication 1995). Some of the damage in Florida by Andrew has been attributed to transient mesoscale vortices in the eyewall (P. Black, personal communication 1995).

${ }^{3} \mathrm{Rau}$ (1993) has also suggested Andrew's second intensification coincided with the diurnal $1-2^{\circ} \mathrm{C} \mathrm{SST}$ maximum over the shallow Great Bahama Bank, and that the 2-5 m ocean depth precluded significant upwelling of cooler SSTs.
} 
in the form of middle level spinup, eye-wall formation..., and reduction of vertical shear due to strong heating (by convection)." While such an opinion cannot be totally discounted, it seems fairly clear in this case that the trough severely damaged Andrew, and the hurricane was revitalized only after the trough propagated away leaving the storm in a low VWS environment (with some extra positive feedback from increasing SSTs). Furthermore, the notion that convection dissipated the trough also seems incorrect - Andrew was virtually devoid of clouds on 19-20 August, and the trough was forced eastward by an approaching upper-level ridge.

All these facets contributing to Andrew's intensification will be covered in this paper. As a starting point, the Air-Sea Interaction by which TCs derive their energy will be described. This most fundamental factor to TC development will be discussed in the next section.

\subsection{The Fading of CISK and the Emergence of Air-Sea Interaction (ASI) Theories}

This title is actually a bit misleading, as the basic Air-Sea Interaction (ASI) from which TCs obtain their energy has been known for many decades (Byers 1944; Riehl 1954; Miller 1958a; Malkus and Riehl 1960). Unfortunately, ASI was temporarily overlooked by misinterpretations of the Conditional Instability of the Second Kind (CISK) theory as advanced by Charney and Eliassen (1964) and Ooyama (1969). Related topics like "wave-CISK" (Lindzen 1974) also served to complicate the issue." Furthermore, ASI theories have been muddled by the currently popular ASI-related Carnot Cycle Theory (Emanuel 1986, 1988, 1991), and a debate over whether Convective Available Potential Energy (CAPE) exists in the hurricane eyewall (Betts 1982; Xu and Emanuel 1989).

\subsubsection{The Air-Sea Interaction (ASI) process}

The ultimate energy source for the $\mathrm{TC}$ is the latent and sensible heat flux from the ocean. As air spirals into a TC, it is subjected to sea-level pressure reductions that would

\footnotetext{
"For an excellent discussion on CISK, a "finite-amplitude" treatment of the CISK equations, and a nice review of TCs in general, the reader is referred to Handel (1990).
} 
decrease the air temperature substantially due to adiabatic expansion; however, because of ocean fluxes [which are at least proportional to $\left|V_{s f c}\right|$ (Ooyama 1969; Willoughby et al. 1984) and more likely to $\left|V_{s f c}\right|^{2}$ (Moss and Rosenthal 1975; Powell 1980)] an air parcel is able to (mostly) preserve its temperature. For example, an air parcel in the West Indies with $T=26.3^{\circ} \mathrm{C}$ and a mixing ratio $q=18.2 \mathrm{~g} \mathrm{~kg}^{-1}$ (Jordan 1958) entering a $\mathrm{TC}$ of $930 \mathrm{mb}$ would experience a cooling to $21.0^{\circ} \mathrm{C}$ and a mixing ratio of $17.5 \mathrm{~g} \mathrm{~kg}^{-1}$ after condensing to fog at $970 \mathrm{mb}$ (Riehl 1954). But this kind of temperature and mixing ratio reduction is not observed, and the eyewall cloud base remains 500 to $1000 \mathrm{~m}$ from the surface (P. Black, personal communication 1995). ${ }^{5}$

In fact, until recently, inflowing air parcels were assumed isothermal. New results suggest that the eyewall boundary layer actually experiences a cooling of $2-4^{\circ} \mathrm{C}$ instead of remaining isothermal. This author believes this cooling is due to ocean fluxes not (totally) compensating adiabatic expansion (see Chapter 7). However, some of this cooling might also be due to a combination of sea spray evaporation and eyewall rainfall/downdraft evaporation (Betts and Simpson 1987; Pudov 1992; Pudov and Holland 1994; Fairall et al. 1994; Kepert 1995), as well as upwelling/mixing of cooler water below the thermocline to the ocean surface (Brand 1971; Black 1983; Black et al. 1989; Shay et al. 1992; Bender et al. 1993; Black and Holland 1995). Downdrafts and rainfall evaporation under spiral bands can also cause cooling of inflowing air on the inside (downstream) edge of the squall line so that "recovery" by surface fluxes to an isothermal value cannot occur before reaching the eyewall, thereby affecting TC intensity (Barnes et al. 1983; Barnes and Stossmeister 1986; Powell 1990). As a further complication, several researchers have hypothesized that surface fluxes are not the dominant mechanism contributing to isothermal inflow, but that mixing (mass recycling) of high potential temperature air from above the boundary layer may supply most of the heat instead (Frank 1977a; Anthes and Chang 1978; Betts and Simpson 1987; Powell 1990; Barnes and Powell 1995). For this discussion, an isothermal expansion is assumed to clarify the ASI process. Evidence of

\footnotetext{
${ }^{5}$ Riehl (1954) states the cloud base is 30 to $300 \mathrm{~m}$, but this is probably too low.
} 
boundary layer cooling, its consequences, and a modified ASI theory accounting for this surface cooling will be discussed in Chapter 7.

ASI is mathematically described most succinctly by Holland (1995), and the process is as follows: as an air parcel flows isothermally towards lower pressure, it experiences an increase in both potential temperature $\theta$ and the saturated mixing ratio $q^{*}$. Furthermore, if one assumes a constant surface relative humidity $\mathrm{RH}, q$ also increases. Both processes sustain buoyancy so that a TC can continue to develop. Some of the latent heat released in the clouds is retained and increases the TC's inner-core temperature, while the rest of the heating propagates away as gravity waves. The computational procedure for computing surface pressure, $\theta_{e}$, and temperature along a moist adiabat will now be described. These equations will be used to describe the ASI process, and are also used in Chapter 7.

\section{Calculation of surface pressure}

Assuming that a level in the upper atmosphere exists where height and pressure anomalies are negligible $\left(p_{t o p}\right){ }^{6}$ the hydrostatic change in surface pressure $p_{s f c}$ associated with a temperature anomaly aloft is (Hirschberg and Fritsch 1993):

$$
\Delta p_{s f c}=\frac{p_{s f c}}{T_{v}\left(p_{s f c}\right)} \int_{p_{s f c}}^{p_{t o p}} \Delta T_{v} \frac{d p}{p}
$$

where $T_{v}=T(1+0.61 q)$ is the virtual temperature, ${ }^{7}$ and $\Delta T_{v}(p)=T_{v c}(p)-T_{v e}(p)$. $T_{v c}(p)$ is the virtual temperature profile of the cloud environment, while $T_{v e}(p)$ is the virtual temperature profile of the environment outside the TC. $p_{\text {top }}$ is chosen to be $100 \mathrm{mb}$ in this study. The new $p_{s f c}$ is found as

$$
p_{s f c}=p_{e n v}+\Delta p_{s f c}
$$

\footnotetext{
${ }^{6}$ This assumption is based on the existence of a stratospheric level of insignificant dynamics (LID). Models and observations generally show a lack of stratospheric intrusions above $100 \mathrm{mb}$ in TCs, making a LID level of $100 \mathrm{mb}$ a proper upper-boundary condition for a TC (Bender et al. 1993).

${ }^{7}$ This expression is actually an approximation to the true definition of virtual temperature given by:$$
T_{v}=T \frac{1+q / 0.622}{1+q}
$$

but both equations give nearly the same value of $T_{v}$. More details are contained in Chapter 7 .
} 
where $p_{\text {env }}$ is the environmental surface pressure. Most of the hydrostatic contribution to the pressure drop is from the upper troposphere because, for a given $\Delta T_{v}$, more pressure drop can occur aloft due to the $d p / p$ term in Eq. (3.2). This sensitivity of hydrostatic pressure fall to the height of a temperature anomaly was first noted by Haurwitz (1935).

\section{Calculation of $\theta_{e}$}

Assuming (for the moment) all the liquid water condensate is removed the instant it forms in an ascending moist air parcel (the pseudoadiabatic assumption), the combined effects of increased $\theta$ and $q^{*}$ may be incorporated into a single parameter, equivalent potential temperature $\theta_{e} . \theta_{e}$ is conserved during moist ascent, and therefore $\theta_{e}$ remains constant during a parcel's ascent although $\theta$ and $q^{*}$ will change above the Lifting Condensation Level (LCL). $\theta_{e}$ is commonly computed assuming a pseudoadiabatic process as (Wallace and Hobbs 1977):

$$
\theta_{e}=\theta(z \geq L C L) \exp \left(\frac{L_{v} q^{*}(z \geq L C L)}{c_{p} T(z \geq L C L)}\right)
$$

where $L_{v}$ is the latent heat of vaporization (condensation), and $c_{p}$ is the specific heat at constant pressure. $T, q^{*}$, and $\theta$ are the LCL values if the parcel is located below the LCL (unsaturated). Once $\theta_{e}$ is known from the LCL values, $\theta_{e}$ is held constant, and $T$ and $q^{*}$ may be found through iterative techniques as the parcel rises moist adiabatically above the LCL.

As can be seen, an isothermal increase in $\theta$ and $q^{*}$ below the LCL leads to an increase of $\theta_{e}$. As an alternative notation, $\theta_{e}$ is sometimes referred to as "moist entropy" $\left(s^{*}\right)$ since to a close approximation $d s^{*}=c_{p} d\left(\ln \theta_{e}\right)$ (Holton 1992)..$^{8}$

Since Eq. (3.4) can lead to errors of several degrees in the tropics (Simpson 1978), an empirical formula by Bolton (1980) for $\theta_{e}$ should be used instead of the conventional

\footnotetext{
${ }^{8}$ Some tropical meteorologists also prefer using moist static energy, defined as $h=c_{p} T+g z+L q$, in describing the ASI process. This is because $h$ is approximately conserved when $\theta_{e}$ is conserved, since $d h \approx c_{p} T d\left(\ln \theta_{e}\right)$ (Holton 1992) - however, the analogy is confused by the inclusion of $T$. Furthermore, static energies are based on the assumption that any kinetic energy is locally dissipated into heat, while potential temperature definitions do not make this assumption (Stull 1988).
} 
formula (Eq. (3.4)): ${ }^{9}$

$\theta_{e}=\theta(z \geq L C L) \exp \left[10^{3} q^{*}(z \geq L C L)\left(1+0.81 q^{*}(z \geq L C L)\right)\left(\frac{3.376}{T(z \geq L C L)}-0.00254\right)\right]$

\section{Calculation of temperature along a moist adiabat}

To compute moist adiabatic ascent in the eyewall, LCL values corresponding to surface relative humidity and surface temperature need to be calculated first. The temperature ${ }^{10}$ at the LCL can be computed by an empirical relationship accurate to within $0.1^{\circ} \mathrm{K}$ (Bolton 1980; Holland 1995):

$$
T_{L C L}=\frac{2840}{2.5 \ln T_{s f c}-\ln \left(\frac{0.01 p_{s f c} q_{s f c}}{0.622+q_{s f c}}\right)-4.805}+55
$$

and the LCL pressure level is found by the conservation of potential temperature principle:

$$
p_{L C L}=p_{s f c}\left(\frac{T_{L C L}}{T_{s f c}}\right)^{c_{p} / R}
$$

Surface mixing ratio $q_{s f c}$ is computed from the surface relative humidity $\mathrm{RH}_{\mathrm{sfc}}$

$$
q_{s f c}=\mathrm{RH}_{\mathrm{sfc}} q^{*}\left(p_{s f c} ; T_{s f c}\right)
$$

${ }^{9}$ For a pseudoadiabatic process, the following thermodynamic relationship applies:

$$
c_{p} \ln T+c_{w} \int_{T_{\text {top }}}^{T_{L C L}} q^{*} d \ln T-R \ln \left(p-e^{*}\right)+q^{*} L_{v} / T=\text { constant }
$$

where $c_{w}$ is the specific heat of water at constant pressure and $R$ is the dry gas constant. The mixing ratio must be retained in the integral since it is a function of temperature, and complicates calculations associated with the pseudoadiabatic process. In the conventional derivation of $\theta_{e}$ (which also usually neglects the partial pressure of $e^{*}$ on atmospheric pressure), the term with $c_{w}$ is neglected. When $c_{w}$ is included the equation for $\theta_{e}$ has the following form (Simpson 1978):

$$
\theta_{e}=\theta \exp \left(\frac{L_{v} q^{*}}{c_{p} T}\right)\left(\frac{c_{w}}{c_{p}} \int_{T_{\text {top }}}^{T_{L C L}} q^{*} d \ln T\right)
$$

where $T_{\text {top }}$ is a region aloft in which $q^{*}$ is sufficiently small. In the mid-latitudes, the neglect of the $c_{w}$ term is of little consequence, but in the tropics is relevant. Not considering this effect results in an underestimation of buoyancy with $\theta_{e}$ values incorrect up to $4^{\circ} \mathrm{K}$. Dutton (1986) suggests $c_{w} \int_{T_{\text {top }}}^{T_{L C L}} q^{*} d \ln T \approx c_{w} \overline{q^{*}} \ln \left(T_{L C L} / T_{\text {top }}\right)$ where $\overline{q^{*}}$ is a suitable mean value. Simpson (1978) devises a slightly more accurate linear approximation to this term, and Bolton (1980) describes how it can be solved numerically. In addition, Bolton (1980) computes an empirical equation for $\theta_{e}$ which takes this effect into account, and is used in this paper. It should be noted that most tephigrams igrore the contribution of specific heat of water (Simpson 1978).

${ }^{10}$ All equation variables in Section 3.2 .1 conform to the SI convention of $\mathrm{Pa}, \mathrm{g} \mathrm{g}^{-1}$ and ${ }^{\circ} \mathrm{K}$. 
with the saturated mixing ratio ${ }^{11}$ computed as:

$$
q^{*}=\frac{0.622 e^{*}(T)}{p-e^{*}(T)}
$$

and the saturation vapor pressure $e^{*}$ is computed using the empirical formula from Bolton (1980) with an accuracy of $0.1 \%$ for $-30^{\circ} \leq T \leq 35^{\circ} \mathrm{C}$ :

$$
e^{*}=611.2 \exp \left[\frac{17.67(T-273.15)}{T-29.65}\right]
$$

Combining the definition of $\theta=T\left(p_{o} / p\right)^{\frac{R}{c_{p}}}$ where $p_{o}=10^{5} \mathrm{~Pa}$, the empirical formula for $e^{*}$ (Eq. (3.10)), $q^{*}$ from Eq. (3.9), and the empirical $\theta_{e}$ formulation (Eq. (3.5)), an iterative solution for the temperature along the moist adiabat above the LCL $\left(T_{m}(p)\right)$ may be found by computing $\theta_{e}$ at the LCL and holding it constant with height. Although $q_{s f c}$ should be the same as $q_{L C L}^{*}$, sometimes these are different by an order of $0.01 \mathrm{~g} \mathrm{~kg}^{-1}$ due to the small errors associated with Eq. (3.6). Therefore, to maintain numerical consistency with the iteration, $q_{L C L}^{*}$ is computed from Eq. (3.10) and Eq. (3.9) after $p_{L C L}$ is found from Eq. (3.7). $T_{m}(p)$ is solved using a standard bisection technique (Press et al. 1988), ${ }^{12}$ and the iteration is stopped when the absolute value of the residual $|R e s|$ is less than $0.001^{\circ} \mathrm{C}$ for the following equation:

$$
R e s=T_{m}\left(\frac{p_{o}}{p}\right)^{\frac{R}{c_{p}}} \exp \left\{\left[\frac{622 e^{*}\left(T_{m}\right)}{p-e^{*}\left(T_{m}\right)}\right]\left[1+\frac{0.50382 e^{*}\left(T_{m}\right)}{p-e^{*}\left(T_{m}\right)}\right]\left[\frac{3.376}{T_{m}}-0.00254\right]\right\}-\theta_{e}
$$

where the full $e^{*}\left(T_{m}\right)$ expression from Eq. (3.10) must also be explicitly included in the iteration for $T_{m}(p)$. The ASI process will now be qualitatively illustrated.

\footnotetext{
${ }^{11}$ Some meteorologists drop the $e^{*}$ term in the denominator of $q^{*}$. This approximation will yield errors in the tropics where RH and temperatures are high, and will also gives a $q_{L C L}$ inconsistent with the LCL temperature empirical formula. As a result, the errors in the hydrostatic pressure calculations in the ASI process grow as large as $10-20 \mathrm{mb}$ !

${ }^{12}$ All iterations in this manuscript are solved using the bisection method, since a solution can always be obtained, unlike other techniques (i.e., Newton's method) which are computationally faster but do not always converge. Today's computers are fast enough that the difference in computational speed is negligible.
} 


\section{Qualitative description of ASI process}

The ASI feedback is illustrated schematically in Fig. 3.8 using the Jordan (1958) hurricane season sounding as a reference environment. To emphasize some of the key elements in the ASI process, several assumptions are made. These suppositions are a constant surface temperature, a pseudoadiabatic process, no latent heat of fusion. no latent heat of sublimation, and no entrainment. The path the parcels follows during ascent is also not considered (i.e., vertical or slantwise ascent). As will be discussed in Chapter 7, proper treatment of boundary layer cooling, entrainment, water loading, ice microphysics, buoyancy, eyewall temperature adjustment, and a slanted eyewall are needed for a realistic demonstration of the ASI process.

The ASI process begins with an air parcel lifted dry adiabatically to the LCL, and moist adiabatically upon saturation (which is manifested as a constant $\theta_{e}$ value) to $100 \mathrm{mb}$. $T_{m}(p)$ above the LCL is computed from Eq. (3.11). There is a slight contribution to buoyancy from moisture $\left(q^{*}\right)$ as $T_{m}$ is converted to $T_{v m}$. A small percentage of the buoyancy is converted to eyewall warming (increasing the cloud temperature $T_{c}$ ), while the rest propagates away in gravity waves. Upon computing the corresponding virtual temperature for $T_{c}\left(T_{v c}\right)$, this warmer inner-core gives a new $p_{s f c}$ which is computed from Eq. (3.2) and Eq. (3.3). For this new surface pressure, a parcel is lifted dry adiabatically again with the same surface temperature to saturation, upon which it follows the moist adiabat - but since the parcel's ascent begins at a lower pressure relative to the tropical environment than computed during the last moist ascent, the parcel's moist entropy $\left(\theta_{e}\right)$ will increase. Physically, this means that $T_{v m}$ along the entire column will increase, maintaining buoyancy despite the development of the warm core aloft, and the TC can continue to intensify. In other words, since $\theta_{e}$ and $p_{s f c}$ are functions of each other, for an isothermal process and constant $\mathrm{RH}$, a feedback mechanism begins in which pressure falls lead to increased $\theta_{e}$ and hence to further pressure falls etc. In a sense, the ASI process results from a continuously elevated heat source. Figure 3.8 qualitatively shows "snapshots" of the ASI process for eyewall surface pressures of 1015, 985, and $955 \mathrm{mb}$ with a constant surface temperature of $28^{\circ} \mathrm{C}$ and relative humidity of $90 \%$. 


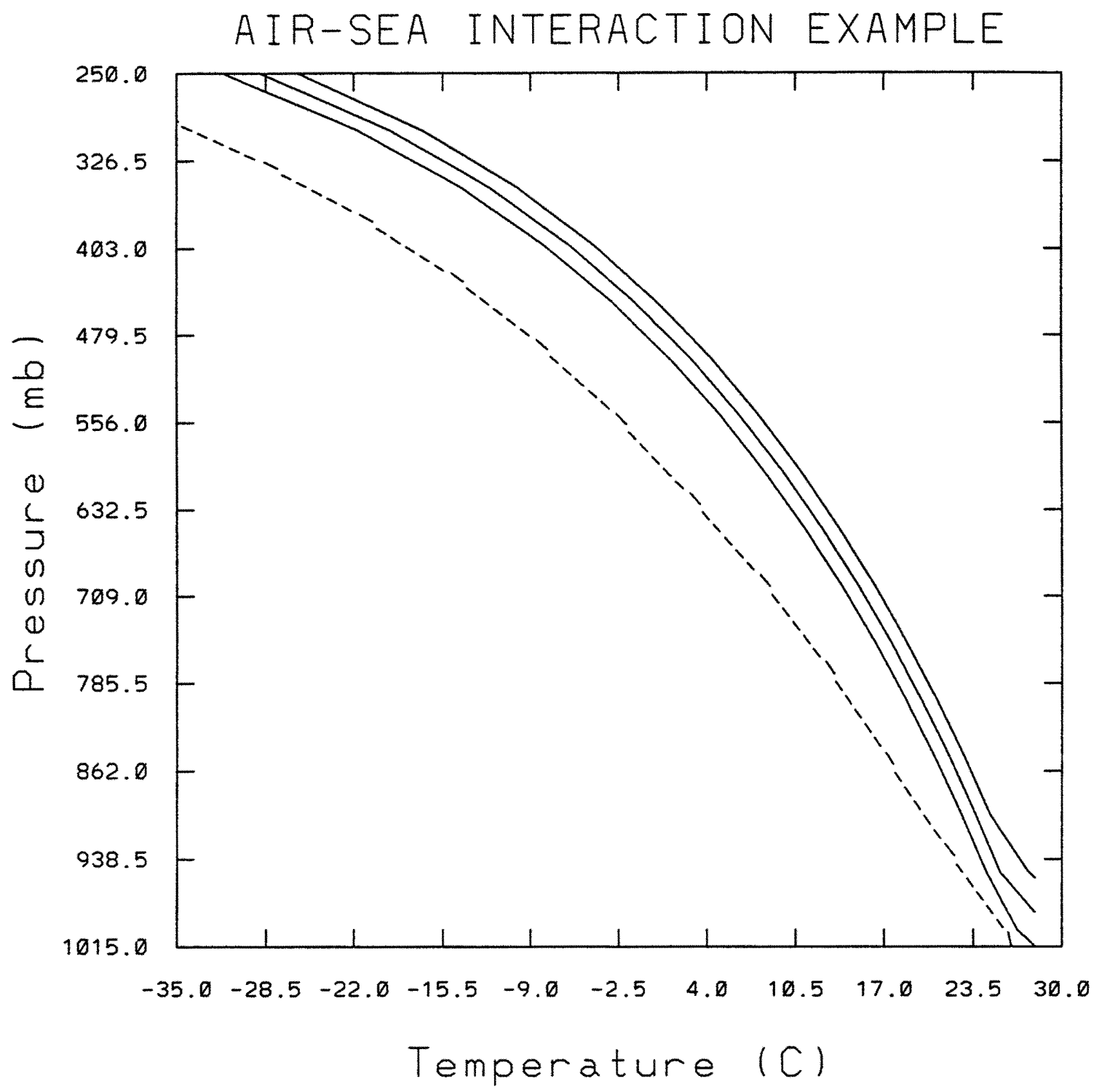

Figure 3.8: Example of the Air-Sea Interaction (ASI) process for a surface temperature which remains constant and is $1^{\circ} \mathrm{C}$ colder than an SST of $29^{\circ} \mathrm{C}$. It is assumed that the ascending parcel follows a pseudoadiabatic process with no latent heat of fusion. Dashed line is the environmental temperature profile (Jordan 1958). The solid lines represent the temperature along a moist adiabat $\left(T_{m}(p)\right.$, or more precisely, a constant $\theta_{e}$ value) corresponding to successive pressure falls at a constant surface temperature of $T=28^{\circ} \mathrm{C}$ and eyewall relative humidity of $90 \%$. The following surface pressure values are used: 1015,985 , and $955 \mathrm{mb}$. The corresponding $\theta_{e}$ values are $363.6,369.0$, and $374.8^{\circ} \mathrm{K}$. Due to the ASI process, buoyancy can be maintained in an eyewall despite the development of the warm-core aloft. 
Implicit in this discussion is that a small portion of the latent heating is converted to increase the kinetic energy of the axisymmetric circulation. The rest of this heating is used to drive the secondary circulation and then radiated away as gravity waves. ${ }^{13}$ This conversion rate is a complicated function of inertial stability and static stability which may be quantified by the length-scale of the TC compared to the the Rossby Radius of Deformation $\lambda_{R}$ (Eliassen 1951; Cotton and Anthes 1989):

$$
\lambda_{R}=\frac{N H}{(\zeta+f)^{1 / 2}\left(2 v_{t} / r+f\right)^{1 / 2}}
$$

where $N$ is the Brunt-Väisälä frequency, $H$ is the scale height of the circulation, $\zeta$ is the relative vorticity, and $f$ is the Coriolis parameter. $\lambda_{R}$ was first defined by Rossby (1937) in Cartesian coordinates for the mutual adjustment of mass and momentum fields to an impulsively generated current. Here Eq. (3.12) is a more generalized form of $\lambda_{R}$ in which cylindrical coordinates is now considered, and $\lambda_{R}$ is the length-scale relevant to adjustments between $p$ and $v_{t}$ with a background vorticity field. A shallow water derivation of Eq. (3.12) is included in Appendix A, and a more in-depth discussion of $\lambda_{R}$ is performed in Chapter 6. Briefly, the conversion rate increases for decreasing static stability (in the numerator) and increasing inertial stability (in the denominator), manifested as a smaller $\lambda_{R}$. In other words, a nonlinear geostrophic adjustment feedback exists in which a developing TC (due to increasing rotation) can convert more and more of the available heating into kinetic energy as it strengthens. In an intensifying TC, an increased proportion of the heating is retained to develop the warm core as the pressure falls.

Assuming enough eyewall ascent exists to continue the intensification, the ASI process can continue until: 1) its Maximum Potential Intensity (MPI) is reached (to be discussed in more detail later); 2) the storm loses its energy source via decreasing SSTs or by

\footnotetext{
${ }^{13}$ Satellite images one minute apart (recorded by rapid scan techniques) and looped clearly show these gravity waves. The generation of gravity waves may be envisioned as follows: a steady heat source disrupts the mass field, exciting a gravity wave. This wave includes only sinking motions since the heat source does not decay, and propagates away from the disturbance. If the heat source decays to zero, then another wave propagates away from the disturbance with an equal but opposite amplitude. Therefore, pulsing convection will launch a family of gravity waves whose horizontal wavelength is proportional to the scale of the convective disturbance.
} 
landfall; or 3) the storm's convective environment is disrupted by environmental or external influences, such as wind shear, dry air intrusion, or insufficient low-level inflow to maintain development (i.e., during a concentric eyewall cycle).

\section{Sensitivity of ASI process to relative humidity and surface temperature}

The importance of SST may now be explored. As shown in Fig. 3.9, what appears as small boundary layer temperature differences is manifested as large buoyancy differences aloft. This is because buoyancy increases nonlinearly for increased $T_{s f c}$. At constant

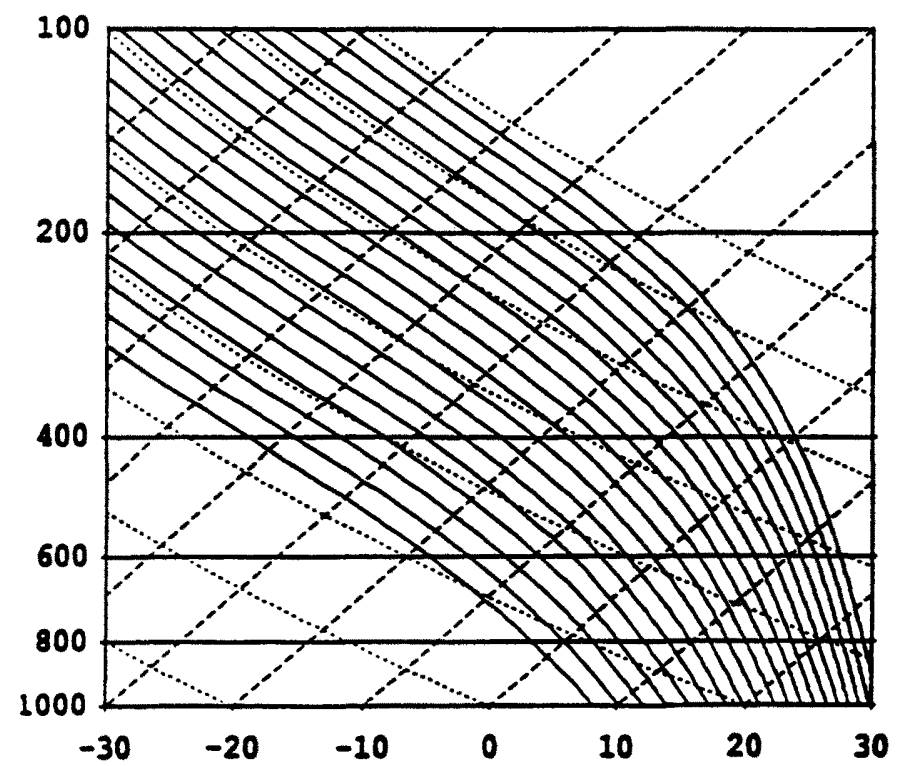

Figure 3.9: Skew- $\mathrm{T}$ diagram showing moist adiabats from $300^{\circ} \mathrm{K}$ to $390^{\circ} \mathrm{K}$ with $5^{\circ}-\mathrm{K}$ intervals. The left axis is pressure in $\mathrm{mb}$, the bottom axis and the long dashes are for temperature with a $10^{\circ}-\mathrm{C}$ interval, and the short dashes are for potential temperature with a $20^{\circ}$-K interval. Buoyancy increases nonlinearly aloft for increases of surface temperature. From Handel (1990).

$\mathrm{RH}_{\text {sfc }}$, small changes in surface temperature lead to much larger changes in $\theta_{e}$, with the slopes of $\theta_{e}$ decreasing aloft for increasing $T_{s f c}$. Furthermore, $\theta_{e}$ increases nonlinearly at the surface as a function of $T_{s f c}-$ assuming surface saturation, $\Delta \theta_{e}=21.6^{\circ} \mathrm{K}$ for an increase of $T_{s f c}$ from 20 to $25^{\circ} \mathrm{C}$, while $\Delta \theta_{e}=28.0^{\circ} \mathrm{K}$ for an increase of $T_{s f c}$ from 25 to $30^{\circ} \mathrm{C}$.

Pressure drop is further accelerated by the " $d p / p$ effect" from the thickness equation (Eq. (3.2)) (Holland 1995; Haurwitz 1935). Consider one TC eyewall which contains 
a small amount of buoyancy due to marginal SSTs while another TC contains a larger amount of eyewall buoyancy due to very warm SSTs. Should (hypothetically) the conversion rate in the geostrophic adjustment process be equal in both cases, the warm core will develop faster in the latter case and lead to faster eyewall pressure falls.

These combined nonlinear processes also explain the $26.5^{\circ} \mathrm{C}$ SST threshold for TC development (Palmén 1948). As noted by Emanuel (1986) and Holland (1995), for typical surface air temperatures above oceans cooler than $26.5^{\circ} \mathrm{C}$, buoyancy does not exist, and therefore a disturbance cannot intensify. ${ }^{14}$ This SST sensitivity explains: 1) why tropical disturbances (depressions) do not form into TCs over colder water; and 2) why TCs rapidly lose convection as they move over colder waters, although the rotation will persist for a few days due to their inertial stability.

The ASI process is very sensitive to $\mathrm{RH}_{\text {sfc }}$, and this is one of the difficulties in resolving precise buoyancy values. For example, for $\mathrm{p}=1015 \mathrm{mb}$ and $T_{s f c}=26.3^{\circ} \mathrm{C}, \theta_{e}$ for $\mathrm{RH}$ values of 80,90 , and $100 \%$ is $348.7,355.1$, and $361.5^{\circ} \mathrm{K}$, respectively (a $6.4^{\circ} \mathrm{K}$ increase for a $5 \%$ $\mathrm{RH}$ change). This sensitivity is even greater for higher temperatures. For $\mathrm{p}=1015 \mathrm{mb}$, $T_{s f c}=28.0^{\circ} \mathrm{C}$, and $\mathrm{RH}$ values of 80,90 , and $100 \%, \theta_{e}$ is $356.4,363.6$, and $370.9^{\circ} \mathrm{K}$, respectively (a $7.2^{\circ} \mathrm{K}$ increase for a $5 \% \mathrm{RH}$ change, and with larger $\theta_{e}$ values than for $T_{s f c}=26.3^{\circ} \mathrm{C}$ ). . The $\mathrm{RH}$ sensitivity also explains why landfalling TCs weaken so quickly, even if land $T_{s f c}$ is relatively the same - due to lack of moisture fluxes $q$ decreases through dry air mixing of continental air and downdrafts. ${ }^{15}$ Usually $T_{s f c}$ also decreases over land, which also accelerates the disintegration of TCs.

\footnotetext{
${ }^{14}$ The SST sensitivity - and the ASI process in general - can also be described in terms of a moist static energy $(h)$ budget. Tropical systems tend to export more $h$ at the top of the troposphere than they import in the lower levels, hence tropical cloud clusters tend to dissipate over colder waters. In the same context, tropical cyclones can intensify only if the enhanced surface fluxes associated with the ASI process can overcompensate the large export of $h$ from the upper eyewall (Gray 1982).

${ }^{15}$ Hurricane Andrew actually weakened very little upon passage over south Florida. Mayfield et al. (1994) speculate that a combination of low friction and high surface moisture from the Everglades helped maintain the storm until it reentered the Gulf of Mexico. Modeling results of a TC passage over a wetlands area support this reasoning (Tuleya 1994).
} 
The Maximum Potential Intensity (MPI) issue

An intriguing and controversial issue is what determines the MPI of a TC for a given SST. A view held by some researchers (Holland 1995), and by this author until recently, is that as $T_{v c}$ warms in the eyewall, an air parcel following the moist adiabat $\left(T_{v m}\right)$ becomes less and less buoyant, even with isothermal warming. In other words, the ASI process is able to overcome eyewall stabilization but the rate of compensation decreases for each pressure fall. Eventually intensification halts, and the MPI is reached. In Fig. 3.8, this would be manifested as converging $\theta_{e}$ lines as the storm approaches its MPI.

However, a closer scrutiny of this ASI approach - and results to be shown in Chapter 7 - reveals flaws to the stabilization theory. The most contentious issue is the evolution of the eyewall temperature field. Preliminary calculations show that $T_{v c}$ only slightly increases in the lower eyewall as a TC develops, and that most of the warming occurs aloft during the geostrophic adjustment process (see Chapter 7). Furthermore, the eyewall slopes outwards, especially above $300 \mathrm{mb}$, and an ascending air parcel can therefore avoid much of the warming above the radius of maximum wind. Because of these two features, an air parcel following the moist adiabat actually remains buoyant throughout its ascent, even in a very intense TC. It would appear that the MPI must be determined by a dynamic limitation, not a thermodynamic restriction.

It is hypothesized that sufficient eyewall vertical motion is required to balance friction and the outflow of cyclonic tangential momentum aloft for development to continue. In other words, a TC can continue to intensify only when sufficient eyewall ascent (as determined by boundary layer forcing and buoyancy) exists such that friction and upper-level $v_{t}$ export are balanced, with enough vertical momentum remaining to intensify the TC (Gray 1995). Furthermore, although buoyancy is maintained in the eyewall, it fails to increase sufficiently to balance this requirement of increased vertical motion as the TC develops. Therefore, the MPI occurs when eyewall ascent does not increase enough to sustain intensification. This theory is discussed in Chapter 7.

Other problems exist in the ASI process described in this section. The effects of waterloading and ice processes need to be included. How the ASI process can be maintained 
with boundary layer cooling also needs to be explained. Furthermore, the lateral extension of the eye above a sloping eyewall is a crucial component in the ASI process, especially with boundary layer cooling, and needs to be considered. Finally, entrainment must also be accounted for. All of these elements will be discussed in this paper, and a revised description of the ASI process will be presented in Chapter 7.

\subsubsection{The role the eye plays in TC intensity change}

The extraordinary pressure drop observed in TCs once they attain hurricane status cannot be explained by conversion of latent heat to eyewall warming alone. An eye must also be included, which induces large pressure drops by strong subsidence warming in the upper-troposphere. Typically an eye forms near hurricane strength (Dvorak 1984). Figure 3.10 shows the large temperature anomalies in the eye of Hurricane Inez. The maximum temperature deviation occurs aloft, and decreases downward since the sinking of warm air is an indirect, energy consuming process, and due to mixing with eyewall air (Miller 1958a; Holland 1995). ${ }^{16}$ Using Eq. (3.2) for the temperature deviations in Inez gives a MSLP of $935 \mathrm{mb}$, close to the observed MSLP of $927 \mathrm{mb}$.

Also of interest is the temperature perturbation in the eyewall area (approximately 30 n.m.). Little to no temperature deviation exists below $500 \mathrm{mb}$, with the warming increasing above $500 \mathrm{mb}$ to $7^{\circ} \mathrm{C}$ at $300 \mathrm{mb}$. As will be discussed later, the warming between 500 and $300 \mathrm{mb}$ occurs because the geostrophic adjustment is most efficient in this region. The lack of a temperature anomaly in the lower eyewall region might also be due to mixing processes and evaporative cooling by rainfall. Vertical motion cross-sections (not shown) indicate that above $300 \mathrm{mb}$ is the lateral extension of the eye over a sloping eyewall, which from hydrostatic arguments would greatly enhance the ASI process below the eyewall. As will be seen in Chapter 7, the eyewall slope, and the superposition of the edge of the eye directly above, is a crucial factor in maintaining the ASI process in the presence of surface boundary layer cooling under the eyewall.

\footnotetext{
${ }^{16}$ Holland (1995) explains how to simulate eye subsidence using the method of Miller (1958a).
} 


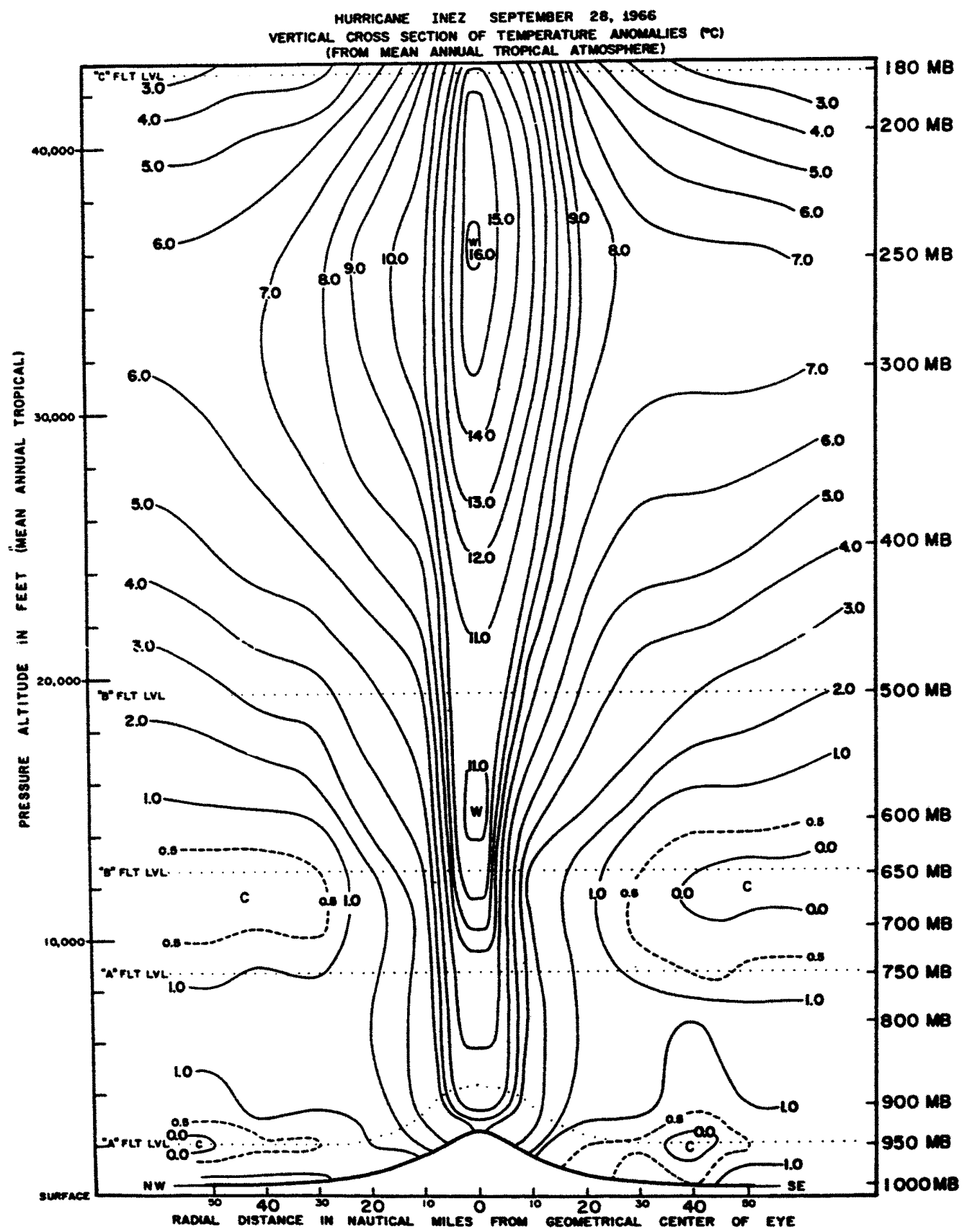

Figure 3.10: Vertical cross-section of temperature anomaly for Hurricane Inez on 19 UTC 28 September 1966. From Hawkins and Imbembo (1976). 
The formation of an eye is still the subject of much research, and the reason why ascent starts to occur away from the center when $V_{\max } \approx 55 \mathrm{kts}$ (near hurricane strength) is still controversial. ${ }^{17}$ Once the eyewall forms, subsidence in the center occurs as a response to the loss of mass. ${ }^{18}$ The rate of subsidence increases - and the level to which subsidence penetrates in the eye decreases in altitude - in intensifying storms when compared to steady state or weakening storms (Jordan 1961; Franklin et al. 1988). After an eye forms, eyewall surface pressure is estimated to be $50-67 \%$ (Holland 1995) to 85\% (Gray 1995) of central MSLP. The eyewall generally tilts away from the eye, and this slope increases with height.

\footnotetext{
${ }^{17}$ Malkus (1958), Kuo (1959), and Shea and Gray (1973) attribute eye formation to supergradient wind flow in which the centrifugal force $\left(v_{t}^{2} / r\right)$ becomes strong due to increasing winds and contracting radius of maximum wind; eventually the pressure gradient force can no longer balance the centrifugal force and air is forced to ascend away from the center. Thereafter, eye subsidence is maintained by mixing of the low momentum air in the eye with the high momentum air in the eyewall, in which further subsidence occurs to satisfy continuity requirements. Other researchers attribute eyewall formation to the "quadratic stress law." Defining friction at the top of the boundary layer as $\frac{1}{\rho} \frac{\partial \tau}{\partial z}$, this law states that in a vortex maximum vertical motion $w_{\max }$ occurs in the center for linear stress $\tau=\rho k v_{t}$ (where $k$ is a drag coefficient), and $w_{\max }$ will become a ring displaced from the center for quadratic stress $\tau=\rho C_{D}\left|v_{t}\right| v_{t}$ where $C_{D}$ is another drag coefficient (Eliassen and Lystad 1977). Handel (1990) suggests that linear stress dominates in the initial stages of TC development, but as the hurricane stage approaches quadratic stress is dominant since Ekman pumping and cross isobar flow increase faster than linearly. In the latter theory, gradient balance above the boundary layer can still exist. Much disagreement exists over whether symmetrically averaged gradient balance exists (Willoughby 1990, 1991; Gray 1991). However, if one rejects supergradient winds as the cause then one must still explain why subsidence occurs in the center rather than weaker ascent or stagnant vertical motion. Anthes (1982) suggests deep eyewall convection causes air to sink on the side (forming an eye), after quadratic stress forces boundary layer convergence away from the center. This explanation is supported by modeling results which show subsidence occurring due to radial gradients of heating (Willoughby 1979).
}

${ }^{18}$ Subsidence in other rapidly rotating vortices has been observed or inferred. Laboratory vortex simulations (Ward 1972; Church et al. 1979) exhibit axial downdrafts when the "swirl" (approximately the ratio of rotation strength to updraft strength) is sufficiently large. Sinclair (1973) observed sinking air in the center of dust devils. Sufficient evidence of axial subsidence in tornadoes is still lacking, but indirect circumstantial evidence does exist. Lemon et al. (1982) observed a radar reflectivity minimum at the core of the Binger, Oklahoma tornado of 22 May 1981. Wylie and Anderson (1983) presented satellite images and photographs showing a warm temperature anomaly and a depressed area at the storm top, implying possible subsidence in the Binger tornado's core. Smith and Howells (1983) developed subsidence in a numerical vortex model on the scale of a tornado when the swirl ratio becomes large, as did Walko (1988). Walko (1988) concludes that an imbalance of the centrifugal force with the pressure gradient initiated central subsidence, and that momentum diffusion maintained the subsidence, in agreement with the theories of Malkus (1958), Kuo (1959), and Shea and Gray (1973). Since Walko (1988) uses a free slip surface boundary condition, this implies that the quadratic stress law is not the main cause of eye subsidence, but a similar experiment by Walko (1988) using a no-slip surface boundary condition implies that the quadratic stress law can modify the development of a sinking core. Therefore, indirect modeling and observational evidence implies that a "tornado eye" may exist, which may play an important role in determining the vortex's maximum wind. 
Eye formation is a key component in the rate of intensity change. Mundell (1990) showed that most rapid intensification occurs right before or at the onset of eye formation. The sudden pressure fall associated with eye formation dictates a sudden increase in the secondary circulation. The stronger boundary layer convergence would also create intense eyewall convection which could cause transient intensity changes. This combination can lead to rapid intensification. Eye formation, however, can also hurt intensification. The strong central warming aloft can stabilize the inner edge of the eyewall. Powerful updrafts can overcome this negative effect by creating strong inner-core upper-tropospheric winds. From thermal wind arguments, building a vertically stacked vortex can rearrange the temperature field such that instability is maintained in the eyewall. Furthermore, as just mentioned, a tilted eyewall also can avoid most of the stabilizing effects of the eye while benefiting from the eye's contribution to low surface pressure and enhancing the ASI process. These ideas will be discussed in Chapters 6 and 7 .

\subsubsection{Original Conditional Instability of the Second Kind (CISK)}

Possibly no theory quite captured the inquisitiveness of tropical meteorologists as CISK. It is interesting that a short (2 page) linear analysis in a 40 page modeling paper (Ooyama 1969) became the basis for most genesis and intensification TC research for 15 years. There are actually two versions of the general CISK idea: the linear stability analysis version and "conceptual nonlinear" CISK. ${ }^{19}$ Both are subjects of criticism today, though the conceptual version still warrants merit.

The flaws of linear CISK are easy to identify. Linear CISK simply predicts that, for a small disturbance to grow, the perturbation must retain enough initial rotation so that strong enough frictional convergence will drive convection out of a nearly saturated boundary layer. However, without internal friction, the fastest growth occurs for the smallest disturbances. Hack and Schubert (1986b) have also shown that realistic intensification

\footnotetext{
${ }^{19}$ Other versions of CISK theory exist. However, these have mostly served to distort the original intent of CISK. Handel (1990) discusses these theories, as well as "CISK parameterizations," in more detail.
} 
can only occur when the nonlinear momentum and thermodynamic terms are retained in the governing equations.

However, the linear version never was meant to explain TC development. As Ooyama (1982) writes, "Linear mathematics necessitated that the theoretical demonstration be confined to the growth of small-amplitude disturbances... This is technically inconsistent with the physical reasoning (of CISK)... The true concept of CISK... is appreciated only through the nonlinear model."

Another problem is that the ASI process is not often included in conceptual nonlinear CISK nomenclature by the general meteorology community. Conceptual nonlinear CISK usually is explained as follows:

1. A weak, rotating disturbance develops from some external process.

2. Organized convection occurs in the region of frictionally induced ascent.

3. A warm core gradually develops, and lowers surface pressure. As an instantaneous response, the secondary circulation increases to bring the system back into gradient and hydrostatic balance.

4. The transverse circulation advects more angular momentum inwards than is lost to friction, and to conserve angular momentum, the tangential wind increases.

5. The faster wind creates higher boundary layer convergence, which enhances convection. A feedback mechanism from steps 3 to 5 is initiated. ${ }^{20}$

The main flaw of this argument is obvious - the ASI process is not included. Indeed, this traditional conceptual CISK argument never increases the moist entropy of the boundary layer.

\footnotetext{
${ }^{20}$ Several additional points need to be made. First, steps 1 to 5 encompass both the genesis and intensification stages. Therefore, the transition from a depression to a tropical storm is confusing in this discussion. Second, as pointed out by Gill (1982), the hydrostatic assumption may not be valid in the genesis stage, which is governed by deep convection. Surface pressure may actually be determined by buoyancy forces instead (called "buoyancy pressure" by Bluestein (1993)). In this context, the secondary circulation in tropical disturbances and depressions is driven by buoyancy, and surface pressure is related to the vertical derivative of buoyancy, not an existing warm core.
} 
The ASI process may be easily incorporated into conceptual CISK by appending step 5 with " $\theta_{e}$ needs to increase enough to maintain convective instability," and indeed Ooyama (1969) noted this as well. The importance of ASI was fully understood by Ooyama, who devoted a whole section of his classic 1969 modeling paper to sensitivity studies on ASI. However, when most introductory (Ahrens 1991) textbooks - as well as many meteorologists - speak of CISK, they fail to take ASI into account, indicating a fundamental lack of understanding of its importance in TC development. Instead, a TC's development is often explained by meteorologists in a frictional forcing context in which Ekman convergence in the boundary layer creating clouds which results in a slight warming during the geostrophic adjustment process; this warming intensifies the TC and increases the Ekman pumping which generates a larger mass of clouds etc. This explanation, which many label as "CISK," is not incorrect but misses the fundamental link that buoyancy is maintained mostly by the ASI process, not increased Ekman pumping. It is doubtful stronger boundary layer convergence alone can overcome the stabilizing effect of the developing warm-core. Therefore, in the most general sense, the CISK theory is fading from acceptance among tropical meteorologists.

In a way, this is a shame. ASI is mostly a thermodynamic theory with implicit dynamics, while conceptual CISK explicitly incorporates dynamics and geostrophic adjustment processes. CISK can help explain some internal processes affecting intensity change and a storm's MPI if ASI is properly included. In particular, CISK can account for the intensification feedback processes of eyewall ascent generated by boundary layer forcing and buoyancy if the secondary circulation is strong enough to overcome frictional dissipation of $v_{t}$ and upper-level removal of cyclonic $v_{t}$. Without sufficient eyewall vertical velocity, a steady-state TC will result, even if the ASI process appears favorable, since $v_{t}$ budgetary requirements are not met. This process, called Internally Forced Convergence (IFC) by Gray (1995), helps sustain and enhance the secondary circulation in cooperation with the ASI process, and is analogous to the CISK ideas that sufficient boundary layer forcing is required for development. In turn, by lowering the surface pressure, radial inflow is enhanced which increases boundary layer forcing, and so on. The IFC concept will be 
expanded on in the next paragraph. Also included in CISK dynamics might be some answers to eye formation and the eye's role in intensification, although traditional conceptual CISK ignores the role of the eye in a TC's development (another often-mentioned CISK criticism).

\subsubsection{CISK with ASI and Internally Forced Convergence (IFC)}

The IFC component of intensification is best described by Gray (1995):

The amount of mass flux which can be processed by the eye-wall clouds...is the primary factor specifying how intense (a TC) can become. A TC can continue to intensify only while (eyewall) vertical motion causes enough inward radial convergence and down pressure gradient flow such that the requirements of surface frictional dissipation and upper-level tangential wind export can be met, and still have enough additional momentum that wind increase...can occur.

Gray (1995) conceptually demonstrates this in Fig. 3.11 for a steady-state situation in which the surface $v_{t}$ is twice as fast as in the upper-level (outflow) region. In this demonstration, only half of the inflow is available to balance surface frictional dissipation - the other half must balance the outflow $v_{t}$. This analysis shows that there must be an additional process to drive the TC's inflow into the eyewall. This internal mechanism (IFC) is boundary layer convergence and buoyancy which, by continuity arguments, supplements the rest of the inflow (mass flux). For intensification to occur, slightly greater than one unit of IFC must occur in this situation.

This IFC concept may enhance understanding of what ultimately bounds MPI. It is hypothesized that MPI is dynamically determined by restrictions that limit the necessary mass flux which sustains the IFC and ASI processes. One possible restriction is that a TC's inertial stability may increase to the point where the secondary circulation becomes too weak to sustain sufficient eyewall ascent. Another possible terminating factor is friction. The exact contribution of friction to the limiting process is not well-understood, but according to turbulence theory friction is proportional to $V_{\max }^{2}$ in a well-mixed boundary layer (Holton 1992). Therefore, friction is likely a partial limiting source. Another potential restrictive agent is that surface fluxes may not be able to sufficiently compensate expansional cooling in the boundary layer. 


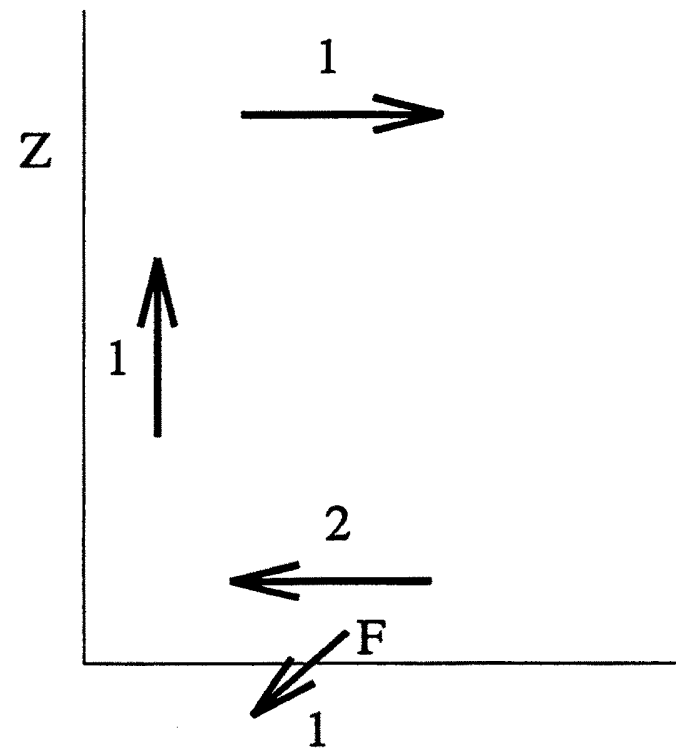

Figure 3.11: Idealized inner-core TC tangential wind budget where two units of low-level $v_{t}$ import by the radial wind $u_{r}$ must occur to balance one unit of frictional dissipation $(F)$ and one unit of upper-tropospheric outflow of $v_{t}$. Quantitatively, the arrows represent a $u_{r} \times v_{t}$ budget. The eyewall $v_{t}$ vertical distribution is such that the the upper-level $v_{t}$ is half of the surface $v_{t}$. Adapted from Gray (1995).

A positive factor to IFC (and thus MPI) is Ekman pumping, which propels mass upwards at some unknown proportionality with TC intensity. The rate at which boundary layer forcing offsets restrictive agents is unclear. As can be seen, the MPI issue is an area which needs more research. Nevertheless, despite these other possible candidates which may explain MPI, it will be hypothesized in Chapter 7 that the ultimate determining force behind MPI is that buoyancy cannot increase enough to sustain the vertical motion required to balance friction and upper-level eyewall outflow.

As discussed previously, many researchers believe MPI is determined by eyewall stabilization through latent heating being converted to upper-level eyewall warming and by eye subsidence infringing on the inner edge of the eyewall. However, because the eyewall slopes with height, rising air parcels can avoid much of the eye warming. Furthermore, a theory by which a TC attempts to circumvent eyewall stabilization is discussed in this paper. This theory, first proposed by Mundell (1990), is based on thermal wind arguments and will be expanded upon in Chapter 6. It will be argued that a TC can overcome upper-level eyewall stabilization by building a vertically stacked vortex to $300 \mathrm{mb}$ such 
that $v_{t}$ does not decrease much with height. From thermal wind theory, the warming is confined to the uppermost layers of the eyewall, thereby maintaining instability in most of the eyewall. Furthermore, by placing the warming as high as possible, the ASI processes is maximized via eyewall surface pressure drops.

\subsubsection{The Carnot Cycle ASI theory}

The Carnot Cycle theory by Emanuel (1986) and refined in later papers (Emanuel $1988,1991)$ is a modified ASI theory in which the TC energy source is moist entropy in the boundary layer and cooling by radiation in the storm's outflow. This theory qualitatively explains the TC as a Carnot cycle "engine," and quantitatively produces MPI values based on a number of factors. The mathematical derivation is quite provocative, but recently has been criticized by Holland (1995) and Gray (1995).

To fully understand the Carnot Cycle theory, and its criticisms, a brief derivation will be shown here which roughly follows Emanuel (1988) and Emanuel (1991). ${ }^{21}$ Assuming a steady-state TC in which mechanical-energy generation balances boundary layer frictional dissipation, Emanuel (1991) has idealized the TC as a Carnot engine by combining Bernoulli's equation and the first law of thermodynamics. The former states that along a streamline:

$$
d \frac{1}{2}|\vec{V}|^{2}+d(g z)+\alpha d p+\vec{F} \cdot d \vec{l}=0
$$

where $\vec{V}$ is the vector velocity, $z$ the height above the surface, $\alpha$ the specific volume, $\vec{F}$ the frictional force per unit mass, $d \vec{l}$ an incremental distance along a streamline, and the other variables have been previously defined. For a moist system the thermodynamic equation is:

$$
T d s^{*}=c_{p} d T+L_{v} d q^{*}-\alpha d p
$$

where the variables have been previously defined and the effects of water loading and specific heat of water have been neglected. The latter two terms are retained to investigate

\footnotetext{
${ }^{21}$ Emanuel $(1988,1991)$ includes an energy removal term due to an upper-level anticyclone; $p_{s f c}$ is not very sensitive to this term (Holland 1995) and is excluded from the derivation.
} 
fully reversible thermodynamics in Emanuel (1988), and produces qualitatively similar Carnot cycle results but with smaller pressure drops.

The TC idealized as a Carnot cycle is shown in Fig. 3.12. For inflowing air in which the surface temperature $T_{s f c}$ remains constant, the moist entropy increases from point $a$ to $c$ (isothermal warming) due to decreasing pressure, is approximately conserved during moist adiabatic ascent from point $c$ in a sloping eyewall (slantwise convection) which parallels a constant angular momentum surface, and flows outwards to long distances (point $o$ ). Energy is lost by infrared radiation, and the parcel slowly subsides to compensate the cooling through compressional warming. Hence, from point o to $o^{\prime}$, the "mean outflow temperature" $\bar{T}_{\text {out }}$ remains constant. The outflow temperature is defined as the temperature along constant angular momentum surfaces at radii displaced far from the storm center (where the angular momentum surfaces are quasi-horizontal). The mean outflow temperature is an entropy weighted average defined by:

$$
\bar{T}_{\text {out }}=\frac{1}{s_{c}^{*} / s_{o}^{*}} \int_{o}^{c} T_{o u t} d s^{*}
$$

which is not the same value as the tropopause temperature as some tropical meteorologists believe. Finally, air sinks dry adiabatically back to point $a$, closing the loop. This represents a modified Carnot engine in which air flowing inward acquires moist entropy from the sea, ascends, and ultimately gives off heat at the colder mean outflow temperature. Furthermore, since for a cyclic process the internal energy does not change, the amount of work done by a system is equal to the net heat absorbed by the system - therefore, the work done by a steady-state TC maintains the kinetic energy of the storm against frictional dissipation.

This balance of friction and generation can be shown by substituting Eq. (3.14) into Eq. (3.13) and integrating around the loop which yields:

$$
\oint T d s^{*}=\oint \vec{F} \cdot d \vec{l}
$$

By assuming all the frictional dissipation is confined to the boundary layer such that $\int_{c}^{a} \vec{F} \cdot d \vec{l}=\oint \vec{F} \cdot d \vec{l}$ and assuming $\vec{V}(a)=\vec{V}(c)=0$, from Eq. (3.13):

$$
\int_{c}^{a} \vec{F} \cdot d \vec{l}=-\int_{c}^{a} \alpha d p=R T_{s e a} \ln \left(\frac{p_{\text {env }}}{p_{s f c}}\right)
$$




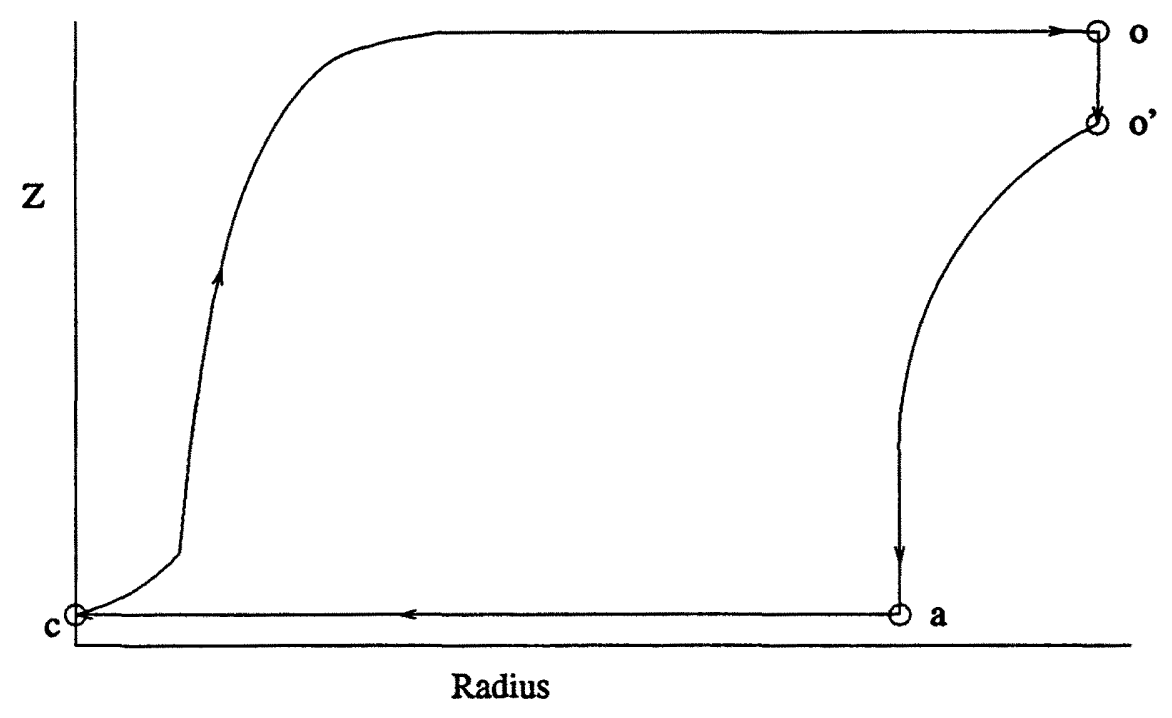

Figure 3.12: The hurricane Carnot Cycle. Air spirals in toward the storm center from point $a$, acquiring moist entropy from the ocean surface at fixed $T_{\text {sea }}$. It then ascends adiabatically from point $c$ flowing out near the storm top to some large radius (point $o$ ). The excess entropy is lost by isothermal radiational cooling between point $o$ and $o^{\prime}$ at a much cooler mean outflow temperature $\bar{T}_{\text {out }}$. The cycle is closed by traveling along the path dry adiabatically from point $o^{\prime}$ to $a$. The adiabatic paths are also assumed to be along constant absolute angular momentum lines, and therefore the eyewall motion represents slantwise convection. Adapted from Emanuel (1991).

Meanwhile, using the fact that $s^{*}$ is conserved along the adiabatic branches so that $\oint T d s^{*}=\left(T_{\text {sea }}-\bar{T}_{\text {out }}\right)\left(s_{c}^{*}-s_{a}^{*}\right)$ with $\left(s_{c}^{*}-s_{a}^{*}\right)=\frac{L_{v}}{T_{\text {sea }}}\left(q_{c}^{*}-q_{a}^{*}\right)+R T_{\text {sea }} \ln \left(\frac{p_{\text {env }}}{p_{s f c}}\right)$, one obtains:

$$
\oint T d s^{*}=\eta L_{v}\left(q_{c}^{*}-q_{a}^{*}\right)+\left(T_{\text {sea }}-\bar{T}_{\text {out }}\right) R \ln \left(\frac{p_{\text {env }}}{p_{s f c}}\right)
$$

where $\eta$ is the thermodynamic efficiency given by:

$$
\eta=\frac{T_{\text {sea }}-\bar{T}_{\text {out }}}{T_{\text {sea }}}
$$

By combining Eqs. (3.16), (3.17), and (3.18), and with some algebraic manipulation of mixing ratio and $\mathrm{RH}$ definitions, the final result is:

$$
\ln \frac{p_{\text {env }}}{p_{s f c}}=\frac{\eta L_{v}}{R \bar{T}_{\text {out }}} \frac{\mathrm{RH}_{\mathrm{sfc}}}{\mathrm{RH}_{\mathrm{env}}}\left(\frac{p_{\text {env }}}{p_{\text {sfc }}}-\frac{\mathrm{RH}_{\mathrm{env}}}{\mathrm{RH}_{\mathrm{sfc}}}\right)
$$

which may be rewritten as:

$$
\ln y=\frac{A}{B}(y-B)
$$


where $A=\frac{\eta L_{v}}{R T_{\text {out }}}, B=\frac{\mathrm{RH}_{\text {env }}}{\mathrm{RH}_{\text {sfc }}}$, and $y=\frac{p_{\text {env }}}{p_{s f c}}$. Since this is a complicated transcendental equation, the solution of $p_{s f c}\left(T_{\text {sea }}, \bar{T}_{\text {out }}, \mathrm{RH}_{\text {env }}, \mathrm{RH}_{\text {sfc }}, p_{\text {env }}\right)$ is not obvious and must be iterated. By setting $\mathrm{RH}_{\mathrm{env}}=75 \%, \mathrm{RH}_{\mathrm{sfc}}=100 \%$, and $p_{\text {env }}=1015 \mathrm{mb}$, Emanuel (1991) produced solutions of $p_{\text {sfc }}$ for $20^{\circ} \leq T_{\text {sea }} \leq 40^{\circ} \mathrm{C}$ and $-135^{\circ} \leq \bar{T}_{\text {out }} \leq 0^{\circ} \mathrm{C}$ (Fig. 3.13). One

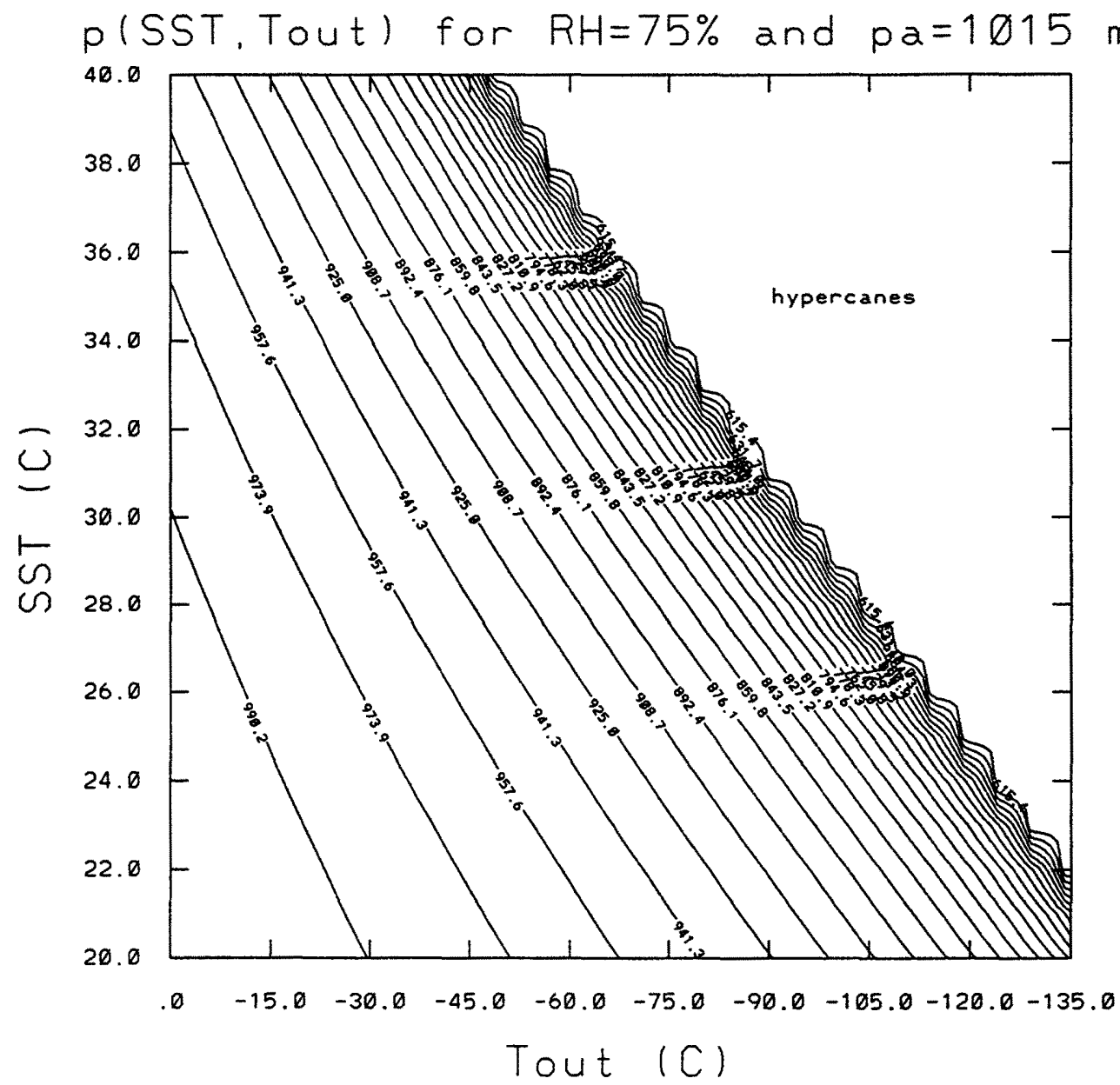

Figure 3.13: The maximum potential intensity (in $\mathrm{mb}$ ) as a function of SST and mean outflow temperature $T_{\text {out }}$ with $\mathrm{RH}_{\text {env }}=75 \%, \mathrm{RH}_{\text {sfc }}=100 \%$, and $p_{\text {env }}=1015 \mathrm{mb}$. Contour interval is $16.5 \mathrm{mb}$. The region labeled "hypercanes" represents a runaway surface pressure fall. Adapted from Emanuel (1991).

of the reasons for the initial popularity of this theory is the reasonable trend it produces in $p_{s f c}$ for a given range of $T_{\text {sea }}$ and $\bar{T}_{\text {out }}$ with the chosen values of $\mathrm{RH}_{\text {env }}, \mathrm{RH}_{\text {sfc }}$, and $p_{e n v}$, although in general the MPI values are too intense for cold SSTs compared to typical values (see Fig. 2.12). Emanuel (1986) states that a typical mean outflow temperature is $-70^{\circ} \mathrm{C}$, although the methodology from which this number is determined is not clear, 
nor should it be valid for all SSTs. Nevertheless, using a $\bar{T}_{\text {out }}$ of $-70^{\circ} \mathrm{C}$ for cold SSTs produces an MPI of $950 \mathrm{mb}$, while for a typical tropical surface temperature of $28^{\circ} \mathrm{C}$ an MPI of $920 \mathrm{mb}$ is obtained. A most remarkable finding is the large MPI sensitivity to $\bar{T}_{\text {out }}$ in this derivation.

Another intriguing result is a "hypercane" region for a sufficiently cold $\bar{T}_{\text {out }}$ and very warm $T_{s f c}$ combination (high efficiency $\eta$ ). In this region of the graph, no MPI solution exists. Emanuel (1991) interprets the hypercane region as a runaway instability in which isothermal warming and radiational cooling is so strong that friction cannot balance the energy input, and $p_{s f c}$ accelerates to $0 \mathrm{mb}$ !

However, Holland (1995) and Gray (1995) question the validity of the Carnot Cycle theory. First of all, Fig. 3.12 does not include a fundamental feature of a TC - the eye. The cycle explicitly incorporates air ascending and condensing in the center of the storm. Furthermore, the arbitrary choice of $\mathrm{RH}_{\text {sfc }}=100 \%$ in the storm center yields the maximum potential intensity, but does not have an observational justification. Frank (1977b) and Holland (1995) observe surface humidities of $95 \%$ and $85 \%$, respectively. As was discussed in the ASI section, pressure drop is very sensitive to surface relative humidity, and this dependency is also displayed in the Carnot Cycle theory. However, the Carnot cycle approach seems to be too sensitive to $\mathrm{RH}$ changes. When the same parameters used to produce Fig. 3.13 are substituted into Eq. (3.20) but with a reduced, more realistic, $\mathrm{RH}_{\text {sfc }}$ of $85 \%$, MPI values are too weak. For example, an SST of $28^{\circ} \mathrm{C}$ and $\bar{T}_{\text {out }}$ of $-70^{\circ} \mathrm{C}$ gives an MPI of $960 \mathrm{mb}$.

Emanuel (1986) understood that $100 \% \mathrm{RH}_{\text {sfc }}$ was an overestimate, but explained "the error works to compensate the neglect of subsidence warming in the eye, though there is no apparent reason to believe this compensation is exact." Indeed, the direct exclusion of an eye alone makes the validity of the Carnot Cycle theory suspect. Emanuel (1995) recently included partial eye dynamics in a revised set of equations.

There are other problems with the Carnot Cycle theory. The MPI in the Carnot Cycle equation is very sensitive to environmental RH (Holland 1995). Such pressure sensitivity to variations of ambient $\mathrm{RH}$ is not observed. The assumptions in deriving Eq. (3.20) 
are also questionable. The secondary circulation is not cyclic as assumed in Fig. 3.12. Therefore, the adiabatic compression branch ( $o^{\prime}$ to a) is very suspect. The other steps in this loop are also full of oddities, such as assuming a parcel is parallel to both moist entropy and angular momentum lines over a very long distance (c to o). The assumption that radiative cooling balances adiabatic compression such that $\bar{T}_{\text {out }}$ remains constant in the $o$ to $o^{\prime}$ isothermal loop is questionable indeed. The only loop that seems to be physically sound is the isothermal warming from a to $\mathrm{c}$ in which the parcel obtains moist entropy, and even that assumption falls apart in the light of new evidence of boundary layer cooling.

Finally, Holland (1995) shows that the ASI process incorporated with eye subsidence and some eyewall entrainment does not contain a runaway intensification solution (the "hypercanes") except under extreme, unrealistic conditions. Even for an outrageous SST of $40^{\circ} \mathrm{C}$, while using an environmental sounding in equilibrium with a $29^{\circ} \mathrm{C} \mathrm{SST}$, an MPI of $619 \mathrm{mb}$ is computed. Such an MPI is impressive but not a hypercane by definition. Even the latter non-hypercane example is flawed, because the environmental sounding would be in convective/radiative adjustment with a $40^{\circ} \mathrm{C} \mathrm{SST}$, producing less of a pressure drop. However, this dramatic example illustrates how fictitious hypercanes actually are. The Carnot Cycle, on the other hand, produces a hypercane for an SST of $40^{\circ} \mathrm{C}$ and $\bar{T}_{\text {out }}=-70^{\circ} \mathrm{C}$. (Fig. 3.13). ${ }^{22}$

\subsection{The debate over eyewall buoyancy}

One of the more surprising recent debates is whether buoyancy exists in the eyewall. Judging from Fig. 3.8, as well as the $v_{t}$ budget requirements dictated by the IFC mechanism, the answer would seem to be a convincing yes. A surface parcel with $85-95 \% \mathrm{RH}$ lifted moist-adiabatically has a certain amount of "CAPE," defined as (Ludlam 1980;

\footnotetext{
${ }^{22}$ The Carnot Cycle has captured the attention of environmental groups concerned about how TC intensity would be affected in a global warming world. It is important that these organizations and the general public understand the apparent major flaws in this theory.
} 
Bluestein 1993):

$$
\mathrm{CAPE}=\int_{L F C}^{E L} g \frac{T_{v m}(z)-T_{v c}(z)}{T_{v c}(z)} d z
$$

where $E L$ is the equilibrium level $\left(T_{v m}=T_{v c}\right)$ and $L F C$ is the level of free convection where a parcel is warmer than the surrounding cloud environment ("positive" buoyancy does not necessarily occur at the $L C L$ ). Since CAPE is the buoyancy force integrated with respect to height, it represents the work done on the parcel by the environment as the parcel is accelerated upwards. According to Bluestein (1993), for a parcel with an initial vertical velocity forced by boundary layer convergence $\left(w_{o}\right)$ in the vicinity of the $L F C$, and neglecting entrainment, water loading, precipitation loading, and nonhydrostatic vertical pressure gradient forces, this acceleration gives a maximum vertical velocity $w_{\max }$ of:

$$
w_{\max }=\left[2 \mathrm{CAPE}+w_{o}^{2}\right]^{1 / 2}
$$

However, some researchers have questioned whether such vertical instability exists in the tropics (Betts 1982; Xu and Emanuel 1989; Emanuel 1989a). They argue that including condensed liquid water $\left(q_{l}\right)$ substantially reduces buoyancy in tropical convection, and have extended this argument to the TC eyewall. While the effect of condensation on density has been known for quite some time, it had been thought until recently that most condensed water rained out before it could accumulate to the point of having a substantial effect on buoyancy. However, this conflicts with the appearance of echoes in all but the very highest levels in tropical convection and TCs, indicating that the density of suspended water must counteract buoyancy to some degree (Williams and Renno 1993). By including this "water-loading" effect and defining a "modified" CAPE as MCAPE (Stricherz 1989; Seitter and Kuo 1983):

$$
\mathrm{MCAPE}=\int_{L F C}^{E L}\left[g \frac{T_{v m}(z)-T_{v e}(z)}{T_{v e}(z)}-q_{l}\right] d z
$$

vertical velocities would be substantially reduced. As a rule of thumb, $3 \mathrm{~g} \mathrm{~kg}^{-1}$ liquid condensate is equivalent to $1^{\circ} \mathrm{C}$ negative buoyancy (Cotton and Anthes 1989). Furthermore, Emanuel (1989a) says that rain may increase the water content in the lower portion of the cloud, further enhancing the water-loading process. Another consideration, according to 
Emanuel (1989a), is that the evaporation of water during mixing reduces buoyancy. Xu and Emanuel (1989) indicate that cloud measurements usually indicate some dilution. ${ }^{23}$ Because of these three reasons, some researchers believe a parcel's ascent is nearly neutral with respect to the eyewall environment. In this context, eyewall convection is viewed as a passive response to intensification (Elsberry et al. 1992).

Even if one doesn't believe that a neutral convective state exists in the tropics, vertical velocities in tropical convection (including $\mathrm{TC}$ convection) are usually significantly smaller than indicated by Eq. (3.23), hence CAPE must be an overestimate (Williams and Renno 1993). However, aside from the fact that a neutral convective environment could not sustain the ASI process due to the $v_{t}$ budget requirements, there is still ample evidence that eyewall buoyancy exists. While eyewall vertical velocities are generally weaker than observed in mid-latitude convection (Jorgensen et al. 1985), strong updrafts have been documented on numerous occasions by observations (Burpee et al. 1989; Marks and Black 1990; Marks et al. 1992; Black 1993; Black et al. 1994), satellite inference (Gentry et al. 1980; Black et al. 1986; Steranka et al. 1986; Black 1987; Ebert and Holland 1992; Mundell 1990), and lightning inference (Lascody 1993; Lyons et al. 1989; Venne et al. 1989; Lyons and Keen 1994; Molinari et al. 1994).

Furthermore, tilted convection can circumvent the negative effects of precipitation by raining on the edge of the main updraft. Also, if condensation is accumulating at the bottom of the cloud then liquid water content must diminish aloft (which is a positive buoyancy effect). Another consideration is latent heat of fusion from ice formation - if liquid condensate is retained in a rising parcel then it must eventually freeze and increase

\footnotetext{
${ }^{23}$ Some researchers argue that the best measurement of CAPE for an ensemble of diluted cumulus clouds of different height is using the cloud work function $A(\Upsilon)$ as defined by Arakawa and Schnbert (1974) where $\Upsilon$ is a parameter that identifies a particular cumulus ensemble. The "quasi-equilibrium" postulate states that the a spectrum of entraining, diluted cumulus clouds which are nearly neutrally buoyant to the environment is more representative of the tropical atmosphere. The cloud work function is given by:$$
A(\Upsilon)=\int_{z_{b}}^{z_{t}(\Upsilon)} g \eta(z, \Upsilon) \frac{T_{v m}(z, \Upsilon)-T_{v e}(z)}{T_{v e}(z)} d z
$$

where $z_{b}$ and $z_{t}(\Upsilon)$ are the cloud base and cloud top heights, and $\eta(z, \Upsilon)$ is a cloud subensemble mass flux normalized at the cloud base. For nonentraining clouds, $\Upsilon=0, \eta(z, \Upsilon)=1$, then $A(\Upsilon)$ is equivalent to CAPE.
} 
the buoyancy in the upper portions of the clouds. Some research indicates that freezing increases the intensity of both updrafts and downdrafts (Lord et al. 1984; Wada 1989). Williams and Renno (1993), upon including ice processes in their CAPE calculations, state, "To a good approximation the negative buoyancy caused by condensate loading is compensated by the positive buoyancy caused by latent heat of fusion." Calculations in Chapter 7 also support this assertion. It is also important to realize that latent heat of fusion becomes important where latent heat of condensation is greatly reduced, since at colder temperatures $e^{*}$ with respect to water diminishes substantially. Finally, any conversion of latent heat of fusion and sublimation to warming which occurs high in the troposphere can accelerate hydrostatic pressure drops due to the "dp/p" effect.

Hence, observations seem to indicate that eyewall buoyancy exists, and there are several ways in which "water-loading" can be avoided or partially balanced such that CAPE still exists. And while it is acknowledged that water-loading and entrainment reduce buoyancy, it will be shown in Chapter 7 that including ice and a slanting eyewall are important effects in the ASI process, especially when boundary layer cooling is considered. Furthermore, the resulting intense updrafts are an indication of future strengthening. These findings, and their application to forecasting, will be discussed in the next chapter and in Chapter 8.

This concludes the discussion on $\mathrm{TC}$ intensity change theories not requiring trough interactions. Particular emphasis was placed on a variety of ideas related to the Air-Sea Interaction (ASI) process. Clearly, a TC is a self-sustaining entity which requires no assistance from upper-level forcing, although trough interaction probably still occurs to some degree. However, upper-level forcing is by no means necessary for TC development. 


\section{Chapter 4}

\section{TROPICAL CYCLONE INTENSITY FORECAST SCHEMES AND FOCUS OF THIS STUDY}

This chapter presents evidence relating inner-core convective bursts to future intensification, and cites previous forecast applications of satellite technology to this idea. Also discussed are some of the subjective and statistical forecast techniques developed in the past 15 years.

The focus of this research is also outlined in Section 4.6. This study encompasses an observational, theoretical, and statistical approach to understanding and predicting TC intensity change. Section 4.6 describes the research methodology in Chapter 6, 7, and 8.

\subsection{Evidence of buoyancy, and the relationship of strong convection to future intensification}

This section confirms the existence of CAPE in at least a few TCs. Generally speaking, convective bursts only occur in developing tropical storms or maturing TCs, since secondary circulations are restricted in very intense TCs due to large inertial stability. Furthermore, the formation of potent convection and eyewall "supercells" tends to portend future intensification.

Studies of lightning data have established this technology as a possibly valuable forecast and analysis tool. Lightning flash rate is proportional to CAPE in tropical convection (Williams et al. 1992), and quite possibly in the TC eyewall, although further research is needed to confirm a relationship between eyewall convection and lightning. For now, it 
will be assumed that vigorous convection may be indirectly deduced from lightning data. ${ }^{1}$ Examples of storms with significant eyewall electrical activity include:

1. Diane (1984), the "unnamed tropical storm of 1987," and Florence (1988) (Lyons and Keen 1994; Venne et al. 1989)

2. Emily (1987) (Black et al. 1994)

3. Andrew (1992) (Lascody 1993; Molinari et al. 1994)

Furthermore, these storms intensified at a fast rate, with a time lag of 12 to $36 \mathrm{~h}$ after the peak lightning activity. Another study (Samsury and Orville 1994) shows no lightning activity within the eyewall region for Hugo (1989) which maintained its Category 4 intensity during the period of study, and no eyewall lightning in a weakening Jerry (1989). These observations of lightning also support the assertion that latent heat of fusion could be playing an important role in the rate of intensification, since ice must be present for lightning to occur.

Observational evidence of eyewall vertical velocity also supports this convective signal for future intensification. Black (1993) stratified vertical velocities for intensifying and weakening storms, and found that $43 \%$ of the intensifying storms contained updrafts greater than or equal to $10 \mathrm{~m} \mathrm{~s}^{-1}$ compared to $20 \%$ of the weakening storms; in addition, $32 \%$ of the filling storms contained weak updrafts (less than $7 \mathrm{~m} \mathrm{~s}^{-1}$ ) compared to $14 \%$ of the intensifying ones. Some of the updraft velocities were as high as 10 to $20 \mathrm{~m} \mathrm{~s}^{-1}$, and extended throughout the troposphere. In a case study by Black et al. (1994) of one

\footnotetext{
${ }^{1}$ Lightning is confined to convective regions in TCs. Black and Hallet (1986) found that supercooled water at upper levels existed only in very strong updrafts. As Lyons and Keen (1994) state, "This is because conditions supportive of electrical charge generation and separation leading to lightning discharges include intense vertical motions with a complex, mixed-phase distribution of supercooled droplets, ice, and graupel at temperatures from $-5^{\circ}$ or $-10^{\circ} \mathrm{C}$ or colder" (Williams 1988, 1989; Jayaratne et al. 1983). The results of Black and Hallet also may discredit the Project Stormfury hypothesis that seeding a TC away from the eyewall would weaken the storm - since outside convective regions little supercooled water exists above the melting level in the presence of abundant ice, seeding would be ineffective. Willoughby et al. (1985) discusses Project Stormfury and its possible theoretical flaws in greater detail. Another good review of Project Stormfury intended for the general public, with comments from believers and dissenters on the merit of this weather modification experiment, is presented by Posey (1994).
} 
of these TCs (Emily 1987), unusually strong updrafts of $24 \mathrm{~m} \mathrm{~s}^{-1}$ were observed during its rapid deepening phase. ${ }^{2}$

Many satellite studies also have construed intense vertical velocities. Ebert and Holland (1992) observed cloud-top temperatures as cold as $-100^{\circ} \mathrm{C}$ in Hilda (1990) during its intensifying and mature stage. Such cold temperatures dictate cloud terrets overshooting into the stratosphere as high as $19 \mathrm{~km}$. Ebert and Holland estimated updraft$\mathrm{s}$ at the tropopause level between 15 and $38 \mathrm{~m} \mathrm{~s}^{-1}$ for Hilda. "Hurricane supercells," defined as large overshooting cloud bursts which remain quasi-stationary in the same storm quadrant over a 12-18 h period, have also been observed (Black et al. 1986; Black 1987). In the case of Gladys (1975), this event marked the onset of rapid intensification (RI). Ebert and Holland (1992), Black et al. (1986), and Black (1987) all noted that an expanding, circular anvil resulted from the overshooting tops, sometimes obscuring the eye.

As previously mentioned in the discussion about the Rossby radius of deformation, another process contributing to development in a maturing convective $\mathrm{TC}$ is the efficien$t$ conversion of latent heat to kinetic energy through geostrophic adjustment processes (Rossby 1937; Schubert and Hack 1982; Hack and Schubert 1986b). Due to its inertial stability, a tropical storm or hurricane can more effectively transform heating to kinetic energy with less of the energy being radiated away as gravity waves. Therefore, it is not surprising that, through efficient ASI, geostrophic adjustment, and IFC processes, potent convection portends future intensification. From the theoretical and observational evidence presented here, it is almost undeniable that CAPE exists in TCs despite some water-loading, entrainment, and rainfall cooling. Indeed, several satellite forecast schemes have been developed on this premise.

\footnotetext{
${ }^{2}$ Lightning activity was intense during these periods. The NOAA P-3 aircraft was struck at least twice by lightning.
} 


\subsection{Satellite TC intensity forecast schemes}

Despite today's accessibility to satellite data, few objective TC intensity forecasting techniques exist based on this technology. The one exception is the "Dvorak scheme" (Dvorak 1975, 1984). Also, a scheme by Mundell (1990) is currently being tested at JTWC. Otherwise, the satellite currently only serves as a tool through which subjective forecasts are made. However, some research suggests that quantitative relationships between TC intensity change and satellite convection exist which would be very useful operationally.

\subsubsection{Early research studies on brightness temperature relationship to inten- sity change}

Gentry et al. (1980) found the cooler the mean brightness (equivalent blackbody) temperatures $T_{b}$ of the cloud tops over an 8-deg area, the stronger and more persistent is the convection and the more likely that maximum winds will increase with time. They found the time lag between $T_{b}$ and future intensification to be about 24-36 h. From these results, regression equations were developed using $T_{b}$ parameters which outperformed persistence at $24 \mathrm{~h}$ on independent data.

Steranka et al. (1986) expanded on Gentry et al.'s research by investigating areaaverage $T_{b}$ (within $2 \mathrm{deg}$ of TC center) from 309 observations of 12 TCs. It was observed that prolonged surges ( $9 \mathrm{~h}$ or more) of intense convection near the storm center (manifested as $6 \mathrm{~h}$ area-averaged $T_{b}$ running means colder than $-35^{\circ} \mathrm{C}$ ) was followed by intensification of $5 \mathrm{~m} \mathrm{~s}^{-1}$ within $24 \mathrm{~h}$ or more $71 \%$ of the time. However, when intense convection was not present, similar wind increases occurred only $37 \%$ of the time. Steranka et al. (1986) defined these prolonged surges of overshooting cloud-tops as "convective bursts," and these findings are consistent with this section. Multiple regression equations were devised which incorporated $T_{b}, T_{b}^{2}$, and current intensity. The predictive equations produced smaller errors than persistence and SHIFOR (a climatology/persistence multiple regression model (Jarvinen and Neumann 1979) which will be defined and discussed more in Section 4.4 and Chapter 8). 


\subsubsection{The Dvorak technique}

The Dvorak (1975) method is the primary satellite technique for estimating and forecasting the intensity of TCs. Its fundamental basis is climatology, based on hundreds of Atlantic hurricanes and tropical storms which were given an intensity range. This intensity range is given a "tropical number," or "T-number." These T-numbers are related to the TC life cycle, with T-number 1 (T1) and T-number 2 (T2) generally designating the depression stage with $V_{\max }$ of 25 and $30 \mathrm{kts}$ respectively, T3 and T4 in the tropical storm stage with $V_{\max }$ of 45 and $65 \mathrm{kts}$ respectively, and T5, T6, T7, and T8 in the hurricane stage with $V_{\max }$ of $90,115,140$, and $170 \mathrm{kts}$ respectively. "Half" T-numbers are also possible, such as $\mathrm{T} 2.5$ corresponding to roughly $35 \mathrm{kts}$.

The Dvorak technique employs a step-by-step procedure to estimate intensity. A TC is given an initial T-number based on pattern recognition, since clouds of TCs evolve through recognizable stages as the intensity increases. These features include: 1) the amount of curvature of the feeder band around the storm center, with the degree to which it "coils" around the center being proportional to intensity; 2) the amount of shear disrupting the cloud pattern; 3) the formation of an eye; and 4) the appearance of a large mass of clouds, known as the "Central Dense Overcast" (CDO), which is typically proportional to TC intensity. In a later version using an Enhanced InfraRed (EIR) technique, the temperature of the eye and the temperature of the coldest cloud band that surrounds the eye are also used to compute an initial T-number (Dvorak 1984).

After an initial T-number is determined, it is modified based on a set of rules which consider the past 24-h trend, the "expected" T-number based on the climatological lifecycle of a TC, and constraints within which the final T-number is expected to fall (i.e., the final T-number is not allowed to change by more than 2 numbers between T-numbers T2 and T6). One problem with these constraints is that fast intensifying TCs can intensify at greater than 2 T-numbers per day. Hence, the intensity analysis is sometimes wrong for fast intensifiers using this technique. There are other problems with the intensity analysis using Dvorak technique which will be discussed in Chapter 5. 
The final step involves converting the final T-number to a Current Intensity (CI) number. A CI number typically is the same as the T-number during the developing stage, but CI is larger than TI during the weakening phase since since there is a lag between the TC's actually intensity and a decrease in T-number.

The Dvorak intensity forecasting procedure uses extrapolation of the "expected" Tnumber based on the climatological life-cycle of a TC, and is modified if the cloud pattern is changing significantly or when the storm will be entering or leaving an environment of strong vertical wind shear, stratocumulus clouds (cold SSTs), land, or southward moving cirrus (also indicative of shear). The procedure only allows a maximum 24-h change of 1.5. This constraint is used to prevent over-forecasting since most storms are not fast intensifiers. Obviously, fast developing TCs will be poorly predicted. Furthermore, even though the Dvorak technique was developed from a large climatological database, both its development and operational implementation is subjective, leading to occasionally large intensity estimate errors which, as a consequence, must result in erroneous intensity forecasts. Mundell (1990) suggests that modifying the Dvorak scheme for hourly analysis instead of twice a day could reduce these analysis and forecasting errors.

\subsubsection{The Mundell scheme}

Building on the need for a more objective technique to forecast intensity change, Mundell (1990) developed a methodology to distinguish which storms will rapidly intensify and which storms will develop at a slower rate. The theoretical basis of Mundell's scheme relies on the relationship between eyewall (inner-core) winds and convection, outer-core winds and convection, and intensification. According to Weatherford and Gray (1988b), cyclone intensification is more complicated than just $V_{\max }$ increasing. Weatherford and Gray (1988a) and Weatherford $(1985,1989)$ state that the most "intense" typhoons (as defined by minimum sea level pressure) are not necessarily the "strongest" (based on outer-core winds). To clarify these statements, the following definitions are presented:

Cyclone intensity - the central pressure as measured by a dropwindsonde or the minimum 700-mb surface height (which Weatherford and Gray (1988a) found to be highly correlated with MSLP, with $99.4 \%$ variance explained (Weatherford 1985)). 
Table 4.1: Average MSLP, maximum sustained surface wind speed $\left(V_{\max }\right)$, and Outer-Core Strength (OCS - see text for definition) at onset of rapid deepening (pressure drop of at least $21 \mathrm{mb}$ in $12 \mathrm{~h}$ ) versus onset of slow deepening (pressure drop less than $5 \mathrm{mb}$ in $12 \mathrm{~h}$ ). The rapid deepening analysis contains 60 cases, and the slow deepening analysis contains 99 cases. Taken from Weatherford and Gray (1989).

\begin{tabular}{|l|c|c|c|}
\hline Rate of Increase & MSLP $(\mathrm{mb})$ & $V_{\max }\left(\mathrm{m} \mathrm{s}^{-1}\right)$ & OCS $\left(\mathrm{m} \mathrm{s}^{-1}\right)$ \\
\hline Rapid deepening & 945 & 44 & 18 \\
Slow deepening & 971 & 31 & 18 \\
\hline
\end{tabular}

Cyclone strength - an area-weighted average $700-\mathrm{mb}$ tangential wind speed from $1 \mathrm{deg}$ to $2.5 \mathrm{deg}$ with respect to the TC center.

Using these definitions, for rapid deepeners the Outer-Core Strength (OCS) to $V_{\max }$ ratio was $40 \%$ on average, whereas for slow deepeners this ratio was about $60 \%$ on average (Table 4.1). Outer-core winds of both classes were the same $\left(18 \mathrm{~m} \mathrm{~s}^{-1}\right)$. Apparently, as shown in Fig. 4.1, inner-core processes of rapid deepeners concentrate momentum more effectively than slow deepeners.

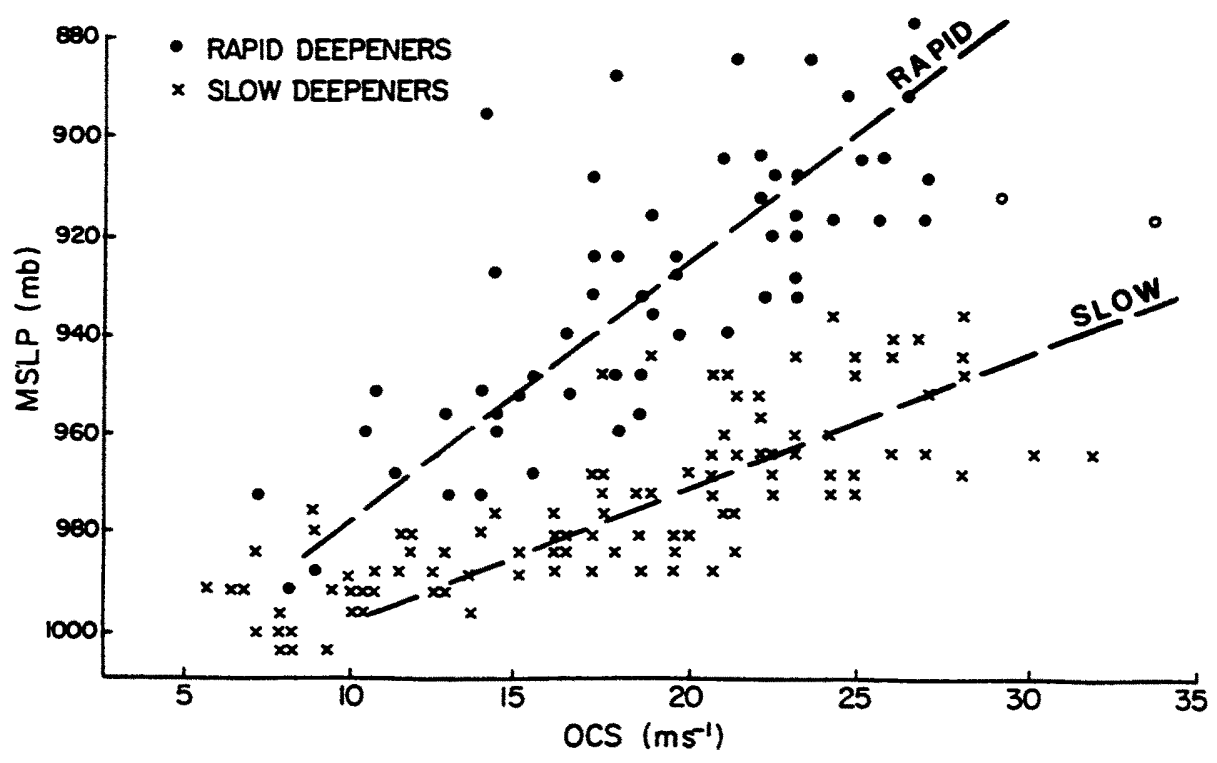

Figure 4.1: Scatter diagram of intensity (MSLP) versus OCS at onset of rapid deepening (denoted by circles) and at onset of slow deepening (denoted by X's). Taken from Weatherford and Gray (1989).

It follows that rapid intensification can be hindered if the OCS is large. In other words, while strong inner-core inertial stability is dynamically favorable (see Chapters 3 
and 6), strong outer-core inertial stability is not. Large horizontal distributions of inertial stability restrain momentum import into the center of the storm, and dampens inner-core convection. This constraint on inner-core convection and intensification by a "stiff" vortex was demonstrated in a meso-scale numerical model by Fitzpatrick (1992). It also follows that, since low-level convergence increases away from the eyewall region in a stiff vortex, convection will increase in the outer-core region. ${ }^{3}$ This may also explain why TCs with $V_{\max }>100 \mathrm{kts}$ generally do not rapidly deepen (Mundell 1990). In general, very intense storms likely have developed large OCS thus precluding RI, although intensification may still occur. These ideas are incorporated in Mundell's forecast scheme that will be discussed next.

Mundell developed a satellite scheme which measures the degree of inner versus outercore convection. Mundell hypothesized that high ratios of inner versus outer-core convection indicate future rapid intensification since inner-core processes would dominate over outer-core inertial stability. A way to measure the degree of convective concentration is to set up a cylindrical grid and count the number of pixels colder than a given brightness temperature threshold for inner radial bands (0-2 deg radius) and outer radial bands (2$4 \mathrm{deg}$ radius). This grid will be described in Chapter 5. An inner/outer convection ratio is computed, and large ratio values indicate concentrated deep convection. The $T_{b}$ threshold value chosen for the inner region was $-75^{\circ} \mathrm{C}$, and $-65^{\circ} \mathrm{C}$ was chosen for the outer area.

This satellite data is then smoothed by averaging the previous $12-\mathrm{h}$ and future $12-\mathrm{h}$ observations. The motive for smoothing satellite data is to remove the natural oscillatory behavior of convection so that the long-term behavior can be ascertained. Another reason for the 24-h smoothing is to eliminate the diurnal TC convective signals (Browner et al. 1977; Gray and Jacobson 1977; Hobgood 1986; Zehr 1987; Hallin 1991). Chapter 8 investigates whether temporal smoothing of satellite data as performed by some researchers (Steran-

\footnotetext{
${ }^{3}$ Wei and Gray (1985) proposed that the "Radius of $30 \mathrm{kts}$ winds" (an important parameter for marine interests) may be inferred from the edge of the intense convection based on this idea. Shoemaker (1989) found that 24-h running means of pixels colder than $-25^{\circ} \mathrm{C}$ within 4 deg of the TC center correlated with the Radius of $30 \mathrm{kts}$ winds.
} 
ka et al. 1986; Mundell 1990) correlates best with intensity change, or if unsmoothed satellite data at the beginning of the forecast period contains a stronger relationship.

When a plot of temporally smoothed inner and outer-core pixel counts is plotted versus time, a predictive signal seems to appear. According to Mundell, when the axes are scaled such that 200 pixels are subtracted in the 0 to 2 -deg region, the intersection of the inner and outer pixel counts is a favorable but not sufficient 12 -h precursor to the onset of RI (Fig. 4.2). The second (and sufficient) condition for RI is favorable climatology.

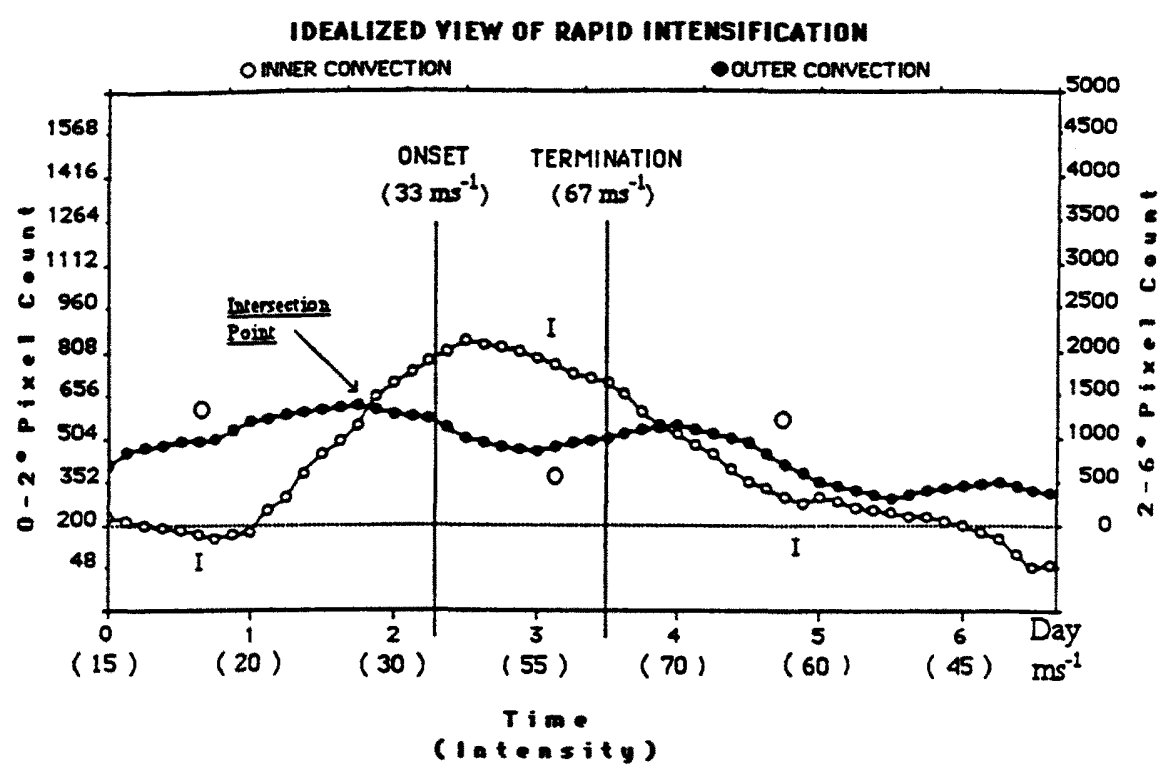

Figure 4.2: Mlustration of an idealized rapid intensification event predicted by the intersection of the relative concentrations of inner ( 0 to 2-deg radii) pixels colder than $-75^{\circ} \mathrm{C}$ versus outer ( 2 to 6 -deg radii) pixels colder than $-65^{\circ} \mathrm{C}$. According to Mundell (1990), as long as all climatological parameters are favorable, the onset of rapid intensity change is likely within $12 \mathrm{~h}$. Taken from Mundell (1990).

Mundell found the following climatological characteristics for rapid deepeners, which show a preference for geographic position, time of year, SST, and initial winds in the western North Pacific. It is very infrequent in the South China Sea and poleward of $22 \operatorname{deg} \mathrm{N}$, and during the off-season of December through June. Rapid deepening is most likely mid-July to mid-August and September to November, with a lull in-between because the western North Pacific monsoon trough shifts too far poleward (Lander 1995) over less warm SSTs. According to Mundell, the minimum SST needed for RI is $28.5^{\circ} \mathrm{C}$. Naturally, the favorable region shifts with the monsoon trough, but RI systems typically 
reach tropical storm and minimal typhoon intensities south of the seasonal mean latitude storm track, and never more than 1-deg north of the seasonal mean track. A minimum of $45 \mathrm{kts}$ is necessary before the onset of a RI event, with most beginning at $65 \mathrm{kts}$. Finally, as previously mentioned, RI is not likely after an intensity of $100 \mathrm{kts}$ is attained. If these conditions are not met, $\mathrm{RI}$ is not forecast even if the inner and outer pixel counts intersect.

Using the intersection technique with a climatology filter, Mundell forecasted 7 out of $10 \mathrm{RI}$ cases correctly. The three that were incorrectly forecasted he attributed to eye contraction, since there was not a concentration of deep convection near the eye and/or the satellite's inability to resolve the the warm temperatures near the small eye. Furthermore, of the 60 non-RI cases, 59 were forecast correctly since their pixel lines did not intersect or the case did not meet climatology.

While Mundell's work is impressive, it possesses several problems that this paper would like to improve upon. One criticism is the axis normalization procedure (Fig. 4.2) seems arbitrary and lacks a physical basis. Perhaps the normalization fits the 7 successful cases examined but may not represent such storms in other years - such is the problem with applying a subjective technique. One may also argue that the 70 total cases investigated represents a marginal data set.

In fact, when the intersection technique was used operationally at JTWC, the scheme more often than not failed in distinguishing RI and non-RI cases, mostly because the intersection did not occur at the proper time (D. Shoemaker, personal communication 1993). However, JTWC did notice that an increase in inner-core convection preceded RI, consistent with the previously cited studies in this paper.

\subsubsection{Analysis of satellite data in this report}

A major focus of this report is twofold: 1) To incorporate inner-core pixel counts in an intensity forecasting scheme by a more objective manner; and 2) To develop a better understanding of the role of convection in intensity change. How this is accomplished will be described in the next sections on intensity forecasting schemes. 


\subsection{Subjective TC intensity forecast schemes}

Although the Dvorak scheme and other satellite schemes are valuable components in intensity forecasting, most operational techniques incorporate a variety of parameters in making their predictions. Sometimes a tree diagram such as displayed in Fig. 4.3 is used which includes an assessment of vertical shear, SSTs, inner-core convection, dry-air intrusion, environmental interaction, proximity to land, etc..... (McBride and Holland 1987). A similar methodology is an "EXPERT" system which involves a decision tree, but the amount of forecasted intensity change is dependent on a balance of positive and negative factors (Zehr and Phillips 1994). The main criticism of these techniques is that both their development and implementation is subjective. In reality, even if a formal decision tree is not used, all forecasters base their decisions on an "if-then-else" process. One exception is a scheme based on statistics. ${ }^{4}$

\subsection{Statistical TC intensity forecast schemes}

The power of multiple regression analysis is the objective selection of predictors. This type of analysis benefits the researcher and forecaster in several ways:

1. Only the predictors which are "statistically significant" influences on the dependent variable (intensity change) are chosen - the other predictors are rejected. To a large degree, this procedure avoids the arbitrary selection of predictors in the decision tree development.

2 The importance of each significant predictor may be quantitatively assessed - in the decision tree development, the wrong amount of "weight" can be assigned to a predictor.

\footnotetext{
${ }^{4}$ It could be argued that this is not entirely true. For example, the researcher picks the variables to be investigated, and chooses a significance level. Therefore, there still is human intuition involved. Nevertheless, a statistical approach certainly reduces the amount of subjectivity involved in developing a forecast scheme.
} 


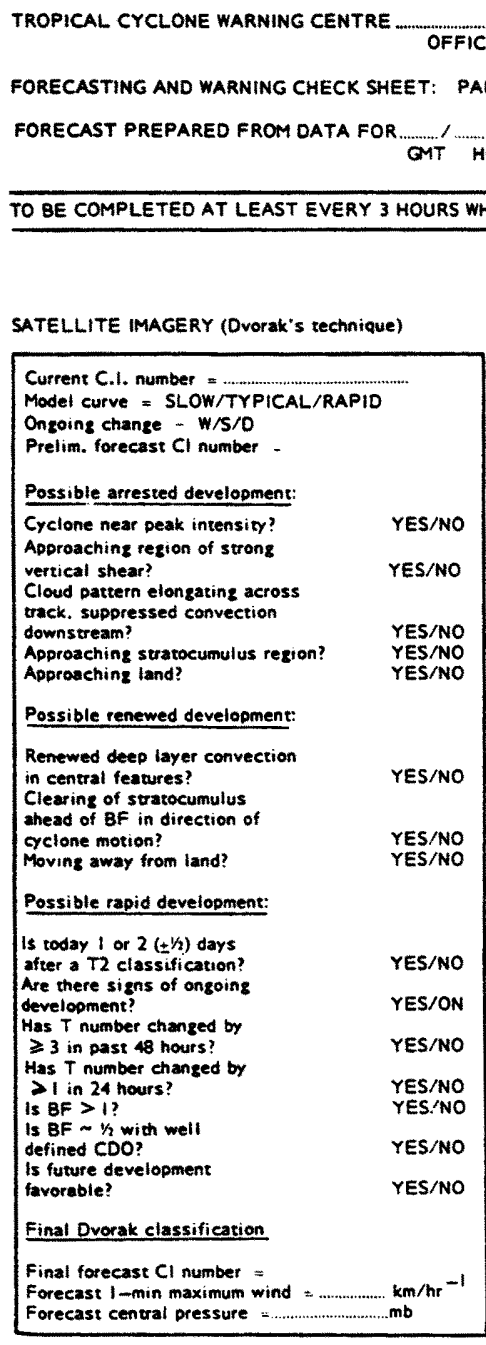

CYCLONE

$$
\text { FFICE }
$$

MOUR/DATE HOUR/DATE AT FORECASTER 
3. Furthermore, the true impact a predictor has on the dependent variable can be better determined since the variables are analyzed together instead of by single linear regression - it is not uncommon for a variable to be statistically insignificant in single regression but be significant in multiple regression because other variables are helping to explain the variance. In some cases, the regression coefficient may even switch signs between single and multiple linear regression analysis - see Figs. 2.14 and 2.15 .

4. The amount of variance explained by the regression is quantitatively determined by the coefficient of determination $R^{2}$, which measures the proportion of the variation in the dependent variable that is explained by the combination of the independent variables in the linear multiple regression model (Aczel 1989). Therefore, one can measure the "goodness of fit" of the regression from $0 \%$ (no linear correlation) to $100 \%$ (perfect linear correlation). The larger the $R^{2}$, the larger the confidence in the regression, and one can assess the explained and unexplained variations in the dependent variable for predictive and research purposes.

In other words, not only does multiple regression provide a forecast equation, but it generates understanding of intensity change processes through objective analysis. Of course, multiple regression suffers from a variety of problems as well, and these shortcomings will be described in Chapter 8.

The first intensity change regression model, developed for the Atlantic basin and based on climatology, is called the Statistical Hurricane Intensity FORecast, or SHIFOR (Jarvinen and Neumann 1979). Generation of the forecast equations is done by stepwise regression using postprocessed ("besttrack") TC data as input (see Chapters 5 and 8 for more details). There are seven significant quadratic predictors which include Julian day, initial storm latitude, initial storm longitude, zonal and meridional component of initial storm movement, initial intensity, and previous 24-h intensity change. As shown in Fig. 1.3, SHIFOR is still competitive with NHC. Recently, Landsea (1995) has slightly improved SHIFOR (dubbed "SHIFOR94") by including climatological shear, sub-surface 
SST, and climatological moist stability. A similar scheme for the western North Pacific was developed by Elsberry et al. (1975), and recently updated by Chu (1994). Chu's model is called the Statistical Typhoon Intensity FORecast model (STIFOR).

The drawback of the climatology/persistence schemes is obvious - without curren$t$ synoptic information available (especially wind shear), the errors are sometimes large. Indeed, the amount of variance explained by SHIFOR for 24-h intensity change is only about $30 \%$. Furthermore, SHIFOR forecasts fast intensifying storms poorly. This motivated researchers to include synoptic parameters.

The first attempt at incorporating synoptic parameters in a multiple regression model was performed by Pike (1985). Pike included the SHIFOR output and the 1000-700-mb thickness with only marginal improvement. Despite these limitations, this scheme was used for operational guidance at NHC under the acronym "SPIKE" until recently (Sheets 1993). Merrill (1987) considered a much broader range of predictors (synoptic, persistence, climatology, and SST) for the Atlantic. Merrill found that using POT (Eq. (2.4)) instead of SST explained more variance. He also concluded that wind shear was a statistically significant quantity and must be included in an intensity change regression scheme. However, Merrill's work was complicated by the inclusion of landfalling storms, which probably exhibit different statistical properties compared to open-ocean TCs. A year later, Elsberry et al. (1988) developed a regression scheme for the western North Pacific which included climatology, persistence, and synoptic predictors. Important synoptic parameters included environmental wind flows and wind shears, but unfortunately they were decomposed into empirical orthogonal functions (EOFs). ${ }^{5}$ A larger flaw was the direct exclusion of SST data, although SSTs were indirectly included in the climatology terms.

\footnotetext{
${ }^{5}$ EOFs extract spatial patterns in the environmental field. They indicate a particular arrangement of adjacent synoptic features. They also have the nice feature of reducing a spatial field with a lot of data to a few "principal components," thus: 1) effectively reducing the size of the data (statistically speaking, its dimensionality has been reduced); 2) retaining different geographic signals for different components; 3 ) explaining the amount of variance each component contributes to the field, with each component being uncorrelated to the other (hence the term orthogonal); and 4) showing which spatial pattern explains the most variance (component 1 ), with components $2,3,4 \ldots$... etc. explaining successively less variance. The reader is referred to Jolliffe (1990) for a nice summary on elementary EOF analysis. However, it is difficult to physically understand what these complex quantitative numbers represent, especially for operational forecasting purposes.
} 
DeMaria and Kaplan (1994b) expanded on Merrill's work with the Statistical Hurricane Intensity Prediction Scheme (SHIPS) for the Atlantic Basin. The sample excluded land cases, and included an updated data set with improved objective analysis (Lord and Franklin 1987; Ooyama 1987). SHIPS is currently used as forecast guidance at NHC.

Important predictors included POT, shear, persistence, Relative Eddy angular momentum Flux Convergence (REFC, see Chapter 3), Julian date, longitude, distance to land, size (similar to OCS), and change of shear along the future path. ${ }^{6}$ One interesting result is that size was positively correlated to intensity change, whereas Weatherford and Gray (1988a) found it to be a negative influence. Another predictor, which theoretically increases the upper-level cyclonic rotation of a TC by fluxing cyclonic earth vorticity into the storm, is the 200-mb Planetary Eddy angular momentum Flux Convergence (PEFC) given by:

$$
\mathrm{PEFC}=-\overline{f^{\prime} u_{r}^{\prime}}
$$

where $\mathrm{f}$ is the Coriolis parameter, $u_{r}$ is the radial wind, the primes indicate eddies, and the overbar denotes an azimuthal average. PEFC represents the effect of nonzero flow across the $\mathrm{TC}$ in the meridional direction. In the presence of a north to south flow, cyclonic Coriolis torque to the north exceeds anticyclonic torque to the south due to the Beta effect, inducing counterclockwise rotation (Molinari and Vollaro 1990). ${ }^{7}$ In the western North Pacific, TCs are generally embedded in southeasterly flow, which theoretically acts to spin down TCs (Frank 1977a).

The amount of variance explained by SHIPS is about $40 \%$ at $24 \mathrm{~h}$, which is a $10 \%$ improvement over SHIFOR. SHIPS was tested in quasi-independent tests using a jack-

\footnotetext{
${ }^{6}$ Since the 1994 paper was written, the data set has increased with the inclusion of more years. Distance to land and change of shear along the future TC path were found to be no longer significant. Environmental 200-mb temperature, however, is significant (M. DeMaria, personal communication 1994; 1995).

${ }^{7}$ It was recently discovered by this author and M. DeMaria (personal communication 1994) that SHIPS contained a coding error in the PEFC term in which a cosine was used in the Coriolis parameter instead of sine. Further inspection showed that this "wrong" term was significant because it was measuring 200$\mathrm{mb}$ zonal flow. As a result, 200-mb zonal wind is now used as a predictor in SHIPS instead of PEFC. Furthermore, the correct formulation of PEFC was found to be statistically insignificant. However, because of its theoretical importance, PEFC will be investigated in Chapter 8.
} 
knife procedure where a given storm is systematically removed from the sample and the regression coefficients are rederived with each removal. SHIPS showed 10-20\% improvement compared with the official forecast and SHIFOR (see Fig. 1.3). However, SHIPS still has trouble anticipating fast intensification.

Because of its promise, the SHIPS' methodology will be used in Chapter 8. A major focus of this paper is to incorporate satellite data into a multiple regression scheme, and investigate if this new information will reduce errors associated with fast intensifiers. This is the only multiple regression scheme which combines climatology, persistence, synoptic, and SST information with intensity change as the dependent variable. Furthermore, no scheme analogous to SHIPS currently exists in the western North Pacific.

\subsection{The current state of TC intensity numerical modeling}

It is natural to ask whether statistical regression is a viable approach to forecasting intensity change when "state of the art" numerical models are being developed. Currently, the Geophysical Fluid Dynamics Laboratory (GFDL) has developed a high-resolution multiply-nested movable mesh hurricane model (Bender et al. 1993). With proper model initialization, Bender et. al showed that the rapid deepening of Hurricane Gloria (1985) was fairly well-anticipated by the GFDL model, although still underforecast. Gloria's weakening was also well-forecast. However, when the GFDL model was run on an operational basis at NHC in 1994, the intensity forecasts were poor (Kurihara et al. 1995). Intensification was overestimated for tropical storms, but underestimated for intensifying hurricanes. The GFDL researchers maintain that higher resolution is needed. Current inner-core resolution is $17 \mathrm{~km}$, which is too large to resolve the eyewall structure. Furthermore, even though numerical model solutions contain Fourier components of varying length-scales, spectral components shorter than $100 \mathrm{~km}$ are not simulated well during time integration due to a variety of numerical reasons, such as (Pielke 1984): 1) short waves

being improperly advected, typically moving slower than reality; and 2) the amplitude of short waves not being conserved, becoming damped with each timestep.

However, this writer suspects the errors are also associated with additional physical factors, such as improper cumulus parameterization. How the cumulative effects of 
convective clouds on the large-scale temperature and moisture fields is properly treated in a numerical simulation of TC development is the source of much debate. TC development is sensitive to the specification of grid-scale condensation and the parameterized condensation. Cumulus parameterization schemes assume there is a spectral gap between resolvable and sub-grid scales which in nature does not exist, hence partitioning their separate contributions to TC intensity in a numerical model is unclear (Cotton and Anthes 1989). For example, in control experiments where only resolved convection is used (no parameterization), the simulation TC becomes considerably less intense than when parameterization is included (Baik et al. 1990a). The issue is further confused by the sensitivity of TC simulations to different control parameters in a particular parameterization scheme. In a sensitivity analysis of the Betts-Miller convective scheme (Betts 1986), Baik et al. (1990b) found that a TC develops faster when an "adjustment time scale" is decreased or if a "stability weight" of the moist adiabat in the lower atmosphere is increased. In turn, adjusting these parameters affect the grid-scale convection and parameterized convection in non-consistent ways. Finally, simulated TCs evolve differently for separate parameterization schemes. Baik et al. (1991) found that TCs develop faster for Kuo parameterization schemes (Kuo 1965, 1974; Anthes 1977) than for the Betts-Miller scheme. Other researchers have reached similar conclusions with regard to control parameters and scheme comparisons (Hobgood and Rayne 1989; Puri and Miller 1990; Kain and Fritsch 1993).

The inconsistent $\mathrm{TC}$ responses to different control parameters and parameterization schemes are quite discouraging should one wish to apply a TC model for operational use. In fact, it is because of TC's sensitivity to cumulus parameterization and the current uncertainty of inner-core convective processes in relation to intensity change that motivated the alternative SHIPS' approach (M. DeMaria, personal communication 1994).

\subsection{Focus of this study and research approaches}

From this discussion, it is clear that more research is needed on improving intensity change prediction as well as comprehending the processes involved in intensity change. 
This study encompasses an observational, theoretical, and statistical approach to understanding and predicting TC intensity change. The description of the data used in this research is presented in Chapter 5. The next three chapters provide the results.

Chapter 6 investigates inner-core processes related to intensity change, with particular attention on the relationship between the vertical distribution of the tangential wind field in the eyewall region and future pressure changes. Theoretical discussions combining thermodynamical and dynamical reasoning clarifies this relationship. ${ }^{8}$ Evidence is presented that fast, symmetrical, inner-core upper-tropospheric tangential winds and symmetrical, strong gradients of inner-core upper-tropospheric temperature fields may portend future intensity change. This evidence is displayed as soundings, cross-sections, and uppertropospheric planviews. Furthermore, it is shown that inner-core, upper-tropospheric tangential winds may distinguish between storms that intensify fast and those that develop more slowly. Balanced Vortex theory computations (Eliassen 1951; Ooyama 1969; Sundqvist 1970; Pfeffer and Challa 1980; Holland and Merrill 1984; DeMaria et al. 1993; Krishnamurti et al. 1994) also will support this claim that TCs of similar intensity but with different upper-level inner-core windspeeds will evolve differently. Finally, 0-15-deg composites of fast and slow developers show their broad-scale differences in terms of tangential winds, radial winds, thickness, and temperature.

Chapter 7 presents a modified ASI theory which incorporates $3-4^{\circ} \mathrm{C}$ boundary layer cooling instead of $T_{s f c}$ remaining $1^{\circ} \mathrm{C}$ cooler than the SST during TC development. A crude reformulation of the bulk sensible heat flux equation is performed which allows an imbalance between heat fluxes and expansional cooling due to decreasing pressure. Other modifications include water-loading during the moist ascent of a parcel in the eyewall by combining pseudoadiabatic and reversible moist processes, and contributions from latent heat of fusion and deposition. It will be shown that ice microphysics generally offsets the negative effects of water-loading. The lateral extension of the eye above a sloping eyewall

\footnotetext{
${ }^{8}$ A quantitative measure of this dynamical process is the Rossby radius of deformation. However, since the derivation of this parameter in cylindrical coordinates is not shown anywhere in the literature, a derivation is provided in Appendix A.
} 
is hypothesized to be a crucial feature in maintaining the ASI process with boundary layer cooling.

Observational evidence based on composites is presented that buoyancy (including water-loading and ice microphysics) increases in the eyewall environment as a TC intensifies, and therefore stabilization is probably not the limiting factor that determines MPI. Instead, it is hypothesized that MPI is determined by dynamical and thermodynamical constraints. It will be proposed that MPI results from CAPE being unable to increase at a rate sufficient to sustain the vertical motion required to balance friction and upper-level $v_{t}$ export (the IFC process). It will also be hypothesized that vertical motion must increase approximately linearly with $V_{\max }$ to sustain the IFC process, but since $w_{\max }$ only increases roughly to the square root of CAPE (Eq. (3.23)), MPI is reached when CAPE no longer increases fast enough to support IFC.

Chapter 8 describes a multiple regression scheme that is analogous to SHIPS, but with two major differences - the ocean basin is different (western North Pacific), and digitized infrared satellite data are used as predictors. To this author's knowledge, this is the first time such satellite information has been combined with other predictors into a multiple regression forecast analysis. The new scheme is called the Typhoon Intensity Prediction Scheme (TIPS), and incorporates climatology, SST, persistence, synoptic winds, and infrared satellite "pixel count" data. Regression is performed on $12,24,36$, and 48-h forecasts of the future change of $V_{\max }\left(\Delta V_{\max }\right)$. Investigated climatology predictors include storm location, storm motion, and Julian date. A modified persistence term with an "eye parameterization" is studied. A storm's maximum potential intensity is explored using the POT formulation as a function of SST (Eq. (2.4)). The investigated synoptic predictors consist of single point and areally averaged vertical wind shear, 200-mb vorticity, and upper-level forcing terms wuch as vorticity advection, planetary eddy fluxes, and relative angular momentum eddy fluxes. The potential satellite predictors are $30 \mathrm{com}$ binations of different radial band and brightness temperatures, their squared (nonlinear) counterparts, their 6,12 , and 24 -h running mean counterparts, and 12-h convective trends.

A stepwise approach is used with a filtering procedure and strict significance levels to limit the number of chosen predictors based on the years 1984-86. Overall errors, as well 
as stratifications by intensity change, are computed on the dependent data set as well as on an independent data set (1983), and compared to JTWC's forecast errors. However, besides yielding a forecast regression equation, TIPS generates understanding of the intensity change process. Normalized regression coefficients will show a predictor's relative importance compared to the other predictors and compared to other forecast periods. This normalization process also gives "threshold" values useful to operational forecasters. An averaging procedure of the predictors for different intensity change regimes reveals how well a predictor distinguishes between these intensity change intervals. As will be seen, satellite data contains vital information which distinguishes between TCs that intensify fast and those that intensify at a slower rate, underscoring the conclusions of Chapters 2-4 that buoyancy and convection are vital elements to intensity change. In contrast, commentary is included on the statistically non-significant predictors, such as the upper-level forcing parameters, also in agreement with the discussions in Chapters 2-3. Pixel count sensitivity tests are performed on different pixel count combinations, different running means, and the nonlinear counterparts. Shear sensitivity tests compare the variance explained by perfectly forecasting single point and areally averaged VWS, as well as shear forecasts based on persistence. Single variable regression analysis is also computed between intensity change and the statistically significant predictors, as well as correlations between some of the potential predictors themselves (i.e., how well does REFC correlate with vorticity advection?). Details of the official TIPS regression scheme and alternate versions are listed in Appendix B.

Chapter 9 summarizes the research findings. Several suggestions for future study are also included in Chapter 9. 


\section{Chapter 5}

\section{DATA SOURCES}

\subsection{Compositing procedure}

Chapters 6 and 7 employ the concept of "composite stratifications." The basic philosophy is to composite rawinsonde observations within $15 \mathrm{deg}$ of the cyclone's center so that a general picture of a TC which meets a specific category may emerge. These categories may be defined such that they meet one stratification (i.e., a composite of all TCs greater than $50 \mathrm{kts}$ ) or a combination of stratifications (i.e., a composite of all TCs greater than $50 \mathrm{kts}$ in August, south of $30 \mathrm{deg} \mathrm{N}$, which intensified the last $12 \mathrm{~h}$ ). Obviously, the possible combinations are endless, and is one of the advantages of such a research approach.

The true benefit of composites, however, is that the problem of scarce observations is circumvented by combining the data so that sufficient resolution is obtained. For example, for one TC, three total measurements may exist within its $15-\mathrm{deg}$ radius at a particular time. However, by combining several hundred uniform TCs, it is possible to accumulate numerous observations at all radii and compute averages within each grid space. In this manner, TCs exhibiting similar behavioral characteristics can be analyzed while unimportant idiosyncrasies and measurement errors/noise are averaged out. The composites are computed on a cylindrical grid fixed on the TC center, divided into eight octants of $45 \mathrm{deg}$ azimuthal extent, and partitioned into eleven radial belts of $0-1,1-2,2-3,3-4,4-5,5-6$, 6-7, 7-9, 9-11, 11-13, and 13-15 deg (Fig. 5.1). Cross-sections are outputted in 0-1, 1-2, 2-3, 3-4, 4-5, 5-6, and 6-7-deg belts for detailed analysis. For broader-scale cross-sections, composite data is outputted in $0-3,3-5,5-7,7-9,9-11,11-13$, and 13-15-deg belts. Sometimes, when few observations exist even after a composite, only the coarser grid yields useful information. 


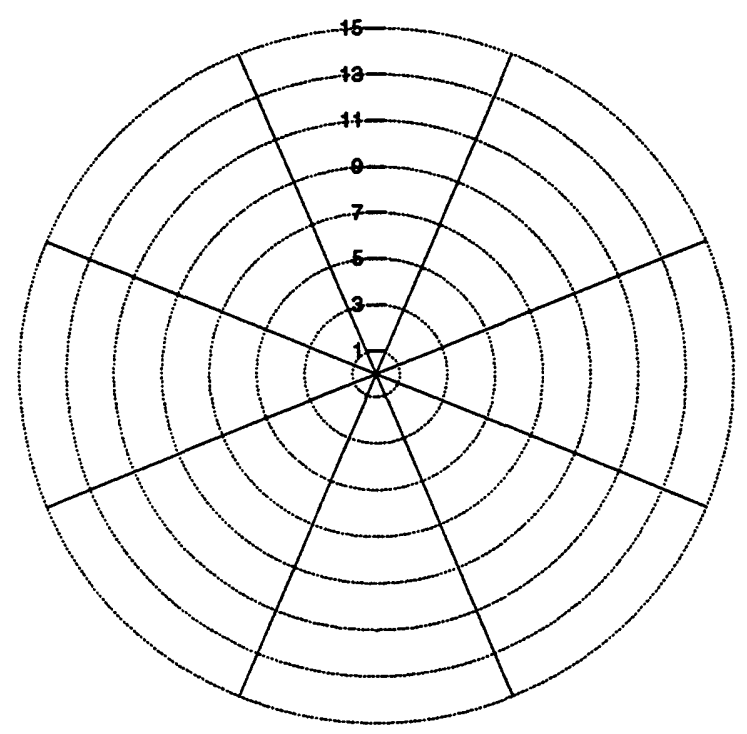

Figure 5.1: Cylindrical coordinate grid from 0-15 deg used to composite rawinsonde data relative to a fixed cyclone at the center. The top portion is octant 1 and oriented north. Other octants are labeled sequentially counterclockwise to octant 8 (Frank 1977b).

In the vertical, data is processed at the surface and at the following pressure levels: $1000,950,900,850,800,700,600,500,400,300,250,200,150,100,80,70,60$, and $50 \mathrm{mb}$. The compositing procedure has recently been improved by the author and project programmer Bill Thorson by correcting the locations of a few Atlantic stations and fixing a few very minor programming bugs. The years the data is available is from 1957 to 1977 . It is possible the data set could be extended to 1987 in the future.

The effect of balloon motion with respect to cyclone motion is accounted for in the composites. In this report, the cyclone motion is subtracted from all wind observations (the "MOT" coordinate system). Composites are performed in the Atlantic and western North Pacific, but generally speaking the Pacific composites are the superior data sets since there are more observations within $15 \mathrm{deg}$ of the TC and fewer continental land influences. The data used in this study is output in cross-sectional radial averages of the composites. A contour program written by the author facilitates the analysis process. Vertical profile composites at a specific radius are also studied, as are individual soundings.

There are some limitations to the compositing procedure. Variable features which could be important are smoothed, the time resolution is seriously degraded, and surrounding regional biases could exist. With regards to the latter, one problem with Atlantic 
composites is that the outer features beyond $10 \mathrm{deg}$ radius often occur over continents and high terrain, which somewhat distorts the wind flow and tremendously alters the temperature field and surface pressure measurements in these outer radii. Another problem is that observations within 1 to $2 \mathrm{deg}$ of the cyclone are rare due to the small inner-core area over data-sparse oceans.

Nevertheless, composites greatly improve our basic knowledge about TCs. It is particularly valuable at showing differences between TCs with different behavioral characteristics. For instance, one can determine differences between fast intensifying and slower intensifying TCs, as is done in this paper.

\subsection{Inner-core hurricane flight data}

Temperature and wind observations from approximately 100 hurricane flight missions (533 radial "legs") for 22 hurricanes on 41 storm days has been assembled for the years 1957-1967 and 1969. ${ }^{1}$ These flights were sponsored by the National Hurricane Research Project (NHRP) ${ }^{2}$ through the National Oceanic and Atmospheric Administration (NOAA).

Data is computed along individual radial flight legs at 2.5 nautical mile (n.m.) intervals from 5 to 50 n.m. Typically 4 to 6 radial legs were flown at one level in and out of a TC during a 4 to 8 -h period. This data is stored at several pressure levels for separate legs and as symmetric averages. Most propeller-driven flights could only be done between 900 and $500 \mathrm{mb}$. Planes used at the low and mid-levels were two TB-50A, four engine, propeller-driven aircraft from 1956 to 1958 , and two DC-6, four-engine, propeller-driven aircraft after 1960 (Sheets 1990). However, upper-tropospheric sampling was accomplished between the 180 and 260 -mb levels by one B-47B turbojet powered aircraft aircraft in 1957

\footnotetext{
${ }^{1}$ After 1958, the Air Force withdrew support for the flights and civilian authorities assumed responsibility. As a result, in 1959 flight data is scarce.

${ }^{2}$ In 1965 the name was changed to the National Hurricane Research Laboratory (NHRL) and is now known as the Hurricane Research Division (HRD) of the Atlantic Oceanographic and Meteorological Laboratory (AOML).
} 
and 1958, and by a WB-57 jet after 1960 (Sheets 1990). Because of limited range, high cost, and instrument difficulties, only 11 upper-level missions were accomplished before upper-tropospheric flights were discontinued in 1971 (41 radial legs). Nevertheless, this remains the only data set with upper-level inner-core flight data. Current aircraft flown into TCs are the Air Force Reserve C-130 reconnaissance planes from Keesler Air Force Base, and the NOAA Orion research WP-3D propeller planes, and both aircraft can only fly to about $400 \mathrm{mb}^{3}$ However, the latter has derived some upper-level winds from an installed Doppler radar, and these measurements are also included in this paper.

The NHRP data has been used for numerous case studies (Colón 1961; Hawkins 1962; Hawkins and Imbembo 1976; Miller 1958b; Staff 1958) and inner-core hurricane research (Shea and Gray 1973; Gray and Shea 1973). Hardcopy is available in the form of a data atlas (Gray and Shea 1976) and in electronic form (from Gray's research project).

In the past, there has been some concern about wind accuracy in this data set (Willoughby 1991). Wind calculations were based on a radio signal that radiates from the aircraft to the ocean surface. Analysis of the Doppler effect through the return signal permits the determination of the drift angle and aircraft motion with respect to the surface. With knowledge of the air speed as given by the aircraft instruments, the wind vector is obtained. This procedure assumes zero motion of the water surface. However, ocean spray, breaking waves, and fast-moving waves can alter the return signal. The radio signal may also bounce back from the precipitation shield below the aircraft. Any of these contingencies will reduce the wind calculations (Colón 1961). The Doppler winds may underestimate the true winds by 5 to 10\% (Gray and Shea 1976). Observations where obvious wind underestimation occurred were removed during data set assembly, but some uncertainties remain. However, these possible errors should not affect the scope of this research (H. Willoughby, personal communication 1994).

\footnotetext{
${ }^{3}$ These planes will be supplemented by faster, high-altitude Gulfstream IV jet aircrafts in 1996 . Not only will upper-tropospheric measurements be obtained again, but flight legs will be traversed quicker than before. This new plane can travel distances of $7550 \mathrm{~km}$ at speeds of $225 \mathrm{~m} \mathrm{~s}^{-1}$ in the upper-troposphere. These jets will also be equipped with 1500 dropsondes, with plans of deploying perhaps 100 dropsondes in the vicinity of a single TC!
} 
Other inner-core wind data were provided by the Hurricane Research Division in the form of cross-sectional plots. These winds were measured from Doppler radar techniques during the WP-3D research flights.

\subsection{Data for statistical analysis}

The years 1983-1986 are chosen for the data sample, coinciding with western North Pacific reconnaissance flights before their termination in 1987. Later years are available for processing, but since there are few "ground-truth" measurements of TC winds over the open ocean, the accuracy of the "besttrack" data prepared by JTWC is uncertain (JTWC 1993) since the cases without aircraft support are primarily estimated using the satellite techniques of Dvorak (1984). ${ }^{4}$ Other sources of wind and position estimation during the post-processing stage are (Guard et al. 1992): 1) the DMSP (Defense Meteorological Satellite Program ) SSM/I (Special Sensor Microwave/Imager) which can estimate surface wind structures, but the observations only arrive once or twice a day (with a $59 \%$ chance of three passes per day) in narrow strips with some data gaps in tropical regions (Alliss and Raman 1992; Elsberry et al. 1992); 2) land-based radar for positions and some Doppler radar installations (i.e, on Guam) for wind estimates; 3) limited weather reconnaissance aircrafts from field experiments; and 4) analysis of surface and 850-mb synoptic data.

However, conventional satellite data provides the main source for post-analysis, and without the plane observations the besttrack data is likely not as accurate after 1986, especially over data-sparse regions. The errors associated with satellite estimates are position errors of $55 \mathrm{~km}$ (with a $10 \%$ chance of $110 \mathrm{~km}$ errors) and 10-20 mb intensity errors with a tendency to underestimate those undergoing rapid intensification, and an overestimate bias of 10-15 mb for strong, mature TCs (Martin 1988; Martin and Gray 1993).

\footnotetext{
"The term "besttrack" refers to post-processed intensity $\left(V_{\max }\right)$ estimates and storm positions at 6-h intervals. MSLP is not included in the JTWC besttrack, but the Navy besttrack does contain pressure values.
} 
Multiple regression will be performed on this data in Chapter 8 for intensity change. The sample consists of the besttrack data for climatological analysis, tropical analysis supplemented by model data for synoptic evaluations, monthly SST data for each of the 4 years, and infrared satellite data.

\subsubsection{SST data}

The SST data is stored by year and month on a 2-deg latitude and longitude grid. The data is computed as "blended SSTs" using the scheme of Reynolds (1988). These fields are blended using in situ data with AVHRR (Advanced Very High Resolution Radiometer) satellite defining the shape of the field in areas of missing data. The in situ ship and buoy data are spatially filtered except where observations are dense to define "benchmark" SSTs in regions of frequent direct observations. The satellite SST retrieval algorithms are "tuned" by regression against quality-controlled drifting buoy data, then linearly smoothed. The blended values are determined by solving a Poisson equation using a relaxation technique which satisfies boundary conditions set by locations with dense observations (either satellite or in situ). This technique effectively eliminates the biases associated with satellite and in situ data. ${ }^{5}$ The SSTs are interpolated to the storm center. Should a storm occur the first or last 5 days of a month, the SSTs are averaged by the current and nearest month.

\subsubsection{Synoptic wind data}

The synoptic wind data is obtained from the Australian Bureau of Meteorology Research Centre (BMRC) for 1984 to July 1986. The BMRC tropical analysis scheme is discussed in detail by Davidson and McAvaney (1981) and summarized in Davidson et al. (1983). The BMRC data was used in preference to initialized model analysis since forecast models are initialized with dynamical constraints such as Nonlinear Normal Mode Initialization (NNMI) (Holton 1992). For 1983 and July-December 1986, synoptic data from

\footnotetext{
${ }^{5}$ For example, ship engine intake values can be too warm, and satellite values can be too cold when cloud top temperatures are accidentally mixed with SSTs. Reynolds (1988) and Reynolds and Smith (1994) describe these biases in more detail.
} 
European Centre for Medium-range Weather Forecasts (ECMWF) is used instead since the project lacks BMRC data for those time periods. Both synoptic data are on 2.5-deg grid spacing analyzed at 00 and 12 UTC. The synoptic parameters are interpolated to the storm center. Occasionally some of the BMRC data is missing, and no statistical analysis is performed for those time periods of no data.

Since it is preferable that regressions be developed on a homogeneous data set, one may question whether introducing two separate analyses is a good idea. However, Hendon (1988) found that the ECMWF wind data and the BMRC analysis depicts large-scale tropical circulations equally well. Besides, one may also argue that including two separate analyses smooths out any biases each may exhibit.

\subsubsection{Satellite data}

The satellite source is three hourly, $10-\mathrm{km}$ resolution infrared "pixels" from three separate Geostationary Meteorological Satellite (GMS) during 1983-1986. Early in 1984 GMS-2 malfunctioned and GMS-1 had to be reactivated. In May-June 1984 GMS-1 failed and GMS-2 was reactivated. In September 1984 GMS-3 replaced GMS-2. The satellites are positioned in geostationary orbit above the equator at $140 \mathrm{deg} \mathrm{E}$.

Dr. Ray Zehr of the National Environmental Satellite, Data, and Information Service (NESDIS) through the Cooperative Institute for Research in the Atmosphere (CIRA) provided the satellite data. The data for 1983-1985 was already processed. This author processed the data for $1986 .^{6}$

Details of the GMS data are contained in Schiffer and Rossow (1983). Zehr (1992) explains the calibration procedure. The IR data is sensed at the 11.2 micrometer wavelength, where the atmosphere is essentially transparent to that channel except for small water vapor absorption and emittance. Therefore, the brightness temperature $T_{b}$ is essentially a measure of cloud-top temperature or the earth's surface in a cloud-free atmosphere. However, inaccurate readings can occur for thin cirrus, whereby $T_{b}$ is a combination of the cirrus emission and the surface or clouds below.

\footnotetext{
${ }^{6}$ CIRA personnel and project programmer Bill Thorson helped in this endeavor.
} 
The data processing procedure is covered extensively in Zehr (1992), and will be summarized here. Akima cubic spline interpolation (Akima 1970) of the besttrack data was used to obtain center positions corresponding to 3 -h satellite intervals. 512 by 512 pixels were then centered on the besttrack data and saved on a grid as shown in Fig. 5.2. This grid contains 15 radial areas $(0-1,0-2,0-4,0-6,0-8,1-2,1-4,1-6,1-8,2-4,2-6,2-8$, $4-6,4-8$, and 6-8 deg) and four quadrants. The number of IR pixels for every $T_{b}$ value was

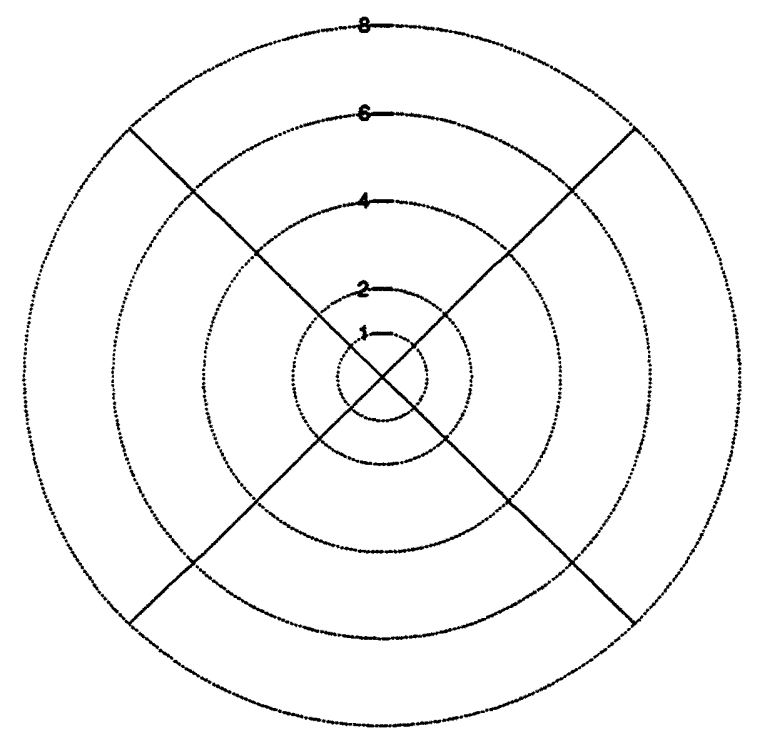

Figure 5.2: Grid used for InfraRed (IR) data processing. Data is available between 0 and $8 \mathrm{deg}$. Top portion is northern quadrant, left is west, bottom is south, and right is east.

tabulated and saved in "count" files for each storm every $3 \mathrm{~h}$. For example, the number of pixels for every $T_{b}$ value for Typhoon Georget on 03 UTC 12 August 1986 was saved in a file called "gt6081203i.cnt". These count files are stored on optical disk and CD-ROM. This data is available from the Gray research project or CIRA. A variety of procedures exist for evaluating the count files.

The routine used by this author is a time-series program for a predefined radial area and $T_{b}$ threshold. The routine accesses the count files for every besttrack position for a particular TC, counts the percentage of pixels colder than the threshold for each image, and outputs a time series. For instance, one could compute the percentage of pixels in a 0-1-deg area colder than $-70^{\circ} \mathrm{C}$, and plot a time series of how the inner-core convective cloudtops increased and decreased during a storm's life cycle. 
Since individually exploring the different $T_{b}$ and radial band combinations was cumbersome, this author decided to create a second data set incorporating a variety of areal and threshold combinations into one "time-series" data set for each storm between 1983 and 1986. The chosen areal and $T_{b}$ combinations are shown in Table 5.1. For example,

Table 5.1: Radial bands and threshold brightness temperature $\left(T_{b}\right)$ combinations investigated in this study. Special time-series data sets have been created with these pixel count combinations for each TC during the years 1983-1986.

\begin{tabular}{|c|c|c|c|}
\hline Radial bands (deg) & $T_{b}\left({ }^{\circ} \mathrm{C}\right)$ & Radial bands (deg) & $T_{b}\left({ }^{\circ} \mathrm{C}\right)$ \\
\hline \hline $0-1$ & -55 & $0-2$ & -70 \\
\hline $0-1$ & -60 & $0-2$ & -75 \\
\hline $0-1$ & -65 & $0-2$ & -80 \\
\hline $0-1$ & -70 & $0-4$ & -55 \\
\hline $0-1$ & -75 & $0-4$ & -60 \\
\hline $0-1$ & -80 & $0-4$ & -65 \\
\hline $1-2$ & -55 & $0-6$ & -55 \\
\hline $1-2$ & -60 & $0-6$ & -60 \\
\hline $1-2$ & -65 & $0-6$ & -65 \\
\hline $1-2$ & -70 & $2-4$ & -55 \\
\hline $1-2$ & -75 & $2-4$ & -60 \\
\hline $1-2$ & -80 & $2-4$ & -65 \\
\hline $0-2$ & -55 & $2-6$ & -55 \\
\hline $0-2$ & -60 & $2-6$ & -60 \\
\hline $0-2$ & -65 & $2-6$ & -65 \\
\hline
\end{tabular}

these combinations for the whole life cycle of Typhoon Georget (1986) are stored in a file called "gt86.ts". A time series plot in 12-h intervals for one of these combinations (percentage of pixels with $T_{b}<-70^{\circ} \mathrm{C}$ in a $0-1-\mathrm{deg}$ area) is shown in Fig. 5.3, which depicts the inner-core convective life cycle of Typhoon Georget. These time-series data sets are also available from the Gray research project.

One of the advantages of digital IR satellite data is that quantitative information can be extracted in an objective manner. Obviously, its potential for regression analysis is tremendous. Furthermore, the timely manner in which the data is stored (3-h intervals) is a godsend for researchers trying to understand TCs in data-void regions. In fact, the major limitation to the regression data set is not the satellite data but the synoptic data which 
TIME SERIES OF GEORGET

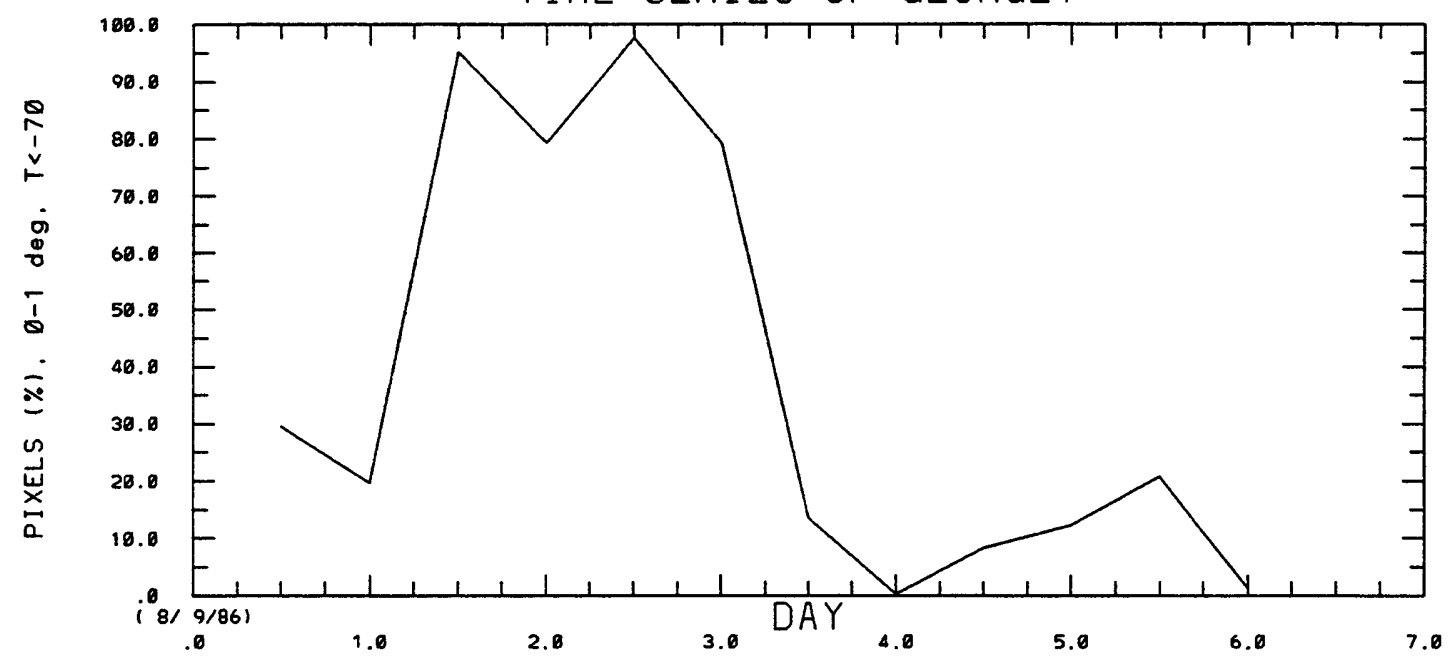

Figure 5.3: Example of pixel count time series expressed as percentage of pixels with $T_{b}<-70^{\circ} \mathrm{C}$ in a $0-1$-deg area. Storm is Typhoon Georget (1986).

is only available every $12 \mathrm{~h}$ - the regression sample could be doubled if the synoptic data were available in 6 -h intervals. Furthermore, the data set could be quadrupled if besttrack and synoptic data could be reasonably interpolated to 3 -h intervals!

Another benefit of using 3-h satellite data is that 6,12, and 24-h running means can be computed. A running mean is an average of current and previous observations. For example, a 6 -h running mean is the average value for the current observation, the $3-\mathrm{h}$ old observation, and the 6-h old observation. Running means are often found useful in meteorological analysis when the data is oscillatory, such as satellite data.

Because of malfunctions, satellite data is occasionally missing. An entire week of imagery in January 1984 and nine days in February were lost during the GMS-2 failure. In late May 1984, the GMS-1 images became cut-off south of 10-15 deg N and completely failed on 29 June 1984. GMS-2 was reactivated but imagery was available only every $6 \mathrm{~h}$ until GMS-3 was activated in September 1984 (Miller and Fritsch 1991). Also, due to a CIRA programming bug, occasionally 03 UTC satellite data was missing. Some of these 03 UTC observations were interpolated by averaging 00 and 06 UTC observations. Other satellite data at the beginning or end of a storm's life cycle also occasionally were missing for unknown reasons. Even with this missing data, over $85 \%$ of the satellite data were available for use. 
Other software was utilized for research purposes. Satellite loops were available on VAX workstations (both geography-fixed and storm-fixed). Color enhancement schemes were used to produce hardcopy. Many other satellite products not used in this study are also available and described in Zehr (1992). The combined satellite capabilities at Colorado State University (CSU) and CIRA is a tremendous resource that no other university has, especially in combination with the rawinsonde and aircraft data.

\subsubsection{Summary of regression sample}

In summary, the four-year regression sample contains 00 and 12 UTC besttrack, synoptic, SST, and satellite data. The satellite data is stored in 30 radial and $T_{b}$ threshold combinations. Furthermore, this satellite data is stored as observed, and as 6, 12, and 24-h running means. This is the first TC data set which contains climatology, SST, synoptic, and satellite data in this extensive a format. 


\section{Chapter 6}

\section{INNER-CORE PROCESSES RELATED TO INTENSITY CHANGE}

\subsection{Inner-core temperature profile for intensifying TCs}

When dealing with intensification issues, one needs to explain the following paradox: convection is needed near the center for intensification, but the conversion of some of this latent heat to upper-level warming needed for the surface pressure drop can potentially stabilize the eyewall. Furthermore, the lateral extension of subsidence warming in the eye can also increase eyewall stability. Somehow, this liberation of latent heat in the eyewall cloud and eye warming must be surmounted so that instability is maintained and intensification can continue. The Air-Sea Interaction (ASI) process is a major agent in circumventing upper-level stabilization, as is a sloping eyewall. But are there other secondary processes working which help destabilize the eyewall to maintain the Internally Forced Convergence (IFC) process and even accelerate intensification? Such is the focus of this chapter.

To investigate possible differences between developing and non-developing TCs, Mer-

rill (1985) stratified two typhoons with $V_{\max }$ between 65 and $125 \mathrm{kts}$ into "intensifying" (increase of $V_{\max }$ by at least $15 \mathrm{kts}$ in the next $24 \mathrm{~h}$ ) and "non-intensifying" (absolute change of $V_{\max }$ by $10 \mathrm{kts}$ or less in the next $24 \mathrm{~h}$ and $5 \mathrm{kts}$ or less in the next $12 \mathrm{~h}$ ). Merrill showed that the intensifying storms were cooler aloft in the inner-core region than the non-intensifying storms (Fig. 6.1). This implies that inner-core static stability is less in intensifying typhoons. The intensifying typhoon and the non-intensifying typhoon composites have similar MSLPs (the former was only $3 \mathrm{mb}$ higher), therefore the temperature difference is not due to the non-developing TCs being more intense (and, therefore, containing a warmer core). The low-level temperatures are nearly identical, hence the 


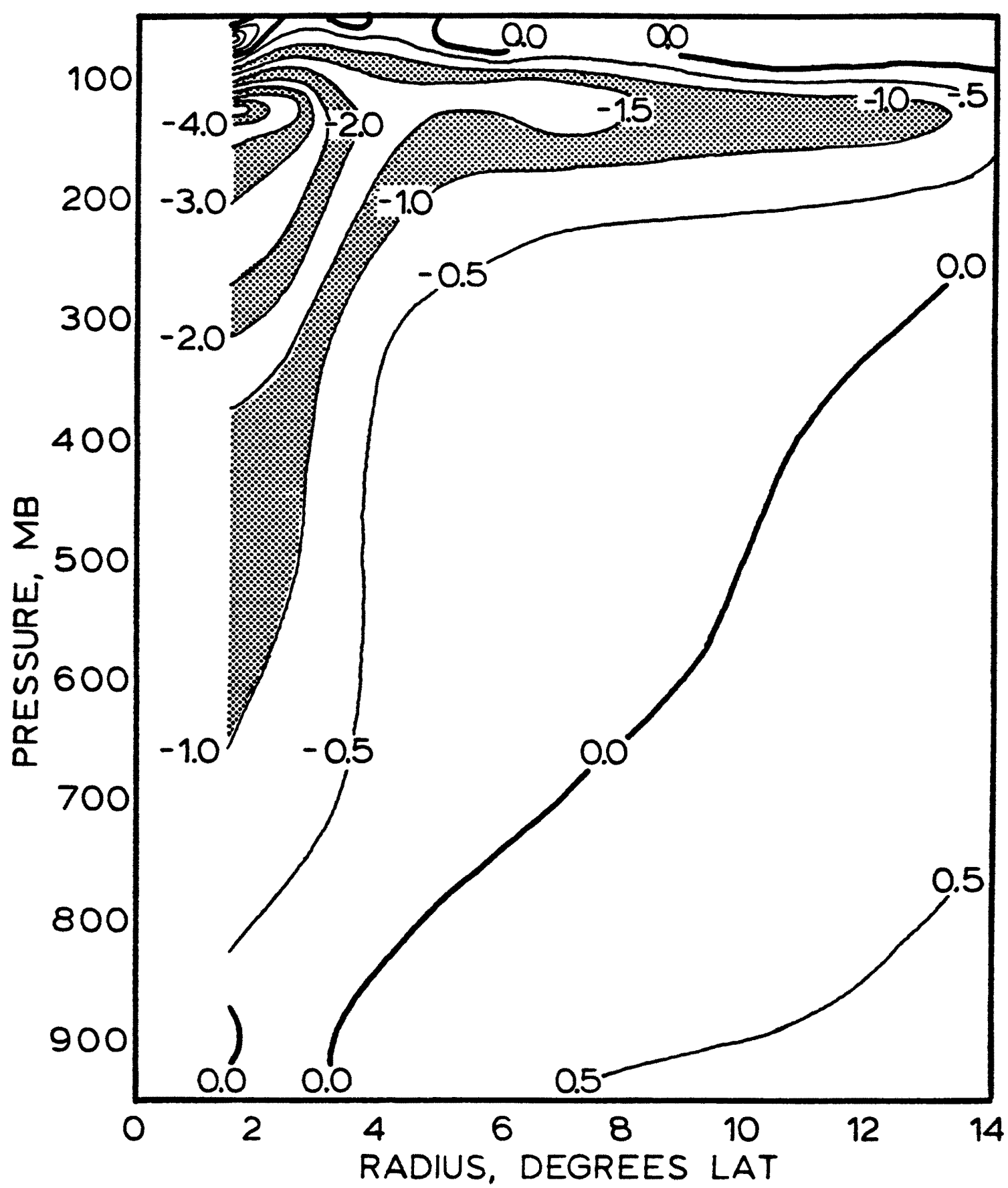

Figure 6.1: Vertical profile of mean temperature difference $\left({ }^{\circ} \mathrm{C}\right)$ between intensifying TCs versus nonintensifying TCs of similar intensity ( $V_{\max }$ of 65 to $125 \mathrm{kts}$ ) in the western North Pacific. The intensifying typhoons are defined as an increase of $V_{\max }$ by at least $15 \mathrm{kts}$ in the next $24 \mathrm{~h}$, and non-intensifying as a change of $V_{\max }$ by $10 \mathrm{kts}$ or less in the next $24 \mathrm{~h}$ and $5 \mathrm{kts}$ in the next $12 \mathrm{~h}$. From Merrill (1985). 
difference is probably not due to a different average location. Indeed, the intensifying TC is located at $16.5 \mathrm{deg} \mathrm{N}$, about $4 \mathrm{deg}$ equatorwards of the non-intensifying TC. However, being further south - and the slightly warmer low-level temperature - does suggest that warmer SSTs might be contributing to the difference. This also suggests that the intensifying TC contains more potential buoyancy via surface fluxes.

As will be discussed, an intensifying TC can transport cyclonic momentum upwards in the eyewall region, which through thermal wind arguments results in most of the warming occurring aloft. As a result, the eyewall maintains buoyancy, and through this feedback process the TC can continue intensifying. Very warm SSTs also facilitate this process. It will be shown that a distinguishing feature between fast intensifying TCs and slower developing TCs is that the former contains a more vertically stacked vortex. But, before discussing this link between the wind profile and eyewall stability, it is necessary that the inner-core tangential wind $\left(v_{t}\right)$ evolution in an intensifying $\mathrm{TC}$ be discussed.

\subsection{The progression of the inner-core upper-level winds}

\subsubsection{Generalization of inner-core $v_{t}$ evolution}

As a TC intensifies, it is observed that the winds at its inner-core (within $2 \mathrm{deg}$ of the TC center) becomes cyclonic through the whole troposphere. Figure 6.2 shows the rawinsonde composites of $v_{t}$ within 1-2 deg radius for a variety of TC intensities in the western North Pacific. As the central pressure decreases, $v_{t}$ increases significantly in the upper levels. Outflow level $v_{t}(300 \mathrm{mb})$ is about one-fourth of 850 -mb $v_{t}$ for a weak tropical storm, increases to one-half as the storm intensifies, and a ratio of two-thirds is possible with intense cyclones. Hawkins (1962) obtained similar results. In situations where vertical wind shear is weak such that the cyclonic vortex is not disrupted, the development of strong inner-core upper-tropospheric $v_{t}$ is not uncommon. This is demonstrated in Fig. 6.3, which shows upper-tropospheric azimuthally averaged tangential winds within $1 \mathrm{deg}$ radius (550 n.m.) for nine hurricanes. These measurements were taken by upper-tropospheric aircraft penetrations along a constant pressure surface in the 1950 s and 1960 s for the National Hurricane Research Project (NHRP). In general, the most intense storms contain 


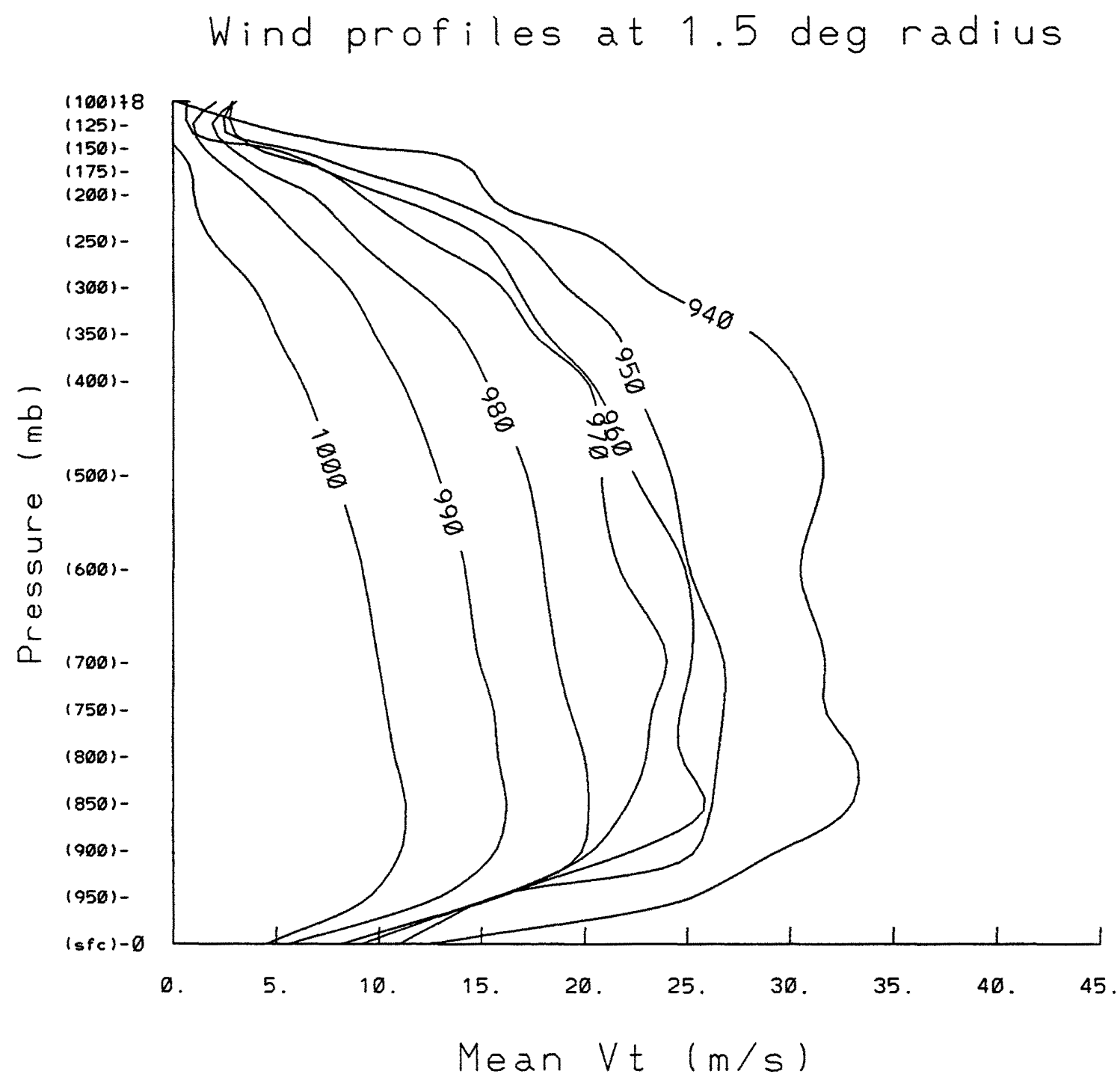

Figure 6.2: Vertical profile of composited tangential winds within 1-2 deg radius for various tropical cyclone intensities in the western North Pacific. The intensity stratifications are for 996 to $1005 \mathrm{mb}$ (denoted as 1000), 986 to $995 \mathrm{mb}$ (denoted as 990 ), etc. Storm motion is removed. 
the strongest winds aloft, and are located at smaller radii. When storms occur in a low shear environment, inner-core convection can transport cyclonic momentum vertically to the upper circulation. It is this chapter's contention that the concentration of upper-level inner-core cyclonic momentum can lead to fast intensification.

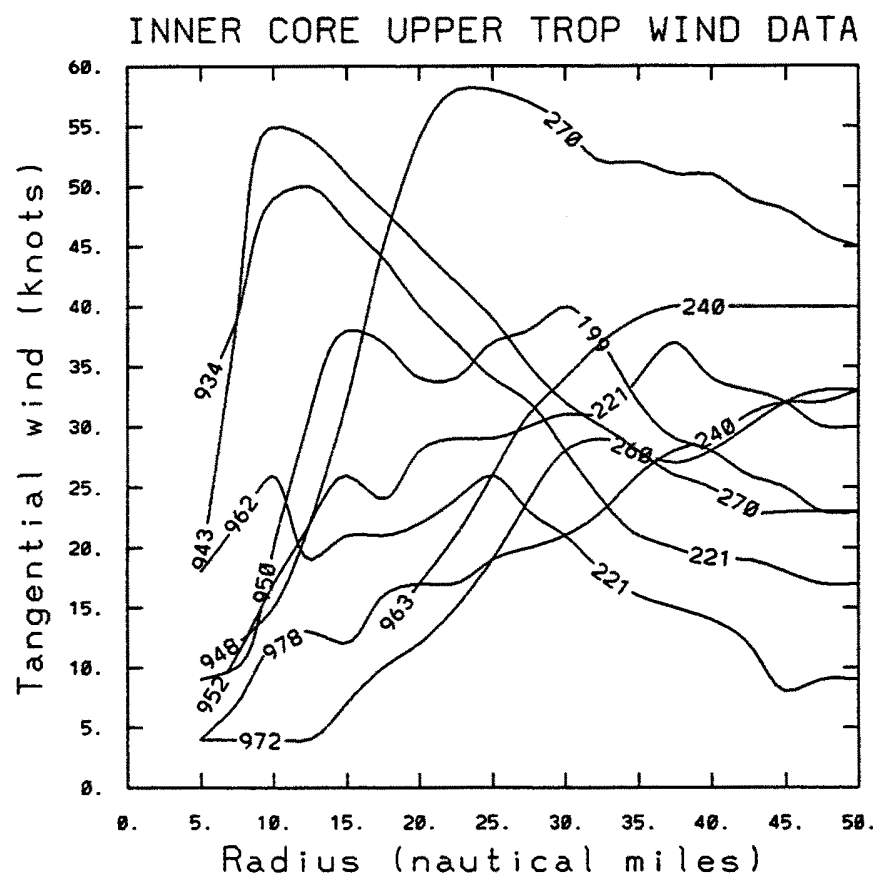

Figure 6.3: Inner-core upper-tropospheric tangential winds as measured by the National Hurricane Research Project reconnaissance flights. Numbers on left side are surface pressures, and on right are pressure flight levels. Storm motion is removed.

In fact, a common characteristic of TCs which intensify at a fast rate compared to storms which intensify at a slow rate is that the inner-core upper-tropospheric $v_{t}$ is stronger for fast developing systems. Figure 6.4 shows the average inner-core wind profile for western North Pacific storms where the change in MSLP in the next $24 \mathrm{~h}$ is $\Delta p \geq 30 \mathrm{mb}$ (profile A), ${ }^{1}$ and Pacific and Atlantic storms where $10 \leq \Delta p \leq 20 \mathrm{mb}$ the next day (profile B). To insure that the two composite storms are relatively the same initial intensity, only storms where $50 \leq V_{\max } \leq 85 \mathrm{kts}$ are considered. The faster developing case is less sheared, with stronger upper-tropospheric (500 to $200-\mathrm{mb}) v_{t}$. Furthermore, the winds

\footnotetext{
${ }^{1}$ No Atlantic storms contained inner-core data for this stratification
} 
are the same near the surface, hence in a composite sense the storms are of equal initial intensity. The composite storms also contain similar $V_{\max }$, MSLP, eye diameters, and radius of observations.

It is postulated that profile $A$ is more conducive to faster development than profile B because, from a thermal wind viewpoint, profile A provides more eyewall buoyancy, and because profile A enhances the ASI process. This theory was introduced in Mundell (1990), and is expanded upon in Section 6.3. The next subsection illustrates the inner-core $v_{t}$ evolution of some individual storms.

\subsubsection{Individual examples of $v_{t}$ evolution}

One of the first successful NHRP studies of inner-core $v_{t}$ evolution is on Hurricane Daisy (Colón 1961; Shea and Gray 1973). The circulation of Daisy has been documented during its deepening stage for 12 UTC 25 August and 18 UTC 27 August 1958. On 25 Aug., Daisy was a minimal hurricane (996 mb MSLP), and was approaching its peak intensity of $935 \mathrm{mb}$ on 27 Aug. (948 mb MSLP). There is little change in $v_{t}$ between the surface and middle levels on 25 Aug. (Fig. 6.5), and probably also on 27 Aug. although no low-level data is available on that date (Fig. 6.6). Daisy's winds are slightly different in magnitude compared to Fig. 6.3 because Figs. 6.5 and 6.6 are cross-sections perpendicular to storm motion, while Fig. 6.3 contains symmetrically averaged winds.

Figure 6.7 shows that the intensification of Daisy was limited inside 20 n.m., with a striking increase of $v_{t}$ in the upper troposphere. On 27 Aug., winds at $13,000 \mathrm{ft}(\approx 637 \mathrm{mb})$ were $115 \mathrm{kts}$, and at $35,000 \mathrm{ft}(\approx 270 \mathrm{mb})$ had reduced to $70 \mathrm{kts}$. This is only a $40 \%$ reduction of $v_{t}$ with height, and is a graphic example of wind preservation with height in an intense tropical cyclone. Radar data found that the eye-wall system extended to $60,000 \mathrm{ft}$, which is normally above the 80 -mb surface. Clearly, inner-core convection transported cyclonic momentum to the upper troposphere. For an additional perspective, planview figures are shown for 27 Aug. at the 270 and $637-\mathrm{mb}$ pressure levels, again showing the conservatism of $v_{t}$ with height in an intense hurricane (Figs. 6.8 and 6.9). Maximum winds range from 80 to $110 \mathrm{kts}$ at $627 \mathrm{mb}$, and only decrease to 55 to $70 \mathrm{kts}$ at $270 \mathrm{mb}$. Note also the symmetry of the $270-\mathrm{mb}$ inner-core winds. Other intense hurricanes in the NHRP 


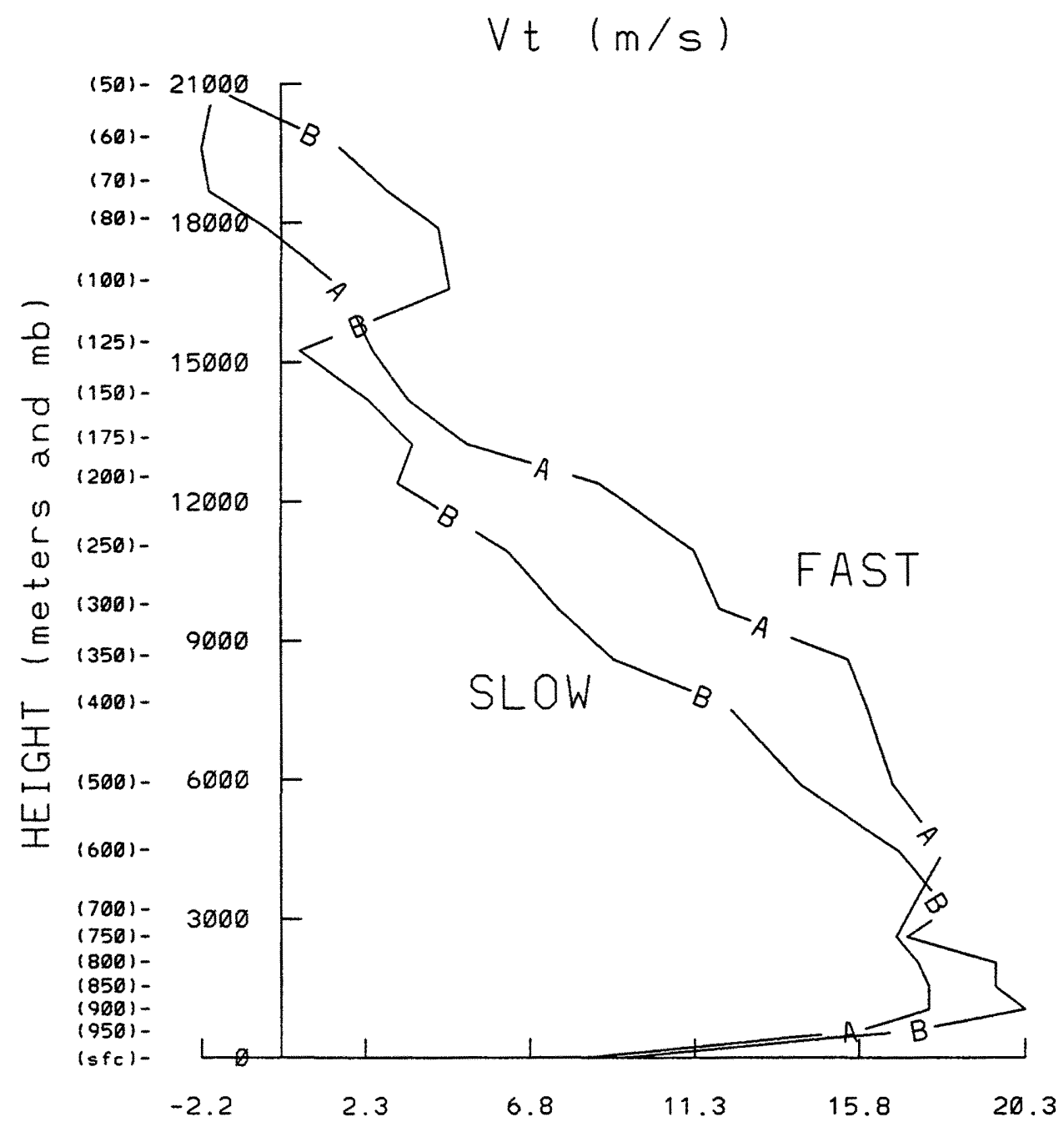

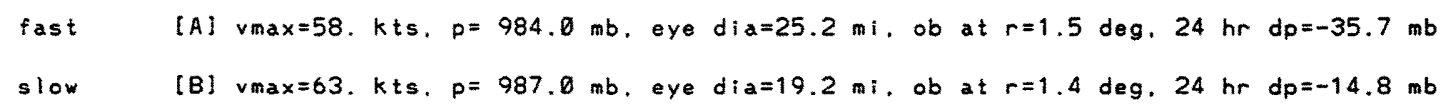

Figure 6.4: Average inner-core $(0.8 \leq r \leq 2 \mathrm{deg}) v_{t}$ profiles for fast developing (A) and slow developing (B) storms at beginning of pressure drop. Fast developing storms are defined as a pressure drop $\geq 30 \mathrm{mb}$ in the next $24 \mathrm{~h}$, and slow developing as $10 \leq \Delta p \leq 20 \mathrm{mb}$ in the next $24 \mathrm{~h}$. Storm motion is removed, and the analysis is restricted to storms whose initial intensity is $50 \leq V_{\max } \leq 85 \mathrm{kts}$. Profile A contains 9 storms, and profile B contains 19 storms. Profile B contains Atlantic and Pacific storms, while A only contains Pacific storms since no data existed in the Atlantic. Only one rawinsonde observation occurred in each storm. Storm information is presented underneath the graph. Shown (in order) is the profile indicator (in brackets), the average maximum winds (vmax), the average MSLP, the average eye diameter (in n.m.), the average sounding radius with respect to the storm center, and the future average $24-\mathrm{h}$ pressure fall. 
data set exhibit similar wind profile trends, such as Hurricanes Helene (1958) and Inez (1966).

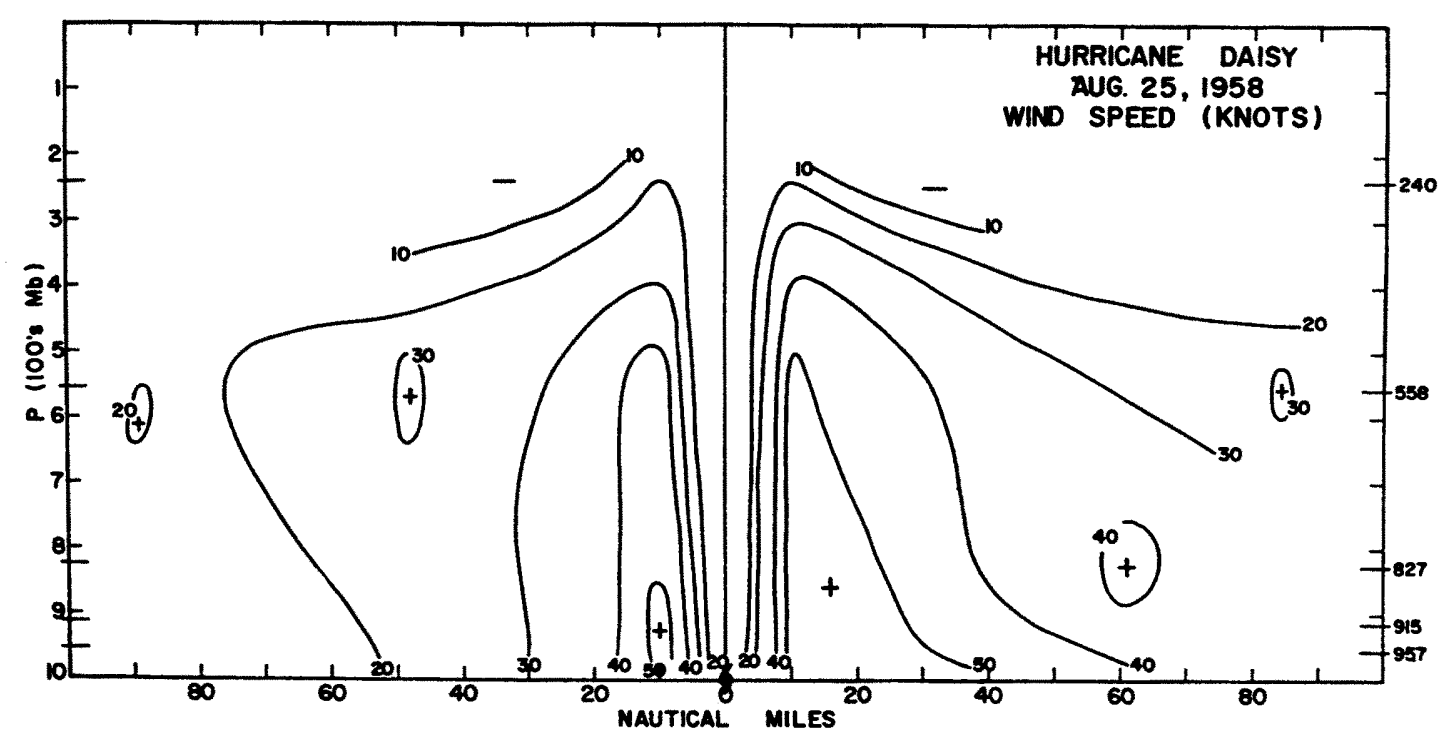

Figure 6.5: Vertical cross-section of $v_{t}$ of Hurricane Daisy on 12 UTC 25 August 1958. Winds include storm motion. Cross-section is perpendicular to direction of storm motion. From Coloń (1961).

A classic example of $v_{t}$ preservation with height in an intense hurricane is shown in Fig. 6.10. This figure depicts Hurricane Gilbert near its record MSLP of $888 \mathrm{mb}$ (Willoughby et al. 1989). The vertical conservatism of $v_{t}$ is striking. The vertical structure of $v_{t}$ for Gilbert versus three hurricanes of different intensities is shown in Fig. 6.11. As was shown in the composites (Fig. 6.2), the weaker the storm, the more $v_{t}$ decreases with height.

Now that the development of upper-level cyclonic vorticity in the inner-core has been established, its relationship to the eyewall temperature will be discussed.

\subsection{Thermal relationship to inner-core wind profile}

Recall Fig. 6.1 which showed that intensifying TCs were cooler aloft in the eyewall than non-intensifying storms, and Fig. 6.3 which showed that the most intense hurricanes contain: 1) the fastest upper-level inner-core winds; and 2) a smaller radius of maximum 


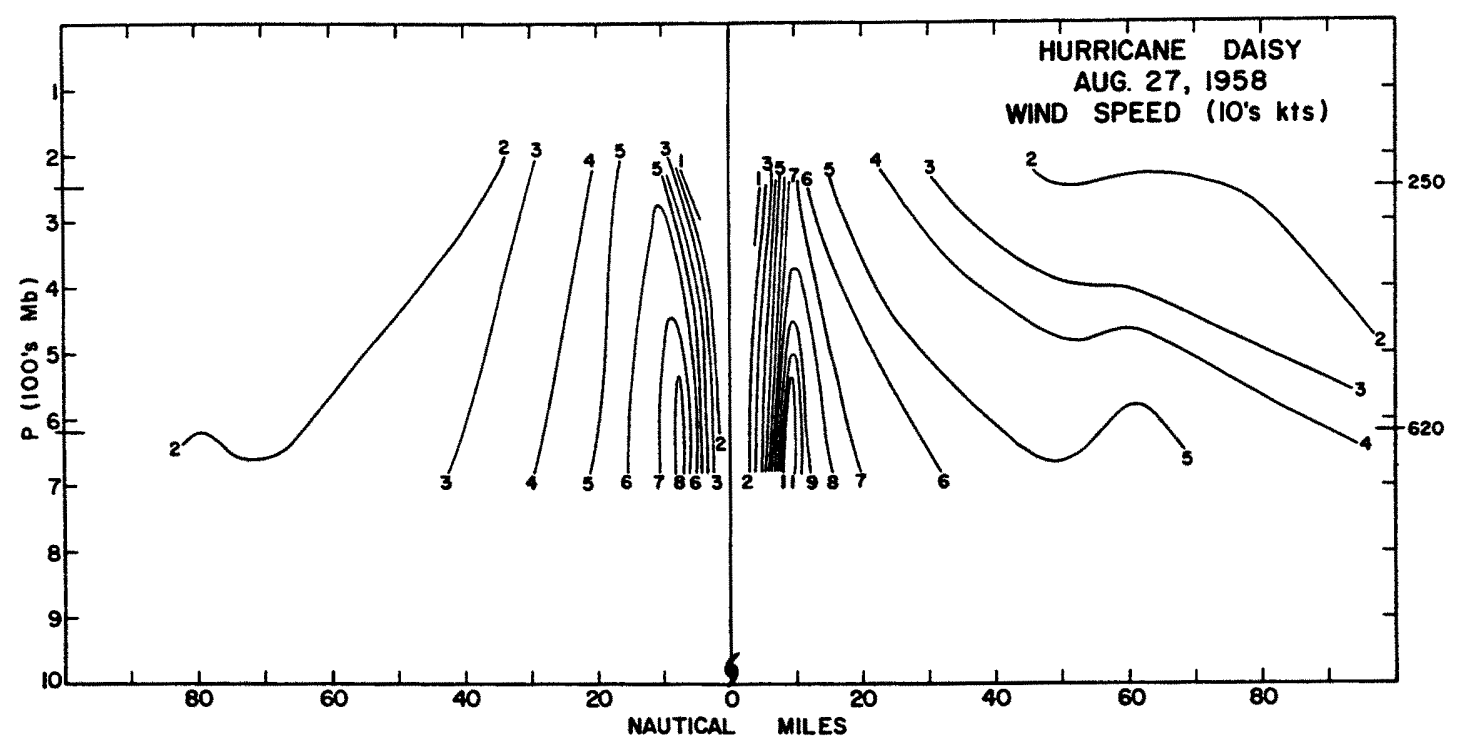

Figure 6.6: Same as Fig. 6.5, but for 18 UTC 27 August 1958. From Coloń (1961).

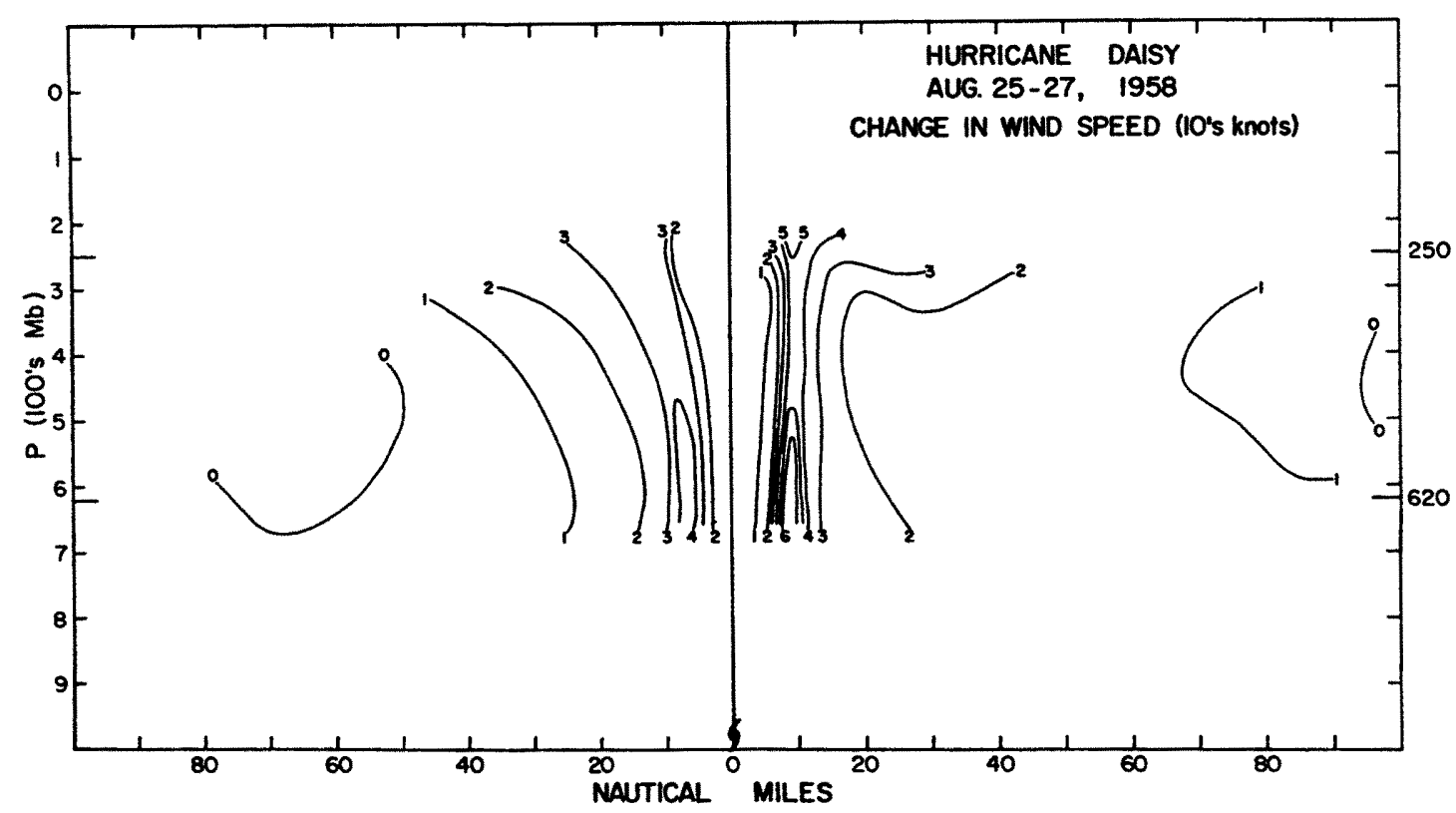

Figure 6.7: Changes in $v_{t}$ between 12 UTC 25 August (Fig. 6.5) and 18 UTC 27 August (Fig.6.6) 1958 for Hurricane Daisy. From Colon (1961). 


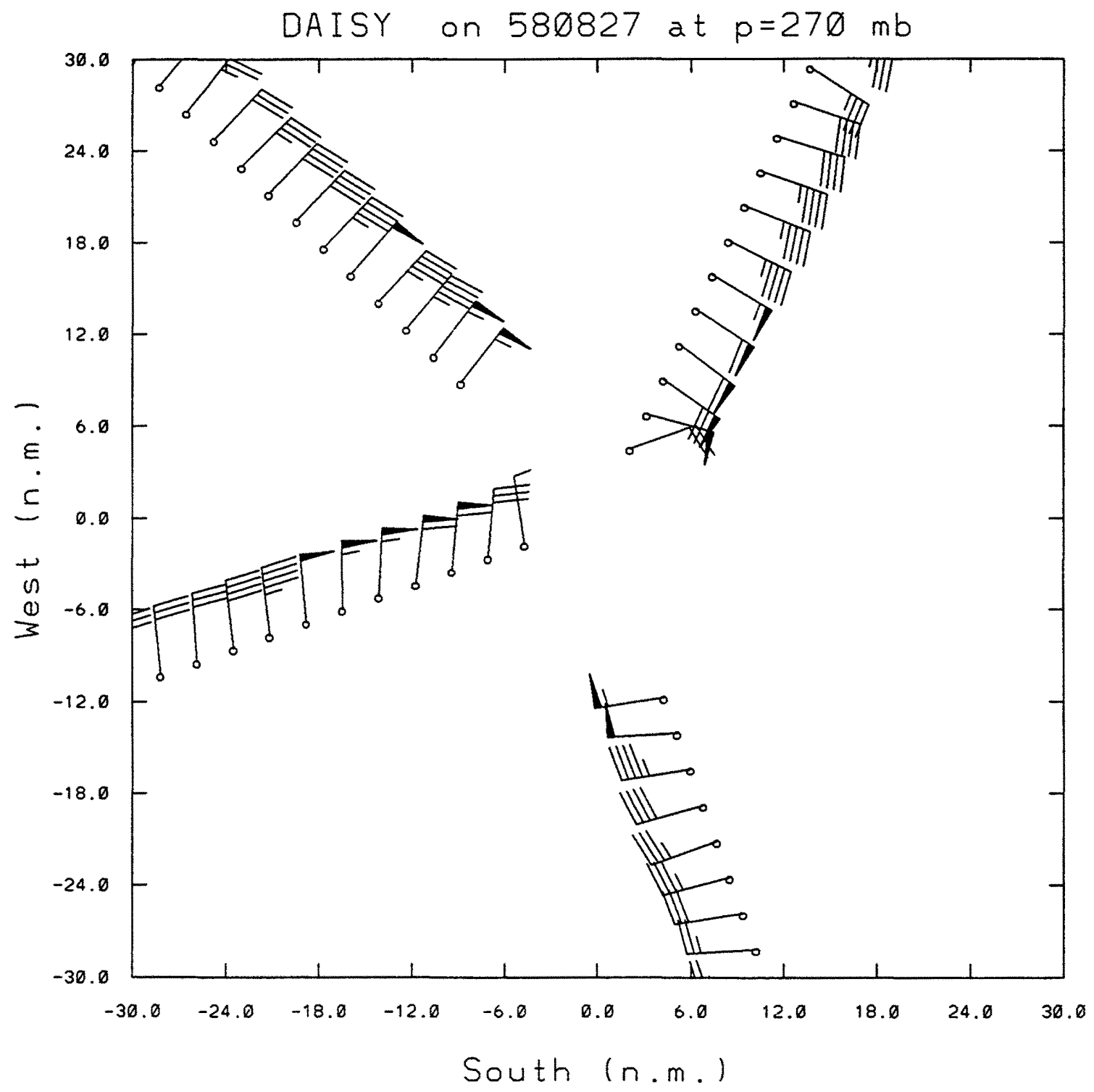

Figure 6.8: Planview of Hurricane Daisy inner-core winds on 18 UTC 27 August 1958. Flags denote $50 \mathrm{kts}$, barbs denote $10 \mathrm{kts}$, half-barbs denote $5 \mathrm{kts}$. Pressure level is $270 \mathrm{mb}$. The storm center is located at the 0 n.m. intersection. Storm motion is removed. 


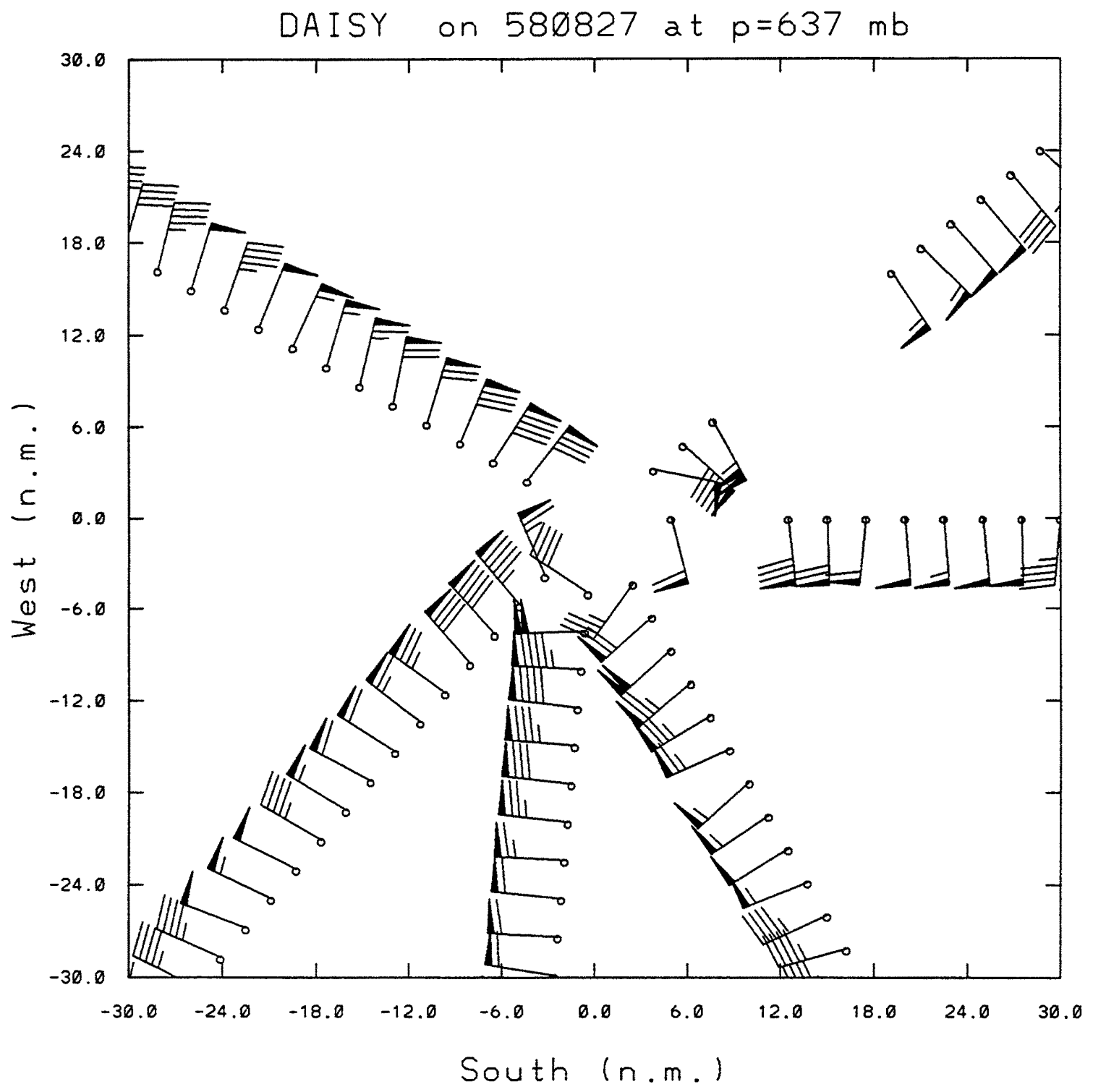

Figure 6.9: Planview of Hurricane Daisy inner-core winds on 18 UTC 27 August 1958. Flags denote $50 \mathrm{kts}$, barbs denote $10 \mathrm{kts}$, half-barbs denote $5 \mathrm{kts}$. Pressure level is $637 \mathrm{mb}$. The storm center is located at the 0 n.m. intersection. Storm motion is removed. 


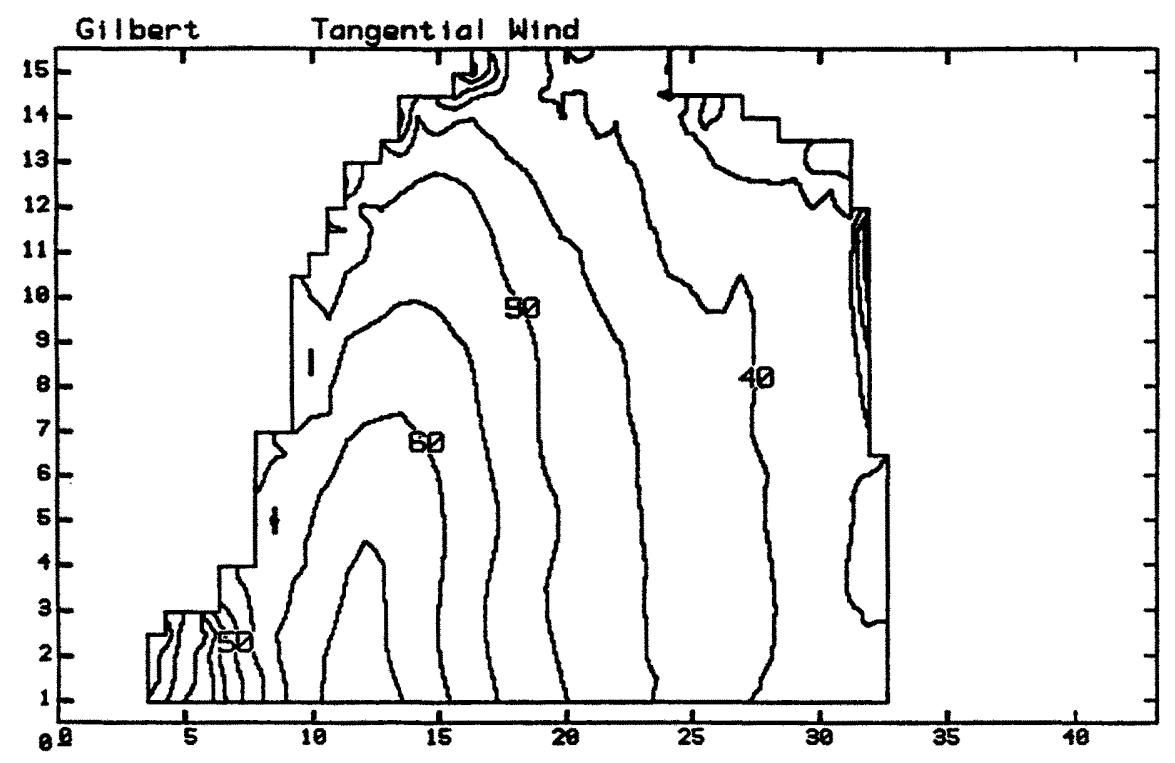

Figure 6.10: Cross-section of Hurricane Gilbert inner-core winds on 06 UTC 14 September 1988. Central pressure is $895 \mathrm{mb}$. Wind analysis is obtained from pseudo-dual Doppler analysis (Marks et al. 1992). Winds include storm motion. Contour intervals is $5 \mathrm{~m} \mathrm{~s}^{-1}$. Figure is courtesy of Peter Dodge, Bob Burpee, and Frank Marks of the Hurricane Research Division. 


\section{COMPARISON OF MEAN TANGENTIAL WINDS}
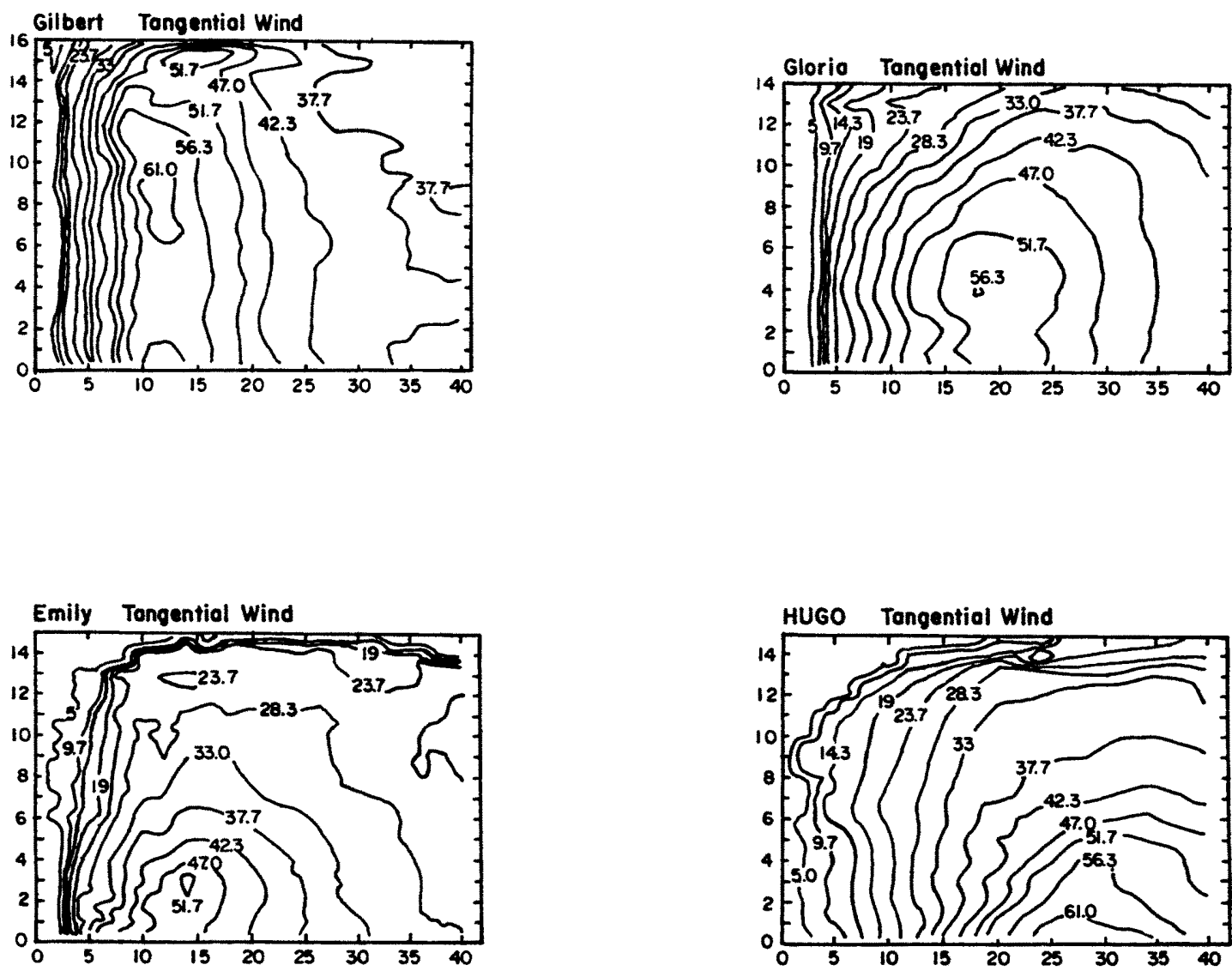

Figure 6.11: Cross-section of hurricanes at different intensities: 1) Hurricane Gilbert on 10 UTC 14 September 1988 with an MSLP of $890 \mathrm{mb}$; 2) Hurricane Gloria on 00 UTC 25 September 1985 with an MSLP of $920 \mathrm{mb}$; 3) Hurricane Emily on 18 UTC 22 September 1987 with an MSLP of $958 \mathrm{mb}$; and 4) Hurricane Hugo on 21 UTC 17 September 1989 with an MSLP of $941 \mathrm{mb}$. Wind analysis is obtained from velocity track display (VTD) Doppler analysis (Lee et al. 1994). Winds include storm motion. Contour interval is $4.7 \mathrm{~m} \mathrm{~s}^{-1}$. Figure is courtesy of Frank Marks of the Hurricane Research Division. 
winds. This trend continues in Fig. 6.12, which shows that the NHRP TCs with the

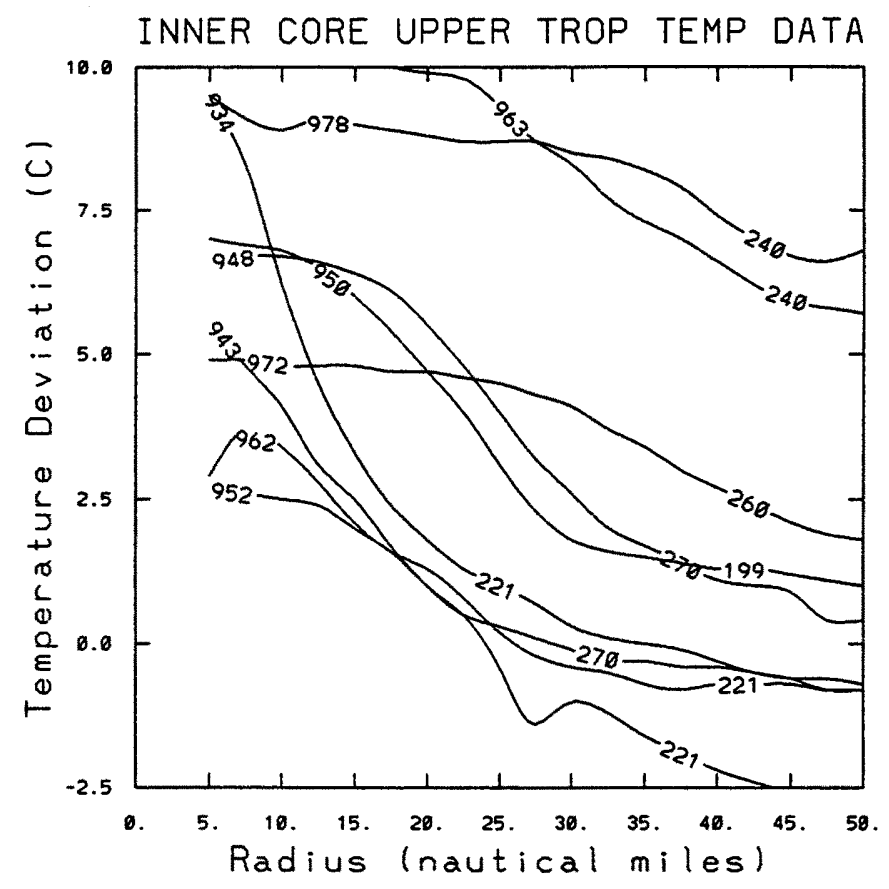

Figure 6.12: Inner-core upper-tropospheric temperature anomalies relative to the mean western North Pacific tropical "clear area" sounding (Frank 1977b; Gray and Frank 1977). Inner-core measurements were done by the National Hurricane Research Project reconnaissance flights. Numbers on left side are MSLPs, and on right are pressure flight levels.

lowest MSLP tend to be warmer on average in the upper troposphere eye region when compared to the weaker storms, but on average cooler in the upper-level eyewall cloud when compared to the weaker storms.

Figure 6.13 shows the inner-core $(1-2 \mathrm{deg})$ temperature difference between the fast developing and slow developing Pacific TCs corresponding to Fig. 6.4. ${ }^{2}$ Unfortunately, temperature is a noisier variable than $v_{t}$ since its diurnally, seasonally and geographically dependent. Lack of inner-core observations also makes analysis difficult. However, Fig. 6.13 presents tentative observational evidence that faster developing TCs have a cooler inner-core aloft. This cooling is probably not due to the $150-\mathrm{mb}$ pressure surface being forced higher by overshooting cloud-tops, as the geopotential height values only differed

\footnotetext{
${ }^{2}$ Atlantic storms were excluded in the temperature comparison since environmental temperatures are different in this basin.
} 
by $6 \mathrm{~m}$ between the composites at $150 \mathrm{mb}$ (not shown). Comparisons between individual TCs in the two categories also do not show much height difference that would explain the temperature difference.

In other words, the most intense TCs tend to develop strong temperature gradients from the eye to eyewall cloud. These data suggest that baroclinicity is a key factor in explaining why deep cumulus convection can be maintained in the eyewall cloud as the eye undergoes strong warming. This baroclinicity can be related to vorticity and vertical variation of $v_{t}$ using the thermal wind equation in cylindrical coordinates: ${ }^{3}$

$$
\left(f+\frac{2 v_{t}}{r}\right) \frac{\partial v_{t}}{R \partial \ln p}=-\frac{\partial T}{\partial r}
$$

For cases under minimal vertical wind shear such that vigorous inner-core updrafts exist, a cyclonic circulation should extend almost to the tropopause. In theory, the vertical variation of $v_{t}$ in most of the troposphere would be relatively small. Most of the large vertical gradient of $v_{t}$ with respect to $\ln p$ would be confined to near the tropopause. To simplify the theoretical discussion, in all wind profiles it will be assumed that the wind velocity goes to zero at $100 \mathrm{mb}$.

A concentration of $v_{t}$ near the top of the troposphere has important thermal consequences. According to Eq. (6.1), thermal wind balance is achieved by an increase in baroclinicity $(-\partial T / \partial r)$ above and within the region of maximum $v_{t}$ decrease with respect to $\ln p$. In a more common type of intensification where moderate amounts of shear exist, the vortex cannot vertically build as high (Fig. 6.14), and the inner-core warming is located in the middle troposphere where the vertical variation of $v_{t}$ with respect to $\ln p$ is greatest. This mid-level stabilization over time tends to reduce further deep convection, and intensification continues at a slower pace. Furthermore, the maximize pressure drop

\footnotetext{
${ }^{3}$ The cylindrical thermal wind equation is an incorporation of the hydrostatic assumption and gradient wind balance assumption. To derive the thermal wind equation in pressure coordinates, one takes $\partial / \partial r$ of the hydrostatic equation $(g \partial Z / \partial \ln p=-R T)$ where $Z$ is the geopotential height, and $\partial / \partial \ln p$ of the gradient wind equation $\left(v_{t}^{2} / r+f v_{t}=g \partial Z / \partial r\right)$, and set the two relationships equal to each other.
} 


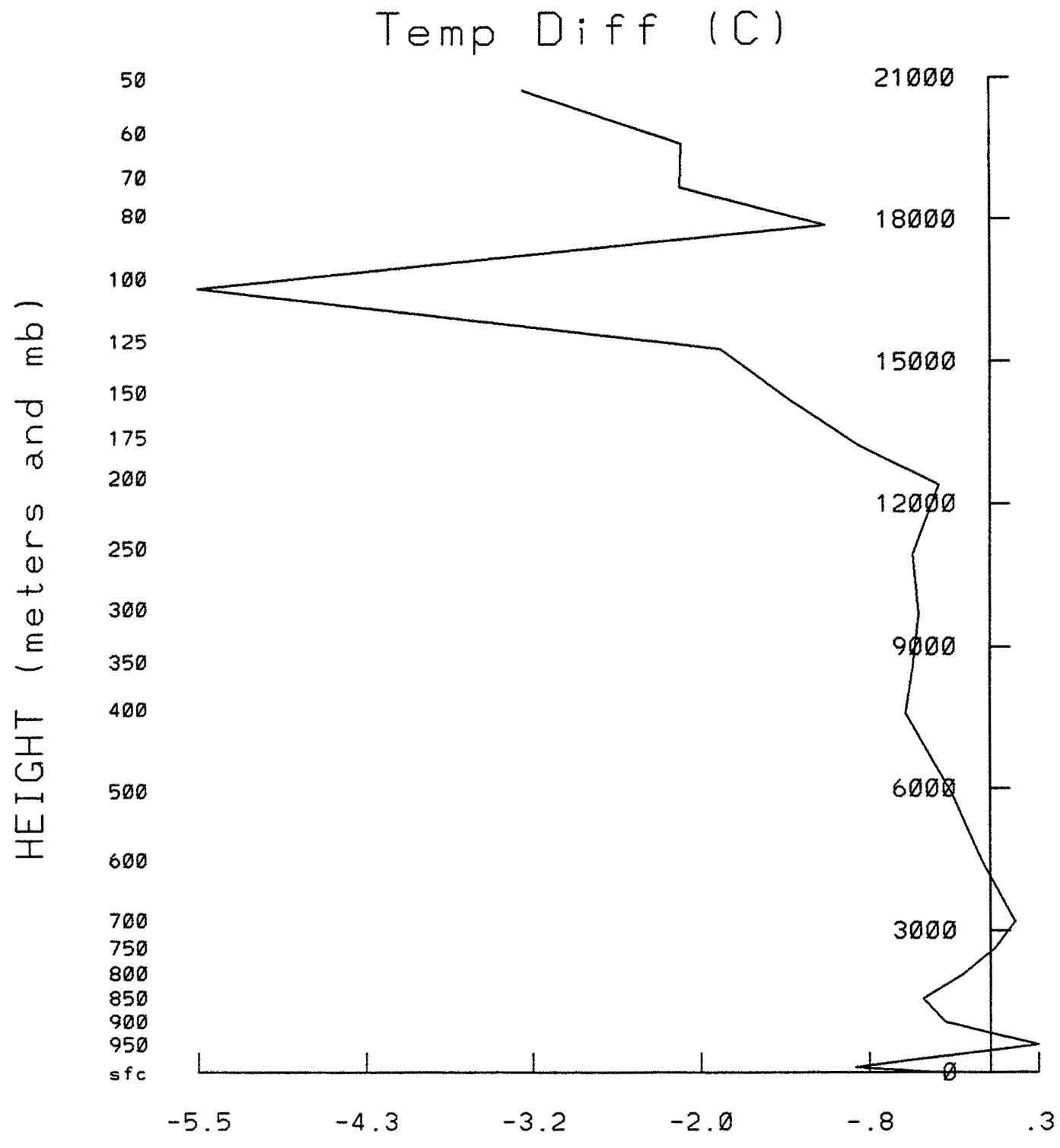

Figure 6.13: Average inner-core $(r \approx 1.5 \mathrm{deg})$ temperature differences for faster developing minus slower developing Pacific storms at beginning of pressure drop. Faster developing storms are defined as a pressure drop $\geq 30 \mathrm{mb}$ in the next $24 \mathrm{~h}$, and slower developing as $10 \leq \Delta p \leq 20 \mathrm{mb}$ in the next $24 \mathrm{~h}$. The analysis is restricted to storms whose initial intensity is $50 \leq V_{\max } \leq 85 \mathrm{kts}$. There are 8 observations for the fast developing storm composite up to $175 \mathrm{mb}$, then decreases to 4 in the stratosphere. There are 9 observations for the slower developing storm composite up to $175 \mathrm{mb}$, then decreases to 4 in the stratosphere. 
cannot be realized since the warmest temperature perturbation is confined to the midtroposphere (recall from Chapter 3 that maximum pressure drop is nonlinearly related to the height of the temperature perturbation).

Figure 6.15 corresponds to a hypothetical fast intensifying case. It is postulated that storms at the onset of fast intensification have developed a vertically stacked vortex such that the vertical variation of $v_{t}$ is maximum near the tropopause and, therefore, so is the maximum warming. In turn, the maximum pressure drop can be realized. Figure 6.16 displays the $v_{t}$ and temperature differences between the two cases (fast intensification minus slower intensification). For two storms of comparable intensity but different wind speed aloft, the fast intensifying storm is generally cooler except near the tropopause.

The importance of a relatively cool inner-core environment at upper levels with subsidence warming concentrated as close as possible to the center is schematically illustrated in Fig. 6.17. It should be noted that some eyewall slope is needed near the tropopause so that ascending air parcels avoid the warmest region of a TC, thereby maintaining their buoyancy. The lateral extension of the eye near the tropopause above the eyewall surface is also a desirable feature, because it accelerates surface pressure falls under the eyewall and therefore also aids eyewall buoyancy through the ASI process. The necessity of the eyewall sloping outwards near the tropopause will be discussed in Chapter 7. Nevertheless, the importance of inner-core $v_{t}$ being vertically conserved to near the tropopause is just as important a feature in fast intensifying systems.

In summary, it is thought that the stabilizing effects are concentrated near the tropopause for a storm about to undergo fast intensification. In this manner:

- The maximum warm core anomaly is close to the tropopause so that eyewall instability (CAPE) is large. In this manner, sufficient vertical mass flux exists to balance $v_{t}$ lost to friction and upper-level export and still contain a surplus to intensify the storm - maybe even accelerate intensification if this IFC process is strongly enhanced by an anomalously cool environmental eyewall.

- Since the warming is placed near the tropopause, surface pressure falls through the ASI process are accelerated. 

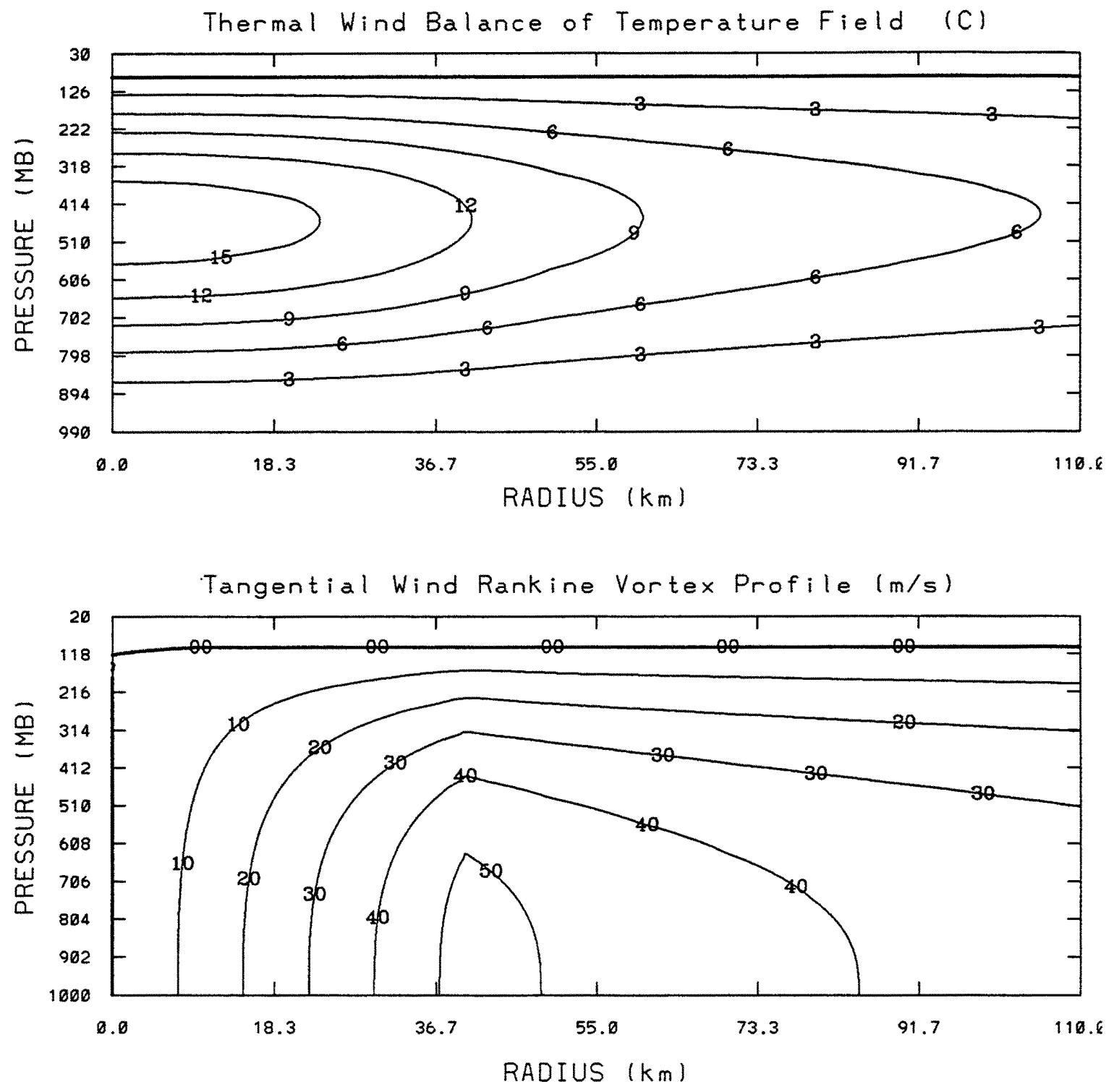

Figure 6.14: Temperature anomaly distribution (displayed in top figure) as dictated by thermal wind balance to the tangential wind distribution shown in the bottom figure. The wind profile represents a hypothetical case of moderate shear such that the vortex does not extend to the tropopause. Therefore, the region of maximum $\partial v_{t} / \partial \ln p$ is in the mid-troposphere. 

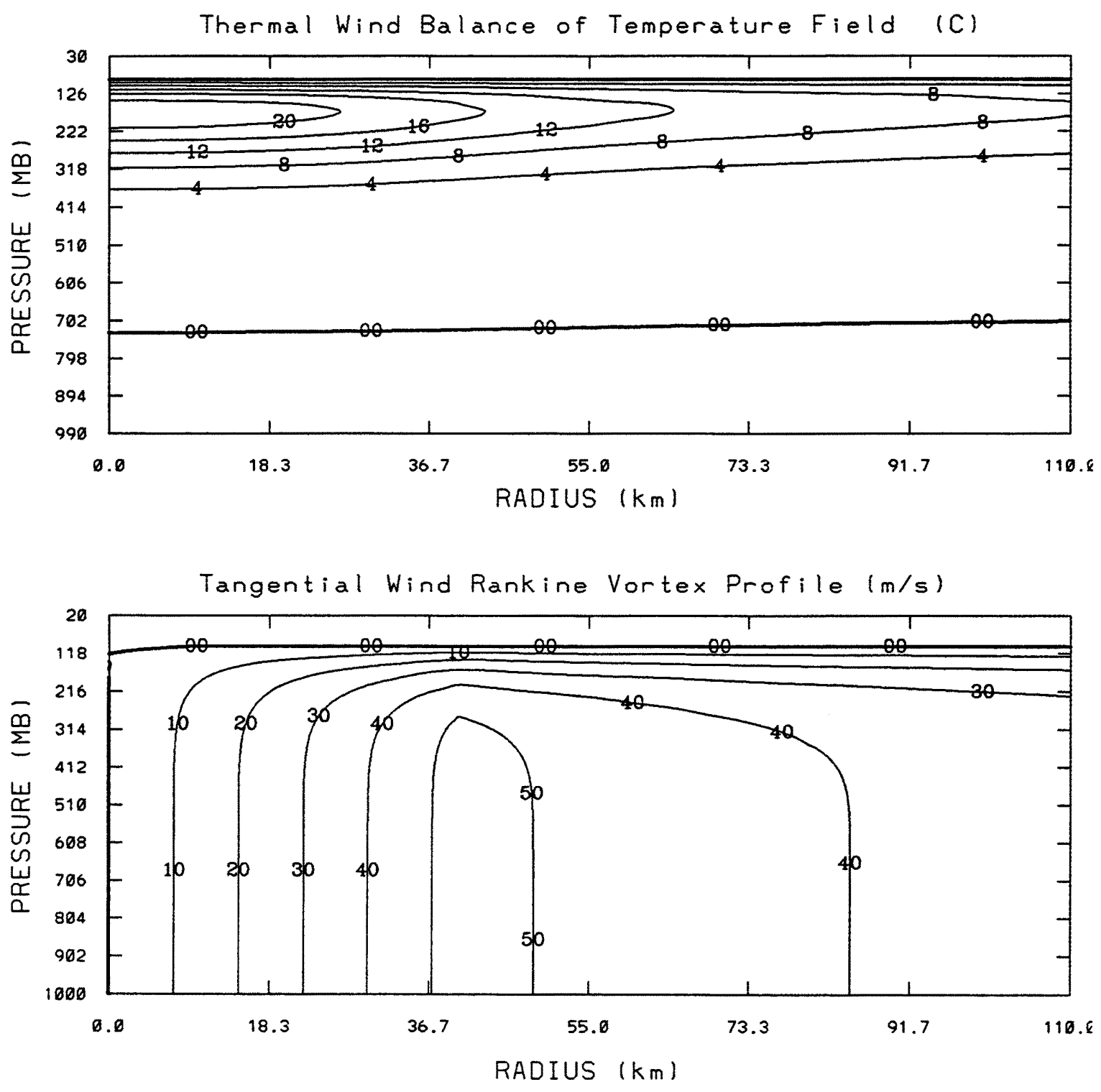

Figure 6.15: Temperature anomaly distribution (displayed in top figure) as dictated by thermal wind balance to the tangential wind distribution shown in the bottom figure. The wind profile represents a hypothetical case of low shear such that the vortex extends to the tropopause. Therefore the region of maximum $\partial v_{t} / \partial \ln p$ is near the tropopause. 

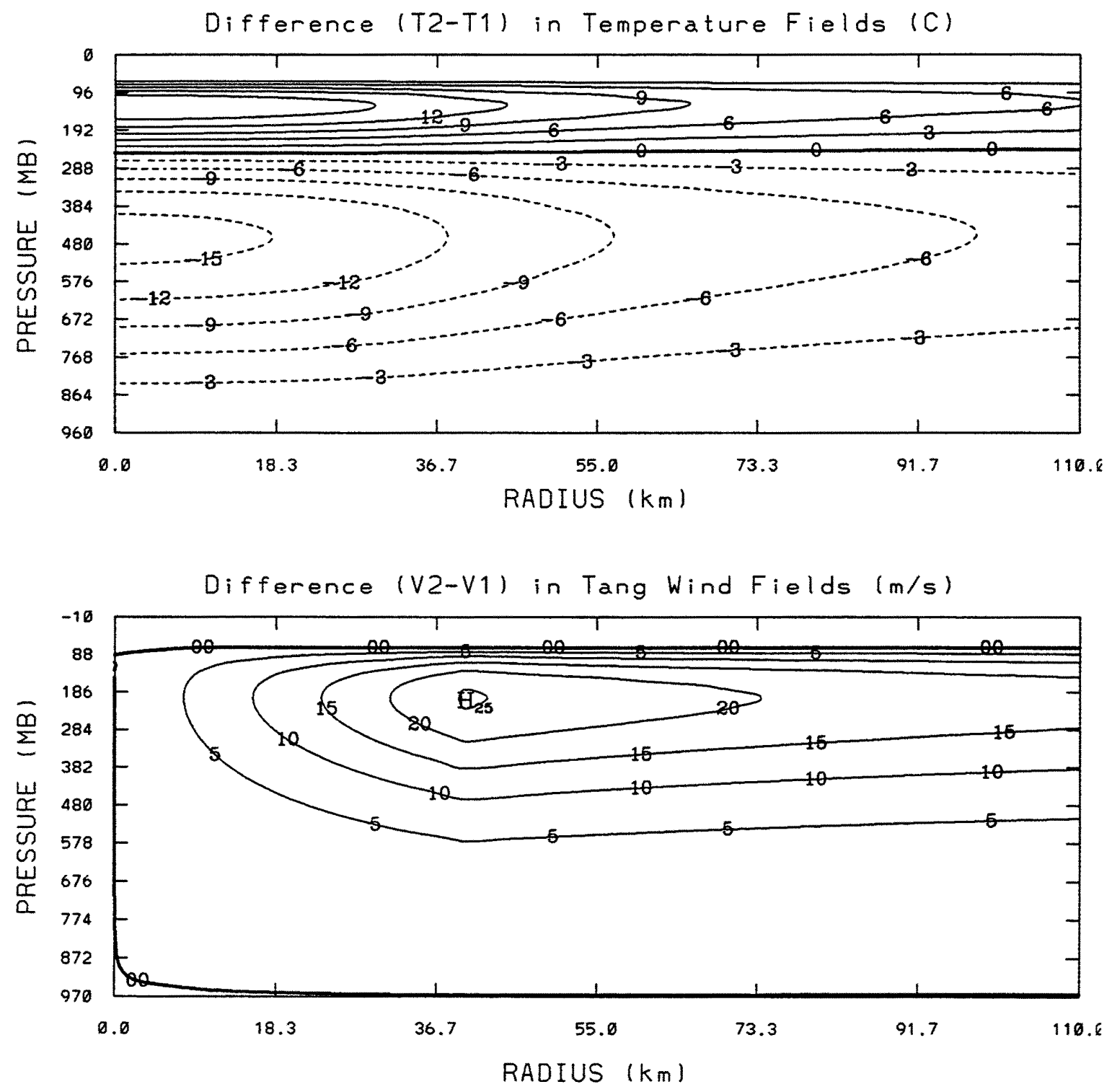

Figure 6.16: Temperature and wind differences between Figs. 6.14 and 6.15 in which the moderate-shear example (case 1) is subtracted from the low-shear example (case 2). 


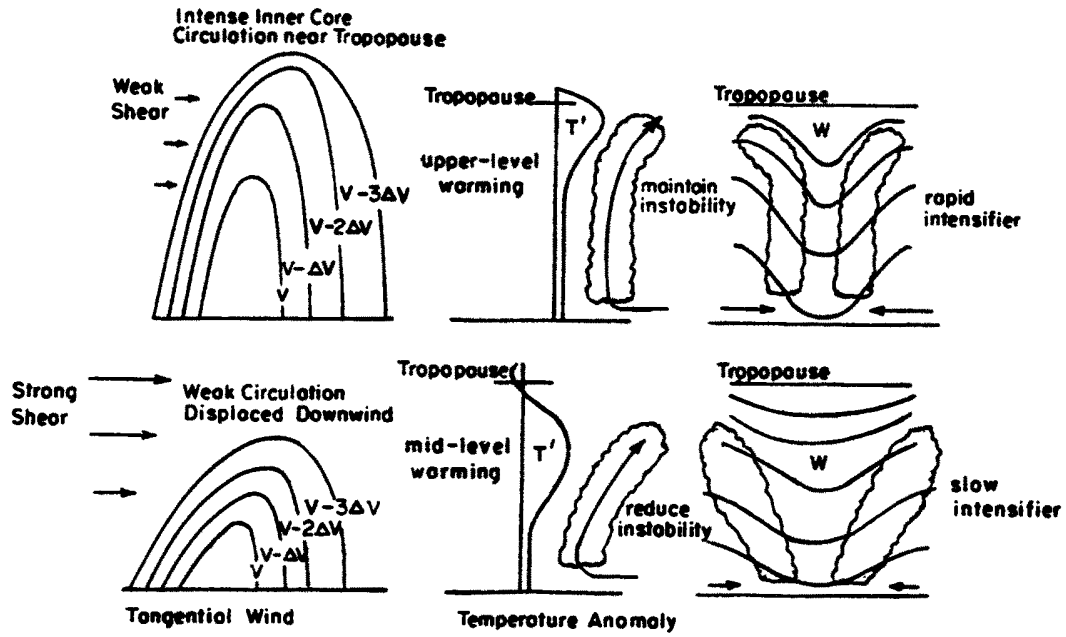

Figure 6.17: Cross-sectional illustration of upper-tropospheric temperatures and tangential winds for fast intensifying storms and slower intensifying storms. The wind profile of the fast intensifier contains a more vertically stacked vortex which only tilts outwards near the tropopause, while the slow intensifier is more tilted and $v_{t}$ decreases more with height. The former is hypothesized to be cooler on average in the eyewall but warmer than average in the eye. Modified from Mundell (1990).

When one combines these processes with the fact that the conversion efficiency of latent heat to kinetic energy increases with: a) inner-core vorticity (inertial stability) and b) vertically stacked vortices, a TC will intensify at a fast rate and possibly even rapidly intensify for very warm SSTs. These geostrophic adjustment processes and other dynamical factors will be discussed in the next section.

\subsection{Dynamical relationships to inner-core wind profile}

Recall from Chapter 3 that faster pressure drops can occur for maturing storms of tropical storm strength or hurricane strength when compared to weak tropical storm strength. This is because the inertial stability is increasing while the static stability is decreasing (or at least held constant), which is manifested as a smaller Rossby Radius of Deformation $\lambda_{R}$ (see Eq. (3.12)). $N H$ is the phase speed of an internal gravity wave while $\left[(\zeta+f)^{1 / 2}\left(2 v_{t} / r+f\right)^{1 / 2}\right]^{-1}$ is the time scale over which gradient balance occurs. Therefore, $\lambda_{R}$ is the length scale that a gravity wave will travel before gradient balance occurs. The horizontal scale of a disturbance $L$, when compared to $\lambda_{R}$, determines how 
much of the gravity wave energy is incorporated into the generation of kinetic energy of the disturbance. ${ }^{4}$

Typically in the tropics, weak convective systems contain large values of $\lambda_{R}$ because the denominator of Eq. (3.12) approaches the Coriolis parameter. Assuming $f \approx 5 \times$ $10^{-5} \mathrm{~s}^{-1}, N=0.01 \mathrm{~s}^{-1}$, and $H=8 \mathrm{~km}, \lambda_{R}$ is on the order of $1500 \mathrm{~km}$. However, the disturbance scale is on the the order of 10 to $100 \mathrm{~km}$. Since $L<<\lambda_{R}$, the gravity waves excited by the heating propagate out of the system before gradient balance can occur. Therefore, the initial pressure perturbation generated by the heating dissipates to its original (environmental) pressure value, and no circulation is generated. In other words, the mass field adjusts to the wind field (which, in this example, consisted of a “calm" background atmosphere).

However, due to its relative vorticity, a maturing TC contains smaller values of $\lambda_{R}$. As $L \rightarrow \lambda_{R}$, more of the gravity wave energy is partitioned to the kinetic energy of the TC. As the system intensifies, $L$ increasingly exceeds $\lambda_{R}$, and the TC's winds increase to bring the system back into thermal wind balance. Stated another way, the wind field adjusts to the

\footnotetext{
Actually, in the stratified atmosphere, a broad range of gravity wave speeds (and therefore $\lambda_{R}$ ) needs to be considered, which considerably complicates a description of the geostrophic adjustment process in terms of $\lambda_{R}$. Solutions of the stratified atmosphere may be reduced to a superposition of solutions corresponding to various "normal modes," which are free modes of oscillation in the atmosphere (Holton 1992). By linearizing the primitive equations with $n$ layers, defining $\Phi_{n}(z)$ such that it satisfies a vertical structure equation for each normal mode, and specifying appropriate boundary conditions, the original $n$ layer system can be transformed to n-shallow-water systems with an "equivalent height" for each "internal mode" (in contrast to an "external mode" which represents the non-stratified shallow-water component). The phase speed is inversely proportional to the internal mode with vertical structure $\Phi_{n}(z)$ - for example, for the first internal mode with vertical structure $\Phi_{1}$, the phase speed may be $50 \mathrm{~m} \mathrm{~s}{ }^{-1}$, and for the fifth internal mode with $\Phi_{5}(z)$, the phase speed may be $10 \mathrm{~m} \mathrm{~s}^{-1}$ (Hack and Schubert 1981). Therefore, $\lambda_{R}$ is inversely proportional to the internal mode, and "shallower modes" can become geostrophic at smaller scales. In other words, the geostrophic adjustment problem consists of a superposition of internal modes, which in turn have different dependencies on the depth of the disturbance, latitude, background vorticity, and horizontal extent of the disturbance.

The use of normal modes in the initialization of models is discussed by DeMaria and Schubert (1984, 1985) for TC models, and by many researchers in regular models (Temperton and Williamson 1981; Bourke and McGregor 1983; Holton 1992). The application of lateral boundary conditions using normal modes in TC models is discussed by Hack and Schubert $(1981,1986)$ and Baik (1989). The use of normal modes in the analysis of atmospheric structure is described in Kasahara (1976). Finally, the transformed shallowwater equations may be reduced into a set of ordinary differential equations with the the amplitude of each normal mode as the dependent variable. This set of equations is dependent on: 1) the normal mode frequency, and 2) the amplitude of the normal mode "projection" of the nonlinear and forcing terms. Such a simplified set of equations has many numerical and computational advantages in models, as discussed by DeMaria and Schubert $(1984,1985)$ and Seager and Zebiak $(1994,1995)$.
} 
hydrostatically induced pressure (mass) field. Physically, because of the increased inertial stability horizontal displacements of air parcels are more restricted. Due to this decreased secondary circulation combined with the increased Coriolis force (which is proportional to wind velocity), gravity waves are retained in the disturbance for a longer period of time. As a result, a larger fraction of the latent heat is able to warm the air column before each gravity wave radiates away from the TC. ${ }^{5}$ Therefore, an increased rate of pressure fall will be generated by the larger retention of latent heat, more low-level air will flow down the pressure gradient to compensate, and results in a larger rate of $V_{\max }$ increase compared to a weaker system.

Hack and Schubert (1986b) derived a quantity known as "dynamic efficiency" which measures the percent of latent heating being converted to kinetic energy generation at a given location. Obviously, the larger the dynamic efficiency, the quicker the rate of intensification. But in addition to understanding these fundamental geostrophic adjustment processes, dynamic efficiency computations also help explain how vertically stacked vortices increase the conversion of latent heat to kinetic energy.

Figure 6.18 shows three different wind profiles for a $\mathrm{TC}$ of equal intensity $\left(V_{\max }=\right.$ $15 \mathrm{~m} \mathrm{~s}^{-1}$ at $r=50 \mathrm{~km}$ ). The top panel represents a vertically stacked vortex, while the middle and bottom panel shows a succeedingly weaker vertical structure. Hack and Schubert (1986b) assume that the latent heat release maximum shifts upwards with the

${ }^{5} \mathrm{~A}$ thermodynamic viewpoint for explaining warming in the TC has been proposed by Schubert and Hack (1982). Neglecting horizontal advection, the thermodynamic equation may be written in height coordinates as:

$$
\frac{\partial \ln \theta}{\partial t}=-w \frac{\partial \ln \theta}{\partial z}+\frac{\dot{Q}}{c_{p} T}
$$

where $\theta$ is the potential temperature, $c_{p}$ is the specific heat of dry air at constant pressure, and $\dot{Q}$ is the diabatic heating rate. The first term on the right hand of the equation is the vertical advection of $\theta$, usually called the adiabatic cooling rate. The second term is the diabatic heating rate due to condensation. Schubert and Hack (1982) claim that, for the same diabatic heat source, increasing inertial stability leads to weaker vertical motion and decreased diabatic cooling in the eyewall region. Hence, a smaller fraction of the diabatic heating is being used to compensate for the adiabatic cooling and a larger fraction is used to warm the eyewall. However, according to Holton (1992), $\dot{Q} \approx-w L_{v} \partial q^{*} / \partial z$ where $L_{v}$ is the latent heat of condensation and $q^{*}$ is the saturation mixing ratio. Since diabatic heating is also proportional to vertical velocity, the exact relationship of warming to inertial stability is unclear in the thermodynamic equation. Furthermore, it is uncertain that Schubert and Hack's explanation of inertial stability's role in TC development is similar to the geostrophic adjustment argument presented in this report. This is an area in need of more research, since it directly affects TC intensity change. 
vertical structure. This is a valid assumption, since convection in a vertically stacked vortex will penetrate deeper due to the inner-core being more unstable. An imposed diabatic heating of $18^{\circ} \mathrm{C} \mathrm{d}^{-1}$ is placed at 400,500 , and $600 \mathrm{mb}$ for the top, middle, and bottom panel, respectively - roughly in the area where $\partial v_{t} / \partial z$ is a maximum. The dynamic efficiency is largest for the case where $v_{t}$ is most conserved with height. Therefore, by optimizing $v_{t}$ such that it decreases little with height except near the tropopause, a $\mathrm{TC}$ is able to retain more of the latent heat release, thereby accelerating the pressure fall. Furthermore, if this conversion is more efficient aloft, the decrease of surface pressure is enhanced by the " $d p / p$ " effect. In turn, through thermal wind balance, the kinetic energy of the storm must increase, thereby initiating a feedback process.

A caveat to the geostrophic adjustment theory is that the outer-core inertial stability should not be too strong. This is because faster than average outer-core winds will constrain the secondary circulation too much, and radial inflow will be too weak to generate potent eyewall convection. Recall that Weatherford and Gray (1988a) found weaker outercore inertial stability values for storms which rapidly deepened. Hence, there is a delicate balance between the horizontal distribution of inertial stability and intensity change.

A second consideration is that Ekman pumping increases with intensity, which would enhance the transverse circulation. The interaction of boundary layer forcing with inertial stability, the ASI process, and the IFC process is not well-understood. As noted throughout this paper, the effect of Ekman pumping on intensity change is a complicated process which needs more research.

A final consideration is that the rate of intensification is dependent on the Radius of Maximum Wind (RMW) location and the cross-isobaric inflow angle in the boundary layer. Neglecting the vertical advection of $v_{t}$, the Coriolis parameter, and eddy vorticity fluxes, the $v_{t}$ equation of motion at the RMW simplifies to:

$$
\frac{\partial v_{t}}{\partial t}=\frac{-u_{r} v_{t}}{\mathrm{RMW}}+F
$$

where $\partial v_{t} / \partial r=0$ and $F$ denotes friction. If one assumes a small cross-isobaric inflow angle $\alpha$ such that $u_{r}=-\alpha v_{t}$, and crudely approximates friction in a well-mixed boundary 

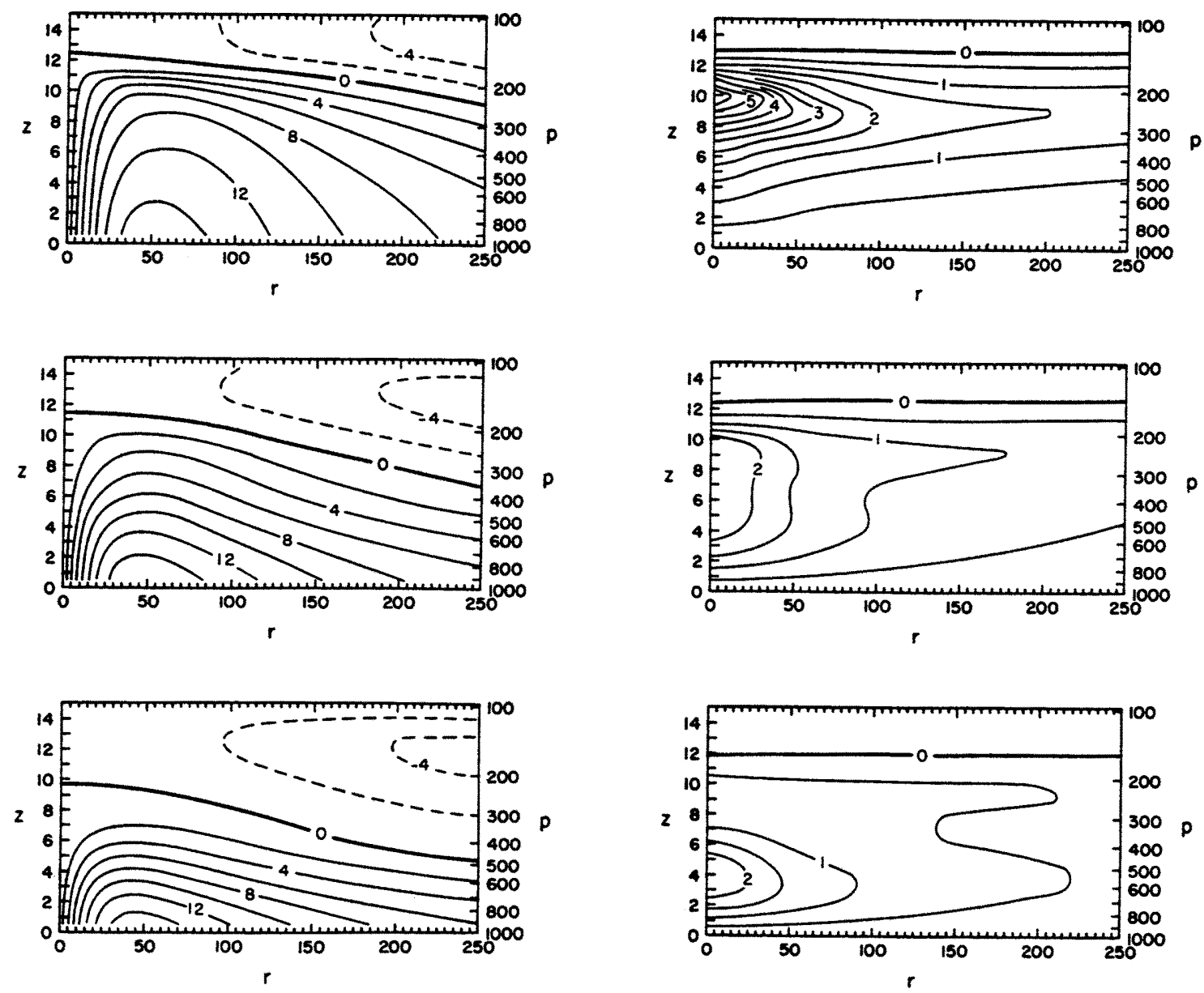

Figure 6.18: Tangential wind profiles (left panels) and the corresponding dynamic effciency factor (right panels) when 400-mb maximum heating (top row), 500-mb maximum heating (middle row), and 600-mb maximum heating (bottom row) are imposed at $r=0$ in a $250-\mathrm{km}$ region. The heating rate in each case is $18^{\circ} \mathrm{C} \mathrm{d}^{-1}$. Dynamic efficiency is defined as the percentage of latent heating being converted to kinetic energy generation at a given location. Each vortex has $V_{\max }=15 \mathrm{~m} \mathrm{~s}^{-1}$ at $50 \mathrm{~km}$, and hence is the same intensity. The contour interval is $2 \mathrm{~m} \mathrm{~s}^{-1}$ for the wind field, and $0.5 \%$ for the efficiency factor. From Hack and Schubert (1986). 
layer as $F=\sim-C_{D} v_{t}^{2} / \Delta z$ (Holton 1992) where the eddy viscosity $C_{D}$ is a constant and $\Delta z$ is the height of the boundary layer, the tangential equation of motion may be written (Gray 1995; Mundell 1990):

$$
\frac{\partial v_{t}}{\partial t}=v_{t}^{2}\left(\frac{\alpha}{\mathrm{RMW}}-\frac{C_{D}}{\Delta z}\right)
$$

which may be rewritten as

$$
\frac{\partial v_{t}}{\partial t}=\text { GENERATION }- \text { DISSIPATION }
$$

Equation (6.4) implies that intensification requires the generation term $\alpha /$ RMW to be greater than frictional dissipation. This is accomplished by an increase of the inflow angle or a decrease in the RMW. Hence, a small RMW and a steep pressure gradient is required to insure a vigorous transverse circulation. Furthermore, the smaller the RMW, the less cross-isobaric inflow is required for intensification. This is another reason rapid intensification is favored by convective processes close to the storm center.

However, there is a limit to how small the RMW can become. As stated by Gray (1995):

(The) tendency for a continuous decrease in...RMW must be terminated when...(it) becomes too small to vertically evacuate all of the converging air... It then becomes necessary for a...(second) eyewall to develop at larger radius. As the inner eye wall becomes encircled by the outer eye wall, its mass source is eliminated and it weakens and dies over a period of a few hours. The cyclone then experiences a weakening of intensity until the newly formed eye wall becomes well-established and it too begins a gradual inward contraction.

This is the concentric eye-wall cycle (Willoughby et al. 1982; Willoughby 1990; Black and Willoughby 1992) which was discussed in Chapter 3. This is yet another consideration in understanding and forecasting TC intensity change.

\subsection{Forecast potential}

This chapter's discussion on the relationship between vertical $v_{t}$ profiles and TC intensity change appears to offer forecast potential, since there seems to be a time lag between the increase in upper-level vorticity in the eyewall and the central pressure fall. Should high tangential winds be observed in the upper eyewall, and the potential intensification 
be large (as dictated by a large difference between MPI and current $V_{\max }$ ), one may predict future deepening at a fast rate. One example is the fast upper-level tangential winds observed during Hurricane Andrew's rapid deepening as shown in Fig. 3.7. Another is Supertyphoon Flo on 07 UTC 16 September 1990.

Figure 6.19 shows a planview of Flo at the onset of a $35 \mathrm{mb}$ pressure drop in the next $24 \mathrm{~h}$. Surface winds are $115 \mathrm{kts}$, yet $200-\mathrm{mb}$ winds are as fast as $90 \mathrm{kts}$, indicating a small $\partial v_{t} / \partial z$. The $200-\mathrm{mb}$ baroclinicity is evident by the $9^{\circ} \mathrm{C}$ temperature decrease from the eye to the eyewall, consistent with the reconnaissance plane observations discussed previously on intense storms (Fig. 6.12). The next day, surface winds are $145 \mathrm{kts}$ and $200-\mathrm{mb}$ winds approach $110 \mathrm{kts}$, indicating that the vortex indeed has deepened (Fig. 6.20). The storm then recurved and began to weaken as it entered a region of increased shear. It should be noted that Flo did not rapidly intensify due to an import of REFC, as eddy flux values were less than $5 \mathrm{~m} \mathrm{~s}^{-1} \mathrm{~d}^{-1}$ (Merrill and Velden 1996).

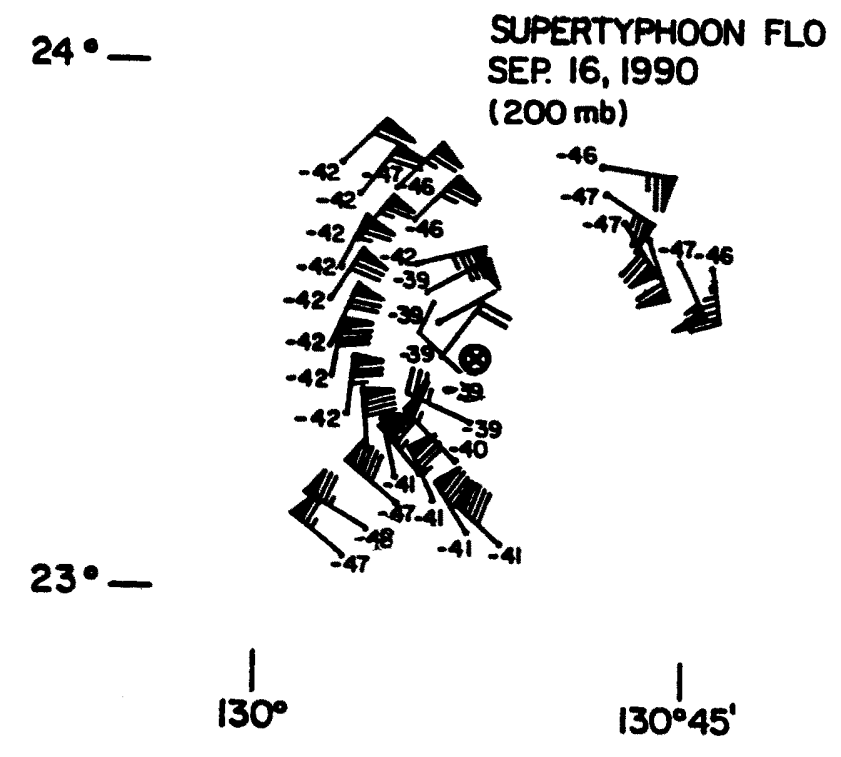

Figure 6.19: Planview of Supertyphoon Flo inner-core winds on 07 UTC 16 September 1990 at $200 \mathrm{mb}$. Winds are in kts, and include storm motion. Temperature is shown next to windbarbs $\left({ }^{\circ} \mathrm{C}\right)$. Surface $V_{\max }$ is $115 \mathrm{kts}$.

\subsubsection{Intensity prediction based on inner-core sounding}

As suggested by Flo and Fig. 6.4, a direct proportionality to the rate of intensification and the preservation of $v_{t}$ with height for storms of similar intensity seems to exist. To 


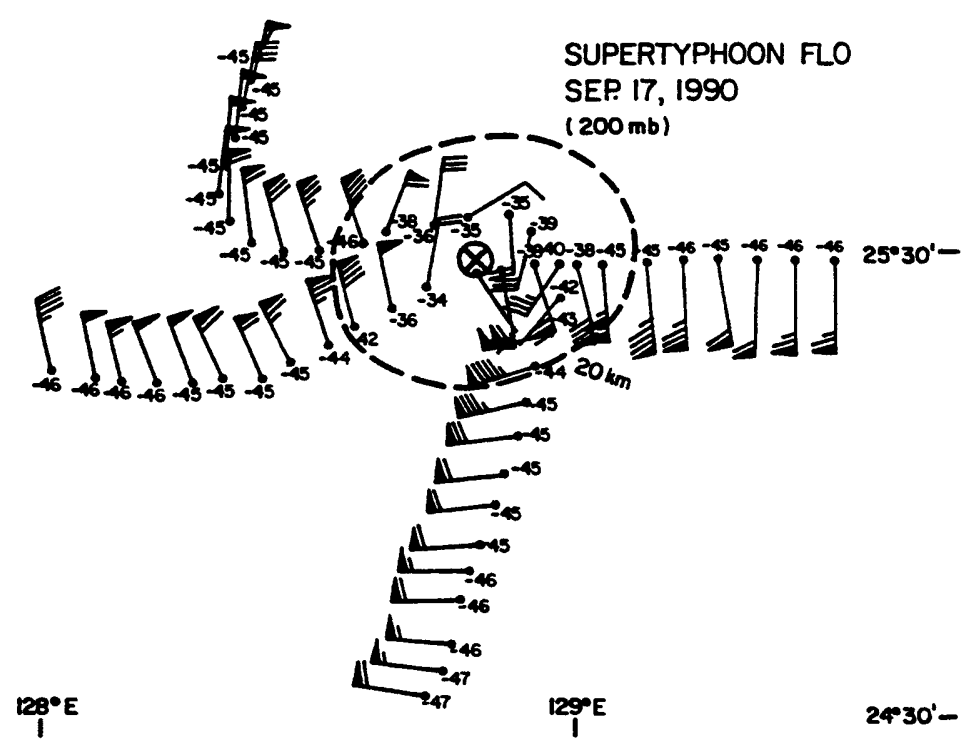

Figure 6.20: Planview of Supertyphoon Flo inner-core winds on 07 UTC 17 September 1990 at $200 \mathrm{mb}$. Winds are in kts, and include storm motion. Temperature is shown next to windbarbs $\left({ }^{\circ} \mathrm{C}\right)$. Surface $V_{\max }$ is $145 \mathrm{kts}$.

investigate this relationship further, the 9 soundings which comprise the fast developers and the 19 soundings which represent the slow developers in Fig. 6.4 are stratified by radial position. This stratification compares fast developers to slow developers within $0.3 \mathrm{deg}$ radius. Since these storms are of similar intensity $\left(50 \leq V_{\max } \leq 85 \mathrm{kts}\right), v_{t}$ profile comparisons at a specific radius should offer additional insight between fast and slow developers. This approach also checks whether an anomalously different sounding is influencing the composites in Fig. 6.4.

The mean 500 to $200-\mathrm{mb}$ tangential wind $\left(\overline{v_{t}}\right)$ is compared between a fast intensifying storm $\left(\overline{v_{t}}(\mathrm{f})\right)$ and slower developing storm $\left(\overline{v_{t}}(\mathrm{~s})\right)$ for similar radii. Three categories are defined: 1) $\left.\left.\overline{v_{t}}(\mathrm{f})>\overline{v_{t}}(\mathrm{~s}) ; 2\right) \overline{v_{t}}(\mathrm{f}) \approx \overline{v_{t}}(\mathrm{~s}) ; 3\right) \overline{v_{t}}(\mathrm{f})<\overline{v_{t}}(\mathrm{~s})$. Table 6.1 shows the results for each of the 9 fast developers compared to slow developers located within $0.3 \mathrm{deg}$ of each fast developer.

Even though the sample is small, Table 6.1 suggests a tendency for vertically stacked TCs to develop faster than TCs with similar $V_{\max }$ but weaker $v_{t}$ aloft. About threefourths $(74 \%$, or 56 out of 76$)$ of the comparisons exhibited stronger upper-tropospheric $v_{t}$ for the fast developing storm. This is quite a remarkable observation given that the 
Table 6.1: Comparison of mean 500 to 200 -mb tangential winds $\left(\overline{v_{t}}\right)$ for each of nine fast developers (pressure drop $\geq 30 \mathrm{mb}$ in the next $24 \mathrm{~h}$; denoted with an "f") against slower developers (10 $\leq \Delta p \leq 20 \mathrm{mb}$ in the next $24 \mathrm{~h}$; denoted with an "s") located within 0.3-deg radial distance of each fast developer. The number (\#) of slow developers within $0.3 \mathrm{deg}$ of each fast developer is shown. Comparisons are expressed in percentage and number of observations for three categories as defined by: 1) $\left.\overline{v_{t}}(\mathrm{f})>\overline{v_{t}}(\mathrm{~s}) ; 2\right) \overline{v_{t}}(\mathrm{f}) \approx \overline{v_{t}}(\mathrm{~s})$; 3) $\overline{v_{t}}(\mathrm{f})<\overline{v_{t}}(\mathrm{~s})$. All fast and slow developers are similar in intensity $\left(50 \leq V_{\max } \leq 85 \mathrm{k}-\right.$ ts). The table is listed by increasing sounding radius. The winds are from rawinsonde measurements. Storm motion is removed. For example, Amy (1971) was a fast developing TC with a rawinsonde measurement taken at $1.2 \mathrm{deg}$, and 6 slow developers contained measurements between $0.9 \mathrm{deg}$ and $1.5 \mathrm{deg}$; Amy's 500 to $200-\mathrm{mb}$ mean $v_{t}$ was stronger in 4 of these cases (67\%), and in the other two cases (33\%) mean upper-level $v_{t}$ was weaker.

\begin{tabular}{|c|c|c|c|c|c|}
\hline $\begin{array}{c}\text { Fast } \\
\text { developer }\end{array}$ & $\begin{array}{c}\text { Radius of } \\
\text { sounding }\end{array}$ & $\begin{array}{c}\text { \# of slow } \\
\text { developers } \\
\leq 0.3 \mathrm{deg}\end{array}$ & $\begin{array}{c}\% \text { \& number } \\
\overline{v_{t}}(\mathrm{f})>\overline{v_{t}}(\mathrm{~s})\end{array}$ & $\begin{array}{c}\% \text { \& number } \\
\overline{v_{t}}(\mathrm{f}) \approx \overline{v_{t}}(\mathrm{~s})\end{array}$ & $\begin{array}{c}\% \text { \& number } \\
\overline{v_{t}}(\mathrm{f})<\overline{v_{t}}(\mathrm{~s})\end{array}$ \\
\hline \hline Gloria (1974) & 0.8 & 3 & $100(3)$ & & \\
\hline Amy (1971) & 1.2 & 6 & $67(4)$ & & $33(2)$ \\
\hline Wendy (1971) & 1.2 & 6 & $67(4)$ & & $33(2)$ \\
\hline Vera (1977) & 1.5 & 13 & $84(11)$ & $8(1)$ & $8(1)$ \\
\hline Wendy (1963) & 1.6 & 12 & $75(9)$ & $25(3)$ & \\
\hline Carla (1967) & 1.8 & 9 & $22(2)$ & $11(1)$ & $67(6)$ \\
\hline Ora (1968) & 1.9 & 9 & $100(9)$ & & \\
\hline Louise (1964) & 1.9 & 9 & $78(7)$ & $22(2)$ & \\
\hline Jean (1968) & 1.9 & 9 & $78(7)$ & $22(2)$ & \\
\hline \hline Total & & 76 & $74(56)$ & $12(9)$ & $14(11)$ \\
\hline
\end{tabular}


0-2 deg horizontal distribution of low-level $v_{t}$ can vary significantly from storm to storm even with the same $V_{\max }$.

Only $14 \%$ of the fast developers did not show stronger upper-level $v_{t}$ compared to the slow developers. Even more striking is that most of this $14 \%$ is associated with one TC (Carla). The reason for this discrepancy is that Carla was apparently a small TC, as the upper-level winds had become strongly anticyclonic at $1.8 \mathrm{deg}$ (not shown).

Figure 6.21 shows the wind profile for one of these fast developing storms (Gloria) at $r=0.8 \mathrm{deg}$ (Curve A) compared to three other slowly intensifying storms at the same general radii (Curves $\mathrm{B}, \mathrm{C}$, and D). Gloria experienced an MSLP change $\Delta p$ in the next $24 \mathrm{~h}$ of $39 \mathrm{mb}$, while $\Delta p$ in the other three was between 11 and $18 \mathrm{mb} \mathrm{d}^{-1}$. Again, the distinction between these two intensification classes is clear, with Gloria containing winds up to $20 \mathrm{~m} \mathrm{~s}^{-1}$ faster in the upper-troposphere than the other storms. Note that the low-level winds are roughly equal in magnitude.

Another wind profile example is shown in Fig. 6.22 for Vera (1977), which experienced a $\Delta p$ of $39 \mathrm{mb}$ in the next day (Profile A). Also shown are wind profiles for 4 storms of similar intensity ( $V_{\max }$ between 50 and $70 \mathrm{kts}$ ) which developed slower ( $\Delta p 10$ to $15 \mathrm{~m}$ $\mathrm{b} \mathrm{d}^{-1}$ ). One should note that the low-level winds are quite different from one another, synonymous with the fact that, in general, individual TCs contain unique low-level horizontal wind distributions. Vera actually contained weaker winds in the lower troposphere compared to the other four storms. Yet, Vera has the fastest 500 to 200 -mb winds - a stark example of wind preservation with height and indicative of potential fast intensification. These upper-level differences are intriguing since the low-level winds are fairly scattered in this example. Hence, even though Vera contains slower low-level winds, the conservation of $v_{t}$ with height results in stronger winds aloft.

Similar findings are shown for Jean (1968) at the onset of $\Delta p=34 \mathrm{mb} \mathrm{d}^{-1}$ (Fig. 6.23) and for rapidly intensifying Wendy (1971) at the onset of $\Delta p=45 \mathrm{mb} \mathrm{d}^{-1}$ (Fig. 6.24). In general, Jean contains stronger or equal 500 to $200-\mathrm{mb}$ winds compared to the slower developers. The 500 to $300-\mathrm{mb}$ winds were all stronger, and comparable to the other storms from 300 to $200 \mathrm{mb}$ in Fig. 6.23 except for Ellen (Profile E). Hence, $\overline{v_{t}}(f)>\overline{v_{t}}(s)$ 


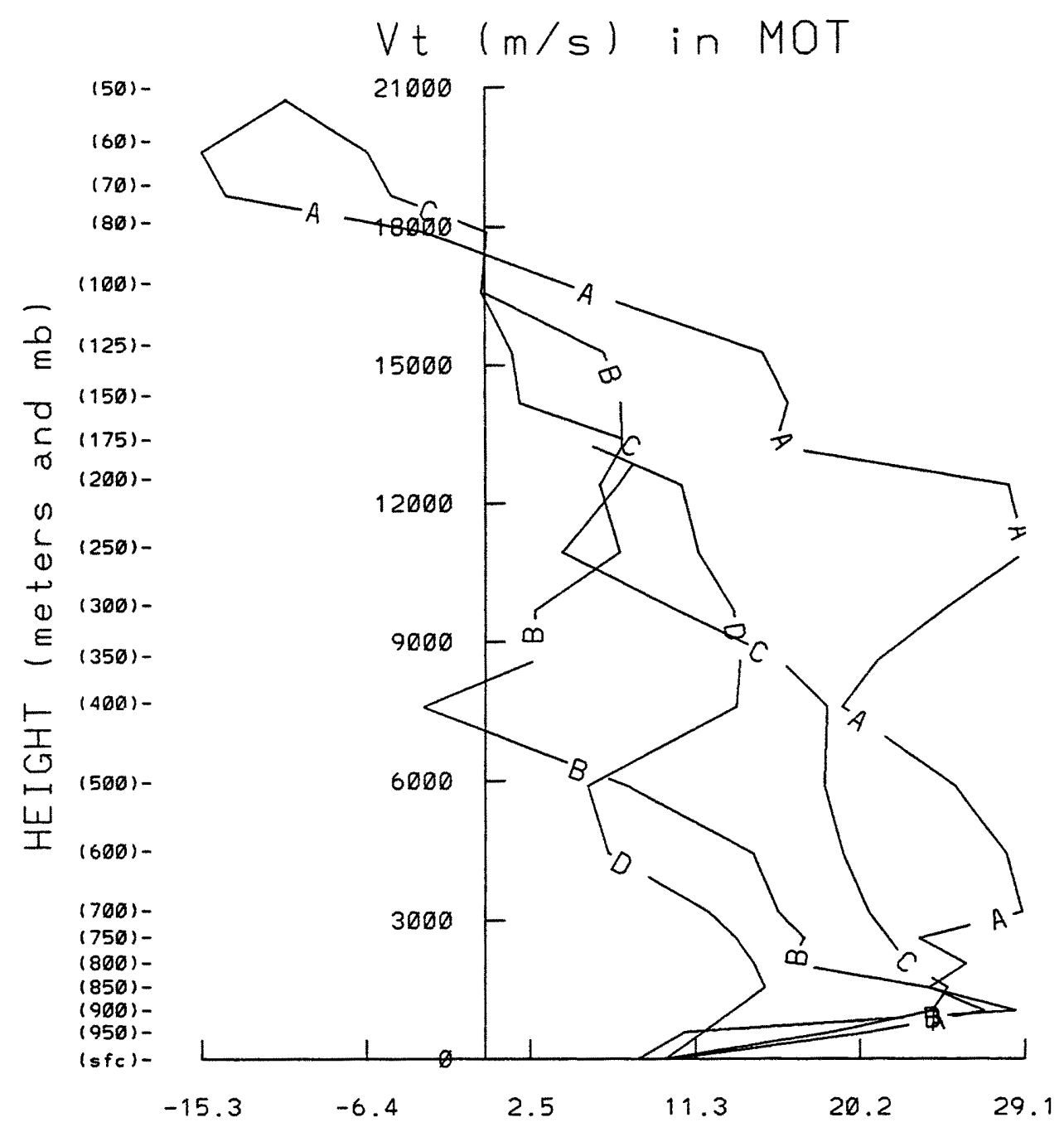

gloria74.pfi

hester57.pnfi

$101075 . p n f i$

susan63.pnfi
[A] $v \max =60$. kts. $p=979.0 \mathrm{mb}$, eye dia $=99.9 \mathrm{~nm}, r=0.8$ deg, $d p=-39.0 \mathrm{mb}$

[B] vmax $=60$. kts, $p=986.0 \mathrm{mb}$, eye dia=99.9 nm, $r=0.8$ deg. $d p=-18.0 \mathrm{mb}$

[C] vmax $=50$. Kts. $p=986.0 \mathrm{mb}$. eye dia=99.9 nm, $r=0.8$ deg, dp=-11.0 mb

[D] vmax $=55$. Kts, $p=1001.0 \mathrm{mb}$, eye dia $=99.9 \mathrm{~nm}, r=0.9$ deg. $d p=-17.0 \mathrm{mb}$

Figure 6.21: Tangential wind sounding for Gloria (1974) at $r=0.8 \mathrm{deg}$, which deepened by $39 \mathrm{mb}$ in the next $24 \mathrm{~h}$ (profile A) compared to three other storms which developed slower - Hester (1957) for profile B, Lola (1975) for profile C, and Susan (1963) for profile D. Storm information is presented underneath the graph. Shown first is the file name, in which the extension ".pf" represents a fast intensifying Pacific storm, a ".pnfi" represents a Pacific storm not intensifying fast, and ".anf" represents an Atlantic storm not intensifying fast. Next shown (in order) is the profile indicator (in brackets), the maximum winds (vmax), the MSLP, the eye diameter (in n.m.), the sounding radius with respect to the storm center, and the future 24-h MSLP fall. Often the eye diameter observation was missing, and is then designated as "99.9 n.m.". Storm motion is removed. For example, profile $\mathrm{C}$ shows the western North Pacific $v_{t}$ rawinsonde observation taken $0.8 \mathrm{deg}$ from TC Lola's center with the storm motion removed; for Lola, $V_{\max }=50 \mathrm{kts}$, MSLP $=986 \mathrm{mb}$, the eye diameter observation was missing, and the future 24-h MSLP fall is $11 \mathrm{mb}$. 


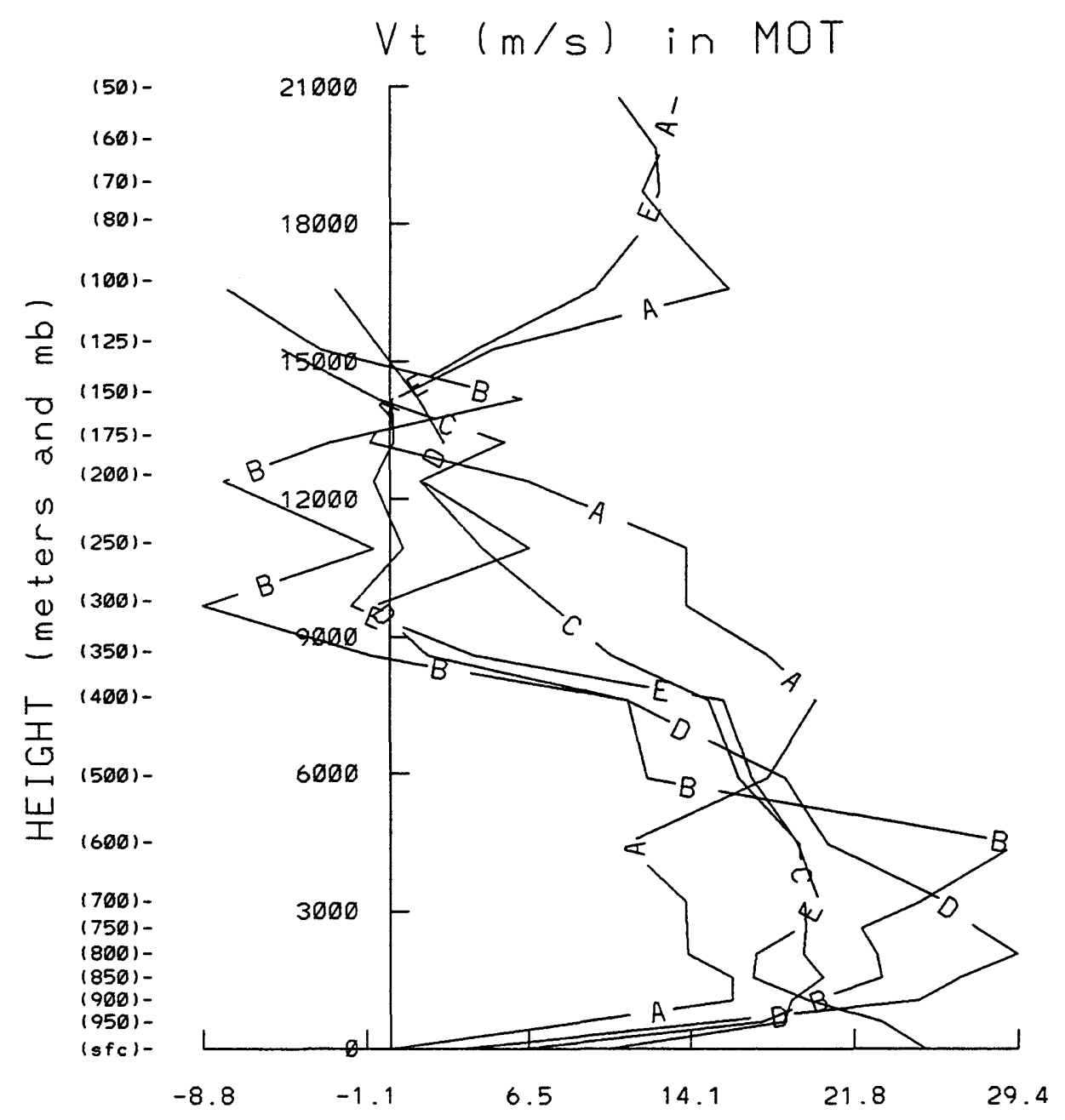

\footnotetext{
vera77.pfi

flora63.anfi

inez66.anfi

agnes 74 .pnfi

beth71.anf ;
}

\begin{abstract}
[A] vmax $=50$. Kts. $p=987.0 \mathrm{mb}$, eye dia $=16.0 \mathrm{~nm}, r=1.5 \mathrm{dog} . d p=-39.0 \mathrm{mb}$
[B] vmax $=70$. Kts, $p=990.0 \mathrm{mb}$, eye dia $=99.9 \mathrm{~nm}, r=1.4 \mathrm{deg} . d p=-15.0 \mathrm{mb}$

[C] vmax=65. kts. $p=995.0 \mathrm{mb}$. eye dia=99.9 nm. $r=1.6 \mathrm{deg} . d p=-10.0 \mathrm{mb}$

[D] $v_{\max }=50 . k \mathrm{ts}, p=994.0 \mathrm{mb}$, eye dia $=99.9 \mathrm{~nm}, r=1.4 \mathrm{deg}, d p=-15.0 \mathrm{mb}$

[E] vmax $=70$. Kts. $p=990.0 \mathrm{mb}$, eye dia $=99.9 \mathrm{~nm}, r=1.2 \mathrm{deg} . d p=-12.0 \mathrm{mb}$
\end{abstract}

Figure 6.22: As in Fig. 6.21, but for Vera (1977) at $r=1.5$ deg, which deepened by $39 \mathrm{mb}$ in the next $24 \mathrm{~h}$ (profile A) compared to four other storms which developed slower Flora (1963) for profile B, Inez (1966) for profile C, Agnes (1974) for profile D, and Beth (1971) for profile E. Storm motion is removed. 
for 3 of the 4 cases, with $\overline{v_{t}}(f) \approx \overline{v_{t}}(s)$ compared to Ellen. Wendy also exhibits stronger upper-tropospheric winds in 3 of the 4 plots in Fig. 6.24, with differences of 5 to $15 \mathrm{~m} \mathrm{~s}^{-1}$. The one exception is Kathy (Profile E), which contains stronger upper-tropospheric winds.

Other examples exist from the NHRP data set. The four storms with the strongest upper-level winds within 25 n.m. in Fig. 6.3 constitute the fastest deepeners. Daisy (1958), with an MSLP of $943 \mathrm{mb}$, was experiencing a pressure fall of $33 \mathrm{mb} \mathrm{d}^{-1}$ during this period. Helene (1958), with an MSLP of $948 \mathrm{mb}$, was in the midst of a $43 \mathrm{mb} \mathrm{d}^{-1}$ pressure drop. Hilda (1964), with an MSLP of $946 \mathrm{mb}$, and Inez (1966), with an MSLP of $934 \mathrm{mb}$, were near the end of 37 and $30 \mathrm{mb} \mathrm{d}^{-1}$ pressure drops, respectively. The other storms with slower $v_{t}$ aloft maintained their current intensity or weakened in the day. The exception is during the early stage of Inez (1966) when the MSLP was $962 \mathrm{mb}$. Inez was crossing the Guadeloupe Islands at this time and slightly weakened for $12 \mathrm{~h}$ (Sugg 1967). Inez resumed intensification upon re-entering the Caribbean Sea.

These upper-tropospheric NHRP results are summarized in Table 6.2, which list the storms from deepening to weakening states. Shown in Table 6.2 is the RMW, the azimuthally averaged temperature difference $(\Delta T)$ from 5 to 50 n.m., and the azimuthally averaged maximum tangential wind $\left(v_{t_{\text {max }}}\right)$ from the NHRP data set. As just discussed, there seems to be a direct correlation between upper-level inner-core $v_{t_{\max }}$ and intensification. Note that $v_{t_{\max }}$ in Table 6.2 steadily decreases from 40-60 kts for developing storms to $10-30 \mathrm{kts}$ for weakening storms. Furthermore, the RMW also tends to be closer to the storm center for the deepening storms, in agreement with Eq. (6.3). Finally, as previously shown in Fig. 6.12, the temperature gradient tends to be stronger in developing storms, with warmer than normal eyes but cooler than average eyewalls. There is a steady temperature decrease from $6-9^{\circ} \mathrm{C}$ for developing storms to $3-5^{\circ} \mathrm{C}$ for weakening storms. A small RMW is also consistent with the large eye-eyewall temperature differential.

\subsubsection{Intensity prediction based on inner-core upper-tropospheric wind and temperature symmetry}

While there seems to be a forecast signal based on vertical soundings of $v_{t}$, the RMW, and the temperature gradient, it is natural to ask if the three-dimensional uppertropospheric wind and temperature structure also yield clues to TC intensification. To 


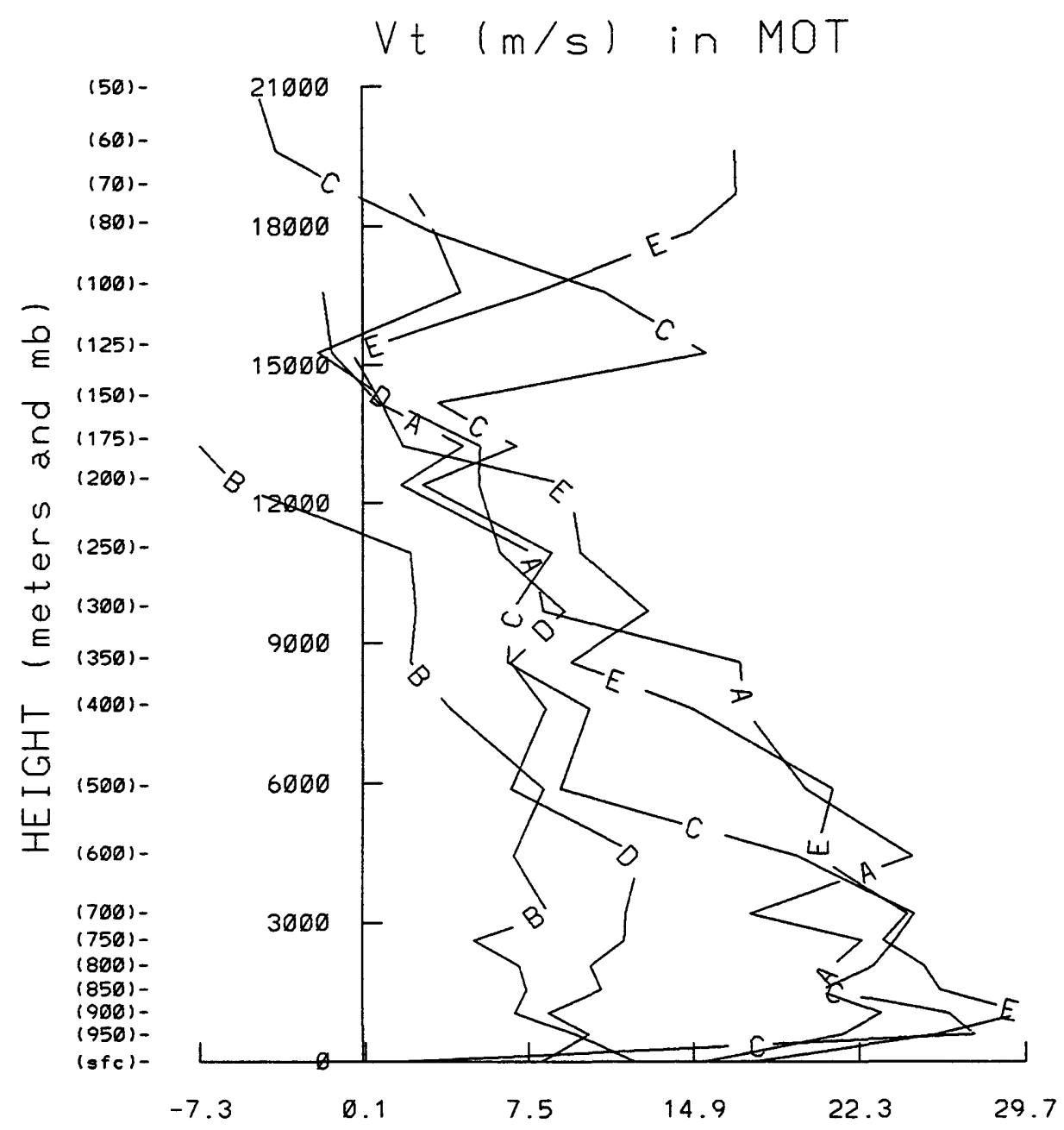

\footnotetext{
jean68.pfi

[A] vmax $=50 . k$ ts. $p=990.0 \mathrm{mb}$. Oye di $a=30.0 \mathrm{~nm}, r=1.9 \mathrm{deg} . d p=-34.0 \mathrm{mb}$

gerda69.anf;

[B] vmax=70. kts. $p=991.0 \mathrm{mb}$. Oye dia $=99.9 \mathrm{~nm}, r=2.0$ deg. $d p=-12.0 \mathrm{mb}$

faye 57 .pnf :

[C] vmax=60. kts. $p=968.0 \mathrm{mb}$, eye dia $=99.9 \mathrm{~nm} . r=1.9$ deg. $d p=-18.0 \mathrm{mb}$

anna61.anf $i$

[D] $v \max =80$. kts, $p=1002.0 \mathrm{mb}$, eye dia $=99.9 \mathrm{~nm}, r=1.6 \mathrm{deg}, d p=-18.0 \mathrm{mb}$

ellen67.pnfi

[E] vmax $=65$. kts. $p=981.0 \mathrm{mb}$. eye dia=15.0 nm, $r=1.8 \mathrm{deg} . d p=-11.0 \mathrm{mb}$
}

Figure 6.23: As in Fig. 6.21, but for Jean (1968) at $r=1.9$ deg, which deepened by $34 \mathrm{mb}$ in the next $24 \mathrm{~h}$ (profile A) compared to four other storms which developed slower Gerda (1969) for profile B, Faye (1957) for profile C, Anna (1961) for profile D, and Ellen (1967) for profile E. Storm motion is removed. 


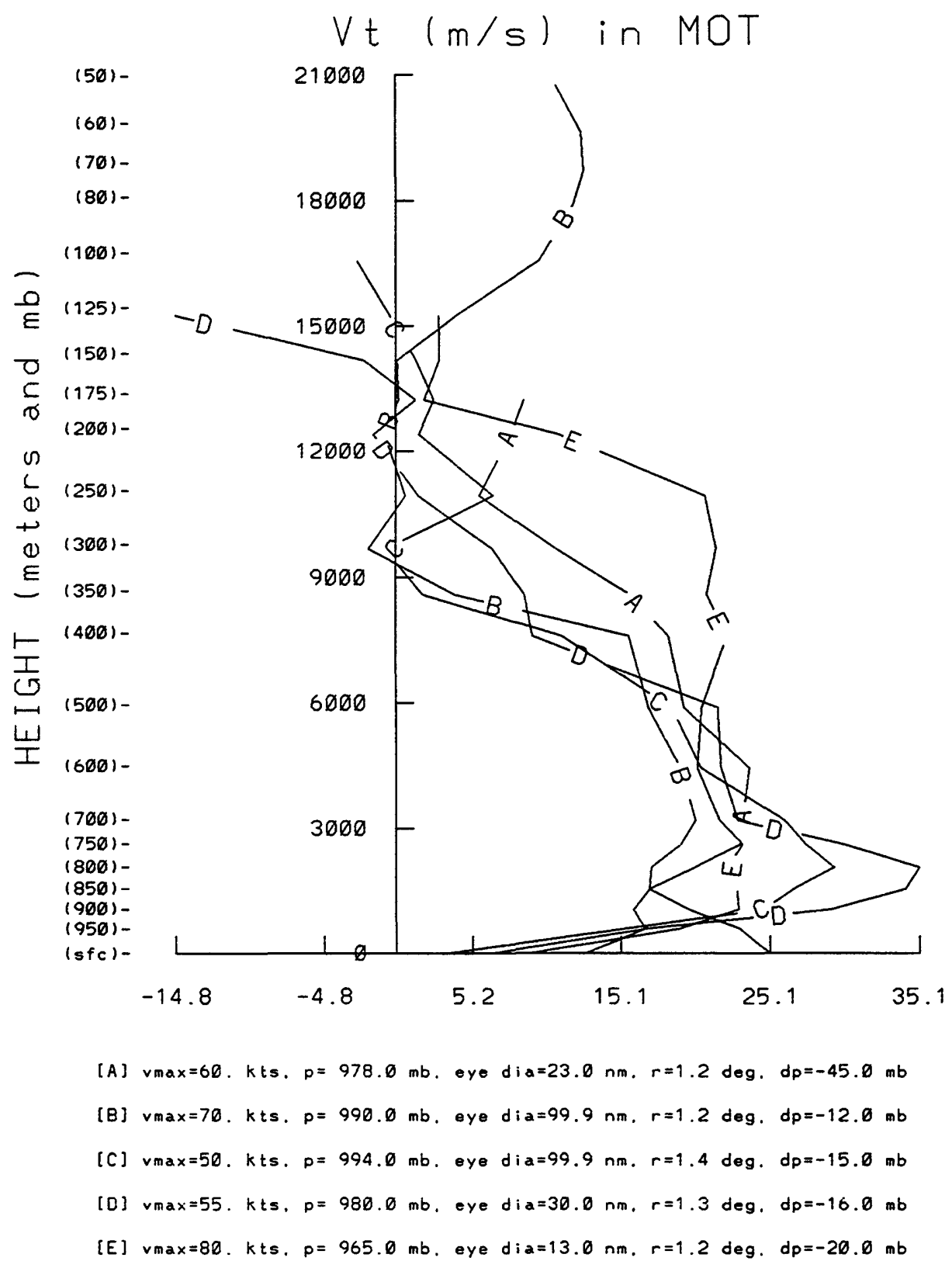

Figure 6.24: As in Fig. 6.21, but for Wendy (1971) at $r=1.2$ deg, which deepened by $45 \mathrm{mb}$ in the next $24 \mathrm{~h}$ (profile A) compared to four other storms which developed slower - Beth (1971) for profile B, Agnes (1974) for profile C, Irma (1968) for profile D, and Kathy (1964) for profile E. Storm motion is removed. 
address this question, planview contour wind plots were performed for NHRP storms with 4 flight legs or more in the upper-troposphere. The contours were performed by interpolating the wind magnitude around each radius using an Akima cubic spline routine (Akima 1970). If an observation was missing in a 2.5 n.m bin, this observation was linearly interpolated along the flight leg.

The storms were subjectively analyzed for temperature and wind symmetry and designated as symmetric (SY), weakly-symmetric (WSY), and asymmetric (ASY). A storm qualified as symmetric if numerous $(>4)$ isolines exhibited circular uniformity, although secondary maximums may exist. A weakly symmetric storm contained just a few (2-3) circular isolines, while asymmetric denoted scattered, non-circular features. The results are shown in Table 6.2.

Table 6.2: List of NHRP flights along an upper-tropospheric pressure surface $\left(p_{u t}\right)$ and their corresponding mean sea-level pressure (SLP). The trend (Tr) of intensification is denoted as deepening (D), steady-state (S), and weakening (W). S/D indicates the storm was experiencing oscillatory, small pressure falls. L denotes land influence. Symmetry classification is performed when at least 4 flight legs $(\mathrm{Lg})$ were performed. Subjective determination of symmetry is denoted as "symmetric" (SY), "weakly symmetric" (WSY), and "asymmetric" (ASY). $\Delta T$ is the radially averaged temperature difference from 5 n.m. to 50 n.m. $\left({ }^{\circ} \mathrm{C}\right)$. RMW is the Radius of Maximum Winds in n.m. $v_{t_{\max }}$ is the symmetrically averaged tangential wind maximum (kts) along $p_{h i g h}$. All times occur between 17 and 20 UTC. The storms are listed from deepening to weakening states. Storm motion is removed.

\begin{tabular}{|l|c|c|c|c|c|c|c|c|c|c|}
\hline Storms & Date & SLP & $p_{\text {ut }}$ & Lg & Tr & $v_{t_{\max }}$ & RMW & $|\vec{V}|$ sym & $\Delta T$ & T sym \\
\hline Helene & $9 / 26 / 58$ & 948 & 270 & 4 & D & 58 & 22.5 & SY & 6 & SY \\
\hline Hilda & $10 / 1 / 64$ & 946 & 199 & 2 & D & 40 & 15.0 & & 6 & \\
\hline Daisy & $8 / 27 / 58$ & 943 & 270 & 4 & D & 55 & 10.0 & SY & 6 & SY \\
\hline Inez & $9 / 28 / 66$ & 934 & 221 & 4 & D & 50 & 12.5 & SY & 9 & SY \\
\hline Betsy & $9 / 3 / 65$ & 952 & 221 & 4 & S/D & 40 & 37.5 & WSY & 6 & SY \\
\hline Carrie & $9 / 15 / 57$ & 964 & 260 & 2 & S & 33 & 42.5 & & 4 & \\
\hline Cleo & $8 / 18 / 58$ & 974 & 260 & 5 & S & 33 & 50.0 & WSY & 3 & WSY \\
\hline Inez & $9 / 27 / 66$ & 962 & 221 & 4 & L & 26 & 25.0 & ASY & 5 & WSY \\
\hline Carrie & $9 / 17 / 57$ & 975 & 260 & 4 & W & 33 & 50.0 & ASY & 3 & ASY \\
\hline Hilda & $10 / 2 / 64$ & 956 & 211 & 2 & W & 27 & 45.0 & & 3 & \\
\hline Betsy & $9 / 5 / 65$ & 970 & 221 & 4 & W & 14 & 40.0 & ASY & 4 & WSY \\
\hline
\end{tabular}

In general, upper-tropospheric symmetry (asymmetry) is correlated with intensification (weakening). Furthermore, an inspection of lower-level data did not show such 
clear distinctions, suggesting that upper-level inner-core data contains crucial predictive intensity change information. This result is not surprising, as developing storms will build a vertically stacked vortex due to eyewall buoyancy and a favorable environment (warm SSTs, low wind shear, etc...) In contrast, weakening TCs cannot sustain upper-level vorticity without sufficient eyewall convection and warm SSTs - furthermore, strong wind shear will advect the upper-level vortex and warm core away from the storm center. These results strongly suggest that symmetric inner-core upper-level features portend intensification. Specific examples now follow.

Recall the $270 \mathrm{mb}$ wind barb plot (Fig. 6.8) for developing Hurricane Daisy which suggested strong inner-core symmetry. Figure 6.25 confirms that the wind magnitude was symmetric, except for a 60 -kts wind maximum in the northeast sector between the symmetric 50-kts isotach. Furthermore, the temperature field also exhibited general circular similarity with a moderate temperature gradient (Fig. 6.26).

Intensifying Helene also contains similar characteristics, although its wind field is not perfectly symmetric with maximum winds in the northeast quadrant (Fig. 6.27). Nevertheless, numerous circular isotachs near the center qualifies it as a symmetric wind field. An examination of the isotherms also strongly suggests symmetry (Fig. 6.28).

In contrast, steady-state Cleo exhibited only weak symmetry in both fields, with a circular 20-kts isotach but distorted 10 and 30-kts isotachs (Fig. 6.29), and a moderate temperature gradient with two circular isotherms but a deformed $5^{\circ} \mathrm{C}$ isotherm (Fig. 6.30). For a weakening storm such as Carrie, both the temperature and wind field is asymmetric (Figs. 6.31 and 6.32).

\subsection{Broad-scale composites of fast and slow developing tropical cyclones}

While this chapter has concentrated on inner-core structure, a general overview of the broad-scale features associated with fast developing and slow developing Pacific storms is also warranted. While inner-core features probably dominate the intensification process, distinctions between the radial winds, tangential winds, and temperature fields in a coarser 2 to 14-deg area may also yield valuable TC information about these two development 


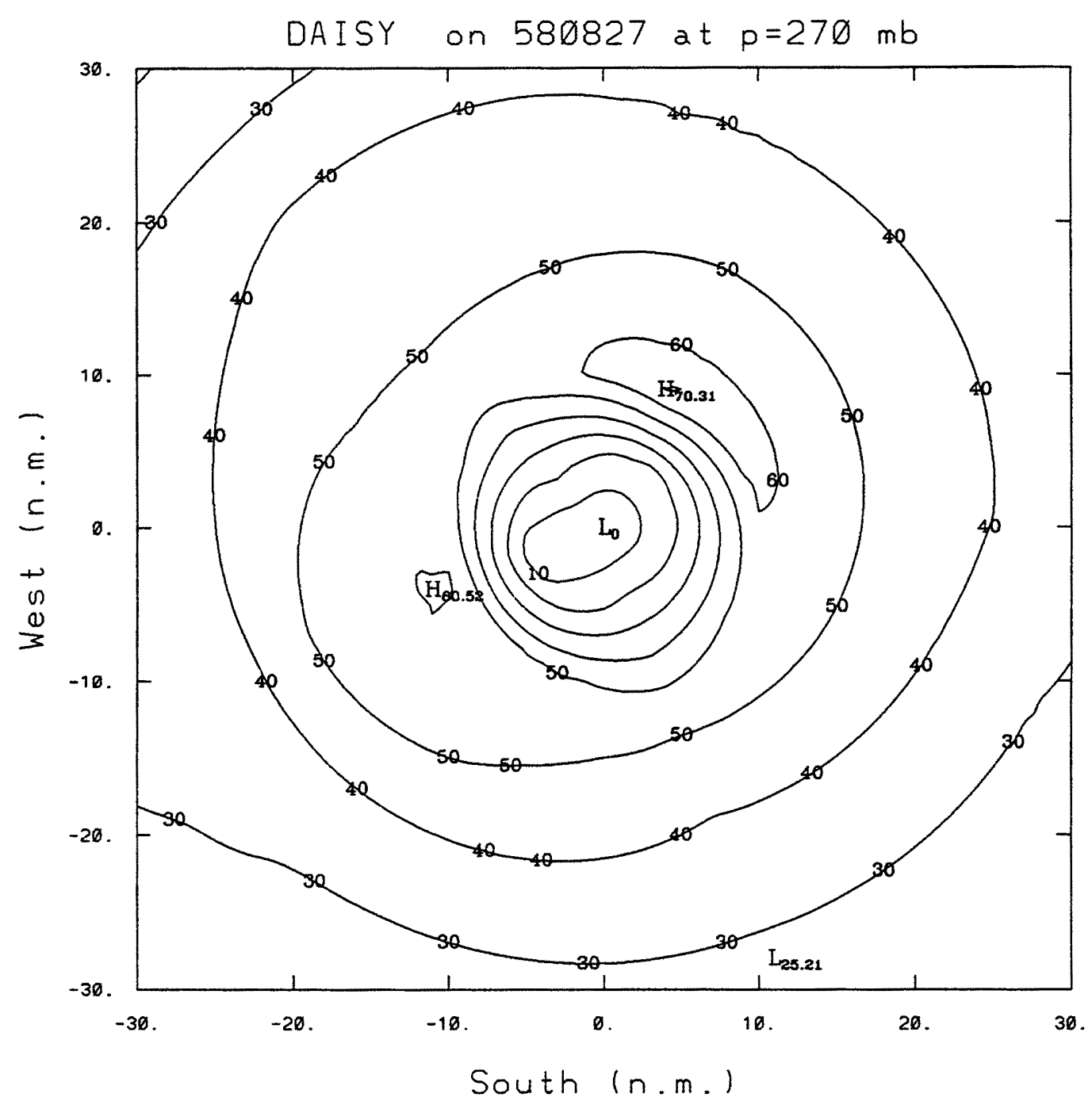

Figure 6.25: Plan-view of wind magnitudes for deepening Hurricane Daisy on 1730 UTC 27 August 1958. Pressure level is $270 \mathrm{mb}$. Contour interval is $10 \mathrm{kts}$. Storm motion is removed. 


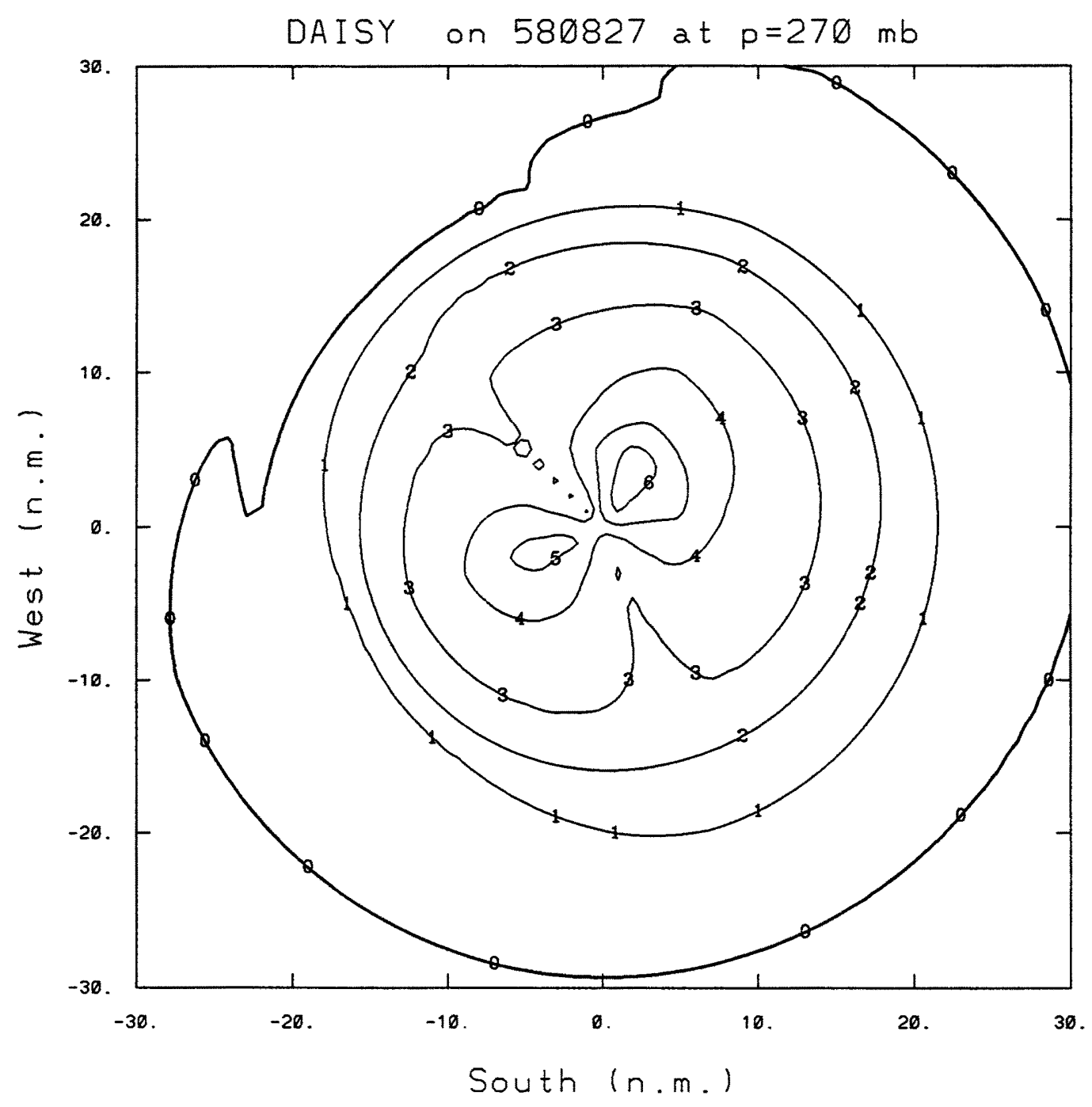

Figure 6.26: Plan-view of temperature deviations for deepening Hurricane Daisy on 1730 UTC 27 August 1958. Pressure level is $270 \mathrm{mb}$. Contour interval is $1^{\circ} \mathrm{C}$. 


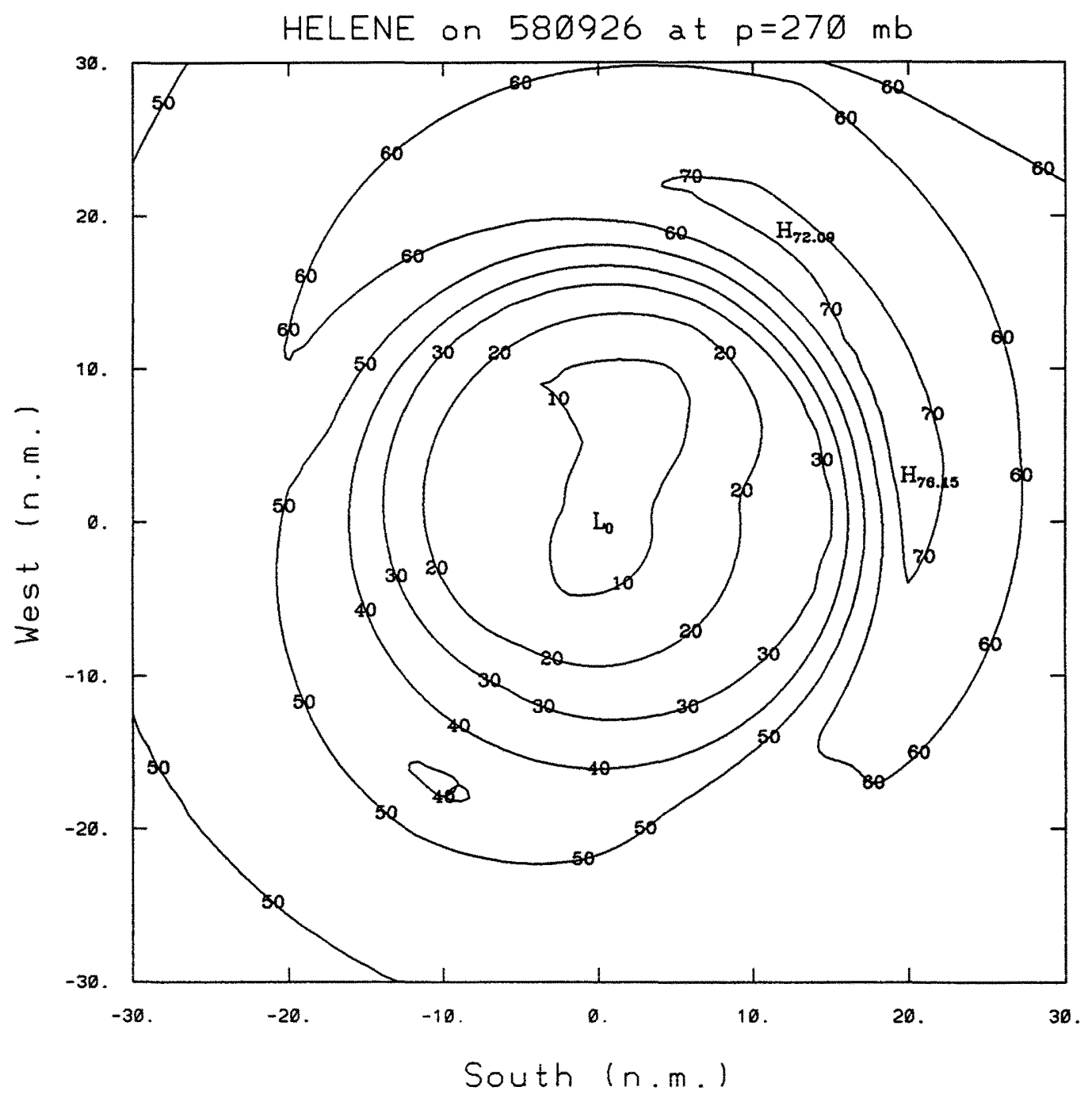

Figure 6.27: Plan-view of wind magnitudes for deepening Hurricane Helene on 18 UTC 26 September 1958. Pressure level is $270 \mathrm{mb}$. Contour interval is $10 \mathrm{kts}$. Storm motion is removed. 


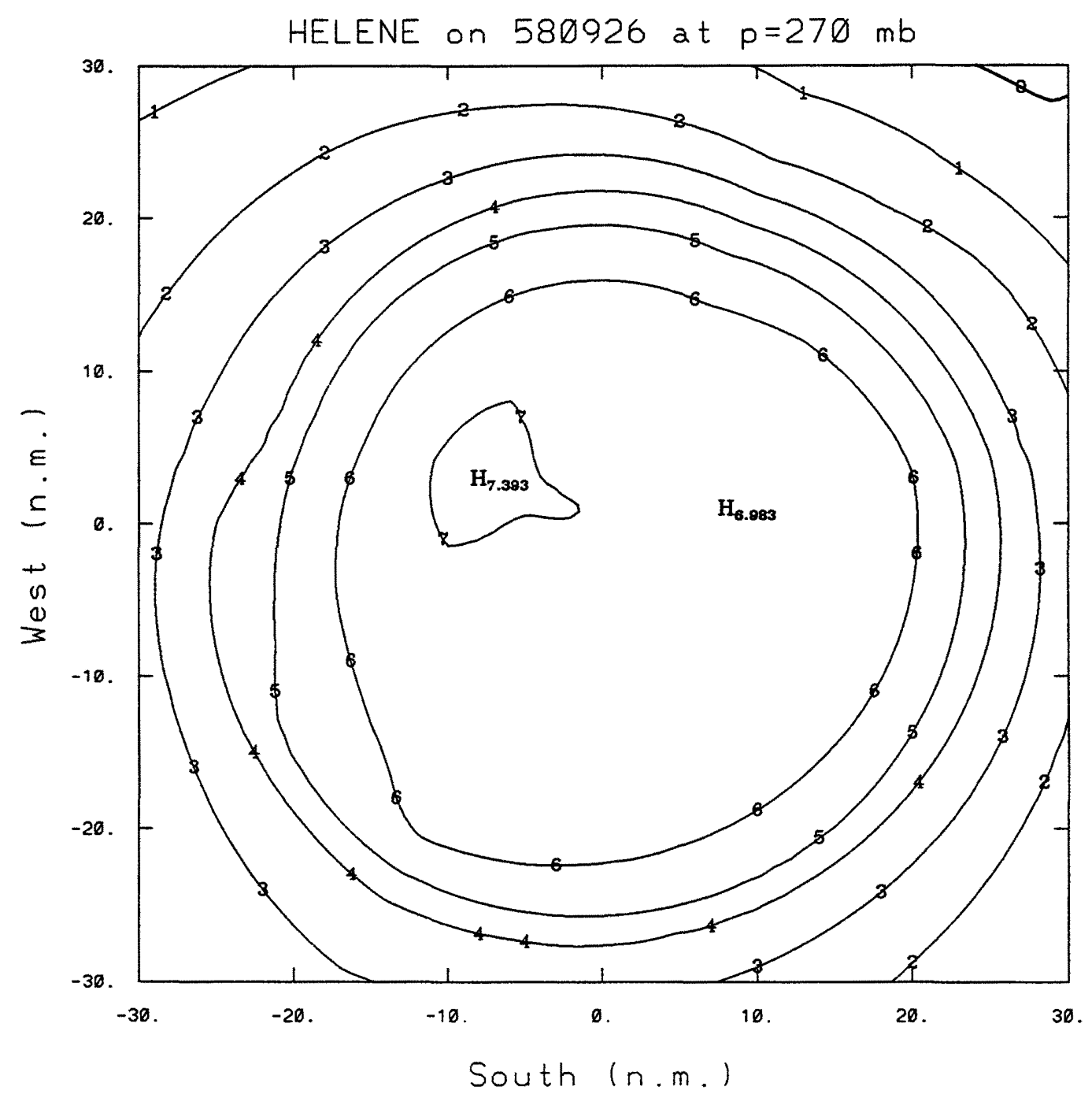

Figure 6.28: Plan-view of temperature deviations for deepening Hurricane Helene on 18 UTC 26 September 1958. Pressure level is $270 \mathrm{mb}$. Contour interval is $1^{\circ} \mathrm{C}$. 


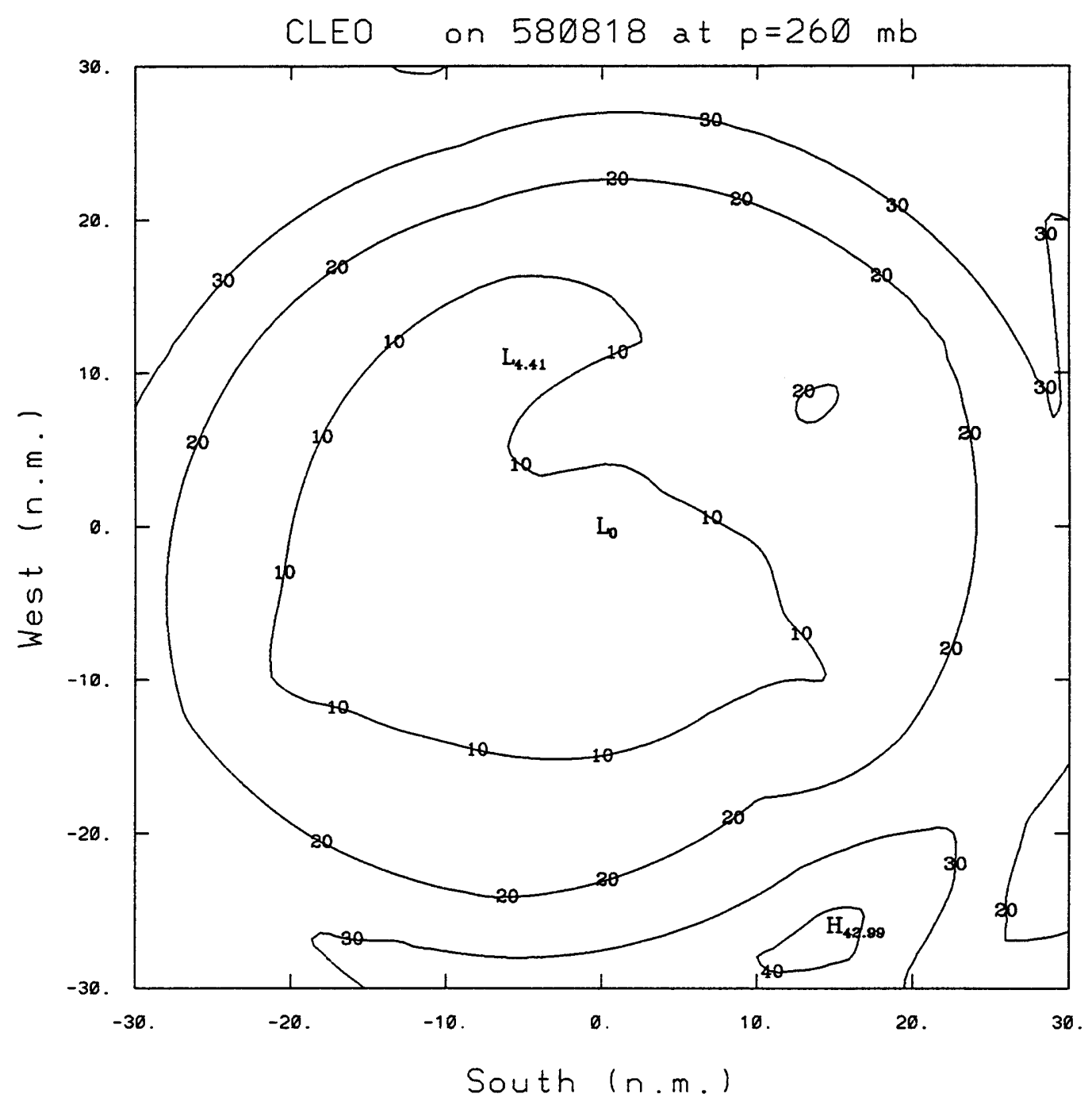

Figure 6.29: Plan-view of wind magnitudes for steady-state Hurricane Cleo on 17 UTC 18 August 1958. Pressure level is $260 \mathrm{mb}$. Contour interval is $10 \mathrm{kts}$. Storm motion is removed. 


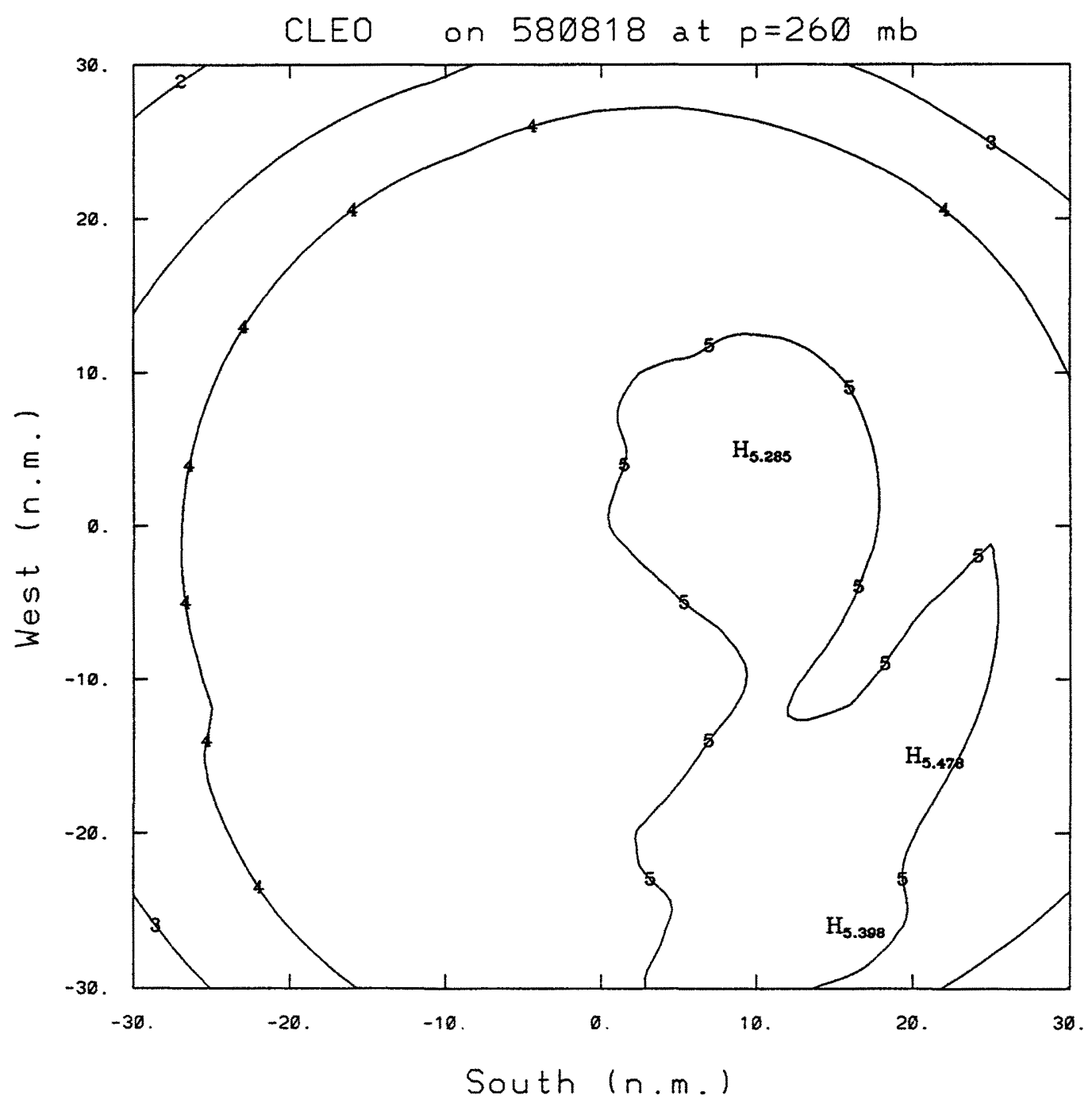

Figure 6.30: Plan-view of temperature deviations for steady-state Hurricane Cleo on 17 UTC 18 August 1958. Pressure level is $260 \mathrm{mb}$. Contour interval is $1^{\circ} \mathrm{C}$. 


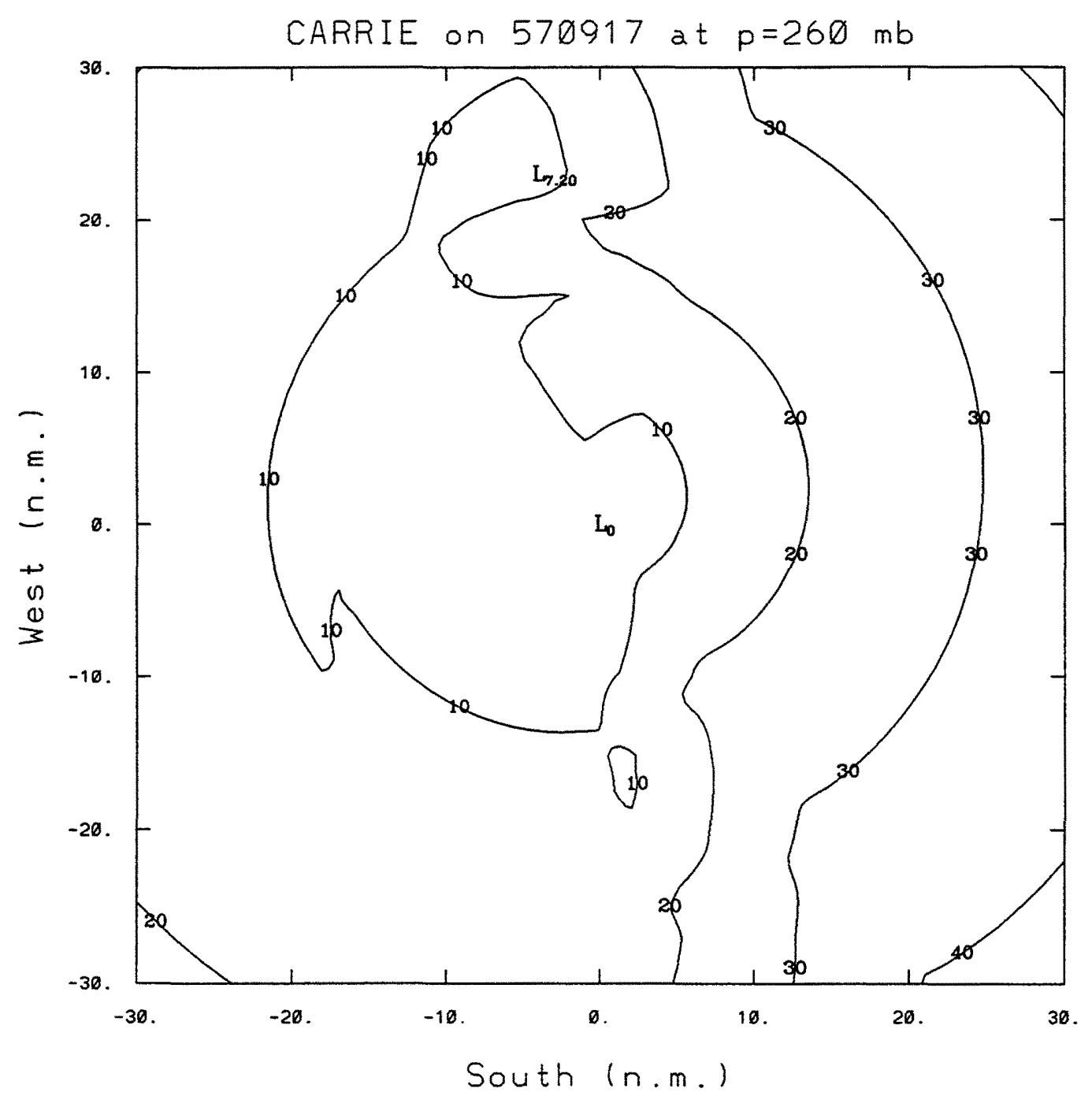

Figure 6.31: Plan-view of wind magnitudes for weakening Hurricane Carrie on 19 UTC 17 September 1957. Pressure level is $260 \mathrm{mb}$. Contour interval is $10 \mathrm{kts}$. Storm motion is removed. 


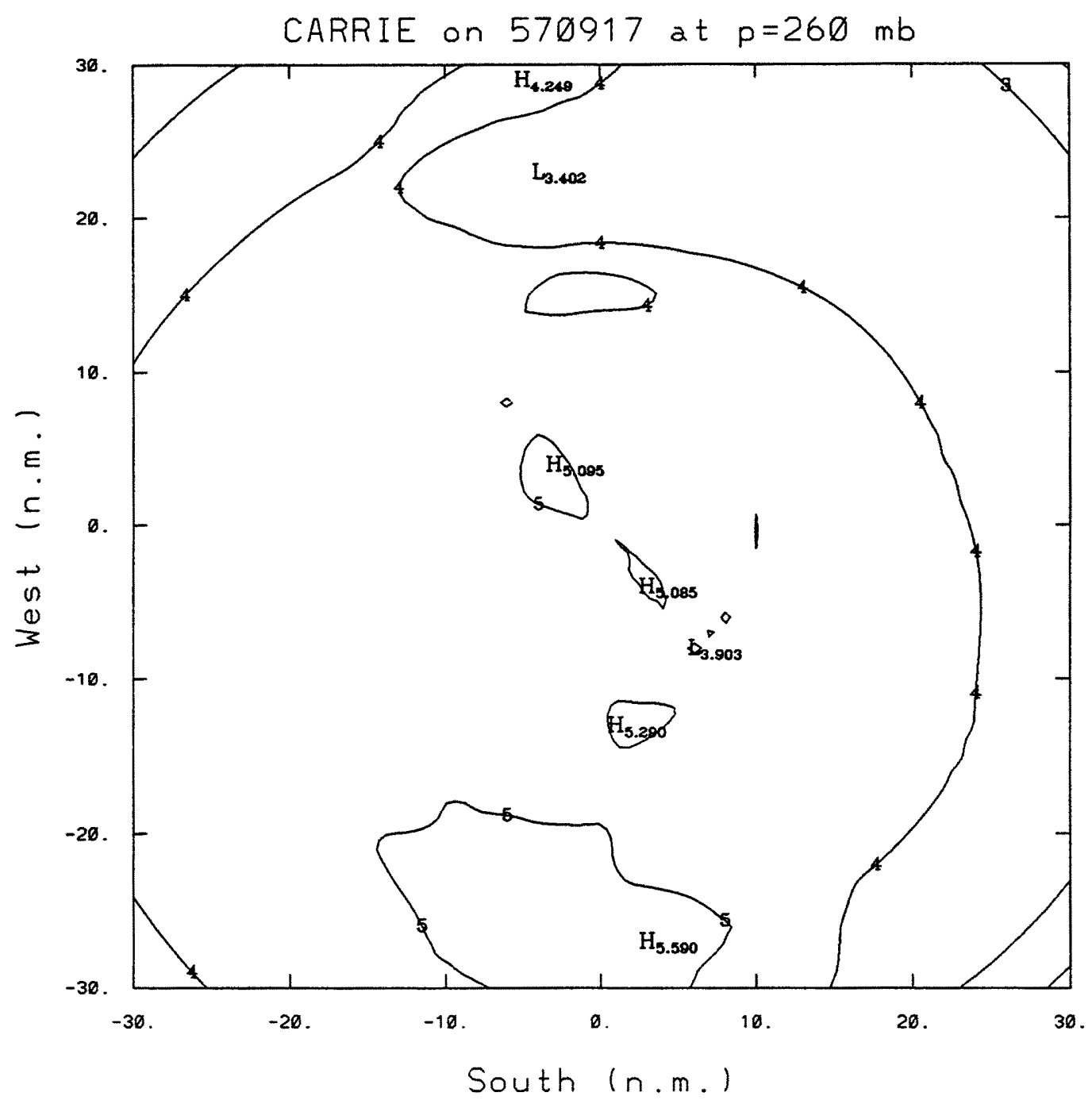

Figure 6.32: Plan-view of temperature deviations for weakening Hurricane Carrie on 19 UTC 17 September 1957. Pressure level is $260 \mathrm{mb}$. Contour interval is $1^{\circ} \mathrm{C}$. 
categories. Composites at 2 deg include all observation between 0 and 3 deg, composites at 4 deg include all observations between 3 and 5 deg, etc. The number of rawinsonde observations in each radial area is shown in Table 6.3. The amount of data should be sufficient to compare the two intensification composites.

Table 6.3: Number of rawinsonde observations at a given radial band (0-3 deg, 3-5 deg, 5-7 deg, etc.) for the fast developing storm composite (central pressure drop $\geq 30 \mathrm{mb}$ in the next $24 \mathrm{~h}$ ) against the slow developing storm composite (10 $\leq \Delta p \leq 20 \mathrm{mb}$ in the next $24 \mathrm{~h}$ ). The former composite is denoted as \# Fast obs, and the latter as \# Slow obs. The number of rawinsonde observations generally decreases with height, so the full observational range is shown. For example, between 0 and $3 \mathrm{deg}$, the fast intensifying composite contains 14 observations at the surface which decreases to 6 observations at $100 \mathrm{mb}$. Both sets of storms are similar in intensity $\left(50 \leq V_{\max } \leq 85 \mathrm{kts}\right)$.

\begin{tabular}{|l|c|c|c|c|c|c|c|}
\hline Radii & $0-3$ & $3-5$ & $5-7$ & $7-9$ & $9-11$ & $11-13$ & $13-15$ \\
\hline \hline \# Fast obs & $6-14$ & $20-33$ & $33-55$ & $58-83$ & $62-97$ & $80-149$ & $104-180$ \\
\hline \# Slow obs & $12-28$ & $31-59$ & $51-94$ & $83-148$ & $125-211$ & $197-313$ & $184-343$ \\
\hline
\end{tabular}

The radial and tangential wind cross-sections for a fast developing TC are shown with the storm motion removed in Fig. 6.33. The appearance is typical of a TC, with lowlevel cyclonic winds decreasing outward, and upper-level anticyclonic winds increasing in magnitude away from the center. The cyclonic winds extend vertically to $150 \mathrm{mb}$ at $r=2 \mathrm{deg}$, but decrease in height radially outwards to be replaced by the anticyclone. The secondary circulation consists of radial inflow with a maximum of $-3 \mathrm{~m} \mathrm{~s}^{-1}$ at $950 \mathrm{mb}$ between 0 and $3 \mathrm{deg}$ ( $r=2 \mathrm{deg}$ in the composite), and outflow aloft with a maximum between 2 and $4 \mathrm{deg}$ at $150 \mathrm{mb}$ of $7 \mathrm{~m} \mathrm{~s}^{-1}$, decreasing in speed outwards as it is deflected by the Coriolis force to form the anticyclone.

Figure 6.34 displays similar $u_{r}$ and $v_{t}$ profiles for the slower developing TC. However, there are differences in the two composites, which is shown in Fig. 6.35 as fast developing TCs minus slow developing TCs. These distinctions are:

1.) The faster developing composite contains weaker low-level (900 to $700 \mathrm{mb}$ ) outer-core tangential winds, particularly between 2-6 deg radius. This agrees with Weatherford (1989) who shows that rapid intensifiers tend to have weaker outer-core winds than non-rapid intensifiers (see also Fig. 4.1). 

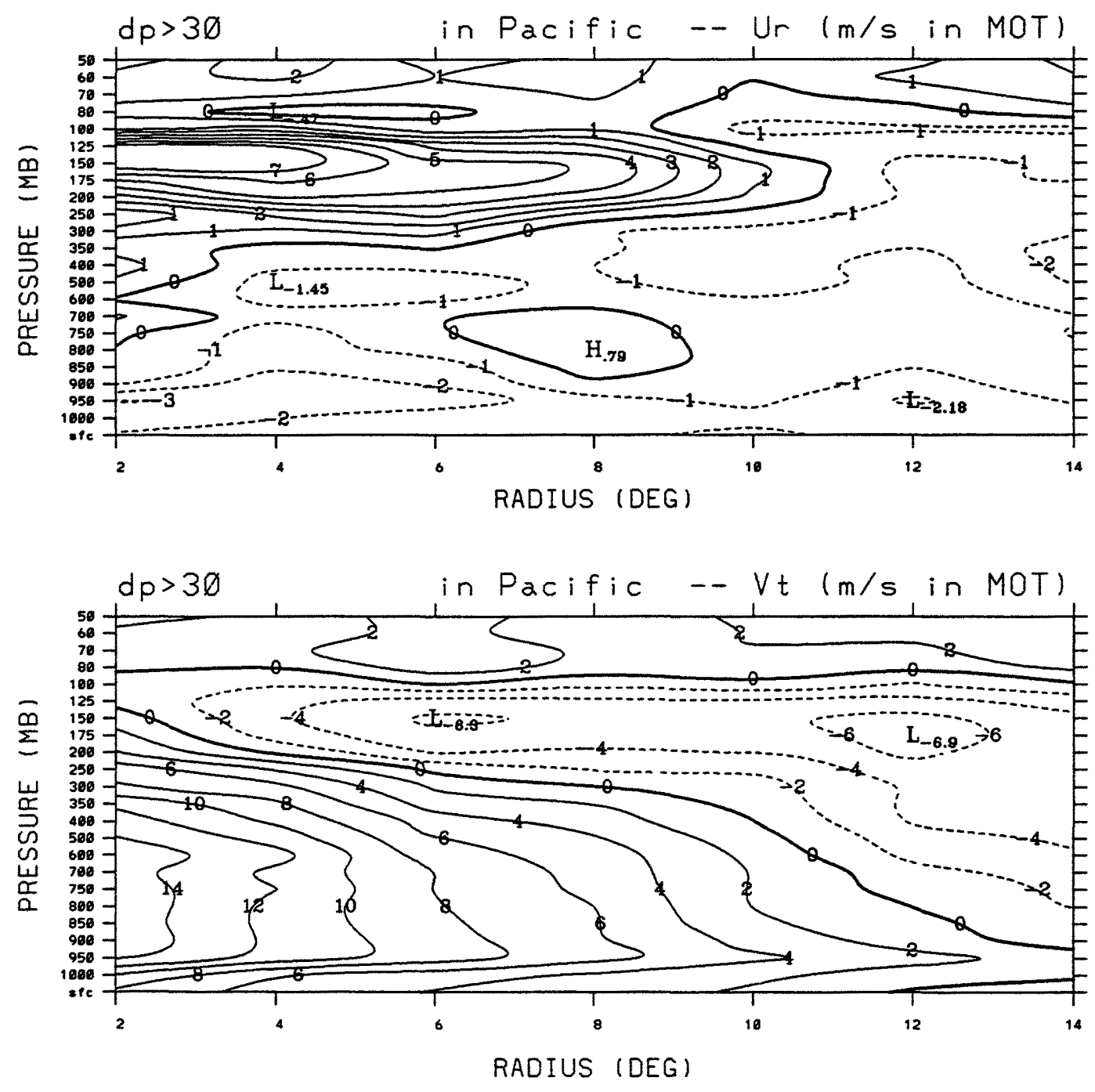

Figure 6.33: Composite cross-section of radial winds (top figure) and tangential winds (bottom figure) for western North Pacific TCs at onset of MSLP drop greater than or equal to $30 \mathrm{mb}$. All storms contain $V_{\max }$ between 50 and $85 \mathrm{kts}$. Contour interval is $1 \mathrm{~m} \mathrm{~s}^{-1}$ for the top figure, and $2 \mathrm{~m} \mathrm{~s}^{-1}$ for the bottom figure. Storm motion is removed. 

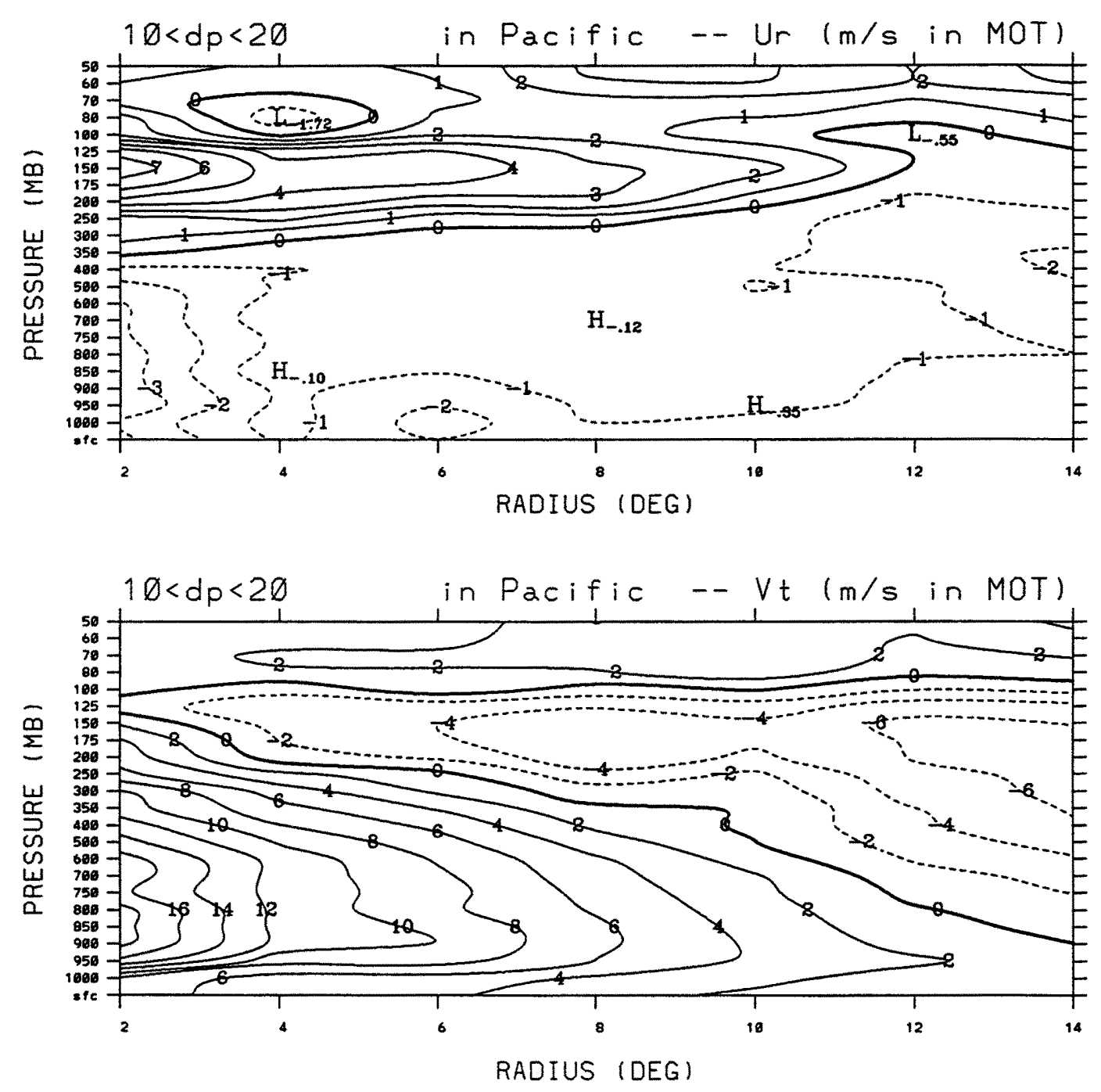

Figure 6.34: Composite cross-section of radial winds (top figure) and tangential winds (bottom figure) for western North Pacific TCs at onset of MSLP drop between 10 and $20 \mathrm{mb}$. All storms contain $V_{\max }$ between 50 and $85 \mathrm{kts}$. Contour interval is $1 \mathrm{~m} \mathrm{~s}^{-1}$ for the top figure, and $2 \mathrm{~m} \mathrm{~s}^{-1}$ for the bottom figure. Storm motion is removed. 

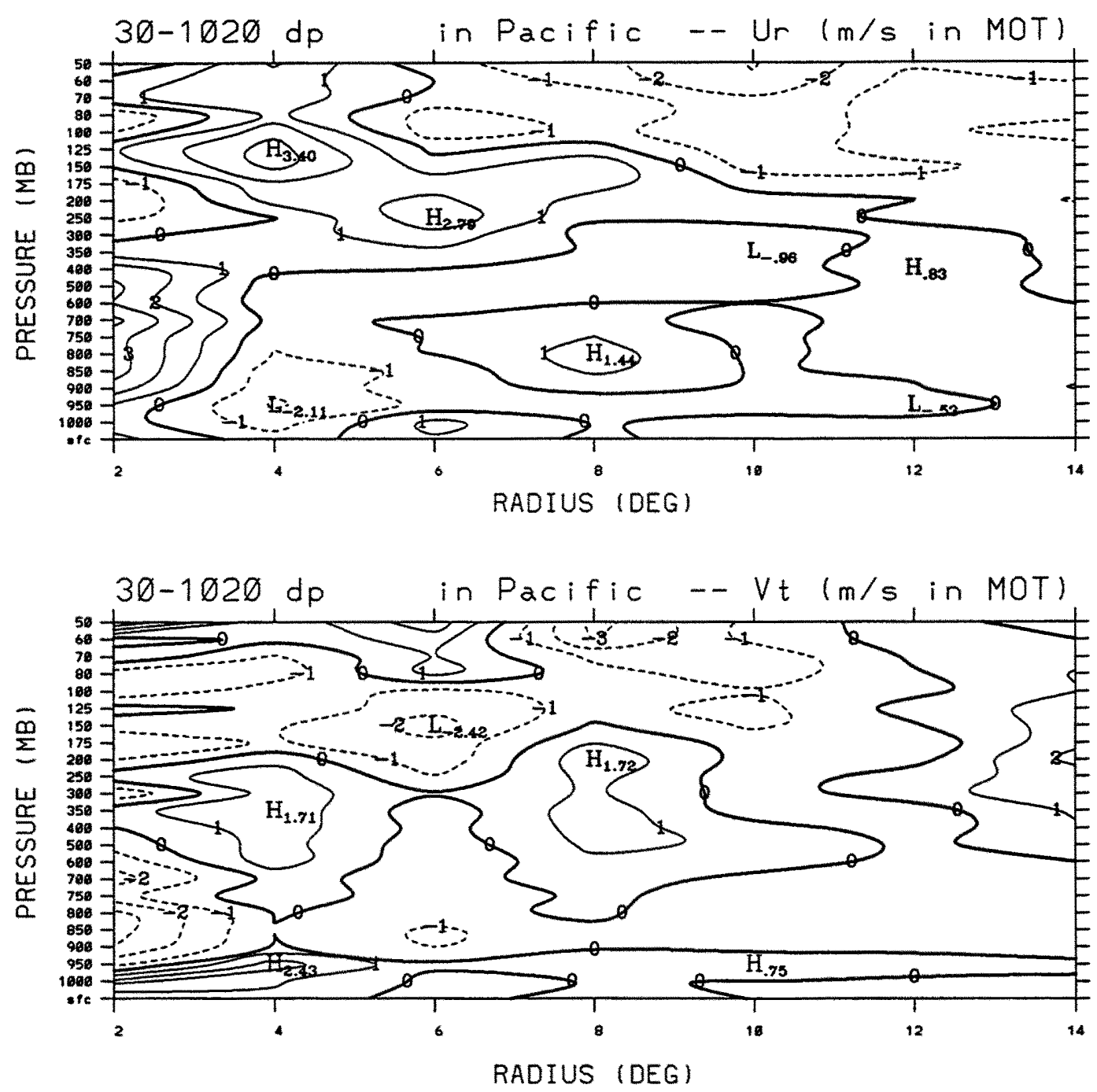

Figure 6.35: Composite cross-section of radial wind (top figure) and tangential wind (bottom figure) differences between western North Pacific TCs at onset of MSLP drop greater than $30 \mathrm{mb}$ (Fig. 6.33) minus MSLP drop between 10 and $20 \mathrm{mb}$ (Fig. 6.34). All storms contain $V_{\max }$ between 50 and $85 \mathrm{kts}$. Contour interval is $1 \mathrm{~m} \mathrm{~s}^{-1}$ for both figures. Storm motion is removed. 
2.) The tangential winds within $1000 \mathrm{~km}$ are preserved more with height for the faster developing composite. Note that the 700 to $200-\mathrm{mb} v_{t}$ is faster from the center out to $12 \mathrm{deg}$, with differences up to $1.7 \mathrm{~m} \mathrm{~s}^{-1}$. This result is consistent with the discussions in this chapter.

3.) There is a tendency for the fast intensifying TC's low-level inflow to be stronger, with a $2 \mathrm{~m} \mathrm{~s}^{-1}$ peak at $4 \mathrm{deg}$. However, the slow developing composite contains stronger inflow between 2 and $3 \mathrm{deg}$ and 7 to $9 \mathrm{deg}$, hence one can only generalize that lowlevel inflow is stronger for the faster developing case. Nevertheless, the outflow of the fast developing TC at $150 \mathrm{mb}$ is stronger and the $u_{r}$ maximum extends further outwards, with wind differences up to $3.4 \mathrm{~m} \mathrm{~s}^{-1}$. In other words, fast developing storms probably contain more convective forcing than slow developing storm. This finding is consistent with the discussions in this report.

4.) The anticyclone is stronger in the fast developing composite compared to the slower developing composite, with differences up to $2.4 \mathrm{~m} \mathrm{~s}^{-1}$ at $r=6 \mathrm{deg}$ and $p=150 \mathrm{mb}$. This relationship is consistent with the results of Chen and Gray (1986) and Merrill (1988a). If the outflow is stronger (see item 3), then the Coriolis force (which is proportional to wind speed) should generate a faster anticyclone. However, it is possible that environmental forcing is also contributing to the negative vorticity. As discussed in Chapter 2, relative eddy angular momentum fluxes may be enhancing the secondary circulation in accordance with the findings of Molinari and Vollaro (1989) and DeMaria et al. (1993); alternatively, the stronger anticyclone may result from a favorable superposition of an environmental trough as discussed by Sadler (1978) and Chen and Gray (1986). However, as stated by Mundell (1990), these processes may be most important in that they maintain the outer-core winds, with no direct relationship to TC intensification. As shown in Chapter 4, inner-core processes (which mostly governs the intensification process) are not well-related to outer-core winds (Weatherford and Gray 1988a). 
Finally, the temperature and height differences between the two composites are presented for these two Pacific cases (Fig. 6.36). Any negative height differences should correspond to colder temperatures. In agreement with this chapter, the mid and uppertroposphere temperatures are colder for the faster developing composite storm, particularly at 2-6 deg. These colder temperatures should also yield lower geopotential heights in the upper-troposphere and stratosphere, which is what is depicted in Fig. 6.36. This is another indication that a large upper-level temperature gradient exists, confining most of the warming near the eye. This can result in a large inward pressure gradient force conducive to fast development. Another interesting feature is that, below $850 \mathrm{mb}$, the temperature is warmer. This might be a reflection that the faster developing storms are occurring over warmer water than the slower developing storms. Indeed, the average latitude for the faster developing storm is $14.8 \mathrm{deg} \mathrm{N}$, compared to $16.0 \mathrm{deg} \mathrm{N}$ for the slower developing case. This also concurs with the findings of Mundell (1990), who found, on average, that rapid intensifiers occur at lower latitudes than slower developing TCs.

\subsection{Diagnostic balanced vortex calculations}

An unresolved issue which needs further research is a more precise relationship between inner-core wind structure and TC intensification. First, some may argue that a vertically developed vortex merely indicates fast intensification is already underway, and hence an obvious "cause and effect" relationship may not exist. However, the limited available evidence presented in this chapter indicates that there probably is a time lag between vortex development and fast intensification. Several theoretical thermodynamic and dynamic arguments, when considered together, also seem to support the plausibility of this relationship.

However, to fully explore these possible relationships, a modelling evaluation needs to be conducted. Complicated, three-dimensional models based on the primitive equations could be used to study the results of this chapter. However, intensity change is mostly associated with inner-core processes, and therefore a two-dimensional, axisymmetric model seems more suitable for the scope of these problems. Not only are these models easier 

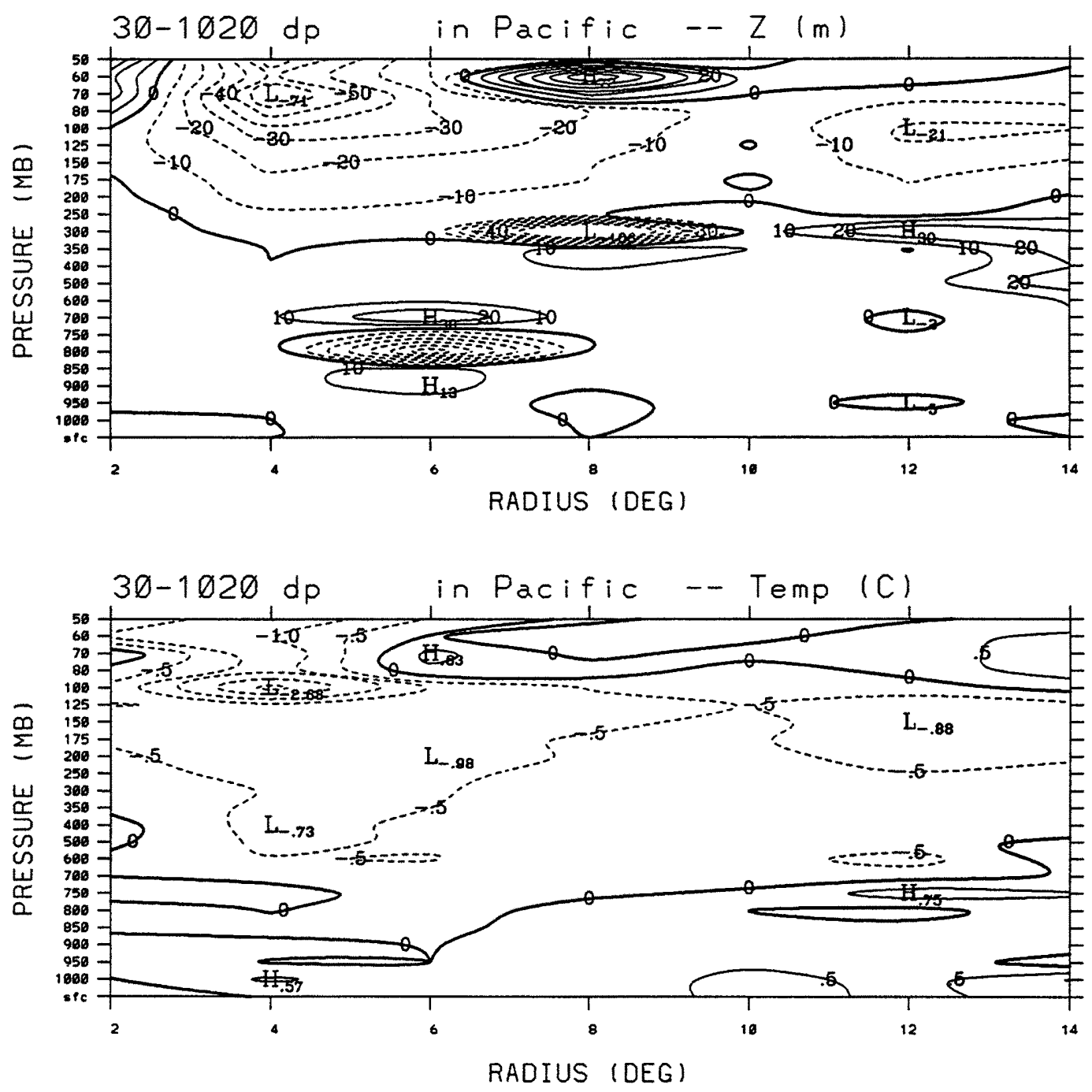

Figure 6.36: Composite cross-section of height (top figure; in $\mathrm{m}$ ) and temperature (bottom figure; in ${ }^{\circ} \mathrm{C}$ ) differences for western North Pacific TCs at onset of MSLP drop greater than $30 \mathrm{mb}$ (Fig. 6.33) and between 10 and $20 \mathrm{mb}$ (Fig. 6.34). All storms contain $V_{\max }$ between 50 and $85 \mathrm{kts}$. Contour interval is $10 \mathrm{~m}$ for the top figure, and $0.5^{\circ} \mathrm{C}$ for the bottom figure. 
to analyze, but they are computationally inexpensive. In addition, the two-dimensional problem may be further approximated by assuming the vortex is in gradient wind balance and hydrostatic balance (and, hence, thermal wind balance). Therefore, due to its relative simplicity and concise treatment of TC evolution, a Balanced Vortex (BV) model is used to investigate inner-core processes.

BV theory was initially formulated by Eliassen (1951). Since Eliassen (1951), several versions of the BV equations have been formulated (Ooyama 1969; Willoughby 1979; Shapiro and Willoughby 1982; Schubert and Hack 1983) which have been used in TC numerical models. Shapiro and Montgomery (1993) have recently introduced a technique to include wavenumber one asymmetries in the BV dynamics. BV dynamics describes the slow evolution of a vortex in gradient and hydrostatic balance responding to weak forcing (in a TC, mostly diabatic forcing). Therefore, balanced vortex theory is a filtered model because the transient aspects of gradient wind adjustment are excluded, and instant thermal wind balance is assumed. Calculations based on BV theory may help validate a possible association between an intense upper-level inner-core vortex and TC intensification, since the diabatic heating rate (buoyancy) increases with a vertically conserved inner-core $v_{t}$ profile.

\subsubsection{Brief review of balanced vortex dynamics}

BV equations consist of a two-dimensional elliptic equation for the streamfunction associated with the secondary circulation in the radial-height plane, and a predictive equation for $v_{t}$ whereby the temperature field can be diagnosed from thermal wind balance. The forcing primarily consists of Ekman pumping, the diabatic heating rate, and diffusion, although eddy fluxes may also be included (see Chapter 2). The BV equations offer several advantages compared to primitive equations.

First of all, the BV equations are easier to apply than primitive equations, since one deals with fewer equations, and because it is a filtered model where the transient aspects of gradient wind adjustment are not included. In other words, the vortex evolves through a sequence of "quasi-equilibrium" states, while the transient unbalanced state associated with gravity waves is bypassed. The balance is only quasi-equilibrium because there is 
an instantaneous ageostrophic response in the secondary circulation when thermal wind balance is achieved (analogous to quasi-geostrophic theory).

Second, understanding the dynamical processes involved in vortex dynamics is conceptually easier to understand in terms of the inertial and static stabilities, baroclinicity, and the forcing under the BV formulation. Air parcels move in the $(r, p)$ plane in response to forcing against two stabilizing forces: static stability, which provides resistance to vertical displacements, and inertial stability, which provides resistance to horizontal displacements. The circulation is favored in the direction of the weakest stability. For example, in a barotropic vortex where static stability is generally weaker than inertial stability, the secondary circulation is elongated in the vertical. When baroclinicity is included, the secondary circulation tilts.

Third, one can perform diagnostic studies of the secondary circulation due to momentum and/or heat forcing either by analytical Green's Function solutions (Shapiro and Willoughby 1982; Eliassen 1951) or through numerical solutions of the elliptic equation (Willoughby 1979; Holland and Merrill 1984; Hack and Schubert 1986b). The elliptic equation may be numerically solved using Successive Over-Relaxation (SOR) techniques (Hack and Schubert 1986b; Press et al. 1988), Alternating-DIrection (ADI) techniques (Sundqvist 1970; Press et al. 1988), multigrid techniques (Ciesielski et al. 1986; Fulton et al. 1986), and direct solvers (Lindzen and Kuo 1969; Willoughby 1979). These diagnostic studies disclose some interesting secondary circulation responses to momentum and heat forcing.

Eliassen (1951) showed that the flow through a point heat source follows a (nearly vertical) source of constant angular momentum in a barotropic vortex, while the flow through a point momentum source follows a (nearly horizontal) isentropic surface in a barotropic vortex. In a baroclinic vortex, constant angular momentum lines and isentropic lines tend to approach one another as the motion in the baroclinic region becomes tilted - in a baroclinic vortex, the updraft at a heat source tilts away from the vertical to follow surfaces of slanting angular momentum surfaces, and the outflow at a momentum source tilts away from the horizontal to follow constant $\theta$ surfaces. Finally, the response 
to a horizontal gradient ("jump" in Eliassen's analysis) of the diabatic heating rate, which increases towards the center, is upwards and outwards. The same secondary circulation response will occur when angular momentum forcing increases in the vertical. Given these responses, the local $v_{t}$ and $\theta$ tendency may also be computed (Willoughby et al. 1982; Shapiro and Willoughby 1982; Schubert and Hack 1982).

\subsubsection{Description of the balanced vortex model}

This author has coded a BV routine based on the formulation of Sundqvist (1970) with some modifications. The grid is staggered, with: 1) the streamfunction $(\psi)$, stability coefficients, and baroclinicity coefficients determined on the grid points; 2) the tangential and vertical wind, diffusion term, and diabatic heating term computed at the horizontal "half-grid" points; and 3) temperature and radial wind determined at the vertical "halfgrid" points. The horizontal grid spacing $(\Delta r)$ is $5 \mathrm{~km}$. The vertical grid spacing $(\Delta p)$ is $-50 \mathrm{mb} . \psi$ is computed using SOR. Solving $\psi$ using a direct solver (Lindzen and Kuo 1969; Willoughby 1979) would have been preferable since this eliminates the restrictive requirement of ellipticity, but inverting the coefficient matrix (which contained small values) proved difficult.

Due to the staggered nature of the grid, boundary conditions are straightforward. $\psi$ is set to 0 at $r=0$ and at the top and bottom of the domain. At the lateral boundary condition, $\psi$ needs to be specified so that minimal distortion of the outflow aloft and inflow in the low-levels occurs. Therefore, a Neuman boundary condition is used following (Ooyama 1969) which closely preserves the secondary circulation. Furthermore, this scheme is flexible in that changes in the lateral domain size does not change the vortex simulation in the inner domain, which obviously is a computational advantage. Other momentum values are specified to be consistent with symmetry and the $\psi$ boundary conditions.

\subsubsection{Methodology of the balanced vortex model}

A methodology for investigating the topics discussed in this paper will now be discussed using the BV model. The sensitivity of the secondary circulation in TCs to different 
$v_{t}$ profiles will be investigated using this model in a diagnostic fashion. Given the distribution of $v_{t}$, the warm core structure of the TC may be instantly computed from thermal wind balance. However, the diabatic heating rate, which mostly determines the secondary circulation of a TC, is intrinsically related to its warm core structure.

How to parameterize $\dot{Q}$ is currently the subject of much research and debate in meteorology. However, it is not the purpose of this research to use or design an elaborate cumulus parameterization scheme. Rather, a diabatic heating scheme should be used which is consistent with convective maximums in TCs. In this manner, tractable comparisons can be made between developing TCs with different vertical $v_{t}$ profiles.

In the past, the diabatic heating rate has been computed using the "Kuo cumulus parameterization scheme" in the following form (Kuo 1965; Sundqvist 1970; Anthes 1968, 1972, 1977): :

$$
\frac{\dot{Q}(p)}{c_{p}}=\frac{\left(\breve{T}_{\rho}(p)-T_{v}(p)\right)}{\tau}
$$

where $\breve{T}_{\rho}$ is the density temperature which includes partial water-loading and ice microphysics as described in Chapter 7. $T_{v}$ is the environmental virtual temperature. Modifications are needed to include an entrainment rate such as done by Anthes (1977) or Zhang and McFarlane (1991). Furthermore, the parcel's path needs to be modified so that it follows sloping convection (roughly a constant angular momentum surface).

The relaxation time constant $\tau$ represents the time it takes for $T_{v}$ to adjust to $\breve{T}_{\rho}$. Many different formulations exist to compute $\tau$, but generally it is controlled by: 1) the influx of moisture at the surface by Ekman pumping, which represents the amount of moisture available for convection (Kuo 1965; Anthes 1968,1972$){ }^{6}$ and 2) the integrated buoyancy difference in that column.

Rather than compute $\tau$ or $\breve{T}_{\rho}, \dot{Q} / c_{p}$ will be specified based on inferences from Eq. (6.5), TC structure, and values used by Hack and Schubert (1986b). On the horizontal plane, $\dot{Q} / c_{p}$ is typically zero in the eye region, and is a maximum in the eyewall region in the

\footnotetext{
${ }^{6}$ Some schemes specify moisture availability by moisture convergence in the entire column (Anthes 1977; Kuo 1974). This parameter is called moisture accession.
} 
vicinity of $V_{\max }$ where $\tau$ is smallest due to strong moisture convergence and buoyancy is enhanced by the ASI process. $\dot{Q} / c_{p}$ then decreases exponentially outwards from the eyewall region.

In the vertical, $\dot{Q} / c_{p}$ will be a maximum in the lower to mid-troposphere. For a moderately sheared vortex, the warm core will be located in the lower troposphere since $v_{t}$ will decrease most rapidly with respect to $\ln p$ there. This implies that maximum $\dot{Q} / c_{p}$ will be located in the mid-troposphere where the warm core is less in magnitude, since diabatic heating is proportional to the temperature difference of an air parcel and its environment. However, for a weakly sheared vortex of similar intensity, the warm core will be elevated, and hence $\dot{Q} / c_{p}$ should be a maximum in the lower troposphere below the warm core. As will be shown, such a configuration will result in faster low-level inflow (and hence faster TC development) compared to the moderately sheared TC.

\subsubsection{Results}

Figure 6.37 shows a TC experiencing moderate shear such that $v_{t}$ decreases with height above $900 \mathrm{mb}$. The hypothesized diabatic heating profile (shown in Fig. 6.38) is maximum at $650 \mathrm{mb}$, above the main warm core region located at $850 \mathrm{mb}$ (Fig. 6.38) where $\partial v_{t} / \partial \ln p$ is maximum. This is because the difference between an ascending air parcel and the environment will be reduced in the warm core region.

The secondary circulation response is shown in Fig. 6.37 broken into radial and vertical velocity components, and the streamfunction field is shown in Fig. 6.38. Low-level inflow is apparent with maximum values of $-0.5 \mathrm{~m} \mathrm{~s}^{-1}$ in the boundary layer. Radial inflow decreases within $60 \mathrm{~km}$ of the center as the inertial stability increases. Ascent occurs within $150 \mathrm{~km}$, and is maximum in the eyewall region and across the $650 \mathrm{mb}$ isobaric surface. Outflow occurs in the upper troposphere.

This is to be contrasted with a TC of similar intensity in which $v_{t}$ is preserved with height to $750 \mathrm{mb}$ since it is experiencing less shear (Fig. 6.39). The hypothesized diabatic heating profile (not shown) is maximum at $800 \mathrm{mb}$, below the main warm core region located at $700 \mathrm{mb}$ (not shown) where $\partial v_{t} / \partial \ln p$ is maximum. Low-level inflow is faster, and is closer to the center than in Fig. 6.37. Vertical ascent in Fig. 6.39 encompasses a 

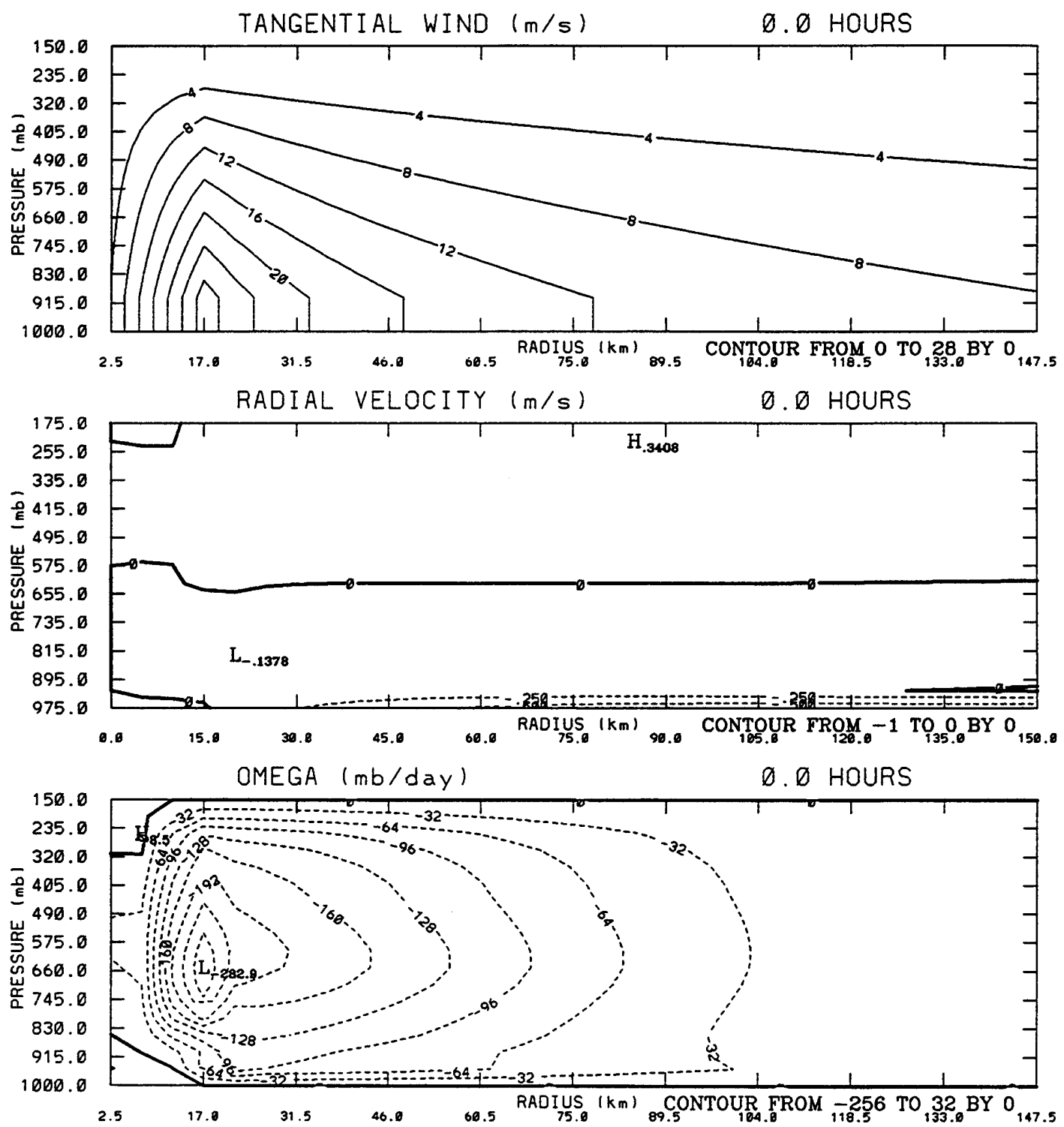

Figure 6.37: A moderately sheared $v_{t}$ profile (top figure; in $\mathrm{m} \mathrm{s}^{-1}$ ) with $V_{\max }$ of $30 \mathrm{~m} \mathrm{~s}^{-1}$ and contour intervals of $4 \mathrm{~m} \mathrm{~s}^{-1}$. The hypothesized diabatic heating profile (shown on Fig. 6.38) is maximum at $650 \mathrm{mb}$, consistent with the warm core located at $850 \mathrm{mb}$ (Fig. 6.38) where $\partial v_{t} / \partial \ln p$ is maximum. The secondary circulation response is shown in the next two figures. The middle figure shows the radial circulation $u_{r}$ (in $\mathrm{m} \mathrm{s}^{-1}$ ) with contour intervals of $0.25 \mathrm{~m} \mathrm{~s}^{-1}$, and the bottom figure shows vertical motion $\omega$ (in $\mathrm{mb} \mathrm{d}^{-1}$ ) with contour intervals of $32 \mathrm{mb} \mathrm{d}^{-1}$. The radial extent is shown out to $150 \mathrm{~km}$, although the calculations are done out to $1000 \mathrm{~km}$. Dashed contours depict negative values. 

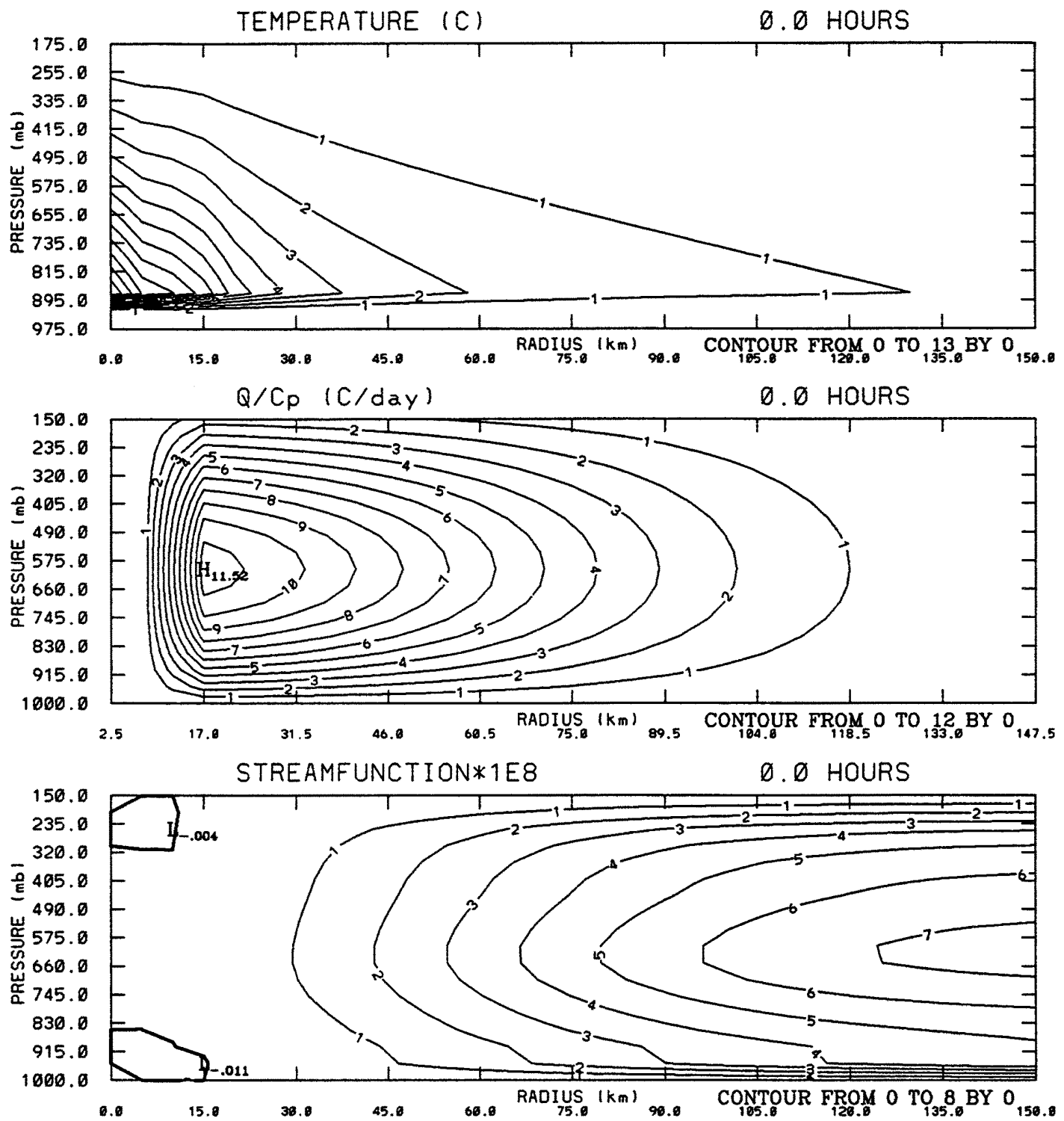

Figure 6.38: The temperature perturbation consistent with thermal wind balance of the vortex shown in Fig. 6.37 is shown in the top figure in $1^{\circ} \mathrm{C}$ intervals. The hypothesized diabatic heating profile consistent with this warm core and assumed maximum heating in the eyewall region is shown in the middle figure with contour interval $2^{\circ} \mathrm{C} \mathrm{d}^{-1}$. The bottom figure shows the streamfunction as a result of the $v_{t}$ and $\dot{Q} / c_{p}$ profile with contour interval $1 \times 10^{8} \mathrm{~kg} \mathrm{~m} \mathrm{~s}^{-3}$. 

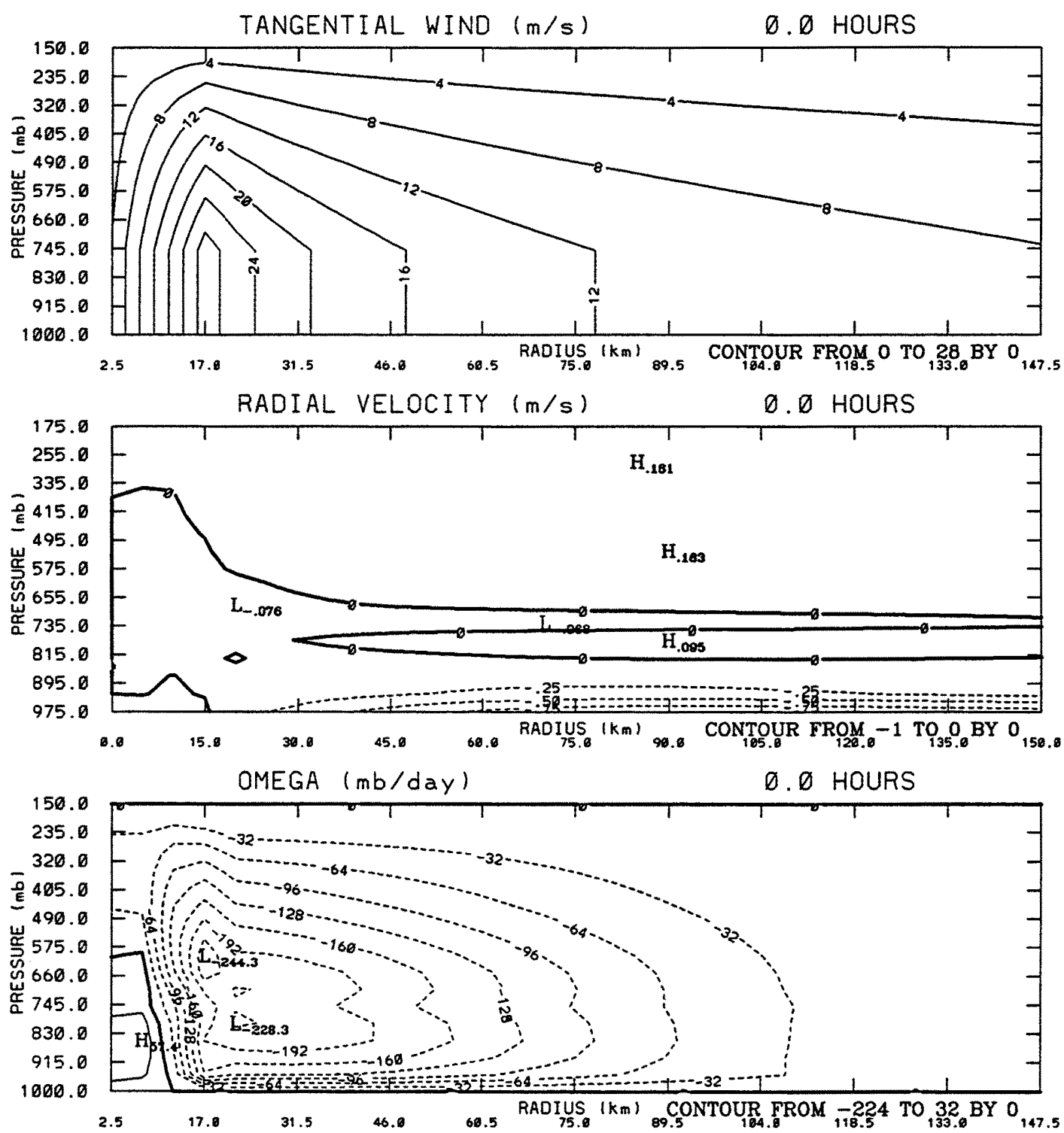

Figure 6.39: A weakly sheared $v_{t}$ profile (top figure; in $\mathrm{m} \mathrm{s}^{-1}$ ) with $V_{\max }$ of $30 \mathrm{~m} \mathrm{~s}^{-1}$ and contour intervals of $4 \mathrm{~m} \mathrm{~s}^{-1}$. The hypothesized diabatic heating profile (not shown) is maximum at $800 \mathrm{mb}$, consistent with the warm core located at $750 \mathrm{mb}$ (not shown) where $\partial v_{t} / \partial \ln p$ is maximum. The secondary circulation response is shown in the next two figures. The middle figure shows the radial circulation $u_{r}$ (in $\mathrm{m} \mathrm{s}^{-1}$ ) with contour intervals of $0.25 \mathrm{~m} \mathrm{~s}^{-1}$, and the bottom figure shows vertical motion $\omega$ (in $\mathrm{mb} \mathrm{d}^{-1}$ ) with contour intervals of $32 \mathrm{mb} \mathrm{d}^{-1}$. The radial extent is shown out to $150 \mathrm{~km}$, although the calculations are done out to $1000 \mathrm{~km}$. Dashed contours depict negative values. 
larger area in the eyewall region than Fig. 6.39. Interestingly enough, $\omega_{\max }$ is actually weaker than the moderately sheared case, since the inertial stability in the low shear case is stronger above $900 \mathrm{mb}$ and restricts low-level inflow above the boundary layer. Still, with the faster boundary layer inflow, larger areally averaged eyewall ascent, and closer proximity of $\omega_{\max }$ to the surface, the weakly sheared case will have larger inner-core $\partial v_{t} / \partial t$ tendencies. This TC will develop faster than the moderately sheared case.

As discussed in Chapter 4, inertial stability may strongly influence intensity change. To investigate this relationship, the calculations for the low shear case are repeated on a TC of similar intensity, but with weaker outer-core $v_{t}$ (Fig. 6.40). Compared to Fig. 6.39, the radial inflow is almost twice as fast, the fast inflow penetrates very close to the center, and $\omega_{\max }$ is larger than the low shear case with stronger inertial stability. The TC in Fig. 6.40 should intensify faster than the TC with stronger inertial stability. This is consistent with the findings of Weatherford (1989), who found that rapid intensifying TCs contained less Outer-Core Strength than slow developing TCs (Fig. 4.1). This is also consistent with the results of Fitzpatrick (1992).

It is interesting that subsidence is produced at $r=0 \mathrm{~km}$ in all these calculations. Anthes (1982) suggests eyewall convection forces air to sink on the side, possibly contributing to eye formation. This explanation is supported by these results, and also by the BV calculations of Willoughby (1979), which show subsidence occurring due to radial gradients of diabatic heating.

Obviously, TC development is sensitive to both the vertical and horizontal distribution of $v_{t}$. Furthermore, the distribution of $\dot{Q} / c_{p}$ is still unclear. Finally, the evolution of the TC's response needs to be done in addition to the diagnostic calculations performed here. Future modelling research will be conducted on these topics to further explore the relationship between inner-core wind structure and TC intensification. Due to a variety of numerical difficulties, the current BV equations (Sundqvist 1970) will be abandoned, and a BV code in the potential radius coordinate system (Hack and Schubert 1986b; Schubert and Hack 1983) will be used instead. The BV system in potential radius coordinates contains more resolution in the TC's inner-core, and a simpler set of equations to solve. 

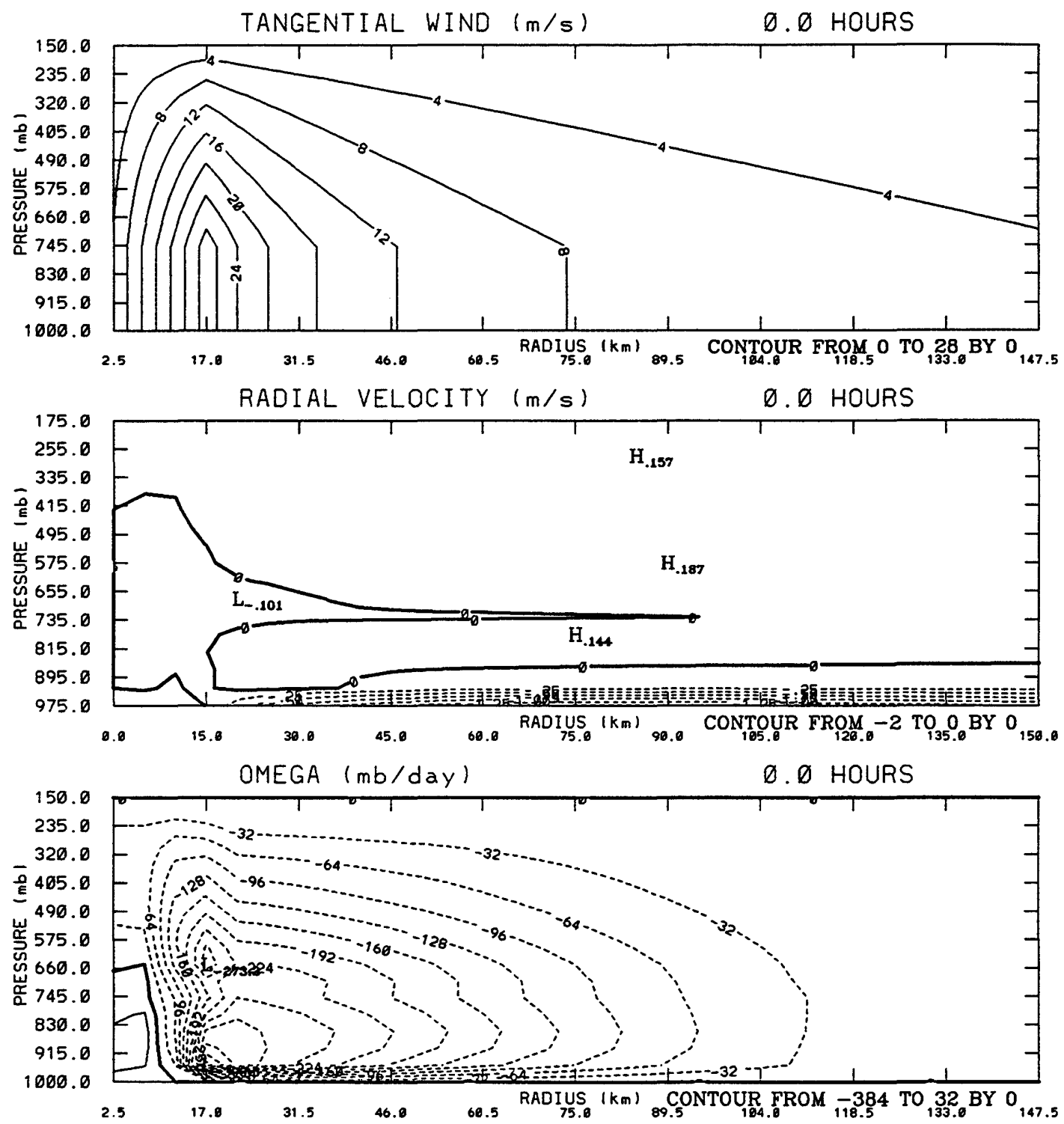

Figure 6.40: A weakly sheared $v_{t}$ profile (top figure; in $\mathrm{m} \mathrm{s}^{-1}$ ) with $V_{\max }$ of $30 \mathrm{~m} \mathrm{~s}^{-1}$ and contour intervals of $4 \mathrm{~m} \mathrm{~s}^{-1}$, but with less Outer-Core Strength (OCS; or weaker inertial stability) compared to the previous page. The hypothesized diabatic heating profile (not shown) is maximum at $800 \mathrm{mb}$, consistent with the warm core located at $750 \mathrm{mb}$ (not shown) where $v_{t}$ begins to decrease with height. The secondary circulation response is shown in the next two figures. The middle figure shows the radial circulation $u_{r}$ (in $\mathrm{m} \mathrm{s}^{-1}$ ) with contour intervals of $0.25 \mathrm{~m} \mathrm{~s}^{-1}$, and the bottom figure shows vertical motion $\omega$ (in $\mathrm{mb} \mathrm{d}^{-1}$ ) with contour intervals of $32 \mathrm{mb} \mathrm{d}^{-1}$. The radial extent is shown out to $150 \mathrm{~km}$, although the calculations are done out to $1000 \mathrm{~km}$. Dashed contours depict negative values. 


\subsection{Summary}

Due to sparse data, little research previously has been conducted on the inner-core upper-tropospheric TC structure. An attempt has been made in this chapter to consolidate the available temperature and wind fields from flight and rawinsonde data so that their evolution in a developing TC can be inferred. Furthermore, possible links between upperlevel inner-core $v_{t}$ and TC intensification have been deduced, and implores that more research and data is needed on this subject.

Documentation of the inner-core tangential wind evolution has been presented which shows upper-tropospheric tangential winds increase significantly as a TC intensifies, and at a greater than one-to-one ratio with respect to low-level $v_{t}$ increase. One importance of this upper-level eyewall spin-up is that sufficient buoyancy must exist to compensate both frictional dissipation and the export of cyclonic $v_{t}$, and still contain sufficient extra momentum available to intensify the TC (the IFC concept). Another important consequence of strong upper-level $v_{t}$ is that, from thermal wind balance and geostrophic adjustment theory, strong eyewall winds aloft can circumvent eyewall stabilization by constraining warming as close to the tropopause and eye region as possible. In this manner: 1) the eyewall environment is held relatively cool so that CAPE remains large, creating vertical velocities which can balance the $v_{t}$ budget requirements against friction and cyclonic $v_{t}$ export while enhancing the low-level boundary layer forcing and inflow for intensification (perhaps even rapid intensification); 2) with warming confined near the tropopause, the Air-Sea Interaction process is enhanced; and 3) the conversion of latent heat to intensification is increased due to higher inertial stability and a vertically stacked vortex. NHRP and rawinsonde data supports this idea that a vertically stacked vortex signifies future fast intensification.

By stratifying the rawinsonde data into future fast and slow developers, it is also shown that, for TCs of similar intensity, the fast developers contained a more vertically stacked inner-core vortex. The NHRP data also suggests a direct correlation between upper-level $v_{t}$ and whether a TC is experiencing intensification, with the rate of intensification proportional to the magnitude of upper-level $v_{t}$. A three-dimensional analysis 
of the NHRP upper-level inner-core winds and temperature field suggest that symmetry (asymmetry) and a strong (weak) $\nabla T$ is correlated with intensification (weakening).

Inspection of low and mid-level fields do not show as clear a relationship to intensity change, suggesting that monitoring of upper-level eyewall temperature and wind fields may yield the most important forecast information about intensity change. F. Marks of HRD, who has much personal experience with Doppler radar observations during hurricane research flights, asserts a similar opinion (personal communication 1995). Based on the results of this report - and the need for more inner-core upper-level data it is recommended that upper-tropospheric jet aircrafts be employed in critical intensity change forecast situations. It is strongly advised that the new HRD jet research plane (to be deployed in 1996) should monitor upper-level inner-core tangential winds in TCs. Gray (1993) even recommends that idle commercial jet aircrafts with cancelled flights due to an approaching TC could be used with proper (and relatively inexpensive) financial reimbursement from the US reconnaissance program.

Despite the hypothesis that a stacked vortex is needed for rapid intensification, it should be noted that some eyewall slope is needed near the tropopause so that ascending air parcels avoid the warmest region of a TC, thereby maintaining their buoyancy. The lateral extension of the eye near the tropopause above the eyewall surface is also a desirable feature, because it accelerates surface pressure falls under the eyewall which also aids eyewall buoyancy. The necessity of the eyewall sloping outwards near the tropopause will be discussed in the next chapter (Chapter 7). Nevertheless, the importance of inner-core $v_{t}$ being vertically conserved to near the tropopause may be just as important a feature in fast intensifying systems as upper-level eyewall structure. 


\section{Chapter 7}

\section{A MODIFIED AIR-SEA INTERACTION THEORY AND NEW MAXIMUM POTENTIAL INTENSITY THEORY}

\subsection{Eyewall processes which affect the ASI process}

This chapter develops an alternative Air-Sea Interaction (ASI) process which incorporates boundary layer cooling of $2-4^{\circ} \mathrm{C}$ as a function of decreasing pressure. As shown in Chapter 3, such cooling cannot sustain buoyancy with regards to the mean tropical sounding (Jordan 1958) for typical environmental surface pressures. However, it will be shown that, with the lateral extension of the eye above a sloping eyewall, the hydrostatically determined surface pressure under the eyewall is sufficiently low, and the ASI process can continue even with surface cooling.

Furthermore, Chapter 3 assumed a pseudoadiabatic process in which all condensate immediately fell out so that no negative effects of water-loading are included. In addition, these calculations assumed undilute ascent with no rain drag. It is likely that such an idealization is a bit optimistic. As a crude approximation, a reversible process represents these detrimental effects on buoyancy (to be explained shortly). However, it will be shown that the inclusion of latent heating by ice processes (which must occur when condensate is retained in a rising parcel above the freezing level) mostly offsets these negative effects. In this manner, CAPE values between a pseudoadiabatic process with no ice and a reversible process with ice are substantially the same. However, it will be shown that the vertical distribution of buoyancy is altered, which could still affect intensification processes. Since

a reversible and pseudoadiabatic approach represents two extreme situations (all condensate instantly removed versus no rain), a simple approach for adapting both methods in buoyancy calculations is proposed. 
Finally, as mentioned in Chapter 3, some researchers have hypothesized that the limiting factor to intensification is the stabilization of the eyewall. This conclusion assumes that the development of the warm core affects the entire ascending branch of the eyewall. However, observations will be presented that suggest the warming only occurs in the upper troposphere, and that low-level eyewall warming is slight. Therefore, buoyancy actually increases during the ASI process, and stabilization cannot be the restrictive factor.

Since eyewall stabilization is probably not the restrictive factor which determines the Maximum Potential Intensity (MPI), the limiting factor must be a dynamical constraint. It is hypothesized this dynamical constraint is insufficient vertical motion to sustain the Internally Forced Convergence (IFC) process (discussed in Chapter 3). The reasons for inadequate ascent can be one or more of the following: 1) the increase of friction (roughly proportional to $V_{\max }^{2}$ ) with TC development; 2) CAPE not increasing enough to sustain the vertical motion necessary for further intensification; and 3) increasing inertial stability as the TC develops restricting the secondary circulation. On the other hand, Ekman pumping increases as the TC intensifies, and the degree to which this compensates these negative influences is unclear. Another complex factor is the evolution of the eye (both above the eyewall and in the center itself) and how it interacts with these processes. Despite these complications, it is hypothesized that the main prohibitive agent that sets the MPI is an insufficient growth of CAPE to balance the momentum budget requirements of increased vertical motion.

The rest of Section 7.1 explains these ideas in more detail. Section 7.2 details the numerical procedures for computing surface cooling due to adiabatic expansion, and moist adiabatic ascent with water-loading and ice microphysics. Section 7.3 compares buoyancy values for reversible and pseudoadiabatic ascent, both with and without ice processes. A simple methodology is proposed for including these processes. Section 7.4 documents the evolution of the eyewall temperature anomaly from composite data, and presents a modified ASI theory which includes boundary layer cooling. Section 7.5 presents numerical results of this modified ASI process. Section 7.6 describes the dynamical constraint on MPI. Section 7.7 summarizes this chapter. 


\subsubsection{Evidence of boundary layer cooling}

As mentioned in Chapter 3, with the exception of a few skeptical researchers in the early 1970s (Carrier 1971; Fendell 1974), analysis of limited hurricane data indicated that the surface fluxes maintained a temperature difference between the ocean and the surface air of about $1^{\circ} \mathrm{C}$ (Anthes 1982). However, some of these surface temperature computations were performed by assuming a location of the LCL from $800-900$-mb flight data and extrapolating downwards (Hawkins and Rubsam 1968; Moss and Rosenthal 1975). Other inferences of $1^{\circ} \mathrm{C}$ cooling were obtained from 200-year records of Jesuit priests over the Philippine and Caribbean Islands (Deppermann 1947; DeAngelis 1989; Udias and Stauder 1991) and eye dropwindsondes (Simpson 1952; Franklin et al. 1988), neither of which may be representative of surface temperatures under an eyewall. In fact, recent research suggests these differences increase to $3-4^{\circ} \mathrm{C}$ under the eyewall as the TC develops.

Figure 7.1 shows ship measurements of SST minus air temperature in two TCs and the corresponding wind speed. For weak winds, the SST minus $T_{s f c}$ difference is about $1^{\circ} \mathrm{C}$, which is typical in the tropics. However, the difference increases nonlinearly with wind speed to $5^{\circ} \mathrm{C}$. This cooling is not due to wet-bulb cooling, as considerable care was used in preventing the thermometers from getting wet by the use of "droplet traps," and when moisture did contaminate the instruments the data was discarded (Fairall et al. 1994). The determination of moisture contamination was aided by a photocell which could detect this process in one thermometer. Therefore, this boundary layer cooling appears not to be an artifact of instrument error. One sounding showed this cooling to extend to a height of $100 \mathrm{~m}$.

Unfortunately, a frontal passage during these observation may have contributed to some of the measurements in Fig. 7.1 (P. Black, personal communication 1995). However, other observations also support eyewall boundary layer cooling as a TC develops, including ship measurements in Hurricane Alma (1966) (Beckerle 1974), buoy data in Hurricane Andrew (1992) (Breaker et al. 1994), and other ship/buoy observations (P. Black, personal communication 1995). Further evidence will be presented from composite data in Section 7.4.2. 


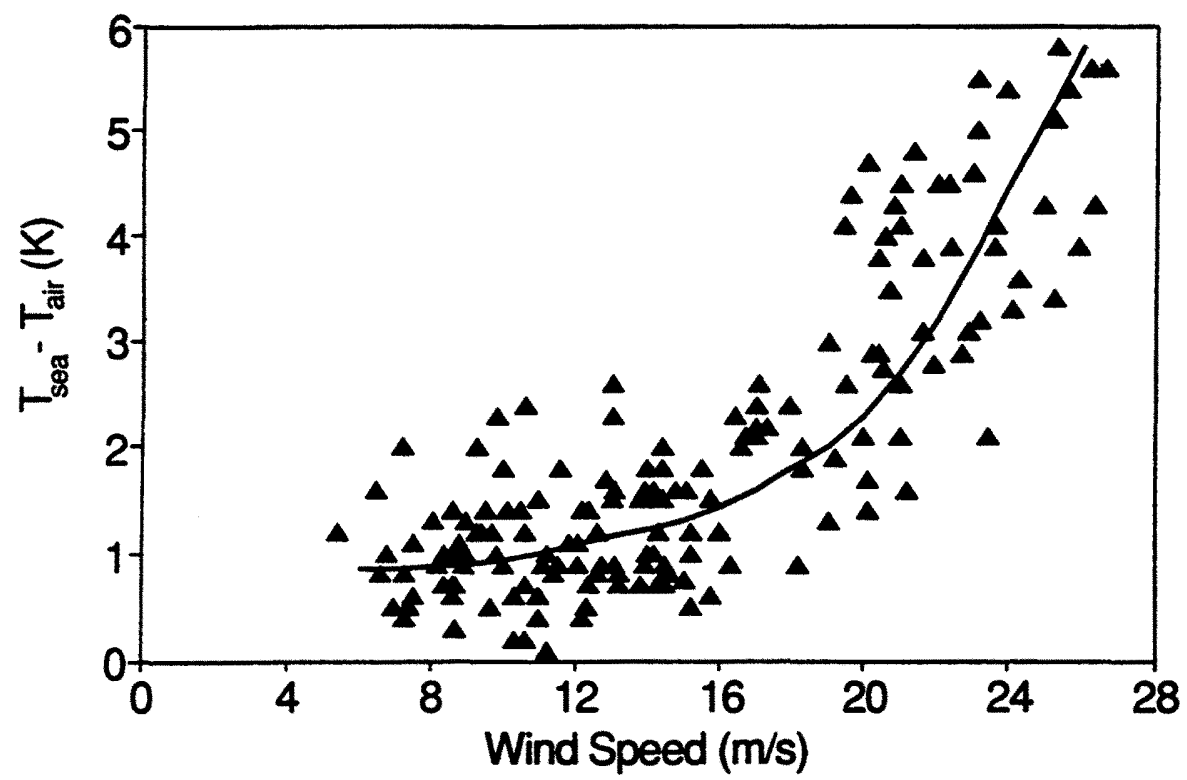

Figure 7.1: Measurements taken aboard the research ship Priliv in 1988. The plot shows the SST minus air temperature difference $\left({ }^{\circ} \mathrm{K}\right)$ versus wind speed $\left(\mathrm{m} \mathrm{s}^{-1}\right)$ in TCs Tess and Skip. Taken from Fairall et al. (1994), who adapted the figure from Pudov (1992).

If this boundary layer cooling is representative of all surface temperatures beneath eyewalls, then what causes this reduced surface air temperature? Several possibilities exist: 1) mixing of cooler subsurface water to the surface where a shallow thermocline is present; 2) upwelling of cooler water due to oceanic Ekman pumping; 3) evaporative cooling of sea spray; 4) evaporative cooling due to rain entering the boundary layer; 5) advection of cool, dry air (downdrafts) into the subcloud layer; and 6) adiabatic expansion of air flowing toward lower pressure not being totally compensated by sensible heat fluxes. None of these processes can be totally discounted, and much more research is needed on the subject. However, Gray (1995) surmises that adiabatic expansion is the dominant mechanism for the surface cooling under the eyewall - a supposition this writer tentatively supports.

Oceanic forcing of boundary layer cooling has been proposed by several researchers, since observations and modeling studies show cooler SSTs in the wake of TCs as a the result of upwelling/mixing (Black 1983; Black et al. 1989; Shay et al. 1992; Bender et al. 1993; Black and Holland 1995). Advection of colder air from the TC's rear into the eyewall could contribute to the boundary layer cooling if the thermocline is shallow enough to support upwelling/mixing processes. Indeed, Holliday and Thompson (1979) found SSTs 
greater than $28^{\circ} \mathrm{C}$ at a depth of $30 \mathrm{~m}$ are necessary for rapid intensification, suggesting that oceanic forcing in shallow thermoclines precludes rapid intensification even when the surface SSTs are very warm. However, oceanic forcing should only affect slow-moving TCs, since most TCs probably move too fast to develop cold water around the center. Furthermore, it will be shown in Chapter 8 that storm speed is a statistically insignificant parameter in correlation calculations with TC intensity change. Therefore, advection of cool air resulting from oceanic processes into the eyewall probably cannot explain the SST minus $T_{s f c}$ differences of $4^{\circ} \mathrm{C}$ in most cases.

Fairall et al. (1994) and Kepert (1995) investigated evaporative processes which may cause boundary layer cooling by using bulk formulas to parameterize the effects of heat and moisture fluxes in a slab model of the TC boundary layer. In addition, they partitioned the cooling and moistening of evaporative processes into contributions by sea spray and rainfall. Fairall et al. (1994) performed simulations in a simple mixed layer model which omitted cloud fluxes but included sea spray evaporation, rain evaporation and "clear sky" fluxes from above the boundary layer. Without sea spray evaporation, the eyewall boundary layer becomes warmer than the environmental boundary layer $500 \mathrm{~km}$ from the center, contrary to observations - with sea spray, the temperature decreased within $200 \mathrm{~km}$ of the center, and $3-4^{\circ} \mathrm{C}$ cooling occurs under the eyewall. This unrealistic warming is due to an inversion at the top of the boundary layer, which fluxes heat downwards. ${ }^{1}$ The addition of rain evaporation did not alter the overall surface temperature evolution, but produced slightly more cooling than with sea spray alone. Kepert (1995) extended this model by including an evolving boundary layer depth, cloud base fluxes (updrafts and downdrafts), and a momentum budget. Kepert also found that, while all processes are important, sea spray is the dominant cooling component as wind speed increases, producing twice as much cooling as rainfall.

\footnotetext{
${ }^{1}$ Several other researchers have hypothesized that surface fluxes are not the dominant mechanism attempting to balance boundary layer cooling, but that mixing (mass recycling) of high potential temperature air from above the boundary layer may supply most of the heat instead (Frank 1977a; Anthes and Chang 1978; Betts and Simpson 1987; Powell 1990; Barnes and Powell 1995).
} 
These results are quite interesting and deserve further research, but the $3-4^{\circ} \mathrm{C}$ evaporative cooling arguments based on evaporation of sea spray seem suspect to this author given the observations of high relative humidity at the surface. As noted by Gray (1995), wet-bulb depressions are quite small in high humidity conditions. ${ }^{2}$ Another problem is that the model of Fairall et al. (1994) and Kepert (1995) contains an inversion above the TC boundary layer, and the model results are quite sensitive to its presence. Since eyewall soundings are rare, it is not clear that inversions occur under eyewalls at all, nor is it certain that boundary layer processes should be so sensitive to its existence. Therefore, while sea spray evaporation may be important, it is still this writer's tentative opinion that cooling by adiabatic expansion is the primary agent of eyewall boundary layer cooling.

\subsubsection{Water-loading}

As discussed in the introductory chapter, some cloud physicists have recently advocated the inclusion of water-loading in calculations of moist ascent. The effect of suspended condensate it to reduce the buoyancy of the air parcel. To include the density of liquid-phase condensate, a modified version of virtual temperature $T_{v}$ is used (density temperature $T_{\rho}$, where $T_{\rho}<T_{v}$ ) which will be defined soon (Emanuel 1994). Ironically, including water loading actually increases the air temperature due to the heat capacity of liquid water (Saunders 1957; Ooyama 1990; Emanuel 1994).

Furthermore, some researchers believe the most representative calculations of $T$ and $T_{\rho}$ during moist ascent is through a reversible process as opposed to an irreversible (pseudoadiabatic) process (Betts 1982; Emanuel 1989b; Xu and Emanuel 1989). This is the other extreme of the pseudoadiabatic process (which assumes all liquid condensate is instantly removed) in that as liquid-phase condensate forms it not only is retained in the parcel, but is carried along the parcel's entire trajectory with no rainfall occurring (Williams and Renno 1993).

\footnotetext{
${ }^{2}$ The wet-bulb temperature $T_{w}$ is defined as the temperature to which a parcel of air is cooled by evaporating water into it at constant pressure until the air is saturated (Wallance and Hobbs 1977). Therefore, $T_{w}$ is bounded by the dewpoint temperature $T_{d}$ and the air temperature $T\left(T_{d} \leq T_{w} \leq T\right)$. Under high relative humidity conditions, the dewpoint depression $\left(T-T_{d}\right)$ will be small, and thus it is difficult to cool an air parcel by water evaporation in near-saturated conditions.
} 
While such a drastic premise seems ridiculous since precipitation is observed to occur in the TC eyewall, these researchers argue that the reversible $T$ and $T_{\rho}$ values are representative once entrainment and the effects of precipitation (by evaporation and a further reduction in buoyancy by rain falling from above) are also included. Indeed, this is the supposition by which Emanuel (1989b) and Xu and Emanuel (1989) base their conditional neutrality theory for tropical convection, and have extended it to the TC eyewall. Of course, this argument ignores the fact that buoyancy will increase aloft due to fallout of rain. Also ignored is: 1) the contribution of latent heating by ice processes which must occur if condensate is retained to the freezing level; 2) the observations of CAPE presented in Chapters 3 and 4 which contradicts the concept of convective neutrality; and 3 ) the fact that a sloping eyewall can circumvent the effects of "precipitation drag" through slantwise convection. An additional problem with the calculations of Betts (1982) and Xu and Emanuel (1989) is that parcels are lifted from the top of the subcloud layer rather than the surface. Renno and Williams (1995) show, from boundary layer observations taken by a remotely piloted sailplane, that convective plumes originate near the surface. Therefore, it is likely that the buoyancy calculations in Betts (1982) and Xu and Emanuel (1989) are underestimated.

Because of these arguments, some researchers dispute the "convective neutrality" arguments. In particular, as first noted by Williams and Renno (1993) and will be confirmed in this chapter, including ice processes restores the buoyancy lost to water-loading, thus rendering the supposition of a "convectively neutral" eyewall (and tropical convection!) very questionable.

\subsubsection{Latent heating by ice processes}

There is little doubt that liquid condensate eventually freezes in deep convection, and deserves inclusion in buoyancy calculations. Yet, ice has rarely been included in such computations. This is because ice formation is a complex process involving a phase transition between vapor and ice (sublimation, also called deposition) in a freezing layer

of unknown depth, which is also related to great uncertainty involving the temperature at 
which any supercooled water freezes to ice (fusion). As explained by Cotton and Anthes (1989):

If the cloud is water saturated and contains a substantial amount of supercooled cloud droplets...the full latent heat is not absorbed by the cloudy air. This is because ice crystals grow at the expense of cloud droplets (the Bergeron-Findeisen process), which must evaporate as the saturation vapor pressure is lowered locally below water saturation. As a result, the evaporating droplets absorb the latent heat of condensation. The net result of ice crystals growing by vapor deposition and of cloud droplets evaporating is that the cloud experiences heating only in proportion to the latent heat of fusion (defined as latent heat of sublimation minus latent heat of condensation) and the water mass deposited on the ice crystals.

Furthermore, Cotton and Anthes (1989) explain the sensitivities which can result from ice processes:

The latent heating (by ice) can be rather smoothly released if ice crystals grow by vapor deposition...In contrast, it can take place as a burst of energy release in convective towers if large quantities of supercooled water suddenly freeze.

In turn, the quantity of supercooled water is related to the updraft speed - a very intense updraft may carry liquid water upward so rapidly that freezing does not occur until very cold temperatures aloft are reached. On the other hand, in weak updrafts the level of freezing may be near $0^{\circ} \mathrm{C}$. As mentioned in Chapter 4, only a little supercooled water exists outside the eyewall where ascent is weak, but in the eyewall liquid water concentrations are as high as several $\mathrm{g} \mathrm{m}^{-3}$ (Willoughby et al. 1985). The existence of icing on reconnaissance aircrafts (Simpson 1963), and the evidence of occasional lightning activity cited in Chapter 4 also supports this assertion.

Additional complications using ice processes include: 1) how ice processes affect updrafts and downdrafts, which are further confounded by ice melting (Lord et al. 1984); 2) the temperature at which freezing nuclei are activated; 3) the growth of ice crystals by riming (ice crystals colliding with supercooled water and becoming graupel) and aggregation (ice crystals colliding and sticking together) (Wallace and Hobbs 1977); 4) secondary ice-crystal production by the rime-splintering mechanism (Hallett and Mossop 1974) which is believed to dominate ice production outside the eyewall (Black and Hallett 1986). The horizontal and vertical transport of ice created by secondary processes also quickly converts any supercooled water located in other portions of the storm to ice; and 
5) how the buoyancy gained by ice processes impacts the low-level inflow - a positive feedback is the essence of the IFC hypothesis discussed earlier (and also the basis for the dynamic seeding hypothesis in weather modification experiments (Cotton and Anthes 1989)). Despite these complex issues, this chapter will approximate the inclusion of ice in the ASI process.

To imitate the effects of freezing, the method of Iribarne and Godson (1981) and Williams and Renno (1993) will be used, which is similar to the approach of other researchers (Saunders 1957; Orville and Hubbard 1973; Stephens 1979). It is assumed that at some temperature, $T_{f}$, the existing liquid condensate $q_{l}$ freezes to ice condensate. However, since the the air initially saturated with respect to water at $T_{f}$ will be supersaturated with respect to the frozen droplets, sublimation will modify the temperature change. This change due to sublimation can lead to slight warming or cooling, with the latter being more common for high liquid water contents. This is because during fusion the parcel equilibrium vapor pressure changes, and it is possible for ice saturation vapor pressure to be greater than the water saturation vapor pressure, causing a moisture transfer from ice to water (MacReady and Skutt 1967; Orville and Hubbard 1973). In contrast, at very cold temperatures where vapor pressures are low, substantial heating from both fusion and sublimation can occur.

Upon reaching equilibrium, a final temperature $T^{\prime}$ is achieved, and the frozen condensate $q_{f}$ will be different than $q_{l}$. In reality, the freezing does not occur at one level (isobaric freezing), but gradually over a temperature interval, but as a first approximation $T^{\prime}$ will be calculated at a specified freezing level of $T_{f}$.

Subsequent ascent above the freezing level takes place along a reversible (assuming ice-loading) or pseudoadiabatic (assuming no ice-loading) path relevant to deposition. In terms of $T_{\rho}$, the increase of air temperature by latent heat of fusion and sublimation mostly offsets the density of ice-loading, even during the extreme case of a reversible process (Williams and Renno 1993). As will be seen, the additional buoyancy due to ice formation offsets the lost of buoyancy due to water-loading in the lower troposphere. Furthermore, any contribution of this latent heat to the TC temperature perturbation 
during geostrophic adjustment enhances the pressure fall, since the thickness equation (Eq. (3.2)) is sensitive to such perturbations due to the "dp/p effect." It should also be noted that ice processes contribute buoyancy in the region where buoyancy production by condensation becomes less important, and thus augments diminishing cloud buoyancy.

The resulting vertical profiles of $T$ and $T_{\rho}$ for reversible and pseudoadiabatic processes with and without ice are shown in Fig. 7.2 (Ooyama 1990). The air temperature with
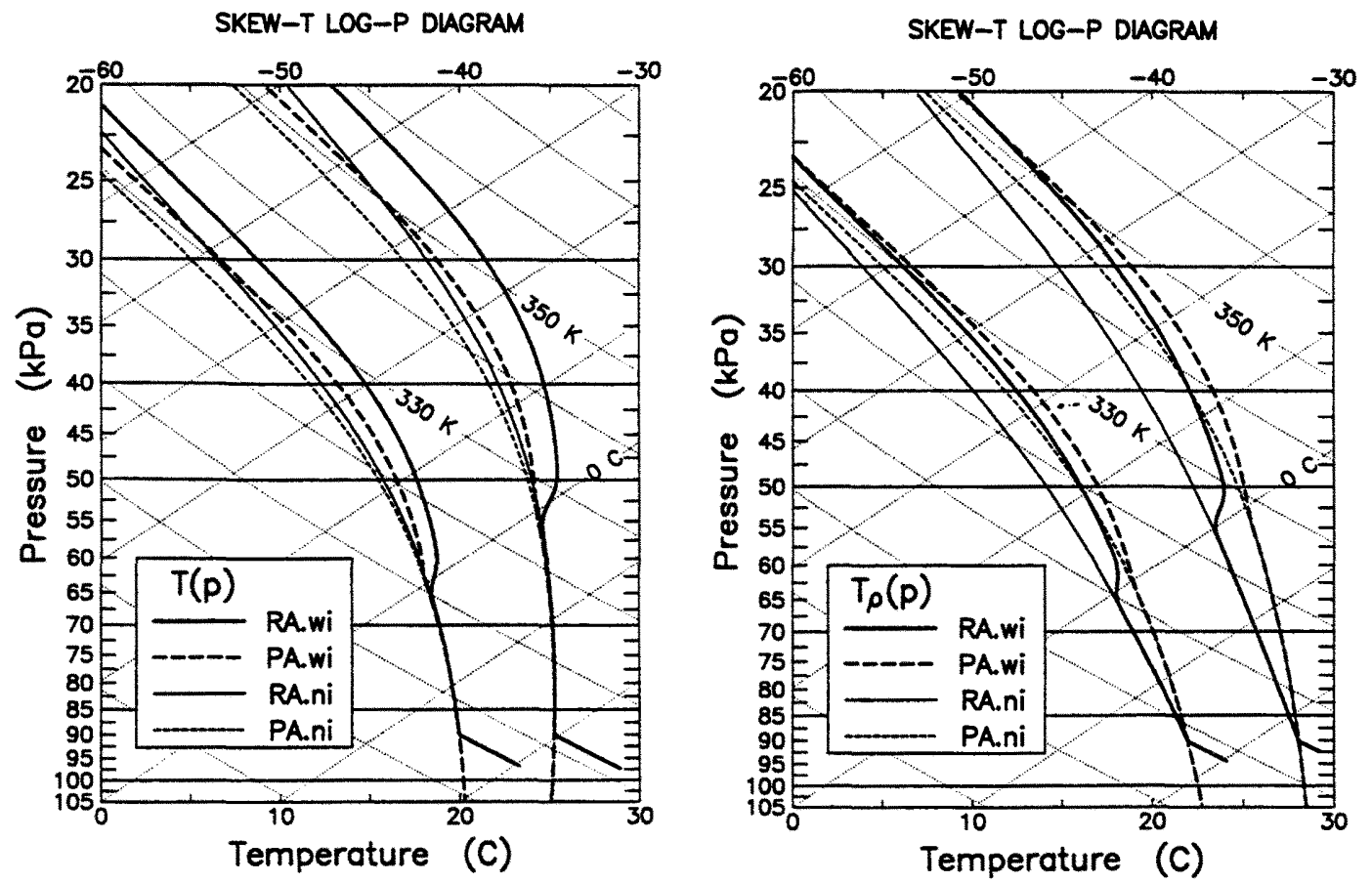

Figure 7.2: Temperature, $(T(p))$ and density temperature $\left(T_{\rho}(p)\right)$ of selected adiabats on a Skew T-Log P diagram. The label "RA" is for reversible adiabats, "PA" for pseudoadiabats, "wi" for with ice, and "ni" for no ice. The $\theta_{e}$ values (as represented by PA.ni) are $330^{\circ} \mathrm{K}$ and $350^{\circ} \mathrm{K}$. Taken from Ooyama (1990).

no ice is warmer for the reversible process than the pseudoadiabatic process due to the specific heat of liquid water. The same pattern holds with ice, except that both are warmer than their non-ice counterparts. However, the non-ice reversible adiabat is more dense due to water-loading compared to the non-ice pseudoadiabat, except above $200 \mathrm{mb}$ (not shown in figure) where $T_{\rho}>T_{v}$ since the reversible adiabat becomes quite warm and $q^{*}$ is negligible in $T_{v}$. However, with ice the buoyancy is substantially greater than the non-ice pseudoadiabat above $400 \mathrm{mb}$. Naturally, the pseudoadiabat with ice is the most buoyant, although the largest difference is confined to the mid-troposphere. 


\subsubsection{Eyewall slope}

As first proposed by Fendell (1974) (who rejected the notion that surface fluxes totally compensated cooling by adiabatic expansion), allowing the eyewall to slope vertically outwards, with the lateral extension of the eye directly above the low-level eyewall, is a critical factor in maintaining the ASI process in the presence of boundary layer cooling. The eyewall may slope up to a $45^{\circ}$ angle relative to the vertical near the tropopause (P. Black, personal communication 1995). As shown in Fig. 7.3, with a portion of the eye temperature perturbation vertically aligned with the lower eyewall, hydrostatic pressure falls (which is nonlinearly related to the height of temperature anomalies) can be sustained under the eyewall even with $2-4^{\circ} \mathrm{C}$ surface cooling. Without the support of the eye, the

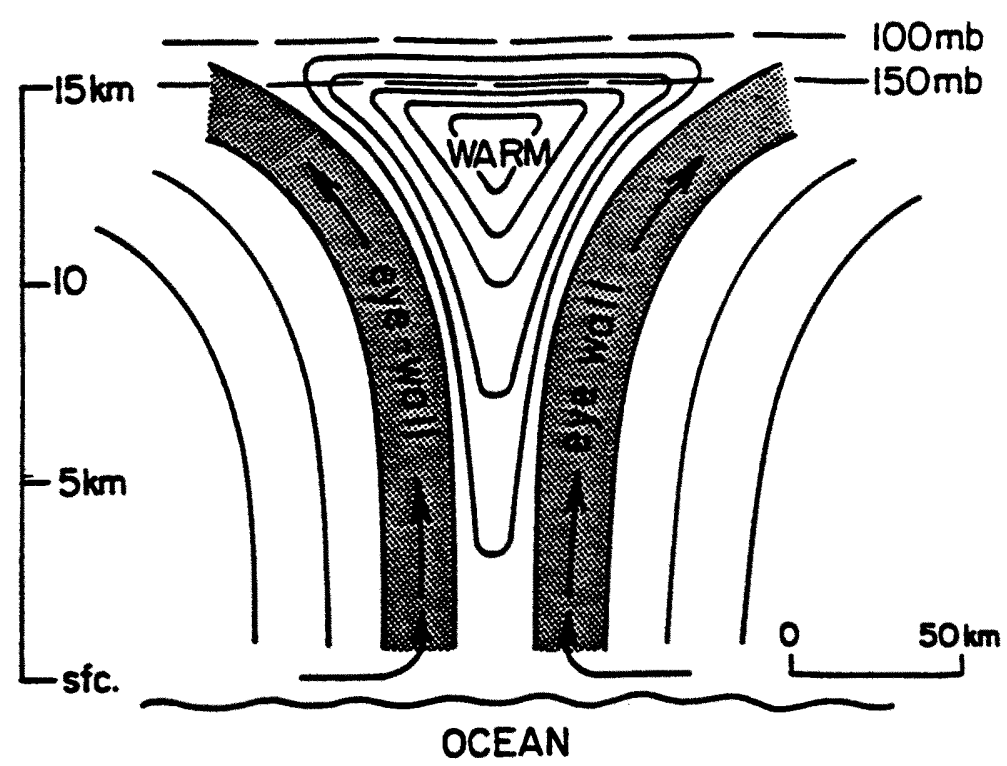

Figure 7.3: Idealized vertical cross-section of eye and eyewall temperature anomalies relative to the outward sloping eyewall. The air-sea interaction process can only be sustained in the presence of $2-4^{\circ} \mathrm{C}$ boundary layer cooling with the lateral extension of the eye aloft contributing to the hydrostatic pressure falls under the eyewall. Taken from Gray (1995).

ASI process with boundary layer cooling will collapse, even with a contribution from ice processes. Recall from Chapter 3 that fast intensification coincides with eye formation. However, in the past researchers have treated the eyewall and the eye as two separate features, with the low MSLP of the eye accelerating radial inflow and its associated positive intensity dynamical feedbacks but independent of the ASI process. It now appears that, 
with boundary layer cooling, both features must be coupled in terms of the ASI process and the various dynamical processes discussed earlier in this manuscript. Not coupling eye and eyewall characteristics causes one to question other theoretical results which do not include this interactive process.

\subsection{Mathematical treatment of cooling under eyewall, reversible ascent, pseu- doadiabatic ascent, and ice microphysics}

This section presents the mathematical framework for cooling under the eyewall, and buoyancy calculations. A crude formulation of boundary layer cooling due to adiabatic expansion with partial compensation by sensible heat fluxes is derived. The computational procedures for reversible moist ascent and latent heat release during ice formation are presented.

\subsubsection{Representation of surface cooling by adiabatic expansion}

The starting point for cooling of surface air by adiabatic expansion as it spirals toward lower pressure is expressed by the First Law of Thermodynamics:

$$
\frac{d \dot{Q}}{c_{p} T}=\frac{d T}{T}-\frac{R}{c_{p}} \frac{d p}{p}
$$

where $d \dot{Q}$ is an incremental rate of heating supplied by the ocean. In an adiabatic process, Eq. (7.1) reduces to the Poisson equation which represents the adiabatic expansion process. Upon integration from the environment to the eyewall, and neglecting the contribution of surface flux in the environment compared to that under the eyewall, Eq. (7.1) may be approximated as:

$$
\frac{\dot{Q}_{e w}}{c_{p} T_{e w}}=\ln \left[\frac{\left(T_{e w} / T_{e n v}\right)}{\left(p_{e w} / p_{e n v}\right)^{R / c_{p}}}\right]
$$

where the subscript $e w$ represents surface eyewall values. The sensible heat flux $h$ is often calculated using the bulk formula (Anthes 1982):

$$
h=\rho C_{D} c_{p}|\vec{V}|\left(T_{s e a}-T_{e w}\right)
$$

where the drag coefficient $C_{D}$ is weakly dependent on wind speed (Powell 1980):

$$
C_{D}=1.0236 \times 10^{-3}+5.366 \times 10^{-5}|\vec{V}|
$$


Therefore, according to Eq. (7.3), the heat flux increases as the temperature difference between the surface air and ocean increases, and is nonlinearly dependent on the wind speed as well. In other words, the ASI process is a result of a positive feedback between surface winds and surface fluxes which (mostly) compensates for boundary layer cooling by adiabatic expansion. Emanuel (1991) labels this feedback mechanism the Wind Induced Surface Heat Exchange (WISHE) instability.

Neglecting advective processes, and (for the moment) adiabatic expansion, the local rate of eyewall temperature change by sensible heat fluxes may be written (Anthes 1982):

$$
\frac{\partial T}{\partial t}=-\frac{1}{\rho c_{p}} \frac{\partial h}{\partial z}
$$

and therefore, assuming heat fluxes are negligible at a distance $\delta z$ from the ocean surface, $\dot{Q}_{e w} / c_{p}$ may be approximated as:

$$
\frac{\dot{Q}_{e w}}{c_{p}}=\frac{C_{D}\left|\vec{V}_{e w}\right|\left(T_{s e a}-T_{e w}\right) \delta t}{\delta z}
$$

where $\delta t$ is the local time scale for the sensible heat flux $\overline{w^{\prime} T^{\prime}}$ to operate. By substituting the effect of adiabatic expansion Eq. (7.2) into Eq. (7.6), the final result is:

$$
\frac{C_{D}\left|\vec{V}_{e w}\right|\left(T_{\text {sea }}-T_{e w}\right) \delta t}{T_{e w} \delta z}=\ln \left[\frac{\left(T_{e w} / T_{e n v}\right)}{\left(p_{e w} / p_{e n v}\right)^{R / c_{p}}}\right]
$$

Since $T_{e w}$ occurs on both sides of Eq. (7.7), the solution must be iterated. Choosing a $\delta t$ of $45 \mathrm{~s}$, a $\delta z$ of $10 \mathrm{~m}, T_{\text {sea }}$ to be $29^{\circ} \mathrm{C}, p_{\text {env }}$ to be $1020 \mathrm{mb}$ (a typical Atlantic value), $T_{\text {env }}$ to be $1^{\circ} \mathrm{C}$ cooler than $T_{\text {sea }}$, and approximating $\left|\vec{V}_{\text {ew }}\right|$ by the empirical relationship of Atkinson and Holliday (1977):

$$
V_{\max }=3.4\left(p_{e n v}-p_{e w}\right)^{0.644}
$$

where pressure has units of $\mathrm{mb},{ }^{3} T_{e w}$ as a function of $p_{e w}$ is shown in Fig. 7.4. The formulation of Eq. (7.7) is crude with some questionable assumptions, but captures the essential features of an increasing air-sea temperature difference to $4^{\circ} \mathrm{C}$ as the pressure

\footnotetext{
${ }^{3}$ Technically speaking, $p_{e w}$ should be the eye pressure, but this is unknown. Besides, using $p_{e w}$ serves as a correction to the overestimate of $\left|\vec{V}_{e w}\right|$ in the integration between the environment and the eyewall.
} 
EYEWALL SURFACE COOLING

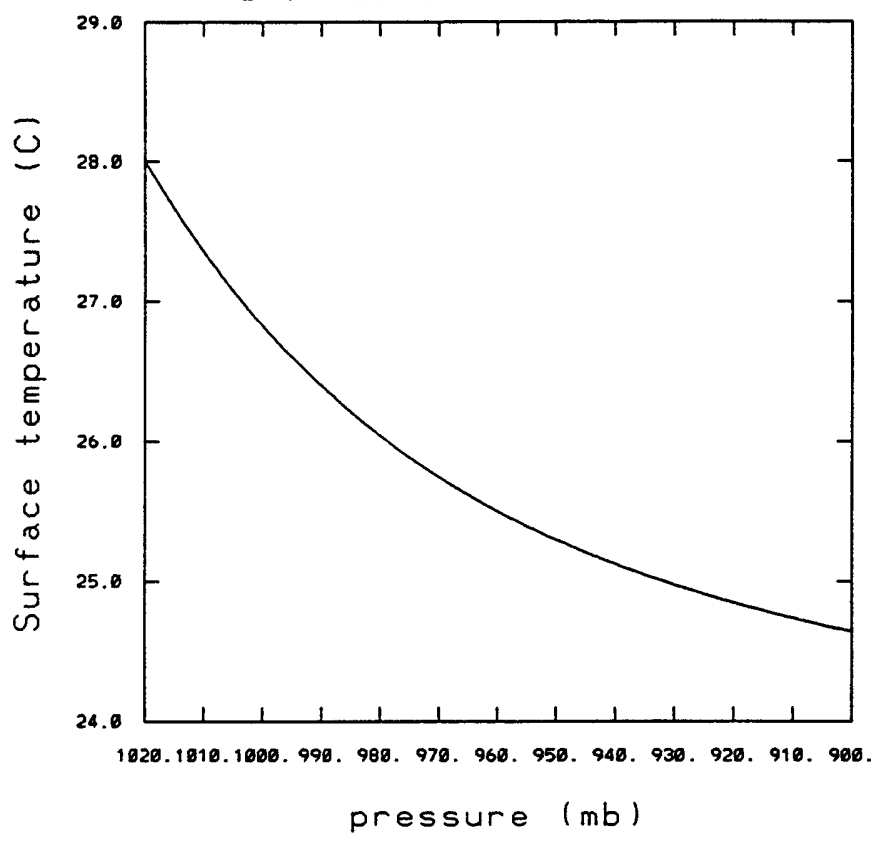

Figure 7.4: The eyewall surface temperature $\left(T_{e w}\right)$ as a function of the eyewall surface pressure $\left(p_{e w}\right)$ for a SST of $29^{\circ} \mathrm{C}$. See text for details of calculation.

decreases, then a leveling off as the WISHE process compensates for the adiabatic cooling. Other SST values depict a similar pattern (not shown). Further research is required of surface cooling processes either through empirical relationships of $T_{e w}\left(p_{e w}\right)$ (Pudov 1992; Pudov and Holland 1994) or by boundary layer models (Kepert 1995; Fairall et al. 1994).

\subsubsection{Representation of water loading}

The thermodynamic relationship for a reversible moist process is (Iribarne and Godson 1981):

$$
c_{p} \ln T+q_{t o t} c_{w} \ln T-R \ln \left(p-e^{*}(T)\right)+q(T) L_{v}(T) / T=s^{*}
$$

where $q_{t o t}=q+q_{l}$ is the sum of the water vapor and liquid water mixing ratios, respectively, and the moist entropy $s^{*}$ is computed at the LCL and held constant during moist ascent. The difference between a pseudoadiabatic process and the reversible process is manifested by the second term in which $q_{l}$ is assumed zero in the former process but retained in the latter. The temperature dependence of latent heat of condensation needs to be retained in the equation, since keeping $L_{v}$ constant can give an error of $0.5-1^{\circ} \mathrm{C} . L_{v}(T)$ is empirically 
computed with $99.9 \%$ explained variance as (Bolton 1980):

$$
L_{v}=(2.501-0.00237[T-273.15]) \times 10^{6}
$$

Because no rain occurs, $q_{t o t}$ is a conserved quantity throughout moist ascent. In other words, $q_{t o t}$ is calculated at the LCL and held constant during the iterative process up to $100 \mathrm{mb}$. Naturally, $q_{l}$ increases during ascent as $q$ decreases, amplifying the effect of negative buoyancy by water-loading with height. Quantitatively, this effect is included in the definition of density temperature $T_{\rho}$ by: ${ }^{4}$

$$
T_{\rho}=T\left(1+0.61 q-q_{l}\right)
$$

and yields less buoyancy. When $q_{l}$ is zero, $T_{\rho}$ reduces to the virtual temperature $T_{v}$.

\subsubsection{Representation of ice processes}

The inclusion of ice processes is done for both an irreversible process and the pseudoadiabatic process, and follows the procedures outlined by Iribarne and Godson (1981) and extended by Williams and Renno (1993). The isentropic quantity for temperatures below freezing $\left(T_{f}\right)$ is done as outlined in Chapter 3 (Eq. (3.5)) for the pseudoadiabatic calculation, and as done in Eq. (7.9) for the reversible process. At the freezing level, (which is assumed to be at $-10^{\circ} \mathrm{C}$ in all calculations), isobaric freezing is postulated with an increase in parcel temperature by latent heat of fusion modified by deposition of water vapor. ${ }^{5}$ The solution for the temperature resulting from this isobaric freezing process $\left(T^{\prime}\right)$

'This expression is actually an approximation to the true definition of density temperature given by:

$$
T_{\rho}=T \frac{1+q / 0.622}{1+q_{\text {tot }}}
$$

but both equations give nearly the same value for $T_{\rho}$. Stull (1988) shows in detail how the approximate form of $T_{\rho}$ is obtained by applying long division between the numerator and denominator and neglecting terms which contain products of mixing ratio values. The definition of $T_{\rho}$ can be extended to ice processes through the mixing ratio summation term $q_{T O T}=q+q_{f}+q_{i c e}$ where $q_{f}$ is frozen condensate and $q_{i c e}$ is the saturation mixing ratio over ice (Emanuel 1994). More detail is contained in Section 7.2.3.

${ }^{5}$ As noted by Williams and Renno (1993), while the buoyancy calculations are affected by the choice of $T_{f}$, the calculations are reasonably bounded by 0 and $-20^{\circ} \mathrm{C}$. Sensitivity calculations show the choice of freezing level not to be crucial. However, using a freezing level introduces a discontinuity which may cause problems in numerical models. To eliminate this discontinuity, Ooyama (1990) proposed that a "freezing zone" be used in which ice effects are gradually included and liquid water progressively removed. The procedure is outlined in more detail by Wang and Randall (1994). 
is given by (Orville and Hubbard 1973; Iribarne and Godson 1981):

$$
T^{\prime}-T_{f}=\frac{L_{f}\left(T_{f}\right) q_{l}}{c_{t o t}\left(T_{f}, T^{\prime}\right)}+\frac{L_{s}\left[q\left(T_{f}\right)-q_{i c e}\left(T^{\prime}\right)\right]}{c_{t o t}\left(T_{f}, T^{\prime}\right)}
$$

where $L_{f}$ and $L_{s}$ are the latent heats of fusion and sublimation, respectively, and $q_{i c e}$ is the saturation mixing ratio over ice. Equation (7.12) is iterated for $T^{\prime}$, although an approximate analytical solution could be used instead (Orville and Hubbard 1973; Stephens 1979; Iribarne and Godson 1981). $L_{s}$ remains fairly constant for temperatures between 0 and $-80^{\circ} \mathrm{C}\left(2.83 \times 10^{6} \mathrm{~J} \mathrm{~kg}^{-1}\right)$ (Iribarne and Godson 1981) while applying a least square fit for $L_{f}(T)$ to the tabular data in Iribarne and Godson (1981) gives:

$$
\begin{aligned}
L_{f} & =\left(0.3337+0.00216[T-273.15]-0.981 \times 10^{-6}[T-273.15]^{2}\right. \\
& \left.+0.159 \times 10^{-6}[T-273.15]^{3}\right) \times 10^{6}
\end{aligned}
$$

which explains $99.9 \%$ of the variance. For the pseudoadiabatic calculations, $q_{l}$ (and hence latent heat of fusion) is set to zero in Eq. (7.12). The "total heat capacity" $c_{\text {tot }}$ at constant pressure is computed as:

$$
c_{t o t}\left(T_{f}, T^{\prime}\right)=c_{p}+q_{i c e}\left(T^{\prime}\right) c_{v}+\left(q_{l}+q\left(T_{f}\right)-q_{i c e}\left(T^{\prime}\right)\right) c_{i c e}
$$

where $c_{v}$ and $c_{i c e}$ are the specific heats at constant pressure for vapor and ice, respectively. $q_{i c e}$ is computed in typical fashion:

$$
q_{i c e}=0.622 \frac{e_{i c e}}{p-e_{i c e}}
$$

with the saturation vapor pressure over ice $\left(e_{i c e}\right)$ calculated using an empirical equation (Rogers and Yau 1989):

$$
e_{i c e}=3.41 \times 10^{10} \exp \left(-6.13 \times 10^{3} / T\right)
$$

As noted previously, the presence of supercooled water can be a significant buoyancy factor, especially at low freezing temperatures and high liquid water content. Consider the limiting case of Eq. (7.12) when $q$ and $q_{i c e}$ is negligible, then $T^{\prime}-T_{f}=q_{l} L_{f} / c_{p}$; for $T_{f}=-30^{\circ} \mathrm{C}$ (where $q$ and $q_{i c e}$ are very small) and a high liquid water content of $q_{l}=10 \mathrm{~g} \mathrm{~kg}^{-1}, T^{\prime}-T_{f}=3.3^{\circ} \mathrm{C}$ (Iribarne and Godson 1981). 
Above the freezing level, the expression for ice entropy $s_{i c e}$ is

$$
c_{p} \ln T+\Lambda q_{T O T} c_{i c e} \ln T-R \ln \left(p-e^{*}(T)\right)+q_{i c e}(T) L_{s} / T=s_{i c e}
$$

where $q_{T O T}=q_{f}+q+q_{i c e}$ (note that a capitalized subscript is used above the freezing level so as to differentiate from $q_{t o t}$ below the freezing level). Again, $q_{T O T}$ is a conserved quantity in the reversible process where ice condensate is retained in the parcel and carried upward along. $q_{f}$ increases in a reversible process as an air parcel ascends above the freezing level, since $q$ and $q_{i c e}$ decrease at smaller temperatures. In the pseudoadiabatic process, ice condensate is assumed to instantly fall out $\left(q_{f}=0\right)$. Since the second term presents computational problems during a pseudoadiabatic process, it is neglected for this calculation $(\Lambda=0)$. For a reversible process, $\Lambda=1$. This term is not negligible in the pseudoadiabatic process, but contributes less than the other terms. Furthermore, $\int\left(q+q_{i c e}\right) c_{i c e} d \ln T$ is difficult to compute (recall from Chapter 3 that including the $c_{w}$ term in the $\theta_{e}$ definition suffers the same problem). Neglecting this term underestimates $T$ and buoyancy, but is not detrimental to the research results in this paper.

The new constant $s_{i c e}$ is computed at the freezing level as:

$$
s_{i c e}=c_{p} \ln T^{\prime}+\Lambda q_{T O T} c_{i c e} \ln T^{\prime}-R \ln \left(p-e^{*}\left(T^{\prime}\right)\right)+q_{i c e}\left(T^{\prime}\right) L_{s} / T^{\prime}
$$

Finally, the approximation to density temperature above the freezing level becomes:

$$
T_{\rho}=T\left(1+0.622 q-\Lambda q_{f}-q_{i c e}\right)
$$

where $q_{f}=q_{T O T}-q-q_{i c e}$. Again, for a reversible process $\Lambda=1$, and for a pseudoadiabatic $\Lambda=0$. The "ice-loading" effect $\left(q_{f}\right)$ would reduce $T_{\rho}$ by itself, but is more than compensated by the increase of $T$ due to latent heat of fusion and sublimation. Hence, ice ultimately increases $T_{\rho}$ and is a positive buoyancy factor. The contribution to $T_{\rho}$ by $q_{i c e}$ is minimal.

\subsection{Buoyancy results with ice microphysics and water-loading}




\subsubsection{Buoyancy comparisons between ice, no ice, reversible, and pseudoadi- abatic calculations}

This section ascertains the sensitivity of buoyancy to four different possible adiabatic ascents: 1) Water-loading (or Reversible), no ice (RA.ni); 2) No water-loading (or Pseudoadiabatic), no ice (PA.ni); 3) Water-loading (or Reversible), with ice (RA.wi); and 4) No water-loading (or Pseudoadiabatic), with ice (PA.wi). In all computations, the LCL is determined given a temperature and relative humidity surface value, and a moist entropy value is computed at the LCL. Then, assuming the moist entropy value is conserved (no entrainment), a temperature value is determined as a function of height along the characteristic curve of moist entropy. When ice processes are included, a new ice entropy value is determined after latent heat of fusion and sublimation occurs, and temperature above the level of freezing is computed along this characteristic curve of ice entropy. After solving for $T(p)$, the density temperature $T_{\rho}(p)$ is computed to measure buoyancy. A detailed account of this procedure now follows.

A moist entropy value is computed at the LCL for a given surface temperature and relative humidity for both a reversible (Eq. (7.9)) and pseudoadiabatic (Eq. (3.5)) process. For the non-ice calculations, once $T(p)$ is known, $T_{\rho}(p)$ is computed in RA.ni from Eq. (7.11), and in PA.ni using the definition of virtual temperature $T_{v}(p)$ (since $q_{l}$ is zero).

For the ice calculations, the same procedure is used as the non-ice up to the freezing level of $T_{f}=-10^{\circ} \mathrm{C}$. At $T_{f}$, liquid water (which is zero for the pseudoadiabatic calculations) is frozen during an isobaric process which releases latent heat as a function of the availability of supercooled droplets, water vapor, and ice. The resulting warmer temperature $T^{\prime}$ is computed from Eq. (7.12). Once this is known, a new ice entropy value is computed from Eq. (7.18), and above the freezing level $T(p)$ is iterated along this characteristic curve which includes latent heat of sublimation to $100 \mathrm{mb}$ (Eq. (7.17)). For PA.wi, $q_{f}$ is assumed to be zero, and is retained in RA.wi. $T_{\rho}(p)$ is computed in both cases from Eq. (7.19).

Assuming a surface temperature of $28^{\circ} \mathrm{C}$ with a surface relative humidity of $90 \%$, the four possible buoyancy profiles are shown relative to the mean Jordan (1958) "hurricane 
season" sounding (Fig. 7.5). These plots are labeled "potential buoyancy" since undilute ascent is assumed. These results are similar to Fig. 7.2, but the differences in the vertical

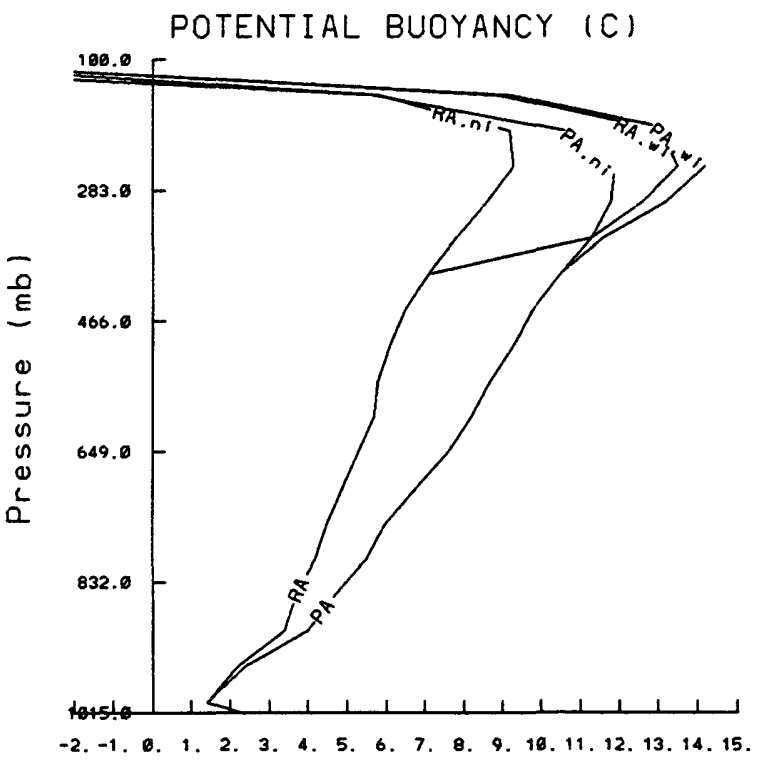

Figure 7.5: Potential buoyancy profiles (defined as $T_{\rho}-T_{v_{\text {env }}}$ ) relative to the Jordan (1958) mean "hurricane season" sounding. A surface temperature of $28^{\circ} \mathrm{C}$ is assumed with a surface relative humidity of $90 \%$. RA.ni represents the reversible process with no ice, and PA.ni represents the pseudoadiabatic process with no ice. The processes with "wi" contain ice. The freezing level is assumed at $T=-10^{\circ} \mathrm{C}$. These profiles are labeled "potential buoyancy" because undilute ascent is assumed.

distribution of buoyancy is clearer to see in Fig. 7.5. As expected, RA.ni is less buoyant than PA.ni except above $200 \mathrm{mb}$ where the contribution of specific heat of water to $T$ overcomes the negative effects of water-loading. However, the boost to an ascending air parcel is striking when ice is included. At $350 \mathrm{mb}, T_{\rho}$ for RA.wi increases $3.5^{\circ} \mathrm{C}$ relative to RA.ni. The increase of $T_{\rho}$ for PA.wi with respect to PA.ni is less since no supercooled water exists (and hence no latent heat of fusion), but the contribution by latent heat of sublimation is still considerable.

The most pertinent result is that, although the reversible adiabatic is less buoyant than the pseudoadiabat without ice, when ice is included RA.wi is more buoyant than PA.ni above the freezing level. Three important facts emerge from this finding:

1. By including the effects of water-loading and ice, the vertical distribution of buoyancy is altered. There is less buoyancy in the lower-troposphere with retained liquid 
water, but these droplets contain "hidden" buoyancy which is released above the freezing level. This sudden burst of energy may play an important role in the maintenance of the secondary circulation, the IFC process, and certainly intensity change.

2. This extra release of latent heat in the upper-troposphere by ice processes occurs in the region where the conversion of latent heat to kinetic energy is most efficient (see Chapter 6 and Fig. 6.18). This is another reason latent heat of fusion and sublimation may be important to intensity change.

3. CAPE in the tropics is roughly the same for a reversible process with ice and a pseudoadiabatic process without ice. CAPE in the tropics is only slightly greater than both of these cases for a pseudoadiabatic process with ice.

Item 3 is interesting because it contradicts the notion of conditional neutrality in the tropics and TC eyewalls (Betts 1982; Xu and Emanuel 1989; Emanuel 1989a). Conditional neutrality is suspect because this concept was obtained using reversible thermodynamic calculations without ice. The RA.ni case in Fig. 7.5 does show reduced buoyancy, but is not realistic because if liquid condensate is retained in deep convection it must eventually freeze somewhere above the $0^{\circ} \mathrm{C}$ isotherm. Therefore, conditional neutrality is invalid in areas of deep convection in the tropics, although it may be reasonable in shallow cumulii or when the equilibrium level occurs below the freezing level. Table 7.1 shows CAPE values computed from Eq. (3.22) for RA.ni, PA.ni, RA.wi, and PA.wi, assuming surface temperatures $1^{\circ} \mathrm{C}$ cooler than SSTs of $27,28,29$, and $30^{\circ} \mathrm{C}$. CAPE values for RA.ni are

Table 7.1: CAPE values $\left(\mathrm{J} \mathrm{kg}^{-1}\right.$ ) assuming a surface temperature $1^{\circ} \mathrm{C}$ cooler than SST and a $\mathrm{RH}$ of $90 \%$ for the four investigated moist adiabatic processes. RA.ni represents the reversible process with no ice, and PA.ni represents the pseudoadiabatic process with no ice. The processes labeled "wi" contain ice. Undilute ascent is assumed.

\begin{tabular}{|l|rrrr|}
\hline SST & RA.ni & PA.ni & RA.wi & PA.wi \\
\hline \hline 1) 27 & 622 & 1494 & 1549 & 1642 \\
\hline 2) 28 & 1593 & 2560 & 2612 & 2723 \\
\hline 3) 29 & 2615 & 3681 & 3580 & 3890 \\
\hline 4) 30 & 3652 & 4823 & 4729 & 4979 \\
\hline
\end{tabular}


much less than the other three cases, as expected. However, PA.ni and RA.wi contain virtually the same CAPE values for their respective SST category. Similar conclusions were obtained by Williams and Renno (1993). A little more CAPE exists in PA.wi compared to PA.ni and RA.wi, but the difference is not large. In reality, entrainment will decrease these CAPE values, but does not alter the conclusion that, even with dilution, PA.ni, RA.wi, and PA.wi roughly contain the same amount of vertically integrated buoyancy. This property allows one to approximate $T(p)$ and $T_{\rho}(p)$ for deep, undilute convection, and will be described in the next section.

\subsubsection{Computational procedure for buoyancy}

As mentioned in Chapter 3, radar echoes in deep tropical convection indicate that the density of suspended droplets must be counteracting buoyancy to some degree. Therefore, pseudoadiabatic thermodynamics is too extreme an approximation. However, observations of heavy rainfall in tropical convection and TCs prove that reversible thermodynamics is too severe a restriction on the buoyancy computations. Since CAPE values are fairly well-bounded when ice is included, it seems reasonable to take the average of the $T(p)$ and $T_{\rho}(p)$ values from ascending parcels following the characteristic curves of RA.wi and PA.wi. This procedure is used for the buoyancy calculations in the next section, and these average values will be denoted as $\breve{T}(p)$ and $\breve{T}_{\rho}(p)$.

\subsection{Methodology for modified ASI process}

\subsubsection{Representation of eyewall warming}

Before discussion can proceed on the ASI process in a TC, the evolution of the eyewall warming must be discussed. Eyewall temperature observations are sparse of the entire life cycle of a TC, so the same composite philosophy discussed in Chapter 5 is used, in which rawinsonde composites for a variety of tropical cyclone intensities in the western North Pacific are done. Figure 7.6 shows the temperature deviations, relative to the mean western North Pacific tropical cluster sounding (Gray and Frank 1977; Frank 1977b), of progressively more intense TCs within 1-deg radius of the storm center. The most striking feature is the disproportionate upper-level inner-core warming relative to the mid 


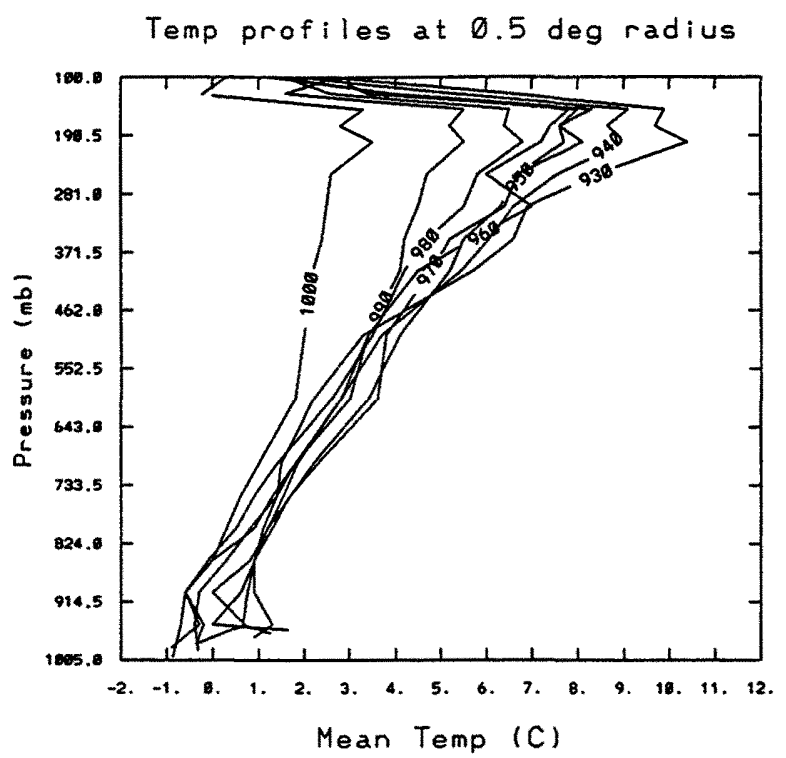

Figure 7.6: Vertical profile of mean temperature deviation within 1-deg radius of the storm center, relative to the mean western North Pacific tropical cluster sounding (Frank 1977b; Gray and Frank 1977), for various tropical cyclone intensities in the western North Pacific Ocean. The intensity stratifications are for 996 to $1005 \mathrm{mb}$ (denoted as 1000), 986 to $995 \mathrm{mb}$ (denoted as 990), etc.

and lower troposphere. For MSLPs ranging from $1000 \mathrm{mb}$ to $930 \mathrm{mb}$, the warming is $0-2^{\circ} \mathrm{C}$ between the surface and $700 \mathrm{mb}, 1-4^{\circ} \mathrm{C}$ between 700 and $500 \mathrm{mb}, 1-6^{\circ} \mathrm{C}$ between 500 and $300 \mathrm{mb}$, and $2-10^{\circ} \mathrm{C}$ between 300 and $150 \mathrm{mb}$. At $100 \mathrm{mb}$, the temperature range with intensity is $0-3^{\circ} \mathrm{C}$.

This upper-level warming can be attributed to two factors. First, as discussed in Chapter 6, the upper-eyewall is the most efficient region for converting latent heat to the kinetic energy of the TC (which dictates an increasingly warm upper-level through thermal wind balance). Second, above the sloping eyewall is the lateral extension of the eye. The contribution from the eye becomes apparent for the composited cases of MSLP $980 \mathrm{mb}$ or lower, consistent with observations that an eye forms when the MSLP falls $30 \mathrm{mb}$ or more relative to the environmental pressure, near hurricane strength (Dvorak 1984; Holland 1995). As will be seen later, eye formation is a crucial component in the ASI process. Furthermore, the fact that the temperature deviation does not vary much with intensity in the mid and lower levels - and the fact that the eyewall slopes away from the 
region of maximum warming - implicitly shows eyewall buoyancy to not decrease with TC intensification.

Given these observations, the maturation of the eyewall temperature perturbation may be constructed from the minimal tropical storm stage to its MPI stage. Four hypothetical curves are shown in Fig. 7.7 with eyewall surface pressures (as computed from Eq. (3.2) and Eq. (3.3)) of 994.3, 979.1, 965.9, and $949.7 \mathrm{mb}$.

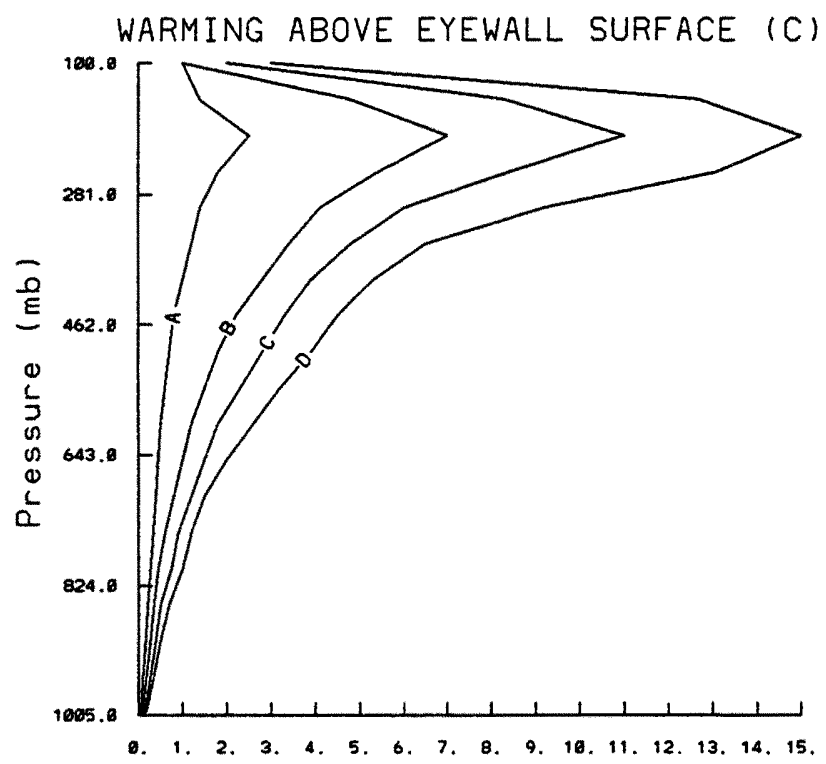

Figure 7.7: Hypothetical development of the eyewall temperature perturbation relative to the western North Pacific mean tropical sounding (Frank 1977b; Gray and Frank 1977). The corresponding surface pressures under the eyewall are 994.3 (A), 979.1 (B), 965.9 (C), and $949.7 \mathrm{mb}(\mathrm{D})$. These temperature profiles represent the TC from its minimal tropical storm stage to its MPI stage.

\subsubsection{Evidence of boundary layer cooling}

Another interesting feature is that the surface temperatures for the intense composite TCs (MSLPs of 940,930 , and $920 \mathrm{mb}$ ) are typically $26^{\circ} \mathrm{C}$ (not shown). Such intense TCs must be located over warm SSTs, and the average locations of these composite storms is 19-20 deg $\mathrm{N}$ where the water temperature is $29^{\circ} \mathrm{C}$ or more most of the TC season (Reynolds 1988). This implies a surface cooling of $3-4^{\circ} \mathrm{C}$, consistent with earlier discussions in this chapter. The idealized eyewall warming is incorporated into the ASI calculations with surface cooling, and this methodology is described next. 


\subsubsection{Computational procedure for modified ASI process}

A modified ASI process is hypothesized which includes an external feature that compensates boundary layer adiabatic expansional cooling. This adjustment factor is the lateral extension of the eye above a sloping eyewall. In this manner, buoyancy is maintained by increasing the relative humidity in conjunction with large pressure falls, and moist entropy increases despite the subcloud cooling. The following methodology is employed.

An SST of $29^{\circ} \mathrm{C}$ with a surface temperature $1^{\circ} \mathrm{C}$ cooler is initially assumed with a relative humidity of $82 \%$ typical of depressions (Frank 1977b). The LCL temperature and pressure level is determined using Eq. (3.6) and Eq. (3.7). Above the LCL, the average temperature and potential buoyancy values relative to a mean tropical atmosphere are computed as an average of the characteristic entropy lines for RA.wi and PA.wi $(\breve{T}(p)$ and $\breve{T}_{\rho}(p)$; see Section 7.3.2).

A temperature anomaly is then assumed to develop as in Fig. 7.7, and an ASI computation is performed for each warm core profile. For each temperature anomaly, the corresponding hydrostatic pressure is computed from Eq. (3.2) and Eq. (3.3). For this surface pressure, the surface temperature cooling as shown in Fig. 7.4 is calculated from Eq. (7.7). Observations of a decreasing cloud base with TC intensity (Riehl 1954) and composite data (Frank 1977b) - as well as a decreasing surface temperature - dictate that the surface relative humidity increases with $\mathrm{TC}$ intensity. In this demonstration, $\mathrm{RH}$ progressively increases in $3.3 \%$ increments from $82 \%$ in the depression stage to $95 \%$ at the

hypothetical MPI stage. Above the LCL, $\breve{T}(p)$ and $\breve{T}_{\rho}(p)$ are computed relative to the eyewall environment of the developing TC. Undilute ascent is assumed, but the effect of entrainment should not qualitatively alter the results of this calculation. Entrainment is considered in a later section.

\subsection{Results of the modified ASI process}

Figure 7.8 shows the results of the modified ASI approach. The surface temperature decreases from 28.0 to $25.4^{\circ} \mathrm{C}$ for a tropical depression of $1005 \mathrm{mb}$ to a mature TC with an eyewall surface pressure of $952 \mathrm{mb}$. The MSLP in the latter is unknown, but is quite 
possible $900 \mathrm{mb}$ or lower, near its MPI for an SST of $29.0^{\circ} \mathrm{C}$. Fig. 7.8 demonstrates that the ASI process can sustain buoyancy even in the presence of sub-cloud cooling below the eyewall. A slight decrease in the slope of $\breve{T}$ above $400 \mathrm{mb}$ indicates that ice processes assist the ASI process. The contributor to the needed increase of $\theta_{e}$ is the development of upperlevel warming in the inner edge of the eyewall where the geostrophic adjustment process is most efficient, and the subsidence warming above the sloping eyewall due to the lateral extension of the eye. Without the contribution of the eye to maintain the ASI process, the TC will collapse in the presence of boundary layer cooling since no buoyancy will exist. This also emphasizes the importance of placing the warming as high as possible due to the surface pressure's nonlinear dependence on the height of the temperature anomaly (Eq. (3.2)).

As alluded to throughout this chapter, buoyancy does not significantly decrease in the evolution of the TC, and actually increases in its mature stages. This is because, as shown in Figs. 7.7 and 7.8, the lower-level of the eyewall does not experience a significant warming as the TC develops. This is because thermal wind theory dictates the warming to occur where $v_{t}$ decreases the most with height (or relative to $\ln p$ ). This lack of low-level warming might also be due to cooling by downdrafts and evaporative processes.

This buoyancy increase is shown in Fig 7.9, where $T_{v}(p)$ of the eyewall environment is subtracted from $\breve{T}_{\rho}(p)$ for the five stages shown in Fig. 7.8. The computations are only shown to $300 \mathrm{mb}$, since generally above this level the eyewall slopes outwards and is not representative of the buoyancy in the eyewall in the upper-troposphere.

The TC in the depression stage contains the most buoyancy since little temperature anomaly exists to counter the parcel's lighter density. Thereafter, buoyancy slowly decreases in the TC's early stages as the surface temperature decreases without an adequate pressure drop to counter the eyewall warming, especially in the middle troposphere. However, as the upper-level warming exponentially increases aloft, and as the surface relative humidity increases, the ASI process is able to replenish the buoyancy in the lower levels up 


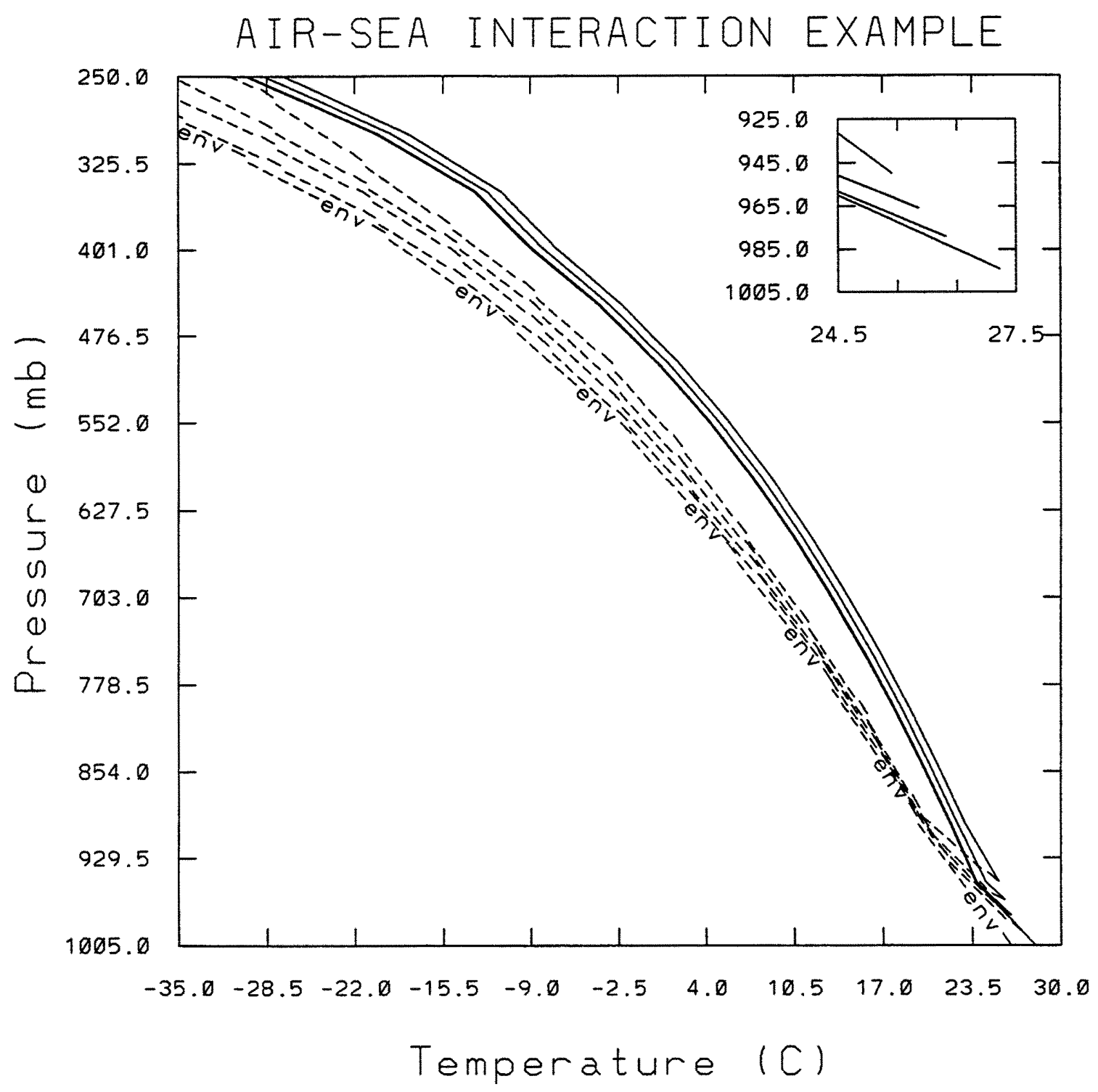

Figure 7.8: Example of the Air-Sea Interaction (ASI) process for a surface temperature initially $1^{\circ} \mathrm{C}$ cooler than an SST of $29^{\circ} \mathrm{C}$, but decreases $2-4^{\circ} \mathrm{C}$ as the surface pressure decreases (see inset in the top right corner). The dashed line labeled "env" represents the mean tropical environment outside the TC, and the other four dashed lines symbolize the evolution of the eyewall warming as shown in Fig. 7.7. The solid lines represent the temperature of each undilute parcel ascent for a given surface temperature, surface pressure, and surface relative humidity. The parcel calculations include water-loading and ice microphysics (see text for details). The first parcel ascent is relative to the mean tropical cluster sounding ("env") representing a tropical depression with $T_{s f c}=28^{\circ} \mathrm{C}$, $p_{s f c}=1005 \mathrm{mb}$, and $\mathrm{RH}=82 \%$. The next four calculations represent an evolving $\mathrm{TC}$ to a hypothetical MPI stage. $T_{s f c}$ decreases, respectively, as $27.2,26.3,25.9$, and $25.4^{\circ} \mathrm{C}$. Eyewall $p_{s f c}$ decreases, respectively, as $994.3,979.1,965.9$, and $949.7 \mathrm{mb}$. RH increases, respectively, as $85.3,88.5,91.8$, and $95.0 \%$. Including the depression stage, $\theta_{e}$ changes with intensity as $367.9,367.6,368.0,370.3$, and $373.3^{\circ} \mathrm{K}$. This demonstrates that eyewall buoyancy can be sustained with the positive feedback of surface pressure fall (due to the lateral extension of the eye above a sloping eyewall), latent heat of fusion and deposition, and increasing relative humidity despite surface cooling. 


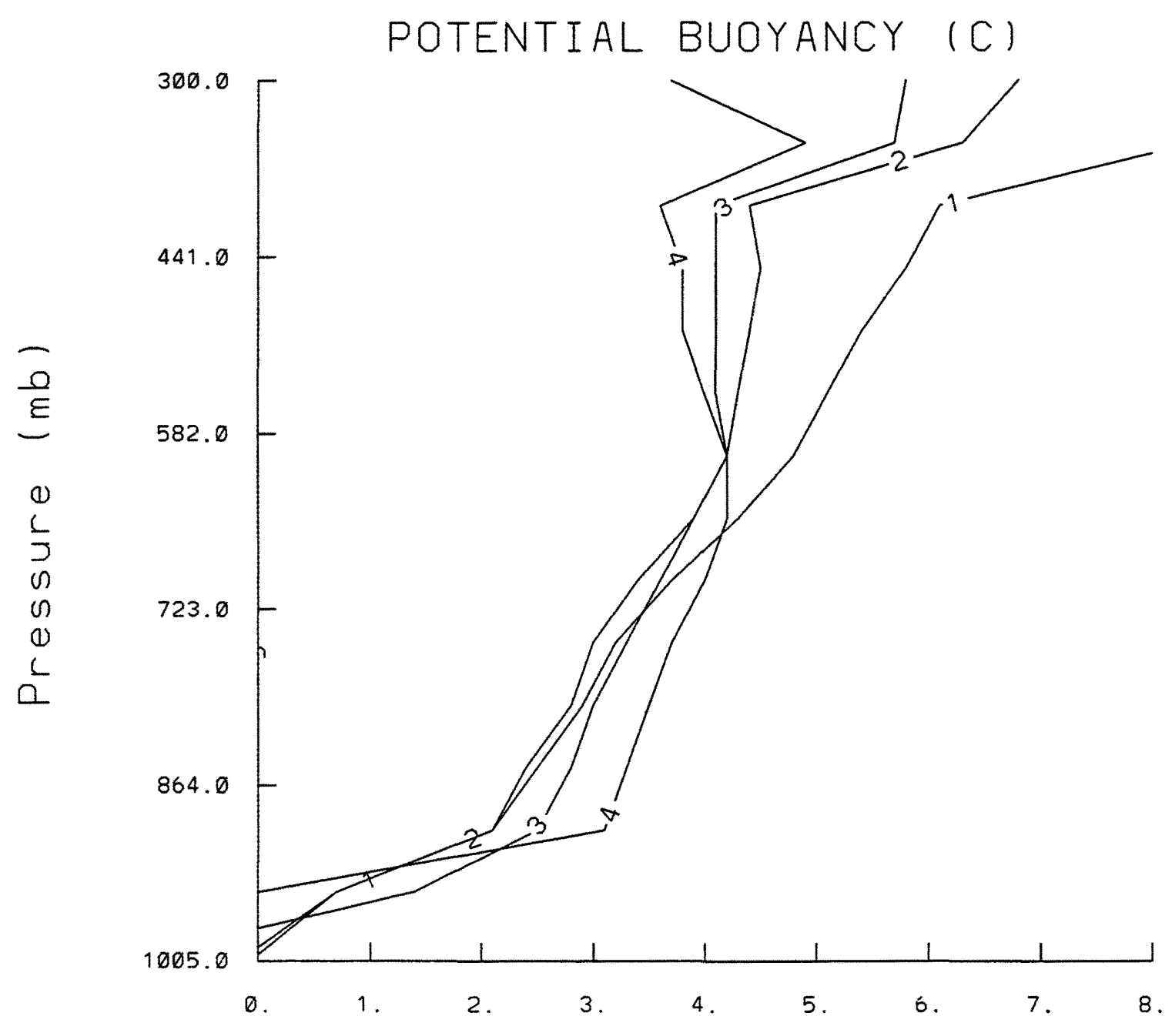

Figure 7.9: Potential buoyancy (defined as $\breve{T}_{\rho}(p)$ minus $T_{v}(p)$ of the eyewall environment) for the four stages shown in Fig. 7.8. Stages 1-4 represent buoyancy calculations from the weak to strongest TC intensity regimes. CAPE values upon integration from the surface to $300 \mathrm{mb}$ are $864,753,764$, and $782 \mathrm{~J} \mathrm{~kg}^{-1}$. Computations are shown only to $300 \mathrm{mb}$, since above this level the eyewall slopes outwards away from the temperature anomaly, and calculations based on vertical ascent of air parcels would not be representative of buoyancy in the eyewall. These profiles are labeled "potential buoyancy" because undilute ascent is assumed. 
to $650 \mathrm{mb}{ }^{6}$ Upper-level buoyancy decreases due to increased upper-level warming during TC development. However, above $400 \mathrm{mb}$ latent heat of fusion and sublimation partially offsets the eyewall temperature perturbation. Therefore, the vertical integral of buoyancy throughout the troposphere actually increases in the later stages. CAPE values initially decrease from 864 to $753 \mathrm{~J} \mathrm{~kg}^{-1}$ as the eyewall surface pressure falls from $994.3 \mathrm{mb}$ to $979.1 \mathrm{mb}$, then slowly increases to $764 \mathrm{~J} \mathrm{~kg}^{-1}$ when the eyewall surface pressure falls to $965.9 \mathrm{mb}$, and in the final stage (eyewall surface pressure of $949.7 \mathrm{mb}$ ) CAPE is $782 \mathrm{~J} \mathrm{~kg}^{-1}$.

The implication of this finding is that eyewall stabilization is not the restrictive agent that determines MPI, especially when Ekman forcing (which increases with TC intensity) is also considered. This is consistent with the satellite observations of Dvorak (1984), and results to be presented in Chapter 8, that cloud tops are coldest at a TC's maximum intensity. Therefore, another factor must bound the ultimate intensity of a TC. This topic will be discussed in the next section.

\subsection{A new Maximum Potential Intensity (MPI) theory}

Recall from Chapter 3 the Internally Forced Convergence (IFC) theory which hypothesizes that sufficient eyewall vertical motion is required to balance friction and the outflow of cyclonic tangential momentum aloft to maintain a TC's intensity (Gray 1995). In other words, a TC can continue to intensify only when sufficient eyewall ascent (as determined by boundary layer forcing and buoyancy) exists such that friction and upperlevel $v_{t}$ export are balanced with enough vertical momentum remaining to intensify the TC. However, as friction increases proportionally with $V_{\max }^{2}$, and as upper-level export of cyclonic momentum also amplifies, the required eyewall vertical motion $\left(w_{\text {req }}\right)$ needed to meet IFC budgetary requirements also increases. It is further hypothesized that, although buoyancy and Ekman pumping increase with intensity, an equilibrium state eventually results when the resulting vertical motion can no longer increase fast enough to balance the

\footnotetext{
${ }^{6}$ It should be noted that the buoyancy calculations are sensitive to relative humidity. This author believes that RH should increase under the eyewall as the TC develops for reasons stated earlier in the chapter. However, if RH is held constant, CAPE does not increase but remains constant. Nevertheless, the conclusion that CAPE does not decrease holds.
} 
IFC budget intensification requirements for even faster eyewall ascent. This equilibrium balance delineates the MPI of a TC. A more detailed discussion follows.

It is postulated that $w_{r e q}$ increases approximately linearly with intensity. However, the discussion from Chapter 3 showed that the maximum eyewall vertical velocity $w_{\max }$ is equal to $\left(2 \mathrm{CAPE}+w_{o}^{2}\right)^{1 / 2}$ (Eq. (3.23)), where $w_{o}$ is the vertical velocity forced by Ekman pumping. In other words, $w_{\max }$ is roughly proportional to the square root of CAPE, with an extra boost from increasing $w_{o}$. It is this disparity between $\partial w_{\text {req }} / \partial t$ and $\partial w_{\max } / \partial t$ as a TC intensifies which ultimately determines the MPI of a TC.

Figure 7.10 schematically shows this idea. In an incipient $\mathrm{TC}, w_{\max }$ is greater in magnitude than $w_{\text {req. }}$. Since there is a residual vertical velocity after budgetary IFC requirements are met, the TC can intensify. However, as the TC develops, $\partial w_{\text {req }} / \partial t$ is more in magnitude than $\partial w_{\max } / \partial t$. Although $w_{0}$ also increases with intensity and partially compensates for this disparity, boundary forcing likely only partially nullifies $w_{\max }$ 's square root dependence on CAPE. In other words, CAPE and $w_{0}$ cannot increase as rapidly as the requirement for faster eyewall vertical velocity. Eventually an equilibrium state is achieved where $w_{\max }$ equals $w_{\text {req }}$, denoted by the dot in Fig. 7.10 , and the MPI has been achieved since intensification can no longer occur.

In reality, the effect of entrainment also needs to be considered in these discussions. Undilute ascent will give a $w_{\max }$ of about 30 to $40 \mathrm{~m} \mathrm{~s}^{-1}$ without boundary layer forcing (Eq. (3.23)). However, the magnitude of eyewall vertical motion is typically 5 to $15 \mathrm{~m} \mathrm{~s}^{-1}$ (Black 1993), with some values as high as 20 to $25 \mathrm{~m} \mathrm{~s}^{-1}$ (Ebert and Holland 1992; Black et al. 1994). Therefore, the effect of dilution by dry air needs to be qualitatively considered, as shown in Fig. 7.11. Entrainment will change where this equilibrium state occurs, but should not alter the essence of this MPI theory.

This hypothesis can also explain MPI's sensitivity to SST (Fig. 7.12), since the ASI process is sensitive to the surface temperature. With an SST of $29^{\circ} \mathrm{C}$ and an eyewall surface temperature of $25^{\circ} \mathrm{C}$ ( $4^{\circ} \mathrm{C}$ sea-air difference), a sloping eyewall, a relative humidity of $95 \%$, and ice microphysics, substantial buoyancy exists to maintain an MSLP of approximately $880 \mathrm{mb}$, but no lower. Likewise, for an SST of $28^{\circ} \mathrm{C}\left(24^{\circ} \mathrm{C}\right.$ surface temperature), a less 


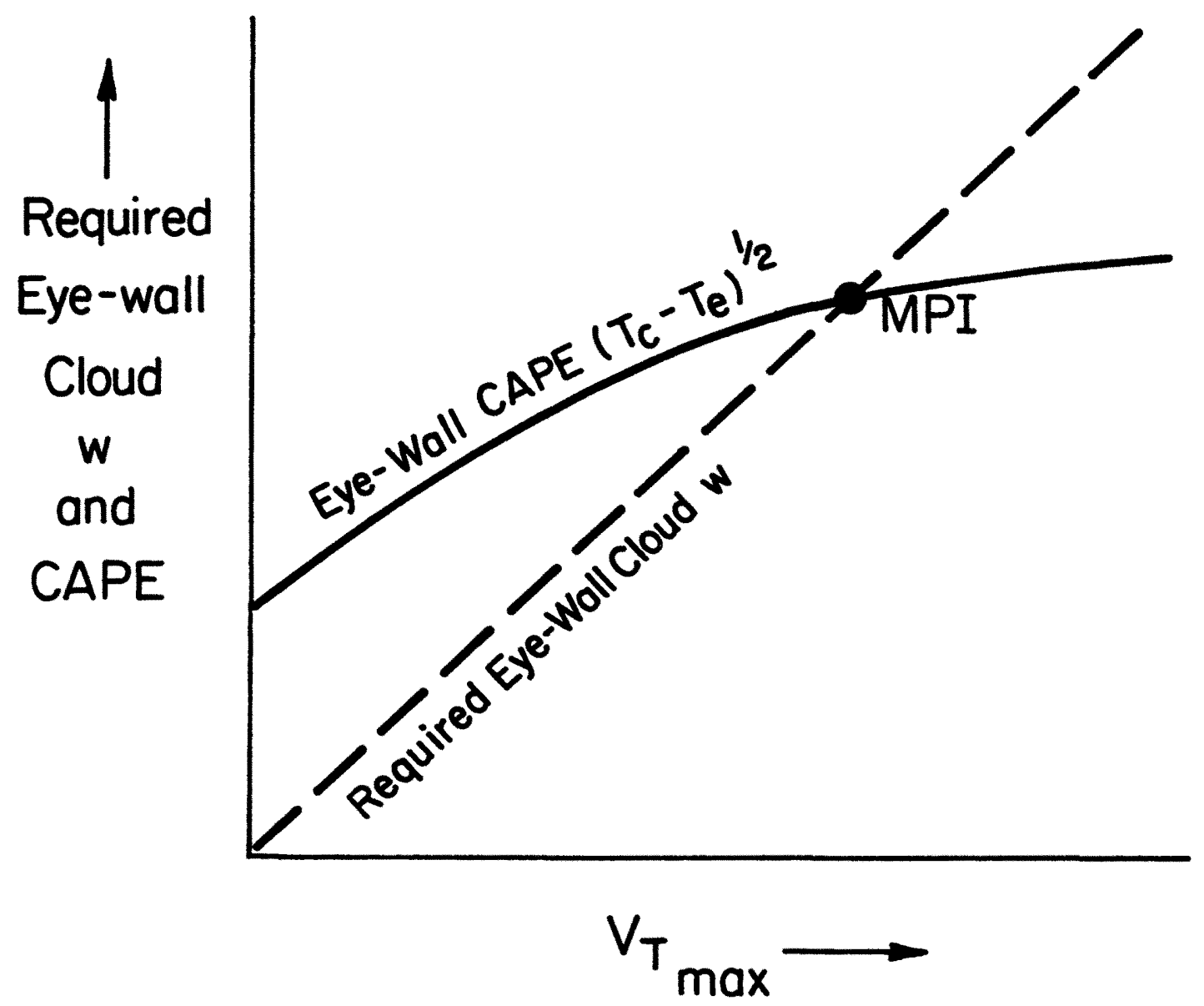

Figure 7.10: Illustration of how the Maximum Potential Intensity (MPI) occurs. The dotted line represents the required vertical velocity $\left(w_{\text {req }}\right)$ to balance friction and the export of cyclonic $v_{t}$ in the upper eyewall and maintain a TC's intensity (the Internally Forced Convergence (IFC) theory described in Chapter 3). The solid line denotes the maximum eyewall vertical velocity $\left(w_{\max }\right)$ due to CAPE. TC intensification proceeds as long as $w_{\max }>w_{\text {req }}$. It is postulated that $w_{\text {req }}$ increases approximately linearly with intensity $\left(V_{\max }\right)$ since friction is proportional to $V_{\max }^{2}$ and since the export of cyclonic $v_{t}$ aloft increases. However, $w_{\max }$ is approximately proportional to the square root of CAPE. As a TC develops, $w_{\text {req }}$ increases faster than $w_{\max }$. In other words, CAPE cannot increase as rapidly as $w_{\max }$. Eventually an equilibrium state is achieved where $w_{\max }=w_{\text {req }}$, denoted by the dot. This is the MPI. CAPE is insufficient to sustain the amount of required vertical motion beyond the dot. Boundary layer forcing only partially offsets $w_{\text {max }}$ 's square root dependence on CAPE. 


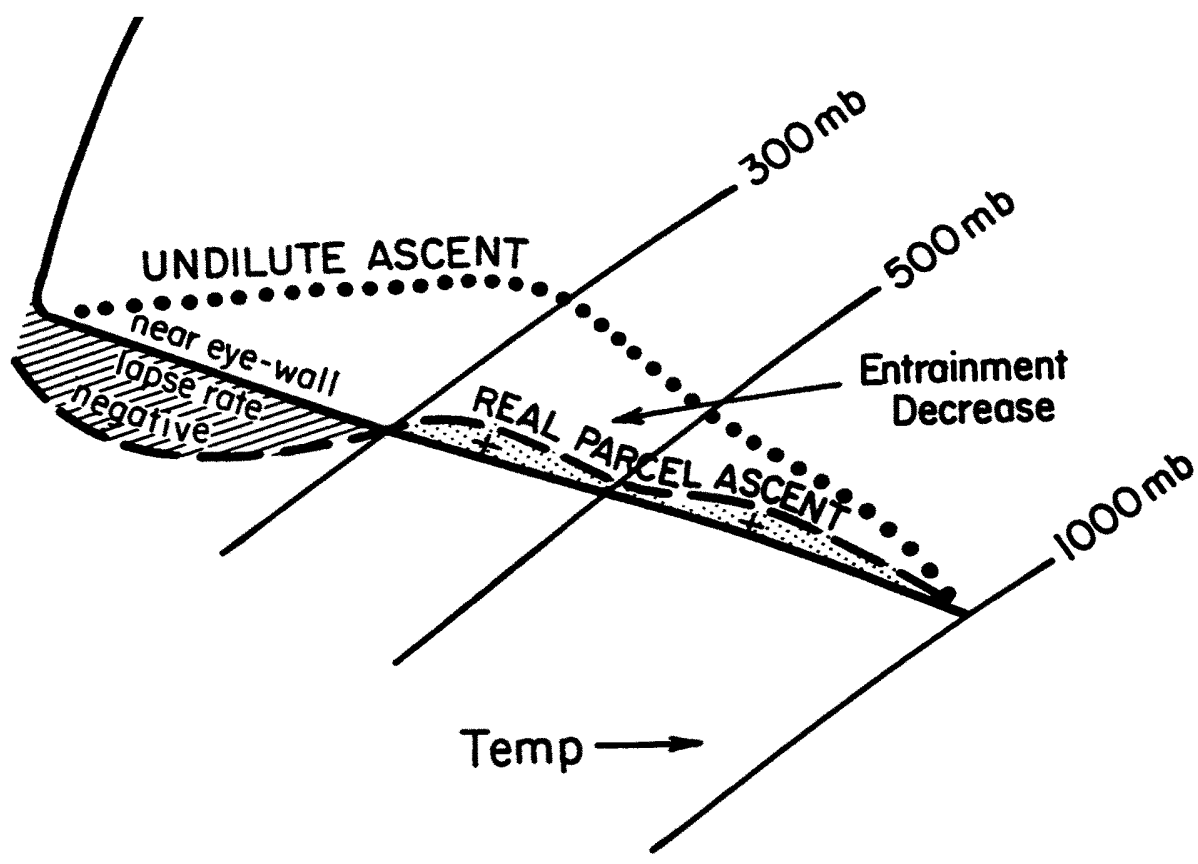

Figure 7.11: Mlustration of the effect of entrainment on parcel ascent. The parcel receives a secondary boost due to latent heat of fusion and sublimation between 500 and $300 \mathrm{mb}$. Undilute ascent is shown as a comparison.

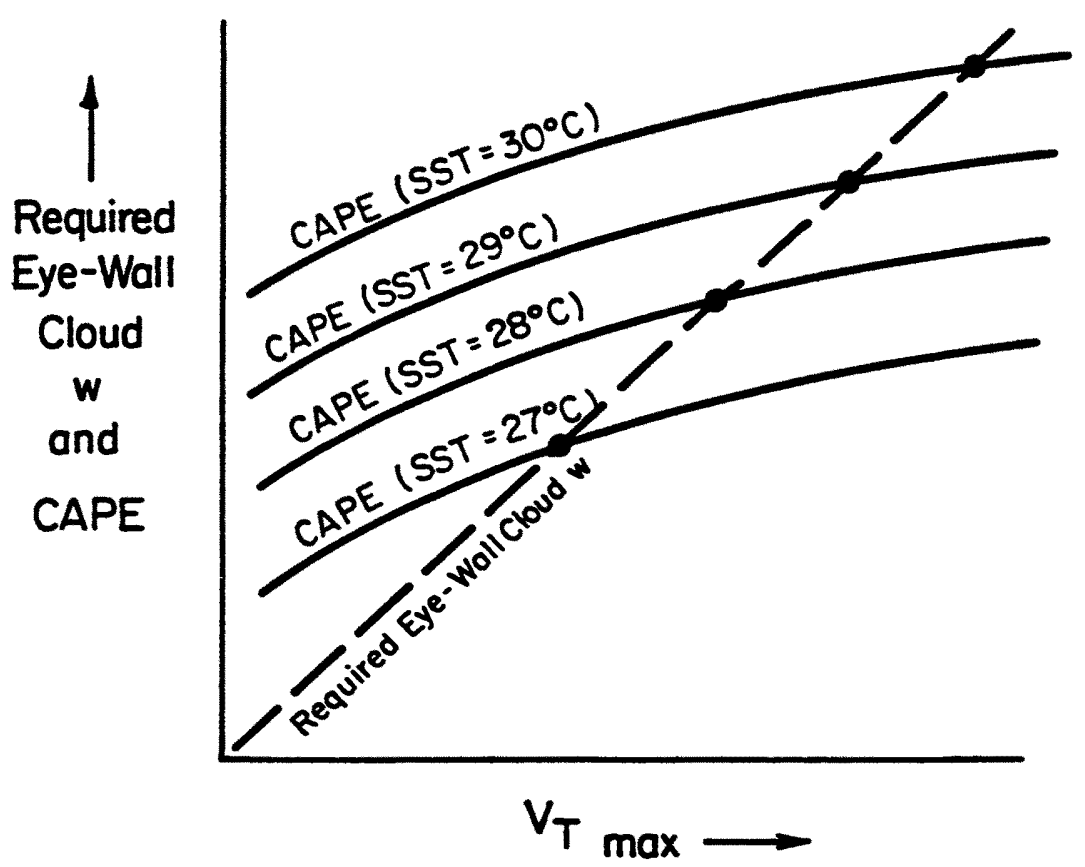

Figure 7.12: Similar to Fig. 7.10, but showing how the CAPE variations with warmer values of SST are associated with larger values of MPI. 
intense TC with an MPI of approximately $920 \mathrm{mb}$ can be obtained. MPI is more restricted for a cooler SST because less CAPE is available at a given intensity, and the increase with intensity of this smaller amount of CAPE to the square root power imposes a faster convergence of $w_{\max }$ and $w_{\text {req. }}$. With an SST of $27^{\circ} \mathrm{C}\left(23^{\circ} \mathrm{C}\right.$ surface temperature $)$, only a moderate TC can be achieved.

\subsection{Summary}

Increasing evidence shows that the air-sea temperature difference increases to $3-4^{\circ} \mathrm{C}$ under the eyewall as a TC intensifies, in contrast with many TC studies which assume a $1^{\circ}$ difference. The possible processes contributing to this cooling were discussed. The most plausible (although still tentative) conclusion was that the adiabatic expansion of air as it flows toward lower surface pressure is not totally compensated by sensible heat fluxes, hence the air parcel experiences a temperature drop. A crude derivation of this surface cooling was presented, in which a positive feedback between surface winds and surface fluxes mostly compensates the adiabatic expansion, but a $3-4^{\circ} \mathrm{C}$ air-sea temperature difference develops with the pressure fall. This boundary layer cooling demands a re-examination of how the Air-Sea Interaction (ASI) process operates, since it is normally difficult to maintain cloud buoyancy relative to the mean tropical atmosphere with cool surface temperature values.

The importance of including water-loading and ice microphysics in buoyancy calculations was discussed. The mathematical treatment of these processes was described, and buoyancy calculations for pseudoadiabatic and reversible adiabatic ascent (with and without ice microphysics) were presented. It was shown that, although the reversible adiabatic without ice is less buoyant (due to water-loading) than the pseudoadiabat without ice, the reversible process with ice is more buoyant than the pseudoadiabatic process without ice above the freezing level. Several conclusions about buoyancy were thus made.

First, by including the effects of water-loading and ice, the vertical distribution of buoyancy is altered. There is less buoyancy in the lower-troposphere with retained liquid water, but these droplets contain potential buoyancy which is released above the freezing 
level. This sudden release of buoyancy above the freezing level can play an important role in TC intensity change, since this release of latent heat will increase the secondary circulation and because this extra source of diabatic heating occurs in a region where geostrophic adjustment processes are more efficient. Second, CAPE in the tropics is roughly the same for a reversible process with ice and a pseudoadiabatic process without ice. This result is interesting because it contradicts the notion of conditional neutrality (which is based on reversible thermodynamics with no ice) in the tropics and TC eyewalls. It was concluded that the conditional neutrality hypothesis for deep convection is invalid in the tropics because if liquid condensate is retained it must eventually freeze somewhere above the $0^{\circ} \mathrm{C}$ isotherm.

Based on these results, a simple methodology for buoyancy calculations was proposed, incorporating the fact that a reversible and pseudoadiabatic process represent extreme adiabatic ascent situations (all condensate retained in a parcel versus all condensate falling from the parcel the instant it appears). Therefore, a reversible and pseudoadiabatic process delineate upper and lower bounds on $T(p)$ and $T_{\rho}(p)$. Furthermore, it was found that CAPE in a pseudoadiabatic process with ice was only slightly greater than a reversible process with ice, because the pseudoadiabatic process contains no supercooled water droplets and therefore the ice microphysics contains only latent heat of deposition. Since CAPE is quasi-conserved including ice microphysics despite the chosen parcel path (reversible or irreversible), it was proposed that buoyancy calculations average the $T$ and $T_{\rho}$ values resulting from these two processes.

Documentation based on composites of TCs in various stages of intensity presents a picture of the eyewall temperature anomaly evolution. It was shown that most of the warming occurs in the upper eyewall, while the middle and (especially) the lower levels experience little temperature increase. Furthermore, this upper-level warming increases disproportionally relative to the low-level warming. This upper-level warming can be attributed to two factors. First, the upper-eyewall is the most efficient region for converting latent heat to the kinetic energy of the TC (which dictates an increasingly warm upperlevel through thermal wind balance). Second, above the sloping eyewall is the lateral extension of the eye, which becomes stronger with intensity. 
Based on all these results, a modified Air-Sea Interaction (ASI) theory was presented. This theory incorporated boundary layer cooling with eyewall pressure fall, and buoyancy calculations which includes partial water-loading and ice microphysics. The eyewall temperature evolves similar to the composite study, and an associated eyewall surface pressure was computed. The surface relative humidity was postulated to increase with intensity, thus also providing a buoyancy source. It was shown that the ASI process can be sustained with the positive feedback of surface pressure fall (due to the lateral extension of the eye above a sloping eyewall), latent heat of fusion and deposition, and increasing relative humidity despite the surface cooling.

Finally, it was shown that eyewall buoyancy remains the same, and quite likely increases, as a TC develops. Buoyancy is sustained even with boundary layer cooling because most of the eyewall warming is confined to the upper troposphere, which enhances a parcel's density difference with the low-level eyewall environment, and maximizes the increase in moist entropy. Furthermore, the eyewall slopes outward aloft above $300 \mathrm{mb}$. In this manner, updrafts travel upwards through regions of lesser warming than would occur if an air parcel traveled vertically through the region of maximum warming. This maintenance of buoyancy suggests that the limiting factor to intensification is not eyewall stabilization.

The Internally Forced Convergence (IFC) hypothesis states that a TC can continue to develop only when sufficient eyewall ascent (as determined by boundary layer forcing and buoyancy) exists such that friction and upper-level $v_{t}$ export are balanced, with enough vertical momentum remaining to intensify the TC. However, since friction increases proportionally with $V_{m a x}^{2}$, and as upper-level export of cyclonic momentum also amplifies, the required eyewall vertical motion needed to meet IFC budgetary requirements also increases. It was hypothesized that, although buoyancy and Ekman pumping increase with intensity, an equilibrium state eventually results where the vertical motion can no longer increase fast enough to balance the IFC budgetary requirements. This equilibrium delineates the MPI of a TC.

The results of this chapter suggest that some numerical simulations of TC development need re-examination. A few models set the surface temperature to a constant value 
of $1^{\circ} \mathrm{C}$ cooler than the SST (Yamasaki 1977) or extrapolate the surface temperature from the first grid point above the surface (Anthes 1972). Most other models use a prognostic equation for surface temperature with a contribution from surface fluxes (Ooyama 1969; Sundqvist 1970; Baik et al. 1990a; Bender et al. 1993). Some simulations have also included ocean-TC interaction with a prognostic ocean mixed layer (Bender et al. 1993). However, to this author's knowledge, no documentation of the modelled surface temperature evolution below the eyewall has been presented in any of these papers. Since TC intensification is critically dependent on boundary layer values, not knowing what these parameters were in the simulations casts considerable uncertainty in the validity of the model runs. 


\section{Chapter 8}

\section{THE TYPHOON INTENSITY PREDICTION SCHEME (TIPS)}

\subsection{Introduction}

This chapter describes a recently developed multiple regression scheme with TC intensity change as the dependent variable. This research was motivated by a multiple regression analysis of Atlantic hurricane intensity change performed by DeMaria and Kaplan (1994b). This forecast scheme, called the Statistical Hurricane Intensity Prediction Scheme (SHIPS), is currently used operationally at NHC.

It was decided that a similar scheme should be developed for the the western North Pacific basin. The new scheme is titled the Typhoon Intensity Prediction Scheme (TIPS), and unlike SHIPS, includes digitized infrared satellite data as predictors - the first time such information has been included explicitly in any multiple regression analysis. Other investigated predictors are based on climatology, SST, persistence, and synoptic winds. Regression is performed on $12,24,36$, and 48 -h forecasts. ${ }^{1}$ It is shown that satellite information contains vital information which distinguishes between TCs that intensify fast and those that intensify at a slower rate.

\subsection{Possible predictors}

The individual potential predictors investigated by the statistical scheme are described in this section. For a description of the data set itself, the reader is referred to Chapter 5 . Since tropical cyclogenesis and TC intensification are separate problems (Zehr 1992), only

\footnotetext{
${ }^{1}$ Unlike SHIPS, no regression is done for 72 - $h$ forecasts since evaluating more than four $12-\mathrm{h}$ forecast intervals made the statistical analysis cumbersome, and because it wasn't clear that 72-h regressions with potentially large errors on a smaller sample size (since more storms dissipate or move over land in this forecast interval) was worth the effort.
} 
observations in which a TC has achieved a minimum of tropical storm strength ( $V_{\max } \geq$ $35 \mathrm{kts}$ ) are included. ${ }^{2}$ This is different from SHIPS, which includes the depression stage of named TC cases.

As will be seen, most of these potential predictors are statistically insignificant. However, determining which predictors are unimportant with regards to TC intensity change is just as enlightening as deducing the significant predictors. Furthermore, some of these possible variables, which some researchers and forecasters believe to be important intensity change prognosticators, are shown to actually be statistically unimportant.

\subsubsection{Climatology}

The climatology parameters are computed in terms of storm location, storm speed, storm direction, and time of year. Many of these parameters are used in climatology and persistence regression models for intensity change forecasts (Elsberry 1974, 1975; Jarvinen and Neumann 1979; Chu 1994; Landsea 1995) and track forecasts (Neumann 1972, 1991). Although parameters related to physical processes (such as warm SSTs, wind shear, and convection) generally contain stronger forecast signals, it will be shown that climatology explains a proportion of the variance which these physical processes cannot.

\footnotetext{
${ }^{2}$ According to Zehr (1992), tropical cyclogenesis consists of a two-stage process. In Stage 1, convection flares up in an area of synoptic-scale cyclonic vorticity, usually in a monsoon trough region and/or an easterly wave. This stage may last 6-24 h, upon which the convection ceases. This cessation of cloud activity may be related to downdrafts transporting low entropy air to the boundary layer (Emanuel 1991). During Stage 1, a mesoscale vortex is generated by the convection. After Stage 1, a period of inactivity occurs, sometimes lasting several days, in which a mesoscale vortex persists with little change in MSLP and often little convection in the center. At Stage 2, significant convection reappears in the center, with slightly curved outer-core cloud bands appearing. At the end of Stage 2, MSLP begins to fall, $v_{t}$ at all radii increases, and a tropical storm is born. It has been proposed by Emanuel (1991) that at this stage the entropy in the middle troposphere has increased enough such that downdrafts no longer overcome the enhanced surface fluxes, and the vortex intensifies. As pointed out in Chapter 3, tropical systems export moist static energy, and if surface fluxes cannot compensate this removal of energy, a tropical system will dissipate (Gray 1982). This writer has personally witnessed this two-stage process many times, and believes it to be representative of most genesis cases. It should also be noted that Stage 2 doesn't always occur after Stage 1 - often, convection fails to reappear for reasons which still need research, but some reasons are increased wind shear, colder SSTs, or the lack of a forcing mechanism to regenerate the convection (termed a "wind surge" by Zehr). Tropical cyclogenesis is a very different and more impulsive process than TC intensification after Stage 2, and therefore the tropical depression stage is excluded from the regression analysis.
} 


\section{Storm location}

From Air-Sea Interaction (ASI) theory, it is reasonable to assume that TCs located over land contain different statistical properties than those over water. When the storm center is located over land during the forecast period, or when land influences are clearly present during the forecast period, these storms are excluded from the data set. Hence, landfalling TCs in China and Vietnam, and TCs interacting with the Philippines, Taiwan, and Japan are excluded from the sample. SHIPS also only includes TCs over the ocean. ${ }^{3}$

Mundell (1990) found that rapid intensifiers occur south of $22 \mathrm{deg} N$ and between 125 and $158 \mathrm{deg}$ E. Hence, latitude (LAT) and longitude (LONG) are included as potential predictors.

\section{Storm motion}

Tropical cyclones have been observed to cool SSTs by as much as $4^{\circ} \mathrm{C}$ in their wakes due to upwelling (Black et al. 1989; Pudov 1992; Pudov and Holland 1994). As a result, several researchers hypothesize that slow-moving TCs weaken because of their proximity to the extracted sub-surface colder waters (Black 1983; Ginis et al. 1992; Shay et al. 1992). Therefore, the storm speed (SPD) is investigated.

Furthermore, fast-moving storms are often related with recurvature, and tend to weaken due to wind shear and colder SSTs. Therefore, since fast and stationary TCs might be associated with weakening, the storm speed anomaly is also investigated as:

$$
\operatorname{SPDAN}=|S P D-5.0|
$$

where SPDAN is the storm speed anomaly (in terms of absolute value) and $5.0 \mathrm{~m} \mathrm{~s}^{-1}$ is the average TC speed (Weatherford 1989).

Intensifying storms tend to contain a more westerly direction $\left(\mathrm{DIR}=310^{\circ}\right)$ of storm motion than filling storms $\left(\mathrm{DIR}=335^{\circ}\right)$ (Weatherford 1989). Furthermore, Weatherford (1989) also noted that rapid deepeners propagate more westerly than slow deepeners $\left(303^{\circ}\right.$

\footnotetext{
${ }^{3}$ Recently, Kaplan and DeMaria (1995) have developed an empirical model for predicting $V_{\max }$ of landfalling TCs.
} 
compared to $320^{\circ}$ ). Since a negative departure from $310^{\circ}$ might portend intensification and a more northerly or easterly direction weakening, the TC direction anomaly (DIRAN) is studied:

$$
\text { DIRAN }=\left\{\begin{array}{cc}
-50-\mathrm{DIR} & \text { for } 0^{\circ} \leq \mathrm{DIR}<130^{\circ} \\
310-\mathrm{DIR} & \text { for } 130^{\circ} \leq \mathrm{DIR}<360^{\circ}
\end{array}\right.
$$

where DIRAN is defined as departures of DIR from $310^{\circ}$, yielding a positive or negative value (between 0 and $\pm 180^{\circ}$ ) with respect to $310^{\circ}$. When DIR is between $130^{\circ}$ and $310^{\circ}$, DIRAN is positive. Otherwise, DIRAN is negative. Southeast propagating storms create spurious values of DIRAN, but fortunately these are rare.

Finally, the zonal component of storm motion (USPD) and meridional component (VSPD) are also investigated as potential predictors. In summary, storm motion variables studied are SPD, SPDAN, DIR, DIRAN, USPD, and VSPD.

\section{Time of year}

DeMaria and Kaplan (1994b) found the absolute value of the Julian date (JD) minus day 253 to be an important long-term predictor, since day 253 (10 September) is the peak of the Atlantic hurricane season in terms of the probability of a TC existing in this basin. Because, the typhoon season is distinctly different in terms of length and activity, and since one of the research goals is to improve RI forecasts, it was decided to investigate Julian date in terms of rapid intensifiers in the western North Pacific. Mundell (1990) found two peaks in the onset of rapid deepeners - day 190 to 230 and 250 to 345, with a lull between these dates. The reason for the inactivity in late August to early September is that the western North Pacific monsoon trough moves too far north (Lander 1995), and is in a region climatologically unfavorable for rapid intensification.

The Julian date anomalies from peak RI onset (JDAN) are computed as follows:

$$
\operatorname{JDAN}= \begin{cases}|\mathrm{JD}-220| & \text { for } \mathrm{JD}<230 \\ |\mathrm{JD}-280| & \text { for } 230 \leq \mathrm{JD}\end{cases}
$$

where the dates are chosen to impose some symmetry on the absolute values of the Julian day departures from peak RI onset. 


\subsubsection{Persistence with an eye parameterization}

Persistence always receives much use in an operational forecast setting, since in general the atmosphere does not contain large temporal disruptions. If a storm is strengthening (weakening) in the past few hours, there is a relatively decent chance it will continue strengthening (weakening). Persistence also tends to be one of the most important predictors in all regression models such as SHIFOR, SHIPS, and the statistical hurricane track forecasting packages CLIPER (CLImatology and PERsistence model; Neumann 1972) and NHC90. ${ }^{4}$ Common sense dictates, however, that persistence is not always a wise approach, especially in situations where the predictor is (or will be) experiencing rapid changes. In addition, the use of persistence aggravates the problem of autocorrelation which occurs in all regression models to some degree. ${ }^{5}$ Sensitivity tests showed that too much weight was applied to persistence - for instance, if the past $12-\mathrm{h} \Delta V_{\max }$ was very positive, there was a tendency for the model to continue this trend and often overforecast future wind changes. Likewise, if the TC did not intensify much in the past, the regression predicted slow intensification in the future when in fact the TC may undergo rapid intensification.

Because of these concerns with autocorrelation and past $\Delta V_{\max }$, a "weaker" form of persistence is sought. One technique is to invoke an "on-off" switch in which an indicator variable is used. An indicator variable (also called a dummy variable) indicates whether

\footnotetext{
${ }^{4} \mathrm{NHC} 90$ is a statistical dynamical model which uses output from CLIPER and forecasted deep-layermean geopotential heights from the National Meteorological Center's (NMC's) Medium-Range Forecast model (MRF) (Neumann 1991).

${ }^{5}$ Autocorrelation (also called serial correlation) is the correlation of a variable with values of the same variable lagged one or more time periods back or in the future (Aczel 1989). This violates one of the assumptions of the regression model, which states that the errors (measured in terms of the regression predicted value minus the observed future value) are independent from observation to observation. In other words, noise from the current forecast is correlated with noise from previous or future forecasts. One consequence of autocorrelation is that a variable may be statistically significant when in fact it is not - as a result the true unexplained variations will be underestimated which inflates measures of significance such as the t-test and F-test (Mendenhall and Reinmuth 1982). According to Neumann et al. (1977) and Shapiro (1984), this inflation is a result of the degree of freedom being too large, and suggest that an "effective" sample size be used instead where the number of forecasts cases are reduced by a factor depending on the autocorrelation between individual cases. Abraham and Ledolter (1983) describe techniques to detect autocorrelation. Aberson and DeMaria (1994) describe a technique to determine the proper "time separation" between observations, and also how to estimate an effective sample size. Merrill (1987) presents an "equivalent" F-test which accounts for autocorrelation. As a first approximation, one may just use observations every $24-36 \mathrm{~h}$ if this allows enough data for the statistical analysis.
} 
or not some condition holds (Aczel 1989). It has the value 1 when a condition holds and the value 0 when a condition does not hold. Therefore, one may assign a binary number given the past intensity trend.

However, it is desirable to apply some physics to this coding as well. Most storms intensify steadily from tropical storm strength to hurricane strength, upon which the rate of intensification increases. Mundell (1990) found that rapid intensification does not occur until $V_{\max }$ is $55 \mathrm{kts}$. Hence, there is an abrupt transition from steady development to fast development which corresponds to the onset of eye formation. This is consistent with the discussions in Chapters 3,6 and 7. It is also advantageous to incorporate the effects of a weakening or dissipating eye, and the concentric eyewall cycle (Willoughby et al. 1982; Willoughby 1990; Black and Willoughby 1992) which corresponds to temporary weakening.

Therefore, persistence with an "eye parameterization" (EYEPER) is investigated as:

$$
\mathrm{EYEPER}=\left\{\begin{array}{cc}
1 & \text { if } V_{\max } \geq 55 \mathrm{kts} \text { and } \Delta V_{\max }(t=-12)>0 \\
0 & \text { otherwise }
\end{array}\right.
$$

where $\Delta V_{\max }(t=-12)>0$ is the past 12-h intensity trend. Equation (8.4) may be interpreted as follows: if a TC's $V_{\max }$ is less than $55 \mathrm{kts}$, then other factors such as warm SSTs and weak wind shear must compensate for intensification. Once an eye forms (typically at $55 \mathrm{kts}$ ), the rate of intensification increases - however, this intensification depends on the recent intensity trend. A positive trend generally indicates that the TC is early in its life cycle, and that the eyewall is contracting with time. As shown by Weatherford (1989), proper consideration of the TC life cycle is important, and Eq. (6.3) shows the need for a contracting RMW. However, a negative tendency indicates one or more of the following possibilities: 1) the TC is in the later stages of its life cycle; 2) the eye is weakening due to colder SSTs, high shear, or other adverse effects; and/or 3) the eye is experiencing a concentric eyewall cycle, and temporary weakening is possible.

This parameterization contains some obvious flaws. However, several different conditions with expanded indicator coding (with 0,1 , and 2) were investigated, but these degraded forecast results. Hence this simple parameterization was chosen. Certainly the 
use of indicator variables warrants further research. Autocorrelation probably still exists, although with the combination of an indicator variable and "eye physics" it is hoped the effect of autocorrelation is sufficiently reduced.

\subsubsection{Sea Surface Temperature}

As mentioned in Chapter 4, Merrill (1987) found that using POT (Eq. (2.4)) instead of SST explained more variance. DeMaria and Kaplan (1994a) derived the following empirical MPI relationship from a 31-year sample of Atlantic TCs:

$$
\mathrm{MPI}(\mathrm{kts})=A+B \exp \left[-C\left(\mathrm{SST}_{\mathrm{o}}-\mathrm{SST}\right)\right]
$$

where $A=66.5 \mathrm{kts}, B=108.5 \mathrm{kts}, C=0.1813^{\circ} \mathrm{C}^{-1}$, and $\mathrm{SST}_{\mathrm{o}}=30.0^{\circ} \mathrm{C}$. Equation (8.5) is formulated by DeMaria and Kaplan (1994a) such that the average translation speed is included in the estimate of MPI. Older versions of MPI (DeMaria et al. 1993) did not contain this empirical contribution of storm motion to a fast-moving, recurved TC over colder water, and sometimes the MPI was less than $V_{\max }$. Since MPI has not been empirically defined in the western North Pacific, Eq. (8.5) will be used in this study. Kubat (1995) has done some qualitative research on MPI in the western North Pacific, but more quantitative studies on this subject are needed.

In SHIPS, MPI is averaged along the future storm track during the forecast interval $(\overline{\mathrm{MPI}})$ to account for SST variations along the track. Besttrack positions are used to determine the storm positions during the forecast period. Commentary on using postprocessed data, such as besttrack positions for future forecast positions, will be done later in this section and in Section 8.4.5. The following version of POT is used (DeMaria and Kaplan 1994b):

$$
\mathrm{POT}=\overline{\mathrm{MPI}}-V_{\max }(t=0)
$$

This formulation of POT is used in TIPS. Should POT become negative (rare), it is set to zero. As mentioned in Chapter 5, one difference between TIPS' SST and SHIPS is that the latter uses climatology while the former uses yearly observed values. 


\subsubsection{Synoptic winds}

In this study, several wind parameters are investigated which some researchers consider important to intensity change. These include 850 to 200 -mb vertical wind shear, 200-mb vorticity, 200-mb vorticity advection, and $200-\mathrm{mb}$ eddy fluxes. All wind values are bilinearly interpolated from ECMWF and BMRC grid fields.

\section{Vertical wind shear}

As discussed previously, vertical wind shear (VWS) is a crucial factor in TC development. However, the best procedure for measuring VWS has received little investigation. DeMaria and Kaplan (1994b) found that shear averaged around a 600-km circle explained slightly more variance of intensity change than traditional "single-point" shear (VWSPT) (see Eq. (3.1)) which is rewritten here as:

$$
\operatorname{VWSPT}=\left[(\Delta u(r=0))^{2}+(\Delta v(r=0))^{2}\right]^{1 / 2}
$$

where the winds are measured over the center of the storm $(r=0)$ at 850 and $200 \mathrm{mb}$ with $\Delta u=u_{200}-u_{850}$ and $\Delta v=v_{200}-v_{850}$.

To investigate whether areally averaged VWS is a better predictor, shear is computed over a 5.0 deg circle (VWS5) as:

$$
\operatorname{VWS5}=\left[(\overline{\Delta u})^{2}+(\overline{\Delta v})^{2}\right]^{1 / 2}
$$

Denoting the radii $r=2.5 \mathrm{deg} \& 5.0 \mathrm{deg}$ with $i=1 \& 2$, and circle angle $\phi=0,90,180$, $\& 270^{\circ}$ with $j=1$ to $4, \overline{\Delta u}$ is computed as:

$$
\overline{\Delta u}=\frac{1}{9}\left[\Delta u(r=0)+\sum_{i=1}^{2} \sum_{j=1}^{4} \Delta u_{i, j}\right]
$$

and likewise for $\overline{\Delta v}$. As an intermediate choice, areally averaged wind shear over a 2.5-deg area (VWS2) is also computed.

Like MPI, VWS is averaged along the future storm track during the forecast interval to account for shear changes along the track. (The same approach is applied for shear in SHIPS.) Besttrack positions are used to determine the storm positions during the forecast 
period. A "perfect-prog" of VWS is assumed. ${ }^{6}$ Using perfect-prog shear is a bit unfair, as operational forecasts of shear by numerical models can be very inaccurate, especially in the vicinity of the Tropical Upper-Tropospheric Trough (TUTT). The Atlantic TUTT tends to be artificially weakened by the Aviation (AVN) model (Fitzpatrick et al. 1995), and TUTTS in other basins also are dissipated by numerical models (L. Leslie and L. Carr, personal communication 1995). Furthermore, some TCs create a region of low shear with a well-developed upper-level anticyclone which can drive away a high shear environment, and this situation cannot be easily anticipated.

Since one of the goals of this research is to understand TC intensity change, not just develop a forecast scheme, perfect-prog shear will be mostly investigated in this paper. Besides, a poor forecast of wind shear will result in poor intensity change forecasts, hence building a regression model based on bad model shear forecasts is a questionable exercise. M. DeMaria (personal communication 1995) attempted building SHIPS regression on AVN shear output and abandoned it because of poor 200-mb wind forecasts.

Instead, SHIPS uses the shear analysis field at the beginning of the forecast period, and interpolates these values over future storm positions. In other words, the shear field is not allowed to evolve in the forecast period, but the TC may be assigned different shear values as it moves into different shear regimes based on $t=0$ computations. Obviously, not allowing the shear field to evolve with time will introduce errors into the analysis. However, DeMaria and Kaplan (1994b) have shown this type of shear computation to be sufficiently representative and simple to implement operationally. Therefore, to compliment this study, VWS values based on "persistence" are also investigated, and these values are denoted as $\mathrm{VWSPT}_{\text {per }}, \mathrm{VWS}_{\text {per }}$, and VWS5 $5_{\text {per }}$. Commentary on using VWS based on persistence is done at the end of this chapter.

However, since the time evolution of VWS is a crucial consideration on TC intensity change, it is recommended that the forecaster use the VWS perfect-prog version. In using

\footnotetext{
${ }^{6}$ Perfect-prog refers to the use of observed future fields rather than forecast fields (which may be incorrect) in the model development phase.
} 
TIPS, a forecaster needs to carefully scrutinize model output of shear, and subjectively alter VWS values based on satellite loops, climatology, persistence, and forecast experience if the numerical guidance is judged to be poor. Fitzpatrick et al. (1995) recommend persistence and climatology for predicting VWS in the vicinity of the Atlantic TUTT as an alternative to the model output, and this procedure is likely to be useful in the Pacific TUTT as well. Satellite loops can also indicate whether a TC is developing a strong anticyclone which strong upper-level winds may not be able to penetrate.

\section{Angular momentum eddy fluxes}

As previously discussed, upper-level angular momentum eddy fluxes impinging on the circulation of the TC and traversing inward with time may intensify the TC in 1 to 2 days. Since it is the outside fluxes which may yield predictive information, fluxes are inspected in the middle and outer radii. For reasons discussed in Chapter 2, these fluxes are computed at $200 \mathrm{mb}$.

DeMaria and Kaplan (1994b) found that radially averaging eddy fluxes was the best procedure for statistical analysis. From Chapter 2, it was shown that a time lag may exist between eddy fluxes impinging on the outside fringes of the TC and intensification 24-48 h later. Therefore Relative Eddy angular momentum Flux Convergence (REFC) is averaged between 7 to $10 \mathrm{deg}\left(\mathrm{REFC}_{\mathrm{mid}}\right)$ and 11 to $15 \mathrm{deg}\left(\mathrm{REFC}_{\text {outer }}\right)$. Equation (2.1) may be rewritten for more accurate computational purposes as:

$$
\mathrm{REFC}=-\frac{2 \overline{u_{r}^{\prime} v_{t}^{\prime}}}{r}-\frac{\partial \overline{u_{r}^{\prime} v_{t}^{\prime}}}{\partial r}
$$

and finite differencing is employed from radii 0 to $15 \mathrm{deg}$. To calculate the eddy terms, one would perform an azimuthal average for any desired radial distance from the TC center (after removing the storm motion) by computing $\overline{u_{r}^{\prime} v_{t}^{\prime}}$ at $1-\mathrm{deg}$ radii increments in the following manner:

$$
\overline{u_{r}^{\prime} v_{t}^{\prime}}=\frac{1}{n} \sum_{n}\left(u_{r}-\bar{u}_{r}\right)\left(v_{t}-\bar{v}_{t}\right)
$$

where the summation is an azimuthal average of observations spaced $60^{\circ}$ apart on a circle, and $\bar{u}_{r}$ and $\bar{v}_{t}$ are the average wind values along a circle. 
The Planetary Eddy angular momentum Flux Convergence (PEFC) is also computed, and simply approximates Eq. (4.1) as:

$$
\mathrm{PEFC}=-2 \Omega \frac{1}{n} \sum_{n}[\sin (\mathrm{LAT})-\sin (\operatorname{LAT}(r=0))]\left[u_{r}-\bar{u}_{r}\right]
$$

with LAT corresponding to the latitude along the circle in $60^{\circ}$ increments, and $\Omega$ is the earth's angular velocity given by $7.292 \times 10^{-5} \mathrm{~s}^{-1}$. PEFC is averaged at the middle $\left(\mathrm{PEFC}_{\mathrm{mid}}\right)$ and outer $\left(\mathrm{PEFC}_{\text {outer }}\right)$ radii similar to REFC. Storm motion is not removed from the calculation (unlike REFC) because in a storm-relative coordinate system (Lagrangian transformation) only the terms in the total derivative have the storm motion removed (Holland 1983; Molinari and Vollaro 1990; Molinari et al. 1993).

\section{Vorticity terms}

$200-\mathrm{mb}$ vorticity is calculated in a $2.5-\mathrm{deg}$ area (VORT2) and 5-deg area (VORT5) over the center of the storm. It was thought that the detection of small-scale cyclonic vorticity or large-scale anticyclonic vorticity might portend intensification. This was computed by finite differencing the conventional vorticity equation $\partial v / \partial x-\partial u / \partial y$. Inner-core

cyclonic vorticity (within $1 \mathrm{deg}$ ) would have been most desirable but such detailed analysis does not currently exist.

Since research indicates that upper-level forcing may contribute to intensification, vorticity advection (VORTADV) is calculated as:

$$
\begin{aligned}
\text { VORTADV }= & -u(r=0) \frac{(\zeta(x=+2.5)-\zeta(x=-2.5))}{\Delta x} \\
& -v(r=0) \frac{(\zeta(y=+2.5)-\zeta(y=-2.5))}{\Delta y}
\end{aligned}
$$

where $\Delta x=\Delta y=5 \mathrm{deg}$, or $555.5 \mathrm{~km}$, and relative vorticity $\zeta$ is computed at zonal and meridional components along a circle $2.5 \mathrm{deg}$ from the storm center.

\subsubsection{Satellite data}

The advantage of digitized, 3 -h satellite data is that its potential uses are almost limitless. As shown in Table 5.1, 30 combinations of radial band and brightness temperature $T_{b}$ are investigated. These radial bands are $0-1,0-2,0-4,0-6,1-2,2-4$, and 2-6 deg. $T_{b}$ 
values from $-55^{\circ}$ to $-80^{\circ} \mathrm{C}$ in $5^{\circ} \mathrm{C}$ increments are investigated. This full temperature range is explored for radii within $2 \mathrm{deg}$, while $-55^{\circ}$ to $-65^{\circ} \mathrm{C}$ is studied for radial legs outside 2 deg.

\section{Linear pixel counts}

The 30 combinations are outputted as percentage of pixels in a radial area colder than a specified $T_{b}$. These combinations are computed at $t=0$, and as 6,12 , and 24-h running means, thus giving 120 possible pixel count variables. The percentages at $t=0$ are coded as PXRdTb, where $R d$ is the radial increment and $T b$ is the brightness temperature designation without the minus sign. For example, the percentage of pixels in a 0-2-deg circular area colder than $-65^{\circ} \mathrm{C}$ is coded PX0265.

Running means are also computed, and coded as RM\#RdTb where \# represents a 6,12 , or 24 -h running mean. These running means are computed in 3 -h intervals as:

$$
\begin{aligned}
\operatorname{RM} 6 R d T b= & {[\operatorname{PXRdTb}(t=0)+\operatorname{PXRdTb}(t=-3)+\operatorname{PXRdTb}(t=-6)] / 3 } \\
\operatorname{RM} 12 R d T b= & {[\operatorname{PXRdTb}(t=0)+\operatorname{PXRdTb}(t=-3)+\operatorname{PXRdTb}(t=-6)+} \\
& \operatorname{PXRdTb}(t=-9)+\operatorname{PXRdTb}(t=-12)] / 5 \\
\operatorname{RM} 24 R d T b= & {[\operatorname{PXRdTb}(t=0)+\operatorname{PXRdTb}(t=-3)+\operatorname{PXRdTb}(t=-6)+} \\
& \operatorname{PXRdTb}(t=-9)+\operatorname{PXRdTb}(t=-12)+\operatorname{PXRdTb}(t=-15)+ \\
& \operatorname{PXRdTb}(t=-18)+\operatorname{PXRdTb}(t=-21)] / 8
\end{aligned}
$$

Some pixel counts are only available every $6 \mathrm{~h}$, and these corresponding running means are done in 6 -h intervals.

Running means are frequently used in forecast procedures because it smooths convective pulses for easier analysis, and because the diurnal oscillation is removed (Browner et al. 1977; Gray and Jacobson 1977; Hobgood 1986; Zehr 1987; Hallin 1991) However, it is possible that some of the forecast signal at $t=0$ might be lost using running means. Therefore, this study will investigate which procedure is best through statistical analysis. 


\section{Nonlinear pixel counts}

Since the interaction between convection and TC development is nonlinear because of the ASI and IFC processes, and due to geostrophic adjustment (Hack and Schubert 1986b), it is conceivable that nonlinear pixel counts are more appropriate predictors (Steranka et al. 1986). Therefore, squared pixel counts (PXRdTb**2, RM6RdTb**2, RM12RdTb**2, and RM24RdTb**2) are investigated.

\section{2-h pixel count trends}

Convective change could signify future intensity change. Therefore, $12-\mathrm{h}$ trends are computed for the pixel counts at $t=0$ (DPXRdTb) and for the running means (DRM6RdTb, DRM12RdTb, and DRM24RdTb).

\section{The "Mundell" technique}

Mundell (1990) developed a technique for predicting rapid deepening. This scheme forecasts RI onset within $12 \mathrm{~h}$ if the inner-core convection $\left(0-2 \mathrm{deg}\right.$ with $\left.T_{b}<-75^{\circ} C\right)$ increases and/or the outer-core convection $\left(2-6 \mathrm{deg}\right.$ with $\left.T_{b}<-65^{\circ} \mathrm{C}\right)$ decreases such that a time series plot intersects these lines at certain specified pixel count percentages (see Chapter 4). Mundell used a 24-h running mean of PX0275 for the inner-core convection and PX2665 for the outer-core. A similar version of this scheme (coded as MUND), but without Mundell's scaling procedure, is investigated as:

$$
\text { MUND }=\frac{\text { PX0275 }}{\text { PX2665 }}
$$

Should PX2665 be less than $5 \%$, it is set equal to $5 \%$ to avoid an artificially high ratio, and in case PX2665 is zero. A similar procedure is done for the running means (RM6MUND, RM12MUND, and RM24MUND).

\section{Horizontal distribution of pixel counts}

Its been suggested by Handel (1990) that more consistent results between pixel counts and intensity change might be obtained by "examining temperature gradient changes rather than looking for locations with infrared temperatures below some fixed temperature 
cutoff." This interesting idea (coded as DIFFPX) is examined by measuring the difference between cold inner-core cloud tops (PX0270) and outer-core cloud tops (PX2665). This is calculated as:

$$
\text { DIFFPX }=\text { PX0270 }- \text { PX2665 }
$$

A similar procedure is done for running means (RM\#DIFFPX) with differences between

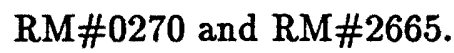

\subsubsection{The plausibility of regression development on besttrack data}

Some may question whether using besttrack data is a realistic method for developing a forecast regression scheme, since such data is not available in real-time. However, DeMaria and Kaplan (1994b) compared SHIPS' results developed on track forecasts based on VICBAR to those developed on besttrack data, and found little difference in the forecast errors. They reasoned that since MPI and VWS are averaged along the track, and because shear is areally averaged as well, the impact of track errors is reduced. Hence, the use of besttrack may not degrade TIPS' intensity forecasts very much. Besides, it seems somewhat unreasonable to develop regression on a storm which is forecast to move west over warm waters but moves north instead over colder waters - a busted track forecast of this type will yield poor intensity predictions regardless which research technique is employed. Finally, besides developing a forecast scheme, an equally important scope of this research is to better understand TC intensity change, which is best analyzed using perfect-prog.

\subsubsection{Alternatives to averaging along the track}

It is desirable to include time and spatial variations along the forecast track, but the best approach may not be averaging shear and MPI. It was thought that including the forecast tendency of MPI, SST, and shear might improve the regression results. However, sensitivity tests found this not to be the case, as replacing averaging with tendency yielded either statistically insignificant results or significant but not equally good results.

It was also thought that shear and MPI values might contain more information at the initial forecast time than at the end of the forecast period. To investigate this premise, 
different "weighted averaging" schemes along the track were done. These results either explained less or equal amounts of variance than non-weighted averaging. Hence, for simplicity non-weighted averaging along the track was the chosen procedure. More research is required on this topic.

\subsubsection{Summary of possible predictors}

The number and variety of possible predictors are quite extensive. For the reader's benefit, the possible climatology, persistence, and SST predictors are summarized in Table 8.1, the possible synoptic wind predictors are listed in Table 8.2, and the possible pixel count formulations are included in Table 8.3. The statistical methodology for assessing the best predictors will now be discussed.

Table 8.1: Potential climatology, persistence, and SST regression predictors. Details of predictors are contained in the text. Only storms over open water with $V_{\max } \geq 35 \mathrm{kts}$ are considered.

\begin{tabular}{|l|l|}
\hline PREDICTOR & DESCRIPTION \\
\hline \hline LAT & Initial storm latitude. \\
\hline LONG & Initial storm longitude. \\
\hline SPD & Observed storm speed. \\
\hline SPDAN & $\begin{array}{l}\text { Storm speed anomaly, defined as the absolute value of SPD } \\
\text { minus average storm speed of } 5 \mathrm{~m} \mathrm{~s}^{-1} \text {. }\end{array}$ \\
\hline DIRAN & $\begin{array}{l}\text { Direction anomaly, defined as the TC direction anomaly from } \\
\text { average storm direction of } 310^{\circ} .\end{array}$ \\
\hline USPD & Zonal component of storm motion. \\
\hline VSPD & $\begin{array}{l}\text { Meridional component of storm motion. } \\
\text { Anomaly from peak onset of rapid deepeners, defined as the } \\
\text { obsolute value of observed Julian date minus climatological } \\
\text { onset of rapid intensifiers. }\end{array}$ \\
\hline EYEPER & $\begin{array}{l}\text { Indicator variable that combines persistence with the param- } \\
\text { eterization of a well-formed, contracting eye. }\end{array}$ \\
\hline POT & $\begin{array}{l}\text { Maximum Possible Intensity (MPI) for a given SST minus } \\
\text { initial intensity }\left(V_{\text {max }}\right) . \text { MPI is averaged over the forecast } \\
\text { track. }\end{array}$ \\
\hline
\end{tabular}

\subsection{Regression methodology}

The statistical procedure consists of several phases. First, one needs to check if the least square assumptions are met. Second, an approach in which a few "statistically sig- 
Table 8.2: Potential synoptic regression regression predictors. Details of predictors are contained in the text. Only storms over open water with $V_{\max } \geq 35 \mathrm{kts}$ are considered.

\begin{tabular}{|c|c|}
\hline PREDICTOR & DESCRIPTION \\
\hline VWSPT & $\begin{array}{l}\text { Magnitude of } 200-850 \mathrm{mb} \text { "single point" vertical wind shear } \\
\text { over the storm center. VWSPT is averaged over the forecast } \\
\text { track, and a perfect shear forecast is assumed. }\end{array}$ \\
\hline VWS2 & Same as VWSPT, but averaged over a 2.5-deg circle. \\
\hline VWS5 & Same as VWSPT, but averaged over a 5-deg circle. \\
\hline VWSPT $_{\text {per }}$ & $\begin{array}{l}\text { Same as VWSPT, but no shear forecast is used. Instead, } \\
\text { persistence from the initial shear field is performed along the } \\
\text { forecast track. }\end{array}$ \\
\hline VWS2 & 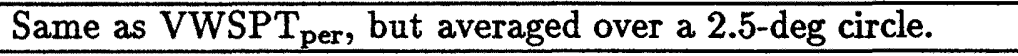 \\
\hline VWS5 $_{\text {per }}$ & Same as VWSPT $_{\text {per }}$, but averaged over a 5 -deg circle. \\
\hline$\overline{\mathrm{REFC}_{\text {mid }}}$ & $\begin{array}{l}200 \text {-mb Relative Eddy angular momentum Flux Convergence } \\
\text { averaged between } 7 \text { and } 10 \mathrm{deg} \text {. }\end{array}$ \\
\hline $\mathrm{REFC}_{\text {outer }}$ & $\begin{array}{l}200 \text {-mb Relative Eddy angular momentum Flux Convergence } \\
\text { averaged between } 11 \text { and } 15 \mathrm{deg} \text {. }\end{array}$ \\
\hline $\mathrm{PEFC}_{\text {mid }}$ & $\begin{array}{l}\text { 200-mb Planetary Eddy angular momentum Flux Conver- } \\
\text { gence averaged between } 7 \text { and } 10 \mathrm{deg} \text {. }\end{array}$ \\
\hline PEFC $_{\text {outer }}$ & $\begin{array}{l}\text { 200-mb Planetary Eddy angular momentum Flux Conver- } \\
\text { gence averaged between } 11 \text { and } 15 \mathrm{deg} \text {. }\end{array}$ \\
\hline VORT2 & $\begin{array}{l}200-\mathrm{mb} \text { vorticity in a } 2.5 \text {-deg circle over the center of the } \\
\text { storm. }\end{array}$ \\
\hline VORT5 & 200 -mb vorticity in a 5 -deg circle over the center of the storm. \\
\hline VORTADV & nb vorticity advection over the center of the storm. \\
\hline
\end{tabular}


Table 8.3: Potential pixel count regression predictors. Details of predictors are contained in the text. Only storms over open water with $V_{\max } \geq 35 \mathrm{kts}$ are considered.

\begin{tabular}{|l|l|}
\hline PREDICTOR & DESCRIPTION \\
\hline \hline PXRdTb & $\begin{array}{l}\text { Percent of infrared pixels (PX) in a radial area (Rd) colder } \\
\text { than a specified brightness temperature (Tb) at } t=0 . \text { All } \\
\text { Tb are negative. 30 combinations are investigated. }\end{array}$ \\
\hline \hline PXRdTb**2 & Same as PXRdTb, but squared. \\
\hline RM6RdTb & $\begin{array}{l}\text { Same as PXRdTb, but the pixel counts are a 6-h running } \\
\text { mean (RM). }\end{array}$ \\
\hline RM12RdTb & Same as RM6RdTb, but squared. \\
\hline RM12RdTb**2 & $\begin{array}{l}\text { Same as PXRdTb, but the pixel counts are a 12-h running } \\
\text { mean (RM). }\end{array}$ \\
\hline RM24RdTb & $\begin{array}{l}\text { Same as PXRdTb, but the pixel counts are a 24-h running } \\
\text { mean (RM). }\end{array}$ \\
\hline RM24RdTb**2 & Same as RM24RdTb, but squared. \\
\hline \hline DPXRdTb & $12-h$ trend of PXRdTb. \\
\hline DRM6RdTb & $12-h$ trend of RM6RdTb. \\
\hline DRM12RdTb & 12-h trend of RM12RdTb. \\
\hline DRM24RdTb & 12-h trend of RM24RdTb. \\
\hline \hline MUND & Ratio of PX0275 to PX2665. \\
\hline RM6MUND & Ratio of RM60275 to RM62665. \\
\hline RM12MUND & Ratio of RM120275 to RM122665. \\
\hline RM24MUND & Ratio of RM240275 to RM242665. \\
\hline \hline DIFFPX & PX0270 minus PX2665. \\
\hline RM6DIFFPX & RM60270 minus RM62665. \\
\hline RM12DIFFPX & RM120270 minus RM122665. \\
\hline RM24DIFFPX & RM240270 minus RM242665. \\
\hline
\end{tabular}


nificant" predictors are chosen from many possible predictors needs to be devised. In this approach, the detrimental effects of artificial skill, autocorrelation, and multicollinearity (all of which will be defined) need to be considered. Third, the multiple regression equation should be arranged so that the predictors (which all contain different units) can be equally compared against each other and compared at different forecast intervals. Finally, a fair procedure to validate the model performance needs to be done. These will all be described in this section.

\subsubsection{Validity of linear least squares multiple regression}

While linear least squares multiple regression is the most popular regression analysis, it is not always the best choice. Certain criteria must be met before least squares analysis can be applied. Should the data fail these rules, other statistical methods must be used (to be discussed in Chapter 9). The assumptions for which least square regression is valid are (Aczel 1989):

1. For each observation, the error (residual) term is independent of the error terms associated with all other observations.

This assumption, which states that autocorrelation should not exist, has already been discussed in the previous section.

2. The residuals are normally distributed with mean zero and standard deviation $\sigma$.

The method of least squares has desirable characteristics when the errors are normally distributed. But if this assumption is severely violated, alternative regression techniques are needed which are developed for specific error distributions (i.e., a Laplace distribution or a Poisson distribution) and desired sensitivity to outliers (IMSL 1987). A sufficiently accurate method for checking assumption 2 is to divide the observed residuals by their standard deviation (Aczel 1989). Histograms of these normalized residuals were plotted, and a Gaussian distribution was evident (not shown). Generally speaking, the errors were 
concentrated around the center, with less than $5 \%$ of the standard residuals over 2 in absolute value. The mean residual value was zero. While residuals can never be perfectly Gaussian, small deviations from normality do not severely violate this assumption. Hence, the TIPS formulation is valid for Criterion 2.

3. The variance of the residuals for each independent variable is constant.

Criterion 3 states that the spread of the errors for each predictor is uniform. For example, if the residual scatter increases as a predictor increases in value, this assumption is violated. This problem is called heteroscedasticity, and when it occurs either another least squares approach must be used (like "weighted" least squares), another variable needs to be added to the regression, or the variable needs to be transformed (Mendenhall and Reinmuth 1982; Aczel 1989). A technique for detecting heteroscedasticity is to plot residuals against each individual predictor to see if the residuals appear randomly with no pattern. ${ }^{7}$ These residual plots showed that all the predictors were randomly distributed with no pattern and equal variance (not shown), therefore these variables are probably specified well-enough to validate Criterion 3 .

4. The independent variables are measured without error.

Of course, in data-void regions with limited temporal continuity $(12 \mathrm{~h})$, the data accuracy is somewhat questionable. This is complicated by the fact that $V_{\max }$ is stored in 5-kts increments, which automatically restricts the maximum theoretical wind forecast accuracy to within 5 to $10 \mathrm{kts}$. Besttrack data is rounded to the nearest $5 \mathrm{kts}$ because often it is a satellite estimate (see Chapter 5), and because dictating a representative $V_{\max }$

\footnotetext{
${ }^{7}$ Residual plots are an effective way to find new variables, or to see if a variable is nonlinear (which violates the assumption of a linear relationship between the dependent variable and independent variable) and should be transformed to linearize the relationship. For instance, when the residual scatter increases with the predictor, a logarithmic transformation often remedies the nonconstant variance. If the residuals exhibit curvature, this suggests a quadratic term should be added. Residual plots of observations as a function of time can detect trend in the data set.
} 
is difficult in a large, often asymmetrical TC. The areal pixel counts may also be skewed in situations when the TC is far from the satellite subpoint (Miller and Fritsch 1991). Unfortunately, no better alternatives exist to perform this study. All one can do is check for suspicious data to minimize the influence of faulty outliers.

To check for bad data, maxima and minima for all potential predictors were outputted. This was beneficial as it revealed coding errors, such as missing data points (designated as "999.9") accidentally included in the sample which the program should have removed. As a secondary precaution, "unusual" predictor values or an "unusual" dependent value were detected by computing a statistical value called the leverage (IMSL 1987) using IMSL routine "RCASE." This revealed additional questionable satellite data for TC Thad (1984). Upon inspection it was discovered that the pixel counts were zero during its rapidly deepening phase, which is clearly incorrect - apparently the wrong storm track over a cloud-free atmosphere where no TC existed was used for the satellite processing of Thad, hence this storm was deleted from the sample. Otherwise, all the normalized standard deviations were within 2.5. While it was quite possible some of these were bad data, inspection showed that most were reasonable and likely contained important predictive information (such as very low shear cases or highly convective cases), hence these were retained.

Therefore, within the threshold of accuracy which is currently possible for synoptic, besttrack, and satellite data, the best possible data is used in this study, and (hopefully) all bad data have been removed from the sample. But even though the multiple regression criteria here are reasonably satisfied, some researchers argue that generalized least squares, weighted least squares, and nonlinear multiple regression are a more optimal approach (IMSL 1987; Aczel 1989). Mielke et al. (1995) suggest that even for small portions of contaminated data, schemes based on Least Absolute Deviations (LAD) are superior to least square schemes because the former minimizes absolute errors while the latter minimizes the square of the errors - therefore, LAD is less sensitive to outliers which could be bad data. Research on the feasibility of alternative regression schemes for TIPS is strongly encouraged. 


\subsubsection{Screening of potential predictors - the hazards of multicollinearity and artificial skill}

The preselection of a large number of potential variables to pick a small number of statistically significant predictors is a delicate problem which has only been earnestly addressed the last fifteen years. This is because the greater the number of available predictors for significance test screening, the greater the artificial skill (Neumann et al. 1977; Merrill 1987; Shapiro and Neumann 1984). Neumann et al. (1977) states, "The practice of making a large number of predictors available and selecting a few adds another play of chance not ordinarily accounted for....in the F-test. The manifestation of this additional play of chance is an increased value" of the F-test. (The meaning and statistical application of the F-test will be discussed later.) In layman's terms, Merrill (1987) explains, for 20 potential predictors "which were actually random variables, one (variable) would be expected to test significant at the $95 \%$ level by chance alone." Another complicating factor is that, as mentioned earlier, autocorrelation can inflate F-test values.

Furthermore, including many potential variables in the preselection process increases the chance that some will be correlated with each other. This problem is called multicollinearity, and is an ill-posed mathematical problem if two variables are strongly correlated with each other (Aczel 1989).$^{8}$ Even if severe multicollinearity does not exist so that regression computations are possible, moderate degrees of multicollinearity present other problems. Effects of moderate multicollinearity are (Aczel 1989):

1. The magnitudes of the regression coefficients are inflated (variance inflation), and sometimes different from logical reasoning.

2. The signs of regression coefficients may be different than what is expected.

\footnotetext{
${ }^{8}$ Denoting the potential predictors as a matrix $\mathcal{X}$ and the dependent variables as $\vec{Y}$, the slope parameters $\vec{b}$ can be solved using numerical linear algebra techniques (i.e., QR decomposition or Cholesky factorization) as $\vec{b}=\left(\mathcal{X}^{T} \mathcal{X}\right)^{-1} \mathcal{X}^{T} \vec{Y}$ where $\mathcal{X}^{T}$ is the transpose of the predictor matrix (Maindonald 1984). If two columns of the matrix contains variables perfectly linearly dependent on each other, $\left(\mathcal{X}^{T} \mathcal{X}\right)^{-1}$ cannot be inverted.
} 
3. The inclusion of two or more correlated predictors makes understanding their individual contributions to the dependent variable difficult.

Ideally, the chosen predictors should be uncorrelated with each other so that each variable contains a unique piece of information about the dependent variable. In reality, multicollinearity always occurs to some extent in multiple regression, since the forecast variables likely share some of the same predictive information. In situations where two of the predictors are moderately correlated, it is difficult to assess the impact of a particular variable on the dependent variable, and sometimes confounded by bewildering regression coefficients. For example, if in $y=\beta_{1} x_{1}+\beta_{2} x_{2}$ the predictors $x_{1}$ and $x_{2}$ are moderately correlated with each other, $\beta_{1}$ may be much greater than 0 and $\beta_{2}$ may be much less than 0 , even though $x_{2}$ by itself is positively correlated to $y$. This situation also inflates the amount of variance (the multiple coefficient of determination $R^{2}$ ) actually explained by these relationships. Therefore, understanding each predictor's contribution to $y$ can be confusing, and the variance explained can be overestimated.

Given these factors, Mendenhall and Reinmuth (1982) state that, for forecasting applications, moderate multicollinearity still should not present any problems, and may actually increase forecast accuracy despite the difficulties in interpreting the predictor's contributions. However, sensitivity experiments by this author found that not to be true. Including two terms correlated with each other actually decreased forecast accuracy on an independent data set in TIPS. Perhaps some of the least square assumptions listed above were violated with multicollinearity, or maybe the data was "overfit".

Procedures exist to calculate "equivalent" F-tests which account for autocorrelation and artificial skill (Merrill 1987; Aberson 1994), but the commercial package used in this study (IMSL) does not contain this flexibility. Furthermore, these techniques may not totally eliminate moderate multicollinearity. Hence, it was decided to minimize the effect of multicollinearity and artificial skill by using a three-step process.

\section{Step 1}

The first step involves reducing the large number of possible predictors and eliminating some repetitious variables so that the occurrence of artificial skill is reduced. For example, 
the pixel count input should be restricted to one of the four generic possibilities: PXRdTb, RM6RdTb, RM12RdTb, or RM24RdTb. The same exclusions apply to the pixel count trends. The wind shear is restricted to one of the three perfect-prog possibilities: VWSPT, VWS2, or VWS5.

Sensitivity runs (using the total 3 step procedure) found that VWS5 explained slightly more variance than VWS2 or VWSPT, similar to the results of SHIPS. While certainly there were occasions when single point shear might be better than areally averaged shear (especially for small TCs), statistically speaking VWS5 is the best choice. A more surprising finding was that observed pixel counts were the best choice, instead of running means. This is a bit shocking considering how oscillatory convection is, but apparently too much of the forecast signal is lost during the smoothing process. These sensitivity runs will be discussed in more detail later in the chapter.

It was also decided that, except for the pixel counts, only linear predictors would be investigated. It is possible to include a wide variety of cross-terms (i.e., USPD $\times$ LONG) and quadratic terms (i.e., $\mathrm{LAT}^{2}$ ) which may be statistically significant, and help explain some nonlinear interactions with intensity change (Jarvinen and Neumann 1979; Landsea 1995). Such terms are difficult to interpret, however. Furthermore, as mentioned previously, it is unwise to indiscriminately use too many possible predictors since this increases the possibility of artificial skill.

Therefore, the preselected variables for regression screening are: LAT, LONG, SPD, SPDAN, DIRAN, USPD, VSPD, JDAN, EYEPER, POT, VWS5, REFC mid $_{\text {, }}$ REFC $_{\text {outer }}$,

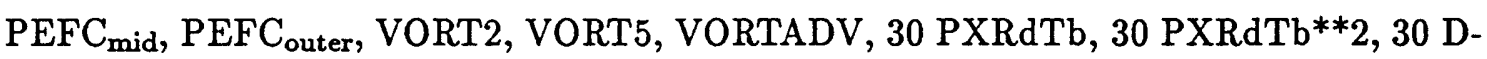
PXRdTb, MUND, and DIFFPX. This gives a total of 110 potential predictors.

\section{Step 2}

For this empirical scheme, the future change of maximum wind $\left(\Delta V_{\max }\right)$ is chosen as the dependent variable (consistent with SHIPS). For example, $\Delta V_{\max }$ in a 24-h period would be $V_{\max }(t=24)-V_{\max }(t=0)$, and for a 48-h forecast it is $V_{\max }(t=48)-V_{\max }(t=$ $0)$. Some schemes, such as STIFOR, use the future $V_{\max }$ as the dependent variable in coordination with the current $V_{\max }$ (Chu 1994), but this approach skews the reference 
frame for variance calculations by clustering the data between widely ranging wind values of 35 to $160 \mathrm{kts}$, thus inflating $R^{2}$. A comparison of SHIPS and SHIFOR (which uses $\Delta V_{\max }$ ) and STIFOR reveals similar forecast absolute errors of $10 \mathrm{kts}$, causing one to question whether the higher $R^{2}$ values reported by the latter are representative of an improvement in a practical sense.

In the development of a multiple regression model, it is desirable to pick the fewest number of significant predictors, as "overfitting" the sample data will lead to degradation of skill on the independent data (Aczel 1989). Therefore, stepwise regression is used to optimize the number of significant predictors. IMSL routine "RSTEP" is the chosen algorithm.

To ascertain whether variables are significant, a combination of F-tests and partial F-tests are performed. The F-test is used to determine if a linear relationship between the dependent variable and a set of one or more independent variables exists for a userspecified level of significance. ${ }^{9}$ As a rule of thumb, an F-value greater than 5 leads to rejection of the null hypothesis, and the higher the F-value, the stronger the regression relationship (Aczel 1989).

A partial F-test determines whether adding additional predictors to a regression equation with predetermined significant variables will bestow additional information to the dependent variable. For example, if for $y=\beta_{1} x_{1}+\beta_{2} x_{2}$, variables $x_{1}$ and $x_{2}$ have been determined to be significant, will adding $x_{3}$ and $x_{4}$ to the regression equation significantly increase its predictive power? If one is testing whether including a single variable yields additional information, then the partial F-test approximately reduces to a t-test (Aczel 1989), which is what the IMSL package computes.

The stepwise procedure is as follows, and is summarized in Fig. 8.1: All possible predictors are included in the first step, and the most significant single-variable regression is

\footnotetext{
${ }^{9}$ Statistically speaking, the "null hypothesis" is made that there is no linear relationship between $y$ and any $x_{i}$ such that the regression coefficients $\beta_{1}=\beta_{2}=\beta_{3}=\ldots . \beta_{k}=0$. If, on the other hand, it is determined that not all $\beta_{k}$ are zero, then the null hypothesis is rejected, and there is a regression relationship between $y$ and at least one of the independent variables.
} 


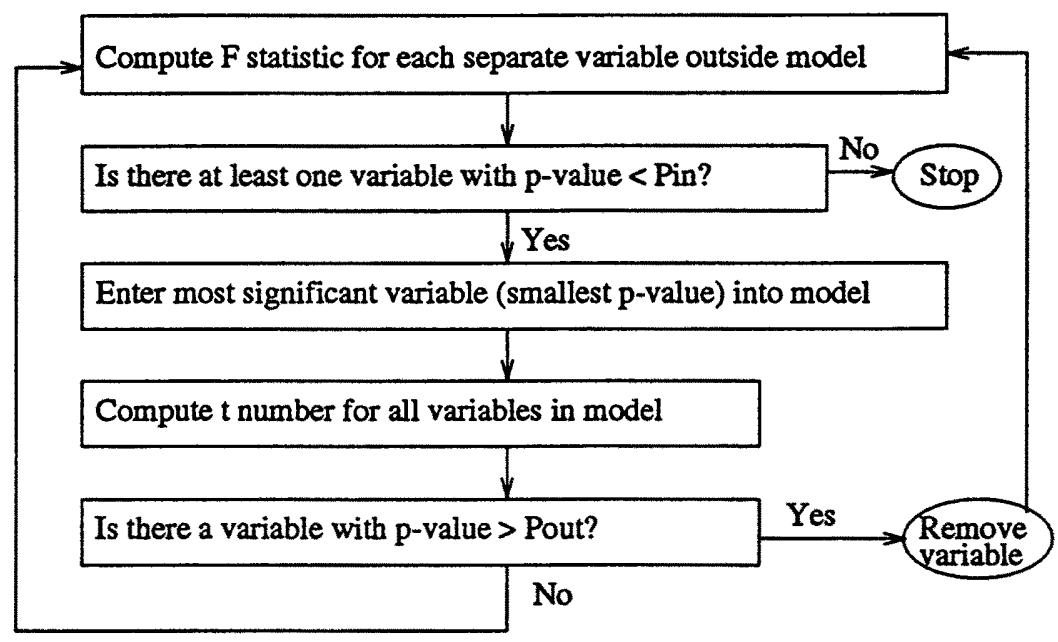

Figure 8.1: Flowchart showing the stepwise variable selection procedure. $P_{i n}$ is the significance level for a variable to enter a multiple regression model, and $P_{\text {out }}$ is the significance level for a selected variable to stay in the model. Adapted from Aczel (1989).

found through the largest $\mathrm{F}$-number. If none of the variables are significant, the procedure stops and multiple regression is not possible. Should one significant variable exist, the remaining variables are checked with a t-test, and the most significant variable, assuming it meets the "entry" significance requirement, is added to the model. At this point, a "backward glance" is employed in which the original variable in the model is reevaluated to see if it meets the "staying" significance standard for remaining in the model once the new variable has been added - if not, the original variable is dropped. Then variables still outside the model are screened for entry requirement, and the most significant one, if found, is added. All variables in the model are then checked again for staying-significance once the new variable has been added. The procedure continues until no variables outside the model can be added, and no variables inside the model can be removed.

The minimum significance $t$-test requirements to enter and to stay in the model are called $P_{\text {in }}$ and $P_{\text {out }}$, respectively. As mentioned previously, the t-test values may be inflated by artificial skill and autocorrelation. Shapiro and Neumann (1984) suggest setting strict significance values to compensate artificially high t-test values. Therefore the entry significance value level of acceptance is $99 \%\left(P_{i n} \leq 0.01\right)$. Typically, the staying significance is set to twice $P_{\text {in }}$ (IMSL 1987), so $P_{\text {out }} \leq 0.02$. 
It is important to note that stepwise regression does not necessarily find the best model, and that there may be several possibilities that are functions of evaluation criteria and order dependence in the selection process. Unfortunately, another IMSL routine which finds the best number of predictors based on $R^{2}$ ("RBEST") contains a bug discovered by this author which precludes input of more than 24 variables at once. Improved stepwise algorithms, and alternatives to stepwise regression, are encouraged for further refinements of TIPS.

\section{Step 3}

Subroutine RBEST allows two variables which are moderately correlated with each other to be chosen as predictors. As a result, several pixel count values were picked which contained correlations of 0.5 or higher with each other. Regression at the $99 \%$ level also allows the possibility that one variable out of 110 could enter the model by chance alone. Finally, the regression exhibited a tendency to pick different inner-core pixel counts for different forecast periods - however, many of the inner-core pixels are highly correlated with each other, so therefore it is prudent to pick one inner-core PXRdTb to represent all the forecast intervals.

Step 3 involves a filtering process in which multicollinearity is reduced in the model by keeping only one of the variables, and removing the other variables related to the retained variable. Part of the multicollinearity reduction involves the selection of one PXRdTb for all forecast times. Then, stepwise regression is run again on the remaining variables.

With the second stepwise application, sometimes additional variables retained in step 2 are rejected in step 3 . Perhaps these variables were kept the first time due to artificial skill. It was observed that the IMSL routine exhibited oscillatory behavior in which an extra variable or variables would be added to compensate for residuals introduced with multicollinearity or for a variable which marginally passed the level of significance. This oscillatory behavior increased if the user-specified level of significance decreased, and is one reason the strict level of $99 \%$ was used, as a 90 or $95 \%$ level allowed these random residuals to be explained by additional but probably unimportant predictors. 
Multicollinearity was checked by computing a correlation matrix of the chosen predictors from the first stepwise regression. ${ }^{10}$ It is assumed that multicollinearity existed when the linear correlation coefficient is $\geq 0.5$ between two chosen predictors. This often occurs when several pixel count data or climatology variables are selected. Under these circumstances, one of the correlated variables was chosen and the rest rejected. The retention of a variable was based on the order it was chosen in the stepwise procedure, its individual correlation to $\Delta V_{\max }$, and how often the predictor was chosen for different forecast intervals.

The selection of one representative pixel count was made clearer by noticing that the 0-4 deg linear pixel counts were picked the most in the four forecast frames, although linear and nonlinear 0-1, 0-2, and 0-6 deg pixels were also picked. Further inspection showed that PX0455 was picked first in the 12 and 24-h regression, and that the correlation between the pixels chosen first in the 36 and 48 -h and PX0455 was greater than 0.9. Finally, the variance explained by PX0455 alone with regards to $\Delta V_{\max }$ varies from 5 to $9 \%$ for all timeframes, which is one of the highest correlation values for all the pixel counts. Sensitivity runs (to be discussed later) also implied that PX0455 is the best predictor for this sample. Therefore, PX0455 is the chosen representative pixel count variable for all four forecast periods. This is a bit surprising, as one would suspect the $0-1$ or $0-2$ pixels would contain more skill. Apparently the 0-4 deg pixel contains convection, cloud symmetry, and shear information, as well as compensates for errors in locating the TC center. These ideas will be expanded on shortly. The ability to extract such unexpected information underscores the power of multiple regression analysis.

${ }^{10}$ A correlation matrix has the form:

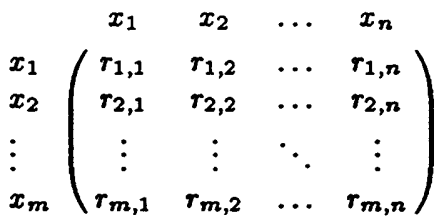

where $r_{m, n}$ is the correlation coefficient between $x_{m}$ and $x_{n}$. If $m=n$, then $r_{m, n}=1$. 


\subsubsection{Normalization of regression coefficients}

It is desirable to compare the chosen predictors to ascertain their importance, yet this is difficult to accomplish when the predictors and the dependent variable contain different units. Therefore, a normalization procedure is needed for equal comparison between the variables. Denoting $\sigma$ as the standard deviation of a variable, $y=\Delta V_{\max }, \bar{x}$ as the predictor mean, and $\bar{y}$ as the mean $\Delta V_{\max }$, a number $k$ statistically significant predictors are normalized by the following regression:

$$
(y-\bar{y}) / \sigma_{y}=\sum_{i=1}^{k} c_{i}\left(x_{i}-\bar{x}_{i}\right) / \sigma_{i}
$$

The advantage of this approach is that the importance of a predictor may be assessed by the regression coefficient $c_{i}$ between different variables and different forecast intervals (DeMaria and Kaplan 1994b), and that the y-intercept is zero (Edwards 1984). As a simplistic example, if $c_{i}=0.5$ for a variable and if $\left(x_{i}-\bar{x}_{i}\right) / \sigma_{i}=1.0$ for 24 -h regression, then that independent variable's contribution to $24-\mathrm{h}$ intensity change will be one-half of $\Delta V_{\text {max }}$ 's standard deviation plus $\bar{y}$. Furthermore, if the same variable's $c_{i}$ is larger for $48-\mathrm{h}$ regression, that variable is more important for 48 -h forecasts than 24 -h forecasts (relative to other chosen predictors).

In addition, $\bar{x}_{i}$ may be interpreted (to a first approximation) as a "threshold" value which distinguishes between intensification and weakening. Assuming $\bar{y}$ is small (it ranges from 1 to $\left.3 \mathrm{~m} \mathrm{~s}^{-1}\right)$, and a predictor is positively correlated $\left(c_{i}>0\right)$, then for $x_{i}>\bar{x}_{i}$ intensification is likely to occur, and vice versa for $x_{i}<\bar{x}_{i}$. Similarly, if a predictor is

negatively correlated $\left(c_{i}<0\right)$, values of $x_{i}$ greater (less) than $\bar{x}_{i}$ correspond to weakening (intensification).

\subsubsection{Verification of model performance}

During the past decade, regression model performance often has been evaluated by cross-validation. Examples include the forecast validations of SHIPS (DeMaria and Kaplan 1994b), Atlantic seasonal hurricane activity (Hess et al. 1995; Gray et al. 1992, 1993, 1994), and numerous studies summarized in Barnston and den Dool (1993). Crossvalidation (also called the jackknife technique) involves the systematic removal of a single 
observation (i.e., one year from a climate record stored in yearly slots) or a block of observations (i.e., one TC from several years of besttrack data), whereupon the regression coefficients are recomputed from the remaining data and the forecast errors for the removed case is computed. By repeating this process until all possible combinations are exhausted, cross-validation can produce error estimates that represent the level of skill expected on independent data.

However, several authors have questioned the validity of cross-validation. Barnston and den Dool (1993) illustrates an example in which four pairs of predictor-predictand $(x, y)$ values - $(1,1),(1,-1),(-1,1)$, and $(-1,-1)$ - have no relationship, but through crossvalidation an apparent correlation exists. In this example, cross-validation indicates some skill in the relationship when in the whole sample no skill exists. The linear correlation would show $r=-0.5$ when in fact $r=0.0$. This degeneracy arises not because of sample size but because when any point is withheld, the remaining points no longer have zero correlation. The degeneracy can occur to some degree in larger data samples, and the effect becomes severe when relationships are weak.

Despite these problems, most researchers are not fortunate enough to have enough data which may represent an entire population, ${ }^{11}$ therefore cross-validation is often used. Elsner and Schmertmann (1994) and Barnston and den Dool (1993) describe methods to minimize the effects of degradation, and how to avoid misapplications of cross-validation.

A better approach when the data set is sufficiently large is to break the sample into a "training period" data set for which the prediction model is developed, and a "forecasting period" data set to test the prediction equations on. Some researchers apply this validation technique in climate forecasts by developing predictive equations on several decades of data, then testing the equations on separate years not included in the development of the equations (Hastenrath 1995). For example, Hastenrath et al. (1995) developed their

\footnotetext{
${ }^{11} \mathrm{~A}$ population is the set representing all measurements of interest to the sample collector. A sample is a subset of measurements selected from the population of interest. It is hoped that the data sample adequately represents the population, but this often is not the case due to data sparsity.
} 
equations for predicting summer rainfall over South Africa on the years 1954-78, and tested their scheme on the years 1979-1993.

This "grouping" verification approach is done for TIPS, with regression applied on $\Delta V_{\max }$ in $12,24,36$, and 48 - $\mathrm{h}$ forecast intervals in a three-year period. The validation of the scheme is done in a separate one-year period. The regression equations are built on the years 1984-1986, and the accuracy of the empirical scheme is tested on independent data for 1983. 1984-1986 was chosen because these years contain the most data compared to other three-year subsets. It is very likely that these three years do not represent a population, and that the forecast validation from one independent year may not represent other independent years, but this approach avoids the many criticisms of cross-validation which currently exist. The forecast errors will be compared to the 24 and 48 -h errors from JTWC as a means to assess TIPS' accuracy against an operational setting.

\subsubsection{Summary remarks on the regression technique}

Stepwise regression with normalized coefficients is a robust technique, as subjective interpretation of important predictors is minimized through strict quantitative assessment of "significant" variables. The stepwise technique, modified to reduce artificial skill, autocorrelation, and multicollinearity, yields a few important predictors so as to not "over-fit" the regression. However, the statistical formulation doesn't just give a forecast equation. Equally as important, the normalization scheme allows understanding of each predictor's importance relative to other predictors and other forecast periods. The normalization also generates "threshold values" useful to operational forecasters which may be used as "rules of thumb." The regression equations are derived from three years of data, and the forecast validation is tested on an independent year, thereby avoiding the questions surrounding cross-validation. TIPS' 24 and 48-h predictions are compared to JTWC's forecast errors.

\subsection{Regression results}

\subsubsection{The significant predictors}

After the filtering and stepwise procedures are completed, 5 to 6 significant predictors are chosen. Table 8.4 shows the normalized regression coefficients $c_{i}$ from Eq. (8.16) for 
the selected predictors during the $12,24,36$, and 48 -h forecast intervals. The chosen predictors are schematically shown in Fig. 8.2.

Table 8.4: Normalized regression coefficients for the combined climatological, persistence, satellite, and synoptic data for the TIPS forecast periods of $12,24,36$, and $48 \mathrm{~h}$. Variables not important at the $99 \%$ level are labeled NA (not applicable); $R^{2}$ (the coefficient of determination) is the percent of the total variance explained by the regression; $\mathcal{R}^{2}$ is the estimated population variance (see text); $n$ is the sample size for 1984-1986.

\begin{tabular}{|l|rrrr|}
\hline Variable coefficients $\left(c_{i}\right)$ & 12 & 24 & 36 & 48 \\
\hline \hline 1) POT & +0.46 & +0.60 & +0.67 & +0.68 \\
\hline 2) PX0455 & +0.27 & +0.30 & +0.30 & +0.26 \\
\hline 3) EYEPER & +0.31 & +0.28 & +0.25 & +0.21 \\
\hline 4) VWS5 & -0.18 & -0.26 & -0.29 & -0.27 \\
\hline 5) DPX0165 & +0.16 & NA & NA & NA \\
\hline 6) VSPD & +0.09 & +0.14 & +0.13 & NA \\
\hline 7) LONG & NA & NA & NA & +0.10 \\
\hline \hline$R^{2}(\%)$ & 46.5 & 52.2 & 59.0 & 60.0 \\
\hline $\mathcal{R}^{2}(\%)$ & 46.5 & 52.2 & 59.0 & 59.9 \\
\hline $\mathrm{n}$ & 611 & 530 & 459 & 397 \\
\hline
\end{tabular}

Predictors

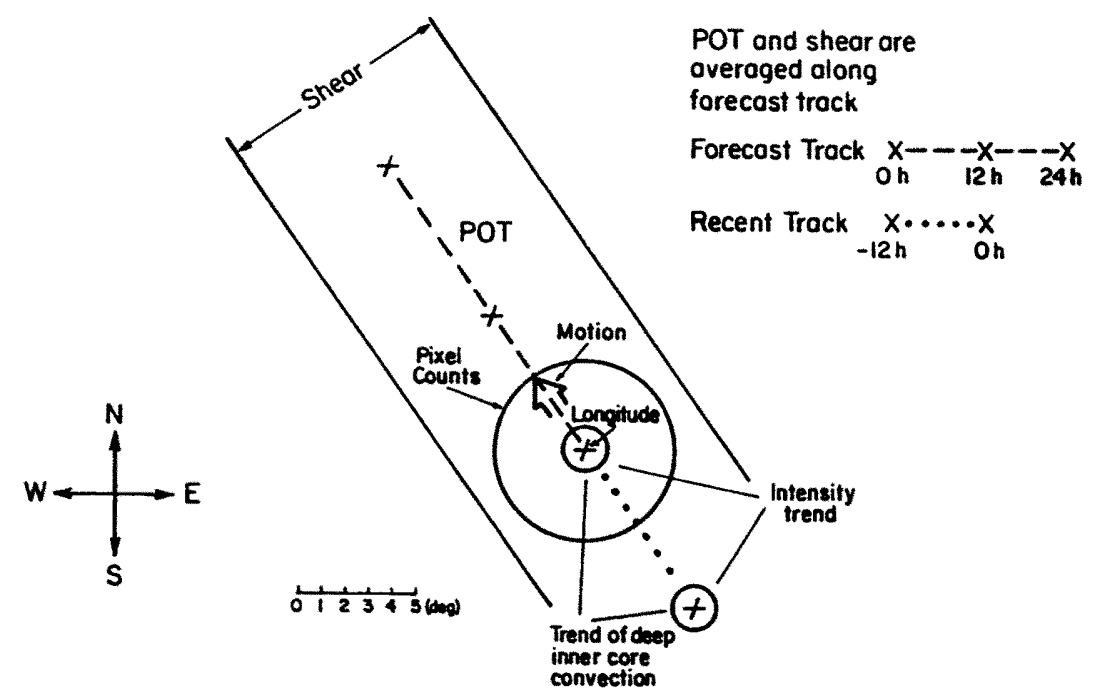

Figure 8.2: General schematic of chosen predictor for TIPS, which are defined in Tables 8.1, 8.2, 8.3, and 8.4. Example 12 and 24-h tracks are shown. Persistence, POT, 5-deg areally averaged wind shear, and 0-4 deg convection are used for all forecast periods $(12,24,36$, and $48-\mathrm{h})$. Inner-core (0-1 deg) convective trend is used in the a 12 -h forecast, meridional storm motion is used in the 12,24 , and $36-\mathrm{h}$ forecasts, and longitude is used in the $48-\mathrm{h}$ prediction. 
Correlation matrix calculations show that multicollinearity is minimal, as correlations between predictors are 0.34 or less (not shown). A statistical quantity called the variance inflation factor (VIF) was also computed, which measures the inflation factor of the regression coefficient due to multicollinearity. For example, if VIF $=6$, then the coefficient is six times what it would be if no multicollinearity exists. VIF values for the chosen TIPS' variables are 1.2 or less (not shown). Therefore, each predictor indeed contains a unique relationship to $\Delta V_{\max }$.

The predictors in Table 8.4 are ordered by the magnitude of the regression coefficient. The four most critical predictors are (listed in importance): POT, PX0455, EYEPER, and VWS5. This shows that including satellite information is a crucial component in forecasting intensity change. The fact that the SST term is most important is not surprising, but now its significance has been quantitatively measured. POT is 1.5 times more important than EYEPER and PX0455 at $12 \mathrm{~h}$, and 2 to 3 times more important than the other parameters. POT becomes more important for the longer forecast times (from +0.46 at $12 \mathrm{~h}$ to +0.68 at $48 \mathrm{~h}$ ), and its coefficient is 2 to 5 times larger than other predictors. In general, it can be stated that SST is 2 to 3 times more important than any other predictor. DeMaria and Kaplan (1994b) reached similar conclusions with SHIPS, although TIPS' coefficients tend to be larger. This suggests that POT might be more important in the western North Pacific than the Atlantic. However, it might also be an artifact of SHIPS using climatological SSTs rather than observed SSTs, and hence SHIPS might be missing some valuable information on SST anomalies.

The importance of PX0455 does not change much with forecast interval, and it is the second most important predictor except for the eye/persistence term at $12 \mathrm{~h}$ (which becomes less consequential with time). It is hypothesized that convection out to $4 \mathrm{deg}$ embodies several influential processes which relate to intensity change. First, it is a measure of deep inner-core convection (within 0-2 deg). Second, it is a measure of cloud symmetry - asymmetric clouds or lack of large-scale cloudiness are signs of an unhealthy storm, usually due to an unfavorable environment (shear or dry air intrusion) or due to movement over colder water. Third, the "spreading out" of cloudiness is a sign of a vigorous 
secondary circulation. Dvorak (1984) shows that a Central Dense Overcast (CDO) tends to form right before hurricane intensity is reached, and persists during the storm's evolution as long as other conditions are favorable. In addition to these physical processes, the correlation with 4 deg may also be due to occasional errors in the location of the TC center.

Wind shear averaged over a 5-deg area is equally as important as convection from 24 to $48 \mathrm{~h}$. The importance of areally average shear dictates that shear on the edge of a TC apparently affects intensity change. It probably also signifies that a TC is about to enter a $200-\mathrm{mb}$ westerly (or strong easterly) wind regime, or that westerly (or strong easterly) winds are about to traverse over a TC. Furthermore, area averaging reduces the chance of one erroneous wind observation contaminating the true shear environment, such as a bad data point or outflow from the outflow misrepresenting the calculation. Using area averaging also removes the circulation of an anticyclone above a well-developed TC, which is beneficial because the anticyclone could contaminate shear calculations.

Other predictors are only significant at certain forecast times. At $12 \mathrm{~h}$, the DPX0165 term is the fifth most important term. Since DPX0165's components are the 12-h tendency of $T_{b}<-65^{\circ} \mathrm{C}$ in a $0-1-\mathrm{deg}$ area, it is a measure of deep inner-core convective change. The other two predictors are climatology terms. At $48 \mathrm{~h}$, the storm longitude is a useful intensity change indicator. This is opposite of SHIPS, which finds LONG only to be important at 12 and $24 \mathrm{~h}$, which indicates that the Pacific and Atlantic basins contain different climatologies.

A surprising finding is that the other climatology variable - meridional storm speed (VSPD) - is a significant and positive contributor to intensity change. This somewhat bewildering discovery contrasts SHIPS, which finds VSPD to be insignificant in the Atlantic. Once again, the basins exhibit different climatology. Furthermore, one would expect VSPD to be a negative influence if it is significant, since a fast northward motion takes a TC over colder waters and high shear - but this is not the case in the western North Pacific.

A review of the best-track data and the annual JTWC TC summaries helps explain the positive relationship. The predicted intensity variable $V_{\max }$ contains the storm motion 
component, and in some recurving situations may strongly counteract the weakening influence of colder SSTs and wind shear if the storm is moving fast. One example is Typhoon Marge (1983), which achieved unusually fast movement upon recurvature (JTWC 1992a). $V_{\text {max }}$ on 2254 UTC 6 November was $90 \mathrm{kts}$, but its speed of movement was $54 \mathrm{kts}$ ! Marge was actually barely a tropical storm, but due to its motion contribution still contained hurricane-force winds. Why VSPD is chosen instead of SPD in TIPS is a bit perplexing, but perhaps the zonal component is insignificant, hence SPD is also rejected. A correlation analysis between SPD and VSPD reveals a variance of only $13 \%$.

To compliment these findings, the partial correlations for each significant variable are shown in Table 8.5. The partial correlation $\left(r_{p}\right)$ measures how much variance is explained

Table 8.5: Partial correlations for the TIPS forecast periods of 12, 24, 36, and $48 \mathrm{~h}$. Partial correlations measure how much variance is explained by one predictor when the other predictors are "held constant." Variables not important at the $99 \%$ level are labeled NA (not applicable).

\begin{tabular}{|l|rrrr|}
\hline Partial correlations & 12 & 24 & 36 & 48 \\
\hline \hline 1) POT & +0.50 & +0.63 & +0.69 & +0.71 \\
\hline 2) PX0455 & +0.33 & +0.39 & +0.42 & +0.37 \\
\hline 3) EYEPER & +0.37 & +0.36 & +0.34 & +0.31 \\
\hline 4) VWS5 & -0.22 & -0.33 & -0.39 & -0.38 \\
\hline 5) DPX0165 & +0.20 & NA & NA & NA \\
\hline 6) VSPD & +0.11 & +0.18 & +0.19 & NA \\
\hline 7) LONG & NA & NA & NA & +0.16 \\
\hline
\end{tabular}

by one predictor when the other predictors are "held constant" (Edwards 1984). ${ }^{12}$ The same patterns in Table 8.4 persist in Table 8.5. This alternate perspective shows that,

\footnotetext{
${ }^{12}$ In this report, IMSL subroutine "PCORR" is used to compute partial correlations. This statistical approach can be explained by first defining the "total" correlation coefficient $r_{12}$ as the correlation between variables $x_{1}$ and $x_{2}$. When a third variable $x_{3}$ is included in the analysis, a "partial" correlation coefficient can be computed between $x_{1}$ and $x_{2}$ when the effect of $x_{3}$ has been removed, and is denoted $r_{12.3}$. This coefficient is obtained by correlating $\tilde{x}_{1}-\tilde{b}_{13} \tilde{x}_{3}$ with $\tilde{x}_{2}-\tilde{b}_{23} \tilde{x}_{3}$ where $\tilde{x}_{1}, \tilde{x}_{2}$, and $\tilde{x}_{3}$ are the deviations of the three variables from their respective mean values and $\tilde{b}_{i j}$ is the slope of the regression line between $\tilde{x}_{i}$ and $\tilde{x}_{j}$ (Brooks and Carruthers 1978). In this manner, the effect of $x_{3}$ on $x_{2}$ and $x_{1}$ has been removed. Substituting these expressions into standard correlation and slope equations gives

$$
r_{12.3}=\frac{r_{12}-r_{13} r_{23}}{\left[\left(1-r_{13}^{2}\right)\left(1-r_{23}^{2}\right)\right]^{0.5}}
$$

Other combinations are possible. For example, $x_{2}$ can be "held constant" while the partial correlation between $x_{1}$ and $x_{3}$ is computed $\left(r_{13.2}\right)$. Similarly, if $r_{12.34}$ is the partial correlation coefficient between $x_{1}$
} 
for the same persistence, convection, wind shear, and climatology values, a change in SST along the tracks will have the most profound effects, with $r_{p}$ ranging from 0.5 to 0.71 ( 25 to $49 \%$ variance). Similarly, for other predictors held fixed, the variance explained by EYEPER is about $9-13 \%$, for PX0455 11-18\%, and for VWS5 5-15\% while other variables affect the partial variance less.

The total variance $\left(R^{2}\right)$ of intensity change explained by TIPS is $46.3 \%$ at $12 \mathrm{~h}$, and steadily increases with each forecast interval to $60 \%$ (Table 8.4). The variance increase is due to several reasons. First, this variance increase with forecast time represents the growing importance of the SST along the storm track in explaining $\Delta V_{\max }$. Second, this increase might result from the 5-kts discretization of $V_{\max }$ in the besttrack data set (DeMaria and Kaplan 1994b). As seen in Appendix B, $\sigma_{y}$ is $10 \mathrm{kts}$ for intensity change in $12 \mathrm{~h}$, and increases to $28 \mathrm{kts}$ at $48 \mathrm{~h}$. However, the mean $\Delta V_{\max }$ only slightly increases from $1.5 \mathrm{kts}$ to $5 \mathrm{kts}$, respectively. This $5 \mathrm{kts}$ truncation makes explaining $R^{2}$ more difficult at shorter forecast intervals since $\sigma_{y}$ is within the noise level. ${ }^{13}$ Finally, the lower $R^{2}$ may indicate that inner-core processes which cannot be resolved on the synoptic scale complicate short-term intensity changes. It should be noted that less explained variance at $12 \mathrm{~h}$, when compared to $48 \mathrm{~h}$, does not correspond to larger wind forecast errors at $12 \mathrm{~h}$, as will be seen in a moment.

Since TIPS is developed on a data sample where the true population is not known, it is possible that the variance explained is overestimated. The estimated "population $R$ " $(\mathcal{R})$ has been derived by Mielke et al. (1995) as:

$$
\mathcal{R}=\min \left[R, \max \left\{0,1-\left(\frac{\ln R}{\min \left(-10^{-50}, \mu_{1} d f^{0.27}+\mu_{2} d f^{0.32}+\mu_{3} d f^{0.37}\right)}\right)^{\frac{1}{1.32}}\right\}\right]
$$

and $x_{2}$ while keeping $x_{3}$ and $x_{4}$ "constant," then

$$
r_{12.34}=\frac{r_{12.4}-r_{13.4} r_{23.4}}{\left[\left(1-r_{13.4}^{2}\right)\left(1-r_{23.4}^{2}\right)\right]^{0.5}}=\frac{r_{12.3}-r_{14.3} r_{24.3}}{\left[\left(1-r_{14.3}^{2}\right)\left(1-r_{24.3}^{2}\right)\right]^{0.5}}
$$

The partial correlation calculations involving more than 4 variables requires somewhat complicated linear algebra, and is explained by Maindonald (1984) and Brooks and Carruthers (1978).

\footnotetext{
${ }^{13}$ Since $\sigma_{y}$ is slightly smaller in the Atlantic than the western North Pacific, this implies that $R^{2}$ should automatically be a little higher in TIPS than SHIPS since there is more $\Delta V_{\text {max }}$ variance to explain in the western North Pacific (M. DeMaria, personal communication 1994).
} 
where $\mu_{1}=155.60230, \mu_{2}=-279.97996$, and $\mu_{3}=124.79452$. The degrees of freedom $d f$ is computed as $d f=n-p-1$, where $n$ is the number of cases and $p$ is the number of predictors. As can be seen in Table 8.4, the true variance explained by TIPS is approximately $R^{2}$.

A discouraging finding is that $55 \%$ to $40 \%$ of the variance is still unexplained by TIPS. This accentuates the difficulty of forecasting intensity change. There is still much room for improvement in TC intensity forecasting. However, the utilization of quantitative satellite data seems promising, and its potential will become more evident as this chapter continues.

Furthermore, multiple regression is a better approach than single variable linear regression. This is intuitively obvious, as this report shows that several components must be considered when making a TC intensity forecast. To highlight these differences, Table 8.6 shows the variance explained in a linear, one variable regression model $\left(r^{2}\right)$. The variance explained by a single variable is astonishing low. Even POT only explains $15-38 \%$ by itself.

Table 8.6: Variance explained in a linear, one independent variable regression model for the forecast periods of $12,24,36$, and $48 \mathrm{~h}$. Whether a variable is a positive or negative correlation is indicated. Variables not important at the $99 \%$ level in the multiple regression analysis are labeled NA (not applicable).

\begin{tabular}{|l|rrrr|}
\hline Single predictor variance & 12 & 24 & 36 & 48 \\
\hline \hline 1) POT & +15.4 & +23.7 & +32.1 & +38.1 \\
\hline 2) PX0455 & +10.8 & +7.9 & +5.6 & +3.0 \\
\hline 3) EYEPER & +11.2 & +7.2 & +4.1 & +1.6 \\
\hline 4) VWS5 & -9.9 & -11.1 & -12.9 & -13.0 \\
\hline 5) DPX0165 & +9.2 & NA & NA & NA \\
\hline 6) VSPD & +2.8 & +1.3 & +0.9 & NA \\
\hline 7) LONG & NA & NA & NA & +1.4 \\
\hline
\end{tabular}

The other variables explain even less variance. In fact, some values of $r^{2}$ are so low (such as for LONG, VSPD, EYEPER, and PX0455) they would be dismissed as unimportant if considered by themselves. This is one of the true benefits of multiple regression - in the stepwise procedure, a variable is chosen which explains a certain amount of variance, and in the next step another variable is chosen which helps explain a percentage of the 
remaining variance, and so on. Therefore, a variable which by itself appears to not account for much variance may actually explain "leftover" variance once other predictors are included.

\subsubsection{Threshold values}

Table 8.7 shows the mean predictor value $\bar{x}_{i}$ from Eq. (8.16) which can be interpreted (to a first approximation) as threshold values. Unlike $c_{i}$, these do not vary much for

Table 8.7: Mean value of predictors for the TIPS forecast periods of $12,24,36$, and $48 \mathrm{~h}$. Variables not important at the $99 \%$ level are labeled NA (not applicable). The units are $\mathrm{m} \mathrm{s}^{-1}$ for POT, VWS5, and VSPD, percent for PX0455 and DP0165, and deg E for LONG.

\begin{tabular}{|l|rrrr|}
\hline Variable mean & 12 & 24 & 36 & 48 \\
\hline \hline 1) POT & 38.9 & 38.8 & 38.9 & 39.0 \\
\hline 2) PX0455 & 24.6 & 25.5 & 26.2 & 26.8 \\
\hline 3) EYEPER & 0.37 & 0.38 & 0.39 & 0.40 \\
\hline 4) VWS5 & 8.5 & 8.4 & 8.4 & 8.5 \\
\hline 5) DPX0165 & -0.9 & NA & NA & NA \\
\hline 6) VSPD & 2.2 & 2.0 & 1.8 & NA \\
\hline 7) LONG & NA & NA & NA & 137.3 \\
\hline
\end{tabular}

different forecast intervals. Therefore, as "rules of thumb," the following statements can be made:

1. If $\mathrm{POT}$ is greater (less) than $39 \mathrm{~m} \mathrm{~s}^{-1}$, intensification (weakening) is favored.

2. If PX0455 is greater (less) than 25\%, intensification (weakening) is favored.

3. If VWS5 is less (greater) than $8.5 \mathrm{~m} \mathrm{~s}^{-1}$, intensification (weakening) is favored.

4. If deep convection within 0-1-deg of the TC center has increased (decreased) the past $12 \mathrm{~h}$, then intensification (weakening) is favored the next $12 \mathrm{~h}$.

5. If the meridional component in a recurving storm is greater than $2 \mathrm{~m} \mathrm{~s}^{-1}$, then $\Delta V_{\max }$ will be less negative in the next $36 \mathrm{~h}$ due to the translation speed offsetting the weakening tendency. 
6. If a TC is east (west) of $137.3 \mathrm{deg} \mathrm{E}$, then intensification is favored (less likely) in the next $48 \mathrm{~h}$.

These conclusions will be discussed in more detail in the next subsection. However, there are some interesting issues here.

First, the 5.0-deg areally averaged wind shear threshold of $8.5 \mathrm{~m} \mathrm{~s}^{-1}$ is less than the $12.5 \mathrm{~m} \mathrm{~s}^{-1}$ threshold for "point shear" used by Zehr (1992) in his tropical cyclogenesis study. It should be mentioned that Zehr's threshold was determined observationally, and that a statistical analysis may yield a different shear threshold for genesis to be possible. Furthermore, the SHIPS' threshold shear value is virtually the same $8.5 \mathrm{~m} \mathrm{~s}^{-1}$ as TIPS' (M. DeMaria, personal communication 1995). Therefore, it is safe to suggest that this shear criteria for TC intensity change is probably representative of all tropical basins.

Second, the fact that TCs east of $137.3 \mathrm{deg}$ E are favored to develop is consistent with the climatology of rapid deepeners discussed by Mundell (1990). Third, the POT threshold of $39 \mathrm{~m} \mathrm{~s}^{-1}$ is a statement that most storms do not reach their MPI, as has been discussed in Chapter 2. However, this also presents forecast problems, as this term will tend to underforecast fast developers unless the other terms compensate. As will be seen, TIPS is competitive with JTWC in predicting intensification, but still underforecasts fast intensification changes, perhaps because POT skews $\Delta V_{\max }$ toward lesser values. Kubat (1995) has recently computed MPI values as a function of location and time of year, and it is recommended that his stratified MPI values be investigated for predictive potential in the future, and to assess whether these values might lessen the underforecasting problem.

It also needs to be emphasized that threshold values should not be considered separately, but collectively as a group. This point has already been discussed in the comparison of single and multiple regression, but this issue will be stressed again. Just because POT is favorable, intensification will not occur unless a majority of the other threshold values (like favorable shear and 0-4 deg convection) are either neutral or surpassed.

\subsubsection{Complete information on TIPS}

The full TIPS package, complete with the sample coefficients, means, and standard deviations, are shown in Appendix B. Also shown are variations of TIPS with single point 
shear (VWSPT), and shear based on persistence, not perfect-prog. These versions may be more applicable under operational forecasting situations, although the variance explained decreases a little (to be discussed later).

It is also possible that TIPS could be used in other basins, but its suitability is questionable due to different tropopause heights which might require different pixel count predictors. Furthermore, other basins contain different climatologies and different storm histories. The BMRC data was limited to a region bounded by $45 \mathrm{deg} \mathrm{N}$ and 70-180 deg $\mathrm{E}$, and using VWS5 further restricts the area of analysis to $40 \mathrm{deg} \mathrm{N}$ and 75-175 deg E. As pointed out by Aczel (1989), extrapolating outside the regime for which the regression is developed is risky, as the estimated relationship may not be appropriate outside this range.

TIPS should be used as guidance only, as there is still considerable unexplained variance. However, as will be discussed, this writer will conservatively claim that TIPS is competitive with other TC intensity change forecast products. It is hoped that with refinements using improved predictors and other statistical techniques, a more polished version of TIPS will result in the future.

\subsubsection{Predictor contributions to intensity change stratifications}

Another feature that can be studied is how a predictor contributes to different magnitudes of $\Delta V_{\max }$. To study these stratifications, $\Delta V_{\max }$ will be divided into 5 categories: 1) Fast intensifying; 2) Slowly intensifying; 3) Quasi steady-state; 4) Slowly weakening; and 5) Fast weakening. The magnitude of $\Delta V_{\max }$ which qualifies for these categories have been intuitively chosen, and are dependent on the forecast interval of interest - for example, the designation for a 12 - $\mathrm{h}$ fast intensifying storm is $\Delta V_{\max } \geq 15 \mathrm{kts}$, and for $48 \mathrm{~h}$ is $\Delta V_{\max } \geq 45 \mathrm{kts}$. The contributions of the predictors to different intensification classes are measured in three ways:

1. average predictor value for a class (designated as $\overline{x c}$ );

2. average Normalized Standardized Deviation $([\overline{x c}-\bar{x}] / \sigma)$ for a class, designated as $N S D ;$ and 
3. whether $\overline{x c}$ is a positive influence ( $\mathrm{P}$; (sign of regression coefficient) $\times N S D \geq 0.2$ ), negative influence $(\mathrm{N}$; ( sign of regression coefficient) $\times N S D \leq-0.2)$, or "zero" influence $(\mathrm{Z} ; N S D<|0.2|)$ in a class.

This methodology is shown in Tables $8.8,8.9,8.10$, and 8.11 for the $12,24,36$, and 48 -h analysis, respectively. Of course, to fully understand a variable's contribution to each intensification class, one needs to multiply $N S D$ by the magnitude of the corresponding regression coefficient, but this type of analysis is more confusing, and inspection by the author showed that the following conclusions were not altered.

Table 8.8: Normalized predictors (and their true numerical values in parenthesis) for different intensification regimes (kts) during the 12-h periods for 1984-1986. "P" denotes a positive contribution to intensification for that stratification, while " $\mathrm{N}$ " denotes a negative contribution. " $\mathrm{Z}$ " denotes zero contribution, defined as when the normalized standard deviation is less than the absolute value of 0.2 . Details are contained in the text. The units are $\mathrm{m} \mathrm{s}^{-1}$ for POT, VWS5, and VSPD, while PX0455 and DP0165 are in percent.

\begin{tabular}{|l|c|c|c|c|c|}
\hline Variables & $\Delta V \geq 15$ & $10 \geq \Delta V \geq 5$ & $\Delta V=0$ & $-5 \geq \Delta V \geq-10$ & $\Delta V \leq-15$ \\
\hline 1) POT & $+0.23(42.2) \mathrm{P}$ & $+0.42(44.8) \mathrm{P}$ & $+0.10(40.4) \mathrm{z}$ & $-0.47(32.3) \mathrm{N}$ & $-1.04(24.1) \mathrm{N}$ \\
\hline 2) PX0455 & $+0.70(35.7) \mathrm{P}$ & $+0.16(27.2) \mathrm{z}$ & $-0.09(23.1) \mathrm{z}$ & $-0.33(19.4) \mathrm{N}$ & $-0.51(16.6) \mathrm{N}$ \\
\hline 3) EYEPER & $+0.53(0.62) \mathrm{P}$ & $+0.24(0.48) \mathrm{P}$ & $-0.12(0.31) \mathrm{z}$ & $-0.33(0.21) \mathrm{N}$ & $-0.54(0.11) \mathrm{N}$ \\
\hline 4) VWS5 & $-0.33(6.9) \mathrm{P}$ & $-0.24(7.4) \mathrm{P}$ & $+0.04(8.7) \mathrm{z}$ & $+0.21(9.6) \mathrm{N}$ & $+0.95(13.2) \mathrm{N}$ \\
\hline 5) VSPD & $-0.03(2.1) \mathrm{z}$ & $-0.14(1.8) \mathrm{z}$ & $-0.10(1.9) \mathrm{Z}$ & $+0.10(2.5) \mathrm{Z}$ & $+0.69(4.0) \mathrm{P}$ \\
\hline 6) DPX0165 & $+0.31(8.1) \mathrm{P}$ & $+0.31(8.1) \mathrm{P}$ & $-0.26(-8.5) \mathrm{N}$ & $-0.22(-7.3) \mathrm{N}$ & $-0.69(-20.8) \mathrm{N}$ \\
\hline
\end{tabular}

Table 8.9: Normalized predictors (and their true numerical values in parenthesis) for different intensification regimes (kts) during the 24 -h periods for 1984-1986. "P" denotes a positive contribution to intensification for that stratification, while " $\mathrm{N}$ " denotes a negative contribution. " $\mathrm{Z}$ " denotes zero contribution, defined as when the normalized standard deviation is less than the absolute value of 0.2. Details are contained in the text. The units are $\mathrm{m} \mathrm{s}^{-1}$ for POT, VWS5, and VSPD, while PX0455 is in percent.

\begin{tabular}{|l|c|c|c|c|c|}
\hline Variables & $\Delta V \geq 25$ & $20 \geq \Delta V \geq 10$ & $5 \geq \Delta V \geq-5$ & $-10 \geq \Delta V \geq-20$ & $\Delta V \leq-25$ \\
\hline 1) POT & $+0.38(44.2) \mathrm{P}$ & $+0.54(46.6) \mathrm{P}$ & $+0.04(39.3) \mathrm{z}$ & $-0.54(31.0) \mathrm{N}$ & $-1.33(19.6) \mathrm{N}$ \\
\hline 2) PX0455 & $+0.78(37.9) \mathrm{P}$ & $+0.03(26.0) \mathrm{z}$ & $-0.12(23.7) \mathrm{z}$ & $-0.27(21.3) \mathrm{N}$ & $-0.19(22.6) \mathrm{N}$ \\
\hline 3) EYEPER & $+0.51(0.63) \mathrm{P}$ & $+0.07(0.41) \mathrm{z}$ & $+0.02(0.39) \mathrm{z}$ & $-0.32(0.23) \mathrm{N}$ & $-0.26(0.26) \mathrm{N}$ \\
\hline 4) VWS5 & $-0.33(7.0) \mathrm{P}$ & $-0.28(7.2) \mathrm{P}$ & $+0.01(8.5) \mathrm{z}$ & $+0.25(9.6) \mathrm{N}$ & $+0.90(12.5) \mathrm{N}$ \\
\hline 5) VSPD & $-0.04(1.8) \mathrm{z}$ & $-0.04(1.9) \mathrm{z}$ & $-0.07(1.8) \mathrm{z}$ & $+0.00(2.0) \mathrm{z}$ & $+0.53(3.2) \mathrm{P}$ \\
\hline
\end{tabular}

By the nature of regression, one would expect that, on average, there would be positive contributions to the intensification classes, and negative influences on the weakening 
Table 8.10: Normalized predictors (and their true numerical values in parenthesis) for different intensification regimes (kts) during the 36 -h periods for $1984-1986$. "P" denotes a positive contribution to intensification for that stratification, while "N" denotes a negative contribution. " $\mathrm{Z}$ " denotes zero contribution, defined as when the normalized standard deviation is less than the absolute value of 0.2. Details are contained in the text. The units are $\mathrm{m} \mathrm{s}^{-1}$ for POT, VWS5, and VSPD, while PX0455 is in percent.

\begin{tabular}{|l|c|c|c|c|c|}
\hline Variables & $\Delta V \geq 35$ & $30 \geq \Delta V \geq 15$ & $10 \geq \Delta V \geq-10$ & $-15 \geq \Delta V \geq-30$ & $\Delta V \leq-35$ \\
\hline \hline 1) POT & $+0.56(47.1) \mathrm{P}$ & $+0.50(46.1) \mathrm{P}$ & $+0.12(40.7) \mathrm{z}$ & $-0.71(28.4) \mathrm{N}$ & $-1.67(14.3) \mathrm{N}$ \\
\hline 2) PX0455 & $+0.78(38.5) \mathrm{P}$ & $+0.04(26.8) \mathrm{Z}$ & $-0.18(23.4) \mathrm{Z}$ & $-0.13(24.1) \mathrm{z}$ & $-0.24(22.3) \mathrm{N}$ \\
\hline 3) EYEPER & $+0.43(0.60) \mathrm{P}$ & $+0.09(0.44) \mathrm{Z}$ & $-0.04(0.38) \mathrm{Z}$ & $-0.22(0.28) \mathrm{N}$ & $-0.29(0.25) \mathrm{N}$ \\
\hline 4) VWS5 & $-0.43(6.6) \mathrm{P}$ & $-0.32(7.1) \mathrm{P}$ & $+0.01(8.4) \mathrm{z}$ & $+0.38(10.0) \mathrm{N}$ & $+0.93(12.2) \mathrm{N}$ \\
\hline 5) VSPD & $-0.17(1.5) \mathrm{z}$ & $+0.05(1.9) \mathrm{Z}$ & $-0.08(1.6) \mathrm{Z}$ & $+0.01(1.8) \mathrm{z}$ & $+0.67(3.2) \mathrm{P}$ \\
\hline
\end{tabular}

Table 8.11: Normalized predictors (and their true numerical values in parenthesis) for different intensification regimes (kts) during the $48-\mathrm{h}$ periods for 1984-1986. "P" denotes a positive contribution to intensification for that stratification, while " $\mathrm{N}$ " denotes a negative contribution. " $\mathrm{Z}$ " denotes zero contribution, defined as when the normalized standard deviation is less than the absolute value of 0.2 . Details are contained in the text. The units are $\mathrm{m} \mathrm{s}^{-1}$ for POT and VWS5, percent for PX0455, and deg E for LONG.

\begin{tabular}{|l|c|c|c|c|c|}
\hline Variables & $\Delta v \geq 45$ & $40 \geq \Delta V \geq 20$ & $15 \geq \Delta V \geq-15$ & $-20 \geq \Delta V \geq-40$ & $\Delta V \leq-45$ \\
\hline \hline 1) POT & $+0.69(49.2) \mathrm{P}$ & $+0.56(47.2) \mathrm{P}$ & $+0.05(39.8) \mathrm{z}$ & $-0.84(26.7) \mathrm{N}$ & $-2.1(7.8) \mathrm{N}$ \\
\hline 2) PX0455 & $+0.69(37.6) \mathrm{P}$ & $+0.04(27.5) \mathrm{z}$ & $-0.15(24.4) \mathrm{z}$ & $-0.04(26.2) \mathrm{z}$ & $-0.18(24.0) \mathrm{z}$ \\
\hline 3) EYEPER & $+0.23(0.51) \mathrm{P}$ & $+0.05(0.42) \mathrm{z}$ & $+0.02(0.41) \mathrm{z}$ & $-0.14(0.33) \mathrm{z}$ & $-0.48(0.17) \mathrm{N}$ \\
\hline 4) VWS5 & $-0.53(6.4) \mathrm{P}$ & $-0.35(7.1) \mathrm{P}$ & $+0.06(8.7) \mathrm{z}$ & $+0.47(10.3) \mathrm{N}$ & $+0.66(11.1) \mathrm{N}$ \\
\hline 5) LONG & $+0.41(143.2) \mathrm{P}$ & $+0.04(137.9) \mathrm{z}$ & $-0.09(136.1) \mathrm{z}$ & $-0.12(135.6) \mathrm{z}$ & $+0.27(141.3) \mathrm{N}$ \\
\hline
\end{tabular}


classes, with zero inducements for the quasi steady-state classes. Furthermore, one would expect large $N S D$ differences between fast and slow developing classes. Indeed, these are the general patterns, but there are some notable exceptions which reveal much about TC intensity change.

Recall that a major focus of this study is to discern differences between TCs which develop at a fast rate and those that develop more slowly. Note that the POT $N S D$ and $\overline{x c}$ values are not very different in these classes. For example, at $24 \mathrm{~h}$ (Table 8.9) the fast and slow POT $\overline{x c}$ values are 44.2 and $46.6 \mathrm{~m} \mathrm{~s}^{-1}$, respectively. The slower 12 and 24-h cases have slightly higher POT values because they tend to represent TCs in their formative tropical storm, non-eye stage. At 36 and $48 \mathrm{~h}$ (Tables 8.10 and 8.11), the faster developing cases have slightly larger $\overline{x c}$ POT values than the slow intensification classes. This is because, on average, fast developers are usually over warm water longer than slow developers.

Nevertheless, the key point is that since average POT is not very different between the fast and slow developing cases, POT cannot distinguish whether a TC will develop fast or slowly. To elaborate this point more, bar graphs are plotted in Fig. 8.3 for the 24-h classes, with the 24-h POT sample mean shown as reference. Again, POT is a positive contributor to both classes, but cannot differentiate between the classes.

In stark contrast, the average pixel count values are very different between fast and slow intensification cases. At $24 \mathrm{~h}$, the $N S D$ for PX0455 is 0.75 greater $(x c=37.9 \%$ compared to 26\%), and in fact, on average PX0455 makes zero contribution to intensity change for the slow class. This trend is evident for the other forecast periods as well. Therefore, including pixel count information can distinguish whether a TC will develop at a fast or slow rate, and is a valuable component of TIPS.

This conclusion is further demonstrated in Fig. 8.4 for the 24-h classes, with the 24-h PX0455 sample mean shown as reference. It is clear that abundant CAPE is an integral part in fast developing TCs, while slower developing storms contain less CAPE on average. This supports the arguments presented throughout this report.

Opposite conclusions are reached for the slow and fast weakening cases. While both predictors contribute negatively to the weakening classes, POT is the key predictor 


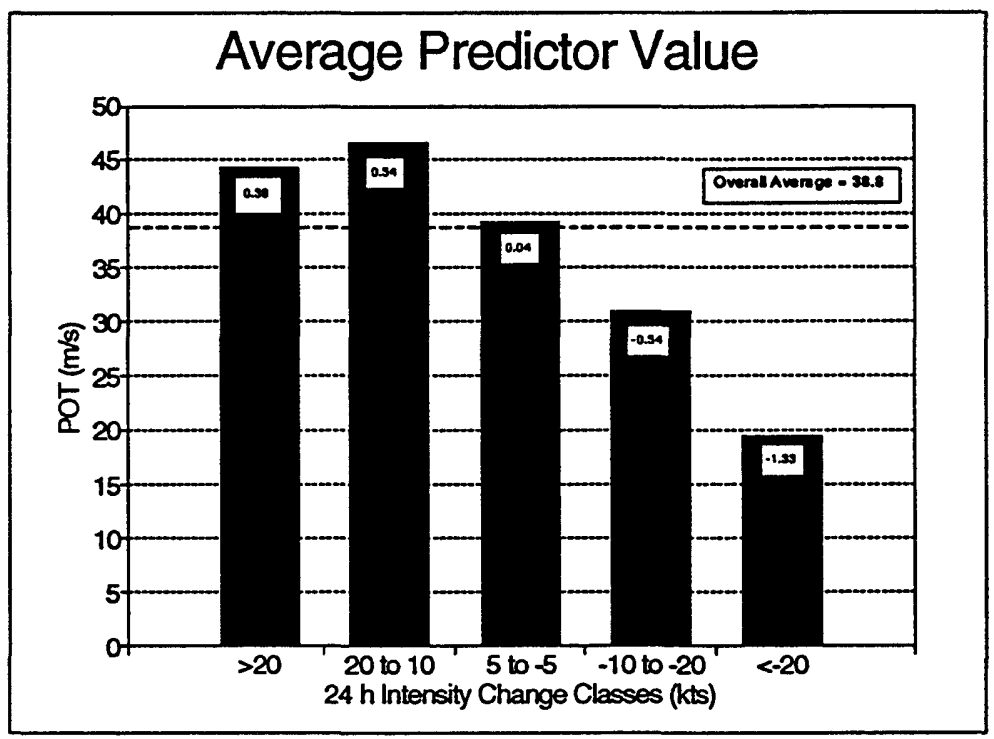

Figure 8.3: Average POT values for different 24-h $\Delta V_{\max }$ classes. Numbers on the bars are normalized standard deviations $(N S D)$ defined by $(\overline{x c}-\bar{x}) / \sigma$, where $\overline{x c}$ is the average POT value for each class and $\sigma$ is POT's standard deviation in the sample. The sample average ( $\bar{x}$; denoted by the dashed line) is shown for comparison.

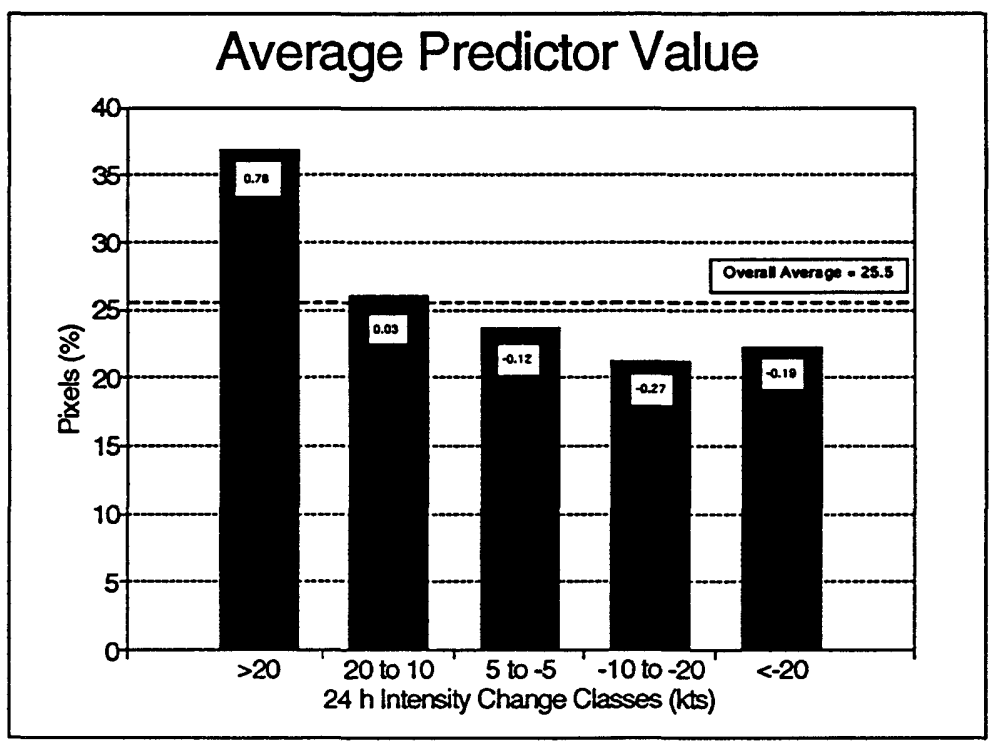

Figure 8.4: Average PX0455 values for different 24-h $\Delta V_{\max }$ classes. Numbers on the bars are normalized standard deviations $(N S D)$ defined by $(\overline{x c}-\bar{x}) / \sigma$, where $\overline{x c}$ is the average PX0455 value for each class and $\sigma$ is PX0455's standard deviation in the sample. The sample average ( $\bar{x}$; denoted by the dashed line) is shown for comparison. 
for differentiating between a fast and slow disintegrating TC; PX0455 does not make this distinction. The average NSD POT difference between fast and slow weakening regimes is dramatically different, with differences of 0.57 for $12 \mathrm{~h}(-0.47$ in slow weakening class compared to -1.04 in fast weakening class) and steadily increasing with the forecast interval to a $N S D$ difference of 1.26 at $48 \mathrm{~h}$ in Table $8.11(-0.84$ in slow weakening class compared to -2.1 in fast weakening class).

Interesting trends can also be inferred about the other predictors. As can be expected, the binary eye/persistence term makes a valuable distinction to all the fast and slow classes at $12 \mathrm{~h}$ and the weakening classes at $48 \mathrm{~h}$. However, EYEPER suffers oscillatory problems at other periods, as evident from the fact that the class mean hovers between 0.4 and 0.5 . It is because of this oscillatory fashion that EYEPER drops in importance from $2 \mathrm{nd}$ to $4 \mathrm{th}$ place after $24 \mathrm{~h}$, and only makes slight contributions in the extreme $\Delta V_{\max }$ classes afterwards.

Wind shear adheres to the same trend as POT, in that the mean values for the fast and slow intensification classes are not appreciatedly different, yet the $N S D$ is markedly different between the fast and slow weakening classes. The following $N S D$ values are observed for slow and fast weakening, respectively: 0.21 compared to 0.95 at $12 \mathrm{~h}, 0.25$ compared to 0.90 at $24 \mathrm{~h}, 0.38$ compared to 0.93 at $36 \mathrm{~h}$, and 0.47 compared to 0.66 at $48 \mathrm{~h}$. Therefore, while low shear is a vital component for intensification, on average it does not distinguish between fast and slow developing TCs. On the other hand, shear is a crucial parameter for differentiating between fast and slow weakeners on average.

The pixel count trend information at $12 \mathrm{~h}$ (DPX0165) portrays a behavior similar to POT and wind shear when stratified by average $N S D$. The fast and slow developing classes contain the same $N S D$ value of 0.31 , but the fast and slow weakening classes exhibit markedly different $N S D$ values. On average, the pixel count trend of deep innercore convection does not differentiate between fast and slow 12-h developers, but does convey knowledge about when fast $V_{\max }$ decrease might occur. If PX0165 has decreased by at least $20 \%$ in the past $12 \mathrm{~h}$, then $V_{\max }$ might 
decrease by $15 \mathrm{kts}$ or more in the next $12 \mathrm{~h}$. It might seem contradictory that DPX0165 supplies no additional insight into intensification behavior given the strong signal of PX0455, but it's not contradictory. This is because the PX0455 term must be considered in conjunction with the pixel count trend - an PX0455 increase from slight cloudiness to moderate cloudiness will correspond to a slow $V_{\max }$ increase, but an increase from moderate cloudiness to heavy cloudiness will correspond to a fast $V_{\max }$ increase. Yet, the inner-core pixel count trend in both cases might be the same (recall that the inner-core signal is embedded in the PX0455 term).

The two climatology predictors also exhibit informative trends once stratified. As suspected earlier, the meridional storm motion only contributes significantly in the fast weakening class, and its role is to retard the rate of weakening during recurvature by superimposing its fast motion on $V_{\max }$. At $48 \mathrm{~h}$, the initial storm longitude makes zero contribution to the slow and steady-state classes on average. The initial storm longitude only contributes significantly to fast developers, with a mean location of $143.2 \mathrm{deg}$ E. Given that other conditions are favorable, TCs in the general vicinity of Guam will experience a $V_{\max }$ increase of at least $45 \mathrm{kts}$ in the next two days.

In summary, except for EYEPER at 12 and $24 \mathrm{~h}$, the observed pixel count information is the critical element in differentiating fast and slow developing TCs, and, as a corollary, rapid intensifiers. Certainly warm SSTs and weak shear provide the necessary environment for fast development, but only after intense convection over a large circular area has been generated - so that the Air-Sea Interaction and Internally Forced Convergence processes are more efficient and eye formation initiated - will accelerated development occur. Large CAPE (convective bursts) indeed appears to be the required agent for fast development. On the other hand, cold SSTs and strong shear appear to be the main factors which cause fast disruption of a TC - a finding which comes as no surprise. 


\subsubsection{Evaluation of TIPS intensity forecasts}

The relative and absolute errors for TIPS on the dependent (1984-86) and independent (1983) data set will be discussed in this section. Relative errors measure forecast bias, whereas absolute errors reflect overall forecast inaccuracy. These errors/biases are computed for different $\Delta V_{\max }$ classes, and as an overall average. The errors are computed as $\Delta V_{\max }$ (observed) minus $\Delta V_{\max }$ (forecast).

These errors will be compared to JTWC's 24 and 48-h official forecast errors. Unfortunately, no 12 and $36-\mathrm{h}$ forecasts are made by JTWC for a similar comparison. Therefore, the 24 and 48-h errors will be concentrated on, and the others will be briefly discussed at the end of the section. The comparisons are qualitatively similar anyway.

Tables 8.12 and 8.13 show the 24 -h relative and absolute errors, respectively for both 1984-86 and 1983. Comparing how TIPS performs against JTWC in 1984-86 is not an accurate assessment between the two forecasts, since TIPS was developed on the dependent data set - and because TIPS uses perfect-prog forecasts of shear and track - but it does serve as a baseline comparison.

Table 8.12 indicates that both forecasts (JTWC and TIPS) suffer the same biases in 1984-86. Fast intensification is underforecast, slow intensification and quasi steady-state are generally well-anticipated (residuals $3 \mathrm{kts}$ or less), forecast $\Delta V_{\max }$ is less in magnitude than observed $\Delta V_{\max }$ for slow weakening, and is much less in magnitude for fast weakening. On the independent data set, this general trend in the biases continues, although TIPS fairs better in the fast intensification, slow intensification, and fast weakening classes and worse in the other classes - than JTWC.

Table 8.13 presents a slightly different perspective, since this shows the total error. The absolute error between JTWC and TIPS in the fast intensification class is equal in 1984-86, and JTWC is slightly better in 1983 for the slow weakeners. Otherwise, all other parameters in 1984-86 and 1983 show less error for TIPS, indicating that TIPS is closer than JTWC, on average, to the observed $\Delta V_{\max }$ despite the biases. It is interesting that TIPS' errors are less than JTWC on the independent set compared to the dependent data set, as usually such contrasts are less clear on the independent set. This indicates that 
1983 was a tougher forecast year, which is also suggested by the much higher errors compared to 1984-86. Indeed, in 1983 Typhoons Abby and Marge experienced wind increases of $60 \mathrm{kts} \mathrm{d}^{-1}$, Wayne underwent deepening of $75 \mathrm{kts} \mathrm{d}^{-1}$, and Forrest deepened at an incredible rate of $85 \mathrm{kts} \mathrm{d}^{-1}$ on 00 UTC 22 September 1983. 1984-86 contained several rapid intensifiers but the majority contained wind increases of 45-50 $\mathrm{kts} \mathrm{d}^{-1}$ (Mundell 1990). The fact that TIPS beats JTWC in 1983 indicates the regression scheme may be a valuable forecast aid, especially for identifying potentially fast (or even rapid) intensification situations.

Table 8.12: Average 24-h relative errors (in kts) for the regression model and JTWC forecast for the dependent data set (1984-1986) and independent data set (1983). The errors are stratified by intensity change regimes. Number of cases for all stratification is also shown. Since JTWC released fewer forecasts than was empirically analyzed, there are fewer cases in the error analysis (514) than in Table 8.4 (530). Errors are computed as $\Delta V_{\max }$ (observed) minus $\Delta V_{\max }$ (forecast). TIPS is developed on perfect-prog forecasts of shear and track, so this table does not imply that TIPS can outperform JTWC.

\begin{tabular}{|l|c|c|c|c|c|c|}
\hline Years & $\Delta v \geq 25$ & $20 \geq \Delta v \geq 10$ & $5 \geq \Delta v \geq-5$ & $-10 \geq \Delta v \geq-20$ & $\Delta v \leq-25$ & Overall \\
\hline \hline 84-86 TIPS & +16.1 & +3.8 & -2.1 & -7.2 & -13.4 & 0.0 \\
84-86 JTWC & +16.3 & +2.1 & -2.9 & -6.7 & -13.0 & -0.6 \\
\hline 84-86 Cases & 66 & 146 & 148 & 119 & 35 & 514 \\
\hline \hline 83 TIPS & +21.1 & +2.0 & -6.2 & -8.8 & -14.2 & -1.6 \\
83 JTWC & +25.5 & +8.7 & 0.7 & -4.6 & -17.7 & +3.2 \\
\hline 83 Cases & 20 & 35 & 28 & 39 & 13 & 135 \\
\hline
\end{tabular}

These 24-h 1983 error reductions are shown graphically in Fig. 8.5. In general, the regression errors are fairly high, but constitute a $19 \%$ improvement on average compared to JTWC, a $35 \%$ improvement for the slower intensifiers, and a $20-23 \%$ refinement to fast rates of $\Delta V_{\max }$. On the other hand, TIPS faired worst than JTWC for the slow weakeners in 1983 , indicating that TIPS often misses the transition from an intensifying to weakening storm. The persistence term is partially responsible for this error.

The error reductions are larger at $48 \mathrm{~h}$, as indicated in Tables 8.14 and 8.15. The same error patterns as $24 \mathrm{~h}$ are exhibited for the $48 \mathrm{~h}$ forecasts. However, in all cases except the slow weakeners, TIPS' biases and absolute errors are less than JTWC. Many of TIPS' biases are on the order of 5-10 kts. Furthermore, the fact that the bias is $17 \mathrm{kts}$ less 
Table 8.13: Average 24-h absolute errors (in kts) for the regression model and JTWC forecast for the dependent data set (1984-1986) and independent data set (1983). The errors are stratified by intensity change regimes. Number of cases for all stratification is also shown. Since JTWC released fewer forecasts than was empirically analyzed, there are fewer cases in the error analysis (514) than in Table 8.4 (530). TIPS is developed on perfect-prog forecasts of shear and track, so this table does not imply that TIPS can outperform JTWC.

\begin{tabular}{|l|c|c|c|c|c|c|}
\hline Years & $\Delta v \geq 25$ & $20 \geq \Delta v \geq 10$ & $5 \geq \Delta v \geq-5$ & $-10 \geq \Delta v \geq-20$ & $\Delta v \leq-25$ & Overall \\
\hline \hline 84-86 TIPS & 16.7 & 7.2 & 6.6 & 9.3 & 13.4 & 9.2 \\
84-86 JTWC & 16.7 & 8.8 & 10.5 & 11.0 & 15.3 & 11.3 \\
\hline 84-86 Cases & 66 & 146 & 148 & 119 & 35 & 514 \\
\hline \hline 83 TIPS & 21.1 & 8.4 & 9.0 & 11.3 & 14.2 & 11.8 \\
83 JTWC & 27.5 & 13.0 & 11.8 & 10.5 & 17.7 & 14.6 \\
\hline 83 Cases & 20 & 35 & 28 & 39 & 13 & 135 \\
\hline
\end{tabular}

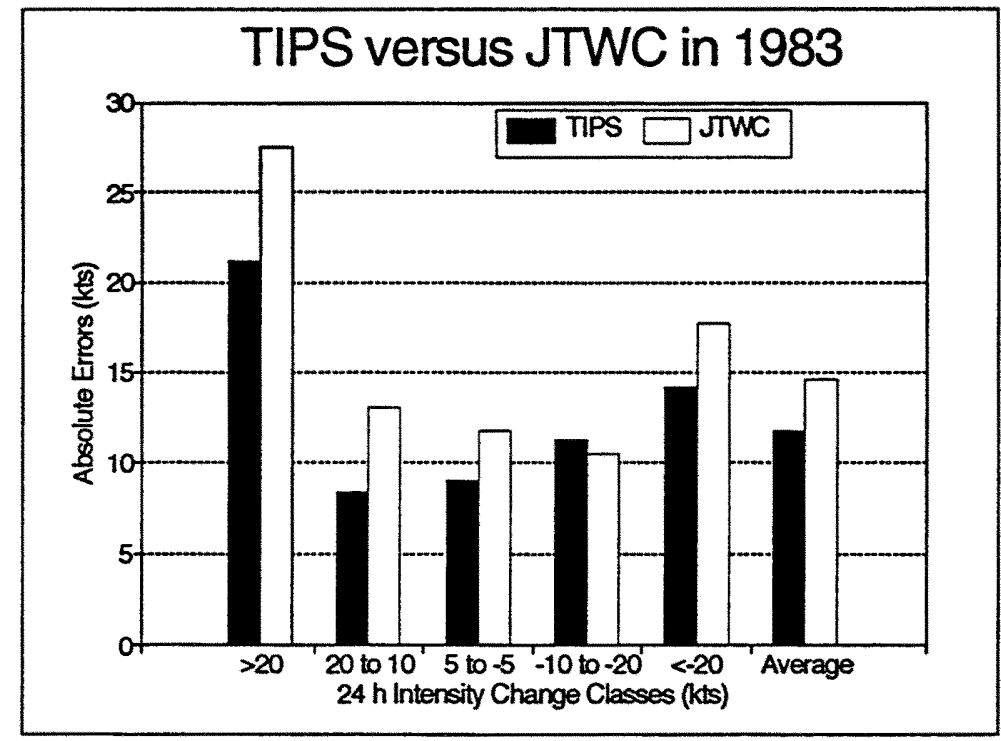

Figure 8.5: Absolute 24-h errors for TIPS and JTWC on the independent data set (1983) for different $\Delta V_{\max }$ classes and overall average. TIPS is developed on perfect-prog forecasts of shear and track, so this figure does not imply that TIPS can outperform JTWC. 
for both 1983 fast and slow intensifying cases, with a $4.3 \mathrm{kts}$ bias for the slow intensifier, is very encouraging. Tables 8.14 and 8.15 show an overall improvement in intensifiers and fast weakeners compared to JTWC. Again, TIPS portrays a tendency to lag behind the transition to the weakening stage, with JTWC experiencing smaller errors than TIPS in this class.

Table 8.14: Average 48-h relative errors (in kts) for the regression model and JTWC forecast for the dependent data set (1984-1986) and independent data set (1983). The errors are stratified by intensity change regimes. Number of cases for all stratification is also shown. Since JTWC released fewer forecasts than was empirically analyzed, there are fewer cases in the error analysis (375) than in Table 8.4 (397). Errors are computed as $\Delta V_{\max }$ (observed) minus $\Delta V_{\max }$ (forecast). TIPS is developed on perfect-prog forecasts of shear and track, so this table does not imply that TIPS can outperform JTWC.

\begin{tabular}{|l|c|c|c|c|c|c|}
\hline Years & $\Delta v>40$ & $40 \geq \Delta v \geq 20$ & $15 \geq \Delta v \geq-15$ & $-20 \geq \Delta v \geq-40$ & $\Delta v<-40$ & Overall \\
\hline \hline 84-86 TIPS & +27.0 & +7.7 & -4.6 & -10.5 & -10.2 & 0.0 \\
84-86 JTWC & +31.8 & +6.0 & -8.9 & -9.4 & -23.3 & -1.7 \\
\hline 84-86 Cases & 36 & 97 & 159 & 71 & 12 & 375 \\
\hline \hline 83 TIPS & +30.5 & +4.3 & -6.8 & -12.9 & -21.2 & -1.4 \\
83 JTWC & +47.1 & +21.2 & -1.2 & -7.9 & -35.8 & +6.8 \\
\hline 83 Cases & 14 & 21 & 32 & 24 & 6 & 97 \\
\hline
\end{tabular}

Table 8.15: Average 48-h absolute errors (in $\mathrm{kts}$ ) for the regression model and JTWC forecast for the dependent data set (1984-1986) and independent data set (1983). The errors are stratified by intensity change regimes. Number of cases for all stratification is also shown. Since JTWC released fewer forecasts than was empirically analyzed, there are fewer cases in the error analysis (375) than in Table 8.4 (397). TIPS is developed on perfect-prog forecasts of shear and track, so this table does not imply that TIPS can outperform JTWC.

\begin{tabular}{|l|c|c|c|c|c|c|}
\hline Years & $\Delta v \geq 45$ & $40 \geq \Delta v \geq 20$ & $15 \geq \Delta v \geq-15$ & $-20 \geq \Delta v \geq-40$ & $\Delta v \leq-45$ & Overall \\
\hline \hline 84-86 TIPS & 27.0 & 10.8 & 11.4 & 14.5 & 13.2 & 13.4 \\
84-86 JTWC & 32.1 & 15.0 & 17.0 & 16.8 & 25.0 & 18.1 \\
\hline 84-86 Cases & 36 & 97 & 159 & 71 & 12 & 375 \\
\hline \hline 83 TIPS & 31.1 & 10.5 & 19.1 & 16.5 & 21.2 & 18.4 \\
83 JTWC & 47.1 & 23.1 & 21.6 & 15.4 & 35.8 & 25.0 \\
\hline 83 Cases & 14 & 21 & 32 & 24 & 6 & 97 \\
\hline
\end{tabular}

The 48-h 1983 error reductions are shown graphically in Fig. 8.6, and indicate a $26 \%$ overall improvement compared to JTWC. The following error reductions are noted: for fast intensifiers, $34 \%$; for slow intensifiers, $55 \%$; for steady-state, $12 \%$; and fast weakeners, 
41\%. The errors are still unsatisfactory, but since it's been demonstrated that the satellite information is the key factor in discriminating between the fast and slow intensifiers, the prospects for future error reductions are encouraging. Furthermore, with refinements to the other predictors, perhaps intensity forecasting can be improved in the future.

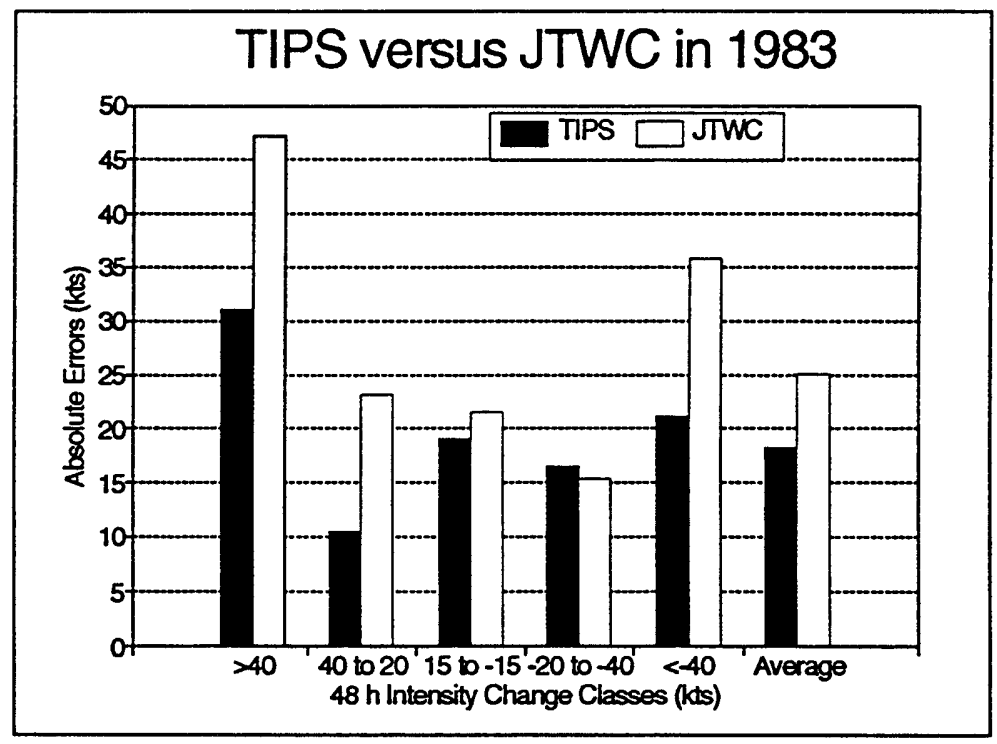

Figure 8.6: Absolute 48-h errors for TIPS and JTWC on the independent data set (1983) for different $\Delta V_{\max }$ classes and overall average. TIPS is developed on perfect-prog forecasts of shear and track, so this figure does not imply that TIPS can outperform JTWC.

\subsubsection{Overall assessment of TIPS compared to JTWC}

It is tempting to claim that TIPS outperformed JTWC on the independent data set (1983) and, to a lesser extent, on the dependent data set (1984-86), but such a statemen$t$ is unwise. Surely some of the JTWC errors resulted from bad track forecasts and to uncertainty in the wind shear evolution. As discussed earlier, forecasting wind shear is a daunting problem. As mentioned throughout this chapter, TIPS is developed assuming perfect-prog forecasts of storm motion and shear. Finally, JTWC doesn't have access to best-track data in real-time - indeed, sometimes the position and motion of a TC is uncertain, and operational data may contain errors which affect the forecaster's judgment. ${ }^{14}$

\footnotetext{
${ }^{14}$ One source of uncertainty is the location of a TC's center at night. Upon sunrise, visible imagery may indicate that the night-time estimate of a TC's center based on infrared imagery to be quite incorrect,
} 
However, it is fair to conclude that TIPS is at least competitive with JTWC, since all the errors were similar or less in magnitude. TIPS can certainly play a valuable role in forecast guidance, especially when its biases are understood by the forecaster.

To complete this chapter, biases and total errors for 12 and 36-h are displayed in Tables $8.16,8.17,8.18$, and 8.19 . The same characteristics discussed in the previous tables are evident in these forecast intervals as well.

Table 8.16: Average 12-h relative errors (in kts) for the regression model for the dependent data set (1984-1986) and independent data set (1983). The errors are stratified by intensity change regimes. Number of cases for all stratification is also shown. Errors are computed as $\Delta V_{\max }$ (observed) minus $\Delta V_{\max }$ (forecast). TIPS is developed on perfect-prog forecasts of shear and track, so this table does not imply that TIPS can outperform JTWC.

\begin{tabular}{|l|c|c|c|c|c|c|}
\hline Years & $\Delta v \geq 15$ & $10 \geq \Delta v \geq 5$ & $\Delta v=0$ & $-5 \geq \Delta v \geq-10$ & $\Delta v \leq-15$ & Overall \\
\hline \hline 84-86 TIPS & +11.0 & +1.9 & -0.8 & -4.2 & -9.8 & 0.0 \\
\hline 84-86 Cases & 69 & 226 & 104 & 175 & 37 & 611 \\
\hline \hline 83 TIPS & +16.0 & +1.6 & -0.2 & -5.8 & -11.3 & -0.3 \\
\hline 83 Cases & 18 & 56 & 24 & 45 & 14 & 157 \\
\hline
\end{tabular}

Table 8.17: Average 12-h absolute errors (in kts) for the regression model for the dependent data set (1984-1986) and independent data set (1983). The errors are stratified by intensity change regimes. Number of cases for all stratification is also shown. TIPS is developed on perfect-prog forecasts of shear and track, so this table does not imply that TIPS can outperform JTWC.

\begin{tabular}{|l|c|c|c|c|c|c|}
\hline Years & $\Delta v \geq 15$ & $10 \geq \Delta v \geq 5$ & $\Delta v=0$ & $-5 \geq \Delta v \geq-10$ & $\Delta v \leq-15$ & Overall \\
\hline \hline 84-86 TIPS & 11.1 & 4.1 & 4.2 & 5.2 & 9.9 & 5.6 \\
\hline 84-86 Cases & 69 & 226 & 104 & 175 & 37 & 611 \\
\hline \hline 83 TIPS & 16.0 & 5.0 & 4.4 & 6.3 & 11.3 & 7.1 \\
\hline 83 Cases & 18 & 56 & 24 & 45 & 14 & 157 \\
\hline
\end{tabular}

with position errors of $100 \mathrm{~km}$ or more. This error is most common for weak, ill-defined TCs undergoing wind shear in which the low-level center is displaced from the convection. This situation is sometimes called the "sunrise surprise" (J. Martin, personal communication 1995). Night-time storm intensity estimates and storm motion estimates may also have been wrong during such a circumstance (Mielke et al. 1995). 
Table 8.18: Average 36-h relative errors (in kts) for the regression model for the dependent data set (1984-1986) and independent data set (1983). The errors are stratified by intensity change regimes. Number of cases for all stratification is also shown. Errors are computed as $\Delta V_{\max }$ (observed) minus $\Delta V_{\max }$ (forecast). TIPS is developed on perfect-prog forecasts of shear and track, so this table does not imply that TIPS can outperform JTWC.

\begin{tabular}{|l|c|c|c|c|c|c|}
\hline Years & $\Delta v \geq 35$ & $30 \geq \Delta v \geq 15$ & $10 \geq \Delta v \geq-10$ & $-15 \geq \Delta v \geq-30$ & $\Delta v \leq-35$ & Overall \\
\hline \hline $84-86$ TIPS & +20.6 & +5.3 & -4.0 & -8.6 & -11.3 & 0.0 \\
\hline 84-86 Cases & 55 & 121 & 160 & 99 & 24 & 459 \\
\hline \hline 83 TIPS & +24.2 & +2.9 & -4.6 & -14.5 & -14.9 & -2.1 \\
\hline 83 Cases & 16 & 26 & 42 & 28 & 8 & 120 \\
\hline
\end{tabular}

Table 8.19: Average 36-h absolute errors (in kts) for the regression model for the dependent data set (1984-1986) and independent data set (1983). The errors are stratified by intensity change regimes. Number of cases for all stratification is also shown. TIPS is developed on perfect-prog forecasts of shear and track, so this table does not imply that TIPS can outperform JTWC.

\begin{tabular}{|l|c|c|c|c|c|c|}
\hline Years & $\Delta v \geq 35$ & $30 \geq \Delta v \geq 15$ & $10 \geq \Delta v \geq-10$ & $-15 \geq \Delta v \geq-30$ & $\Delta v \leq-35$ & Overall \\
\hline \hline 84-86 TIPS & 20.9 & 9.6 & 10.2 & 12.0 & 12.8 & 11.9 \\
\hline 84-86 Cases & 55 & 121 & 160 & 99 & 24 & 459 \\
\hline \hline 83 TIPS & 25.8 & 9.9 & 12.9 & 15.6 & 16.2 & 14.8 \\
\hline 83 Cases & 16 & 26 & 42 & 28 & 8 & 120 \\
\hline
\end{tabular}




\subsection{Comments on insignificant predictors}

While the significant predictors are of most concern in this paper, it is equally illuminating which predictors were not significant - especially since many of the unchosen potential predictors are considered to be very important to TC intensity change by some researchers. First and foremost, none of the trough forcing terms such as Relative Eddy angular momentum Flux Convergence (REFC) and 200-mb vorticity advection (VORTADV) were considered statistically significant in the regression analysis. This concurs with the discussion in Chapters 2 and 3 , in which it was postulated that any positive benefits associated with these terms are overwhelmed by the corresponding negative effects of vertical wind shear which usually accompanies a trough.

In agreement with DeMaria et al. (1993) (see Fig. 2.15), these terms are actually negatively correlated in TIPS with $\Delta V_{\max }$ by themselves (roughly $r=-0.12$ on average). This is because these terms are positively correlated with VWS (both areal and single point) on average, with $r \approx 0.1$. Furthermore, when forced into multiple regression, each term in TIPS remains very statistically insignificant (generally between the 40 and $70 \%$ significance level), and with negative regression coefficients! Only for $48-\mathrm{h}$ forecasts are

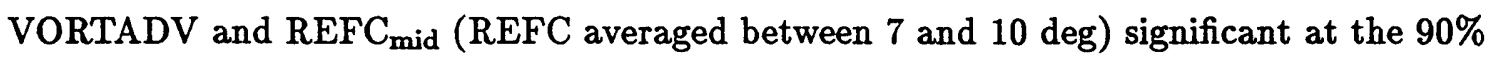
level, and their coefficients are still negative.

The problem with past studies on REFC, with the exception of DeMaria et al. (1993), is that researchers have based their conclusions on a single case study (Molinari and Vollaro 1989) or highly idealized models (Pfeffer and Challa 1980) with little regard to how the general ensemble of TCs respond to upper-level forcing in conjunction with wind shear. These are easy traps to fall into, and this author can easily show a TC (i.e., Typhoon Ike in 1984) which seems to follow the pattern of intensification 2 days after a REFC event (Fig. 8.7), similar to the figures shown in Chapter 2. However, very weak wind shear, warm SSTs, and increased convection (not shown) also preceded the intensification, leading one to believe that the REFC event quite probably was not responsible for the intensification of Ike. REFC plots were done for all TCs in 1983-1986, and to a large degree no REFC 


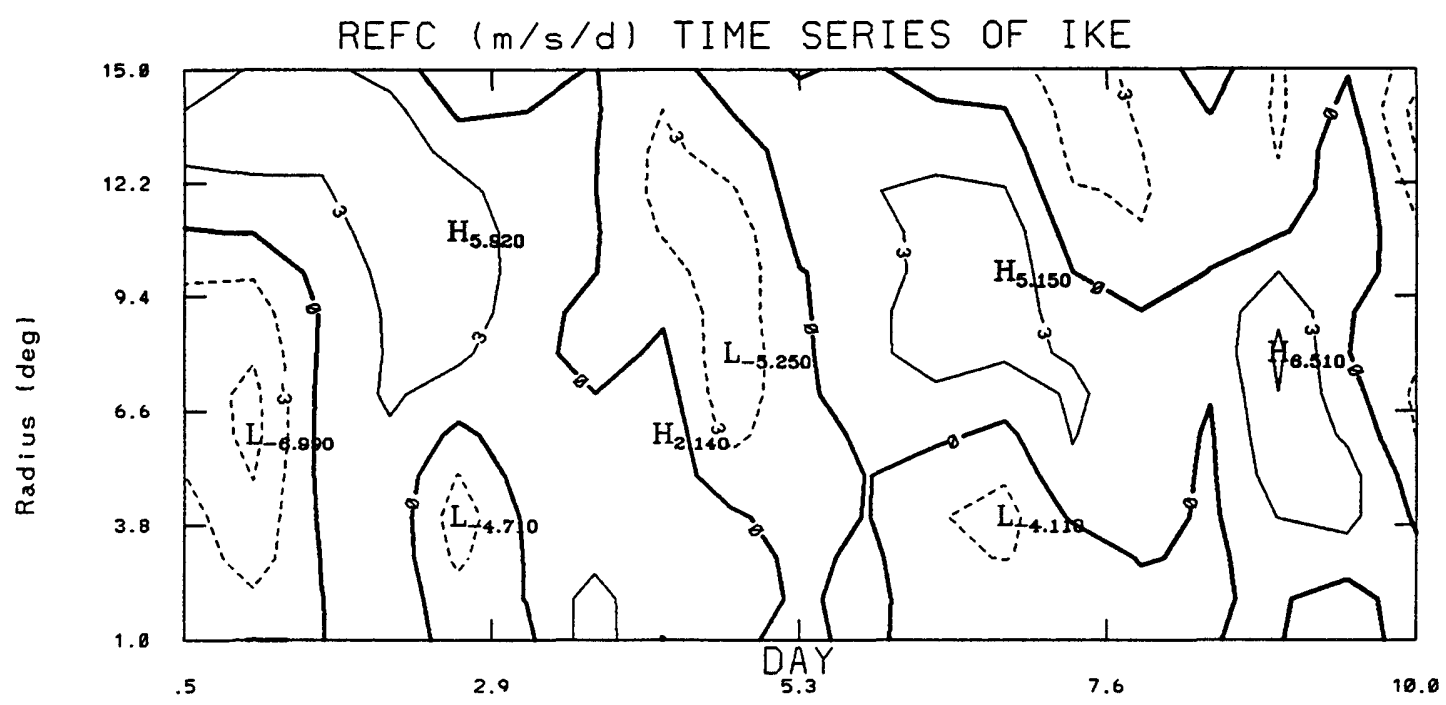

Figure 8.7: Radius-time series (Hovmoeller) diagram of 1-15 deg REFC for Typhoon Ike from Day 0.5 (12 UTC 26 August 1984) to Day 10 (00 UTC 5 September 1984). The REFC event on Day 3 preceded the rapid intensification of Ike on Day 5. However, small vertical wind shear, warm SSTs, and favorable 0-4 deg convection probably played the dominant intensification role. Contour interval is $3 \mathrm{~ms}^{-1} \mathrm{~d}^{-1}$.

pattern as described in Chapter 2 was detected in the analysis. The statistical calculations also support this claim.

Indeed, it would appear that a single case study - or case studies on just a few TCs - is an inappropriate approach to studying upper-level forcing, as are two-dimensional models that include an eddy torque term but no shear torque term. It is not disputed that an occasional TC intensifies under baroclinic forcing influences, but these storms constitute less than $10 \%$ of the total TCs (Gray 1968, 1981), and on a seasonal basis upper-level forcing is usually a negative influence. It is also recognized by the author that REFC is a noisy term which is sensitive to wind accuracy, and given that actual upper-level wind measurements in the tropics are sparse, perhaps a forecast signal is being masked by wind uncertainty. Certainly some more upper-level forcing research with better data is warranted, such as the higher quality data set for Hurricane Emily (1987) which contains combined dropwindsonde, P-3 flight data, commercial aircraft, rawinsonde, and satellite data (Kaplan 1995). It is also possible that upper-level forcing plays a role in TC intensity change in the Atlantic, in agreement with DeMaria et al. (1993) and 
DeMaria and Kaplan (1994b), since this basin contains more baroclinic interactions than the western North Pacific.

However, this author questions the overall current emphasis on upper-level forcing on TC intensity change. Given this author's personal experience at daily tropical discussions held at Colorado State University, and the results in this report, it would be appear that the upper-level forcing hypotheses have been exaggerated. Certainly a TC in a low shear environment can rapidly intensify through the Air-Sea Interaction process without trough interactions.

It is also pertinent that the following variables were not chosen: Planetary Eddy angular momentum Flux Convergence (PEFC), storm speed anomaly (SPDAN), latitude (LAT), storm direction anomaly (DIRAN), radial pixel count gradient (DIFFPX), and the Mundell scheme of inner versus outer radius convection (MUND). The non-significance of PEFC suggests that environmental eddy flux terms are unimportant. The non-significance of SPDA suggests that the effect of upwelling on slow-moving TCs may not be of much consequence to intensification (Black 1983; Black et al. 1989; Shay et al. 1992; Bender et al. 1993; Black and Holland 1995) in the western North Pacific, perhaps because warm SSTs penetrate further down in this basin (Holliday and Thompson 1979). Storm latitude is not important because POT contains a much stronger forecast signal (the correlation between POT and LAT is only -0.22). The deviation from a mean storm track of $310^{\circ}$ is not the best predictor, as is the difference between inner and outer-core pixels - both somewhat surprising findings. The Mundell scheme also is not the optimum pixel count scheme, which reflects that too much subjectivity on a limited data set was introduced by Mundell, although the foundation of the scheme (inner-core convective increase) is valid.

\subsection{Case studies}

To compliment the previous two sections, case studies of typhoons will be shown with the emphasis on $24-\mathrm{h}$ intensity change. Since meridional storm motion is only important for recurving storms, it will not be discussed much in this analysis. Time series plots of $V_{\text {max }}$, MPI, PX0455, and VWS5 are shown. TIPS' forecast errors (relative and absolute) 
are tabulated and compared to JTWC. Five TCs from the dependent years (1984-86) are shown first, then three TCs from 1983. TIPS' performance on rapidly intensifying storms is emphasized in the 1983 analysis.

\subsubsection{Analysis of case studies in 1984-86}

As stimulation for discerning why some TCs rapidly intensify, why some intensify at a slower rate, and why some do not develop much at all, a rapid developer (Lola 1986) will be investigated first (Fig. 8.8). As a reference frame for this and other discussions in this section, the time series are plotted from Day 0 of the TC (usually its depression phase) with the date shown in the figure. The Maximum Potential Intensity (MPI) as a function of SST interpolated to the TC center and $V_{\max }$ are plotted in $0.25 \mathrm{~d}(6-\mathrm{h})$ increments. VWS5 and PX0455 are plotted in $0.5 \mathrm{~d}(12-\mathrm{h})$ increments. Threshold values for VWS5 and PX0455 from Table 8.7 are also included in the plots for reference. Occasionally due to land interaction, missing synoptic data, or missing satellite data, there is no corresponding TIPS forecast.

Typhoon Lola experienced rapid deepening starting Day 1 since all the conditions were extremely favorable: VWS5 approached zero, PX0455 remained high at $62 \%$ (2.3 NSD), warm SSTs of $29^{\circ} \mathrm{C}$ supported large potential for growth (POT $=44 \mathrm{~m} \mathrm{~s}^{-1}$ ), and a history of development (persistence) with eye formation initiated when $V_{\max }$ became faster that $55 \mathrm{kts}$. In fact, Lola managed the rare feat of reaching its MPI due to the favorable environment. Lola then began to weaken on Day 3 as the water became cooler (note decrease in MPI in Fig. 8.8) and shear increased with a marked decrease in convection.

Table 8.20 shows the performance of TIPS and JTWC for Lola. The problem previously discussed of TIPS underforecasting rapid intensification is evident on Day 1 , but the error is $13 \mathrm{kts}$ less than JTWC. TIPS also performed well on Day 0.5 (no error) compared to the $10 \mathrm{kts}$ error of JTWC, and did equally poor on Day 1.5. As will be seen, TIPS, in general anticipates rapid intensification well (although it still underforecasts $\Delta V_{\max }$ ) but has trouble maintaining the intensity trend should the storm approach its MPI. This is because, statistically speaking, a TC reaching its MPI is an unusual occurrence, and hence TIPS tends to flatten out $\Delta V_{\max }$ as POT decreases. 
However, the overall performance of TIPS is an improvement compared to JTWC. Except for Day 2, TIPS' errors are less than JTWC, with an absolute error of $13.3 \mathrm{kts}$ compared to $16.0 \mathrm{kts}$. Although this is the dependent set with perfect-prog forecasts of shear and motion, as well as besttrack (not operational) data, this is an encouraging sign. As will be seen, TIPS also handles rapid intensification cases in 1983 better. As suggested in the previous section, TIPS contains less errors in the weakening stage as well.

Table 8.20: 24-h forecast errors from the observed future $\Delta V_{\max }$ for TIPS and JTWC for Typhoon Lola. Errors are defined as observed minus forecast. Day 0 is 00 UTC 17 May 1986.

\begin{tabular}{|c|c|c|c|}
\hline \multicolumn{4}{|c|}{ 24-h forecast errors for Typhoon Lola (1986) } \\
\hline Day & $\Delta V_{\max }$ & TIPS error & JTWC error \\
\hline \hline 0.5 & 25 & -1 & 10 \\
\hline 1 & 50 & 22 & 35 \\
\hline 1.5 & 60 & 40 & 40 \\
\hline 2 & 25 & 17 & -5 \\
\hline 2.5 & -10 & -8 & -20 \\
\hline 3 & -20 & -4 & 0 \\
\hline 3.5 & -20 & 7 & 5 \\
\hline 4.0 & -40 & -19 & -25 \\
\hline 4.5 & -35 & -11 & -15 \\
\hline 5.0 & -25 & -4 & -5 \\
\hline \hline \multicolumn{2}{|c|}{ Mean Rel. Error } & 3.9 & 2.0 \\
\hline Mean Abs. Error & 13.3 & 16.0 \\
\hline
\end{tabular}

As also discussed in the last sections, the pixel counts are an essential predictor in distinguishing intensity rates. Fig. 8.9 of Vera (1986) is a good example. Even though VWS5 and SSTs are favorable, PX0455 toggles the threshold value of 25\%. As a result, fast intensification occurs but not rapid. In fact, because TIPS detected the lack of strong convection, it forecasted fast intensification at the proper times (Days 2-3) and handled the whole life cycle of Vera rather well, with errors less than or comparable to JTWC except for Day 9.5 (Table 8.21). TIPS' overall absolute error is $7 \mathrm{kts}$ while JTWC's is $11 \mathrm{kts}$.

Of course, fast intensification can be suppressed even with strong convective signals if the shear increases, as in the case of Hope (1985) in Fig. 8.10. Hope is primed for fast, or even rapid, intensification on Days 1-2 with all factors highly favorable. But on Days 

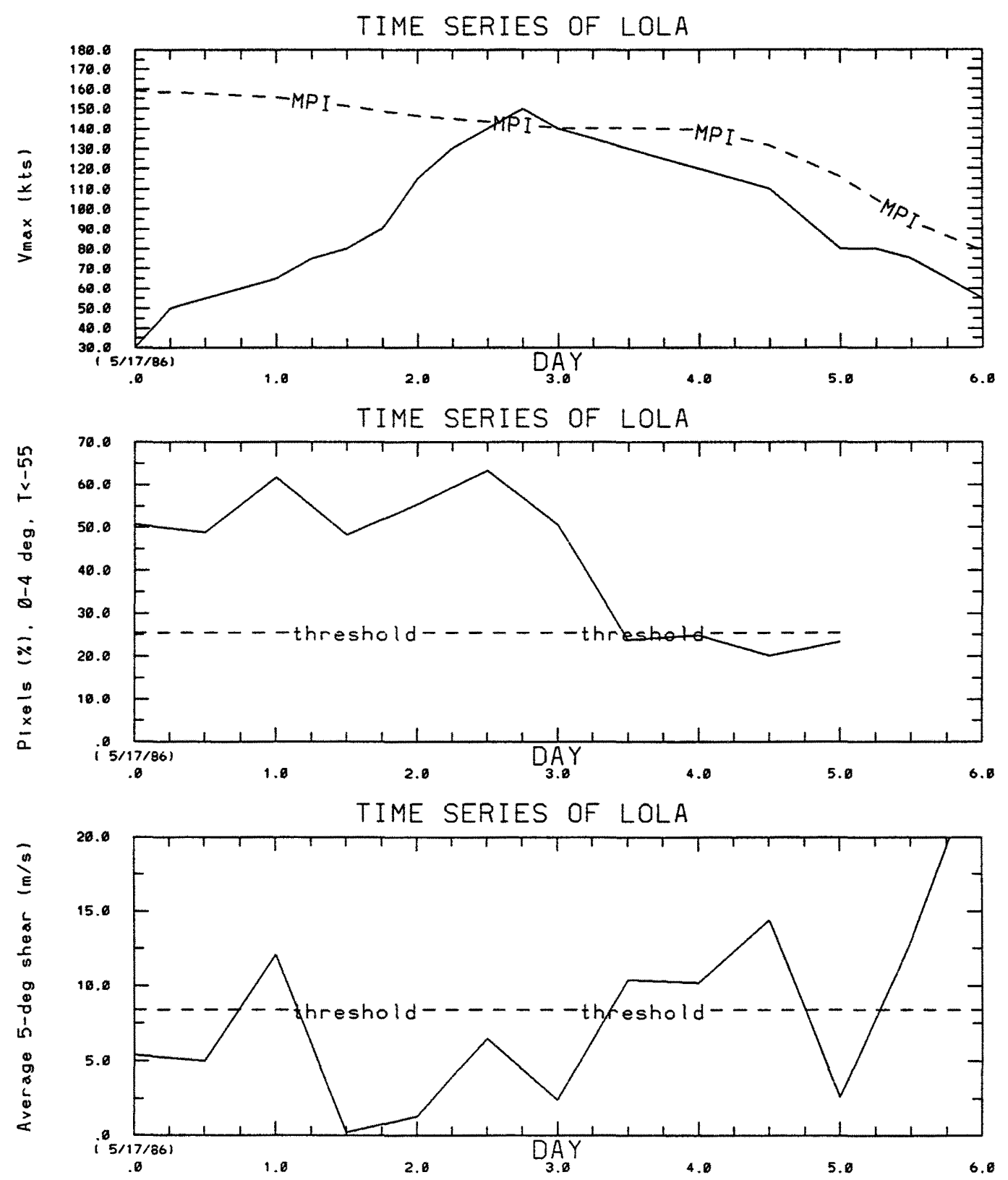

Figure 8.8: Time series of MPI and $V_{\max }$ (kts, top figure), PX0455 with its threshold value for reference (\%; middle figure), and VWS5 with its threshold value for reference ( $\mathrm{m} \mathrm{s}^{-1}$; bottom figure) for Typhoon Lola (1986). 


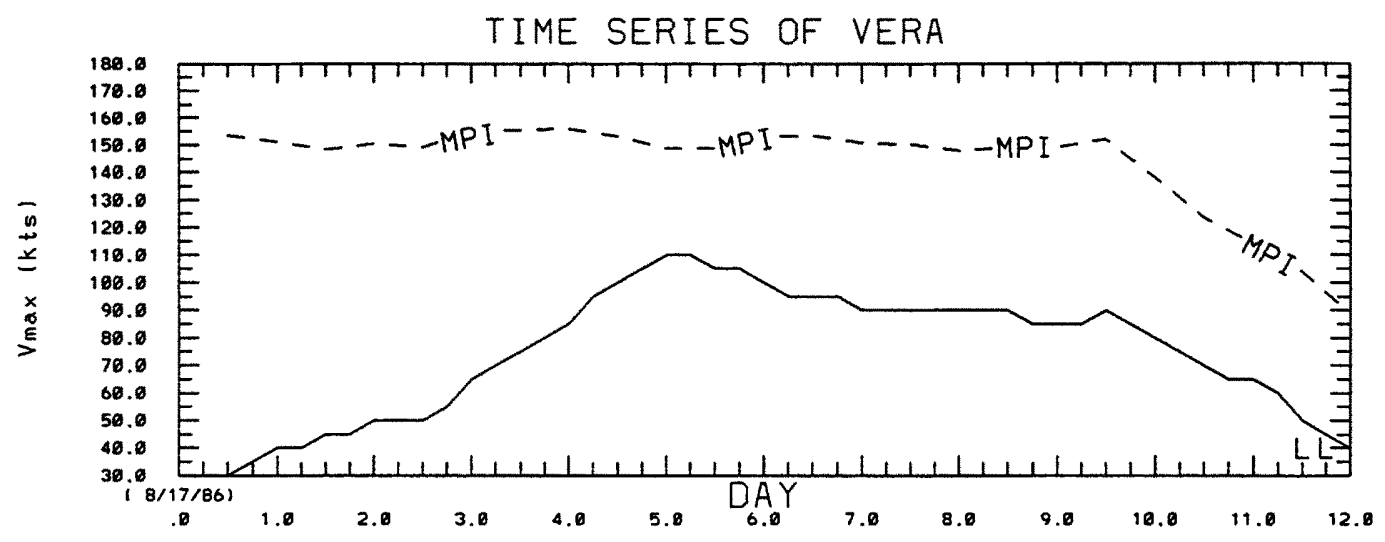

TIME SERIES OF VERA

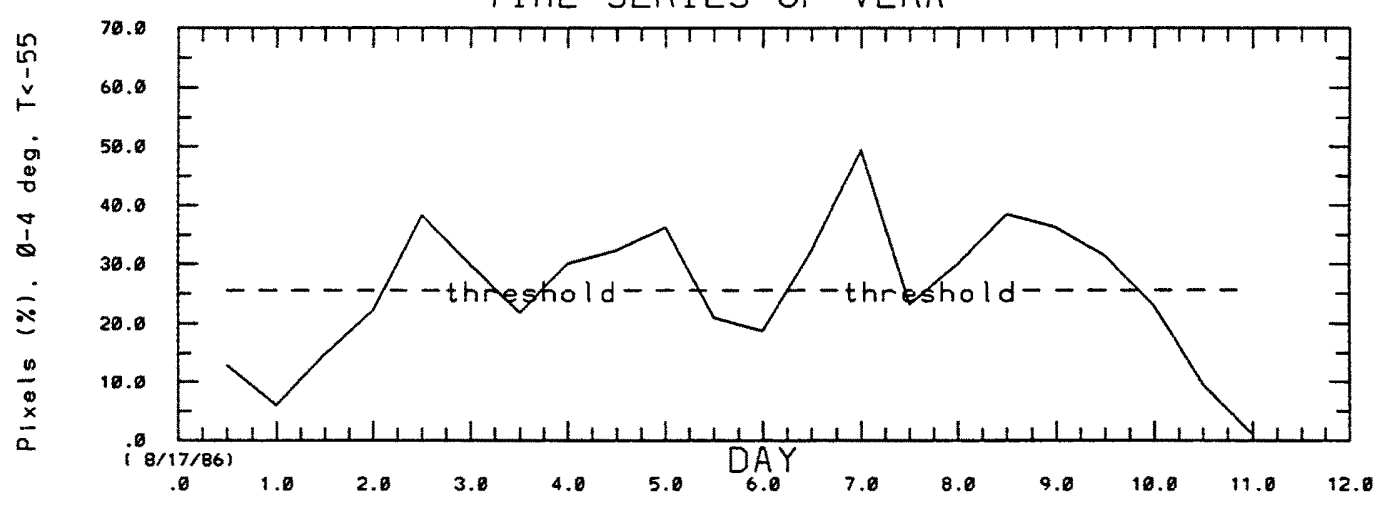

TIME SERIES OF VERA

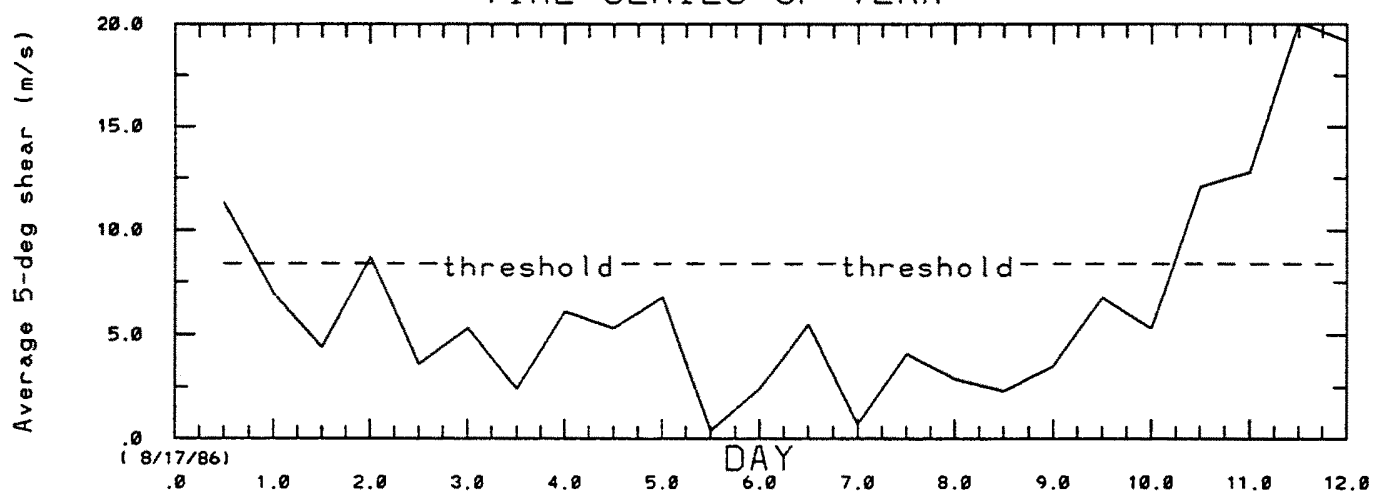

Figure 8.9: Time series of MPI and $V_{\max }$ (kts, top figure), PX0455 with its threshold value for reference (\%; middle figure), and VWS5 with its threshold value for reference $\left(\mathrm{m} \mathrm{s}^{-1}\right.$; bottom figure) for Typhoon Vera (1986). " $L$ " denotes land interaction on Days 11.5 and 11.75 . 
Table 8.21: 24-h forecast errors from the observed future $\Delta V_{\max }$ for TIPS and JTWC for Typhoon Vera. Errors are defined as observed minus forecast. Day 0 is 00 UTC 17 August 1986.

\begin{tabular}{|c|c|c|c|}
\hline \multicolumn{4}{|c|}{ 24-h forecast errors for Typhoon Vera (1986) } \\
\hline Day & $\Delta V_{\max }$ & TIPS error & JTWC error \\
\hline \hline 1 & 10 & 3 & 5 \\
\hline 1.5 & 5 & -3 & -5 \\
\hline 2 & 15 & 10 & 10 \\
\hline 2.5 & 25 & 10 & 20 \\
\hline 3 & 20 & 5 & -5 \\
\hline 3.5 & 25 & 14 & 10 \\
\hline 4 & 25 & 17 & 20 \\
\hline 4.5 & 5 & 0 & -15 \\
\hline 5 & -10 & -14 & -30 \\
\hline 5.5 & -10 & -1 & -35 \\
\hline 6 & -10 & -2 & 5 \\
\hline 6.5 & -5 & -3 & 0 \\
\hline 7 & 0 & -6 & 0 \\
\hline 7.5 & 0 & 3 & -10 \\
\hline 8 & -5 & -6 & 10 \\
\hline 8.5 & 0 & -3 & 15 \\
\hline 9 & -5 & -7 & 5 \\
\hline 9.5 & -20 & -22 & -5 \\
\hline 10 & -15 & -4 & -5 \\
\hline \hline Mean Rel. Error & -0.5 & -0.5 \\
\hline Mean Abs. Error & 7.0 & 11.1 \\
\hline & & & \\
\hline
\end{tabular}


2.5-3.5, VWS5 became greater than $20 \mathrm{~m} \mathrm{~s}^{-1}$, and intensification halted. Then Hope moved over colder waters with marginally unfavorable shear conditions and dissipated. Table 8.22 shows that TIPS anticipates the intensification rates equally or better than JTWC from Days 1-2, but misses the short intensification burst by 20 kts (which mostly occurred in the first $12 \mathrm{~h}$ ) on Day 2.5 because of the anticipated increased shear on Day 3 (JTWC also made this mistake with a $25 \mathrm{kts}$ error). Then, because of the persistence term, TIPS forecasted steady-state conditions despite unfavorable shear and decreasing SSTs, and JTWC outperformed TIPS on Day 3.5. This problem of missing the transition from intensification to weakening due to the persistence term was mentioned in the previous sections. Afterwards, TIPS outperformed JTWC during the weakening phase, and the overall absolute error is $2.5 \mathrm{kts}$ better than JTWC.

Table 8.22: 24 -h forecast errors from the observed future $\Delta V_{\max }$ for TIPS and JTWC for Typhoon Hope. Errors are defined as observed minus forecast. Day 0 is 00 UTC 17 December 1985.

\begin{tabular}{|c|c|c|c|}
\hline \multicolumn{4}{|c|}{ 24-h forecast errors for Typhoon Hope (1985) } \\
\hline Day & $\Delta V_{\max }$ & TIPS error & JTWC error \\
\hline \hline 1 & 25 & -4 & 15 \\
\hline 1.5 & 20 & -10 & 10 \\
\hline 2 & 30 & 6 & 20 \\
\hline 2.5 & 25 & 20 & 25 \\
\hline 3 & -10 & -8 & -5 \\
\hline 3.5 & -35 & -32 & -20 \\
\hline 4 & -20 & -17 & -15 \\
\hline 4.5 & 0 & 2 & 5 \\
\hline 5 & 5 & 4 & 15 \\
\hline 5.5 & 5 & 7 & 15 \\
\hline 6 & -20 & -22 & 15 \\
\hline \hline Mean Rel. Error & -4.9 & 7.3 \\
\hline Mean Abs. Error & 12.0 & 14.5 \\
\hline
\end{tabular}

To further emphasize the importance of convection, Fig. 8.11 shows a case (Pat 1985) where the shear was very low $\left(\approx 3 \mathrm{~m} \mathrm{~s}^{-1}\right)$ throughout the life cycle, but never developed much convection even though SSTs are $28-28.5^{\circ} \mathrm{C}$, and as a result intensified at a slow rate over a three-day period to a maximum of $95 \mathrm{kts}$ on Day 3.5. Pat then weakened as it 

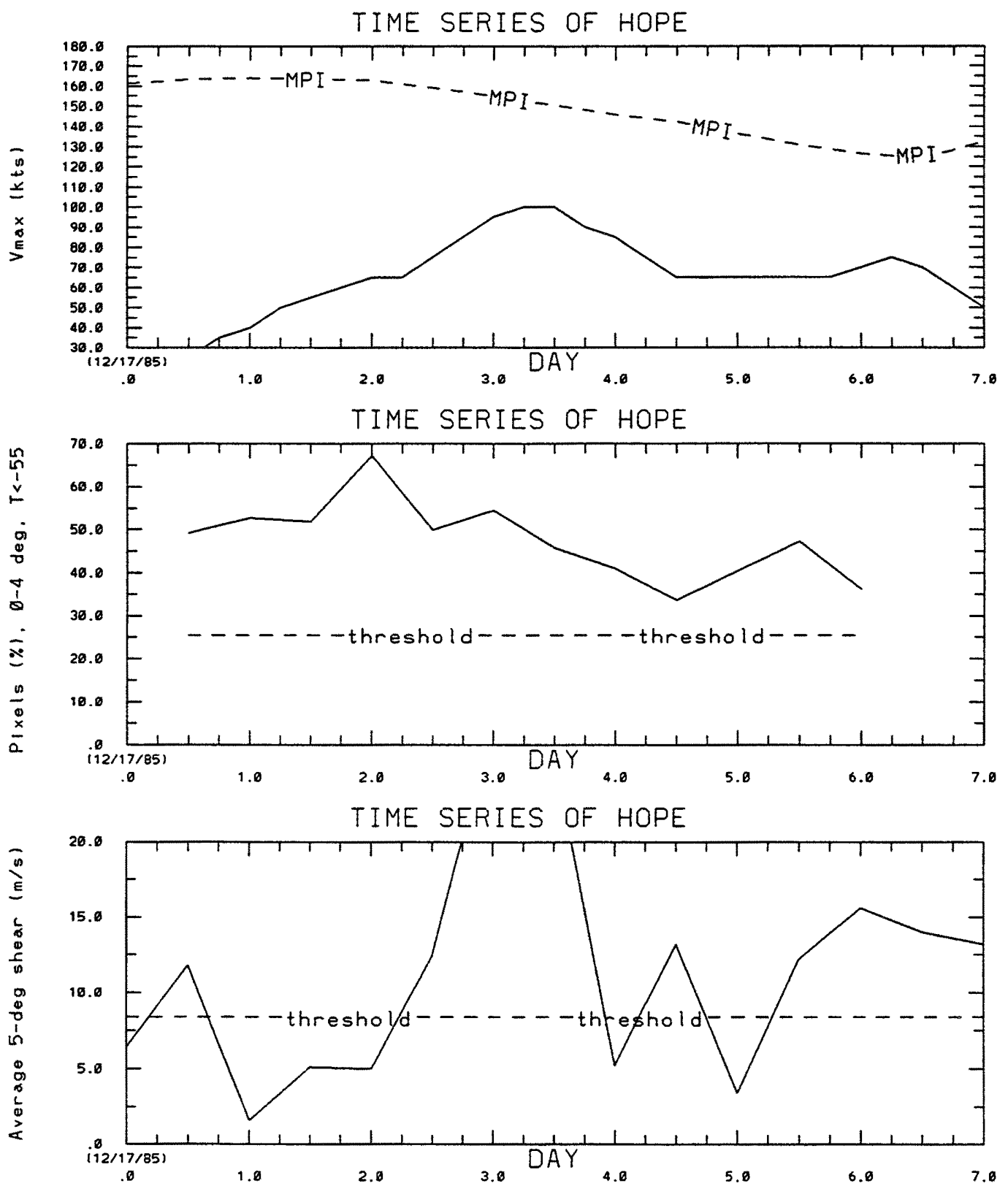

Figure 8.10: Time series of MPI and $V_{\max }$ (kts, top figure), PX0455 with its threshold value for reference (\%; middle figure), and VWS5 with its threshold value for reference (m s ${ }^{-1}$; bottom figure) for Typhoon Hope (1985). 
made landfall over southwestern Japan. Errors from both JTWC and TIPS are low before landfall occurred (Table 8.23).

Table 8.23: 24-h forecast errors from the observed future $\Delta V_{\max }$ for TIPS and JTWC for Typhoon Pat. Errors are defined as observed minus forecast. Day 0 is 00 UTC 27 August 1985.

\begin{tabular}{|c|c|c|c|}
\hline \multicolumn{4}{|c|}{ 24-h forecast errors for Typhoon Pat (1985) } \\
\hline Day & $\Delta V_{\max }$ & TIPS error & JTWC error \\
\hline 0.5 & $\overline{15}$ & $\overline{0}$ & -5 \\
\hline 1 & 20 & 5 & 0 \\
\hline 1.5 & 10 & $\overline{2}$ & -5 \\
\hline 2 & 5 & $\overline{-1}$ & 5 \\
\hline 2.5 & 15 & 19 & 5 \\
\hline \multicolumn{2}{|c|}{ Mean Rel. Error } & $\overline{5.0}$ & $\overline{0.0}$ \\
\hline \multicolumn{2}{|c|}{ Mean Abs. Error } & $\overline{5.4}$ & 4.0 \\
\hline
\end{tabular}

Finally, if a storm encounters strong shear after genesis such that no convection persists, little intensification will ever occur, as shown for Skip (1985) in Fig. 8.12. Skip persists due to warm SSTs of 28.25 to $28.5^{\circ} \mathrm{C}$, but except for Day 3.5 encounters shear much greater than the threshold of $8.5 \mathrm{~m} \mathrm{~s}^{-1}$. As a result, convection always remained below the intensification threshold. This pixel count information clearly helps the TIPS forecast of quasi steady-state conditions (Table 8.24), as its errors are quite a bit lower than JTWC (absolute error of $7 \mathrm{kts}$ compared to $11.4 \mathrm{kts}$ ).

\subsubsection{Analysis of rapidly intensifying case studies in 1983}

For a better test of the validity of TIPS, three of the more challenging typhoons in 1983 (the independent year) will be examined. Two are rapid intensifiers, the other is a fast intensifier.

The onset of rapid intensification (denoted as RI to simplify this discussion) for Marge (1983) was Day 3.5, coinciding with very warm SSTs of $29.1^{\circ} \mathrm{C}$, low shear, the formation of the eye, and PX0455 of 40-50\%, eventually reaching its MPI on Day 5 (Fig. 8.13). The TIPS' forecasts for Marge (Table 8.25) are reasonably close on Days 2.5 and 3, as well as at the onset of RI - and the errors are much less than JTWC, especially at the onset (9 kts compared to $40 \mathrm{kts}$ ). On Day 4 during RI, TIPS' errors are large (39 kts) but less 
TIME SERIES OF PAT

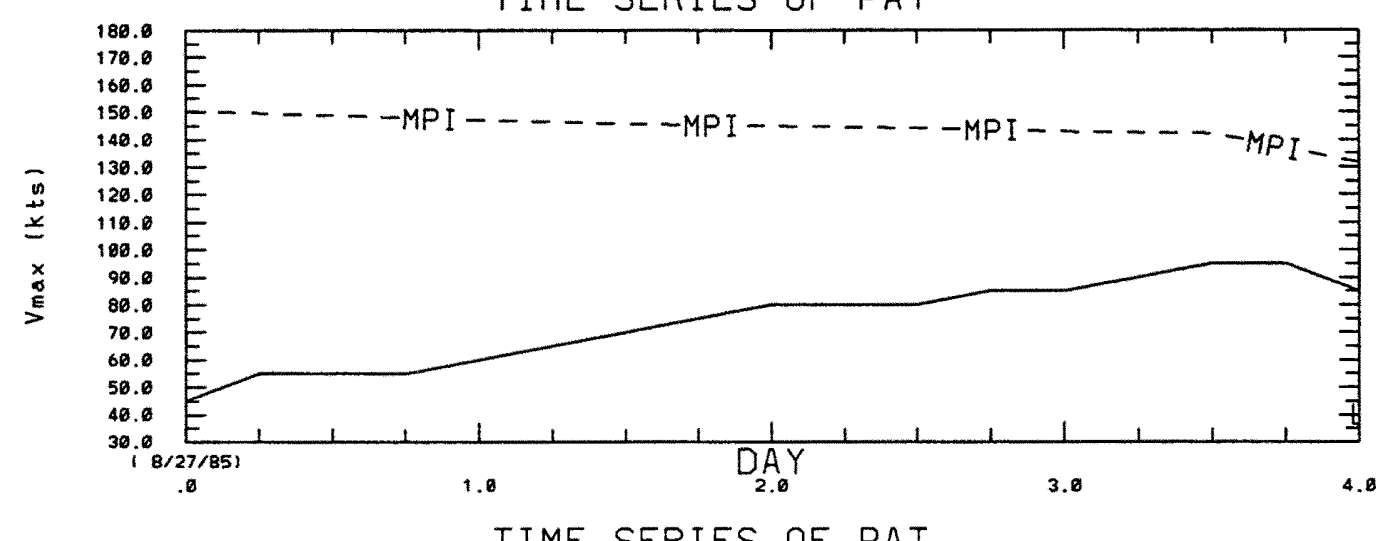

TIME SERIES OF PAT

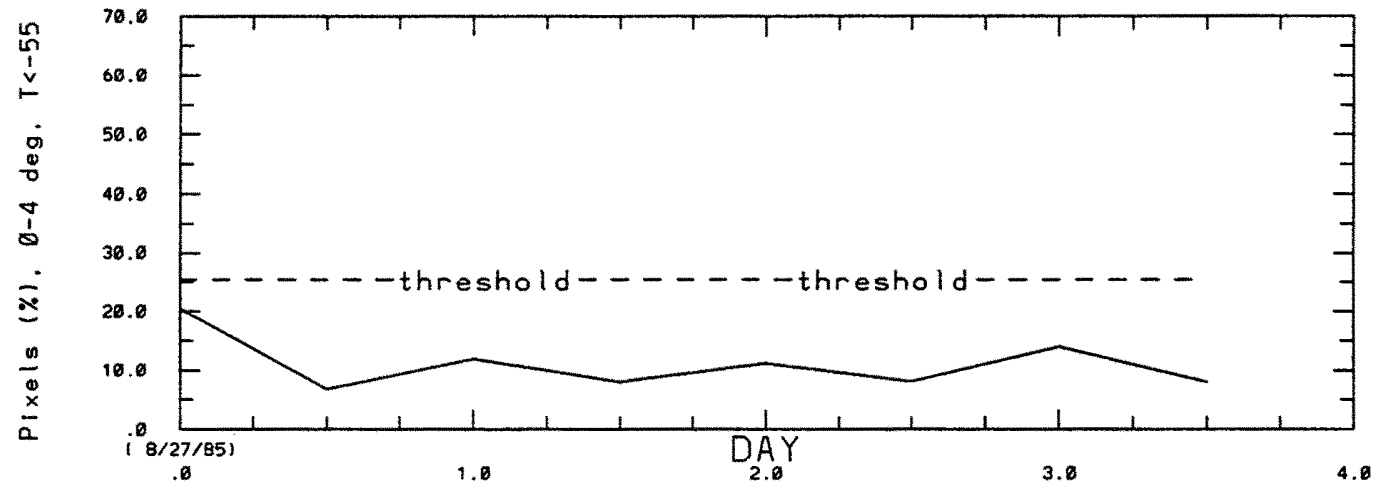

TIME SERIES OF PAT

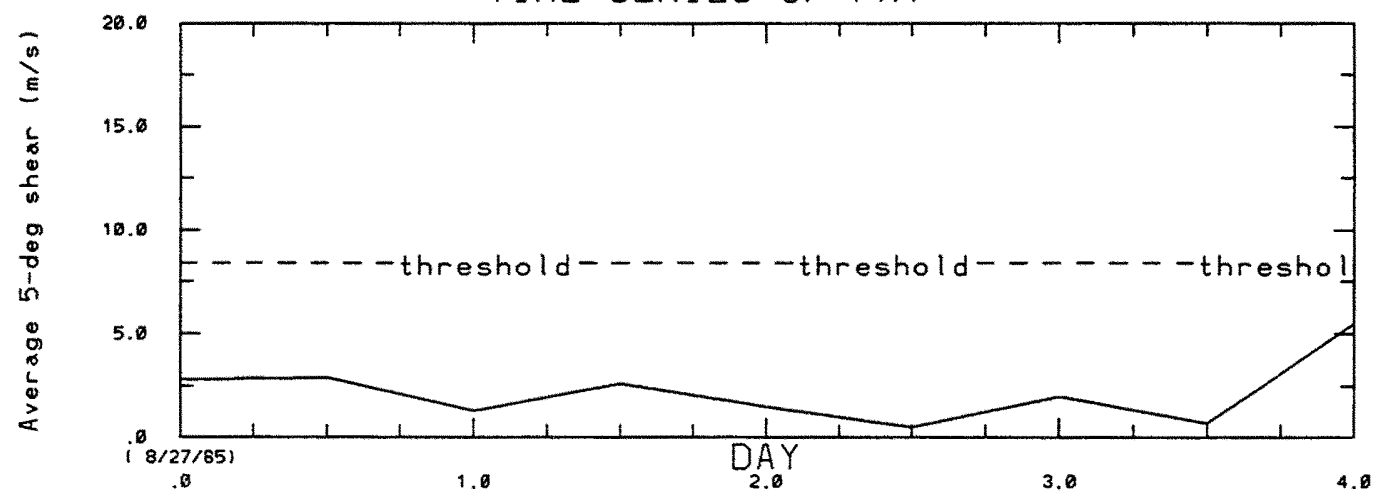

Figure 8.11: Time series of MPI and $V_{\max }$ (kts, top figure), PX0455 with its threshold value for reference (\%; middle figure), and VWS5 with its threshold value for reference $\left(\mathrm{m} \mathrm{s}^{-1}\right.$; bottom figure) for Typhoon Pat (1985). "L" denotes land interaction on Day 4. 


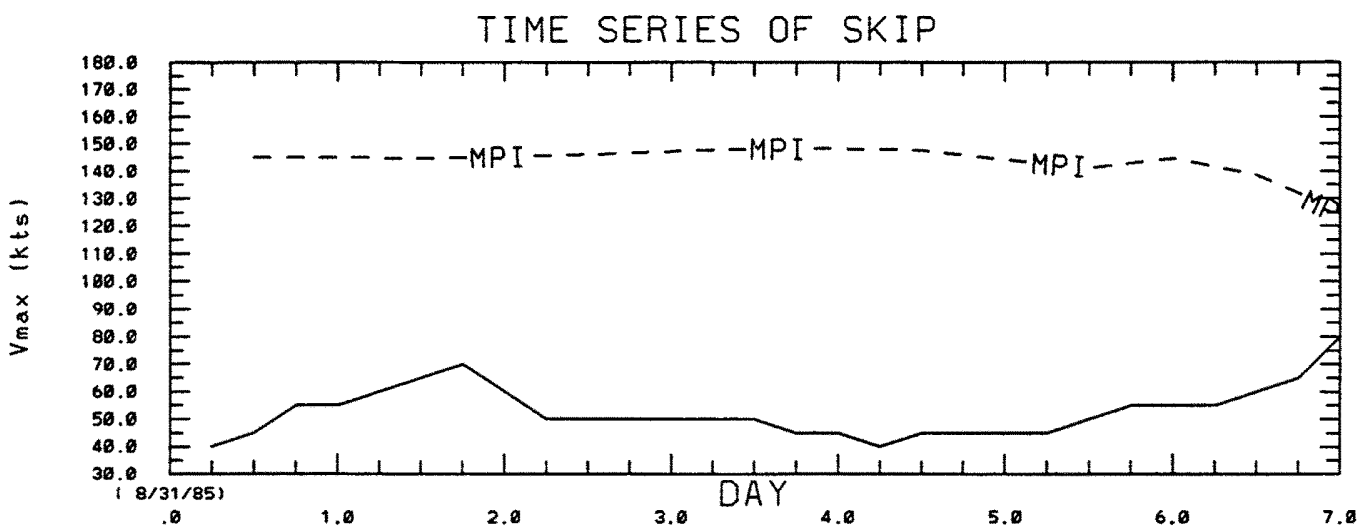

TIME SERIES OF SKIP

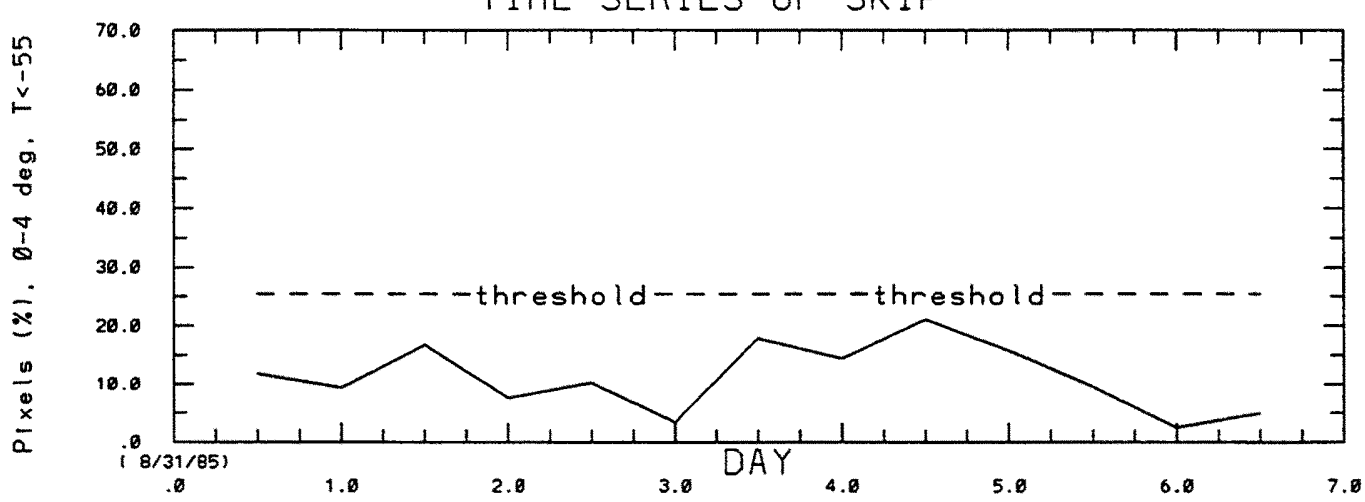

TIME SERIES OF SKIP

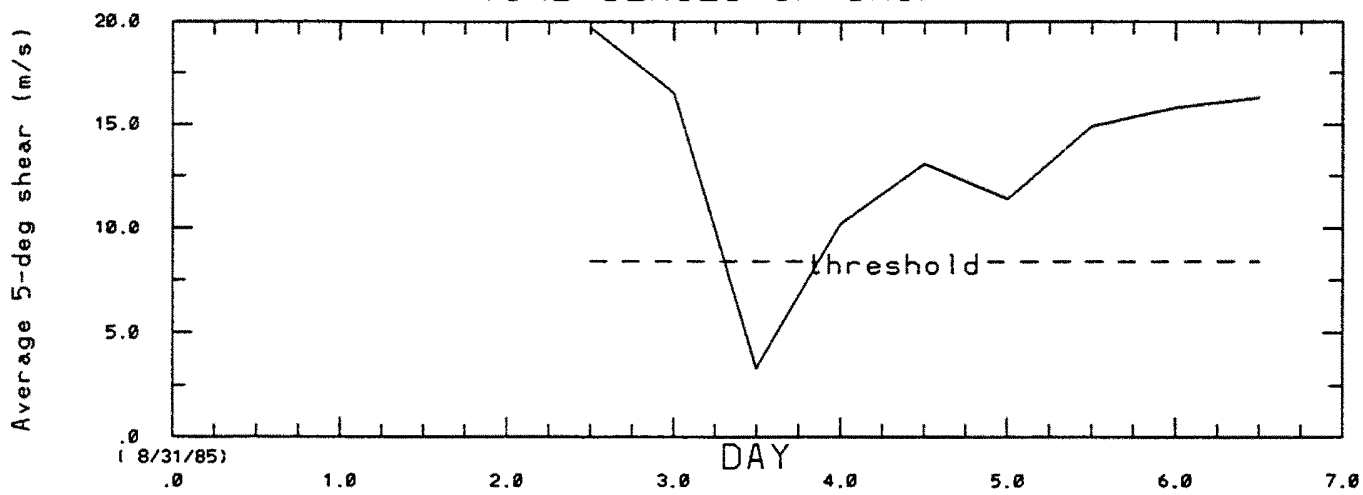

Figure 8.12: Time series of MPI and $V_{\max }$ (kts, top figure), PX0455 with its threshold value for reference (\%; middle figure), and VWS5 with its threshold value for reference ( $\mathrm{m} \mathrm{s}^{-1}$; bottom figure) for Typhoon Skip (1985). VWS5 was not available on Days 0-2.0 and on Day 7 since the TC was located east of $175 \mathrm{deg} \mathrm{E}$, and 5-deg areally averaged shear could not be computed in the BMRC data set (which is restricted to $180 \mathrm{deg} \mathrm{E}$ ). 
Table 8.24: 24 -h forecast errors from the observed future $\Delta V_{\max }$ for TIPS and JTWC for Typhoon Skip. Errors are defined as observed minus forecast. Day 0 is 00 UTC 31 August 1985. No forecast is issued on Days 0-2.0 and on Day 6 since VWS5 was not available. On Days 0-2.0 and on Day 7, the TC was located east of $175 \mathrm{deg}$ E, and 5-deg areally averaged shear could not be computed in the BMRC data set (which is restricted to $180 \mathrm{deg} \mathrm{E}$ ).

\begin{tabular}{|c|c|c|c|}
\hline \multicolumn{4}{|c|}{ 24-h forecast errors for Typhoon Skip (1985) } \\
\hline Day & $\Delta V_{\max }$ & TIPS error & JTWC error \\
\hline 2.5 & $\overline{0}$ & $\overline{4}$ & -25 \\
\hline 3 & -5 & -3 & -5 \\
\hline 3.5 & -5 & -10 & -10 \\
\hline$\overline{4}$ & 0 & -2 & -5 \\
\hline 4.5 & 5 & 4 & 10 \\
\hline 5 & 10 & 11 & 15 \\
\hline 5.5 & 10 & 15 & 10 \\
\hline \multicolumn{2}{|c|}{ Mean Rel. Error } & $\overline{2.7}$ & -1.4 \\
\hline \multicolumn{2}{|c|}{ Mean Abs. Error } & 7.0 & 11.4 \\
\hline
\end{tabular}

than JTWC's (55 kts). On Day 4.5, JTWC outforecast TIPS since the latter is reluctant to forecast a TC reaching its MPI. During the weakening phase (associated with cooler SSTs and increased shear), TIPS' errors are generally less than $10 \mathrm{kts}$, partially because TIPS includes the meridional storm motion as a predictor. As mentioned in Section 8.4.1, Marge experienced fast movement after recurvature (not shown), with meridional motion of $11 \mathrm{kts}$ on Day 7 partially balancing the decreasing $V_{\max }$ as the shear increased and SSTs decreased. TIPS' overall absolute error is $4 \mathrm{kts}$ less than JTWC.

Typhoon Forrest (1983) was perhaps one of the strangest storms in the data set, experiencing a wind increase of $60 \mathrm{kts}$ starting Day 1.5 (onset of RI), and a phenomenal wind increase of $85 \mathrm{kts}$ starting Day 2. An inspection of Fig. 8.14 shows why such enormous intensification occurred. The MPI value is about $170 \mathrm{kts}$, corresponding to the very warm SSTs of $29.5^{\circ} \mathrm{C}$. Shear is very low throughout the intensification cycle, and pixel counts are much above normal. All the predictors correspond to Normalized Standard Deviations of about 1 . As a result, even though TIPS will not forecast such large intensification rates, it did forecast a $\Delta V_{\max }$ of $32 \mathrm{kts}$ on Day 1.5 and $35 \mathrm{kts}$ on Day 2, while JTWC predicted less intensification (Table 8.26). TIPS again experienced trouble when Forrest was near its MPI, but starting Day 4 TIPS' errors are comparatively low during its weakening stage. 


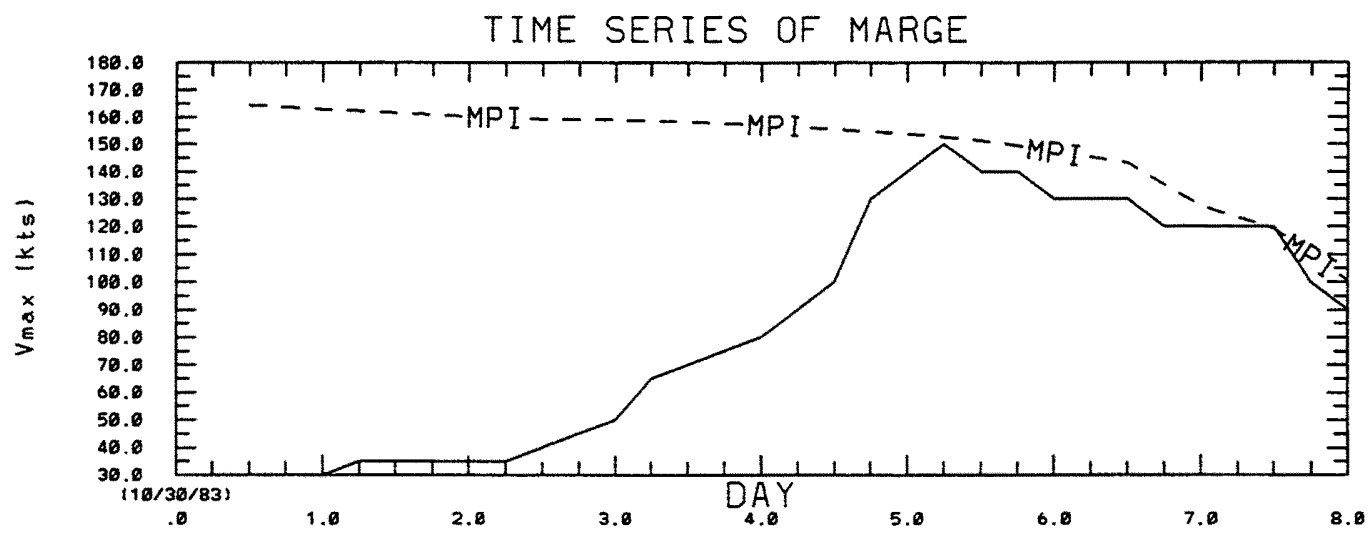

TIME SERIES OF MARGE

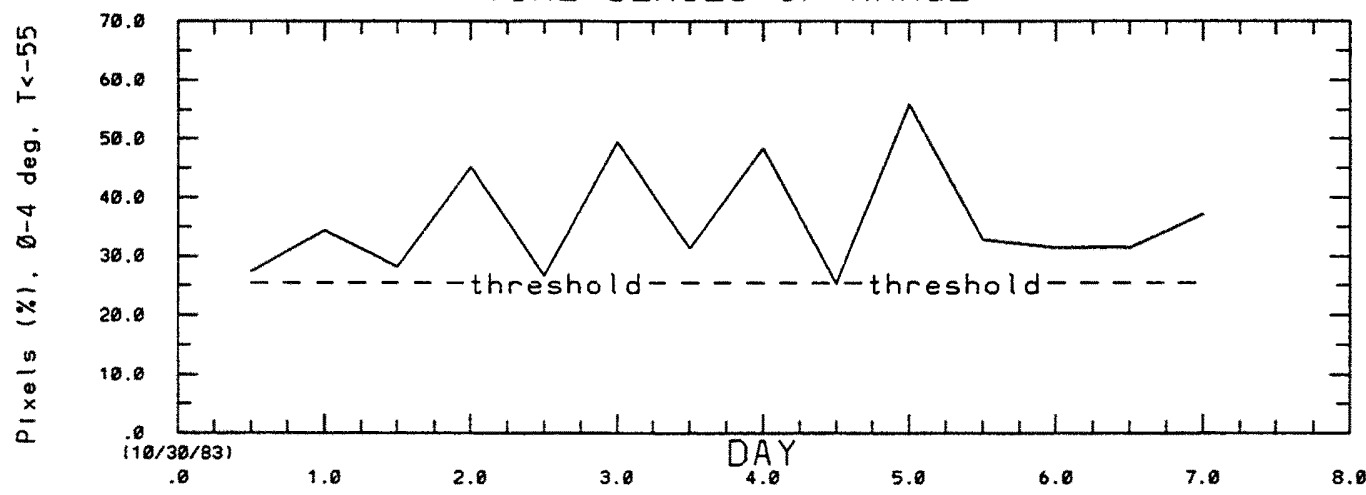

TIME SERIES OF MARGE

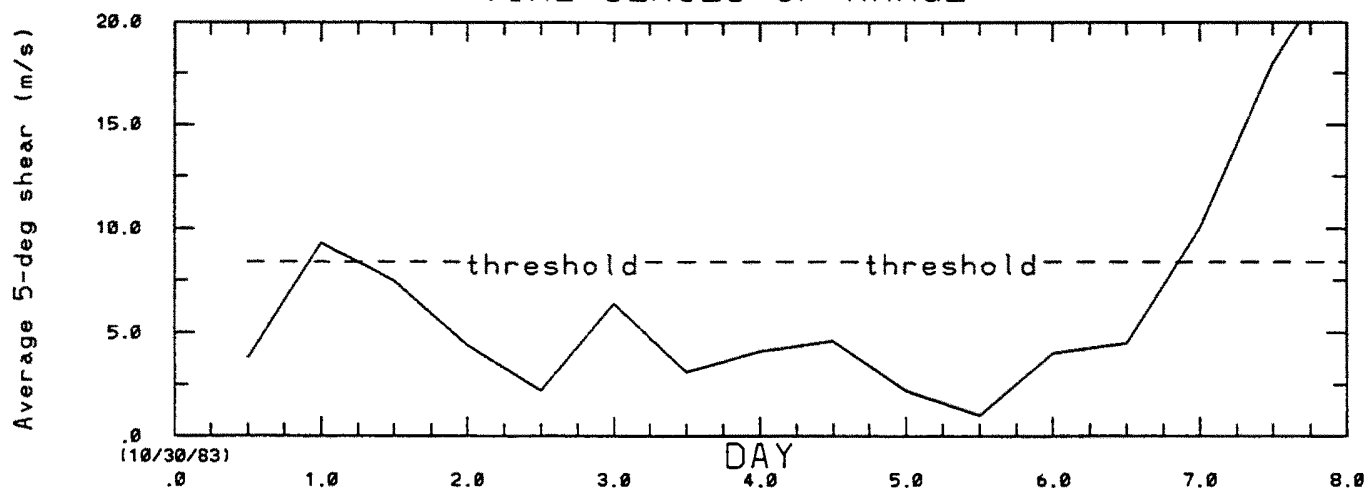

Figure 8.13: Time series of MPI and $V_{\max }$ (kts, top figure), PX0455 with its threshold value for reference (\%; middle figure), and VWS5 with its threshold value for reference ( $\mathrm{m} \mathrm{s}^{-1}$; bottom figure) for Typhoon Marge (1983). 
Table 8.25: 24 -h forecast errors from the observed future $\Delta V_{\max }$ for TIPS and JTWC for Typhoon Marge. Errors are defined as observed minus forecast. Day 0 is 00 UTC 30 October 1983.

\begin{tabular}{|c|c|c|c|}
\hline \multicolumn{5}{|c|}{ 24-h forecast errors for Typhoon Marge (1983) } \\
\hline Day & $\Delta V_{\max }$ & TIPS error & JTWC error \\
\hline \hline 1.5 & 5 & -17 & -5 \\
\hline 2 & 15 & -13 & -15 \\
\hline 2.5 & 30 & 8 & 20 \\
\hline 3 & 30 & 5 & 15 \\
\hline 3.5 & 30 & 9 & 40 \\
\hline 4 & 60 & 39 & 55 \\
\hline 4.5 & 40 & 34 & 20 \\
\hline 5 & -10 & -10 & -30 \\
\hline 5.5 & -10 & 8 & -15 \\
\hline 6 & -10 & 10 & 0 \\
\hline 6.5 & -10 & 17 & 10 \\
\hline 7 & -30 & 0 & -5 \\
\hline \hline Mean Rel. Error & 7.5 & 7.5 \\
\hline Mean Abs. Error & 14.2 & 18.3 \\
\hline
\end{tabular}

During the fast weakening stage when the SSTs became colder, the shear increased, and convection decreased, TIPS' errors are much less than JTWC's -20 kts errors. TIPS' mean absolute error is $12.2 \mathrm{kts}$ compared to JTWC's $16.5 \mathrm{kts}$.

The final 1983 storm is Typhoon Orchid (Fig. 8.15), which intensified at a fast but not rapid pace despite much convective activity (PX0455 of 70\%). The reason is because the shear hovered around the threshold value, thus precluding an RI event. In this example, TIPS' errors are very low (many below $5 \mathrm{kts}$ ), and much less than JTWC in almost all cases, with a mean absolute error of $9.9 \mathrm{kts}$ compared to $18.5 \mathrm{kts}$ (Table 8.27).

\subsection{Miscellaneous results}

\subsubsection{Experiment with assorted pixel count values}

A series of experiment are performed to test TIPS' sensitivity to different types of pixel counts at 24 and $48 \mathrm{~h}$. The first trial replaces PX0455 with its highly correlated PX0255, PX0265, PX0155, and PX0165 counterparts. Since it is possible that nonlinear satellite data will correlate better with $\Delta V_{\max }$, a similar test is performed on their squared 

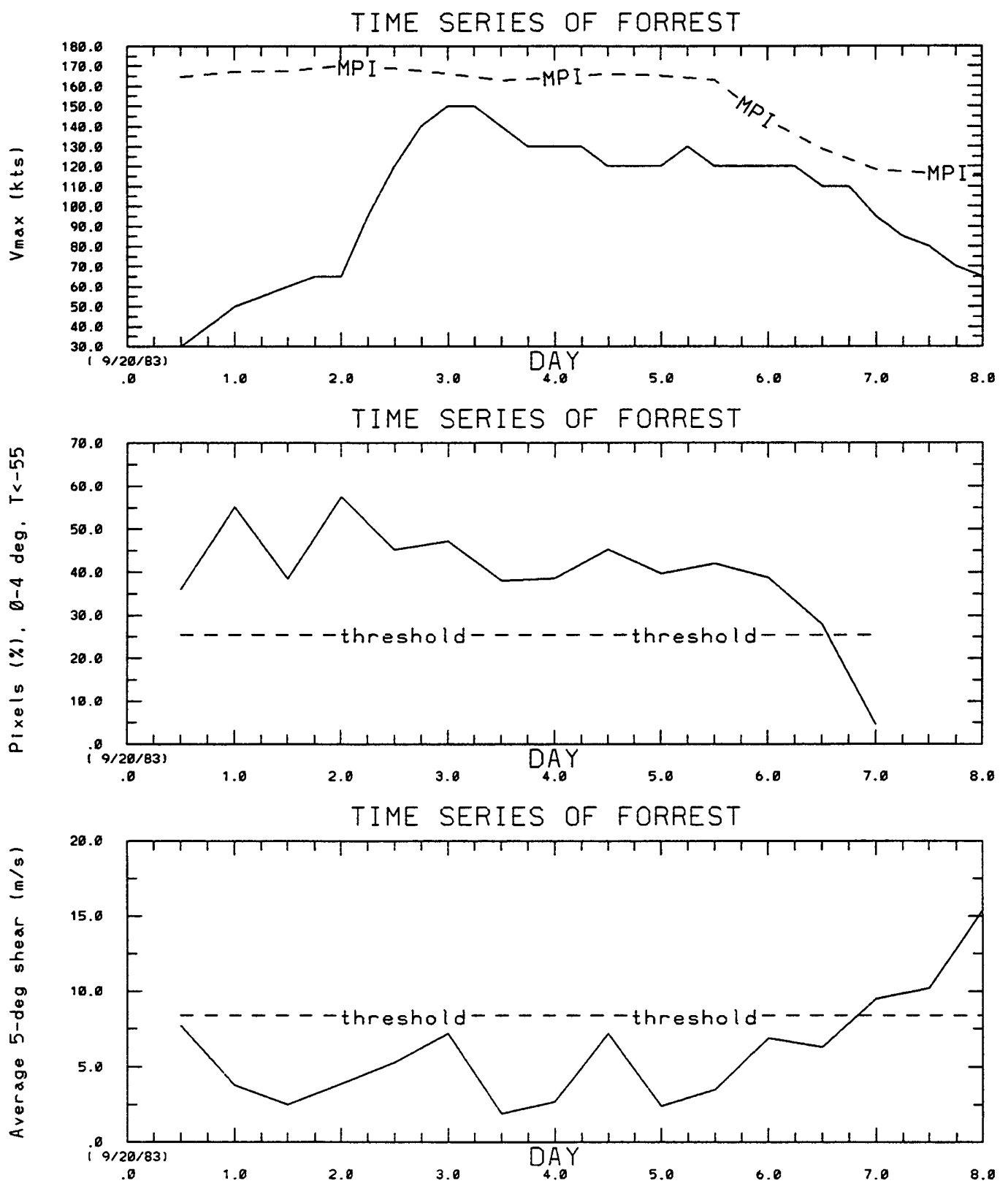

Figure 8.14: Time series of MPI and $V_{\max }$ (kts, top figure), PX0455 with its threshold value for reference (\%; middle figure), and VWS5 with its threshold value for reference ( $\mathrm{m} \mathrm{s}^{-1}$; bottom figure) for Typhoon Forrest (1983). 
TIME SERIES OF ORCHID

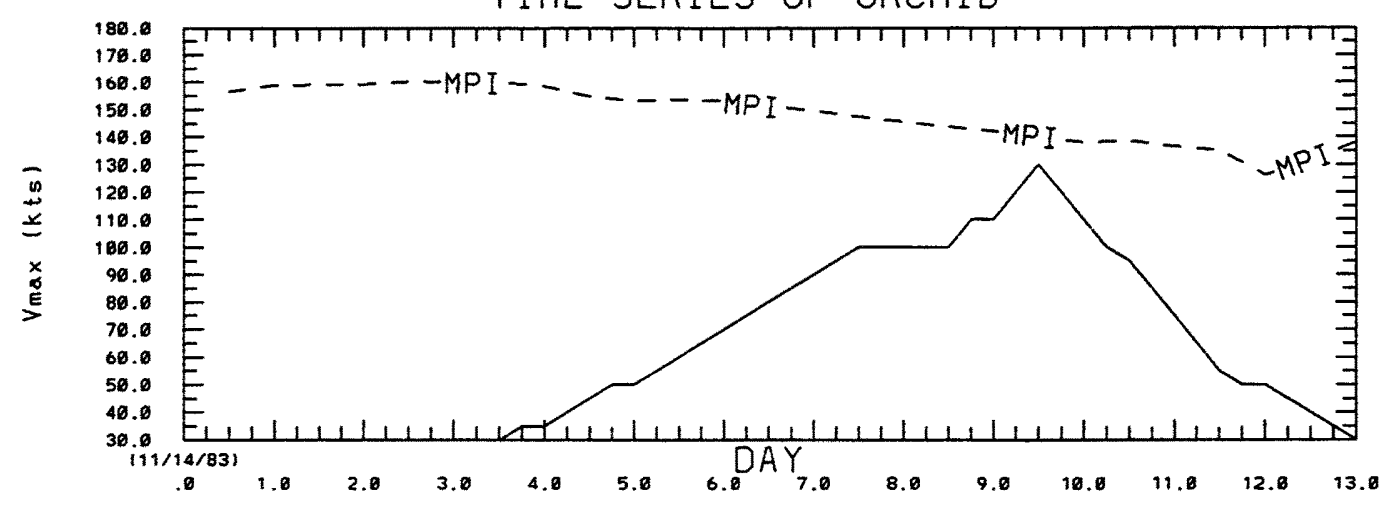

TIME SERIES OF ORCHID

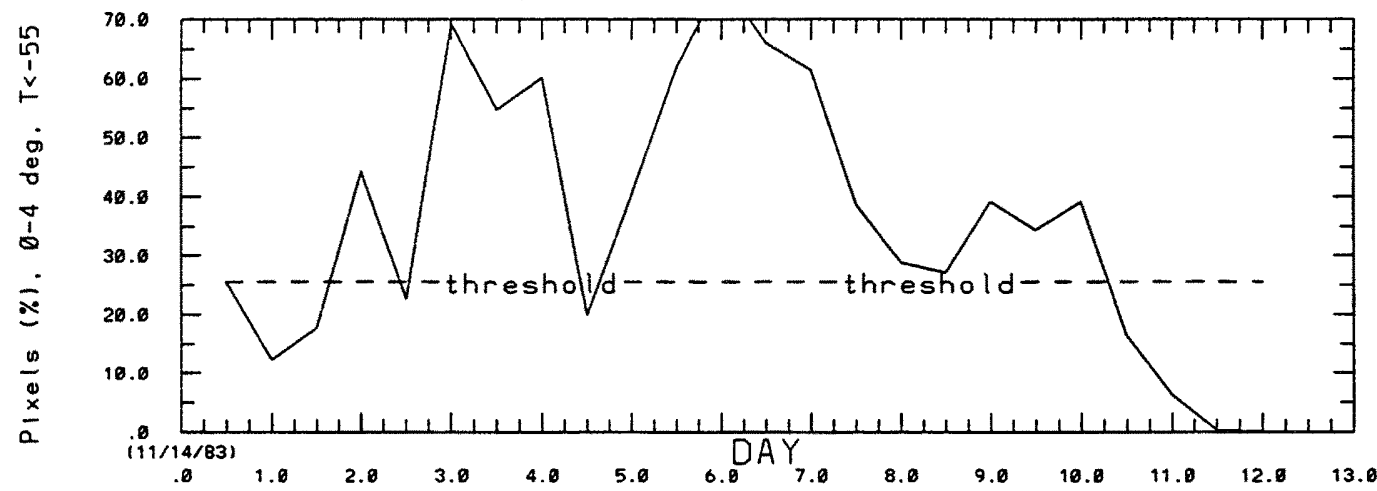

TIME SERIES OF ORCHID

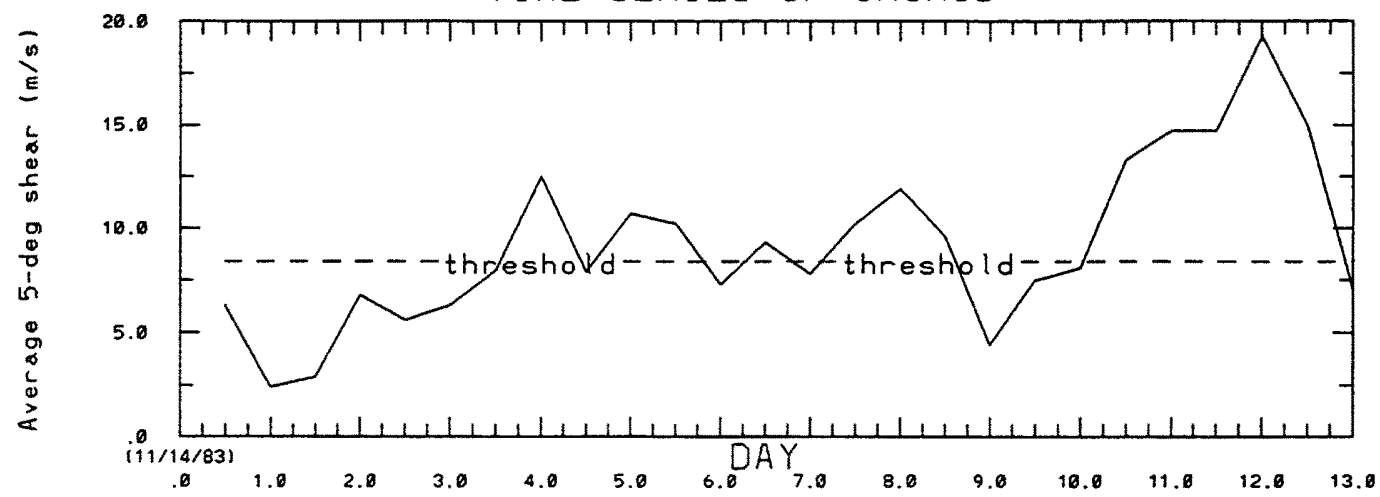

Figure 8.15: Time series of MPI and $V_{\max }$ (kts, top figure), PX0455 with its threshold value for reference (\%; middle figure), and VWS5 with its threshold value for reference ( $\mathrm{m} \mathrm{s}^{-1}$; bottom figure) for Typhoon Orchid (1983). 
Table 8.26: 24 -h forecast errors from the observed future $\Delta V_{\max }$ for TIPS and JTWC for Typhoon Forrest. Errors are defined as observed minus forecast. Day 0 is 00 UTC 20 September 1983.

\begin{tabular}{|c|c|c|c|}
\hline \multicolumn{4}{|c|}{ 24-h forecast errors for Typhoon Forrest (1983) } \\
\hline Day & $\Delta V_{\max }$ & TIPS error & JTWC error \\
\hline \hline 1.0 & 15 & -17 & -25 \\
\hline 1.5 & 60 & 28 & 35 \\
\hline 2 & 85 & 50 & 65 \\
\hline 2.5 & 20 & 8 & -5 \\
\hline 3 & -20 & -19 & -5 \\
\hline 3.5 & -20 & -10 & -5 \\
\hline 4 & -10 & -4 & 10 \\
\hline 4.5 & 0 & 0 & 10 \\
\hline 5 & 0 & 5 & 5 \\
\hline 5.5 & -10 & -1 & 5 \\
\hline 6 & -25 & -8 & -5 \\
\hline 6.5 & -30 & -8 & -20 \\
\hline 7 & -30 & -1 & -20 \\
\hline \hline Mean Rel. Error & 1.8 & 3.5 \\
\hline Mean Abs. Error & 12.2 & 16.5 \\
\hline
\end{tabular}

counterparts (labeled PX0255**2, PX0265**2, etc.), as well as PX0455**2. Finally, since running means are used in some operational settings, PX0455 is replaced by its 6, 12, and 24-h running means (RM6PX0455, RM12PX0455, and RM24PX0455) to assess any forecast differences. The sample size for the running means is less because sometimes no previous satellite observations existed for the averaging procedure. Results are presented in Tables 8.28 and 8.29 , which show $R^{2}$ and the average absolute error for 1983 and $1984-86$.

\section{Different pixel count radii}

In general, there is a $1-3 \%$ reduction in variance for inner-core pixels at $24 \mathrm{~h}, 1-2 \%$ decrease at $48 \mathrm{~h}$, and an increase in absolute error for both forecast periods. PX0255 seems to retain the most forecast signal next to PX0455. The overall increase in 1984-86 24-h errors is slight but not insignificant for a sample size of 530. The error differences at $48 \mathrm{~h}$ are more substantial. In 1983, a larger error spread compared to PX0455 is evident. One cannot reject the possibility that one of these pixel counts could be more important 
Table 8.27: 24 -h forecast errors from the observed future $\Delta V_{\max }$ for TIPS and JTWC for Typhoon Orchid. Errors are defined as observed minus forecast. Day 0 is 00 UTC 14 November 1983.

\begin{tabular}{|c|c|c|c|}
\hline \multicolumn{4}{|c|}{ 24-h forecast errors for Typhoon Orchid (1983) } \\
\hline Day & $\Delta V_{\max }$ & TIPS error & JTWC error \\
\hline \hline 4 & 15 & -9 & 0 \\
\hline 4.5 & 15 & 10 & 10 \\
\hline 5 & 20 & 9 & 30 \\
\hline 5.5 & 20 & -4 & 25 \\
\hline 6 & 20 & -8 & 20 \\
\hline 6.5 & 20 & 1 & 20 \\
\hline 7 & 10 & -2 & 0 \\
\hline 7.5 & 0 & 0 & -5 \\
\hline 8 & 10 & 22 & 15 \\
\hline 8.5 & 30 & 41 & 50 \\
\hline 9 & 0 & 2 & 35 \\
\hline 9.5 & -35 & -21 & -25 \\
\hline 10 & -35 & -16 & -30 \\
\hline 10.5 & -40 & -16 & -35 \\
\hline 11 & -25 & -2 & -15 \\
\hline 11.5 & -15 & 3 & 0 \\
\hline 12 & -20 & -3 & 0 \\
\hline \hline Mean Rel. Error & 0.4 & 5.6 \\
\hline Mean Abs. Error & 9.9 & 18.5 \\
\hline
\end{tabular}


Table 8.28: The 24-h $R^{2}(\%), 1984-86$ absolute error (kts), and 1983 absolute error (kts) for the TIPS variable PX0455 compared to different radii and brightness temperature pixel counts. PX0455 is also compared to their nonlinear (squared) counterparts (labeled with a ${ }^{* *} 2$ extension) as well as PX0455**2. Finally, PX0455 is compared to its 6,12 , and 24-h running means (RM6PX0455, RM12PX0455, RM24PX0455). If the number of cases is different than TIPS, it is also shown.

\begin{tabular}{|c|c|c|c|c|c|}
\hline $\begin{array}{l}\text { Different } \\
\text { Pixel counts }\end{array}$ & $R^{2}$ & $\begin{array}{c}1984-86 \\
\text { Abs. error }\end{array}$ & $\begin{array}{c}1983 \\
\text { Abs. error }\end{array}$ & $\begin{array}{c}1984-86 \\
n\end{array}$ & $\begin{array}{c}1983 \\
n\end{array}$ \\
\hline PX0455 & 52.2 & 9.2 & $\overline{11.6}$ & 530 & 139 \\
\hline 1) PX0265 & 50.4 & $\overline{9.3}$ & 11.7 & & \\
\hline 2) PX0255 & 51.1 & 9.2 & 11.7 & & \\
\hline 3) PX0165 & 49.5 & 9.4 & $\overline{11.9}$ & & \\
\hline 4) PX0155 & 49.2 & 9.4 & $\overline{12.2}$ & & \\
\hline 1) PX0455**2 & $\overline{51.6}$ & $\overline{9.2}$ & $\overline{12.0}$ & & \\
\hline 2) $\mathrm{PX0265^{** } 2}$ & 49.1 & 9.4 & 12.2 & & \\
\hline 3) $\mathrm{PX} 0255^{* *} 2$ & 50.9 & 9.2 & 11.7 & & \\
\hline 4) $\mathrm{PX} 0165^{* *} 2$ & 49.0 & 9.4 & 12.0 & & \\
\hline 5) $\mathrm{PX} 0155^{* * 2}$ & 49.1 & 9.4 & 12.1 & & \\
\hline 1) RM6PX0455 & 50.9 & 9.3 & $\overline{11.7}$ & $\overline{527}$ & \\
\hline 2) RM12PX0455 & 51.4 & 9.2 & 11.6 & 514 & \\
\hline 3) RM24PX0455 & 50.6 & $\overline{9.3}$ & 11.7 & 498 & \\
\hline
\end{tabular}


Table 8.29: The 48-h $R^{2}(\%), 1984-86$ absolute error (kts), and 1983 absolute error (kts) for the TIPS variable PX0455 compared to different radii and brightness temperature pixel counts. PX0455 is also compared to their nonlinear (squared) counterparts (labeled with a ${ }^{* *} 2$ extension) as well as PX0455**2. Finally, PX0455 is compared to its 6,12 , and 24-h running means (RM6PX0455, RM12PX0455, RM24PX0455). If the number of cases is different than TIPS, it is also shown.

\begin{tabular}{|l|c|c|c|c|c|}
\hline $\begin{array}{l}\text { Different } \\
\text { Pixel counts }\end{array}$ & $R^{2}$ & $\begin{array}{c}1984-86 \\
\text { Abs. error }\end{array}$ & $\begin{array}{c}1983 \\
\text { Abs. error }\end{array}$ & $\begin{array}{c}1984-86 \\
n\end{array}$ & $\begin{array}{c}1983 \\
n\end{array}$ \\
\hline \hline \multicolumn{1}{|c|}{ PX0455 } & 60.0 & 13.8 & 18.2 & 397 & 102 \\
\hline \hline 1) PX0265 & 58.9 & 14.2 & 19.0 & & \\
\hline 2) PX0255 & 59.0 & 14.2 & 19.1 & & \\
\hline 3) PX0165 & 58.4 & 14.3 & 19.2 & & \\
\hline 4) PX0155 & 58.2 & 14.3 & 19.6 & & \\
\hline \hline 1) PX0455**2 & 59.9 & 13.7 & 18.6 & & \\
\hline 2) PX0265**2 & 58.3 & 14.0 & 19.4 & & \\
\hline 3) PX0255**2 & 59.2 & 14.0 & 19.1 & & \\
\hline 4) PX0165**2 & 58.4 & 14.3 & 19.3 & & \\
\hline 5) PX0155**2 & 58.6 & 14.3 & 19.4 & & \\
\hline \hline 1) RM6PX0455 & 59.1 & 13.8 & 18.1 & 395 & \\
\hline 2) RM12PX0455 & 59.5 & 13.7 & 17.9 & 383 & \\
\hline 3) RM24PX0455 & 59.3 & 13.8 & 17.8 & 368 & \\
\hline
\end{tabular}


than PX0455 in a different sample. Furthermore, the 0-1 deg and 0-2 deg pixel counts may explain less variance due to errors in positioning the center of a TC to the satellite data. However, positioning errors will occur in an operational setting as well. More pixel count analysis is needed in the future, but for now PX0455 seems to be the best linear choice.

\section{Nonlinear pixel counts}

The squared terms generally explain slightly less variance than their corresponding linear terms at $24 \mathrm{~h}$, but slightly more variance at $48 \mathrm{~h}$, indicating that a long-term nonlinear feedback might exist. In both forecast times, PX0455**2 is generally the best alternate, followed by PX0255**2. It is interesting that in both the linear and nonlinear cases the best $T_{b}$ threshold is $-55^{\circ} \mathrm{C}$, perhaps because the warmer cloud-tops contain a stronger signal due to their longevity compared to higher cloud-tops with a shorter, unresolved lifespan.

The 1984-86 absolute errors are similar to their linear counterparts, but in 1983 all the errors are larger than PX0455. Furthermore, PX0455 still contains the highest $R^{2}$ at 24 and $48 \mathrm{~h}$. The nonlinear terms warrant further research, especially at longer forecast times, but it appears that the linear PX0455 predictor is a better choice.

\section{Tests of different pixel count running means}

Running means also show a degradation in variance compared to the observed pixel counts of $0.5-1.5 \%$. At both 24 and $48 \mathrm{~h}$, the least degradation occurs with the 12-h running means, and at $48 \mathrm{~h}$ there is less overall variance decrease compared to $24 \mathrm{~h}$. The errors are comparable or even less than PX0455, but this might be an artifact of fewer data samples compared to PX0455 in 1984-86. This reduction in sample size also probably inflates $R^{2}$ values for the running means. However, as indicated by the errors in 1983 where no sample size decrease exists, it is possible that running means may slightly improve 48h forecasts compared to PX0455. But the rest of the evidence suggests that temporal smoothing of the satellite data removes vital predictive signals retained in the pixel count data at $t=0$. Furthermore, it is clear that running means do not substantially improve 
the forecasts (with degradation more likely), and since they are harder to implement in an operational setting, observed pixel counts are recommended - for TIPS, PX0455 still is the best choice.

\section{Overall analysis of pixel count sensitivity}

In the previous experiments with different pixel count terms, stepwise regression was not allowed so that easier comparisons could be made. This is because stepwise regression usually adds an additional term (often a climatology term such as LONG or VSM) to help offset the decrease in variance when using other pixel counts besides PX0455. Interestingly enough, when the extra term was added by stepwise regression, $R^{2}$ increased but the absolute errors also increased in almost all cases (not shown). This is a result of "overfitting" the data.

In conclusion, based on the support of $R^{2}$ and absolute error computations, it appears that the chosen predictors are the premier forecast variables until further evidence suggests otherwise. Furthermore, PX0455 is the optimum pixel count predictor for intensity change.

\subsubsection{Comparison of different wind shear parameters}

Sensitivity experiments were performed with point wind shear (VWSPT), areally averaged wind shear in a 2.5-deg circle (VWS2), and areally averaged wind shear in a 5-deg circle (VWS5). The variance decreased consistently from VWS5 to VWS2 by $0.5 \%$ out to $36 \mathrm{~h}$, and $1 \%$ at $48 \mathrm{~h}$ (not shown), indicating a slightly larger dependence at $48 \mathrm{~h}$. The variance decreased consistently by $1.3 \%$ from VWS5 to VWSPT, and $1.7 \%$ at $48 \mathrm{~h}$ (not shown). In other words, there is a distinct favorism for storms to intensify in a region of large-scale low shear, as shear impinging on the edge of the TC: 1) serves to weaken the storm even if shear over the storm center might be low; 2) indicates that the TC is about to move into an unfavorable shear region; and/or 3) indicates that 200-mb westerly winds or accelerating easterly winds or about to traverse over the TC center. Furthermore, area averaging reduces the chance of one erroneous wind observation contaminating the true shear environment, such as a bad data point or outflow from the TC misrepresenting the calculation. Finally, using Eq. (8.8) for area averaging removes the circulation of an 
anticyclone above a well-developed TC - westerly outflow from an anticyclone using single point calculations can introduce unphysically large wind shear.

An interesting result is that the VWS5 threshold of $8.5 \mathrm{~m} \mathrm{~s}^{-1}$ increases to $9.0 \mathrm{~m} \mathrm{~s}^{-1}$ for VWS2, and $9.5 \mathrm{~m} \mathrm{~s}^{-1}$ for VWSPT. This agrees qualitatively with Zehr's (1992) $12.5 \mathrm{~m} \mathrm{~s}^{-1}$ threshold for single point shear in his tropical cyclogenesis work. Since the variance degradation is not very much, and because single point shear is used in operational settings (mostly for convenience), an alternative version of TIPS with VWSPT is presented in Appendix B.

\subsubsection{Comparison of perfect-prog shear forecasts against shear forecasts based on persistence}

As mentioned previously, SHIPS uses shear analysis at the beginning of the forecast period to evaluate VWS at future storm positions. Such a procedure was chosen in SHIPS because forecasting shear based on persistence is easier to implement operationally, and because shear forecast errors are often of the same magnitude as persistence. This author's personal experience shows that anticipating when a trough (or TUTT) will dissipate or retreat into the mid-latitudes - and thus changing the tropical atmosphere from a high shear regime to a low shear regime - is difficult to anticipate. Furthermore, predicting 200-mb westerly wind fluxes from the mid-latitudes into the tropics - and thus inducing a high shear regime (and sometimes forming a TUTT low) - is equally frustrating. Numerical models provide little forecast guidance as well. The AVN model tends to artificially weaken existing westerlies (and TUTTs) in the Caribbean Sea, and therefore the prognosis of a low shear regime often does not verify (Fitzpatrick et al. 1995). TUTTs in other basins also are not well-forecast by models (L. Leslie and L. Carr, personal communication 1995).

Hence, it seems prudent and more realistic to develop an alternative TIPS version based on persistence of shear. Alternative versions of TIPS based on VWS5 forecasts based on persistence (VWS5 $5_{\text {per }}$ ) and VWSPT forecasts based on persistence (VWSPT per $_{\text {) have }}$ been developed, and the statistical information is provided in Appendix B. The results 
are summarized here, as are the absolute 24- and 48-h forecast errors in Tables 8.30 and 8.31 against JTWC for 1984-86 (development data set) and 1983 (independent data set). As expected, since incorrect shear forecasts are included in the regression, there is a reduction in variance explained for both point shear and areally averaged shear based on persistence (Appendix B). 24-h $R^{2}$ for $\mathrm{VWS}_{\text {per }}$ and $\mathrm{VWSPT}_{\text {per }}$ is $49.5 \%$ and $48.3 \%$, respectively, compared to $52.2 \%$ and $51.0 \%$ for VWS5 and VWSPT, respectively. 48-h $R^{2}$ for $\mathrm{VWS}_{\text {per }}$ and VWSPT $\mathrm{\text {Ver }}$ is $56.3 \%$ and $55.3 \%$, respectively, compared to $60.0 \%$ and $58.3 \%$ for VWS5 and VWSPT, respectively. In other words, shear forecasts based on persistence compared to perfect-prog decreases $R^{2}$ by $2.7 \%$ for 24 -h forecasts and by $3.0-3.7 \%$ for 48 -h forecasts.

Otherwise, the important predictors remain essentially the same as the perfect-prog version. The difference is that the importance of shear forecasts based on persistence (as inferred by the normalized regression coefficient $c_{i}$ ) is less compared to shear forecasts based on perfect-prog for the same forecast interval, and this difference increases as the forecast period extends to $48 \mathrm{~h}$. Of course, this regression analysis indicates the introduction of poor shear forecasts by the persistence method which amplifies with time. Otherwise, $c_{i}$ between the persistence method and perfect-prog remains essentially the same for other predictors.

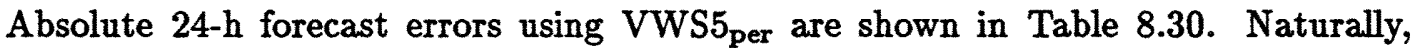
errors compared to the TIPS perfect-prog version (Table 8.13) are larger. Still, in general TIPS' errors based on persistence of shear are less than JTWC. The overall absolute error increases from 9.2 (perfect-prog of shear) to $9.4 \mathrm{kts}$ (persistence of shear) for the dependent data set, but is still less than JTWC's error of $11.2 \mathrm{kts}$. The overall absolute error increases from 11.8 (perfect-prog of shear) to $12.3 \mathrm{kts}$ (persistence of shear) for the independent data set, but is still less than JTWC's error of $14.6 \mathrm{kts}$.

Absolute 48-h forecast errors using VWS5 ${ }_{\text {per }}$ are shown in Table 8.31. Again, errors compared to the TIPS perfect-prog version (Table 8.15) are larger, but still less than JTWC. The overall absolute error increases from 13.4 (perfect-prog of shear) to $14.3 \mathrm{kts}$ (persistence of shear) for the dependent data set, but is still less than JTWC's error of 
Table 8.30: Average 24-h absolute errors (in kts) for an alternative version of TIPS developed on 5-deg areally averaged vertical wind shear based on persistence (VWS5 per). The JTWC forecast for the dependent data set (1984-1986) and independent data set (1983) is shown for comparison. The errors are stratified by intensity change regimes. Number of cases for all stratification is also shown. This alternative version of TIPS is developed on perfect-prog track forecasts, so this table does not imply that this version can outperform JTWC.

\begin{tabular}{|l|c|c|c|c|c|c|}
\hline Years & $\Delta v \geq 25$ & $20 \geq \Delta v \geq 10$ & $5 \geq \Delta v \geq-5$ & $-10 \geq \Delta v \geq-20$ & $\Delta v \leq-25$ & Overall \\
\hline \hline 84-86 TIPS & 17.6 & 7.5 & 6.6 & 9.3 & 14.6 & 9.4 \\
84-86 JTWC & 16.8 & 8.8 & 10.7 & 10.9 & 15.3 & 11.2 \\
\hline 84-86 Cases & 69 & 158 & 154 & 122 & 35 & 538 \\
\hline \hline 83 TIPS & 22.0 & 7.8 & 9.2 & 12.0 & 17.4 & 12.3 \\
83 JTWC & 27.5 & 13.0 & 11.8 & 10.5 & 17.7 & 14.6 \\
\hline 83 Cases & 20 & 35 & 28 & 39 & 13 & 135 \\
\hline
\end{tabular}

$18.2 \mathrm{kts}$. The overall absolute error increases from 18.4 (perfect-prog of shear) to $19.7 \mathrm{kts}$ (persistence of shear) for the independent data set, but is still less than JTWC's error of 25.0 kts.

Table 8.31: Average 48-h absolute errors (in kts) for an alternative version of TIPS developed on 5-deg areally averaged vertical wind shear based on persistence (VWS $5_{\text {per }}$ ). The JTWC forecast for the dependent data set (1984-1986) and independent data set (1983) is shown for comparison. The errors are stratified by intensity change regimes. Number of cases for all stratification is also shown. This alternative version of TIPS is developed on perfect-prog track forecasts, so this table does not imply that this version can outperform JTWC.

\begin{tabular}{|l|c|c|c|c|c|c|}
\hline Years & $\Delta v \geq 45$ & $40 \geq \Delta v \geq 20$ & $15 \geq \Delta v \geq-15$ & $-20 \geq \Delta v \geq-40$ & $\Delta v \leq-45$ & Overall \\
\hline \hline 84-86 TIPS & 30.2 & 12.0 & 11.9 & 14.8 & 16.0 & 14.3 \\
84-86 JTWC & 31.9 & 14.5 & 16.9 & 17.2 & 29.3 & 18.2 \\
\hline 84-86 Cases & 39 & 108 & 175 & 74 & 14 & 410 \\
\hline \hline 83 TIPS & 35.1 & 10.9 & 18.3 & 18.6 & 26.9 & 19.7 \\
83 JTWC & 47.1 & 23.1 & 21.6 & 15.4 & 35.8 & 25.0 \\
\hline 83 Cases & 14 & 21 & 32 & 24 & 6 & 97 \\
\hline
\end{tabular}

Even though persistence of shear is used in this alternative version of TIPS, this regression is still developed on post-processed data (which would contain uncertainties in an operational setting) and perfect-prog track forecasts. Therefore, this writer cannot claim this version of TIPS (with shear forecasts based on persistence) outperforms JTWC. 
However, it does reinforce this writer's claim that TIPS may be competitive with JTWC, and can provide forecast guidance.

It is the forecaster's choice on which TIPS' version should be used. It is this writer's opinion that the version based on perfect-prog of shear should be used since it is such a crucial forecast parameter. As discussed in Fitzpatrick et al. (1995), model output should be carefully analyzed, and subjectively altered if the numerical guidance is judged to be poor. Fitzpatrick et al. (1995) recommend persistence and climatology for predicting VWS in the vicinity of the Atlantic TUTT as an alternative to the model output, and this procedure is likely to be useful in the Pacific TUTT as well. This writer's personal experience also shows water vapor loops supply critical information about the upper-level wind evolution, and recommends its use to supplement shear forecasts.

\subsubsection{Other sensitivity experiments}

Sensitivity runs based on stratifications were pursued to see if the errors could be reduced more than TIPS. Neumann (1991) has shown that deriving separate regression equations for storms equatorward or poleward of $25 \mathrm{deg} \mathrm{N}$ helped distinguish between TCs traversing westward and TCs recurving. Elsberry et al. (1988) stratified TCs by weak, moderate, and strong $V_{\max }$ regimes, and found that the regression forecast errors were reduced on the dependent data set (although this test was not applied on an independent data set). Therefore, several geographical and intensity stratifications were examined for TIPS. As in the other sensitivity experiments, absolute errors increased (not shown). Apparently, including the full TC life cycle is a key ingredient to accurate forecasting of intensity. Furthermore, decreasing the sample numbers by several hundred introduces much uncertainty in the appropriateness of the regression results.

\subsubsection{Relationship of Relative Eddy angular momentum Flux Convergence (REFC) to upper-level vorticity advection}

As mentioned in Chapter 2, DeMaria et al. (1993) suggests that REFC (imposed as a torquing mechanism on an azimuthally averaged system) and 200 -mb vorticity advection (with synoptic ascent downstream of a trough) share the same forcing characteristics 
once allowances for the different coordinate systems are made. To examine this assertion more closely, linear correlations between the two terms are computed. Indeed, a positive correlation of $r=0.25$ is found between VADV and REFC mid $_{\text {(REFC averaged between } 7}$ and $10 \mathrm{deg}$ ), with a smaller positive correlation for REFC outer $_{\text {(REFC averaged between }}$ 11 and $15 \mathrm{deg}$ ) of $r=0.18$. Given that correlations between other potential independent predictors are, in general, much smaller, one can conclude that there is some analogy between these forcing terms. On the other hand, the unexplained variance between the two terms is also quite large, suggesting that REFC contains forcing components not associated with either: 1) a trough; or 2) a trough's influence on the entire azimuthal state of the TC.

\subsection{Summary}

This chapter describes a multiple regression scheme (the Typhoon Intensity Prediction Scheme, TIPS) developed for the West Pacific basin. Out of 110 possible climatology, persistence, satellite, SST, and synoptic predictors, applying stepwise regression (with an autocorrelation "filtering" procedure and a strict 99\% significance level) yields 5-6 final predictors for forecast periods of $12,24,36$, and $48 \mathrm{~h}$. Commentary is included on the apparent insignificance of upper-level forcing, contrary to the results of some researchers.

A normalization procedure allows one to infer the importance of each significant predictor, which shows the SST term (POT) to be two to three times as important as other terms, and the pixel count information to be second in importance followed by wind shear, persistence with an "eye parameterization," pixel count trend, storm motion, and longitude. Sensitivity tests show that pixel counts in a 4-deg area with $T_{b}$ less than $-55^{\circ} \mathrm{C}$ is the best satellite predictor. Wind shear over a 5-deg area is the best VWS predictor, although single point shear explains just slightly less variance. It is shown that nonlinear pixel counts and running means generally explain less variance as well. The normalization procedure also provides operational forecasters threshold values which can be used as "rules of thumb."

Stratifications by intensity change and case studies show that the pixel count information provides essential information to distinguish between TCs which will intensify fast 
and those that will intensify at a slower rate, while water temperature and shear cannot infer these differences. This implies that CAPE is playing an important role in the intensification process. On the other hand, SSTs and shear do provide key information between forecasting slow and fast weakening, while pixel counts do not.

Evaluation of TIPS' performance shows that the regression scheme can still suffer large errors at times, and underforecasts fast intensification and fast weakening situations. However, a comparison against JTWC for 1984-86 (the dependent data set) and 1983 (the independent data set) reveals that TIPS is at least competitive with JTWC, and perhaps a little better, especially in situations where $\Delta V_{\max }$ is large in magnitude. 


\section{Chapter 9}

\section{CONCLUSIONS AND RECOMMENDATIONS}

\subsection{Description of this report}

The following tasks were performed in this report:

1. Documentation of forecast errors associated with TC intensity change was done, using Hurricane Hugo (1989), Hurricane Andrew (1992), and Typhoon Omar (1992) as examples. The implications of poor $\Delta V_{\max }$ forecasting, especially storms undergoing rapid intensification (pressure drop of $42 \mathrm{mb}$ or more in $24 \mathrm{~h}$ ), were presented which motivated this study.

2. Before this report was written, documentation of research on TC intensity change was scattered throughout the literature. An effort was made to consolidate these theoretical and observational studies into Chapters 2, 3, and 4.

3. Chapter 2 summarized research efforts to relate TC intensity change to upper-level forcing. These trough interactions include enhancement of TC outflow channels by favorable positioning of a trough, by eddy angular momentum (and/or eddy heat) fluxes, or by cyclonic vorticity advection.

4. Chapter 3 summarized intensification mechanisms which do not require upper-level forcing. Favorable internal contributors to intensification include warm SSTs, low shear, potent convection and eyewall buoyancy, a strong upper-level vortex, and eye formation. Modulation of TC intensity change by strong outer-core inertial stability and concentric eyewall cycles were noted. Commentary on the questionable validity of upper-level forcing as a positive interaction with TCs was then made, emphasizing that most researchers have made their conclusions based on case studies or highly 
idealized models with little regard on how the general ensemble of TCs respond to upper-level forcing (which is usually associated with wind shear).

5. Current Air-Sea Interactions (ASI) theories were discussed in Chapter 3. Flaws in current ASI theories were recognized, such as the assumption of isothermal warming when in reality some boundary layer cooling is probably occurring with TC intensification. Additional problems associated with the currently popular Carnot Cycle ASI theory were discussed.

6. A new theory called Internally Forced Convergence (IFC) was introduced in Chapter 3 , which states that a TC can continue to intensify only if eyewall vertical motion is strong enough to balance frictional loss and upper-level cyclonic tangential wind export, and still have enough additional momentum that $V_{\max }$ increases. This is a statement that strong eyewall buoyancy is needed for intensification, in contrast to arguments that eyewall ascent is conditionally neutral to the eyewall environment. Chapter 4 presents ample evidence that buoyancy exists in TC eyewalls.

7. Chapter 4 documented a variety of proposed and operational intensity forecast schemes. These formulations are concentrated in five areas: 1) climatology; 2) satellite; 3) EXPERT systems; 4) numerical modeling; and 5) statistical multiple regression. These schemes were briefly described, as were their benefits and disadvantages. The advantage of using statistical multiple regression was emphasized because it not only yields an objectively determined predictive scheme, but generates understanding of what parameters are statistically relevant to intensity change, and quantitatively assesses each significant predictor's importance.

8. Chapter 5 described the inner-core data sets. These include rawinsonde data, as well as sparse but invaluable upper-level inner-core flight data from NHRP and HRD. Analysis of the inner-core data was performed in Chapter 6 . This wind data provided rare analysis of the inner-core vertical structure in terms of soundings, cross-sections, and upper-tropospheric planviews. A variety of composite stratifications were also be done. The inner-core data provided insight - and perhaps predictive information - 
in what distinguishes fast and slowly developing TCs in Chapter 6. A theory, based on thermal wind and geostrophic adjustment, was postulated on the relationship between the vertical structure of the inner-core wind and intensification. Tentative calculations based on balanced vortex supported this theory.

9. Chapter 5 also described the data set for the regression analysis in Chapter 8. The years 1983-1986 are chosen, coinciding with western North Pacific reconnaissance flights before their termination in 1987. The sample consists of SST, synoptic, besttrack, and digitized infrared satellite data. From this data, a multiple regression scheme analogous to the Statistical Hurricane Intensity Prediction Scheme (SHIPS) used at NHC was developed, but with two major differences - the ocean basin is different (western North Pacific), and satellite data is used. Furthermore, to this author's knowledge, this is the first time such satellite information has been combined with other predictors into a multiple regression forecast scheme. This new scheme is called the Typhoon Intensity Prediction Scheme (TIPS), and it was shown that the satellite data contains vital information which distinguishes between fast and slow developing TCs. The importance and contribution of other predictors (such as SSTs and wind shear) to intensity change were also clarified in the statistical analysis.

10. Chapter 7 presented a modified ASI theory which incorporates boundary layer cooling, and therefore is a slight departure from previous ASI theories. The buoyancy calculations included ice microphysics and water-loading, and surface pressures were computed from a rawinsonde composite of the inner-core temperature perturbation evolution in a developing TC. Entrainment was only heuristically considered. The latter demonstrated the importance of the lateral extension of the eye above a sloping eyewall so that the ASI process can be sustained even with boundary layer cooling. Implications of an insufficient increase of CAPE to meet the budgetary requirements of IFC were also discussed, and it was hypothesized that this bounds MPI.

The specific results of this research will now be summarized. 


\subsection{Summary}

\subsubsection{Rapid intensification theory}

Documentation of the inner-core tangential wind evolution was presented which shows upper-tropospheric $v_{t}$ increase significantly as a TC intensifies, and at a greater than one-to-one ratio with respect to low-level $v_{t}$ increase. One importance of this upperlevel eyewall spin-up is that sufficient buoyancy must exist to compensate both frictional dissipation and the export of cyclonic $v_{t}$, and still contain sufficient extra momentum available to intensify the TC (the IFC concept).

It is hypothesized that the vertical transport of eyewall cyclonic momentum plays an important role in TC intensity change through thermal wind balance and geostrophic adjustment processes. A low shear environment allows a vertically stacked vortex to develop, with cyclonic winds almost as fast as the lower troposphere. Such a vertical structure favors fast development. The relationship may be discussed in terms of balanced vortex theory initially formulated by Eliassen (1951), and used in two-dimensional, axisymmetric TC numerical models models (Ooyama 1969; Sundqvist 1970; Pfeffer and Challa 1980; Schubert and Hack 1982; Hack and Schubert 1986b).

Balanced vortex dynamics describes the slow evolution of a vortex in gradient and hydrostatic balance responding to weak forcing (in a TC, mostly diabatic forcing). Therefore, balanced vortex theory is a filtered model because the transient aspects of gradient wind adjustment are excluded, and instant thermal wind balance is assumed.

Consider a TC in the tropical storm stage with vigorous convection in a weakly sheared environment. The resulting diabatic heating will drive fast vertical motions. The resulting inflow increases low-level $v_{t}$, but the upward advection of cyclonic momentum aloft increases $v_{t}$ in the upper-levels at a proportionately faster rate. A composite analysis of TCs in different stages of intensity showed that outflow level $v_{t}(300 \mathrm{mb})$ is about onefourth of 850 -mb $v_{t}$ for a weak tropical storm, increases to one-half as the storm intensifies, and a ratio of two-thirds is possible with intense cyclones. This preservation of $v_{t}$ with height was documented from aircraft data as well. 
Only a small fraction of the heat released in moist convection goes into warming - just so much as is necessary to adjust the temperature field back to thermal wind balance with the new vortex profile. The rest of the heat is transmitted in the form of gravity waves to a wide outside area, and is not considered explicitly in the balanced vortex framework (which is the definition of a filtered model). The maximum inner-core temperature perturbation occurs in the region where $v_{t}$ decreases the most with respect to $\ln p$ (Eq. (6.1)).

Therefore, the balanced vortex model consists of the following steps: 1) compute the radial distribution of Ekman pumping; 2) compute the vertical distribution of the diabatic heating rate, which is parameterized as a function of mass flux from the boundary layer forcing and the amount of buoyancy achieved. The buoyancy itself is related to the surface pressure, surface relative humidity, surface temperature, rate of entrainment, water-loading, latent heat of condensation, and latent heat of sublimation and fusion; 3) compute the resulting secondary circulation due to the the distribution of Ekman pumping and diabatic heating rate; 4) time-step a new $v_{t}$ profile; 5) compute the temperature field which conforms to thermal wind balance; 6) compute the resulting surface pressure field; 7) compute surface temperature and surface relative humidity; and 8) repeat steps 1-7.

For cases under minimal VWS and warm SSTs such that vigorous inner-core updrafts exist, the cyclonic circulation should extend almost to the tropopause. In theory, the vertical variation of $v_{t}$ in the lower-to-middle troposphere would be relatively smal1. Most of the large vertical gradient of $v_{t}$ with respect to $\ln p$ would be confined near the tropopause. Therefore, the maximum temperature perturbation is confined near the tropopause, and instability can be maintained in the updraft region. Furthermore, the maximum pressure drop can be realized, since pressure fall is nonlinearly related to the height of the temperature perturbation (the "dp/p effect" in Eq. (3.2)). Hence, a stacked vortex indirectly enhances the ASI process through these feedback mechanisms. Finally, the geostrophic adjustment process is most efficient for a stacked vortex, thereby maximizing the fastest possible increase of the temperature perturbation per $v_{t}$ time-step. This increased "dynamic efficiency" also enhances intensification. 
When one combines these processes with the formation of an eye (which also enhances the secondary circulation and the ASI process), a rapidly intensifying TC is possible. In a more common type of intensification where moderate amounts of shear exist such that the vortex cannot vertically build as high, the inner-core warming is located in the middle troposphere where the vertical variation of $v_{t}$ with respect to $\ln p$ is greatest. This midlevel stabilization over time tends to reduce further deep convection, and intensification continues at a slower pace. Furthermore, the maximize pressure drop cannot be realized since the warmest temperature perturbation is confined to the mid-troposphere.

Tentative diagnostic calculations based on balanced vortex theory support this chain of events. However, modulation of TC development by strong outer-core inertial stability was also noted, and is consistent with the findings of Weatherford (1989) and Fitzpatrick (1992). Therefore, the horizontal distribution of $v_{t}$ may be just as important as the vertical structure of a TC with regards to intensity change.

\subsubsection{Observational study of possible link between inner-core upper-level tropical cyclone structure and intensification}

These results appear to offer forecast potential, since there seems to be a time lag between the increase in upper-level vorticity in the eyewall and the central pressure fall. Should high tangential winds be observed in the upper eyewall, and the potential intensification based on SST be large (as dictated by a large difference between MPI and current $V_{\max }$ ), one may predict future fast deepening. Examples are given for Supertyphoon Flo (1990) and Hurricane Andrew (1992).

NHRP and rawinsonde data also supports this idea that a vertically stacked vortex signifies future fast intensification. By stratifying the rawinsonde data into future fast (pressure drop $\geq 30 \mathrm{mb}$ in the next $24 \mathrm{~h}$ ) and slow developers (pressure fall between 10 and $20 \mathrm{mb}$ in the next $24 \mathrm{~h}$ ), it was shown that, for TCs of similar intensity ( $50 \leq V_{\max } \leq$ $85 \mathrm{kts}$ ), the fast developers contained a more vertically stacked inner-core vortex. This conclusion was based on 0.8 to $2.0 \mathrm{deg}$ composite analysis of the $v_{t}$ wind profile of fast and slow developers. Individual comparisons of $v_{t}$ soundings between fast developers and slow developers also supported this claim. 
The NHRP data suggested a direct correlation between upper-level $v_{t}$ and whether a TC is experiencing intensification as well, with the rate of intensification proportional to the magnitude of upper-level $v_{t}$. A three-dimensional analysis of the NHRP upperlevel inner-core winds and temperature field suggest that symmetry (asymmetry) and a strong (weak) $\nabla T$ was correlated with intensification (weakening). Inspection of mid-level fields did not show as clear a signal, suggesting that monitoring of upper-level eyewall temperature and wind fields may yield important forecast information about intensity change.

A general overview of the broad-scale features associated with fast developing and slow developing Pacific storms also was discussed. While inner-core features probably dominate the intensification process, distinctions between the radial winds, tangential winds, and temperature fields in a coarser 2 to 14-deg area also yielded valuable $\mathrm{TC}$ information between these two development categories.

The composite of these fast and slow developers showed that the former contains a faster secondary circulation (implying more convective forcing), more preservation of $v_{t}$ with height, weaker outer-core low-level winds, and a cooler troposphere (particularly near the inner-core). This is consistent with the ideas discussed in the previous paragraphs. Anticyclonic outflow past $6 \mathrm{deg}$ was stronger for future fast developers. The surface temperatures were warmer in the fast developers, implying that warmer SSTs are also important for this class of TCs.

\subsubsection{Alternative Air-Sea Interaction (ASI) theory with boundary layer cool- ing, water-loading, and ice microphysics}

Increasing evidence shows that the air-sea temperature difference increases to $3-4^{\circ} \mathrm{C}$ under the eyewall as a TC intensifies, in contrast to the long-time consent among tropical meteorologists that the difference remains $1^{\circ} \mathrm{C}$. The possible processes contributing to this cooling were discussed. The most plausible (although still tentative) conclusion was that the adiabatic expansion of air as it flows toward lower surface pressure is not totally being compensated by sensible heat fluxes, hence the air parcel experiences a temperature drop. A crude derivation of this surface cooling was presented, in which a positive feedback 
between surface winds and surface fluxes mostly compensates the adiabatic expansion, but a $3-4^{\circ} \mathrm{C}$ air-sea temperature difference develops with the pressure fall.

The importance of including water-loading and ice microphysics in buoyancy calculations was discussed. The mathematical treatment of these processes was discussed, and buoyancy calculations for pseudoadiabatic and reversible adiabatic ascent (with and without ice microphysics) were presented. It was shown that, although the reversible adiabatic without ice is less buoyant (due to water-loading) than the pseudoadiabat without ice, the reversible process with ice is more buoyant than the pseudoadiabatic process without ice above the freezing level. It was acknowledged that entrainment will reduce the amount of buoyancy, but since the eyewall entrainment rate is probably fairly constant throughout the TC's life cycle, this doesn't qualitatively alter these results. Several conclusions about buoyancy were thus made.

First, by including the effects of water-loading and ice, the vertical distribution of buoyancy is altered. There is less buoyancy in the lower-troposphere with retained liquid water, but these droplets contain potential buoyancy which is released above the freezing level. This sudden release of buoyancy above the freezing level can play an important role in TC intensity change, since this release of latent heat will increase the secondary circulation, and because this extra source of diabatic heating occurs in a region where geostrophic adjustment processes are more efficient.

Second, CAPE in the tropics is roughly the same for water-loading (reversible process) with ice and no water-loading (pseudoadiabatic process) with no ice. This result is interesting because it contradicts the notion of conditional neutrality in the tropics and TC eyewalls which some researchers claim exist. This is because the conditional neutrality concept is based on reversible thermodynamics with no ice. It was concluded that the conditional neutrality hypothesis for deep convection is invalid in the tropics because if liquid condensate is retained it must eventually freeze somewhere above the $0^{\circ} \mathrm{C}$ isotherm.

Based on these results, a simple methodology for buoyancy calculations was proposed. A reversible and pseudoadiabatic process represent extreme adiabatic ascent situations (all condensate retained in a parcel versus all condensate falling from the parcel the instant it 
appears). Furthermore, it was found that CAPE in a pseudoadiabatic process with ice was only slightly greater than a reversible process with ice, because the pseudoadiabatic process contains no supercooled water droplets and therefore the ice microphysics contains only latent heat of deposition. Since both contain similar CAPE values when ice was included. it was proposed that buoyancy calculations average the $T$ and $T_{\rho}$ values resulting from these two processes.

Documentation based on composites of TCs in various stages of intensity presented a picture of the eyewall temperature anomaly evolution. It was shown that most of the warming occurs in the upper eyewall, while the middle and (especially) the lower levels experience little temperature increase. Furthermore, this upper-level warming increases disproportionally relative to the low-level warming. This upper-level warming can be attributed to two factors. First, the upper-eyewall is the most efficient region for converting latent heat to the kinetic energy of the TC (which dictates an increasingly warm upperlevel through thermal wind balance). Second, above the sloping eyewall is the lateral extension of the eye, which becomes stronger with intensity.

Based on all these results, a modified ASI theory was presented. This theory incorporated boundary layer cooling with eyewall pressure fall, and buoyancy calculations which includes partial water-loading and ice microphysics. The eyewall temperature evolves similar to the composite study, and an associated eyewall surface pressure was computed. The surface relative humidity was postulated to increase with intensity and the boundary layer cooling, thus also providing a buoyance source. It was shown that the ASI process can be sustained with the positive feedback of surface pressure fall (due to the lateral extension of the eye above a sloping eyewall), latent heat of fusion and deposition, and increasing relative humidity despite the surface cooling.

\subsubsection{The increase of CAPE in the eyewall, and a new Maximum Potential Intensity (MPI) theory}

From the modified ASI calculations, it was shown that eyewall buoyancy remains the same, and quite likely increases, as a TC develops. Buoyancy is sustained even with boundary layer cooling because most of the eyewall warming is confined to the upper 
troposphere. This enhances a parcel's density difference with the low-level eyewall environment, and also maximizes the increase in moist entropy. Furthermore, the eyewall slopes outward aloft above $300 \mathrm{mb}$. In this manner, updrafts travel upwards through regions of lesser warming than would occur if an air parcel traveled vertically through the region of maximum warming. This maintenance of buoyancy suggests that the limiting factor to intensification is not eyewall stabilization.

The Internally Forced Convergence (IFC) hypothesis states that a TC can continue to develop only when sufficient eyewall ascent (as determined by boundary layer forcing and buoyancy) exists such that friction and upper-level $v_{t}$ export are balanced, with enough vertical momentum remaining to intensify the TC. However, since friction increases proportionally with $V_{m a x}^{2}$, and as upper-level export of cyclonic momentum also amplifies, the required eyewall vertical motion $\left(w_{\text {req }}\right)$ needed to meet IFC budgetary requirements also increases. It is further hypothesized that, although buoyancy and Ekman pumping increase with intensity, an equilibrium state eventually results where the vertical motion can no longer increase fast enough to balance the IFC budgetary requirements. This equilibrium balance delineates the MPI of a TC.

It was postulated that $w_{r e q}$ approximately increases linearly with intensity. However, the maximum eyewall vertical velocity $w_{\max }$ is equal to $\left(2 \mathrm{CAPE}+w_{o}^{2}\right)^{1 / 2}$ where $w_{0}$ is the vertical velocity forced by Ekman pumping. In other words, $w_{\max }$ is roughly proportional to the square root of CAPE, with an extra boost from increasing $w_{0}$. It is this disparity between $\partial w_{r e q} / \partial t$ and $\partial w_{m a x} / \partial t$ as a TC intensifies which ultimately determines the MPI of a TC. In an incipient TC, $w_{\max }$ is greater in magnitude than $w_{\text {req }}$ Since there is a residual vertical velocity after budgetary IFC requirements are met, the TC can intensify. However, as the TC develops, $\partial w_{r e q} / \partial t$ is more in magnitude than $\partial w_{\max } / \partial t$. Although $w_{o}$ also increases with intensity and partially compensates for this disparity, boundary forcing likely only partially nullifies $w_{\max }$ 's square root dependence on CAPE. Therefore, CAPE and $w_{o}$ cannot increase as rapidly as the requirement for faster eyewall vertical velocity. Eventually an equilibrium state is achieved where $w_{\max }$ equals $w_{\text {req }}$, and the MPI has been achieved since intensification can no longer occur. 
This hypothesis can also explain MPI's sensitivity to SST, since the ASI process is sensitive to the surface temperature. With an SST of $29^{\circ} \mathrm{C}$, substantial buoyancy exists to maintain an MSLP of approximately $880 \mathrm{mb}$, but no lower. Likewise, for cooler SSTS, less intense TCs can be obtained since less CAPE is available at a given intensity, and the increase with intensity of this smaller amount of CAPE to the square root power imposes a faster convergence of $w_{\max }$ and $w_{\text {req }}$.

\subsubsection{Statistical analysis of tropical cyclone intensity change}

A multiple regression scheme with intensity change $\Delta V_{\max }$ as the dependent variable was developed for the western North Pacific Ocean. The new scheme is titled the Typhoon Intensity Prediction Scheme (TIPS). Out of 110 possible climatology, persistence, satellite, SST, and synoptic predictors, stepwise regression was applied with an autocorrelation "filtering" procedure and a strict $99 \%$ significance level. This methodology yielded 5-6 final predictors for forecast periods of $12,24,36$, and $48 \mathrm{~h}$. Several different versions of TIPS are outlined in Appendix B.

The significant predictors for all forecast periods are: 1) an SST term based on the storm's "potential" for intensification (POT) which is averaged along the future storm track; 2) a binary 12-h persistence term with an "eye parameterization" (EYEPER); 3) pixel count terms within $4 \mathrm{deg}$; and 4) wind shear in a 5-deg circle averaged along the future storm track. In addition, the previous 12 -h convective trend $\left(T_{b}<-65^{\circ} \mathrm{C}\right)$ within $1 \mathrm{deg}$ is significant for 12-h forecasts (DPX0165), meridional storm motion (VSPD) is significant except for 48 -h forecasts, and storm longitude is important for 48 -h forecasts. All significant predictors are positively correlated with $\Delta V_{\max }$ except wind shear. The surprising fact that meridional storm motion is positively correlated (which conflicts with the results of SHIPS) may be due to $V_{\text {max }}$ containing the storm motion component. In some recurving situations, a fast northerly component may strongly counteract the TC weakening due to colder SSTs and wind shear.

The total variance $\left(R^{2}\right)$ of intensity change explained by TIPS is $46.3 \%$ at $12 \mathrm{~h}$, and steadily increases with each forecast interval to $60 \%$. The variance increase is due to several reasons. First, this variance increase with forecast time represents the growing importance 
of the SST along the storm track in explaining $\Delta V_{\max }$. Second, this increase might result from the 5-kts discretization of $V_{\max }$ in the besttrack data set. As seen in Appendix B, the standard deviation of $\Delta V_{\max }\left(\sigma_{y}\right)$ is $10 \mathrm{kts}$ for intensity change in $12 \mathrm{~h}$, and increases to $28 \mathrm{kts}$ at $48 \mathrm{~h}$. However, the mean $\Delta V_{\max }$ only slightly increases from $1.5 \mathrm{kts}$ to $5 \mathrm{kts}$, respectively. This $5 \mathrm{kts}$ truncation makes explaining $R^{2}$ more difficult at shorter forecast intervals since $\sigma_{y}$ is within the noise level. Finally, the lower $R^{2}$ may indicate that that inner-core processes which cannot be resolved on the synoptic scale complicate short-term intensity changes. These results imply that there may be a limit to how accurate intensity change forecasting can be without better quantification/measurements of TC intensity, and without a better understanding of convective influences and the ASI process on TCs.

Multiple regression appears to be a much better approach in TC intensity change analysis than single variable linear regression. The variance explained by a single variable is astonishing low, indicating that TC intensity change is dependent on many factors. Even POT only explains $15-38 \%$ by itself. The other variables explain even less variance. In fact, some values of $r^{2}$ are so low (such as for LONG, VSPD, EYEPER, and pixel counts) they would be dismissed as unimportant if considered by themselves. This is one of the true benefits of multiple regression - in the stepwise procedure, a variable is chosen which explains a certain amount of variance, and in the next step another variable is chosen which helps explain a percentage of the remaining variance, and so on.

Sensitivity tests showed that pixel counts in a 4-deg area with a brightness temperature less than $-55^{\circ} \mathrm{C}$ (PX0455) was the best satellite predictor, explaining 2-3\% more variance than other pixel count possibilities. It was hypothesized that convection out to 4 deg embodies several influential processes which relate to intensity change. First, it is a measure of deep inner-core convection (within 0-2 deg). Second, it is a measure of cloud symmetry - asymmetric clouds or lack of large-scale cloudiness are signs of an unhealthy storm, usually due to an unfavorable environment (shear or dry air intrusion) or due to movement over colder water. Third, the "spreading out" of cloudiness is a sign of a vigorous secondary circulation. This Central Dense Overcast (CDO) tends to form right before hurricane intensity and it persists during the storm's evolution as long as other conditions 
are favorable. In addition to these physical processes, the highest correlation to $4 \mathrm{deg}$ may also be due to occasional position errors of the TC center. The sensitivity tests hint that 1 and 2-deg convection may be equally good, or perhaps better, predictors if the TC locations were very accurate, and/or for a different data sample. However, since 4-deg areal convection currently explains more variance - and because TC position errors will occur in reality - this predictor is recommended in TIPS.

It was also shown that nonlinear pixel counts and running means generally explain less variance as well. The somewhat surprising fact that temporal smoothing of highly oscillatory satellite data degrades forecast performance suggests that other predictive schemes which use running means of satellite data need re-evaluation. Apparently, running means remove vital predictive signals retained in the pixel count data at the start of the forecast period.

Wind shear over a 5-deg area was the best shear predictor (VWS5), consistent with the findings of SHIPS by DeMaria and Kaplan (1994b). There is a distinct favorism for storms to intensify in a region of large-scale low shear, as shear impinging on the edge of the TC: 1) serves to weaken the storm even if shear over the storm center might be low; 2) indicates that the TC is about to move into an unfavorable shear region; and/or 3) indicates that $200-\mathrm{mb}$ westerly winds or accelerating easterly winds are about to traverse over the TC center. Furthermore, area averaging reduces the chance of one erroneous wind observation contaminating the true shear environment, such as a bad data point or outflow from the TC misrepresenting the calculation. Finally, using area averaging removes the circulation of an anticyclone above a well-developed TC - westerly outflow from an anticyclone using single point calculations can introduce unphysically large wind shear. However single point shear (VWSPT) explains just 1-2Since the variance degradation was not very much, and because single point shear is used in operational settings (mostly for convenience), an alternative version of TIPS with VWSPT is presented in Appendix B.

While the significant predictors are of most concern in this paper, it was equally illuminating which predictors were not significant - especially since many of the unchosen potential predictors are considered to be very important to TC intensity change by 
some researchers. First and foremost, the trough forcing terms - Relative Eddy angular momentum Flux Convergence (REFC) and $200-\mathrm{mb}$ vorticity advection over the center of the storm (VORTADV) - were statistically insignificant in the regression analysis. This concurs with the discussion in Chapters 2 and 3, in which it was postulated that any positive benefits associated with these terms are overwhelmed by the corresponding negative effects of vertical wind shear which usually accompanies a trough. It is not disputed that an occasional TC intensifies under baroclinic forcing influences, but on a seasonal basis upper-level forcing is usually a negative influence. It is also possible that upper-level forcing plays a role in TC intensity change in the Atlantic, since this basin contains more baroclinic interactions than the western North Pacific. However, this author questions the overall current emphasis on upper-level forcing on TC intensity change. Given this author's personal experience at daily tropical discussions held at Colorado State University, and the results in this report, it would be appear that upper-level forcing research hypotheses have been exaggerated. Certainly a TC in a low shear environment can rapidly intensify through the ASI process without trough interactions.

It is also pertinent that the following variables were not chosen: Planetary Eddy angular momentum Flux Convergence (PEFC), storm speed anomaly (SPDA), latitude (LAT), and the Mundell scheme (MUND). The non-significance of PEFC suggests that environmental eddy flux terms are unimportant to intensity change. The non-significance of SPDA suggests that the effect of upwelling on slow-moving TCs may not be of much consequence to intensification in the western North Pacific, perhaps because warm SST$s$ penetrate further down in this basin. Storm latitude is not important because POT contains a much stronger forecast signal. The Mundell (1990) scheme also is not the optimum pixel count scheme, which reflects that too much subjectivity on a limited data set was introduced by Mundell, although the foundation of the scheme (inner-core convective increase) is valid.

Stratifications by intensity change showed that the pixel count information provides essential information to distinguish between TCs which will intensify fast and those that will intensify at a slower rate, while water temperature and shear cannot infer these differences. This implies that CAPE is playing an important role in the intensification process. 
On the other hand, SSTs and shear do provide key information between forecasting slow and fast weakening, while pixel counts do not. Interesting trends can also be inferred about the other predictors. The binary eye/persistence term makes a valuable distinction to the fast and slow classes at $12 \mathrm{~h}$ and the weakening classes at $48 \mathrm{~h}$. On average, the pixel count trend of deep inner-core convection (DPX0165) does not differentiate between fast and slow 12-h developers, but does convey knowledge about when fast $V_{\max }$ decrease might occur. If PX0165 has decreased by at least $20 \%$ in the past $12 \mathrm{~h}$, then $V_{\max }$ might decrease by $15 \mathrm{kts}$ or more in the next $12 \mathrm{~h}$. The meridional storm motion only significantly contributes in the fast weakening class, and its role is to retard the rate of weakening during recurvature by superimposing its fast motion on $V_{\max }$. The initial storm longitude only contributes significantly to fast 48 -h developers, with a mean location of $143.2 \mathrm{deg}$ E.

An evaluation of TIPS' overall performance - and clarified by case studies of 8 TCs - showed that the regression scheme still suffers large errors, and underforecasts the magnitude of $\Delta V_{\text {max }}$ in fast intensification and fast weakening situations. TIPS, in general, anticipates fast intensification better than JTWC (although it still underforecasts $\Delta V_{\max }$ ) but has trouble maintaining the intensity trend should the storm approach its MPI. This is because, statistically speaking, a TC reaching its MPI is an unusual occurrence, and hence TIPS tends to flatten out $\Delta V_{\max }$ as POT decreases. On the other hand, slow intensification and quasi steady-state situations are generally well-anticipated by TIPS. TIPS faired worst than JTWC for the slow weakeners, indicating that TIPS often misses the transition from an intensifying to weakening storm. The persistence term is partially responsible for this error, as this predictor skews the forecast toward further intensification if a TC has intensified in the past $12 \mathrm{~h}$. TIPS contained less errors than JTWC for fast weakeners.

Therefore, the overall comparison of the official TIPS version (based on perfect-prog of shear) against JTWC for 1984-86 (the dependent data set) and 1983 (the independent data set) revealed that TIPS contained smaller absolute errors in most intensification stratifications except for slow weakeners, and hence the overall absolute error was less than JTWC. This trend continued even when persistence of wind shear is used instead of wind 
shear forecasts based on perfect-prog, although the fast intensification and fast weakening stages were forecast less well than the perfect-prog version. (Alternative versions of TIPS based on persistence of shear are contained in Appendix B.)

It cannot be claimed that TIPS outperformed JTWC because all regression analysis is developed on post-processed data and perfect-prog of the track forecast. Furthermore, the recommended version of TIPS is developed on perfect-prog of shear as well. In an operational setting, there would be occasional uncertainty about storm location and movement, and both shear and track forecasts will occasionally be significantly wrong. However, DeMaria and Kaplan (1994a), using persistence of areally averaged wind shear, compared SHIPS' results developed on track forecasts based on VICBAR to those developed on besttrack data, and found little difference in the forecast errors. He reasoned that since MPI and VWS are averaged along the track, and because shear is areally averaged as well, the impact of track errors is reduced. Hence, the use of besttrack may not degrade TIPS' intensity forecasts very much. TIPS appears to at least be competitive with JTWC. Semantic arguments aside about the true forecast performance of TIPS, it is clear that this regression scheme can provide forecast guidance, especially when its biases are understood by the forecaster.

A discouraging finding is that $55 \%$ to $40 \%$ of the variance is still unexplained by TIPS. This accentuates the difficulty of forecasting intensity change. There is still much room for improvement in TC intensity forecasting. However, the multiple regression approach, and the utilization of quantitative satellite data, seems promising in understanding and forecasting $\mathrm{TC}$ intensity change.

\subsubsection{Forecasting tips and "rule of thumbs"}

A normalization procedure in the multiple regression analysis allows one to infer the importance of each significant predictor. The following statements can be made with regards to TC intensity change:

1. The SST term (POT) is two to three times as important as other predictors. Furthermore, the influence of SST (POT) amplifies for longer forecast periods, consistent with ASI theories. 
2. Satellite pixel count information (convection) is second in importance for $24-48-h$ forecasts.

3. Persistence with an "eye parameterization" is initially important, but decreases in importance with longer forecast periods.

4. Wind shear is 4 th in importance at $12 \mathrm{~h}$, but becomes equally important as the pixel count (convection) information in later forecast periods.

5. Other predictors, such as inner-core convective tendency (significant for $12-\mathrm{h}$ forecasts), meridional storm motion (significant for 12 to 36 -h forecasts), and longitude (significant for 48-h forecasts) need to be considered, but are generally of lesser importance.

The normalization procedure also provides operational forecasters approximate threshold values which help differentiate whether a predictor is favorable, neutral, or unfavorable with regards to intensification. It needs to be emphasized that threshold values should not be considered separately, but collectively as a group. Just because POT is favorable, intensification will not occur unless a majority of the other threshold values (like favorable shear, 0-4 deg convection) are either neutral or surpassed. With that cautionary note, the following statements can be made:

1. If $\mathrm{POT}$ is greater (less) than $39 \mathrm{~m} \mathrm{~s}^{-1}$, intensification (weakening) is favored.

2. If PX0455 is greater (less) than $25 \%$, intensification (weakening) is favored.

3. If VWS5 is less (greater) than $8.5 \mathrm{~m} \mathrm{~s}^{-1}$, intensification (weakening) is favored. If single point shear is used instead, the threshold value increases to $9.5 \mathrm{~m} \mathrm{~s}^{-1}$.

4. If deep convection within $0-1 \mathrm{deg}$ of the TC center has increased (decreased) the past $12 \mathrm{~h}$, then intensification (weakening) is favored the next $12 \mathrm{~h}$.

5. If the meridional component in a recurving storm is greater than $2 \mathrm{~m} \mathrm{~s}^{-1}$, then $\Delta V_{\max }$ will be less negative in the next $36 \mathrm{~h}$ due to the translation speed offsetting the weakening tendency. 
6. If a TC is east (west) of $137.3 \mathrm{deg} \mathrm{E}$, then intensification is favored (less likely) in the next $48 \mathrm{~h}$.

\subsection{Recommendations}

Based on the findings of this report, the following suggestions are included for further research:

1. It is recommended that a modern data set be created which contains upper-tropospheric inner-core winds for as many post-NHRP TCs as possible similar to the data set of Gray and Shea (1976).

2. It is recommended that upper-tropospheric jet aircrafts be employed in critical intensity change forecast situations. It is strongly advised that the new HRD jet research plane (to be deployed in 1996) should monitor upper-level inner-core tangential winds in TCs. Gray (1993) even recommends that idle commercial jet aircrafts with cancelled flights due to an approaching TC could be used with proper (and relatively inexpensive) financial reimbursement from the US reconnaissance program.

3. It is recommended that "rapid-scan" imagery from the GOES-8 be employed in critical intensity forecast situations to assess the inner-core upper-tropospheric vorticity of a TC. Special rapid-scan TC research data sets similar to that done by Zehr (1995) and Rodgers and Gentry (1983) are also encouraged. The usefulness of cloud-tracked winds, and a description of the computer algorithm, are contained in Velden et al. (1992) for the GOES satellites and in Ohshima et al. (1991) for the GMS satellites.

4. It is recommended that numerical models be used to study both the sensitivity of TC intensification to different wind profiles, and to investigate the ASI process.

5. It is recommended that the composite data set (which currently has been processed to 1977 ) be extended to 1987 . 
6. It is recommended that further research be conducted on the cause of boundary layer cooling below the TC eyewall. More eyewall boundary layer observations are needed as well.

7. It is recommended that more observational work be done on the role of CAPE both in tropical convection and in TCs, as well as the influence of water-loading, ice microphysics, entrainment, and downdrafts.

8. It is recommended the relationship between TC intensity change and lightning data be be further investigated. Should a data sample become large enough, perhaps lightning data could be incorporated in a statistical analysis of TC intensity change.

9. It is recommended that an empirical MPI relationship similar to the Atlantic version be defined for the western North Pacific. Furthermore, additional empirical MPI values should be derived based on stratifications, such as storm location and time of year. Kubat (1995) has done some qualitative work on this topic. Since the effect of upwelling may be important, the relationship of TC intensity change to sub-surface SSTs 30 and $60-\mathrm{m}$ deep (with corresponding MPI values) also requires research (Holliday and Thompson 1979; Gray 1979). An MPI based on such stratifications may reduce the tendency of TIPS to underforecast a TC approaching the supertyphoon category.

10. It is recommended that better versions of persistence be investigated for TIPS.

11. It is recommended that synoptic data be estimated at 06 and 18 UTC so that the statistical sample size can be doubled. In fact, since the satellite data is stored in 3-h intervals most of the time, it is feasible that the statistical sample size could be effectively quadrupled if synoptic data is estimated at $03,09,15$, and 21 UTC as well.

12. It is recommended that observed weekly SSTs with better resolution ( $1 \mathrm{deg}$ ) be used in future statistical analyses (Reynolds and Smith 1994). 
13. It is recommended that better methods of forecasting wind shear be investigated. These should include numerical modeling, regression analysis, and EXPERT systems.

14. It is recommended that satellite pixel counts be further investigated in assessing TC intensity change. For example, the distribution of the pixel counts lend themselves to spectral and Fourier analysis, which may reveal pertinent information on convective structure (i.e., rainbands). Automated pattern recognition techniques for pixel count data, such as Bayes decision rule (Ebert 1987), are also worthy research topics. In addition to pixel count applications for predicting TC intensity change, improvements in the "Dvorak technique" (Dvorak 1975, 1984) in estimating TC intensity could also be investigated using these techniques.

15. It is recommended that different empirical models be investigated to assess whether least squares is the best approach for TIPS. Since least squares is formulated on the minimization of the square of errors, it is heavily influenced by outliers. Sometimes these outliers are physically important, but often are just bad data. Furthermore, even though the least square approach appears to meet the criterion listed in Chapter 8, it is always possible one of the assumptions could be violated in a rigorous examination. Finally, even if the least squares approach reasonably satisfies the assumptions listed in Chapter 8 , other scheme might still outperform it. Therefore, other empirical schemes should be studied. These include Least Absolute Deviations (LAD) (Mielke et al. 1995), weighted least squares (IMSL 1987), neural networking (Hastenrath et al. 1995), linear discriminant analysis (Hastenrath et al. 1995), principal-component analysis (also called Empirical Orthogonal Function (EOF) analysis) (Jolliffe 1990), and nonlinear statistical schemes (IMSL 1987; Elsner and Schmertmann 1993; Ramirez-Beltran 1995). 


\section{ACKNOWLEDGEMENTS}

This report results from the knowledge of many leading tropical cyclone researchers. In many respects, this study is an accumulation of many scientists' ideas in which this author has been fortunate enough to be associated with.

The author deeply appreciates the continued support, guidance, and encouragement of Dr. William Gray. Committee members Drs. Wayne Schubert, Michael Montgomery, and Paul Mielke provided insight through office conversations, classroom lectures, and suggestions on older versions of this manuscript. Indeed, most of the meteorology professors at Colorado State University benefited this research through classroom instruction and office discussions. This author also acknowledges his alma mater, Texas A\&M University, as a contributing factor to this research. Finally, Jackson State University and the Mississippi Center for Supercomputing Research at the University of Mississippi provided the computer resources to work on this report, and discussions with Dr. Paul Croft, Dr. Kunal Ghosh, Dr. Shayne Johnston, Dr. Keith Johnson, Dr. R. Reddy, Dr. Ed Stanford, Kathy Gates, and Jimmy Ball were useful.

A solid research project also provided support to this paper. Dr. Chris Landsea, John Knaff, Gary Kubat, Jim Kossin, John Sheaffer, and Carl McElroy all provided tremendous help and insight to this work. Dr. Landsea's careful proofreading of this paper also greatly improved its quality. Bill Thorson and Rick Taft assisted in the data processing and computer guidance. Dr. Ray Zehr and Nan McClurg of the National Environmental Satellite, Data, and Information Service (NESDIS) through the Cooperative Institute for Research in the Atmosphere (CIRA) provided the satellite data and processing programs. In addition, Dr. Zehr had a special perceptiveness into many aspects of this research. J. Sorbie-Dunn drafted some of the figures in this paper. Barbara Brumit's extraordinary 
secretarial talents helped this author in many ways the past few years. Laneigh Walters and Amie Hedstrom also provided secretarial assistance.

Other researchers from which this writer benefited from include Dr. Greg Holland, Jeff Kepert, Steve Worley, George Forristall, Mike Vogal, James Stricherz, Dan Shoemaker, Joel Martin, Chip Guard, the staff at the National Hurricane Center (NHC), and the staff at the Hurricane Research Division (HRD). In particular, I would like to thank Dr. Holland for explaining and clarifying the Air-Sea Interaction process, even though we disagree what determines a tropical cyclones maximum potential intensity. With regards to HRD, this author especially appreciates the help and hospitality of Dr. Hugh Willoughby, Dr. Mark DeMaria, Dr. Frank Marks, Dr. Pete Black, and John Kaplan.

Finally, the completion of this report was made much easier with the love and many sacrifices of Amy, my wife.

This research has been supported by the Office of Naval Research through Robert Abbey, Jr., and the Air Force Phillips Laboratory through Morton Glass. The National Science Foundation through Pamela Stephens also contributed significant support. The author also acknowledges the support of the Air Force Office of Scientific Reserch (AFOSR) under the Department of Defense's Augmentation Awards for Science and Engineering Research Training (ASSERT). 


\section{Bibliography}

Aberson, S. D., 1994: Verification of a nested barotropic hurricane track forecast model (VICBAR). Mon. Wea. Rev., 122, 2804-2815.

Abraham, B. and J. Ledolter, 1983: Statistical Methods for Forecasting. John Wiley \& Sons, $445 \mathrm{pp}$.

Aczel, A. D., 1989: Complete Business Statistics. Richard D. Irwin, Inc., 1056 pp.

Ahrens, C. D., 1991: Meteorology Today. West Publishing Company, 576 pp, 4th edition.

Akima, H., 1970: A new method of interpolation and smooth curve fitting based on local procedures. J. Assoc. Comput. Mach., 17, 589-602.

Alliss, R. J. and S. Raman, 1992: Special Sensor Microwave/Imager (SSM/I) observations of Hurricane Hugo (1989). Mon. Wea. Rev., 120, 2723-2737.

Anthes, R. A., 1968: Generation of available potential energy in Hurricane Hilda (1964). Mon. Wea. Rev., 96, 291-302.

Anthes, R. A., 1972: The development of asymmetries in a three-dimensional numerical model of the tropical cyclone. Mon. Wea. Rev., 100, 461-476.

Anthes, R. A., 1977: Hurricane model experiments with a new cumulus parameterization scheme. Mon. Wea. Rev., 105, 287-300.

Anthes, R. A., 1982: Tropical Cyclones: Their Evolution, Structure and Effects. Meteor. Monogr., volume 19,. No. 41, Amer. Meteor. Soc., 208 pp.

Anthes, R. A. and S. W. Chang, 1978: Response of the hurricane boundary layer to changes in sea surface temperature in a numerical model. J. Atmos. Sci., 35, 12401255. 
Arakawa, A. and W. H. Schubert, 1974: Interaction of a cumulus cloud ensemble with the large-scale environment: Part I. J. Atmos. Sci., 31, 674-701.

Atkinson, G. D. and C. R. Holliday, 1977: Tropical cyclone minimum sea level pressure maximum sustained wind relationship for the western North Pacific. Mon. Wea. Rev., $105,421-427$.

Baik, J., 1989: Tropical cyclone simulations with the Betts convective adjustment scheme. Ph. D. dissertation, North Carolina State University, Raleigh, NC, 27695, 159 pp.

Baik, J., M. DeMaria, and S. Raman, 1990a: Tropical cyclone simulations with the Betts convective adjustment scheme. Part I: Model description and control simulation. Mon. Wea. Rev., 118, 513-528.

Baik, J., M. DeMaria, and S. Raman, 1990b: Tropical cyclone simulations with the Betts convective adjustment scheme. Part II: Sensitivity experiments. Mon. Wea. Rev., 118, 529-541.

Baik, J., M. DeMaria, and S. Raman, 1991: Tropical cyclone simulations with the Betts convective adjustment scheme. Part III: Comparisons with the Kuo convective parameterization. Mon. Wea. Rev., 119, 2889-2899.

Barnes, G. M. and M. D. Powell, 1995: Evolution of the inflow boundary layer of Hurricane Gilbert (1988). Mon. Wea. Rev., 123, 2348-2368.

Barnes, G. M. and G. J. Stossmeister, 1986: The structure and decay of a rainband in Hurricane Irene (1981). Mon. Wea. Rev., 114, 2590-2601.

Barnes, G. M., E. J. Zipser, D. Jorgensen, and F. Marks, 1983: Mesoscale and convective structure of a hurricane rainband. J. Atmos. Sci., 40, 2125-2137.

Barnston, A. G. and H. M. V. den Dool, 1993: A degeneracy in cross-validated skill in regression-based forecasts. J. Climate, 6, 963-977.

Beckerle, J. C., 1974: Air and sea temperatures during traverse of Hurricane Alma 1966. J. Phys. Ocean., 4, 487-492. 
Bender, M. A., I. Ginis, and Y. Kurihara, 1993: Numerical simulations of tropical cycloneocean interactions with a high-resolution model. J. Geophys. Res., 98, 245-263.

Bender, M. A., R. J. Ross, R. E. Tuleya, and Y. Kurihara, 1993: Improvements in tropical cyclone track and intensity forecasts using the GFDL initialization system. Mon. Wea. Rev., 121, 2046-2061.

Betts, A. K., 1982: Saturation point analysis of moist convective overturning. J. Atmos. Sci., 39, 1484-1505.

Betts, A. K., 1986: A new convective adjustment scheme. Part I: Observational and theoretical basis. Quart. J. R. Meteor. Soc., 112, 677-691.

Betts, A. K. and J. S. Simpson, 1987: Thermodynamic budget diagrams for the hurricane subcloud layer. J. Atmos. Sci., 44, 842-849.

Black, M. and H. E. Willoughby, 1992: The concentric eyewall cycle of Hurricane Gilbert. Mon. Wea. Rev., 120, 947-957.

Black, M. L., 1993: Comparisons of tropical cyclone intensity with eyewall vertical velocity. In Preprints, 20th Conf. on Hurricanes and Tropical Meteorology, 520-524.

Black, P. G., 1983: Ocean temperature changes induced by tropical cyclones. Ph. D. dissertation, Penn. State University, State College, PA, 16802, 278 pp.

Black, P. G., 1987: Environmental interactions associated with hurricane supercells. In Preprints, 17th Conf. on Hurricanes and Tropical Meteorology, 416-419.

Black, P. G. and G. J. Holland, 1995: The boundary layer of Tropical Cyclone Kerry (1979). Mon. Wea. Rev., 123, 2007-2028.

Black, P. G. and F. D. Marks, 1991: The structure of an eyewall meso-vortex in Hurricane Hugo (1989). In Preprints, 19th Conf. on Hurricanes and Tropical Meteorology, 579582. 
Black, P. G., F. D. Marks, and R. A. Black, 1986: Supercell structure in tropical cyclones. In Preprints, 23rd Conf. on Radar Meteorology and Conf. on Cloud Physics, JP255JP259.

Black, P. G., L. K. Shay, R. L. Elsberry, and J. D. Hawkins, 1989: Response of the Gulf of Mexico to Hurricane Gilbert. In Preprints, 18th Conf. on Hurricanes and Tropical Meteorology, 226-227.

Black, R. A., H. B. Bluestein, and M. L. Black, 1994: Unusually strong vertical motions in a Caribbean hurricane. Mon. Wea. Rev., 122, 2722-2739.

Black, R. A. and J. Hallett, 1986: Observations of the distribution of ice in hurricanes. $J$. Atmos. Sci., 43, 802-822.

Bluestein, H. B., 1993: Synoptic-Dynamic Meteorology in Midlatitudes Vol. II: Observations and Theory of Weather Systems. Oxford University Press, 594 pp.

Bolton, D., 1980: The computation of equivalent potential temperature. Mon. Wea. Rev., 108, 1046-1053.

Bourke, W. and J. L. McGregor, 1983: A nonlinear vertical mode initialization scheme for a limited area prediction model. Mon. Wea. Rev., 111, 2285-2297.

Brand, P. G., 1972: Changes in the characteristics of typhoons crossing the Philippines. NAVENVPREDRSCHFAC Tech. Paper TP 6-72, Naval Env. Pred. Res. Fac., Monterey, CA, 93943, $38 \mathrm{pp}$.

Brand, S., 1971: The effects on a tropical cyclone of cooler surface waters due to upwelling and mixing produced by a prior tropical cyclone. J. Appl. Meteor., 10, 865-874.

Breaker, L. C., L. D. Burroughs, Y. Y. Chao, J. F. Culp, and N. L. Guinasso, 1994: The impact of Hurricane Andrew on the near-surface marine environment in the Bahamas and the Gulf of Mexico. Wea. Forecasting, 9, 542-556.

Brooks, C. E. P. and N. Carruthers, 1978: Handbook of Statistical Methods in Meteorology. AMS Press, 412 pp. 
Browner, S., W. L. Woodley, and C. G. Griffith, 1977: Diurnal oscillation of the area of cloudiness associated with tropical storms. Mon. Wea. Rev., 105, 856-864.

Burpee, R. W., M. L. Black, and F. D. Marks, 1989: Vertical motions measured by airborne doppler radar in the core of Hurricane Elena (1985). In Preprints, 18th Conf. on Hurricanes and Tropical Meteorology, 69-70.

Burpee, R. W. and H. R. D. Staff, 1994: Real-time guidance provided by NOAA's Hurricane Research Division to forecasters during Emily of 1993. Bull. Amer. Meteor. Soc., 75, 1765-1783.

Byers, H. R., 1944: General Meteorology. McGraw-Hill, 645 pp.

Carrier, G. F., 1971: The intensification of hurricanes. J. Fluid. Mech., 49, 145-158.

Challa, M. and R. Pfeffer, 1990: Formation of Atlantic hurricanes from cloud clusters and depressions. J. Atmos. Sci., 47, 909-927.

Charney, J. G. and A. Eliassen, 1964: On the growth of the hurricane depression. J. Atmos. Sci., 21, 68-75.

Chen, L. and W. M. Gray, 1986: Global view of the upper level outflow patterns associated with tropical cyclone intensification change during FGGE. Dept. Atmospheric Sci. Paper No. 392, Colorado State University, Fort Collins, CO, 80523, 126 pp.

Chu, J., 1994: A regression model for the western North Pacific tropical cyclone intensity forecast. Tech. Report NRL/MR/7541-94-7215, Naval Research Laboratory, Monterey, CA, 93943, $33 \mathrm{pp}$.

Church, C. R., J. T. Snow, G. L. Baker, and E. M. Agee, 1979: Characteristics of tornadolike vortices as a function of swirl ratio. A laboratory investigation. J. Atmos. Sci., 36, 1755-1766.

Ciesielski, P. E., S. R. Fulton, and W. H. Schubert, 1986: Multigrid solution of an elliptic boundary value problem from tropical cyclone theory. Mon. Wea. Rev., 114, 797-807. 
Colón, J. A., 1961: On the structure of Hurricane Daisy. NHRP No. 48, National Hurricane Research Project, Miami, FL, 33146, 102 pp.

Colón, J. A. and W. R. Nightingale, 1963: Development of tropical cyclones in relation to circulation patterns at the 200 millibar level. Mon. Wea. Rev., 91, 329-336.

Cotton, W. R. and R. A. Anthes, 1989: Storm and Cloud Dynamics. Academic Press, 883 pp.

Davidson, N. E., G. J. Holland, J. L. McBride, and T. D. Keenan, 1990: On the formation of AMEX tropical cyclones Irma and Jason. Mon. Wea. Rev., 118, 1981-2000.

Davidson, N. E. and B. J. McAvaney, 1981: The ANMRC tropical analysis scheme. Aust. Meteor. Mag., 29, 155-168.

Davidson, N. E., J. L. McBride, and B. J. McAvaney, 1983: The onset of the Australian monsoon during winter MONEX: Synoptic aspects. Mon. Wea. Rev., 111, 496-516.

DeAngelis, D., 1989: The hurricane priest. Weatherwise, 42, 256-257.

DeMaria, M., J. Baik, and J. Kaplan, 1993: Upper level eddy angular momentum flux and tropical cyclone intensity change. J. Atmos. Sci., 50, 1133-1147.

DeMaria, M. and J. Kaplan, 1991: A statistical model for predicting tropical cyclone intensity change. In Preprints, 19th Conf. on Hurricanes and Tropical Meteorology, 521-526.

DeMaria, M. and J. Kaplan, 1994a: Sea surface temperature and the maximum intensity of Atlantic tropical cyclones. J. Climate, 7, 1324-1334.

DeMaria, M. and J. Kaplan, 1994b: A Statistical Hurricane Intensity Prediction Scheme (SHIPS) for the Atlantic Basin. Wea. Forecasting, 9, 209-220.

DeMaria, M. and W. H. Schubert, 1984: Experiments with a spectral tropical cyclone model. J. Atmos. Sci., 41, 901-924. 
DeMaria, M. and W. H. Schubert, 1985: Axisymmetric, primitive equations, spectral tropical cyclone model. Part II: Normal mode initialization. J. Atmos. Sci., 42, 1225-1236.

Deppermann, C. E., 1947: Notes on the origin and structure of Philippine typhoons. Bull. Amer. Meteor. Soc., 28, 399-404.

Dutton, J. A., 1986: The Ceaseless Wind. Dover Publications, Inc., 617 pp.

Dvorak, V. F., 1975: Tropical cyclone intensity analysis and forecasting from satellite imagery. Mon. Wea. Rev., 103, 420-430.

Dvorak, V. F., 1984: Tropical cyclone intensity analysis using satellite data. NESDIS 11, NOAA Tech. Memo., Washington, D. C., 20233, 47 pp.

Ebert, E., 1987: A pattern recognition technique for distinguishing surface and cloud types in the polar regions. J. Climate Appl. Meteor., 26, 1412-1427.

Ebert, E. E. and G. J. Holland, 1992: Observations of record deep convection in Tropical Cyclone Hilda. Mon. Wea. Rev., 120, 2240-2251.

Edwards, A. L., 1984: An Introduction to Linear Regression and Correlation. W. H. Freeman and Company, 206 pp.

Eliassen, A., 1951: Slow thermally or frictionally controlled meridional circulation in a circular vortex. Astrophys. Norv., 5, 19-60.

Eliassen, A. and M. Lystad, 1977: The Ekman layer of a circular vortex. A numerical and theoretical study. Geophysica Norv., 31, 1-16.

Elsberry, R., G. Holland, H. Gerrish, M. DeMaria, and C. P. Guard, 1992: Is there any hope for tropical cyclone intensity prediction? - A panel discussion. Bull. Amer. Meteor. Soc., 73, 264-275. 
Elsberry, R. L., G. G. Coltrane, and P. L. Krueger, 1974: Typhoon and tropical storm intensity forecasts for 24,48 , and 72 hours using statistical regression equations. ENVPREDRSCHFAC Tech. Note 15, Naval Env. Pred. Res. Fac., Monterey, CA, 93943, 25 pp.

Elsberry, R. L., G. G. Coltrane, and P. L. Krueger, 1975: Statistical forecasts of 24, 48 and $72 \mathrm{~h}$ typhoon and tropical storm intensity changes. J. Appl. Meteor., 14, 445-451.

Elsberry, R. L., E. L. Weniger, and D. H. Meanor, 1988: A statistical tropical cyclone intensity forecast technique incorporating environmental wind and vertical shear information. Mon. Wea. Rev., 116, 2142-2154.

Elsner, J. B. and C. P. Schmertmann, 1993: Improving extended-range seasonal predictions of intense Atlantic Hurricane activity. Wea. Forecasting, 8, 345-351.

Elsner, J. B. and C. P. Schmertmann, 1994: Assessing forecast skill through cross validation. Wea. Forecasting, 9, 619-624.

Emanuel, K. A., 1986: An air-sea interaction theory for tropical cyclones. Part I: Steadystate maintenance. J. Atmos. Sci., 43, 585-604.

Emanuel, K. A., 1988: The maximum intensity of hurricanes. J. Atmos. Sci., 45, 11431155.

Emanuel, K. A., 1989a: Dynamical theories of tropical convection. Aust. Meteor. Mag., 37, 3-10.

Emanuel, K. A., 1989b: The finite-amplitude nature of tropical cyclogenesis. J. Atmos. Sci., 46, 3431-3456.

Emanuel, K. A., 1991: The theory of hurricanes. Ann. Rev. Fluid Mech., 23, 179-196.

Emanuel, K. A., 1994: Atmospheric Convection. Oxford University Press, 580 pp.

Emanuel, K. A., 1995: Sensitivity of tropical cyclones to surface exchange coefficients and a revised steady-state model incorporating eye dynamics. J. Atmos. Sci., 52, 3969-3976. 
Evans, J. L., 1993: Sensitivity of tropical cyclone intensity to sea surface temperature. $J$. Climate, 6, 1133-1140.

Fairall, C. W., J. D. Kepert, and G. J. Holland, 1994: The effect of sea spray on surface energy transports over the ocean. The Atmosphere Ocean System, 2, 121-142.

Fendell, F. E., 1974: Tropical Cyclones, volume 17,. Advances in Geophysics, New York, 100 pp.

Fitzpatrick, P. J., 1992: A numerical study of mesoscale convection in a rotating tropical atmosphere. M. S. thesis, Texas A\&M University, College Station, TX, 77843, 117 pp.

Fitzpatrick, P. J., J. A. Knaff, C. W. Landsea, and S. V. Finley, 1995: Documentation of a systematic bias in the Aviation model's forecast of the Atlantic Tropical UpperTropospheric Trough: Implications for tropical cyclone forecasting. Wea. Forecasting, $10,433-446$.

Frank, W. M., 1977b: The structure and energetics of the tropical cyclone. Part I: Storm structure. Mon. Wea. Rev., 105, 1119-1135.

Frank, W. M., 1977a: The structure and energetics of the tropical cyclone. Part II: Dynamics and energetics. Mon. Wea. Rev., 105, 1136-1150.

Franklin, J. L., S. J. Lord, and F. D. Marks, 1988: Dropwindsonde and radar observations of the eye of Hurricane Gloria (1985). Mon. Wea. Rev., 116, 1237-1244.

Fulton, S. R., P. E. Ciesielski, and W. H. Schubert, 1986: Multigrid methods for elliptic problems: A review. Mon. Wea. Rev., 114, 943-959.

Gentry, R. C., E. B. Rodgers, J. Steranka, and W. E. Shenk, 1980: Predicting tropical cyclone intensity change using satellite measured equivalent blackbody temperatures of cloud tops. Mon. Wea. Rev., 108, 445-455.

Gill, A. E., 1982: Atmosphere-Ocean Systems. Academic Press, 662 pp, 3rd edition. 
Ginis, I., M. A. Bender, and Y. Kurihara, 1992: A numerical study of the tropical cycloneocean interaction. In ICSU/WMO International Symposium on Tropical Cyclone Disasters, 343-355.

Gray, W. M., 1968: Global view of the origins of tropical disturbances and storms. Mon. Wea. Rev., 96, 669-700.

Gray, W. M., 1979: Hurricanes: Their formation, structure, and likely role in the tropical circulation. Meteorology Over the Tropical Oceans. Roy. Meteor. Soc., 155-218 pp.

Gray, W. M., 1981: Recent advances in tropical cyclone research from rawinsonde composite analysis. World Meteorological Organization, Geneva, Switzerland, 407 pp.

Gray, W. M., 1982: Tropical cyclone genesis and intensification. In Topics in Atmospheric and Oceanographic Sciences, Intense Atmospheric Vortices, 3-20.

Gray, W. M., 1991: Comments on "Gradient balance in tropical cyclones". J. Atmos. Sci., 48, 1201-1208.

Gray, W. M., 1993: Proposal for upper tropospheric aircraft reconnaissance. In Preprints, 20th Conf. on Hurricanes and Tropical Meteorology, 244-247.

Gray, W. M., 1995: Tropical Cyclones. World Meteorological Organization, in preparation.

Gray, W. M. and W. M. Frank, 1977: Tropical cyclone research by data compositing. NEPRF Tech. Report TR-77-01, Colorado State University, Fort Collins, CO, 80523, $70 \mathrm{pp}$.

Gray, W. M. and R. W. Jacobson, 1977: Diurnal variation of deep cumulus convection. Mon. Wea. Rev., 105, 1171-1188.

Gray, W. M., C. W. Landsea, P. W. Mielke, and K. J. Berry, 1992: Predicting Atlantic seasonal hurricane activity 6-11 months in advance. Wea. Forecasting, 7, 440-455.

Gray, W. M., C. W. Landsea, P. W. Mielke, and K. J. Berry, 1993: Predicting Atlantic basin seasonal hurricane activity by 1 August. Wea. Forecasting, 8, 73-86. 
Gray, W. M., C. W. Landsea, P. W. Mielke, and K. J. Berry, 1994: Predicting Atlantic basin seasonal hurricane activity by 1 June. Wea. Forecasting, 9, 103-115.

Gray, W. M. and D. J. Shea, 1973: The hurricane's inner core region. Part II: Thermal stability and dynamic characteristics. J. Atmos. Sci., 30, 1565-1576.

Gray, W. M. and D. J. Shea, 1976: Data summary of NOAA's hurricane inner-core radial leg flight penetrations 1957-1967 and 1969. Dept. Atmospheric Sci. Paper No. 257, Colorado State University, Fort Collins, CO, 80523, 219 pp.

Guard, C. P., L. E. Carr, F. H. Wells, R. A. Jeffries, N. D. Gural, and D. K. Edson, 1992: Joint Typhoon Warning Center and the challenges of multibasin tropical cyclone forecasting. Wea. Forecasting, 7, 328-352.

Hack, J. J. and W. H. Schubert, 1986a: Lateral boundary conditions for tropical cyclone models. J. Atmos. Sci., 43, 1559-1573.

Hack, J. J. and W. H. Schubert, 1986b: Nonlinear response of atmospheric vortices to heating by organized cumulus convection. J. Atmos. Sci., 43, 1559-1573.

Hallett, J. and S. C. Mossop, 1974: Production of secondary ice particles during the riming process. Nature, 249, 26-28.

Hallin, S. C., 1991: Diurnal variations in tropical cyclones. M. S. thesis, Colorado State University, Fort Collins, CO, 80523, 99 pp.

Handel, M. D., 1990: Tropical cyclone intensification from finite amplitude disturbances. $\mathrm{Ph}$. D. dissertation, Massachusetts Institute of Technology, Cambridge, MA, 02139, $261 \mathrm{pp}$.

Hastenrath, S., 1995: Recent advances in tropical prediction. J. Climate, 8, 1519-1532.

Hastenrath, S., L. Greischar, and J. V. Heerden, 1995: Prediction of the summer rainfall over South Africa. J. Climate, 8, 1511-1518. 
Haurwitz, H., 1935: The height of tropical cyclones and the eye of the storm. Mon. Wea. Rev., 63, 45-49.

Hawkins, H. F., 1962: Vertical wind profiles in hurricanes. NHRP No. 55, National Hurricane Research Project, Miami, FL, 33146, 16 pp.

Hawkins, H. F. and S. M. Imbembo, 1976: The structure of a small intense hurricane Inez, 1966. Mon. Wea. Rev., 104, 418-442.

Hawkins, H. F. and D. T. Rubsam, 1968: Hurricane Hilda, 1964: II. Structure and budgets of the hurricane on October 1, 1964. Mon. Wea. Rev., 96, 617-636.

Hendon, H. H., 1988: A qualitative assessment of the Australian Tropical Region Analyses. Mon. Wea. Rev., 116, 5-17.

Hess, J. C., J. B. Elsner, and N. E. LaSeur, 1995: Improving seasonal hurricane predictions for the Atlantic basin. Wea. Forecasting, 10, 425-432.

Hirschberg, P. A. and J. M. Fritsch, 1993: On understanding height tendency. Mon. Wea. Rev., 121, 2646-2661.

Hobgood, J. S., 1986: A possible mechanism for the diurnal oscillations of tropical cyclones. J. Atmos. Sci., 43, 2901-2922.

Hobgood, J. S. and J. N. Rayne, 1989: A test of convective parameterizations in a tropical cyclone model. Mon. Wea. Rev., 117, 1221-1226.

Holland, G. J., 1983: Angular momentum transports in tropical cyclones. Quart. J. R. Meteor. Soc., 109, 187-209.

Holland, G. J., 1995: The maximum potential intensity of tropical cyclones. Submitted to J. Atmos. Sci.

Holland, G. J. and R. T. Merrill, 1984: On the dynamics of tropical cyclone structure changes. Quart. J. R. Meteor. Soc., 110, 723-745. 
Holliday, C. R. and A. H. Thompson, 1979: Climatological characteristics of rapidly intensifying storms. Mon. Wea. Rev., 107, 1022-1034.

Holton, J. R., 1992: An Introduction to Dynamic Meteorology. Academic Press, 511 pp, 3rd edition.

Hoskins, B. J., M. E. McIntyre, and A. W. Robertson, 1985: On the use and significance of potential vorticity maps. Quart. J. R. Meteor. Soc., 111, 877-946.

IMSL, 1987: FORTRAN subroutines for statistical analysis. International Mathematical \& Statistical FORTRAN Library, 1232 pp.

Iribarne, J. V. and W. L. Godson, 1981: Atmospheric Thermodynamics. D. Reidel Publishing Company, 259 pp.

Jarvinen, B. R. and C. J. Neumann, 1979: Statistical forecasts of tropical cyclone intensity. NWS NHC-10, NOAA Tech. Memo., Miami, FL, 33146, 22 pp.

Jayaratne, E. R., C. P. R. Saunders, and J. Hallett, 1983: Laboratory studies of the charging of soft-hail during ice crystal interactions. Quart. J. R. Meteor. Soc., 106, $609-630$.

Jolliffe, I. T., 1990: Principal component analysis: A beginner's guide. Weather, 45, 375-382.

Jordan, C. L., 1958: Mean soundings of the West Indies area. J. Meteor., 15, 91-97.

Jordan, C. L., 1961: Marked changes in the characteristics of the eye of intense typhoons between their deepening and filling stages. J. Meteor., 18, 779-789.

Jorgensen, D. P., E. J. Zipser, and M. A. LeMone, 1985: Vertical motions in intense hurricanes. J. Atmos. Sci., 42, 839-856.

JTWC, 1987: Synoptic discussion of tropical cyclone Niña. 1987 annual tropical cyclone report, JTWC, Guam, Mariana Islands, 124-131 pp. 
JTWC, 1992a: Synoptic discussion of tropical cyclone Marge. 1992 annual tropical cyclone report, JTWC, Guam, Mariana Islands, 74-77 pp.

JTWC, 1992b: Synoptic discussion of tropical cyclone Omar. 1992 annual tropical cyclone report, JTWC, Guam, Mariana Islands, 80-87 pp.

JTWC, 1993: Reconnaissance reports. 1993 annual tropical cyclone report, JTWC, Guam, Mariana Islands, $11-18 \mathrm{pp}$.

Kain, J. S. and J. M. Fritsch, 1993: The impact of model physics on numerical simulations of tropical cyclone Irma. In Preprints, 20th Conf. on Hurricanes and Tropical Meteorology, 135-138.

Kaplan, J., 1995: An examination of the role of large-scale forcing on the rapid intensification of Hurricane Emily. In Preprints, 21st Conf. on Hurricanes and Tropical Meteorology, 332-334.

Kaplan, J. and M. DeMaria, 1995: A simple empirical model for predicting the decay of tropical cyclone winds after landfall. J. Appl. Meteor., 104, 669-690.

Kasahara, A., 1976: Normal modes of ultralong waves in the atmosphere. Mon. Wea. Rev., 104, 669-690.

Kepert, J., 1995: Heat and moisture budgets of the tropical cyclone subcloud layer. Submitted to J. Atmos. Sci.

Krishnamurti, T. N., H. S. Bedi, D. Oosterhof, and V. Hardiker, 1994: The formation of Hurricane Frederic of 1979. Mon. Wea. Rev., 122, 1050-1074.

Kubat, G. B., 1995: Tropical cyclone intensity relationships. M. S. thesis, Colorado State University, Fort Collins, CO, 80523, 86 pp.

Kuo, H. L., 1959: Dynamics of convective vortices and eye formation. Rossby Memorial Volume, B. Bolin., Rockefeller Institute Press, 413-424. 
Kuo, H. L., 1965: On formation and intensification of tropical cyclones through latent heat release by cumulus convection. J. Atmos. Sci., 22, 40-63.

Kuo, H. L., 1974: Further studies of the parameterization of the influence of cumulus convection on large-scale flow. J. Atmos. Sci., 31, 1232-1240.

Kurihara, Y., M. A. Bender, and R. E. Tuleya, 1995: Performance evaluation of the GFDL hurricane prediction system in the 1994 hurricane season. In Preprints, 21st Conf. on Hurricanes and Tropical Meteorology, 41-43.

Lander, M. A., 1995: Specific tropical cyclone track types and unusual tropical cyclone motions associated with a reverse-oriented monsoon trough in the western North Pacific. Submitted to Wea. Forecasting.

Landsea, C., 1995: SHIFOR94 - Atlantic tropical cyclone intensity forecasting. In Preprints, 21st Conf. on Hurricanes and Tropical Meteorology, 365-367.

Lascody, R. A., 1993: A different look at Hurricane Andrew - Lightning around the eye wall. Natl. Wea. Dig., 18, 39-40.

Lee, W., F. D. Marks, and R. E. Carbone, 1994: Velocity track display - A technique to extract real-time tropical cyclone circulations using a single airborne Doppler Radar. J. Atmos. Ocean. Technol., 11, 337-356.

Lemon, L. R., D. W. Burgess, and L. D. Hennington, 1982: A tornado extending to extreme heights as revealed by Doppler radar. In Preprints, 12th Conf. Severe Local Storms, 430-432.

Lindzen, R. S., 1974: Wave-CISK in the tropics. J. Atmos. Sci., 31, 156-179.

Lindzen, R. S. and H. L. Kuo, 1969: A reliable method for the numerical integration of a large class of ordinary and partial differential equations. Mon. Wea. Rev., 97, $732-734$.

Lord, S. J. and J. L. Franklin, 1987: The environment of Hurricane Debby. Part I: Winds. Mon. Wea. Rev., 115, 2760-2780. 
Lord, S. J., H. E. Willoughby, and J. M. Piotrowicz, 1984: Role of a parameterized icephase microphysics in an axisymmetric, nonhydrostatic tropical cyclone model. $J$. Atmos. Sci., 41, 2836-2848.

Ludlam, F. H., 1980: Clouds and Storms. Pennsylvania State University Press, 404 pp.

Lyons, W. A. and C. S. Keen, 1994: Observations of lightning in convective supercells within tropical storms and hurricanes. Mon. Wea. Rev., 122, 1897-1916.

Lyons, W. A., M. G. Venne, P. G. Black, and R. C. Gentry, 1989: Hurricane lightning: A new diagnostic tool for tropical storm forecasting? In Preprints, 18th Conf. on Hurricanes and Tropical Meteorology, 113-114.

MacReady, P. B. and R. F. Skutt, 1967: Cloud buoyancy increase due to seeding. J. Appl. Meteor., 6, 207-210.

Maindonald, J. H., 1984: Statistical computation. John Wiley \& Sons, 370 pp.

Malkus, J. S., 1958: On the structure and maintenance of the mature hurricane eye. $J$. Meteor., 15, 337-349.

Malkus, J. S. and H. Riehl, 1960: On the dynamics and energy transformation in steadystate hurricanes. Tellus, 12, 1-20.

Marks, F. D. and P. G. Black, 1990: Close encounter with an intense mesoscale vortex within Hurricane Hugo. In Preprints, 4th Conf. on Mesoscale Processes, 114-115.

Marks, F. D., R. A. Houze, and J. F. Gamache, 1992: Dual-aircraft investigation of the inner core of Hurricane Norbert. Part I: Kinematic structure. J. Atmos. Sci., 49, 919-942.

Martin, J. D., 1988: Tropical cyclone observation and forecasting with and without aircraft reconnaissance. Dept. Atmospheric Sci. Paper No. 428, Colorado State University, Fort Collins, CO, 80523, $114 \mathrm{pp}$. 
Martin, J. D. and W. M. Gray, 1993: Tropical cyclone observation and forecasting with and without aircraft reconnaissance. Wea. Forecasting, 8, 519-532.

Mayfield, M., L. Avila, and E. N. Rappaport, 1994: Atlantic Hurricane Season of 1992. Mon. Wea. Rev., 122, 517-538.

McBride, J. L., 1981: Observational analysis of tropical cyclone formation. Part I: Basic description of data sets. J. Atmos. Sci., 38, 1117-1131.

McBride, J. L. and G. J. Holland, 1987: Tropical cyclone forecasting: A world-wide summary of techniques and verification statistics. Bull. Amer. Meteor. Soc., 68, $1230-1238$.

Mendenhall, W. and J. E. Reinmuth, 1982: Statistics for Management and Economics. Duxbury Press, 902 pp.

Merrill, R. T., 1985: Environmental influences on hurricane intensification. Dept. Atmospheric Sci. Paper No. 394, Colorado State University, Fort Collins, CO, 80523, $156 \mathrm{pp}$.

Merrill, R. T., 1987: An experiment in statistical prediction of tropical cyclone intensity change. NWS NHC-34, NOAA Tech. Memo., Miami, FL, 33146, 34 pp.

Merrill, R. T., 1988a: Characteristics of the upper-tropospheric environmental flow around hurricanes. J. Atmos. Sci., 45, 1678-1687.

Merrill, R. T., 1988b: Environmental influences on hurricane intensification. J. Atmos. Sci., 45, 1678-1687.

Merrill, R. T. and C. S. Velden, 1996: A three-dimensional analysis of the outflow layer of Supertyphoon Flo. Mon. Wea. Rev., 124, 47-63.

Mielke, P. W., K. J. Berry, C. W. Landsea, and W. M. Gray, 1995: Artificial skill and validation in meteorological forecasting. Accepted to Wea. Forecasting.

Miller, B. I., 1958a: On the maximum intensity of hurricanes. J. Meteor., 15, 184-195. 
Miller, B. I., 1958b: The three dimensional wind structure around a tropical cyclone. NHRP No. 15, National Hurricane Research Project, Miami, FL, 33146, 41 pp.

Miller, D. and J. M. Fritsch, 1991: Mesoscale convective complexes in the western Pacific region. Mon. Wea. Rev., 119, 2978-2992.

Molinari, J., P. K. Moore, V. P. Idone, R. W. Henderson, and A. B. Saljoughy, 1994: Cloud-to-ground lightning in Hurricane Andrew. J. Geophys. Res., 99, 16665-16676.

Molinari, J. and D. Vollaro, 1989: External influences on hurricane intensity. Part I: Outflow layer eddy angular momentum fluxes. J. Atmos. Sci., 46, 1093-1105.

Molinari, J. and D. Vollaro, 1990: External influences on hurricane intensity. Part II: Vertical structure and response of the hurricane vortex. J. Atmos. Sci., 46, 19021918.

Molinari, J. and D. Vollaro, 1993: Influences of vertical shear and upper level vorticity on tropical cyclone intensification. In Preprints, 20th Conf. on Hurricanes and Tropical Meteorology, 23-26.

Molinari, J., D. Vollaro, and F. Robasky, 1992: Use of ECMWF operational analyses for studies of the tropical cyclone environment. Meteor. Atmos. Phys., 47, 127-144.

Molinari, J., D. Vollaro, and S. Skubis, 1993: Application of the Eliassen balanced vortex model to real-data tropical cyclones. Mon. Wea. Rev., 121, 2409-2419.

Montgomery, M. T. and B. F. Farrell, 1993: Tropical cyclone formation. J. Atmos. Sci., 50, 285-310.

Moss, M. S. and S. L. Rosenthal, 1975: On the estimation of planetary boundary layer variables in mature hurricanes. Mon. Wea. Rev., 103, 980-988.

Mundell, D. B., 1990: Prediction of tropical cyclone rapid intensification events. M. S. thesis, Colorado State University, Fort Collins, CO, 80523, 186 pp. 
National Disaster Survey Report, 1993: Hurricane Andrew: South Florida and Louisiana, August 23-26, 1992. Technical report, NOAA Tech. Report, Silver Spring, MD, 12345, $131 \mathrm{pp}$.

Neumann, C. J., 1972: An alternate to the HURRAN (Hurricane Analog) tropical cyclone forecast system. NWS SR-62, NOAA Tech. Memo., Miami, FL, 33146, 24 pp.

Neumann, C. J., 1991: A revised National Hurricane Center NHC83 model (NHC90). NWS NHC-44, NOAA Tech. Memo., Miami, FL, 33146, 35 pp.

Neumann, C. J., M. B. Lawrence, and E. L. Caso, 1977: Monte Carlo significance testing as applied to statistical tropical cyclone prediction models. J. Appl. Meteor., 16, $1165-1174$.

Ohshima, T., H. Uchida, T. Hamadam, and S. Osano, 1991: A comparison of GMS cloud motion winds with ship-observed winds in typhoon vicinity. Geophys. Mag., $44,27-49$.

Ooyama, K. V., 1969: Numerical simulation of the life cycle of tropical cyclones. J. Atmos. Sci., 26, 3-40.

Ooyama, K. V., 1982: Conceptual evolution of the theory and modeling of the tropical cyclone. J. Meteor. Soc. Japan, 60, 369-380.

Ooyama, K. V., 1987: Scale-controlled objective analysis. Mon. Wea. Rev., 115, 24792506.

Ooyama, K. V., 1990: A thermodynamic foundation for modeling the moist atmosphere. J. Atmos. Sci., 47, 2580-2593.

Orville, H. D. and K. Hubbard, 1973: On the freezing of liquid water in a cloud. J. Appl. Meteor., 12, 671-676.

Palmén, E., 1948: On the formation and structure of tropical cyclones. Geophysica, 3, 26-38. 
Pfeffer, R. L. and M. Challa, 1980: Effects of eddy fluxes of angular momentum on model hurricane development. J. Atmos. Sci., 37, 1603-1618.

Pfeffer, R. L. and M. Challa, 1991: Formation of Atlantic hurricanes from cloud clusters and depressions. In Preprints, 19th Conf. on Hurricanes and Tropical Meteorology, 214-216.

Pfeffer, R. L. and M. Challa, 1993: Model hurricane formation in the presence of a basic current. Atmósfera, 6, 25-37.

Pfeffer, R. L., M. Challa, N. LaSeur, and W. M. Gray, 1990: Composite Atlantic tropical disturbance structure of developing and non-developing disturbances. Geophysical Fluid Dynamics Institute, $96 \mathrm{pp}$.

Pielke, R. A., 1984: Mesoscale Meteorological Modeling. Academic Press, 612 pp.

Pike, A. C., 1985: Geopotential heights and thicknesses as predictors of Atlantic tropical cyclone motion and intensity. Mon. Wea. Rev., 113, 931-939.

Posey, C., 1988: Hurricanes - reaping the whirlwind. Omni, 16, 34-47.

Powell, M. D., 1980: Evaluations of diagnostic marine boundary-layer models applied to hurricanes. Mon. Wea. Rev., 108, 757-766.

Powell, M. D., 1990: Boundary layer structure and dynamics in outer hurricane rainbands. Part II: Downdraft modification and mixed layer recovery. Mon. Wea. Rev., 118, 918-938.

Press, W. H., B. P. Flannery, S. A. Teukolsky, and W. T. Vetterling, 1988: Numerical Recipes: the Art of Scientific Computing. Cambridge University Press, 818 pp.

Pudov, V. D., 1992: The ocean response to the cyclones influence and its possible role in their track formations. In ICSU/WMO International Symposium on Tropical Cyclone Disasters, 367-376. 
Pudov, V. D. and G. J. Holland, 1994: Typhoon and ocean: Results of experimental investigations. BMRC Research Report No. 45, Bureau of Meteorology Research Centre, Melbourne, Australia, 50 pp.

Puri, K. and M. J. Miller, 1990: Sensitivity of ECMWF analyses-forecasts of tropical cyclones to cumulus parameterization. Mon. Wea. Rev., 118, 1709-1741.

Ramirez-Beltran, N. D., 1995: A statistical model to predict hurricane tracks. In Preprints, 21st Conf. on Hurricanes and Tropical Meteorology, 488-490.

Rappaport, E. N., 1994: Hurricane Andrew. Weather, 49, 51-60.

Rau, R. E., 1993: Physical explanations of intensification at various stages of Hurricane Andrew. In Preprints, 20th Conf. on Hurricanes and Tropical Meteorology, 124-126.

Reilly, D. H., 1992: On the role of upper-tropospheric potential vorticity advection in tropical cyclone formation: Case studies from 1991. M. S. thesis, Massachusetts Institute of Technology, Cambridge, MA, 02139, 124 pp.

Renno, N. O. and E. R. Williams, 1995: Quasi-Lagrangian measurements in convective boundary layer plumes and their implications for the calculation of CAPE. Mon. Wea. Rev., 123, 2733-2742.

Reynolds, R. W., 1988: A real-time global sea surface temperature analysis. J. Climate, 1, 75-86.

Reynolds, R. W. and T. M. Smith, 1994: Improved global sea surface temperature analyses using optimum interpolation. J. Climate, 7, 929-948.

Riehl, H., 1948: On the formation of west Atlantic hurricanes. Studies of upper-air conditions in low-latitudes. Report No. 24, University of Chicago, Chicago, IL, 60637, 67 pp.

Riehl, H., 1950: A model of hurricane formation. J. Appl. Phys., 21, 917-925.

Riehl, H., 1954: Tropical meteorology. McGraw-Hill, 392 pp. 
Rodgers, E. B., 1992: Tropical cyclone/upper-atmosphere interaction as inferred from satellite total ozone observations. Ph. D. dissertation, Colorado State University, Fort Collins, CO, 80523, $269 \mathrm{pp}$.

Rodgers, E. B. and R. C. Gentry, 1983: Monitoring tropical cyclone intensity using environmental wind fields derived from short-interval satellite images. Mon. Wea. Rev., 111, 979-996.

Rogers, R. R. and M. K. Yau, 1989: A Short Course in Cloud Physics. Pergamon Press, $293 \mathrm{pp}$.

Rossby, C. G., 1937: On the mutual adjustment of pressure and velocity distribution in certain simple current systems; Part I. J. Mar. Res., 1, 15-28.

Rossby, C. G., 1938: On the mutual adjustment of pressure and velocity distribution in certain simple current systems; Part II. J. Mar. Res., 2, 239-263.

Sadler, J. C., 1978: Mid-season typhoon development and intensity changes and the Tropical Upper Tropospheric Trough. Mon. Wea. Rev., 106, 1137-1152.

Samsury, C. E. and R. E. Orville, 1994: Cloud-to-ground lightning in tropical cyclones: A case study of Hurricanes Hugo (1989) and Jerry (1989). Mon. Wea. Rev., 122, $1887-1896$.

Saunders, P. M., 1957: The thermodynamics of saturated air: A contribution to the classical theory. Quart. J. R. Meteor. Soc., 83, 342-350.

Schiffer, R. A. and W. B. Rossow, 1983: The international satellite cloud climatology project (ISCCP): The first project of the World Climate Research Program. Bull. Amer. Meteor. Soc., 64, 779-784.

Schubert, W. H. and J. J. Hack, 1982: Inertial stability and tropical cyclone development. J. Atmos. Sci., 39, 1687-1697.

Schubert, W. H. and J. J. Hack, 1983: Transformed Eliassen balanced vortex model. J. Atmos. Sci., 40, 1571-1583. 
Seager, R. and S. E. Zebiak, 1994: Convective interaction with dynamics in a linear primitive equation model. J. Atmos. Sci., 51, 1307-1331.

Seager, R. and S. E. Zebiak, 1995: Simulation of tropical climate with a linear primitive equation model. J. Climate, 8, 2497-2520.

Seitter, K. L. and H. Kuo, 1983: The dynamic structure of squall-line type thunderstorms. J. Atmos. Sci., 40, 2831-2854.

Shapiro, L. J. and J. L. Franklin, 1995: Potential vorticity in Hurricane Gloria. Mon. Wea. Rev., 123, 1465-1475.

Shapiro, L. J. and M. T. Montgomery, 1993: A three-dimensional balance theory for rapidly rotating vortices. J. Atmos. Sci., 50, 3322-3335.

Shapiro, L. J. and C. J. Neumann, 1984: Sampling errors in statistical models of tropical cyclone motion: A comparison of predictor screening and EOF techniques. Mon. Wea. Rev., 112, 1378-1388.

Shapiro, L. J. and H. E. Willoughby, 1982: The response of balanced hurricanes to local sources of heat and momentum. J. Atmos. Sci., 39, 378-394.

Shay, L. K., P. G. Black, A. J. Mariano, J. D. Hawkins, and R. L. Elsberry, 1992: Upper ocean response to Hurricane Gilbert. J. Geophys. Res., 97, 20227-20248.

Shea, D. J. and W. M. Gray, 1973: The hurricane's inner core region. Part I: Symmetric and asymmetric structure. J. Atmos. Sci., 30, 1544-1564.

Sheets, R. C., 1990: The National Hurricane Center - Past, present, and future. Wea. Forecasting, 5, 185-232.

Sheets, R. C., 1993: Catastrophic hurricanes may become frequent events in Caribbean and along the United States East and Gulf Coast. In Hurricanes of 1992 - Lessons Learned and Implications for the Future, 37-51. 
Shoemaker, D. N., 1989: Relationships between tropical cyclone deep convection and the radial extent of damaging winds. Dept. Atmospheric Sci. Paper No. 457, Colorado State University, Fort Collins, CO, 80523, 109 pp.

Simpson, R. H., 1952: Exploring eye of Typhoon Marge, 1951. Bull. Amer. Meteor. Soc., 33, 286-298.

Simpson, R. H., 1963: Liquid water in squall lines and hurricanes at air temperatures lower than $-40^{\circ}$ c. Mon. Wea. Rev., 91, 687-693.

Simpson, R. H., 1974: The hurricane disaster potential scale. Weatherwise, 27, 169-186.

Simpson, R. H., 1978: On the computation of equivalent potential temperature. Mon. Wea. Rev., 106, 124-130.

Sinclair, P. C., 1973: The lower structure of dust devils. J. Atmos. Sci., 30, 1599-1619.

Smith, R. K. and P. Howells, 1983: Numerical simulations of tornado-like vortices. Part II: Two-cell vortices. Geophys. Astrophys. Fluid Dyn., 27, 285-298.

Staff, N., 1958: Details of circulation on the high energy core of Hurricane Carrie. NHRP No. 24, National Hurricane Research Project, Miami, FL, 33146, 15 pp.

Stephens, M. A., 1979: A simple ice phase parameterization. Dept. Atmospheric Sci. Paper No. 319, Colorado State University, Fort Collins, CO, 80523, 122 pp.

Steranka, J., E. B. Rodgers, and R. C. Gentry, 1986: The relationship between satellite measured convective bursts and tropical cyclone intensification. Mon. Wea. Rev., $114,1539-1546$.

Stricherz, J. N., 1989: An analysis of modified available potential energy and Richardson number in mid-latitude squall line environments. M. S. thesis, Texas A\&M University, College Station, TX, 77843, 94 pp.

Stull, R. B., 1988: An Introduction to Boundary Layer Meteorology. Kluwer Academic Publishers, 666 pp. 
Sugg, A. L., 1967: The hurricane season of 1966. Mon. Wea. Rev., 95, 131-142.

Sundqvist, H., 1970: Numerical simulation of the development of tropical cyclones with a ten-level model. Part I. Tellus, 22, 359-390.

Temperton, C. and D. L. Williamson, 1981: Normal mode initialization for a multilevel grid-point model. Part I: Linear aspects. Mon. Wea. Rev., 109, 729-743.

Tuleya, R. E., 1994: Tropical storm development and decay: Sensitivity to surface boundary conditions. Mon. Wea. Rev., 122, 291-304.

Tuleya, R. E. and Y. Kurihara, 1975: The energy and angular momentum budgets of a three-dimensional tropical cyclone model. J. Atmos. Sci., 32, 287-301.

Udias, A. and W. Stauder, 1991: Jesuit geophysical observations. Eos, Trans. Amer. Geophys. Union, 185, 188-189.

Velden, C. S., C. M. Hayden, W. P. Menzel, J. L. Franklin, and J. S. Lynch, 1992: The impact of satellite-derived winds on numerical hurricane track forecasting. Wea. Forecasting, 7, 107-118.

Venne, M. G., W. A. Lyons, C. S. Keen, P. G. Black, and R. C. Gentry, 1989: Explosive supercell growth: A possible indicator for tropical storm intensification? In Preprints, 24th Conf. on Radar Meteorology, 545-548.

Wada, M., 1989: The effects of ice phase on cumulus organization. In Preprints, 18th Conf. on Hurricanes and Tropical Meteorology, 133-134.

Walko, R. L., 1988: Plausibility of substantial dry adiabatic subsidence in a tornado core. J. Atmos. Sci., 45, 2251-2267.

Wallace, J. M. and P. V. Hobbs, 1977: Atmospheric Science: An Introductory Survey. Academic Press, 467 pp.

Wang, J. and D. A. Randall, 1994: The moist available energy of a conditionally unstable atmosphere. Part II: Further analysis of GATE data. J. Atmos. Sci., 51, 703-710. 
Ward, N. B., 1972: The exploration of certain features of tornado dynamics using a laboratory model. J. Atmos. Sci., 29, 1194-1204.

Weatherford, C. L., 1985: Typhoon structure variability. Dept. Atmospheric Sci. Paper No. 391, Colorado State University, Fort Collins, CO, 80523, 77 pp.

Weatherford, C. L., 1989: The structural evolution of typhoons. Dept. Atmospheric Sci. Paper No. 446, Colorado State University, Fort Collins, CO, 80523, 198 pp.

Weatherford, C. L. and W. M. Gray, 1988a: Typhoon structure as revealed by aircraft reconnaissance. Part II: Structure variability. Mon. Wea. Rev., 116, 1044-1056.

Weatherford, C. L. and W. M. Gray, 1988b: Typhoon structure as revealed by aircraft reconnaissance. Part I: Data analysis and climatology. Mon. Wea. Rev., 116, 10321043.

Wei, D. and W. M. Gray, 1985: Estimation of tropical cyclone outer wind strength from satellite imagery. unpublished, copy available from P. Fitzpatrick.

Williams, E. and N. Renno, 1993: An analysis of the conditional instability of the tropical atmosphere. Mon. Wea. Rev., 121, 21-36.

Williams, E. R., 1988: The electrification of thunderstorms. Sci. Amer., 259, 88-99.

Williams, E. R., 1989: The tripole structure of the thunderstorm. J. Geophys. Res., 94, $13151-13167$.

Williams, E. R., S. A. Rutledge, S. G. Geotis, N. Renno, and E. Rasmussen, 1992: A radar and electrical study of tropical "Hot Towers". J. Atmos. Sci., 49, 1386-1395.

Willoughby, H. E., 1979: Forced secondary circulations in hurricanes. J. Geophys. Res., 84, 3173-3183.

Willoughby, H. E., 1990: Temporal changes of the primary circulation in tropical cyclones. J. Atmos. Sci., 47, 242-264.

Willoughby, H. E., 1991: Reply. J. Atmos. Sci., 48, 1209-1212. 
Willoughby, H. E., J. A. Clos, and M. G. Shoreibah, 1982: Concentric eyewalls, secondary wind maxima, and the evolution of the hurricane vortex. J. Atmos. Sci., 39, 395-411.

Willoughby, H. E., H. Jin, S. J. Lord, and J. M. Piotrowicz, 1984: Hurricane structure and evolution as simulated by an axisymmetric, nonhydrostatic numerical model. $J$. Atmos. Sci., 41, 1169-1186.

Willoughby, H. E., D. P. Jorgensen, R. A. Black, and S. L. Rosenthal, 1985: Project STORMFURY: A scientific chronicle, 1962-1983. Bull. Amer. Meteor. Soc., 66, 505514.

Willoughby, H. E., J. M. Masters, and C. W. Landsea, 1989: A record minimum sea-level pressure observed in Hurricane Gilbert. Mon. Wea. Rev., 117, 2824-282.

Wylie, D. P. and C. E. Anderson, 1983: Cloud top temperature anomaly associated with the Binger, OK tornado 22 May 1981. In Preprints, 13th Conf. Severe Local Storms, 150-153.

Xu, K. and K. A. Emanuel, 1989: Is the tropical atmosphere conditionally unstable? Mon. Wea. Rev., 117, 1471-1479.

Yamasaki, M., 1977: A preliminary experiment of the tropical cyclone without parameterizing the effects of cumulus convection. J. Meteor. Soc. Japan, 55, 11-30.

Zehr, R. M., 1987: The diurnal variation of deep convective clouds and cirrus with tropical cyclones. In Preprints, 17th Conf. on Hurricanes and Tropical Meteorology, 276-279.

Zehr, R. M., 1992: Tropical cyclogenesis in the western North Pacific. NESDIS 61, NOAA Tech. Report, Washington, D. C., 20233, 181 pp.

Zehr, R. M., 1995: Improving Geostationary satellite applications for tropical cyclone forecasting. In Preprints, 21st Conf. on Hurricanes and Tropical Meteorology, 628630 .

Zehr, R. M. and R. Phillips, 1994: EXPERT system for tropical cyclone intensity forecasts. In Preprints, 7th Conf. on Satellite Meteorology and Oceanography, 71-74. 
Zhang, G. J. and N. A. McFarlane, 1991: Convective stabilzation in midlatitudes. Mon. Wea. Rev., 119, 1915-1928. 
Appendix A

\section{SHALLOW WATER DERIVATION OF THE ROSSBY RADIUS OF DEFORMATION}

Since this author has not found any documentation on the derivation of the Rossby Radius of Deformation in cylindrical coordinates - a relationship referred to in countless books and papers - it will be included here. This derivation was originally performed by Wayne Schubert.

The axisymmetric shallow water equations in cylindrical coordinates are:

$$
\begin{gathered}
\frac{d u_{r}}{d t}-\left(f+\frac{v_{t}}{r}\right) v_{t}+g \frac{\partial h}{\partial r}=0 \\
\frac{d v_{t}}{d t}+\left(f+\frac{v_{t}}{r}\right) u_{r}=0 \\
\frac{d h}{d t}+h \frac{\partial\left(r u_{r}\right)}{r \partial r}=0
\end{gathered}
$$

where $h$ is the height of the fluid interface. If one assumes gradient wind balance such that $D u_{r} / D t=0$ and linearizes about a nonresting tangential wind basic state but a resting radial wind state:

$$
\begin{aligned}
h & =\bar{h}+h^{\prime} \\
u_{r} & =\bar{u}_{r}+u_{r}^{\prime} \rightarrow u_{r}^{\prime} \\
v_{t} & =\bar{v}_{t}+v_{t}^{\prime} \\
v_{t}^{2} & =\bar{v}_{t}^{2}+2 \bar{v}_{t} v_{t}^{\prime}+\left(v_{t}^{\prime}\right)^{2} \rightarrow \bar{v}_{t}^{2}+2 \bar{v}_{t} v_{t}^{\prime}
\end{aligned}
$$

and assuming that the products of perturbations are negligible yields:

$$
-f v_{t}^{\prime}-f \bar{v}_{t}-\frac{\bar{v}_{t}^{2}}{r}-2 \frac{\bar{v}_{t} v_{t}^{\prime}}{r}+g \frac{\partial h^{\prime}}{\partial r}=0
$$




$$
\begin{gathered}
\frac{\partial v_{t}^{\prime}}{\partial t}+\left(f+\frac{\partial \bar{v}_{t}}{\partial r}+\frac{\bar{v}_{t}}{r}\right) u_{r}^{\prime}=0 \\
\frac{\partial h^{\prime}}{\partial t}+\bar{h} \frac{\partial\left(r u_{r}^{\prime}\right)}{r \partial r}=0
\end{gathered}
$$

Using the equality $\frac{\partial \bar{v}_{t}}{\partial r}+\frac{\bar{v}_{t}}{r}=\frac{1}{r} \frac{\partial r \bar{v}_{t}}{\partial r}$ (which is the basic state relative vorticity $\bar{\zeta}$ ), the linearized tangential momentum equation can be rewritten:

$$
\frac{\partial v_{t}^{\prime}}{\partial t}+(f+\bar{\zeta}) u_{r}^{\prime}=0
$$

This system of equations can be solved for $u_{r}^{\prime}$ by writing Eq. (A.11) and Eq. (A.10) in a manner that is consistent with Eq. (A.8). The first step takes $\partial / \partial t$ of Eq. (A.8) such that:

$$
-\left(f+\frac{2 \bar{v}_{t}}{r}\right) \frac{\partial v_{t}^{\prime}}{\partial t}+g \frac{\partial}{\partial r} \frac{\partial h^{\prime}}{\partial t}=0
$$

while multiplying $\left(f+2 \bar{v}_{t} / r\right)$ times Eq. (A.11) gives:

$$
\left(f+\frac{2 \bar{v}_{t}}{r}\right) \frac{\partial v_{t}^{\prime}}{\partial t}+\left(f+\frac{2 \bar{v}_{t}}{r}\right)(f+\bar{\zeta}) u_{r}^{\prime}=0
$$

Now one substitutes for $\partial h^{\prime} / \partial t$ from Eq. (A.10) into Eq. (A.12), and then substitutes this expression for $\left(f+\frac{2 \bar{v}_{t}}{r}\right) \partial v_{t}^{\prime} / \partial t$ into Eq. (A.13). Upon dividing by $g \bar{h}$ the final result is:

$$
\frac{\partial}{\partial r}\left(\frac{1}{r} \frac{\partial\left(r u_{r}^{\prime}\right)}{\partial r}\right)-\frac{u_{r}^{\prime}}{\lambda_{R}^{2}}=0
$$

where the Rossby radius of deformation $\lambda_{R}$ is

$$
\lambda_{R}=\frac{(g \bar{h})^{1 / 2}}{(\bar{\zeta}+f)^{1 / 2}\left(2 \bar{v}_{t} / r+f\right)^{1 / 2}}
$$

By analogy, in a stratified atmosphere, Eq. (A.15) becomes

$$
\lambda_{R}=\frac{N H}{(\zeta+f)^{1 / 2}\left(2 v_{t} / r+f\right)^{1 / 2}}
$$

where $N$ is the Brunt-Väisälä frequency, $H$ is the scale height of the circulation, $\zeta$ is the relative vorticity, and $f$ is the Coriolis parameter. Rossby (1938) first solved an equation similar to Eq. (A.14), which is a simple 2nd order ordinary differential equation. Steady-state solutions can be obtained after geostrophic adjustment occurs subject to initial conditions of the basic state vorticity field, height displacement, and the length scale of the disturbance with regards to $\lambda_{R}$. While Rossby's version was for geostrophic adjustment of an ocean current (in which $\lambda_{R}=\sqrt{g \bar{h}} / f$ ), this version contains a Rossby radius modified by the local vorticity field $\zeta$ and background vorticity field $2 v_{t} / r$. 


\section{Appendix B}

\section{COMPLETE INFORMATION ON TIPS}

Denoting $\sigma$ as the standard deviation of a predictor, $y=\Delta V_{\max }, \bar{x}$ as the predictor mean, and $\bar{y}$ as the mean $\Delta V_{\max }$, a number $k$ predictors are normalized by the following regression:

$$
y=\bar{y}+\sigma_{y}\left[\sum_{i=1}^{k} c_{i}\left(x_{i}-\bar{x}_{i}\right) / \sigma_{i}\right]
$$

where $c_{i}$ is the normalized regression coefficient. $y$ is the future change of $V_{\text {max }}$. For example, a 24-h forecast would be predicting $V_{\max }(t=24)-V_{\max }(t=0)$, and a 48-h forecast would be predicting $V_{\max }(t=48)-V_{\max }(t=0)$. The predictors $x_{i}$ in Eq. (B.1) are the input variables, such as LONG, VSPD, EYEPER, POT, etc. listed in the tables.

The following tables contain the needed information for: 1) the official version of TIPS (Tables B.2-B.4); and 2) alternate versions using different shear predictors (Tables B.5B.7, Tables B.8-B.10, and Tables B.11-B.13). The official version of TIPS is recommended since the other versions explain a little less variance, but the alternate versions may be more applicable and easier to use in an operational setting (see Chapter 8 for details). The official version uses areally averaged shear over a 5-deg circle in which a perfect shear forecast is assumed (VWS5). Alternative shear parameters include single point shear (VWSPT) in which a perfect shear forecast is assumed, and shear values using persistence from the initial shear field along the forecast track (VWS5 $5_{\text {per }}$ and $V W S P T_{\text {per }}$ ). In other words, TIPS uses the shear analysis field at the beginning of the forecast period, and interpolates these values over future storm positions. In this manner, the shear field is not allowed to evolve in the forecast period, but the TC may be assigned different shear values as it moves into different shear regimes based on $t=0$ computations.

Implicitly required is a forecast track in 12-h intervals for the SST and shear parameters. The Maximum Potential Intensity is averaged along the future storm track, then 
POT is computed. The shear predictors are also averaged along the future storm track. Details are contained in Chapter 8.

The following restrictions apply. Only storms over open water during the forecast interval with $V_{\max } \geq 35 \mathrm{kts}$ at the beginning of the forecast interval can be used. The TIPS' versions with 5-deg average VWS can only be used if the latitude is less than $40.0 \mathrm{deg} N$ and the longitude is between 75.0 and $175.0 \mathrm{deg} \mathrm{E}$, since no statistical analysis was performed outside these regions due to synoptic data limitations. Likewise, the TIPS' versions with single point VWS can only be used if the latitude is less than $45.0 \mathrm{deg} N$ and the longitude is between 70.0 and $180.0 \mathrm{deg} \mathrm{E}$.

Table B.1: Significant regression predictors. Details of predictors are contained in the text. Only storms over open water with $V_{\max } \geq 35 \mathrm{kts}$ are considered. VWS predictors with asterisks are used in alternate TIPS schemes. The versions of TIPS with 5-deg average VWS can only be used if the latitude is less than $40.0 \mathrm{deg} N$ and the longitude is between 75.0 and 175.0 deg E. The versions of TIPS with single point VWS can only be used if the latitude is less than $45.0 \mathrm{deg} N$ and the longitude is between 70.0 and $180.0 \mathrm{deg} \mathrm{E}$.

\begin{tabular}{|c|c|}
\hline PREDICTOR & DESCRIPTION \\
\hline LONG & Initial storm longitude. \\
\hline VSPD & Meridional component of storm motion. \\
\hline EYEPER & $\begin{array}{l}\text { Indicator variable that combines persistence with the param- } \\
\text { eterization of a well-formed, contracting eye. }\end{array}$ \\
\hline POT & $\begin{array}{l}\text { Maximum Possible Intensity (MPI) for a given SST minus } \\
\text { initial intensity }\left(V_{\text {max }}\right) \text {. MPI is averaged over the forecast } \\
\text { track. }\end{array}$ \\
\hline VWSPT $^{*}$ & $\begin{array}{l}\text { Magnitude of } 200-850 \mathrm{mb} \text { "single point" vertical wind shear } \\
\text { over the storm center. VWSPT is averaged over the forecast } \\
\text { track, and a perfect shear forecast is assumed. }\end{array}$ \\
\hline VWS5 & Same as VWSPT, but averaged over a 5-deg circle. \\
\hline VWSPT $_{\text {per }}^{*}$ & $\begin{array}{l}\text { Same as VWSPT, but no shear forecast is used. Instead, } \\
\text { persistence from the initial shear field is performed along the } \\
\text { forecast track. }\end{array}$ \\
\hline VWS5 $5_{\text {per }}^{*}$ & Same as VWSPT ${ }_{\text {per }}$, but averaged over a 5-deg circle. \\
\hline PX0455 & $\begin{array}{l}\text { Percent of infrared pixels in a } 0-4-\text { deg radial area colder than } \\
T_{b}=-55^{\circ} \mathrm{C} \text { at } t=0 \text {. }\end{array}$ \\
\hline DPX0165 & $\begin{array}{l}\text { 12-h trend of infrared pixel counts in a } 0-1-\text { deg radial area } \\
\text { colder than } T_{b}=-65^{\circ} \mathrm{C} \text { measured in percentages. }\end{array}$ \\
\hline
\end{tabular}


Table B.2: Normalized regression coefficients of the official version of TIPS for the forecast periods of $12,24,36$, and $48 \mathrm{~h}$. Variables not significant at the $99 \%$ level are labeled NA (not applicable); $R^{2}$ (the coefficient of determination) is the percent of the total variance explained by the regression; $n$ is the sample size for 1984-1986.

\begin{tabular}{|l|rrrr|}
\hline Variable coefficients $\left(c_{i}\right)$ & 12 & 24 & 36 & 48 \\
\hline \hline 1) POT & +0.46 & +0.60 & +0.67 & +0.68 \\
\hline 2) PX0455 & +0.27 & +0.30 & +0.30 & +0.26 \\
\hline 3) EYEPER & +0.31 & +0.28 & +0.25 & +0.21 \\
\hline 4) VWS5 & -0.18 & -0.26 & -0.29 & -0.27 \\
\hline 5) DPX0165 & +0.16 & NA & NA & NA \\
\hline 6) VSPD & +0.09 & +0.14 & +0.13 & NA \\
\hline 7) LONG & NA & NA & NA & +0.10 \\
\hline \hline$R^{2}(\%)$ & 46.5 & 52.2 & 59.0 & 60.0 \\
\hline $\mathrm{n}$ & 611 & 530 & 459 & 397 \\
\hline
\end{tabular}

Table B.3: Official mean value of predictors $(\bar{x})$ and dependent variable $\Delta V_{\max }(\bar{y})$ for the TIPS forecast periods $12,24,36$, and $48 \mathrm{~h}$. Variables not significant at the $99 \%$ level are labeled NA (not applicable). The units are $\mathrm{m} \mathrm{s}^{-1}$ for POT, VWS5, VSPD, and $\Delta V_{\max }$, percent for PX0455 and DPX0165, and deg E for LONG.

\begin{tabular}{|l|rrrr|}
\hline Variable mean & 12 & 24 & 36 & 48 \\
\hline \hline 1) POT & 38.9 & 38.8 & 38.9 & 39.0 \\
\hline 2) PX0455 & 24.6 & 25.5 & 26.2 & 26.8 \\
\hline 3) EYEPER & 0.37 & 0.38 & 0.39 & 0.40 \\
\hline 4) VWS5 & 8.5 & 8.4 & 8.4 & 8.5 \\
\hline 5) DPX0165 & -0.9 & NA & NA & NA \\
\hline 6) VSPD & 2.2 & 2.0 & 1.8 & NA \\
\hline 7) LONG & NA & NA & NA & 137.3 \\
\hline \hline 6) $\triangle V_{\max }$ & 0.79 & 1.5 & 2.1 & 2.6 \\
\hline
\end{tabular}


Table B.4: Official standard deviations $(\sigma)$ of predictors and dependent variable $\Delta V_{\max }$ for the TIPS forecast periods $12,24,36$, and $48 \mathrm{~h}$. Variables not significant at the $99 \%$ level are labeled NA (not applicable). The units are $\mathrm{m} \mathrm{s}^{-1}$ for POT, VWS5, VSPD, and $\Delta V_{\text {max }}$, percent for PX0455 and DPX0165, and deg E for LONG.

\begin{tabular}{|l|rrrr|}
\hline Variable standard deviation & 12 & 24 & 36 & 48 \\
\hline \hline 1) POT & 14.2 & 14.4 & 14.7 & 14.7 \\
\hline 2) PX0455 & 15.9 & 15.8 & 15.9 & 15.8 \\
\hline 3) EYEPER & 0.48 & 0.49 & 0.49 & 0.49 \\
\hline 4) VWS5 & 4.9 & 4.4 & 4.1 & 4.0 \\
\hline 5) DPX0165 & 29.0 & NA & NA & NA \\
\hline 6) VSPD & 2.7 & 2.3 & 2.1 & NA \\
\hline 7) LONG & NA & NA & NA & 14.4 \\
\hline \hline 6) $\triangle V_{\max }$ & 5.0 & 8.8 & 11.9 & 14.3 \\
\hline
\end{tabular}

Table B.5: Normalized regression coefficients for the alternate version of TIPS with VWSPT for the forecast periods of $12,24,36$, and $48 \mathrm{~h}$. Variables not significant at the $99 \%$ level are labeled NA (not applicable); $R^{2}$ (the coefficient of determination) is the percent of the total variance explained by the regression; $n$ is the sample size for 1984-1986.

\begin{tabular}{|l|rrrr|}
\hline Variable coefficients $\left(c_{i}\right)$ & 12 & 24 & 36 & 48 \\
\hline \hline 1) POT & +0.45 & +0.60 & +0.68 & +0.69 \\
\hline 2) PX0455 & +0.26 & +0.30 & +0.30 & +0.27 \\
\hline 3) EYEPER & +0.32 & +0.30 & +0.26 & +0.22 \\
\hline 4) VWSPT & -0.14 & -0.23 & -0.27 & -0.25 \\
\hline 5) DPX0165 & +0.16 & NA & NA & NA \\
\hline 6) VSPD & NA & +0.11 & +0.11 & NA \\
\hline 7) LONG & NA & +0.10 & +0.11 & +0.14 \\
\hline \hline$R^{2}(\%)$ & 45.1 & 51.0 & 57.7 & 58.3 \\
\hline $\mathrm{n}$ & 617 & 536 & 465 & 403 \\
\hline
\end{tabular}


Table B.6: Mean value of predictors $(\bar{x})$ and dependent variable $\Delta V_{\max }(\bar{y})$ for the alternate TIPS version using VWSPT. Variables not significant at the $99 \%$ level are labeled NA (not applicable). The units are $\mathrm{m} \mathrm{s}^{-1}$ for POT, VWSPT, VSPD, and $\Delta V_{\max }$, percent for PX0455 and DPX0165, and deg E for LONG.

\begin{tabular}{|l|rrrr|}
\hline Variable mean & 12 & 24 & 36 & 48 \\
\hline \hline 1) POT & 38.9 & 38.8 & 38.8 & 39.0 \\
\hline 2) PX0455 & 24.4 & 25.3 & 25.9 & 26.6 \\
\hline 3) EYEPER & 0.37 & 0.39 & 0.39 & 0.40 \\
\hline 4) VWSPT & 9.6 & 9.5 & 9.5 & 9.6 \\
\hline 5) DPX0165 & -0.9 & NA & NA & NA \\
\hline 6) VSPD & NA & 2.0 & 2.1 & NA \\
\hline 7) LONG & NA & 136.1 & 137.0 & 137.8 \\
\hline \hline 6) $\triangle V_{\max }$ & 0.78 & 1.5 & 2.1 & 2.6 \\
\hline
\end{tabular}

Table B.7: Standard deviations $(\sigma)$ of predictors and dependent variable $\Delta V_{\max }$ for the alternate TIPS version using VWSPT. Variables not significant at the $99 \%$ level are labeled NA (not applicable). The units are $\mathrm{m} \mathrm{s}^{-1}$ for POT, VWSPT, VSPD, and $\Delta V_{\max }$, percent for PX0455 and DPX0165, and deg E for LONG.

\begin{tabular}{|l|rrrr|}
\hline Variable standard deviation & 12 & 24 & 36 & 48 \\
\hline \hline 1) POT & 14.1 & 14.4 & 14.6 & 14.7 \\
\hline 2) PX0455 & 15.9 & 15.9 & 15.9 & 15.8 \\
\hline 3) EYEPER & 0.48 & 0.49 & 0.49 & 0.49 \\
\hline 4) VWSPT & 5.2 & 4.7 & 4.2 & 4.0 \\
\hline 5) DPX0165 & 29.0 & NA & NA & NA \\
\hline 6) VSPD & NA & 2.3 & 2.1 & NA \\
\hline 7) LONG & NA & 15.0 & 15.0 & 15.0 \\
\hline \hline 6$) \triangle V_{\max }$ & 5.1 & 8.8 & 11.8 & 14.3 \\
\hline
\end{tabular}


Table B.8: Normalized regression coefficients for the alternate version of TIPS with VWS5 $5_{\text {per }}$ for the forecast periods of $12,24,36$, and $48 \mathrm{~h}$. Variables not significant at the $99 \%$ level are labeled NA (not applicable); $R^{2}$ (the coefficient of determination) is the percent of the total variance explained by the regression; $n$ is the sample size for 1984-1986.

\begin{tabular}{|l|rrrr|}
\hline Variable coefficients $\left(c_{i}\right)$ & 12 & 24 & 36 & 48 \\
\hline \hline 1) POT & +0.43 & +0.58 & +0.65 & +0.68 \\
\hline 2) PX0455 & +0.26 & +0.31 & +0.30 & +0.27 \\
\hline 3) EYEPER & +0.33 & +0.29 & +0.25 & +0.23 \\
\hline 4) VWS5 per & -0.13 & -0.21 & -0.22 & -0.18 \\
\hline 5) DPX0165 & +0.16 & NA & NA & NA \\
\hline 6) VSPD & NA & +0.13 & +0.12 & NA \\
\hline 7) LONG & NA & NA & NA & +0.10 \\
\hline \hline$R^{2}(\%)$ & 44.6 & 49.5 & 55.6 & 56.3 \\
\hline $\mathbf{n}$ & 626 & 555 & 491 & 435 \\
\hline
\end{tabular}

Table B.9: Mean value of predictors $(\bar{x})$ and dependent variable $\Delta V_{\max }(\bar{y})$ for the alternate TIPS version using VWS5 $5_{\text {per. }}$ Variables not significant at the $99 \%$ level are labeled NA (not applicable). The units are $\mathrm{m} \mathrm{s}^{-1}$ for POT, VWS5 $5_{\text {per }}$, VSPD, and $\Delta V_{\max }$, percent for PX0455 and DPX0165, and deg E for LONG.

\begin{tabular}{|l|rrrr|}
\hline Variable mean & 12 & 24 & 36 & 48 \\
\hline \hline 1) POT & 38.9 & 38.9 & 39.0 & 39.1 \\
\hline 2) PX0455 & 24.5 & 25.5 & 26.2 & 26.9 \\
\hline 3) EXEPER & 0.37 & 0.39 & 0.40 & 0.41 \\
\hline 4) VWS5 per & 9.2 & 9.9 & 10.4 & 10.9 \\
\hline 5) DPX0165 & -1.0 & NA & NA & NA \\
\hline 6) VSPD & NA & 1.9 & 1.8 & NA \\
\hline 7) LONG & NA & NA & NA & 137.3 \\
\hline \hline 6) $\Delta V_{\max }$ & 0.81 & 1.6 & 2.2 & 2.8 \\
\hline
\end{tabular}


Table B.10: Standard deviations $(\sigma)$ of predictors and dependent variable $\Delta V_{\max }$ for the alternate TIPS version using VWS $5_{\text {per }}$. Variables not significant at the $99 \%$ level are labeled NA (not applicable). The units are $\mathrm{m} \mathrm{s}^{-1}$ for POT, VWS5 $5_{\text {per, }}$ VSPD, and $\Delta V_{\max }$, percent for PX0455 and DPX0165, and deg E for LONG.

\begin{tabular}{|c|c|c|c|c|}
\hline Variable standard deviation & 12 & 24 & 36 & $\overline{48}$ \\
\hline 1) $\mathrm{POT}$ & $\overline{14.2}$ & $\overline{14.5}$ & $\overline{14.8}$ & 14.8 \\
\hline 2) $\mathrm{PX} 0455$ & 15.8 & 15.7 & 15.7 & 15.7 \\
\hline 3) EYEPER & 0.48 & 0.49 & 0.49 & 0.49 \\
\hline 4) VWS5 $5_{\text {per }}$ & 5.8 & 5.8 & 5.6 & $\overline{5.5}$ \\
\hline 5) DPX0165 & 28.8 & NA & $\mathrm{NA}$ & $\overline{\mathrm{NA}}$ \\
\hline 6) VSPD & $\overline{\mathrm{NA}}$ & 2.3 & 2.1 & $\overline{\mathrm{NA}}$ \\
\hline 7) LONG & $\mathrm{NA}$ & $\overline{\mathrm{NA}}$ & NA & 14.0 \\
\hline 6) $\Delta V_{\max }$ & $\overline{5.0}$ & $\overline{8.7}$ & 11.9 & 14.5 \\
\hline
\end{tabular}

Table B.11: Normalized regression coefficients for the alternate version of TIPS with VWSPT $_{\text {per }}$ for the forecast periods of $12,24,36$, and $48 \mathrm{~h}$. Variables not significant at the $99 \%$ level are labeled NA (not applicable); $R^{2}$ (the coefficient of determination) is the percent of the total variance explained by the regression; $n$ is the sample size for 1984-1986.

\begin{tabular}{|c|c|c|c|c|}
\hline Variable coefficients $\left(c_{i}\right)$ & 12 & 24 & 36 & 48 \\
\hline 1) $\mathrm{POT}$ & +0.43 & +0.58 & +0.66 & +0.68 \\
\hline 2) PX0455 & +0.26 & +0.31 & +0.30 & +0.27 \\
\hline 3) EYEPER & +0.33 & +0.30 & +0.26 & +0.22 \\
\hline 4) $\mathrm{VWSPT}_{\text {per }}$ & -0.10 & -0.19 & -0.20 & -0.15 \\
\hline 5) DPX0165 & +0.16 & $\mathrm{NA}$ & $\overline{\mathrm{NA}}$ & $\mathrm{NA}$ \\
\hline 6) VSPD & $\overline{N A}$ & +0.13 & +0.13 & $\overline{\mathrm{NA}}$ \\
\hline 7) LONG & NA & $\overline{N A}$ & $\overline{\mathrm{NA}}$ & +0.10 \\
\hline$R^{2}(\%)$ & $\overline{43.8}$ & $\overline{48.3}$ & $\overline{54.2}$ & $\overline{55.3}$ \\
\hline $\mathrm{n}$ & 632 & 561 & 497 & 441 \\
\hline
\end{tabular}


Table B.12: Mean value of predictors $(\bar{x})$ and dependent variable $\Delta V_{\max }(\bar{y})$ for the

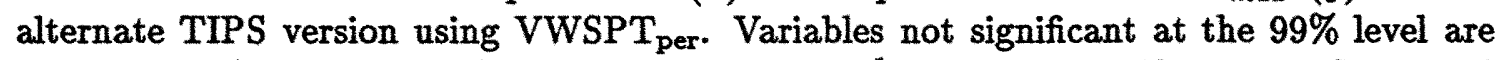
labeled NA (not applicable). The units are $\mathrm{m} \mathrm{s}^{-1}$ for POT, VWSPT $\Delta V_{\text {max }}$, percent for PX0455 and DPX0165, and deg E for LONG.

\begin{tabular}{|c|c|c|c|c|}
\hline Variable mean & 12 & 24 & 36 & 48 \\
\hline 1) $\mathrm{POT}$ & 38.9 & 38.9 & 39.0 & $\overline{39.1}$ \\
\hline 2) PX0455 & 24.4 & 25.3 & 26.0 & 26.7 \\
\hline 3) EYEPER & 0.37 & 0.39 & 0.40 & 0.41 \\
\hline 4) VWSPT $_{\mathrm{per}}$ & 10.5 & $\overline{11.4}$ & 12.0 & 12.6 \\
\hline 5) DPX0165 & -1.0 & NA & NA & $\mathrm{NA}$ \\
\hline 6) VSPD & $\overline{N A}$ & 1.9 & 1.8 & $\overline{\mathrm{NA}}$ \\
\hline 7) LONG & $\mathrm{NA}$ & $\mathrm{NA}$ & $\mathrm{NA}$ & 137.8 \\
\hline 6) $\Delta V_{\max }$ & 0.81 & 1.6 & $\overline{2.2}$ & $\overline{2.7}$ \\
\hline
\end{tabular}

Table B.13: Standard deviations $(\sigma)$ of predictors and dependent variable $\Delta V_{\max }$ for the alternate TIPS version using VWSPT $_{\text {per. }}$. Variables not significant at the $99 \%$ level are labeled NA (not applicable). The units are $\mathrm{m} \mathrm{s}^{-1}$ for POT, VWSPT per, VSPD, and $\Delta V_{\max }$, percent for PX0455 and DPX0165, and deg E for LONG.

\begin{tabular}{|c|c|c|c|c|}
\hline Variable standard deviation & 12 & 24 & 36 & 48 \\
\hline 1) $\mathrm{POT}$ & $\overline{14.2}$ & 14.5 & $\overline{14.7}$ & 14.8 \\
\hline 2) $\mathrm{PX} 0455$ & 15.8 & 15.8 & 15.8 & 15.7 \\
\hline 3) EYEPER & 0.48 & 0.49 & 0.49 & 0.49 \\
\hline 4) $\mathrm{VWSPT}_{\text {per }}$ & 6.3 & 6.3 & 6.1 & 6.1 \\
\hline 5) DPX0165 & 28.8 & $\mathrm{NA}$ & $\overline{\mathrm{NA}}$ & $\overline{\mathrm{NA}}$ \\
\hline 6) VSPD & $\mathrm{NA}$ & 2.3 & 2.1 & $\mathrm{NA}$ \\
\hline 7) $\mathrm{LONG}$ & $\mathrm{NA}$ & $\mathrm{NA}$ & $\overline{\mathrm{NA}}$ & 14.5 \\
\hline 6) $\Delta V_{\max }$ & 5.0 & 8.7 & 11.9 & $\overline{14.5}$ \\
\hline
\end{tabular}

